\title{
Summary of Radioactive Solid Waste Received in the 200 Areas During Calendar Year 1995
}

\author{
J. D. Anderson \\ D. L. Hagel \\ Date Published \\ June 1996
}

Prepared for the U.S. Department of Energy Office of Environmental Restoration and Waste Management

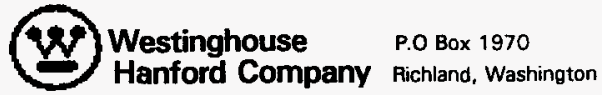

Hanford Operations and Engineering Contractor for the

U.S. Department of Energy under Contract DE-ACO6-87RL10930

Approved for Public Release 


\section{RELEASE AUTHORIZATION}

Document

rumber:

Dooument

Title:
WHC-EP-0125-8

Sumary of Radioactive Solid Waste Received in the 200 Areas During Calendar Year 1995

Release Date: $06 / 06 / 96$

This document was reviewed following the procedures described in WHC-CM-3-4 and is:

APPROVED FOR PUBLIC RELEASE

WHC Information Release Administration specialist:

W. Z. huztiand

V.L. Birkland

$6 \% 196$

Date 
LEGAL DISCLAMER

This report was prepered as an account of work sponsored by an egency of the United States Government. Neither the Urited States Government nor any agency thereof, nor any of their emplovees, nor any of their contractors, subcontractors or their employees, makes any werranty, express or implied. or asaumes any legal liability or responsibility for the accuracy, completeness, or eny third party's use or the results of such use of any information, apparatus, product, or process disclosed, or roprasents that its use would not infringo privately owned rights. Reference heroin to any specific commercial product, process, or service by trade name, trademerk, manufactuger, or otherwise, does not necessarily constitute or imply its endorsement, recommendation, or favoring by the United States Government or any agency therec or its contractors or subcontractors. The views and opinions of euthors expressed herein do not necessarily state or reflect those of the United States Government or any agency thereof.

This report has been reproduced from the best available copy. Available in paper copy and microfiche.

Availabile to the U.S. Department of Energy and its contractors from

Office of Scientific and Technical Intormation

P.O. Box 62

Oak Ridge, TN 37831

(615) 576-8401

Available to the public from the U.S. Depertment of Commerce National Technical Information Service 5285 Port Royal Road

Springfield, VA 22161

(703) $487-4650$

Printed in the United Stetes of Americe 
THIIS PAGE DNTENTIONALLY

LEFr' BLANK

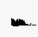




\section{INFORMATION RELEASE REQUEST \\ PUBLIC DOCUMENT CHANGE/REVISION \\ (Short Form No. 1)}

1. Base Document ID Number: WHC-EP-0125

2. Base Document Title:

Summary of Radioactive Solid Waste Received in the 200 Areas During Calendar Year 1995

3. Change/Revision Number: WHC-EP-0125-8

4. Change/Revision Date: $05 / 14 / 96$

5. Unclassified Category: * UC, $, 60 Q, \ldots, \ldots, \ldots$ is

6. Budget \& Reporting Code: ${ }^{*}$ B\&R - EW3130020

I verify this change/revision to the base document indicated above:

- Complies with the provisions of WHC-CM-3-4

- Contains no Limited-Use information

- Contains no classified or Applied Technology references

- Does not change the intent or meaning of the base document

- And, the base document itself is approved for public release.

7. Responsible Manager: K. L. Hladek
MSIN:
T3-01
Telephone Number:
$372-3201$
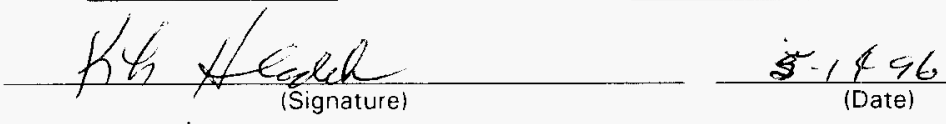

8. Information Release Administration Specialist: *

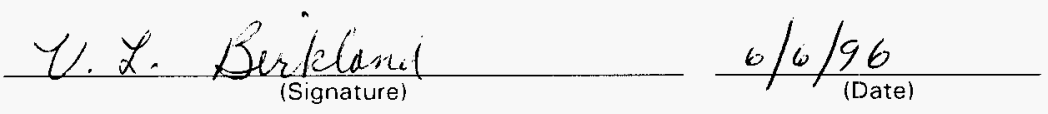

${ }^{*}$ Required only for full revisions transmitted to OSTI.

* * Required only for full revisions. 



$$
\text { WHC-EP-0125-8 }
$$

Document Title: Summary of Radioactive Solid Waste

Received in the 200 Areas During Calendar Year 1995

Prepared by:

Reviewed by:

Approved by:
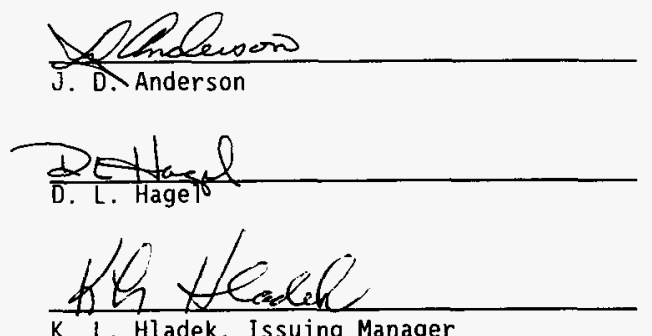

K. L. Hladek, Issuing Manager

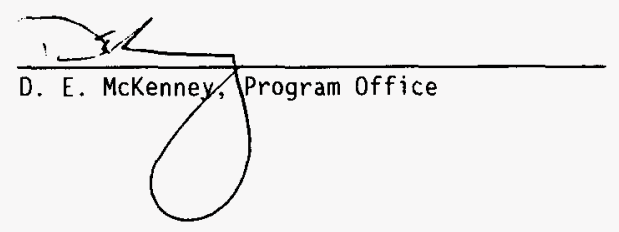

$\frac{5 / 14 / 96}{\text { Date }}$

$\frac{5 / 14 / 96}{\text { Date }}$

$\frac{5-14-96}{\text { late }}$

$\frac{5-21-96}{\text { Date }}$

ii i 
WHC-EP-0125-8

This page intentionally left blank. 
WHC-EP-0125-8

\title{
SUMMARY OF RADIOACTIVE SOLID WASTE \\ RECEIVED IN THE 200 AREAS DURING \\ CALENDAR YEAR 1995
}

\author{
J. D. Anderson \\ D. L. Hagel
}

\begin{abstract}
Westinghouse Hanford Company manages and operates the Hanford Site 200 Area radioactive solid waste storage and disposal facilities for the U.S. Department of Energy, Richland Operations office under contract DE-AC06-87RL10930. These facilities include radioactive solid waste disposal sites and radioactive solid waste storage areas. This document summarizes the amount of radioactive materials that have been buried and stored in the 200 Area radioactive solid waste storage and disposal facilities since startup in 1944 through calendar year 1995. This report does not include backlog waste, solid radioactive wastes in storage or disposed of in other areas, or facilities such as the underground tank farms. Unless packaged within the scope of WHC-EP-0063, Hanford Site Solid Waste Acceptance Criteria," liquid waste data are not included in this document.
\end{abstract}

"WHC, 1988, Hanford Site Solid Waste Acceptance Criteria, WHC-EP-0063, Westinghouse Hanford Company, Richland, Washington. 
WHC-EP-0125-8

This page intentionally left blank. 
WHC-EP-0125-8

\section{CONTENTS}

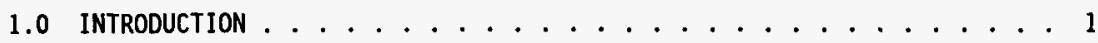

Table 1 Summary of Document Data . . . . . . . . . . . . . 1

Solid Waste Burial Ground Areas Waste Volumes Disposed and Stored and

Nondecayed Curies Content in the 200 Areas from Startup to

December 31, 1995, Including Segregated Transuranic Waste . . . . . . . 3

Solid Waste Burial Ground Areas Decayed Curie Content in the

200 Areas from Startup to December 31, 1995, Including Segregated

Transuranic Waste . . . . . . . . . . . . . . 42

Unsegregated Waste Burial Ground Areas Waste Volumes Buried and

Nondecayed Curie Content from Startup Through December 31, 1995 . . . . . 68

Unsegregated Waste Burial Ground Areas Decayed Curie Content from

Startup through December $31,1995 \ldots \ldots 73$

Post-1970 Nontransuranic Waste Burial Ground Areas Waste Volumes

Buried or Stored and Nondecayed Curie Content through

December 31,1995 ..................... . 76

Post-1970 Nontransuranic Waste Burial Ground Areas Decayed Curie

Content through December $31,1995 \ldots \ldots 131$

Post-1970 Segregated Transuranic Waste Burial Ground Areas Waste Volumes Stored and Nondecayed Curie Content through

December 31,1995 . . . . . . . . . . . . . . . 168

Post-1970 Segregated Transuranic Waste Burial Ground Areas Decayed

Curie Content through December 31, 1995 ............ 185

Transuranic and Nontransuranic Waste Received for Disposal or

Storage for the Calendar Year from January 1 to December 31, 1995 . . . . 193

Status of Individual Burial Grounds and Facilities.

Land Disposal or Storage of Sol id Radioactive Waste from

January 1 to December 31, 1995

Burial Ground 218E12B ................. 219

Burial Ground 218W3A................. . 220

Burial Ground $218 \mathrm{~W} 3 \mathrm{AE} \ldots \ldots . \ldots . \ldots . \ldots 223$

Burial Ground 218W4C . . . . . . . . . . . . . . 239

Burial Ground 218W5 . . . . . . . . . . . . . 254

Transuranic Waste Storage and Assay Facility (TRUSAF) . . . . . 264

Central Waste Complex 2401W Building . . . . . . . . . . 269 
WHC-EP-0125-8

CONTENTS (cont)

Central Waste Complex 2402W Building . . . . . . . . . . . 270

Central Waste Complex 2402WB Building . . . . . . . . . . 272

Central Waste Complex 2402WC Building . . . . . . . . . . 275

Central Waste Complex 2402WD Building . . . . . . . . . 278

Central Waste Complex 2402WE Building ............ 280

Central Waste Complex 2402WG Building . . . . . . . . . . 282

Central Waste Complex 2402WH Building . . . . . . . . . 285

Central Waste Complex 2402WI Building . . . . . . . . . . 287

Central Waste Complex 2402WJ Building . . . . . . . . . . . 289

Central Waste Complex 2403WB Building . . . . . . . . . 291

Central Waste Complex 2403WC Building ............. 303

Central Waste Complex 2403WD Building ............. 311

Alkaline Metal Waste Storage Unit $1 \ldots \ldots 312$

Alkaline Metal Waste Storage Unit $3 \ldots \ldots$. . . . . . 313

Flammable Storage Module 2 . . . . . . . . . . . 314

Flammable Storage Module 3 . . . . . . . . . . . 315

Flammable Storage Module 5 ............... 316

Flammable Storage Module $6 \ldots \ldots$. . . . . . . . 317

Flamnable Storage Module $8 \ldots \ldots 319$

Flammable Storage Module $10 \ldots \ldots 320$

Flammable Storage Module $15 \ldots \ldots 322$

Flammable Storage Module $17 \ldots \ldots 323$

Mixed Waste Staging Pad at the CWC . . . . . . . . . . . 324

Grand Totals for Land Disposal or Storage of Solid Radioactive 325

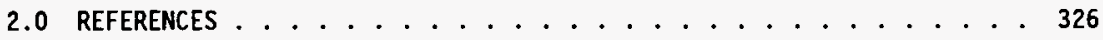

3.0 BIBLIOGRAPHY . . . . . . . . . . . . . . 326 
WHC-EP-0125-8

\section{CONTENTS (cont)}

Appendix A: 200 Area Solid Waste Storage/Disposal Facility Coordinates . A-1

Appendix B: Burial Ground/Storage Facility Land Usage . . . . . . . . B-1 
WHC-EP-0125-8

This page intentionally left blank. 
WHC-EP-0125-8

\section{SUMNARY OF RADIOACTIVE SOLID WASTE \\ RECEIVED IN THE 200 AREAS DURING \\ CALENDAR YEAR 1995}

\subsection{INTRODUCTION}

This document summarizes the amount of radioactive materials buried or stored in the 200 Area solid waste facilities during calendar year (CY) 1995, and lists only those radionuclides as identified by the generator (see Table 1). In addition to the data for CY 1995, cumulative data are presented. This document also lists decayed activity for the plant burial sites. It should be noted that storage locations listed in this report are current only on the run date identified at the top of the data sheets, as these locations are subject to frequent change.

It should be noted that the summation of ${ }^{90} \mathrm{Sr},{ }^{106} \mathrm{Ru},{ }^{137} \mathrm{Cs}$, and other radionuclides does not equal the total curie amount. The difference is the amount of other mixed fission products present in the burials that are not noted in this report. The reported curie values do not include the activities for the radioactive daughters. The data for plutonium for waste disposed before 1970 could be biased low by about $300 \mathrm{~kg}$.

Table 1. Summary of Document Data.

\begin{tabular}{|c|c|}
\hline Pages & Data \\
\hline $3-41$ & $\begin{array}{l}\text { Amount of radioactive materials buried and stored within the } \\
\text { Westinghouse Hanford Company's } 200 \text { Area plateau since startup }\end{array}$ \\
\hline $42-67$ & $\begin{array}{l}\text { Listing of the decayed activities in the various burial ground } \\
\text { facilities from startup through calendar year } 1995\end{array}$ \\
\hline $68-72$ & $\begin{array}{l}\text { Estimate of the radioactive materials in the unsegregated burial } \\
\text { sites }\end{array}$ \\
\hline $73-75$ & Decay status of unsegregated waste from startup to present \\
\hline $76-130$ & $\begin{array}{l}\text { Amount of radioactive material in the nontransuranic disposal or } \\
\text { storage facilities }\end{array}$ \\
\hline $131-167$ & Decay status of nontransuranic waste from 1970 to present \\
\hline $168-184$ & $\begin{array}{l}\text { Amount of radioactive material in the transuranic segregated } \\
\text { storage sites }\end{array}$ \\
\hline $185-192$ & Decay status of transuranic segregated waste from 1970 to present \\
\hline $193-218$ & $\begin{array}{l}\text { Summary of waste received for disposal or storage for calendar } \\
\text { year } 1995\end{array}$ \\
\hline $219-325$ & $\begin{array}{l}\text { Amount of waste received for each burial ground and storage } \\
\text { facility for calendar } 1995\end{array}$ \\
\hline
\end{tabular}


This document was prepared using the Solid Waste Information and Tracking, System database, resulting in more accurate and updated data. Raclioisotopic data are based on the Tabie of Isotopes (Wiley-Interscience 1978). As a result, some of the data are different from previous editions. Some numbers for total decayed curies in the 1992 report (WHC 1992) show an increase in value from the 1991 report (WHC 1991). This increase resulted from previously excluding the presence of daughter products produced in the decay process; thus, the values were low by this amount. Facility names were changed in this document to reflect Hanford Site standard naming conventions. In 1993, I and usage data were removed from the report format and placed in tables in Appendix B for ease of use. To improve the quality of the data, the decay schemes were upgraded in 1994 to more accurately reflect contributions by daughter products.

The computer-generated data in the tables are presented using exponential notation. An example of this form is as follows:

$$
\begin{aligned}
& 2.41 \mathrm{E}+06=2.41 \times 10^{+6}=2,410,000 \\
& 2.41 \mathrm{E}-06=2.41 \times 10^{-6}=0.00000241 .
\end{aligned}
$$

Footnotes for 100 Area waste are offset listed for convenience in the OTHER RADIOACTIVITY (CURIES) column for information purposes, but are not included in the totals for OTHER RADIOACTIVITY radionuclides; the ${ }^{137} \mathrm{Cs}$ is included in the ${ }^{137} \mathrm{Cs}$ column, however. An example follows:

\section{OTHER RADIOACTIVITY (CURIES)}

$\begin{array}{ccc} & 5.660 \mathrm{E}+02 & (\text { OTHER TOTAL) } \\ \mathrm{C}-14 & 1.234 \mathrm{E}+01 & (\text { OTHER) } \\ \text { C0-60 } & 5.537 \mathrm{E}+02 & (\text { OTHER) } \\ \text { CS137 } & 2.356 \mathrm{E}+03 & (105 \mathrm{~N}) \\ \text { CO58 } & 1.343 \mathrm{E}-07 & (105 \mathrm{~N}) \\ \text { CO50 } & 4.566 \mathrm{E}+01 & (105 \mathrm{~N}) \\ \text { EU155 } & 1.846 \mathrm{E}+00 & (105 \mathrm{~N}) \\ \text { C14 } & 1.249 \mathrm{E}+00 & (105 \mathrm{~N}) \\ \text { EU154 } & 8.697 \mathrm{E}+00 & (105 \mathrm{~N}) \\ \text { FE59 } & 4.619 \mathrm{E}-11 & (105 \mathrm{~N}) \\ \text { MN54 } & 1.964 \mathrm{E}-01 & (105 \mathrm{~N}) \\ \text { EU15? } & 9.573 \mathrm{E}-01 & (105 \mathrm{~N})\end{array}$




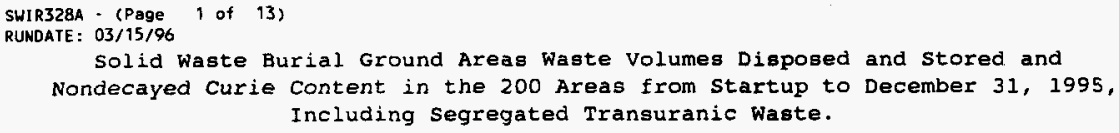

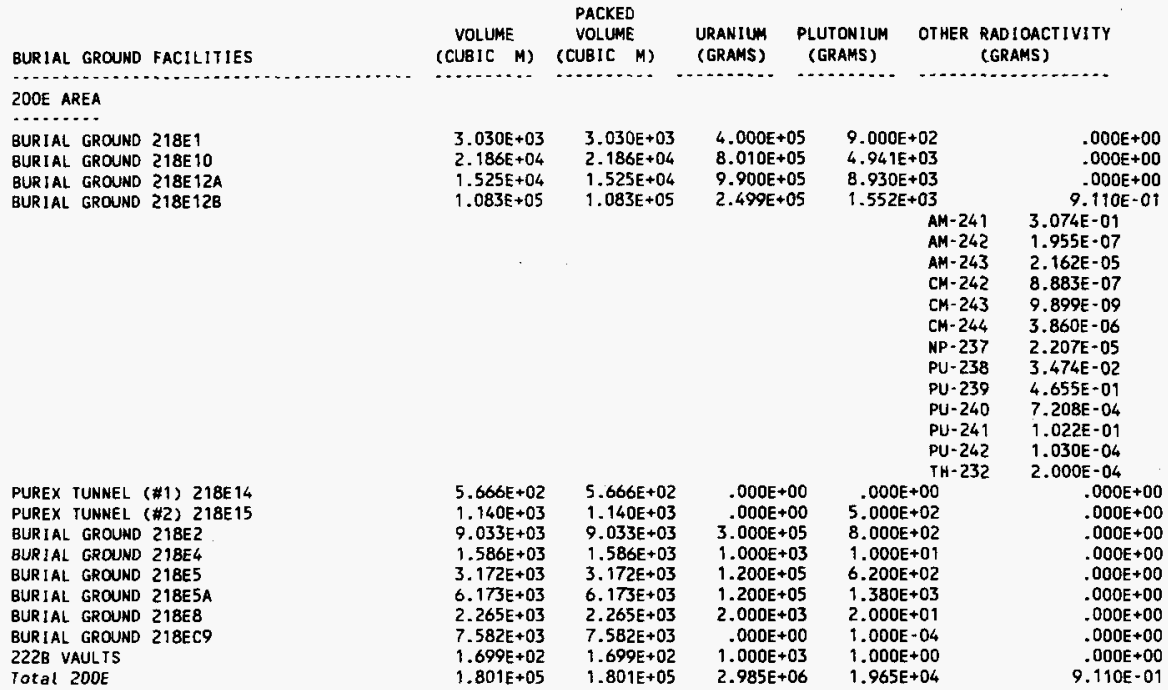




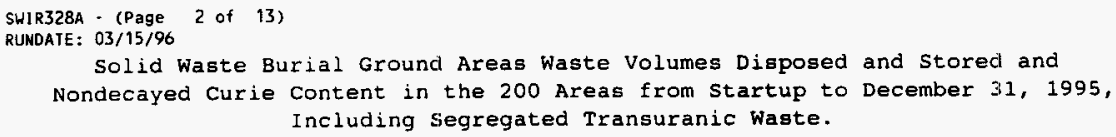

212N BUILOING

$2.167 E+02$

$2.167 E+02$

$1.000 E+02$

4. $280 E+01$

PU- 238

214T FACILITY/TPLANT

$1.041 E+00 \quad 1.041 E+00 \quad .000 E+00$

$.000 E+00$

AN- 241

$\mathrm{CM}-243$

$\mathrm{CM}-244$

NP -237

PU-238

PU-239

TH- 232

u. 233

BURIAL GROUND 218W1

$\begin{array}{lll}7.164 E+03 & 7.164 E+03 & 7.000 E+05 \\ 1.160 E+03 & 1.160 E+03 & .000 E+00 \\ 1.371 E+04 & 1.371 E+04 & 9.000 E+05 \\ 8.240 E+03 & 8.240 E+03 & 1.400 E+06 \\ 2.514 E+04 & 2.514 E+04 & 2.690 E+06 \\ 1.090 E+04 & 1.090 E+04 & 7.000 E+07 \\ 1.009 E+05 & 1.009 E+05 & 7.240 E+07\end{array}$

$9.402 \mathrm{E}+04$

$.000 E+00$

$2,000 \mathrm{E}+03$

$1.260 E+05$

BURIAL GROUNO 218U1A

BURIAL GROUND $218 W 2$

BURIAL GROUND 218W2A

BURIAL GROUND 218W3

BURIAL GROUND 218W3A

BURIAL GROUND 218W3AE

$2.640 \mathrm{E}+04$

$2.640 E+04$

$2.656 E+07$

$1.222 \mathrm{E}+02$

$6.384 \mathrm{E}+03$

$6.800 E+04$

$2.931 E+04$

$\mathrm{TH}-232$

AM- 241

$A M-242$

AM- 243

CF -252

$\mathrm{CM}-242$

$\mathrm{CM}-243$

CM- 244

$\mathrm{H}-2$

LI-6

NP- 237

PU-238

PU- 239

PU-240

PU. 241

PU-242

RA- 226

TH- 228

TH- 230

TH-232

TH- 234

$\mathrm{U}-233$

U-234

$\mathrm{u}-235$

u- 236

U. 238

AM- 241

AM -242

AM- 243

CF -251

CF -252

CM- 242

$\mathrm{CM}-243$

CM- 244

CM- 245

CM-246

CM- 247

$1.000 E+00$
$1.000 E+00$
$4.738 E+00$
$5.555 E-06$
$5.000 E-10$
$9.900 E-09$
$8.581 E-03$
$9.973 E-07$
$1.051 E-03$
$2.284 E-01$
$3.574 E-04$
$4.699 E+00$
$.000 E+00$
$.000 E+00$
$.000 E+00$
$.000 E+00$
$.000 E+00$
$6.000 E+07$

$6.000 \mathrm{E}+07$ $1.440 \mathrm{E}+06$

$2.371 E+01$

$5.908 E-04$

$1.221 \mathrm{E}-01$

3.730E-0B

$3.143 \mathrm{E}-06$

$3.055 \mathrm{E}-06$

$5.550 \mathrm{E}-03$

$2.025 E+03$

$1.636 \mathrm{E}+04$

$2.958 E+01$

5.289E-01

$6.466 \mathrm{E}+00$

$1.908 E+00$

2.511E-01

2.000E-03

$2.596 \mathrm{E}+00$

$1.619 \mathrm{E}-09$

$2.000 E-06$

1. $115 \mathrm{E}+06$

5.672E-09

$2.023 \mathrm{E}+02$

2. $048 \mathrm{E}-01$

$2.136 \mathrm{E}+01$

$2.573 E+00$

$3.060 E+05$ $2.496 E+06$

$2.638 \mathrm{E}+00$

$1.137 \mathrm{E}-03$

2. $400 \mathrm{E}-02$

1.500 E- 07

$6.506 E-08$

$1.909 E-05$

5.516E-06

$1.082 \mathrm{E}-03$

$1.392 E-05$

3. 800 E- 09

3.710E- 04 
SWIR328A - (Page 3 of 13)

RUNDATE: $03 / 15 / 96$

Solid Waste Burial Ground Areas Waste Volumes Disposed and Stored and

Nondecayed Curie Content in the 200 Areas from startup to December 31, 1995, Including segregated Transuranic Waste.

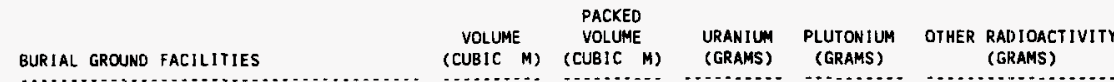

BURIAL GROUND 218 W3AE

(CUBIC M) (CUBIC M) (GRAMS) (GRAMS)

CM-248 $\quad 3.807 E-06$

NP- $237 \quad 6.993 E+00$

PU-238 2.091E-01

PU-239 9.747E+01

PU $-240 \quad 1.045 E+01$

PU-241 8.943E-02

PU-242 1.364E-02

PU-244 2.319E-02

RA-224 7.302E-04

RA-226 5.016E-03

TH-228 $\quad 7.711 \mathrm{E}+02$

TH-230 8.460E-02

TH-232 $6.390 E+04$

TH-234 3.601E-02

U-232 2.479E-05

$\mathrm{U}-233 \quad 5.469 \mathrm{E}+00$

U-234 $4.523 E+01$

$U-235 \quad 1.749 E+03$

$U-236 \quad 1.610 E+01$

BUR IAL GROUND 218W4A

$1.771 E+04 \quad 1.771 E+04 \quad 3.941 E+08$

$3.538 \mathrm{E}+04$

U-238

BUR IAL GROUND 218W4B

$1.046 E+04$

$1.046 E+04$

$5.883 E+06$

$6.634 E+04$

$\mathrm{TH}-232$

AM- 241

II -6

$2.429 E+06$

$2.500 E+08$

$2.500 E+08$

$6.784 E+05$

$1.210 E+00$

NP $-237 \quad 2.140 E+02$

PU-238 $1.432 E+00$

TH-232 $6.685 E+05$

$\mathrm{U}-233 \quad 7.607 \mathrm{E}+03$

BURIAL GROUND 218W4C

$1.754 E+04 \quad 1.754 E+04$

$6.317 E+06$

3. $732 E+05$

AM- 241

AM -242

AM- 243

CF -252

$\mathrm{CM}-242$

$\mathrm{CM}-243$

$\mathrm{CM}-244$

CM-245

$7.607 \mathrm{E}+03$ $1.510 \mathrm{E}+08$

$1.529 E+02$

$1.524 \mathrm{E}-02$

$5.276 E+01$

$2.000 E+00$

1. $182 \mathrm{E}-06$

$2.366 \mathrm{E}-08$

$2.996 \mathrm{E}-06$

$4.822 E+01$

LI 6

$5.900 E+00$

NP -237

PU-238

PU-239

$1.412 E+02$

$\mathrm{PU}-240$

$5.245 \mathrm{E}+03$

$6.479 E+02$

$7.745 E+01$

PU- 241

2.016E+01

PU. 242

PU -244

RA- 224

RA- 226

TH- 228

TH-229

2. $494 E+00$

$1.000 \mathrm{E}-07$

$7.162 E-03$

2.787E-02

4.916E-04

4. $892 \mathrm{E}-04$

$\mathrm{TH}-230$

4. $620 \mathrm{E}-02$

$\mathrm{TH}-232$

IH- 234

4.227E+05

U. 232

8.021E-01

$\mathrm{u}-233$

$7.872 \mathrm{E}-04$

U. 234

$8.376 \mathrm{E}+02$

U -235

$1.755 \mathrm{E}+00$

U- 236

$3.195 E+05$

U- 238

$1.984 \mathrm{E}+00$

$1.503 E+08$ 


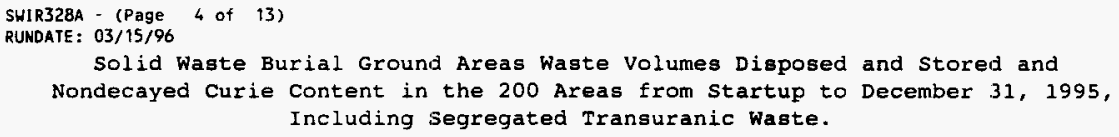

\begin{tabular}{|c|c|c|c|c|c|c|}
\hline BURIAL GROUND FACILITIES & $\begin{array}{l}\text { VOLUME } \\
\text { (CUBIC M) }\end{array}$ & $\begin{array}{c}\text { PACKED } \\
\text { VOLUME } \\
\text { (CUBIC } \quad \text { H) }\end{array}$ & $\begin{array}{l}\text { URANIU⿴囗十 } \\
\text { (GRAMS) }\end{array}$ & $\begin{array}{l}\text { PLUTONIUM } \\
\text { (GRAMS) }\end{array}$ & OTHER & $\begin{array}{l}\text { RAIDIOACTIVITY } \\
\text { (GIRAMS) }\end{array}$ \\
\hline BURIAL GROUND $218 \mathrm{~W} 5$ & $5.029 E+04$ & $5.029 E+04$ & $2.651 E+07$ & 1.681 & $\begin{array}{l}02 \\
A M-241 \\
A M-242 \\
A M-243 \\
C F-250 \\
C F-251 \\
C F-252 \\
C M-242 \\
C M-243 \\
C M-244 \\
C M-245 \\
C M-246 \\
C M-248 \\
C M-250 \\
N P-237 \\
P U-238 \\
P U-239 \\
P U-240 \\
P U-241 \\
P U-242 \\
P U-244 \\
R A-224 \\
R A-226 \\
\text { IH-228 } \\
\text { TH-229 } \\
\text { IH-230 } \\
\text { TH }-232 \\
\text { TH-236 } \\
U-232 \\
U-233 \\
U-234 \\
U-235 \\
U-236 \\
U-238\end{array}$ & $\begin{array}{l}3.764 E+06 \\
6.857 E-01 \\
1.310 E-04 \\
1.833 E-02 \\
2.184 E-05 \\
9.506 E-09 \\
2.503 E-02 \\
4.876 E-03 \\
3.153 E-05 \\
5.195 E-03 \\
1.014 E-05 \\
9.730 E-08 \\
4.970 E-03 \\
1.848 E-20 \\
1.792 E+01 \\
7.375 E+00 \\
1.780 E+00 \\
2.447 E-01 \\
1.756 E-02 \\
9.245 E-02 \\
1.638 E-04 \\
3.525 E-03 \\
2.916 E+00 \\
1.862 E-01 \\
7.400 E-04 \\
9.200 E+01 \\
2.262 E+06 \\
5.633 E-02 \\
1.509 E-03 \\
2.095 E-01 \\
1.678 E+01 \\
1.076 E+04 \\
5.642 E+00 \\
1.491 E+06\end{array}$ \\
\hline $\begin{array}{l}\text { BURIAL GROUND } 218 \mathrm{~W} \\
221 T \text { T PLANT } \\
2225 \text { VAULIS } \\
222 T \text { VAULTS }\end{array}$ & $\begin{array}{l}4.856 \mathrm{E}+02 \\
3.562 \mathrm{E}+00 \\
1.586 \mathrm{E}+02 \\
6.796 \mathrm{E}+01 \\
3.119 \mathrm{~F}+02\end{array}$ & $\begin{array}{l}4.856 E+02 \\
3.562 E+00 \\
1.586 E+02 \\
6.796 E+01 \\
3.119 E+02\end{array}$ & $\begin{array}{r}.000 E+00 \\
.000 E+00 \\
7.000 E+02 \\
3.000 E+02 \\
4.077 F+04\end{array}$ & $\begin{array}{r}.000 \\
.000 \\
7.000 \\
3.000\end{array}$ & $\begin{array}{r}00 \\
+00 \\
.01 \\
-01 \\
01\end{array}$ & $\begin{array}{r}.000 E+00 \\
.000 E+00 \\
.000 E+00 \\
.000 E+00 \\
.774 E+04\end{array}$ \\
\hline $\begin{array}{l}\text { TRANSURANIC WASTE STORAGE \& ASSAY } \\
\text { FACILITY(TRUSAF) }\end{array}$ & $3.119 E+02$ & $3.119 E+02$ & $4.677 E+04$ & 4.1510 & $\begin{array}{l}\text { P4 } \\
A M-241 \\
A M-243 \\
C F-252 \\
C M-243 \\
C M-244 \\
C M-245 \\
N P-237 \\
P U-238 \\
P U-239 \\
P U-240 \\
P U-241 \\
P U-242 \\
P U-244 \\
R A-226 \\
I H-228 \\
I H-232 \\
U-233 \\
U-234 \\
U-235 \\
U-236\end{array}$ & $\begin{array}{l}1.774 E+04 \\
6.877 E+01 \\
4.636 E-02 \\
2.350 E-07 \\
7.204 E-03 \\
3.065 E+00 \\
4.998 E+01 \\
1.848 E+01 \\
9.075 E+00 \\
1.403 E+04 \\
1.237 E+03 \\
1.065 E+02 \\
1.866 E+01 \\
2.885 E-06 \\
4.520 E-02 \\
1.463 E-02 \\
1.000 E+02 \\
2.000 E-01 \\
5.205 E-03 \\
4.128 E+01 \\
5.146 E-03\end{array}$ \\
\hline
\end{tabular}




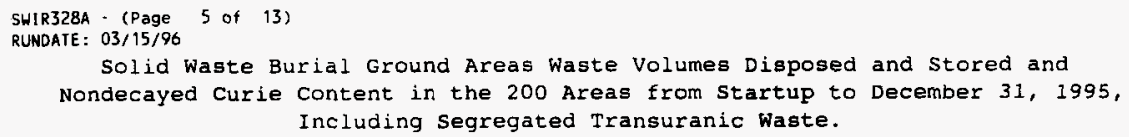

CENTRAL WASTE COMPLEX 2402W BUILOING

$2.969 \mathrm{E}+02$

$2.969 E+02$

$3.142 E+04$

$.000 E+00$

$\mathrm{U}-238$

AM- 241

AM- 243

CM-243

CM- 244

NP -237

PU-238

PU-239

PU- 240

PU- 241

$\mathrm{PU}-242$

TH-228

$\mathrm{u}-233$

$\mathrm{U}-235$

U- 238

CENTRAL WASIE COMPLEX 2602WB BUILDING

$1.526 \mathrm{E}+02$

$1.526 E+02$

$5.240 E+03$

$2.197 E+02$

AM- 241

CM- 243

NP. 237

$\mathrm{PU}-238$

PU-239

PU-240

PU-241

PU-242

PU-244

RA- 224

RA- 226

TH-228

TH-230

TH-232

$\mathrm{TH}-234$

$\mathrm{U}-232$

U. 234

u- 235

U-238

CENTRAL WASTE COMPLEX 2402WC BUILDING
$7.466 E+01$

$5.663 E+02$
$2.530 E+02$

AM- 241

$\mathrm{CH}-242$

$\mathrm{CM}-243$

CM- 244

NP. 237

PU -238

PU- 239

PU- 240

PU- 241

PU -242

RA- 226

$\mathrm{TH}-23 \mathrm{O}$

U-232
$5.830 E-08$ $4.763 E+02$

$1.101 \mathrm{E}+00$

4. $920 E-03$

$6.158 E-04$

3. $067 \mathrm{E}-03$

2.337E-01

$8.558 \mathrm{E}-02$

$2.763 E+02$

$1.746 E+01$

1. $798 \mathrm{E}+00$

4. $001 \mathrm{E}-01$

4.043E-05

$6.730 E+01$

$4.350 E+00$

$1.072 \mathrm{E}+02$

$1.239 E+04$

6. $470 \mathrm{E}-03$

2.590E-07

1. 109E-01

3.194E-03

$7.668 \mathrm{E}+00$

$7.383 E-01$

$6.022 E-02$

$4.915 \mathrm{E}-03$

$1.333 \mathrm{E}+00$

$3.306 \mathrm{E}-09$

6.830E-05

$5.543 E-07$

$4.245 E-02$

7. $143 E+03$

$6.819 E-08$

2.760E-07

$1.728 \mathrm{E}-01$

$1.996 \mathrm{E}+01$

$5.220 E+03$ $4.298 \mathrm{E}+02$

$1.562 \mathrm{E}-01$

1.179E-15

1. $200 \mathrm{E}-10$

$8.481 E-09$

$4.920 E-01$

$3.095 E-02$

$4.506 E+01$

$4.262 E+00$

5. $739 E-01$

6.365 E- 02

5. $500 E-09$

2. $900 E-07$

5. $700 \mathrm{E}-11$ 


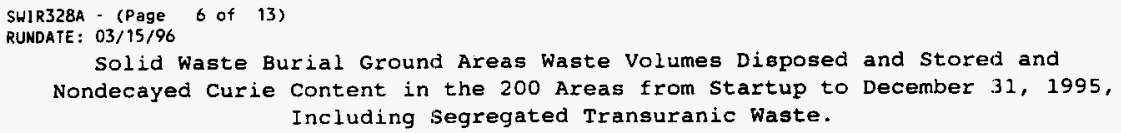

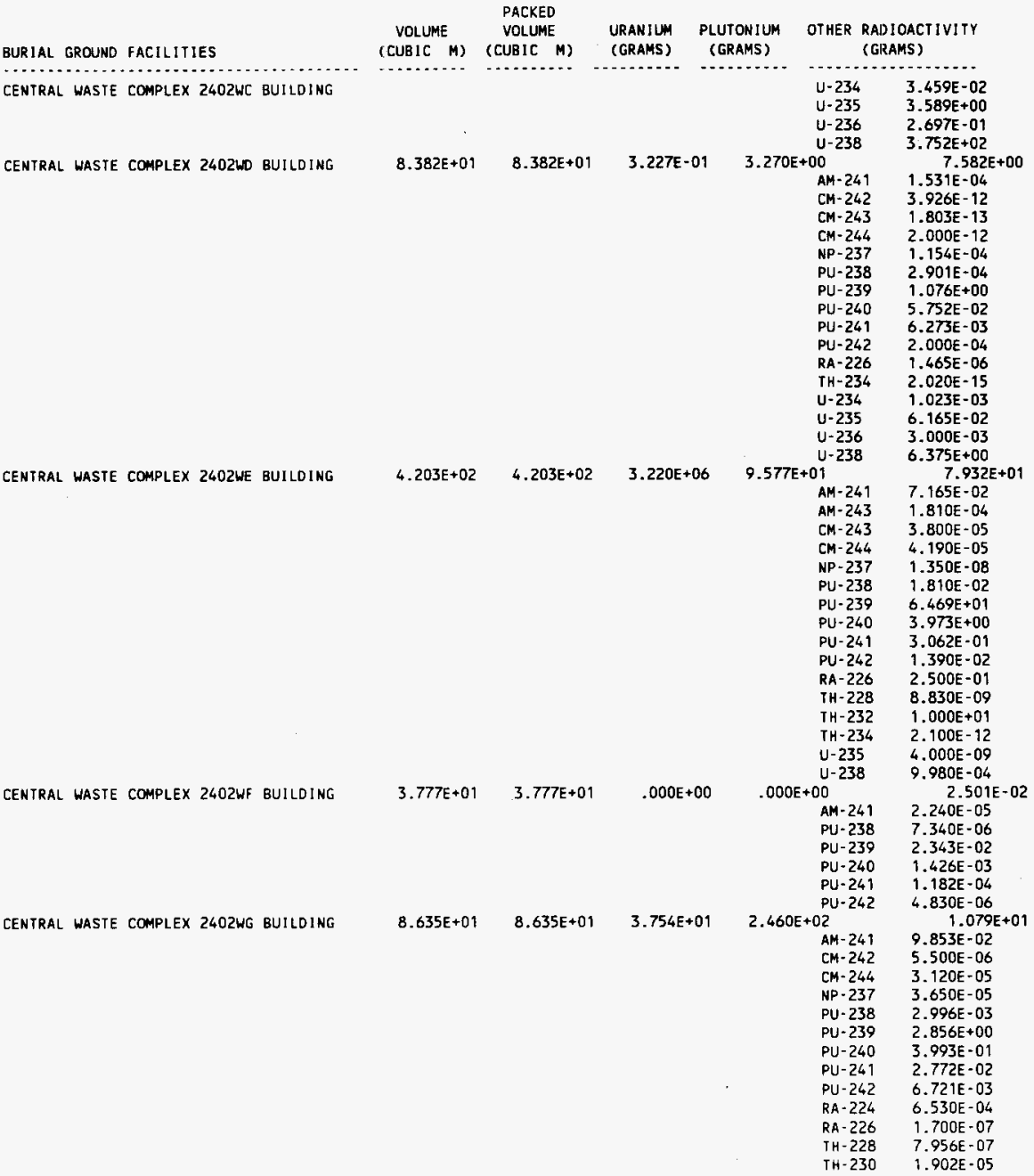




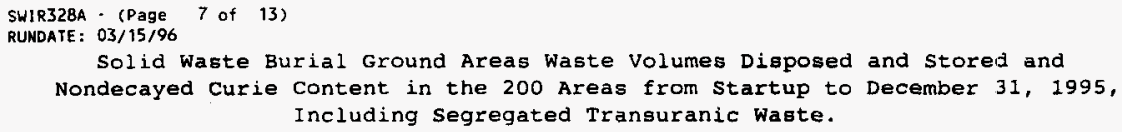

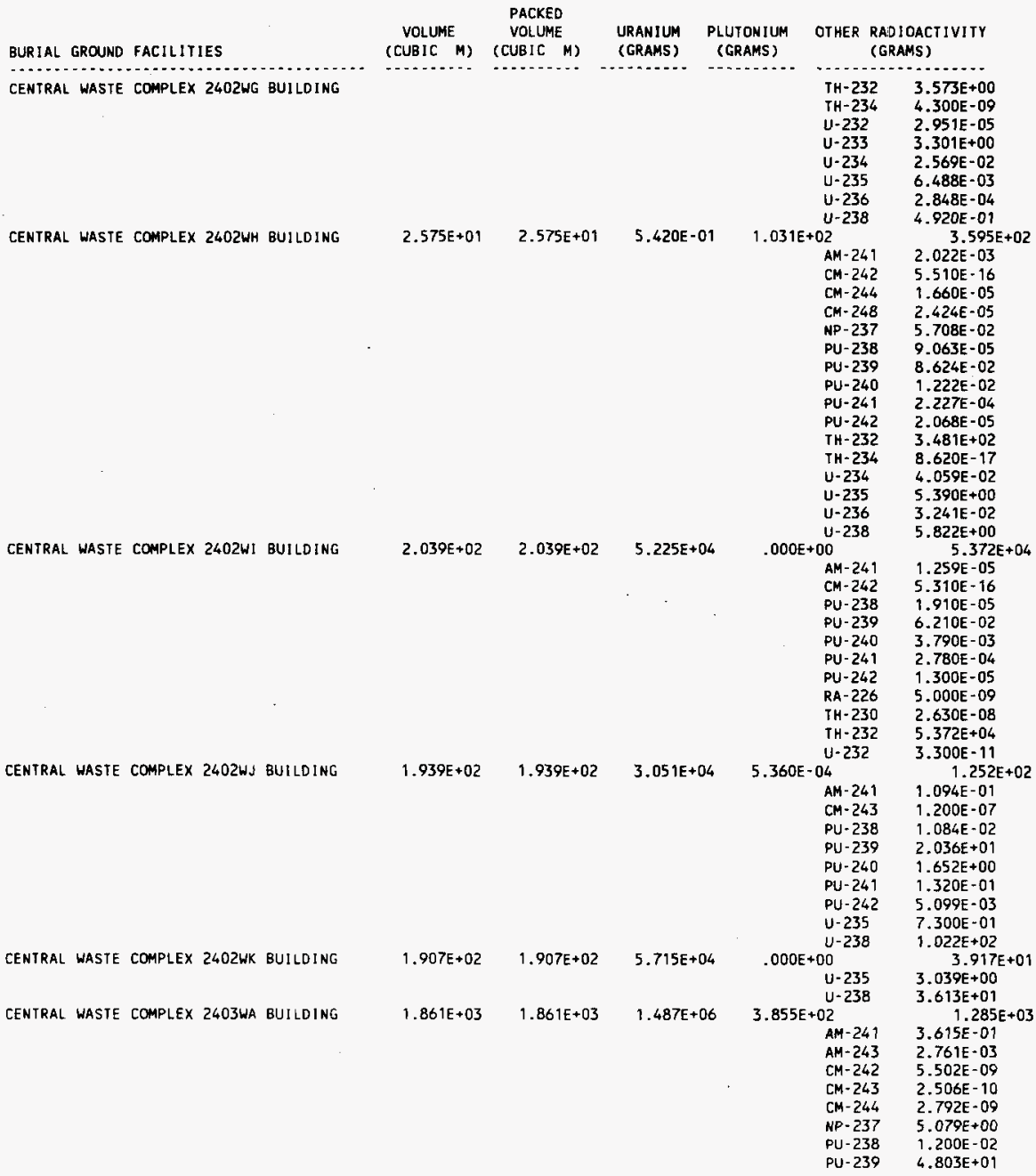


WHC-EP-O $125-8$

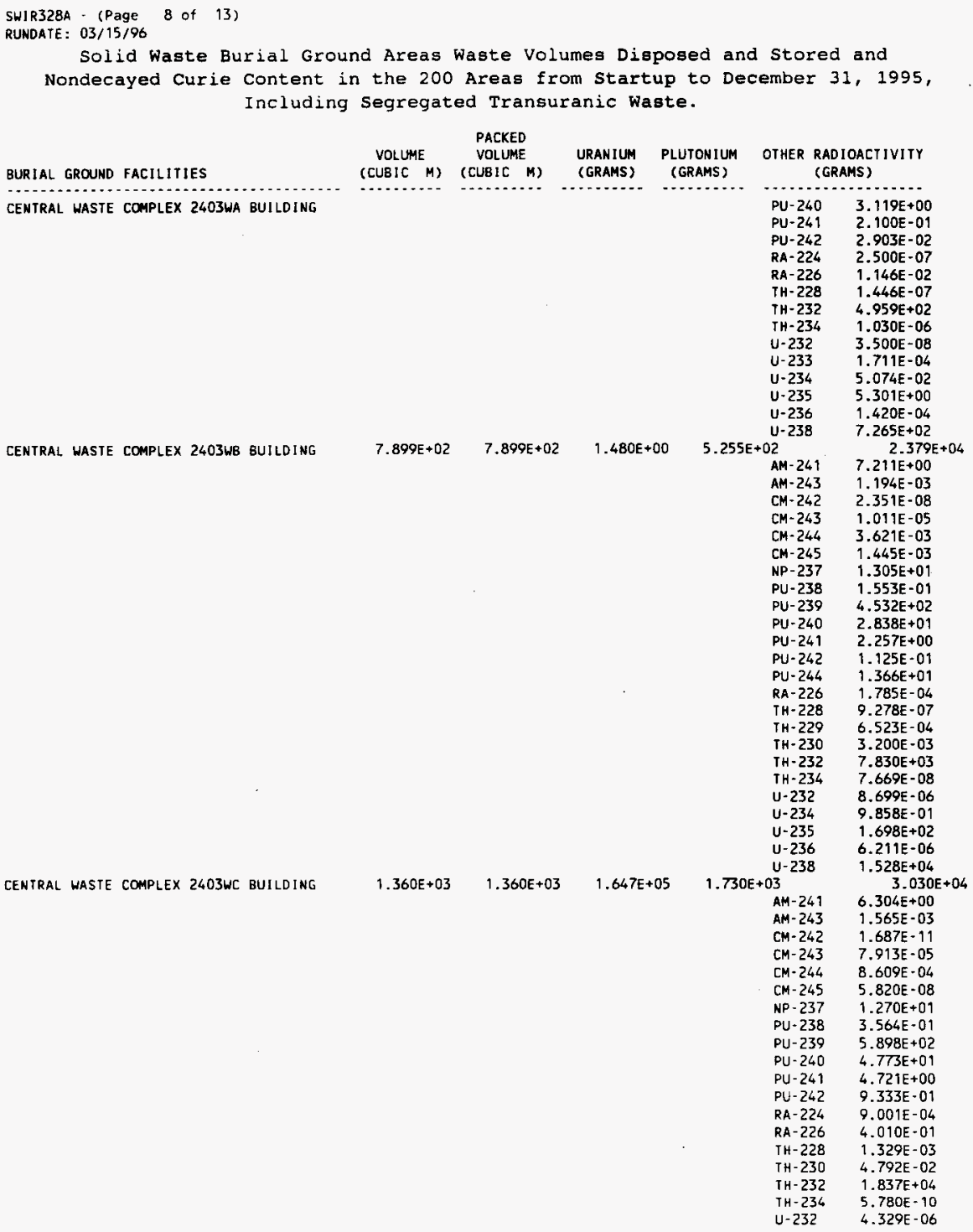


SHIR328A - (Page 9 of 13 )

RUNDATE : $03 / 15 / 96$

Solid Waste Burial Ground Areas Waste Volumes Disposed and Stored and

Nondecayed Curie Content in the 200 Areas from startup to December 31, 1995 , Including Segregated Transuranic Waste.

BURIAL GROUND FACILITIES

CENTRAL WASTE COMPLEX 2403 WC BUILDING

CENTRAL WASTE COMPLEX 2403WD BUILDING

ALKALINE METAL WASTE STORAGE UNIT 1

ALKALINE METAL WASTE STORAGE UNIT 2

ALKALINE METAL WASTE STORAGE UNIT 3 ALKALINE METAL WASTE STORAGE UNIT 4 INVENTORY NOT YEI VERIFIED BY SWO FLAMMABLE STORAGE MOOULE 1

fLAMMABLE STORAGE MODULE 2

FLAMMABLE STORAGE MODULE 3

VOLUME
(CUBIC M) (CUE

PACKED

VOLUME

(CUBIC M) (CUBIC M)

URANIUM

PLUTONIUM

OTHER RADIOACTIVITY

..........

(GRAMS)

(GRAMS)

(GRAMS)

U-233 $1.928 E-01$

$\mathrm{U}-234 \quad 1.460 \mathrm{E}+00$

$\mathrm{U}-235 \quad 9.683 \mathrm{E}+01$

$\mathrm{U}-236 \quad 2.395 \mathrm{E}+00$

$1.336 E+03 \quad 1.336 E+03 \quad 1.111 E+05 \quad 1.000 E-06$

U. 238 1.117E+04

AM-241 9.086E-01

AM- $242 \quad 4.070 E-09$

$A M-243 \quad 2.050 E-07$

CM-242 2.124E-09

$\mathrm{CM}-243 \quad 3.120 \mathrm{E}-07$

CM-244 3.486E-06

NP- $237 \quad 1.676 E+01$

PU- $238 \quad 2.685 E-01$

PU-239 2.826E +02

$P U-240 \quad 1.870 E+01$

PU- $241 \quad 1.673 E+00$

PU-242 1.077E-01

RA- $224 \quad 9.900 E-10$

TH-228 1.200E-12

$\mathrm{TH}-232 \quad 2.231 \mathrm{E}+04$

TH-234 2.960E-08

$\mathrm{U}-232 \quad 4.990 \mathrm{E}-03$

U-233 1.875E-04

$\mathrm{U}-234 \quad 1.108 \mathrm{E}-01$

U-235 $5.630 E+02$

U-236 2.120E+00

$U-238 \quad 2.537 E+06$

$7.469 E+00 \quad 7.469 E+00 \quad .000 E+00 \quad .000 E+00$

$3.331 E+00 \quad 3.331 E+00 \quad .000 E+00 \quad .000 E+00$

AM- 241

PU- 238

PU- 239

PU -240

PU-241

PU-242

$6.371 E+00$

$5.129 E+00$

$6.371 E+00$

$5.129 E+00$

$.000 E+00$

4. $100 \mathrm{E}-01$

4. $100 E-01$

$.000 E+00$

$.000 E+00$

$.000 E+00$

$2.400 E+01$

$.000 \mathrm{E}+00$

$3.358 E+00$

$3.358 \mathrm{E}+00$

2.115E-04

$.000 \mathrm{E}+00$

AM- 241

PU- 238

PU- 239

$3.725 E+00$

$3.725 E+00$

$1.572 E-01$

$.000 E+00$

AM- 241

$\mathrm{CM}-242$

PU- 238

PU- 239

PU-241

RA- 226

TH-234

U- 234

U. 235

$\mathrm{u}-238$

$7.263 E+00$

$7.263 E+00$

$.000 E+00$

$.000 \mathrm{E}+00$

AM- 241

AM- 243

CM- 244

CM- 245 $.000 E+00$

$8.968 E-06$

1.010E-08

$2.620 E-09$

8. $400 \mathrm{E}-06$

5. $120 E-07$

$4.160 \mathrm{E}-08$

$1.760 E-09$ $.000 \mathrm{E}+00$ $-000 E+00$

$.000 E+00$

$9.448 \mathrm{E}-0 \mathrm{~B}$

1.640 E-09

4. $110 \mathrm{E}-11$

9.280E-08

1.095E-01

2.289E-06

$9.240 \mathrm{E}-16$

2. $024 E-08$

$5.819 E-05$

$5.142 E-08$

$1.515 E-08$

$2.210 E-08$

$1.600 \mathrm{E}-04$

$9.910 E-02$

$1.020 E-02$ $1.472 E-02$

8.370E-06

$5.800 E-06$

5. B00E-09

$6.800 E-06$ 


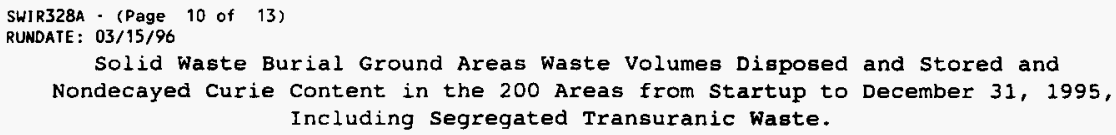
(CUBIC M) (CUBIC M) (GRAMS) (GRAMS) (GRAMS)

FLAMMABLE STORAGE MODULE 4

FLAMMABLE STORAGE MOOULE 5

FLAMMABLE STORAGE MOOULE 6

FLAMMABLE STORAGE MODULE 7

FLAMMABLE STORAGE MODULE 8 FLAMMABLE STORAGE MODULE 10

.......

(n.......

(...........

$\begin{array}{ll}\text { CM-246 } & 3.800 E-06 \\ \text { NP-237 } & 9.885 E-03 \\ \text { PU-238 } & 1.580 E-06 \\ \text { PU-239 } & 2.738 E-04 \\ \text { PU-240 } & 6.700 E-06 \\ \text { PU-242 } & 6.500 E-07 \\ T H-230 & 2.400 E-05 \\ U-232 & 5.400 E-08 \\ U-233 & 3.400 E-03 \\ U-234 & 1.100 E-03\end{array}$

$2.282 E+00 \quad 2.282 E+00 \quad 8.644 E-04 \quad .000 E+00$

$4.7776+00$

$4.777 \mathrm{E}+00$

$1.502 E-02$

$4.789 E+00$

$4.789 E+00$

$.000 E+00$

(2)

$5.411 E+00$

$5.411 E+00$

$1.035 E-01$

4.517E-OS

$.000 E+00$
$1.666 E+00$

$1.666 \mathrm{E}+00$

$.000 E+00$

$.000 E+00$

PU- 242

4. $139 E+00$
$4.139 E+00$
AM- 241

PU-238

PU. 239

TH- 232

U-238

AM- 241

PU- 238

PU-239

$\mathrm{U}-234$

$\mathrm{U}-235$

$\mathrm{U}-236$

$\mathrm{U}-238$

$A M-241$

AM- 243

CM- 242

CM- 244

$\mathrm{CM}-245$

CM- 246

NP- 237

PU -238

Pu-239

PU- 240

PU-241

PU. 242

TH- 230

$\mathrm{TH}-232$

$\mathrm{U}-232$

U. 233

$\mathrm{U} \cdot 234$

$\mathrm{u}-235$

$\mathrm{u}-238$

$$
05
$$

AM- 241

AM- 243

CM- 244

NP -237

PU- 238

PU- 239

PU -240

PU- 241

1.071
$5.460 \mathrm{E}-05$

8. $430 E-11$

1.375E-05

$7.400 E-01$

1. $064 E+02$

$1.090 \mathrm{E}-01$

4. $580 \mathrm{E}-07$

2. $030 \mathrm{E}-09$

$1.050 \mathrm{E}-05$

$9.000 E-05$

$9.200 E-03$

$7.100 \mathrm{E}-04$

$9.900 \mathrm{E}-02$ $1.703 \mathrm{E}+02$

4. $638 \mathrm{E}-06$

3.539E-06

1.585E- 15

3. 100E-09

3. $746 \mathrm{E}-06$

2.116E-06

4. $903 E-03$

$7.936 E-07$

5.020E-04

$3.380 E-06$

4.392E-08

$1.450 E-06$

$9.300 \mathrm{E}-06$

$1.690 \mathrm{E}+02$

$2.937 \mathrm{E}-08$

$1.480 \mathrm{E}-03$

$1.055 \mathrm{E}-02$

$1.220 \mathrm{E}+00$

8.570E-02 $1.309 \mathrm{E}-02$

$6.230 E \cdot 05$

1.060 E-03

2.770E-09

$6.630 \mathrm{E}-03$

$1.049 \mathrm{E}-08$

$5.338 E-03$

1. $144 \mathrm{E}-06$

$6.031 \mathrm{E}-08$

$1.680 \mathrm{E}-09$ $.000 E+00$ 2. $201 \mathrm{E}-01$

AM-241 5.900E-08

PU-239 $3.280 E-06$

RA-226 $1.751 E=05$ 


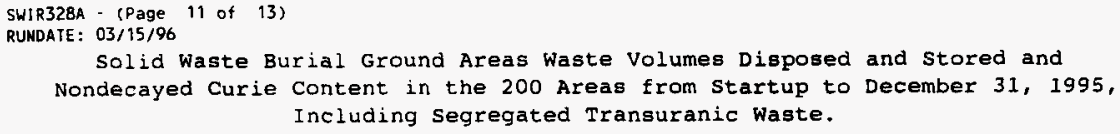

\section{BURIAL GROUMD FACILITIES}

fLAMmABLE STORAGE MODULE 10

fLAMmable STORAGE MODULE 12

FLAMMABLE STORAGE MODULE 13

FLAMmABLE StORAGE MODULE 14

fLAMMABLE STORAGE MODULE 15

\begin{tabular}{cccc}
$\begin{array}{c}\text { VOLUME } \\
\text { (CUBIC M) }\end{array}$ & $\begin{array}{c}\text { PACKED } \\
\text { VOLUME } \\
\text { (CUBIC M) }\end{array}$ & $\begin{array}{c}\text { URANIUM } \\
\text { (GRAMS) }\end{array}$ & $\begin{array}{c}\text { PLUTONIUM } \\
\text { (GRAMS) }\end{array}$ \\
\hline
\end{tabular}

$4.997 E+00$

$.000 E+00$

$3.546 E+00$

$3.546 E+00$

$1.310 E-03$

4.260E-OL $.000 E+00$

AM- 24

MP. 237

PU-238

PU-239

U-233

AM- 241

AM- 243

CM- 242

$\mathrm{CM}-245$

$\mathrm{CM}-246$

NP. 237

PU -238

PU- 239

PU-240

PU-241

PU. 242

$\mathrm{U}-232$

U-233

$\mathrm{u}-234$

U-238

$7.231 E+00 \quad 7.231 E+00 \quad 1.565 E-01 \quad 6.566 E-04$

AM-241

AM -243

CM- 242

$\mathrm{CM}-243$

$\mathrm{CM}-244$

$\mathrm{CM}-245$

$\mathrm{CM}-246$

NP -237

PU -238

PU- 239

PU- 240

PU-241

RA-226

$\mathrm{TH}-234$

$\mathrm{U}-232$

$\mathrm{U}-233$

U-234

$4.383 E+00 \quad 4.383 E+00 \quad 4.054 E-06 \quad .000 E+00$

AM- 241

AM- 243

CM- 244

CM-245

$C M-246$

PU -238

PU- 239

PU. 240

PU- 241

PU- 242

RA- 226

TH -230

$\mathrm{u}-232$

U- 233
RADIOACTIVITY AMS)

$2.630 E-07$

$9.338 \mathrm{E}-10$

1. 130E-07

2. 201E-01

2.383E-03

2.500 E-06

$6.390 \mathrm{E}-05$

1.070E-06

8.384E-04

$1.477 \mathrm{E}-03$

$1.839 E+00$

$1.465 E-06$

$4.650 \mathrm{E}-06$

3. $650 \mathrm{E}-15$

3. $300 E-08$

$1.880 \mathrm{E}-08$

5. $735 \mathrm{E}-02$

8. 500 E- 08

5. $051 E-05$

4.600E-07

$3.399 \mathrm{E}-08$

$5.600 \mathrm{E}-07$

2. $700 \mathrm{E}-10$

2.622E-05

$3.988 E-05$

$1.782 E+00$ $9.782 E-03$

$5.363 E-06$ 4. $000 \mathrm{E}-06$ $6.920 E-16$

$9.530 E-10$

6.070E-10

1. $100 \mathrm{E}-08$

$6.400 E-09$

8.668E-03

$1.388 E-04$

$4.730 E-05$

3. 860 E-06

1.029E-07

8.610E-07

2. $020 E-06$

$9.200 \mathrm{E}-11$

3.606E-04

$5.509 E-04$

$4.170 E+00$

7.937E-07

$2.974 \mathrm{E}-06$

2. $900 \mathrm{E}-09$

3. $486 \mathrm{E}-06$

$1.948 E-06$

$6.500 E-08$

$4.547 E-04$

2. $238 \mathrm{E}-05$

$8.983 E-07$

$6.889 \mathrm{E}-07$

$3.360 \mathrm{E}-08$

1. $200 \mathrm{E}-05$

$2.769 \mathrm{E}-08$

$1.000 E-03$ 


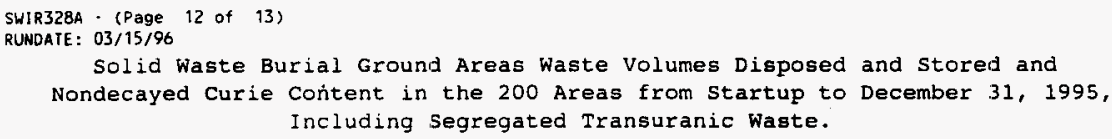

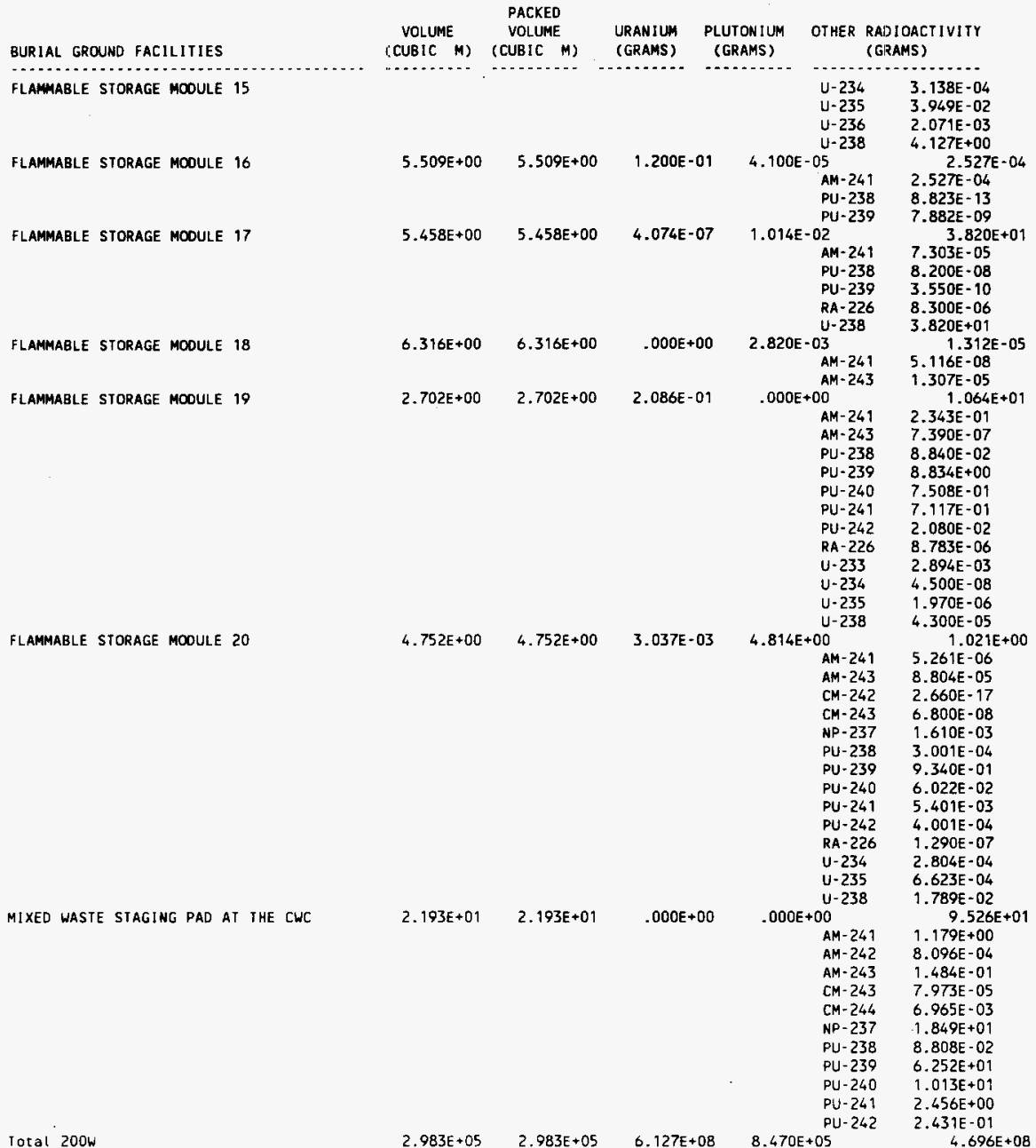




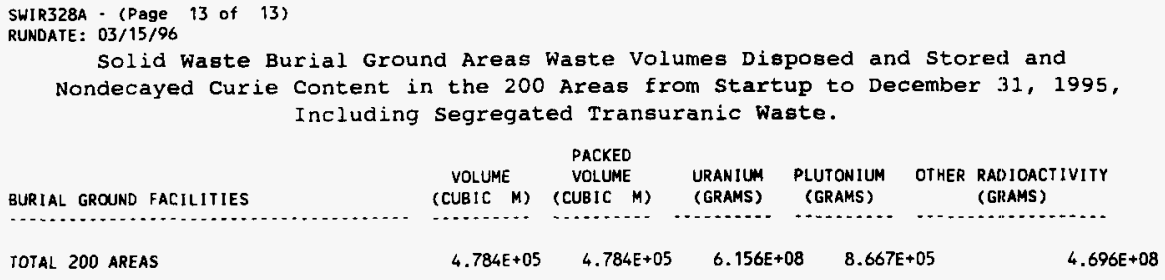

\begin{tabular}{|c|c|c|c|c|c|c|}
\hline CAISSON & - ALPHA 1 & $5.417 E+00$ & $5.417 E+00$ & $5.247 E+04$ & $\begin{aligned} & 1.378 E+ 03 \\
& \text { NP }-237 \\
& \text { TH }-232\end{aligned}$ & $\begin{array}{l}8.862 E+03 \\
1.100 E-02 \\
8.862 E+03\end{array}$ \\
\hline $\begin{array}{l}\text { CAISSON } \\
\text { CAISSON }\end{array}$ & $\begin{array}{l}\text { - ALPHA } 2 \\
\text { - ALPHA } 3\end{array}$ & $\begin{array}{l}6.933 E+00 \\
4.506 E+00\end{array}$ & $\begin{array}{l}6.933 E+00 \\
4.506 E+00\end{array}$ & $\begin{array}{l}3.997 E+03 \\
3.381 E+03\end{array}$ & $\begin{array}{l}1.157 \mathrm{E}+03 \\
1.197 \mathrm{E}+03\end{array}$ & $\begin{array}{r}.000 E+00 \\
6.300 E+00\end{array}$ \\
\hline & & & & & $\begin{array}{l}\text { TH-232 } \\
U-233\end{array}$ & $\begin{array}{l}2.700 \mathrm{E}+00 \\
3.600 \mathrm{E}+00\end{array}$ \\
\hline CAISSON & - ALPHA 4 & $6.491 E+00$ & $6.491 E+00$ & $2.750 E+04$ & $\begin{aligned} & 2.443 E+03 \\
& \text { AM- } 241 \\
& \text { LI }-6 \\
& \text { NP }-237 \\
& \text { TH }-232 \\
& \text { U- } 233\end{aligned}$ & $\begin{array}{l}\quad 9.054 E+03 \\
2.000 E-01 \\
6.940 E+00 \\
1.290 E+00 \\
9.006 E+03 \\
3.940 E+01\end{array}$ \\
\hline CAISSON & - NO. 1 & $6.637 E+00$ & $6.637 \mathrm{E}+00$ & $1.563 \mathrm{E}+05$ & $\begin{array}{r}5.421 E+02 \\
\text { AM-241 } \\
T H-232\end{array}$ & $\begin{array}{l}8.172 E+03 \\
4.000 E-01 \\
8.172 E+03\end{array}$ \\
\hline $\begin{array}{l}\text { CAISSON } \\
\text { CAISSON }\end{array}$ & $\begin{array}{l}\text { NO. } 2 \\
\text { - NO. } 3\end{array}$ & $\begin{array}{l}2.240 E-01 \\
5.677 E+00\end{array}$ & $\begin{array}{l}2.240 E-01 \\
5.677 E+00\end{array}$ & $\begin{array}{l}5.000 E+00 \\
7.727 E+04\end{array}$ & $\begin{array}{l}4.960 E-01 \\
3.791 E+02 \\
P U-238\end{array}$ & $\begin{array}{l}.000 E+00 \\
2.300 E-01 \\
2.300 E-01\end{array}$ \\
\hline CAISSON & - NO. 4 & $4.510 E+00$ & $4.510 E+00$ & $6.389 E+04$ & $\begin{array}{r}8.258 E+02 \\
T H-232\end{array}$ & $\begin{array}{l}5.806 E+04 \\
5.806 E+06\end{array}$ \\
\hline $\begin{array}{l}\text { CAISSON } \\
\text { CAISSON }\end{array}$ & $\begin{array}{l}\text { - NO. } 5 \\
\text { - NO. } 6\end{array}$ & $\begin{array}{l}4.703 E+00 \\
7.163 E+00\end{array}$ & $\begin{array}{l}4.703 E+00 \\
7.163 E+00\end{array}$ & $\begin{array}{r}5.000 E-02 \\
.000 E+00\end{array}$ & $\begin{array}{r}1.000 E-03 \\
.000 E+00\end{array}$ & $\begin{array}{l}.000 E+00 \\
.000 E+00\end{array}$ \\
\hline $\begin{array}{l}\text { CAISSON - } \\
\text { TRENCHES }\end{array}$ & - UNI NO. 1 & $\begin{array}{l}1.501 E+00 \\
1.041 E+04\end{array}$ & $\begin{array}{l}1.501 E+00 \\
1.041 E+04\end{array}$ & $\begin{array}{r}.000 E+00 \\
5.509 E+06\end{array}$ & $\begin{array}{l}.000 E+00 \\
5.841 E+04 \\
\text { AM- }-241 \\
\text { LI -6 } \\
\text { NP- } 237 \\
\text { PU- } 238 \\
T H-232 \\
U-233\end{array}$ & $\begin{aligned} & .000 E+00 \\
& 5.942 E+05 \\
& 6.100 E-01 \\
& 2.000 E+03 \\
& 2.127 E+02 \\
& 1.202 E+00 \\
& 5.844 E+05 \\
& 7.564 E+03\end{aligned}$ \\
\hline
\end{tabular}


SW1R328B - (Page 1 of 26)

RUNDATE: 03/15/96

Solid Waste Burial Ground Areas Waste Volumes Disposed and Stored and

Nondecayed Curie Content in the 200 Areas from startup to December 31, 1995, Including Segregated Transuranic Waste.

\begin{tabular}{llllll} 
BURIAL GROUND FACILITIES & $\begin{array}{c}\text { TOTAL } \\
\text { (CURIES) }\end{array}$ & $\begin{array}{c}\text { SR }-90 \\
\text { (CURIES) }\end{array}$ & $\begin{array}{c}\text { RU-106 } \\
\text { (CURIES) }\end{array}$ & $\begin{array}{c}\text { CS-137 } \\
\text { (CURIES) }\end{array}$ & $\begin{array}{c}\text { OTHER RADIOACTIVITY } \\
\text { (CIJRIES) }\end{array}$ \\
\hdashline
\end{tabular}

\section{OOE AREA}

BURIAL GROUND 218E 1

BURIAL GROUND 218E10

\section{$1.000 E+02$}

$4.698 \mathrm{E}+06$

$1.989 E+00$

$1.002 E+06$

4. $283 \mathrm{E}+00$

$1.772 \mathrm{E}+04$

2. $124 \mathrm{E}+00$

1. $180 \mathrm{E}+06$

CO- 60

60

$\begin{array}{lr}B A 137 & 4.423 E+0 ? \\ C-14 & 4.560 E+01\end{array}$

$\begin{array}{ll}\mathrm{C}-14 & 4.560 \mathrm{E}+01 \\ \mathrm{C} 0-58 & 4.560 \mathrm{E}+01\end{array}$

C0-60 $3.055 E+03$

CS137 4.696E+0?

Eu152 4.560E+01

EU154 $3.876 E+02$

IU155 $3.876 E+02$

FE-59 1.824E+02

$\mathrm{HN}-54 \quad 3.192 \mathrm{H}+02$

$\begin{array}{rrrrr}8.900 E+02 & 1.770 E+01 & 3.812 E+01 & 1.890 E+01 & .000 E+00 \\ 2.812 E+06 & 3.073 E+04 & 7.838 E+02 & 3.102 E+04 & 2.676 E+06\end{array}$

BURIAL GROUND 218E12A

$2.812 E+06 \quad 3.073 E+04$
$\mathrm{BE}-10$

$\mathrm{BE}-7$

C- 14

CE- 141

CE -144

CL. 36

CO-58

Co- 60

CR-51

CS- 134

FE -55

FE. 59

$\mathrm{H}-3$

HF- 181

I -129

K -40

MN -56

MO- 93

NB - $93 \mathrm{M}$

NB- 94

NB -95

NI -59

NI -63

PN- 147

SB-125

SE -79

SM- 151

SN- 119

TA- 182

TC. 99

TE- 125

ZN- 65

2R-93

ZR-95
$1.278 E-06$

$1.300 E-09$

1. $253 \mathrm{E}+02$

4.010E-01

3.477E+01

5.556E-03

$1.323 E+04$

$1.043 E+06$

3. $614 E+02$

1. $062 \mathrm{E}-01$

$7.322 \mathrm{E}+05$

$7.144 E+01$

$1.124 E+03$

$2.320 E+00$

2.274E-02

$9.054 \mathrm{E}-03$

1. $117 E+04$

$6.900 \mathrm{E}-02$

$5.611 E-01$

$1.282 E+01$

$1.076 E+01$

$5.119 E+03$

$8.699 E+05$

$3.004 E+00$

$1.264 E+01$

$2.309 \mathrm{E}-05$

$4.241 \mathrm{E}-01$

3. $020 \mathrm{E}-06$

1. $000 \mathrm{E}-03$

8. $076 \mathrm{E}-01$

$1.314 E+01$

3.316E-01

$5.613 \mathrm{E}-01$

$3.940 E+00$

BA137 $1.166 \mathrm{E}-02$

C- $14 \quad 1.202 E-02$

CO-58 1.202E-02

CO-60 $\quad 8.054 \mathrm{E}-01$

CS137 $1.238 \mathrm{E}-02$

EU152 $1.202 E-02$

EU154 1.022E-01

EU155 1.022E-01

FE-59 4.808E-02

MN-54 8.415E-02 
SWIR328B - (Page 2 of 26)

RUNDATE: $03 / 15 / 96$

Solid Waste Burial Ground Areas Waste Volumes Disposed and Stored and

Nondecayed Curie Content in the 200 Areas from startup to December $31,1995$. Including segregated Transuranic waste.

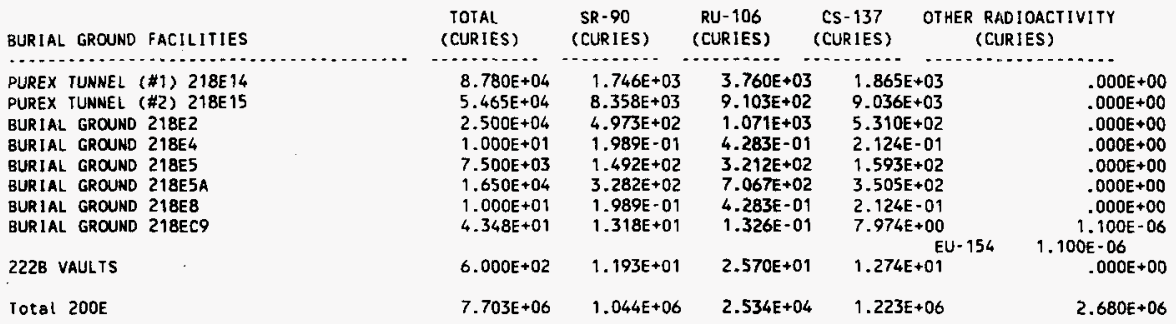


SWIR328B - (Page 3 of 26 )

RUNDATE: 03/15/96

Solid Waste Burial Ground Areas Waste Volumes Disposed and stored and Nondecayed Curie Content in the 200 Areas from startup to December 31, 1995, Including Segregated Transuranic Waste.

BURIAL GROUND FACILITIES

2OOW AREA

TOOH AREA

212N BUILDING

214 T FACILITY/TPLANT

BURIAL GROUND 21BW1

BUR IAL GROUND 218W11

BURIAL GROUND 218W1A

BUR IAL GROUMD 218W2

BURIAL GROUND 218W2A

BURIAL GROUND $218 \mathrm{BH}$

BUR IAL GROUND 218W3A \begin{tabular}{cccc}
$\begin{array}{c}\text { TOTAL } \\
\text { (CURIES) }\end{array}$ & $\begin{array}{c}\text { SR-90 } \\
\text { (CURIES) }\end{array}$ & $\begin{array}{c}\text { RU-106 } \\
\text { (CURIES) }\end{array}$ & $\begin{array}{c}\text { CS-137 } \\
\text { (CURIES) }\end{array}$ \\
\hline
\end{tabular}

OTHER RADIOACTIVITY (CURIES)

(CURIES)

$.000 E+00$

$.000 E+00 \quad .000 E+00$

$2.614 E-03$

$8.027 E-07$

$1.729 \mathrm{E}-06$

$.000 E+00$

8.574E-07

C. 14

EU-152;

$\mathrm{H}-3$

NA-22

$4.248 \mathrm{E}+00$

8. $566 E+00$

$3.978 \mathrm{E}+00$

4. $283 \mathrm{E}-03$

$1.000 E-01$

$1.989 \mathrm{E}-03$

$4.800 \mathrm{E}+04$

$9.547 \mathrm{E}+02$

$5.000 E+02$

$9.945 E+00$

$2.056 \mathrm{E}+03$

$2.142 \mathrm{E}+01$

5. $179 E+03$

$1.065 \mathrm{E}+04$

$2.124 \mathrm{E}-03$

$1.020 E+03$

$1.062 E+01$

$5.537 \mathrm{E}+03$

$9.000 E+02$

1.790E+01

3. $855 \mathrm{E}+01$

$1.912 \mathrm{E}+01$

Co- 60

$1.954 E+06 \quad 1.319 E+05$
3. $581 \mathrm{E}+05$

\section{AG-108}

$A G-110$

$A G-110$

$B A-133$

BE-10

BE-7

BI $-20 \%$

B! -214 ,

C. 14

CA- 45

CD-109)

CD-113

CE-14!

CE-144,

$\mathrm{CL}-36$

CO. 56

CO-57

CO- 58

CO- 60

CR- 51

CS- 134

CS- 13;

EU-15?

EU- 15:

EU-15!

FE -55

FE- 59

GD - 15?

GO- $15 i$

GE -68

H-3

$\mathrm{I} \cdot 125$

$1-129$

1- 131

K-40

KR- 85

MN -54

NA -22

NB $-93 / 4$

NB- 94

N] -59

N] -60

N] -63

P. 32

PN- 147
$.000 E+00$

2.573E-03

9.000E-06

1.000E-O?

2.563E-03

$1.000 E-06$

$.000 \mathrm{E}+00$ $.000 E+00$ $.000 E+00$ $.000 E+00$

$3.400 E+00$

3. $400 E+00$

$.000 \mathrm{E}+00$ $6.083 \mathrm{E}+05$

9. 100E-06

$3.069 E+00$

3. $400 \mathrm{E}-06$

$1.585 E-04$

$6.920 E-10$

$1.748 E-01$

2. $696 \mathrm{E}-03$

$4.400 \mathrm{E}-07$

$1.741 E+00$

$1.000 E-04$

$5.098 \mathrm{E}-04$

3. $61 \mathrm{tE}-05$

$1.746 E+02$

$5.747 \mathrm{E}+04$

2.003E-04

1. 500E-06

2.826E-03

2. $532 \mathrm{E}+01$

$4.980 E+04$

9.964E-03

$1.727 E+04$

1.102E-06

$1.597 \mathrm{E}+00$

5.950E-01

$3.811 \mathrm{E}+01$

1. $706 \mathrm{E}+00$

3. 32\%E-01

$3.390 \mathrm{E}-03$

2.310E-03

2.399E-02

$4.376 \mathrm{E}+05$

$1.510 E-02$

$1.439 E-02$

$1.371 E-03$

$1.245 \mathrm{E}-04$

3. $731 E+03$

4. $357 \mathrm{E}+00$

$6.581 E+00$

7.430E-05

2. $128 E-04$

8. $783 E-04$

2.979E-01

$3.888 E+00$

4.710E-02

$4.024 E+04$ 
WHC-EP-0125-8

SHIR328B - (Page 4 of 26)

RUNDATE : $03 / 15 / 96$

Solid Waste Burial Ground Areas Waste Volumes Disposed and Stored ano

Nondecayed Curie Content in the 200 Areas from startup to December 31 , 1995 , Including Segregated Transuranic Waste.

BURIAL GROUND FACILITIES

$\begin{array}{llll}\text { TOTAL SR } 90 \text { RU-106 } & \text { CS-137 OTHER RADIOACTIVITY }\end{array}$ BURIAL GROUND 21BU3A

(CURIES) (CURIES) (CURIES) (CURIES)
(CIJRIES)

BUR IAL GROUND 218W3AE

$3.997 E+05 \quad 2.444 E+04 \quad 1.264 E+03$

$3.614 E+06$

AC -227

$A C-228$

$A G-108$

AG- 110

AU-195;

$B A-133$

$B E-10$

$B E-7$

BI -207

BI-21?

B] $-21 \%$

C -14

CA -45

CD- 109

CD -113

CE -141

CE- 144

$\mathrm{CL}-36$

Co-56

$\mathrm{CO}-57$

Co- 58

CO-60

CR -51
$1.240 E-05$

5.017E-03

2.000E-04

4.235E-02

$3.000 E-07$

$1.493 \mathrm{E}+03$

$3.993 E+00$

$1.200 E-04$

8.600 -05

$1.116 \mathrm{E}-04$

$1.500 E-05$

2.000E-04

$9.980 \mathrm{E}-04$

$2.867 \mathrm{E}-07$

1.034E-03

2.883E-03

$1.265 \mathrm{E}-03$

2.544E- 01

$3.394 E+02$

$5.000 E-10$

2.000E-04

4. $000 E-08$

$1.400 \mathrm{E}+00$

9.525E-04

$3.982 E+01$

$1.828 \mathrm{E}-05$

2.700E-06

BA $137 \quad 2.807 E+02$

$\mathrm{C}-14 \quad 2.894 \mathrm{E}+02$

CO-58 $2.894 \mathrm{E}+02$

CD- $60 \quad 1.939 E+04$

C5137 2.981E+02

EU152 2.894E+02

EU154 $2.460 E+03$

EU155 $2.460 \mathrm{E}+03$

$\mathrm{FE}-59 \quad 1.158 \mathrm{E}+03$

MN-54 2.026E+03 $2.625 E+05$

$1.000 E-06$

8.075E-04

2.700E-05

$9.850 \mathrm{E}-06$

$9.250 \mathrm{E}-05$

$7.687 \mathrm{E}-06$

$4.600 \mathrm{E}-11$

$1.101 \mathrm{E}-03$

$1.000 \mathrm{E}-03$

$2.975 E-04$

5.697E-05

$3.018 E+01$

3. $121 \mathrm{E}-03$

$6.595 \mathrm{E}-05$

1.260 E-05

$1.000 \mathrm{E}-06$

$9.860 \mathrm{E}-02$

$3.730 \mathrm{E}-08$

$9.630 \mathrm{E}-05$

$3.815 E-03$

$3.916 E+02$

$8.933 E+04$

8.604E-03 
SWIR328B - (Page 5 of 26)

RUNDATE : 03/15/96

Solid Waste Burial Ground Areas Waste Volumes Disposed and Stored and Nondecayed Curie content in the 200 Areas from Startup to December 31, 1995 , Including Segregated Transuranic waste.

BURIAL GROUND FACILITIES

BURIAL GROUND 218U3AE
TOTAL SR-90 RU-106 CS-137 OTHER RADIOACTIVITY $\begin{array}{llll}\text { (CURIES) } & \text { (CURIES) } & \text { (CURIES) (CURIES) } \\ \end{array}$ (CIJRIES)

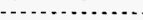

CS-134 $1.862 \mathrm{E}+02$

CS- $135 \quad 3.800 E-07$

E5-254 6.300E-09

EU-152 1.316E+03

EU-153 1.000E-09

EU-154 2.033E+03

EU-155 4.861E+02

FE-55 2.456E+04

FE-59 1.4B9E +02

GE- $68 \quad 1.121 \mathrm{E}-03$

$\mathrm{H}-3 \quad 4.352 \mathrm{E}+04$

$H F-175 \quad 1.000 E-03$

HF- $181 \quad 1.067 \mathrm{~V}-02$

HG-203 1.009E-06

$\mathrm{I}-125 \quad 3.351 \mathrm{E}-02$

I-129 2.346E-06

I- $131 \quad 2.080 E-06$

K-40 $\quad 5.500 E-04$

KR-85 1.302E+01

$M N-54 \quad 8.657 \mathrm{E}+03$

MO-93 5.600E-02

NA-22 9.226E-01

NB-93M 1.326E=03

NB-94 $1.064 E+00$

NB-95 2.090E+03

NI-59 $5.200 E+02$

NI $-60 \quad 1.798 E+03$

$\mathrm{N} 1.63 \quad 6.248 \mathrm{E}+04$

$\mathrm{P}-32 \quad 1.000 \mathrm{E}-01$

$P A-231 \quad 1.729 E-06$

$P B-210 \quad 1.000 E-07$

PB-212 2.951E-04

PB-214 3.661E-05

PM- $147 \quad 1.030 E+00$

PO-210 7.201E-03

RA-228 7.674E-04

RU- $103 \quad 1.000 E-06$

S-35 $\quad 1.605 E-02$

SB-125 $\quad 9.011 E+03$

SB-126 7.200E-08

SC-46 8.071E-03

SE-75 7.400E-07

SE-79 4.819E-05

SM-151 3.514E-04

SN-113 2.000E-06

$\mathrm{SN}-119 \quad 1.280 \mathrm{E}+04$

$S N-121 \quad 1.600 E+01$

$S N-123 \quad 1.080 E+02$

$S N-126 \quad 2.715 E-04$

SR-85 $\quad 1.674 \mathrm{E}-03$

TA-182 1.261E+02

TB-162 1.050E-06

TC. $99 \quad 6.508 E-02$

TE-12S $1.920 \mathrm{E}+03$

$\mathrm{TL}-208 \quad 1.207 \mathrm{E}-06$

IM- $170 \quad 2.001 \mathrm{E}-06$

$V-49 \quad 3.831 E-09$

$Y-88 \quad 4.000 E-06$

$Z N-65 \quad 6.731 E-02$

$2 R \cdot 93 \quad 2.000 E+00$

2R-95 $9.774 E+02$ 
SWIR328B - (Page 6 of 26)

RUNDATE : $03 / 15 / 96$

Solid Waste Burial Ground Areas waste Volumes Disposed and stored and

Nondecayed Curie content in the 200 Areas from Startup to December j1, 1995 , Including Segregated Transuranic Waste.

BURIAL GROUND FACILITIES

TOTAL SR-90 RU-106 CS-137 OTHER RADIOACTIVITY

BURIAL GROUND 218WBAE

(CURIES)

(CURIES) (CURIES) (CURIES) (CLIR\ES)

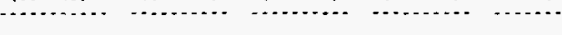

BURIAL GROUND 218W4A

BURIAL GROUND $218 W 49$
$3.815 E+03$

$5.070 E+05$
$6.594 E+01$

$2.238 \mathrm{E}+04$
$1.420 E+02$

$7.450 E+03$

BURIAL GROUND $218 W 4 \mathrm{C}$

$1.865 E+06 \quad 1.015 E+05 \quad 1.165 E+04 \quad 1.544 E+05$

$A C-228$

$A G-110$

$A G-110$

$B A-133$

$B E-10$

$\mathrm{BE}-7$

BI-207

B1-21?

B1-2114

C- 14

CA -45

CD -1017

CD -113

CE-144

$\mathrm{CL}-36$

CO- 56

$\mathrm{CO}-57$

Co- 58

CO- 60

CR- 51

CS- 134

CS -135

EU- 152

EU- 154
EIA 137

$1.092 \mathrm{E}+01$

C- $14 \quad 1.126 \mathrm{E}+01$

Co-58 $\quad 1.126 \mathrm{E}+01$

CO-60 $7.544 E+02$

Cs137 1.160E+01

EU152 1.126E+01

EU154 9.570E+01

EU155 9.570E+01

$\mathrm{FE}-59 \quad 4.504 \mathrm{E}+01$

MN-54 7.882E+01 $5.000 E+02$ 5. $000 E+02$

2. $617 \mathrm{E}+05$

$8.420 E+01$

$2.094 E+04$

$1.000 \mathrm{E}-01$

3. $000 E-01$

2.200E-01

$2.046 \mathrm{E}+00$

$2.311 E+05$

$5.000 E-01$

$3.623 E+00$

$9.586 E+03$

$7.000 \mathrm{E}+00$

$2.000 E-02$

$3.000 E+01$

BA $137 \quad 1.109 E+01$

C- $14 \quad 1.144 E+01$

CO-58 1.144E+01

CO-60 $7.663 E+02$

CS137 1.178E+01

EU152 1.144E+01

EU154 $9.721 \mathrm{E}+01$

EU155 $9.721 E+01$

FE-59 4.575E+01

MN-56 8.006E+0

2.643E-03

$1.000 E-06$

$2.700 E-06$

$5.016 E-04$

5.700E-11

3. 804E-03

2. $117 E-04$

3. $030 \mathrm{E}-03$

3. 303E-04

8. $600 E+00$

3. 200E-07

2.084E-04

$1.570 \mathrm{E}-05$

$1.284 E+03$

$1.007 E-05$

$2.800 \mathrm{E}-05$

4. $519 E-05$

$7.766 \mathrm{E}+03$

$1.028 E+06$

$9.381 E+03$

8. $730 \mathrm{E}+02$

4. $800 \mathrm{E}-07$

2. $094 \mathrm{E}+01$

$3.258 \mathrm{E}+02$ 
SWIR328B - (Page 7 of 26)

RUNDATE: $03 / 15 / 96$

Solid waste Burial Ground Areas waste Volumes Disposed and stored and Nondecayed Curie Content in the 200 Areas from startup to December 31, 1995 , Including segregated Transuranic Waste.

BURIAL GROUND FACILITIES

BURIAL GROUND $218 W 4 \mathrm{C}$ $\begin{array}{llcc}\text { TOTAL } & \text { SR-90 } & \text { RU-106 } & \text { CS-137 } \\ \text { (CURIES) } & \text { (CURIES) } & \text { (CURIES) } & \text { (CURIES) }\end{array}$

(CURIES)

(CURIES)

OTHER F:ADIOACTIVITY (CUR IES)

(C) - . . . . . .

EU-155. 2.491E-0

FE-55 $1.141 E+04$

FE-59 $1.697 E+04$

GA-68 1.600E-12

GE $-68 \quad 1.600 E-12$

$\mathrm{H}-3 \quad 2.575 \mathrm{E}+04$

I-129 1.009E-03

$1-131 \quad 1.000 E-08$

$K=40 \quad 1.885 E-04$

$\mathrm{KR}-85 \quad 2.484 \mathrm{E}+01$

LA-140 1.200 E - 06

$M N-54 \quad 7.679 E+03$

MO-93 $1.256 \mathrm{E}-03$

NA-22 3.917E-03

NB- 93 M $\quad 3.290 \mathrm{E}-05$

NB-94 3.489E-01

NB-95 6.942E-05

N1-59 7.623E+01

N] $-60 \quad 8.433 E-02$

$N 1.63 \quad 9.838 E+03$

P-32 2.016E-02

PA-231 4.450E-03

PB-210 1.103E-06

PB-212 4.240E-03

PB-214. 3.416E-04

PM-14i 2.203E-01

PO-210 1.122E-04

RA-228 4.000E-06

RU-103 2.004E-06

S-35 2.649E-03

SB-125; 1.114E+00

SB-126 $9.000 E-08$

SC-46 5.160E-03

SE-75 5.000E-08

SE-79 2.036E-06

SM-15 $\quad 4.300 E-04$

SN-113 5.000E-07

SN-12। 1.250E-07

SN-126 $\quad 6.400 \mathrm{E}-07$

SR-85 2.445E-04

TA-18? $\quad 8.353 E-01$

TA-183 9.000E-01

TC-99 6.173E-02

TE-125 $5.200 E-04$

$\mathrm{TL}=2013 \quad 4.326 \mathrm{E}-01$

$V-49 \quad 1.700 E \cdot 01$

W-185 2.600E-09

$\mathrm{H}-88$
$\mathrm{H}$

$2 N-65 \quad 1.992 E-04$

$2 R-93 \quad 8.256 E-06$

ZR-95 4.503E-05

$\begin{array}{ll}\text { BA } 137 & 2.616 E+00\end{array}$

$C=14 \quad 2.697 \mathrm{E}+00$

CO-58 2.697E+00

CO-60 $1.807 \mathrm{E}+02$

CS137 2.778E+00

EU152 $2.697 \mathrm{E}+00$

EU154 2.292E+0

EU155 2.292E+01

FE-59 1.079E+01

MN-54 1.888E+01 
SHIR3288 - (Page 8 of 26)

RUNOATE: $03 / 15 / 96$

Solid Waste Burial Ground Areas Waste Volumes Disposed and storecl and

Nondecayed Curie Content in the 200 Areas from startup to December 31 , 1995 , Including Segregated Transuranic waste.

BURIAL GROUND FACILITIES

BURIAL GROUND $278 W 5$ \begin{tabular}{lcc}
$\begin{array}{c}\text { TOTAL } \\
\text { (CURIES) }\end{array}$ & $\begin{array}{c}\text { \$R } 90 \\
\text { (CURIES) }\end{array}$ & $\begin{array}{c}\text { RU-106 } \\
\text { (CURIES) }\end{array}$ \\
\hline
\end{tabular}

(........

3. $162 \mathrm{E}+04$
2. $113 \mathrm{E}+03$

$5.954 \mathrm{E}+01$

CS-137 (CURIES)
3. $008 \mathrm{E}+0$

OTHER RADIOACTIVITY (CURIES)

....--..............

AC -227

AC -228

AG- 108

$A G=110$

$A G-110$

AL -28

$A U-195$

$\mathrm{BA}-133$

$B A-134$

$B A-137$

$B A-140$

BE -7

B I- 207

BI-212

B1- 214

C. 14

CA- 45

CD- 109

CE -141

CE - 144

CL -36

CO-56

co. 57

co- 58

co- 59

Co- 60

CR-51

CS- 133

CS- 134

CU-65M

ES- 254

EU-152

EU- 154

EU-155

FE - 55

FE- 59

GA -68

GD -152

GD -153

GD -154

GE -68

$\mathrm{H}-3$

HF- 175

HF- 181

$\mathrm{HG}-203$.

I -123

I -125

I - 129

I. 131

$k-40$

KR- 85

MN- 54

MO- 93

NA- 22

NB. 91

NB-93M

NB. 94

NB- 95

NB-95M

NO- 14 ?

$2.058 \mathrm{E}+04$

8.436E-05

$1.470 E-04$

$2.843 \mathrm{E}-03$

$2.418 E-04$

5. 514E-04

3.400E-05

$4.780 E-05$

$6.342 E-02$

1.330E-07

2. 090 E- 07

2.800E-06

1. $266 \mathrm{E}+00$

1. $787 \mathrm{E}-03$

?. 035E-03

$6.497 E-04$

$4.442 E+00$

1.693E-02

$1.537 \mathrm{E}-02$

1. 207E-03

6. $758 \mathrm{E}+00$

1. $034 E-03$

5.265E-01

5.659E-01

$6.379 E-01$

4. $000 E-06$

$4.592 E+03$

$4.482 E+00$

$2.800 \mathrm{E}-03$

$1.719 E+00$

7. $020 E-03$

1. $152 E-04$

1. $743 E+00$

1. $273 E+02$

$1.264 \mathrm{E}+01$

2. $126 \mathrm{E}+01$

$3.711 E+00$

$6.209 E-02$

8. $468 E-02$

5.877E-04

4. $420 \mathrm{E}-02$

6. $186 \mathrm{E}-01$

1. $576 E+04$

1. $002 E-06$

3. $360 E-06$

2. 172E-04

5. $610 \mathrm{E}-04$

1.560E-01

3. $266 \mathrm{E}-02$

1. 284E-02

$7.762 E-03$

$9.945 E-02$

$5.821 E+00$

1. $292 \mathrm{E}+00$

1. $350 E+01$

2. $902 E-02$

$9.002 E-05$

$1.443 E-03$

1. $041 E-01$

2. $049 E-07$

2. $406 \mathrm{E}-06$ 
SWIR328B - (Page 9 of 26)

RUNDATE : 03/15/96

Solid Waste Burial Ground Areas Waste Volumes Disposed and stored and Nondecayed Curie Content in the 200 Areas from startup to December 31, 1995, Including Segregated Transuranic Waste.

BURIAL GROUND FACILITIES

BURIAL GROUND 218W5 $\begin{array}{llcc}\text { TOIAL } & \text { SR-90 } & \text { RU-106 } & \text { CS-137 }\end{array}$ (CURIES) - (CURIES) (CURIES) (CURIES)
OTHER RADIOACTIVITY (CURIES)

NI.................

NI $-60 \quad 3.229 E+00$

N1 $-63 \quad 4.068 E+00$

P-32 4.192E-01

$P A-231 \quad 7.735 E-01$

PB- 208 9.203E-08

PB-210 3.148E-02

PB-212 $3.335 E-03$

$\mathrm{PB}-214, \quad 2.402 \mathrm{E}-04$

$P M-147 \quad 3.601 E+00$

PO-210 4.011E-03

PT - 19: $\quad 9.000 E-10$

RA-228 $1.275 E-03$

RB-85N 3.000E-06

RB-86 6.559E-03

RE-18\% 3.000E-06

RU-103 $1.428 \mathrm{E}-01$

S-35 $1.662 E-01$

$S B-124 \quad 1.848 E-04$

SB-125i 2.128E-01

SB-126 $2.500 E-04$

SC-46 $1.537 \mathrm{E}-02$

SE-75 3.527E-03

SE - $79 \quad 4.978 E-03$

SI-31 $1.000 E-04$

SM-15 $1 \quad 8.013 E-01$

$\mathrm{SN}-113 ; \quad 1.886 \mathrm{E}-03$

SN-121 3.300E-07

SN-123 2.000E-05

SN-126, 1.930E-08

SR-82 $4.325 E-04$

SR-85 7.862E-02

SR-89 5.000E-04

TA-182. 2.513E-01

TC- $99 \quad 1.248 \mathrm{E}-01$

TE-121 1.000E-04

IE-125; 4.084E-04

IE-12? 2.500E-04

TE-120 $1.000 E-07$

$\mathrm{TL}-204 \quad 8.500 \mathrm{E}-04$

$T L-2013 \quad 2.116 E-03$

IM-170 $\quad 6.900 E-07$

V-49 2.675E-05

$W=18314 \quad 5.770 E-06$

W.185 2.120E-05

$X E-131 \quad 4.100 E-07$

$Y-87 \quad 3.530 E-03$

$Y-88 \quad 8.872 E-05$

$Y-90 \quad 1.706 E-01$

$2 \mathrm{~N}-65 \quad 2.025 \mathrm{E}+00$

2R-95 1.127E-02

BA $137 \quad 1.065 E+00$

$\mathrm{C}-14 \quad 1.098 \mathrm{E}+00$

Co.58 $\quad 1.098 E+00$

CO.60 $\quad 7.355 E+01$

CS137 1.131E+00

EU152 $1.098 E+00$

EU154 $9.331 \mathrm{E}+00$

EU155 $9.331 E+00$

FE-59 $\quad 4.391 \mathrm{E}+00$

MN-54 $7.685 E+00$ 
SU1R328B - (Page 10 of 26)

RUNDATE : 03/15/96

Solid Waste Burial Ground Areas Waste Volumes Disposed and stored and

Nondecayed Curie Content in the 200 Areas from startup to December 31,1995 , Including Segregated Transuranic Waste.

BURIAL GROUND FACILITIES

BURIAL GROUND $218 \mathrm{H}$

$221 T$ I PLANT
TOTAL SR-90 RU-106 CS-137 OTHER RADIOACTIVITY

(CURIES) (CURIES) (CURIES) (CURIES) (CURIES)

\section{$1.989 E-03$}

$1.028 E-09$
$4.283 E-03$

$1,661 E-13$

6

2.124E-03 $6.888 E-10$

CO- 60

EU- 154

EU- 155

FE- 59

MN -54

N1 -60

N1 -63

$8.016 E+01$

$1.532 \mathrm{E}+01$

$1.726 \mathrm{E}+02$

$3.298 E+01$

5.021E-01

8. $560 E+01$

$1.635 E+01$

$1.530 E+01$
2225 VAULTS

222T VAULTS

IRANSURANIC WASTE SIORAGE \& ASSAY

FACILITY(TRUSAF)

\section{$4.030 E+03$ \\ $7.700 E+02$ \\ $1.889 \mathrm{E}+02$}

$7.536 \mathrm{E}+01$

B1-214

\section{C- 14} CE- 144 Co- 60 c5- 134

\section{EU- 152}

EU- 154

EU-155

$$
H-3
$$

\section{$\mathrm{K}=40$}

MN-54

P- 32

s-35

SB-12:

TE- 125

ZR-95
BA $137 \quad 5.539 E-05$
C-14 5.710E-05
CO-58 $5.710 E-05$
Co-60 3.826E-03
$\operatorname{CS} 137 \quad 5.881 E-05$
EU152 5.710E-05
EU154 4.854E-04
EU155 4.854E-04
FE-59 2.284E-04
MN-54 3.997E-04

CENTRAL WASTE COMPLEX $2401 \mathrm{~W}$ BUILOING

$2.0496+05$

$1.025 E+05$

$1.013 E-04$

$1.696 \mathrm{E}-03$

Co- 60

EU- 154

K. 40

3. $000 E-04$

$4.000 \mathrm{E}-05$

$2.450 E-07$

BA 137 3. $143 \mathrm{E}-08$

C- $14 \quad 3.240 E-08$

CO-58 3.240E-08

C0-60 2.171E-06

CS137 $3.337 E-08$

EU152 3.240E -08

EU154 2.754E-07

EU155 2.754E-07

FE-59 1.296E-07

MN-54 2.268E-07

CENTRAL WASTE COMPLEX $2402 W$ BUILDING

\section{$7.553 E-02 \quad 1.992 E-03$}

9.472E-02

C- 14

CE- 144

CO- 60

CS. 134

EU-152

EU-154

$\mathrm{H}-3$

PM- 147
$1.909 \mathrm{E}-01$

1. $410 \mathrm{E}-12$

6. $000 E-03$

7. $460 E-07$

$5.520 E-06$

3.641E-03

1. $072 \mathrm{E}-03$

$5.650 \mathrm{E}-06$

2. $800 \mathrm{E}-03$ 
SWIR3288 - (Pege 11 of 26)

RUNDATE: $03 / 15 / 96$

Solid Waste Burial Ground Areas Waste Volumes Disposed and stored and Nondecayed Curie Content in the 200 Areas from Startup to December 31, 1995, Including segregated Transuranic Waste.

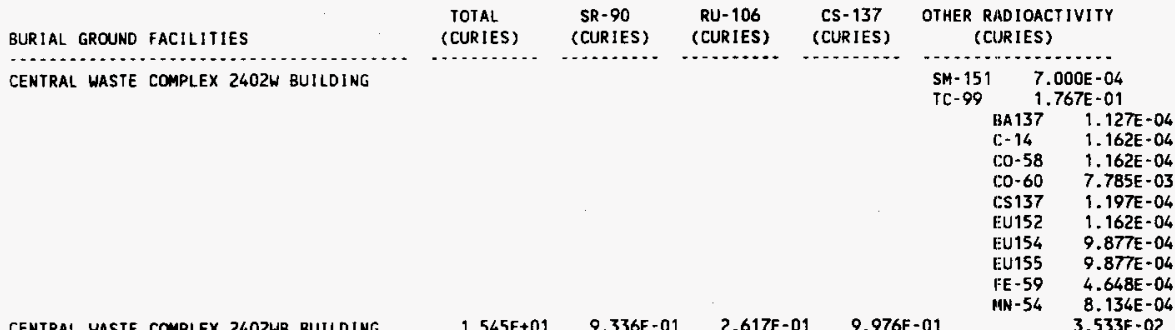

CENTRAL WASTE COMPLEX 2402WB BUILDING

CENTRAL WASTE COMPLEX 2402WC BUILDING

\section{$1.545 E+01$}

$9.336 E-01$
AC -228

$\mathrm{BA}-133$

BI -212

BI -214

C- 14

CE- 144

CO- 60

CS -134

EU- 152

EU- 154

EU- 155

FE- 59

$\mathrm{H}-3$

MN -54

NA- 22

NB-95M

NI-60

PA-231

PB-212

PB- 214

PM- 147

RA- 228

$S M-151$

TC- 99

TL -208

ZR-95

1.755E-08

2.300E- 06

$3.435 E-11$

$1.494 \mathrm{E}-13$

$1.102 E-05$

$1.820 \mathrm{E}-02$

$5.058 \mathrm{E}-03$

$4.455 \mathrm{E}-08$

$4.453 E-05$

8.449E-05

3. $046 \mathrm{E}-05$

4. $200 \mathrm{E}-05$

1. 808E-05

$6.500 E-05$

2. $699 \mathrm{E}-05$

8. $120 E-10$

2. $770 \mathrm{E}-05$

4. $010 E-16$

1.374E-05

8. $110 E-06$

$8.788 \mathrm{E}-03$

$7.497 \mathrm{E}-04$

2.100E-03

$1.524 \mathrm{E}-06$

$4.623 \mathrm{E}-05$

7.626E-06 BA $137^{7}$ 3.159E-07

C-14 3.256E-07 C0.58 3.256E-07

CO-60 2.182E-0S

CS137 3.354E-07

EU152 3.256E-07

EU154 2.768E-06

EU155 2.768E-06

FE-59 $1.302 E-06$

MN-54 2.279E-06

$5.174 E+00 \quad 7.012 E-01 \quad 1.003 E-01 \quad 5.269 E-01$ 9.827E-01

AG- $108 \quad 3.340 E-02$

$A G-110 \quad 1.350 E-06$

BE $-7 \quad 3.200 E-07$

C-14 $1.046 E-05$

$\mathrm{CE}-141 \quad 1.450 \mathrm{E}-08$

CE- $144 \quad 1.851 E-02$

CO-57 7.200E-11

CO-58 $1.260 \mathrm{E}-05$

CO-60 1.779E-01

CR-51 3.252E-06

CS-134 4.192E-05 
SWIR328B - (Page 12 of 26)

RUNDATE : $03 / 15 / 96$

Solid Waste Burial Ground Areas Waste Volumes Disposed and Stored and

Nondecayed Curie content in the 200 Areas from startup to December 31 , 1995 , Including Segregated Transuranic Waste.

BURIAL GROUND FACILITIES

CENTRAL WASTE COMPLEX 2402WC BUILDING
CENTRAL WASTE COMPLEX 2402WD BUILDING
SR-90

(CURIES)
RU- 106

(CURIES) CS-137
(CURIES)
OTHER RADIOACTIVITY (CURIES)

(CIRIES)

EU 152 4.716E-04

EU-154 $\quad 1.708 E-01$

EU-155 3.046E-04

FE-55 4.331E-0?

FE-59 5.340E-04

$\mathrm{H}-3$ 3.100E-03

HF- $181 \quad 2.104 \mathrm{E}-02$

I-129 $6.001 E-06$

MN-56 4.195E-02

M0-93 9.947E-08

NA- $22 \quad 2.620 E-04$

NB-93M 2.306E-08

NB-94 3.000E-05

NB-95 $1.330 \mathrm{E}-08$

NB-95M $\quad 1.150 E-08$

NI-60 1.001E-10

$\mathrm{N} 1-63 \quad 6.008 \mathrm{E}-02$

PB-210 5.500E-09

PM- 147 1.752E-02

PO-210 5.500E-09

SB- $124 \quad 2.700 E-07$

SB-125 5.580E-0S

SE-75 3.000E-10

SE-79 6.000E-06

SM- $151 \quad 2.400 E-03$

SR-85 3.002E-08

TA- $182 \quad 9.009 \mathrm{E}-05$

IC $-99 \quad 6.371 \mathrm{E}-04$

$V-49 \quad 5.349 E-08$

$2 \mathrm{~N}-65 \quad 8.100 \mathrm{E}-10$

$2 R-95 \quad 3.909 E-04$

BA137 $4.819 E-08$

C- $14 \quad 4.968 E-08$

Co-58 4.968E-08

CO-60 3.329E-06

CS137 5.117E-08

EU152 4.968E-08

EU154 4.223E-07

EU155 4.223E-07

FE-59 1.987E-07

MN-54 3.478E-07 $1.268 \mathrm{E}-01$

BE-7 1.200E-07

C-14 $5.731 \mathrm{E}-02$

CA-45 4.039E-07

CE-144, 1.704E-03

CL-36 3.560E-05

CO-57 2.610E-06

CO-60 1.170E-03

CS-134, 6.858E-05

CS-135 5.650E-13

EU-15? 4.564E-08

EU-15i 8.151E-07

EU-15.5 $1.240 E-07$

FE-55 8.300E-07

FE-50 4.311E-12

$\mathrm{H}-3 \quad 6.331 \mathrm{E}-02$

$1.129 \quad 7.429 E-07$

K-40 $1.150 E-07$

MN-54 1.207E-08

NA-22 $5.434 E-04$ 
SWIR328B - (Page 13 of 26)

RUNDATE : 03/15/96

Solid waste Burial Ground Areas Waste Volumes Disposed and stored and Nondecayed Curie Content in the 200 Areas from Startup to December 31, 1995, Including Segregated Transuranic Waste.

\section{BURIAL GROUND FACILITIES}

CENTRAL WASTE COMPLEX 260ZLD BUILDING
TOIAL SR-90 RU-106 CS-137 OTHER RADIOACTIVITY

(CURIES)

(CURIES) (CURIES)

(CURIES)

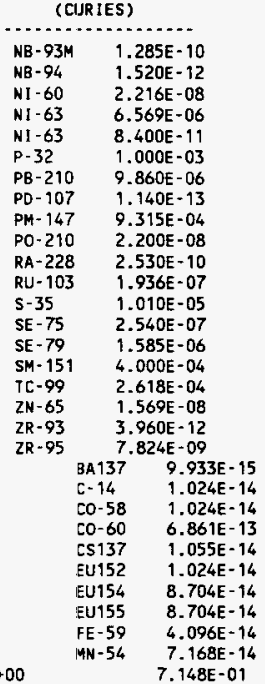

BA -133

C. 14

CE -144

$\mathrm{CO} \cdot 60$

CS -134

EU- 154

EU- 155

FE - 59

$\mathrm{H}-3$

$|-13|$

k-40

MN -54

NI -60

N1 -63

PM- 147

SB- 125

SE -75

SM- 151

TA- 182

TC. 99

TE- 125
3.760E-03

$7.261 \mathrm{E}-04$

$3.855 \mathrm{E}-01$

$5.798 \mathrm{E}-02$

8.968E-03

$1.597 \mathrm{E}-03$

$2.400 \mathrm{E}-05$

$1.359 \mathrm{E}-03$

$6.806 \mathrm{E}-04$

1.600 E- 02

$5.200 E-09$

2. $964 \mathrm{E}-03$

1.200E -07

7.130E-05

$1.769 \mathrm{E}-01$

$1.162 E-04$

$1.410 \mathrm{E}-03$

$5.060 \mathrm{E}-02$

$4.570 \mathrm{E}-03$

$1.570 E-03$

2. $642 \mathrm{E}-05$

BA137 1.627E-06

C-14 1.677E-06

CO-58 $\quad 1.677 \mathrm{E}-06$

CO-60 1.124E-04

CS137 1.727E-06

EU152 $\quad 1.677 E-06$

EU154 1.425E-05

EU155 1.425E-05

FE-59 6.708E-06 
SWIR328B - (Page 14 of 26 )

RUNDATE: $03 / 15 / 96$

Solid Waste Burial Ground Areas waste Volumes Disposed and stored and

Nondecayed Curie Content in the 200 Areas from Startup to December 31, 1995, Including segregated Transuranic Waste.

BURIAL GROUND FACILITIES

CENTRAL WASIE COMPLEX 2402WE BUILDING CENTRAL WASTE COMPLEX $2402 \mathrm{WF}$ BUILDING
TOTAL

(CURIES)

$\mathrm{SR}-90$ (CURIES)

RU-106 (CURIES)

$+$

$1.641 E-01$

$2.801 E-03$

$2.842 E-05$
CS-137 (CURIES)

$3.089 E-03$
Co- 60

EU- 154

EU-155

FE- 59

MN -54

Ni -60

NI -63

(6)

CENIRAL WASTE COMPLEX 2402WG BUILDING

$4.557 E+00 \quad 6.370 E-01 \quad 6.757 E-03$

6.539E- 01

AC -228

BI -212

$\mathrm{C}-14$

CD -113

CE -144

$\mathrm{CL}-36$

CO- 57

Co- 60

CS- 134

CS- 135

EU- 152

EU- 154

EU-155

FE -55

$\mathrm{H}-3$

1- 125

1- 129

I. 131

KR- 85

MN- 54

HA- 22

NB-93N

NB- 94

N] -59

NI -60

N! -63

P-32

PB-212

PM- 147

RA- 228

RB-86

$\mathrm{S} \cdot 35$

SB- 125

SE-79

SM- 151

SN- 121

SN- 126

TA- 182

TC- 99

TE-125

TL- 208

.......

HN-54 1.174E-05

$1.510 \mathrm{E}-01$

1. 150E-01

3.640E-09

$1.568 E-09$

$1.440 \mathrm{E}-02$

2.160E-02

$1.258 E-06$

8.674E-08

$137 \quad 2.409 E-07$

- $14 \quad 2.484 \mathrm{E}-07$

C0-58 2.484E-07

CO-60 1.664E-05

CS137 2.559E-07

EU152 2.484E-07

EU154 2.111E-06

EU155 2.111E-06

FE-59 9.936E-07

MN-54 1.739E-06 2. 102E-01

4.300E-09

6. $530 E-04$

8.788E-04

4.300E-09

2.324E-05

2. $700 E-06$

2.531E-05

4. $066 \mathrm{E}-02$

$1.111 E-06$

2.290E-10

6.500E-05

$1.747 \mathrm{E}-04$

7.539E-05

9. $180 \mathrm{E}-03$

7.136E-02

$6.700 E-06$

7.875E-08

2. $400 \mathrm{E}-03$

4. $420 \mathrm{E}-02$

$5.341 E-05$

$9.226 E-09$

2.410E-09

7.570E-14

$1.741 E-04$

$1.425 E-04$

2.171E-02

3. $150 \mathrm{E}-05$

$6.530 \mathrm{E}-04$

$1.094 \mathrm{E}-02$

$4.300 E-09$

$5.000 E-06$

8.252E-05

$1.200 E-03$

$7.785 \mathrm{E}-10$

4. $880 \mathrm{E}-03$

$5.400 \mathrm{E}-11$

$2.980 E-10$

$2.220 E-07$

$8.492 \mathrm{E}-05$

$2.920 E-04$

2. $250 \mathrm{E}-04$ 
SWIR3288 - (Page 15 of 26)

RUNDATE : 03/15/96

Solid Waste Burial Ground Areas Waste Volumes Disposed and Stored and Nondecayed Curie content in the 200 Areas from Startup to December 31, 1995 , Including segregated Transuranic waste.

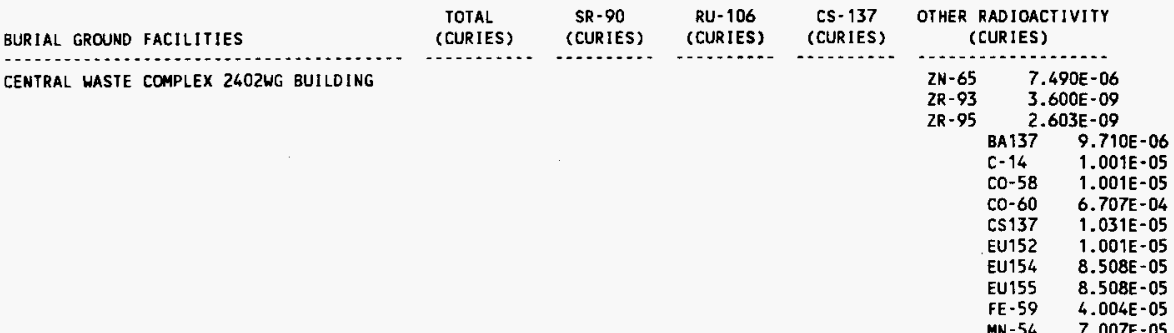

CENTRAL WASTE COMPLEX 2402WH BUILDING

CENTRAL WASTE COMPLEX 2402WI BUILDING

CENTRAL WASTE COMPLEX 2402WJ BUILDING
$1.713 E+00$

$6.593 E-02$

I.7ר3E+00

(6)

$20198-01$

$5.452 E-03$

$2.837 \mathrm{E}-05$

$5.030 \mathrm{E}-03$

$6.906 \mathrm{E}-02$

B1-214

C. 14

CE -144

Co- 60

CS- 135

EU-152

EU-154

EU- 155

FE- 59

$\mathrm{H}-3$

1- 129

] -131

MN-54

NA- 22

NB-94

N] -59

N] -60

NI -63

PB-214

PD -107

PM-14 7

SE - 79

SM- 151

SN-123;

TC- 99

$2 \mathrm{~N} \cdot 65$

ZR-90

$2 R-93$

2R-95

$2.919 E-01$

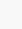

C- 14

CE- 14

CO- 60

$\mathrm{H}-3$

i- 129

NB-94

PM-14?

PO- 210

SE -79

SR- 85

IC -99

3. 216E-01

2. 086 E- 02
4.995E-02

C- 14

CE -14 i

CO-60

CS- 13 :

EU-15?
$1.021 E+00$

1. $140 \mathrm{E}-06$

4. $192 \mathrm{E}-0 \mathrm{~B}$

1. $160 E-02$

$9.098 \mathrm{E}-04$

$1.000 \mathrm{E}-04$

6.694E-05

1. 191E-04

4.816E-04

6.0OOE-OS

$1.000 E+00$

$1.000 E-04$

3. $000 \mathrm{E}-07$

$9.500 E-05$

1. $162 E-05$

$3.044 E-14$

2.500E-10

9.146E-07

2.527E-08

1. $140 \mathrm{E}-06$

1. 000E-04

5.837E-03

3. $730 \mathrm{E}-11$

1.000E-03

$6.000 E-05$

1.012E-04

$1.350 E-10$

1. $000 \mathrm{E}-04$

1. $696 \mathrm{E}-13$

3. $015 \mathrm{E}-06$ $2.691 \mathrm{E}-01$

1.000E-06

1. $122 \mathrm{E}-06$

7.609E-04

$6.776 \mathrm{E}-06$

1.864E-12

$4.270 E-15$

$6.880 E-06$

5. $000 \mathrm{E}-10$

2. $820 E-11$

$1.050 \mathrm{E}-11$

2. $683 E-01$ $1.671 E-01$

$7.390 E-13$

3. $100 \mathrm{E}-03$

7.510E-06

$1.290 E-03$

$1.690 \mathrm{E}-04$ 
SWIR328B - (Page 16 of 26)

RUNDATE: 03/15/96

Solid Waste Burial Ground Areas Waste Volumes Disposed and Stored and

Nondecayed Curie Content in the 200 Areas from Startup to December 31 , 1995 , Including Segregated Transuranic Waste.

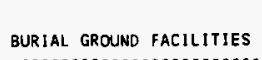

CENTRAL WASTE COMPLEX 2402UJ BUILDING

CENTRAL WASTE COMPLEX 2402WJ BUILDING

CENTRAL WASTE COMPLEX 2402WK BUILDING

CENTRAL WASTE COMPLEX 2403WA BUILDING $\begin{array}{cc}\begin{array}{c}\text { TOTAL } \\ \text { (CURIES) }\end{array} & \text { SR-90 } \\ \text { (CURIES) }\end{array}$

RU-106

(CURIES) (CURIES)

CS- 137
(CUR IES)

OTHER RADIOACTIVITY (CURIES)

EU- $154 \quad 9.370 E-0$

$\begin{array}{ll}\mathrm{H}-3 & 2.960 \mathrm{E}-06\end{array}$

PM-147 1.500E-03

SM- $151 \quad 4.000 E-04$

IC $-99 \quad 1.597 E-01$

BA137 1.940E- 12

C- 14 2.000E- 12

CO-58 2.0OOE-12

CO-60 1.340E-10

CS137 2.060E-12

EU152 2.000E-12

EU154 1.700E -14

EU155 1.700E-11

FE-59 8.000E +12

NIN-54 1.400E-19

$\begin{array}{llll}1.312 E+00 & 1.954 E-02 & 2.280 E-02 & 2.160 E-02\end{array}$

C -14

$1.085 E+00$

EU-155 1.700E-04

H-3 7.001E-01

PM- $147 \quad 4.246 E-02$

SM- $151 \quad 1.350 E-02$

SN-123 4.00OE-05

$\mathrm{T} C-99 \quad 2.394 \mathrm{E}-01$

$3.031 E+01 \quad 5.232 E+00 \quad 6.158 E-01 \quad 5.717 E+00$

AC- 228

$A G=110$

$A G-110$

BE - 7

BI-212

B] -214

C -14

CA- 45

CE -144

Co- 56

co- 57

co. 58

Co- 60

CR- 51

CS- 134

CS- 135

EU. 152

EU- 154

EU- 155

FE- 55

FE- 59

GD -153

GD -154

$\mathrm{H}-3$

HF- 175

HG - 203

1 -125

I- 129

$K-40$

KR- 85

$M N-54$

MO- 93

NA- 22

NB-93N

NB- 94

$4.494 E+00$

8. 200E-0?

$2.235 E-07$

8.526E-08

5.224E-03

2.500E- 07

1. 288E-05

2.389E-03

1. $174 E-03$

8. 929E-01

2.642E-06

$7.318 E-05$

1. 174E- 05

3.604E-01

5. 000 E - 05

$5.593 E-03$

$6.620 E-13$

1. $737 E-03$

3. 306E-03

$6.865 E-04$

3. $516 \mathrm{E}-01$

3.810E-05

5. $000 E-04$

$1.919 E-04$

2. $295 E-02$

3. $200 E-04$

2. 133E-04

2. $050 E-05$

$2.425 E-04$

1. $403 E-02$

$2.486 \mathrm{E}-03$

1. $152 \mathrm{E}-01$

2. $400 E-07$

6. $030 E-04$

2. $292 E-07$

2. $003 E-08$

2.387E-08 
SWIR328B - (Page 17 of 26)

RUNDATE : 03/45/96

Solid Waste Burial Ground Areas Waste Volumes Disposed and stored and Nondecayed Curie content in the 200 Areas from startup to December 31, 1995, Including Segregated Transuranic waste.

BURIAL GROUND FACILITIES

CENTRAL WASTE COMPLEX 2403WA BUILDING

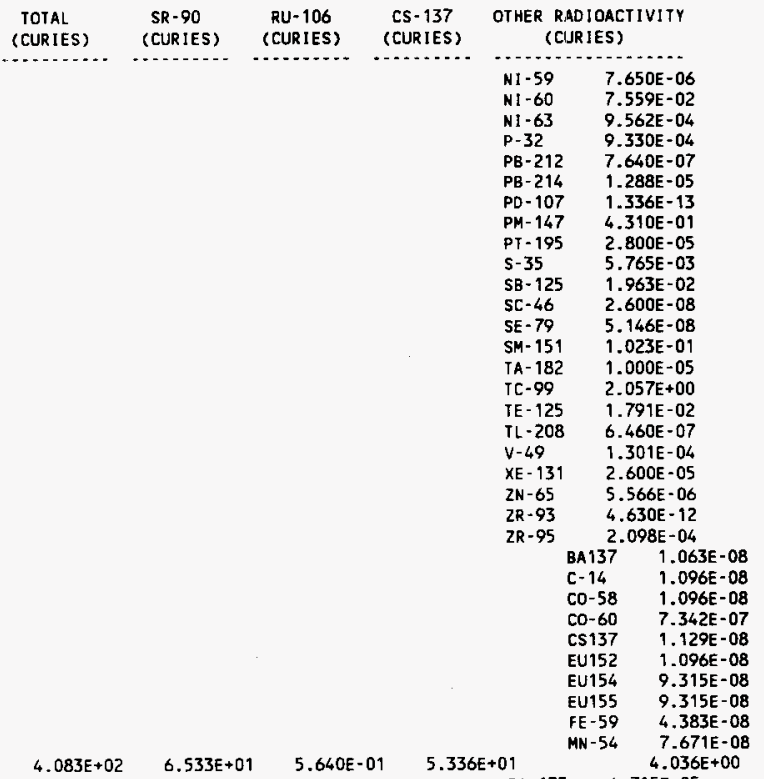

$B A-133$

$B 1-214$

[ -14

CD -105

CE -144

$\mathrm{CO}-60$

CS -134

EU-15:

EU-154

FE -55

FE -59

$\mathrm{H} \cdot 3$

$1-129$

K- 40

$M N-54$

NA -22

NB -93 M

NB- 94

NB- 95

NI -59

NI -60

NI -63

P- 32

PB-210

PM- 147

PO- 2110

RA- 2213
4.715E-05

1. $200 \mathrm{E}-08$

3. $049 \mathrm{E}-09$

$1.000 E-05$

$1.059 E-02$

$1.487 E+00$

6. $795 \mathrm{E}-02$

$1.010 \mathrm{E}-01$

2.894E-01

4. $400 E-08$

$6.800 E-10$

5.382E-01

$3.695 E-05$

3. $113 \mathrm{E}-05$

$2.770 \mathrm{E}-02$

1. 900 E-06

8.670E- 11

$6.230 E-04$

3. $001 \mathrm{E}-02$

2.900E-12

$2.700 E-04$

$1.130 E+00$

$1.000 E-04$

5. $500 \mathrm{E}-07$

2. $73 B E-02$

2. $861 \mathrm{E}-08$

3. $042 E-07$ 
SWIR328B - (Page 18 of 26)

RUNDATE : $03 / 15 / 96$

Solid Waste Burial Ground Areas Waste Volumes Disposed and stored and Nondecayed Curie Content in the 200 Areas from startup to December 31, 1995, Including Segregated Transuranic Waste.

BURIAL GROUND FACILITIES

TOTAL (CURJES)

SR-90

(CUR1ES)

RU- 106

(CURIES)

CENTRAL WASTE COMPLEX 2403WB BUILOING

CENTRAL WASTE COMPLEX 2403WC BUILDING
$6.049 E+01$

$8.569 E-01$
CS- 137
(CUR IES)

-..........

OTHER RADIOACTIVITY (CURIES)

................

SB-125 9.563E-02

SE-79 7.332E-12

SR-85 8.520E-07

SR-89 5.670E-08

TC-99 1.390E-01

TE- $125 \quad 3.825 E-05$

ZN-65 9.000E-02

ZR-95 7.500E-04

BA $137 \quad 3.084 E-04$

C. $14 \quad 3.179 E-04$

CO-58 3.179E-04

CO-60 2.130E-02

CS $137 \quad 3.274 E-04$

EU152 3.179E-04

EU154 2.702E-03

EU155 2.702E-03

FE-59 $1.272 E-03$

MN-54 2.225E-03

$4.463 E+0$

$A C-227$

AC -228

$A G=110$

AG -110

AU- 195

BA -133

BE -7

B] -207

B1-212

B $1-214$

C- 14

$C A-45$

CD- 109

CD -113

CE-141

CE- 144

CL -36

Co- 56

CO- 57

$\mathrm{CO}-58$

CO-60

CR -51

CS- 134

EU- 152

EU- 154

EU- 155

FE- 55

FE- 59

GA -68

GD - 154

GE -68

$\mathrm{H}=3$

HF -175

HG -20

I- 125

I - 129

I -131

$K-40$

KR-85

$M N-54$

MO- 93

NA- 22
$1.200 \mathrm{E}-02$

$1.200 \mathrm{E}-03$

$1.086 \mathrm{E}-04$

$1.791 \mathrm{E}-04$

4. $270 E-07$

$1.060 E-03$

2. $116 E-03$

$1.340 E-06$

1.500E-03

$4.001 E-01$

4. $080 E-03$

7.000E-03

$1.747 \mathrm{E}-01$

8.891E-01

$1.810 \mathrm{E}-06$

$1.841 \mathrm{E}-01$

$1.900 \mathrm{E}-05$

1. $272 \mathrm{E}-06$

$5.128 \mathrm{E}-04$

$2.750 E-03$

$2.309 E-01$

$7.924 E-03$

2.967E-02

1. $146 \mathrm{E}-01$

2. $801 \mathrm{E}-01$

$1.626 \mathrm{E}-01$

3. $045 \mathrm{E}-02$

$7.320 E-04$

7.800E-07

$6.042 E-04$

$5.000 E-06$

7. $274 E-01$

$8.850 E-05$

5.900E-05

2.115E-02

$1.440 \mathrm{E}-03$

1.000E-05

3. $799 E-02$

$4.600 \mathrm{E}-09$

$1.064 E-02$

$3.990 \mathrm{E}-08$

4. $203 E-03$ 
SWIR328B - (Page 19 of 26)

RUNDATE: $03 / 15 / 96$

Solid Waste Burial Ground Areas Waste Volumes Disposed and Stored and Nondecayed Curie Content in the 200 Areas from startup to December 31 , 1995 , Including Segregated Transuranic Waste.

BURIAL GROUND FACILITIES

TOTAL

(CURIES)
$S R-90 \quad R U-106$

(CUR IES)

(CURIES)
CS -137

(CUR IES)

CENTRAL WASTE COMPLEX 2403WC BUILDING

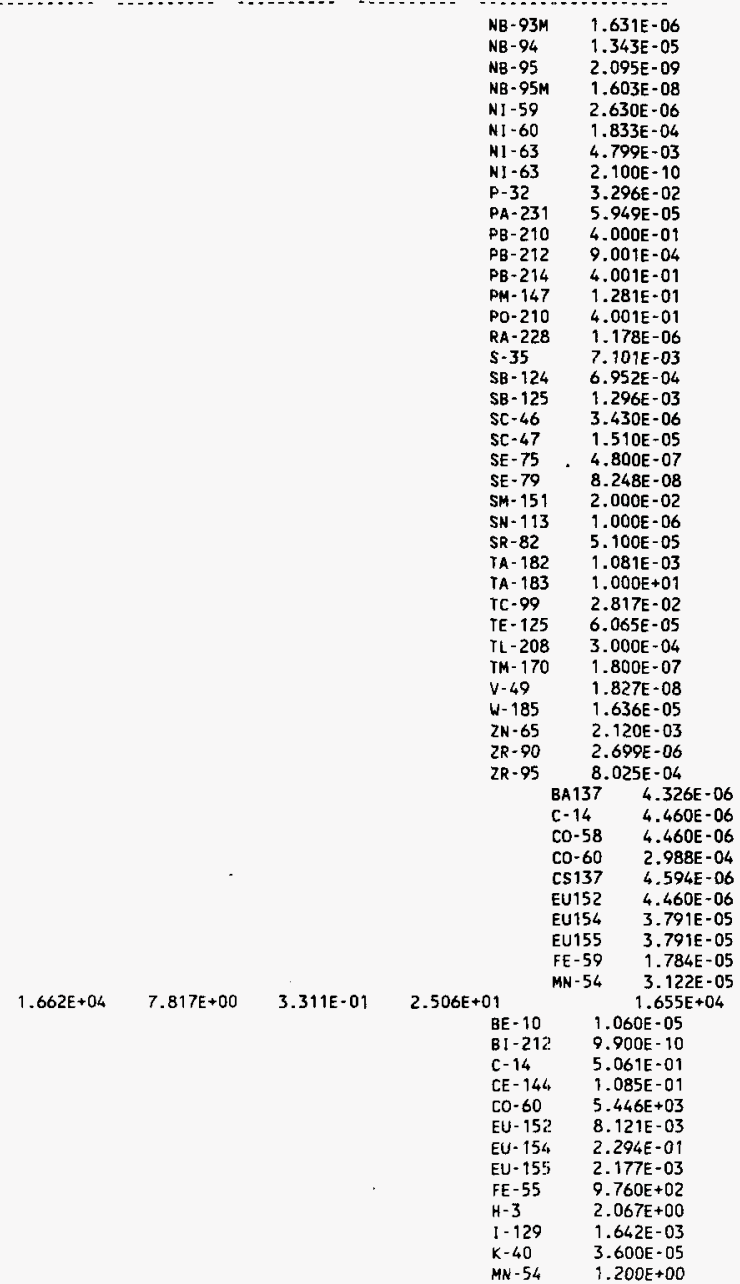

OTHER RMDIOACTIVITY (CURIES) 
SUIR3288 - (Page 20 of 26)

RUNDATE : 03/15/96

Solid Waste Burial Ground Areas Waste Volumes Disposed and stored and Nondecayed Curie content in the 200 Areas from startup to December 31 , 1995 , Including Segregated Transuranic Waste.

BURIAL GROUND FACILITIES

CENTRAL WASTE COMPLEX 2403WD BUILDING $\begin{array}{lccc}\text { TOTAL } & \$ R-90 & \text { RU-106 } & \text { CS-137 } \\ \text { (CURIES) } & \text { (CURIES) } & \text { (CURIES) } & \text { (CURIES) }\end{array}$
OTHER RADIOACTIVITY (ICUR IES)

- . - .

NB-93M 2.230E-04

NB-94 2.610E-01

$N I-59 \quad 8.639 E+01$

HI-60 $2.410 \mathrm{E}-05$

$\mathrm{NI}-63 \quad 1.004 \mathrm{E}+04$

PB-212 9.900E-10

PM- $147 \quad 5.452 E-02$

SE-79 1.873E-05

SM-151 1.361E-02

SN- $123 \quad 1.000 E-04$

TC-99 6.353E-01

2N-65 1.350E-10

2R-93 1.140E-05

BA $137 \quad 4.850 E-06$

$\mathrm{C}-14 \quad 5.000 \mathrm{E}-06$

CO-58 5.000E-06

CO-60 3.350E-04

CS137 5.150E-06

EU152 5.000E-06

EU154 4.250E-05

EU155 4.250E-05

FE- 59 2.000E-05

$M N-54 \quad 3.500 E-05$

$\begin{array}{llll}1.400 E-04 & 8.305 E-07 \quad 4.438 E-09 \quad 7.090 E-05\end{array}$

$1.134 E-01 \quad 1.064 E-04 \quad 2.117 E-06 \quad 5.062 E-02$

$2.310 E-03 \quad 4.152 E-06 \quad 2.219 E-08 \quad 3.545 E-04$

$\begin{array}{llll}7.016 E-02 & 4.368 E-06 & 4.545 E-08 & 1.978 E-02\end{array}$

$1.227 E-04 \quad 6.021 E-06 \quad 7.403 E-11 \quad 5.471 E-06$

$1.348 E-01 \quad 2.990 E-04 \quad 2.300 E-05$

FLAMMABLE STORAGE MODULE 2
$6.885 E-04 \quad 1.284 E-04 \quad 1.956 E-06$
$\mathrm{BA}-133$

NA- 22

$\mathrm{BA}-133$

NA- 22

$B A-133$

NA- 2zi

NA-2?'.

TC- 99

3.211E-04

C -14

CE $-14,4$

$\mathrm{CO}-58$

CO- 60

$C R-51$

FE-50

MN- 5i?

NA- 2?

NB- 95

SC -47

TA- $1 B 2$

TA- 1 B B

$.000 E+00$ $.000 E+00$

$1.460 \mathrm{E}-02$

$1.162 E-03$

1.344E-02 1.610E-03

1. $610 \mathrm{E}-03$ $.000 \mathrm{E}+00$ 3. 165E-02

$3.165 \mathrm{E}-02$ 1. $000 \mathrm{E}-04$

1.00OE- 04 $1.329 \mathrm{E}-01$

$9.420 E-04$

$2.465 E-04$

$6.980 E-03$

$7.671 \mathrm{E}-04$

3. $042 E-03$

1. $000 \mathrm{E}-04$

$2.974 E-03$

1.385E-03

$1.054 E-04$

$4.900 \mathrm{E}-05$

1. $156 \mathrm{E}-01$

4. 390E-04

ZN-65 2.860E-04

$9.057 E-05$

C- 14

CE- 1.44

CS -134

EU- 152

EU-154

EU- 155

$\mathrm{H}-3$

I -129

$x-40$
2.464E-04

4. 300E-05

5.010E-06

$5.813 E-07$

3.080E-05

5. 727E-05

8.620E-06

6. $920 E-05$

1. $097 \mathrm{E}-07$

7.85DE-08 
SWIR328B - (Page 21 of 26)

RUNDATE: $03 / 15 / 96$

Solid Waste Burial Ground Areas Waste Volumes Disposed and stored and

Nondecayed Curie Content in the 200 Areas from startup to December 31, 1995, Including Segregated Transuranic Waste.

BURIAL GROUND FACILITIES

FLAMMABLE STORAGE MODULE 2

flammaBle storage modULE 3

flammable storage module 4

fLAMMABLE STORAGE mODULE 5 \begin{tabular}{lccc}
$\begin{array}{l}\text { TOTAL } \\
\text { (CURIES) }\end{array}$ & $\begin{array}{c}\text { SR-90 } \\
\text { (CURIES) }\end{array}$ & $\begin{array}{c}\text { RU-106 } \\
\text { (CURIES) }\end{array}$ & $\begin{array}{c}\text { CS-137 } \\
\text { (CURIES) }\end{array}$ \\
\hline$\ldots \ldots \ldots \ldots$ & $-\ldots \ldots \ldots \ldots$ & $\ldots \ldots \ldots \ldots \ldots$ & $\ldots \ldots \ldots \ldots$
\end{tabular}

OTHER RADIOACTIVITY (CURIES)

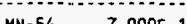

NB-94. $7.420 E-15$

PM- $147 \quad 2.857 E-05$

SE-79 4.900E-11

TA-182 1.110E-07

TC-99 3.017E-06

01

BE -7 $1.538 E-02$

$\mathrm{C}-14 \quad 6.020 \mathrm{E}-03$

CA- $65 \quad 5.000 E-04$

CD- $109 \quad 1.280 E-06$

CO-60 $1.302 \mathrm{E}-03$

EU-152 4.200E-07

EU-156 9.000E-08

FE-55 5.000E-07

FE-59 2.030E-04

$\mathrm{H} \cdot 3 \quad 7.240 \mathrm{E}-03$

N1-63 2.834E-06

P-32 9.800E-05

PM-147 5.000E-07

PO-210 1.180E-06

S-35 1.880E-06

TC-99 1.260E-05

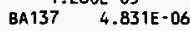

C- $14 \quad 4.980 \mathrm{E}-06$

CO-58 4.980E-06

CO-60 3.337E-04

CS137 5.129E-06

EU152 4.980E-06

EU154 4.233E-05

EU155 4.233E-05

FE-59 1.992E-05

MN-54 3.486E-05

$\begin{array}{llll}1.010 E-01 & 1.750 E-02 & 2.512 E-06 & 8.485 E-03\end{array}$

BE -7

$\begin{array}{ll}B E-7 & 3.493 E-05 \\ C-14 & 5.050 E-04\end{array}$

$3.493 \mathrm{E}-05$

Co-56 1.977E-03

CO-57 7.384E-03

CO.58 $5.219 E-03$

CO-6O $1.776 E-04$

CR-51 2.045E-04

CS-134 2.180E-05

EU-154, 2.460E-05

EU- $15 \xi_{1} \quad 2.770 E-05$

$\mathrm{H}-3 \quad 1.633 \mathrm{E}-02$

$1-129$ 1.210E-10

MN-54 2.367E-03

NA-22 1.490E-06

P. 32 5. $100 \mathrm{E}-04$

$\mathrm{PB}-210 \quad 3.430 \mathrm{E}-05$

$\mathrm{PH}=147 \quad 5.780 \mathrm{E}-08$

$\begin{array}{ll}\mathrm{S}-35 & 3.700 \mathrm{E}-07\end{array}$

SC-46 4.05OE-05

TC-99 9.370E-11

ZN-65 $1.457 \mathrm{E}-02$

$2.602 \mathrm{E}+00 \quad 9.432 \mathrm{E}-06 \quad 1.341 \mathrm{E}-05 \quad 6.834 \mathrm{E}-06$

C- 14

Co- 58

co- 60

EU- 154

EU- 155
$2.601 \mathrm{E}+00$

$4.828 \mathrm{E}-02$

6. $100 E-07$

8. $600 E-08$

1. $600 E-07$

$3.530 E-08$ 


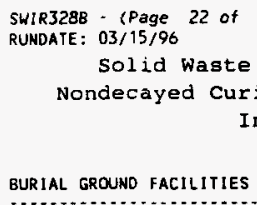

flamMaBle storage mOOULE 6

FLAMMABLE STORAGE MODULE 7

FLAMMABLE STORAGE MODULE 8

FLAMMAGLE StORAGE MODULE 10

FLAMMABLE STORAGE MODULE 12

(CURIES) 1 (CURIES) (CURIES) (CURIES)

OTHER R.ADIOACTIVITY (CIJRIES)

CE-55

$1.200 \mathrm{E}-06$

H-3 $2.553 \mathrm{E}+00$

I-129 S.094E-11

MN-54 6.300E-07

N1-60 8.600E-08

N1 - $63 \quad 1.400 E-07$

PM-147 7.720E-09

TC-99 6.124E-08

$.241 E-02 \quad 8.506 E-04 \quad 1.713 E-03 \quad 8.672 E-04$

$\mathrm{BE}-7$

6. $124 \mathrm{E}-08$
$1.227 \mathrm{E}-02$

BE-7 4.900E-09

C-14 1.373E-06

CA-45 2.500E-04

CD- $109 \quad 1.500 E-06$

CO-60 4.000E-05

EU-152 2.300E-08

FE- $55 \quad 2.500 E-07$

FE-59 9.800E-05

$\mathrm{H}-3 \quad 1.179 \mathrm{E}-02$

N1-63 2.810E-07

P.32 4.900E-05

PM- $147 \quad 2.278 E-05$

PO-210 6.379E-07

S-35 $1.010 E-06$

TC-99 9.150E-06

BA $137 \quad 1.358 E-10$

C-14 1.400E-10

CO-58 $\quad 1.400 E-10$

CO-60 9.380E-09

C\$137 $1.442 E-10$

EU152 1.400E-10

EU154 1.190E-09

EU155 1.190E-09

FE-59 $5.600 E-10$

MN-54 $9.800 E-10$

$1.404 E+00 \quad 6.957 E-04 \quad 3.102 E-05 \quad 6.478 E-04$

C- 14 $1.401 E+00$

CO-60 $\quad 1.601 \mathrm{E}-06$

EU-154. 6.800E-09

EU-155, $\quad 3.260 \mathrm{E}-09$

H-3 $1.392 E+00$

$1-129 \quad 1.815 E-08$

MN-54 1.088E-09

NI $-60 \quad 1.580 \mathrm{E}-06$

NI $-63 \quad 9.840 E-10$

PM- 14i $9.111 \mathrm{E}-05$

TC-99 $4.802 \mathrm{E}-07$

$2 N-65 \quad 5.440 E-10$

$\begin{array}{ccccc}1.125 E-02 & 8.961 E-05 & 6.880 E-06 & 9.605 E-05 & 5.440 E-10 \\ 2 N-65 & 1.075 E-02\end{array}$

C-14 5.960E-03

$\mathrm{H}-3 \quad 4.788 \mathrm{E}-03$

$\begin{array}{lllll}1.134 E-01 & 1.243 E-04 & 2.067 E-04 & 1.282 E-04 & 1.085 E-01\end{array}$

[-14 $1.780 E-04$

C0-60 2.210E-06

$\mathrm{H}-3 \quad 8.495 \mathrm{E}-02$

PB- $210 \quad 5.000 E-09$

PO- 210 B.595E-06

5-35 $\quad 1.090 \mathrm{E}-02$

$1.801 E-04 \quad .000 E+00 \quad .000 E+00 \quad .000 E+00$

C- 14
$1.801 \mathrm{E}-04$

2.587E-05
CE-144 1.080E-05

P-32 $1.246 E-02$ 
SWIR328B - (Page 23 of 26)

RUNDATE: $03 / 15 / 96$

Solid Waste Burial Ground Areas Waste Volumes Disposed and stored and Nondecayed Curie content in the 200 Areas from startup to December 31, 1995 , Including Segregated Transuranic waste.

BURIAL GROUND FACILITIES

Flamagle STORAGE MODULE 12

FLAMmable STORAGE mOOULE 13
TOTAL SR 90 (CURIES)
$R U-106$ (CURIES)
CS- 137 (CURIES)

OTHER RADIOACT IVITY (CLIRIES)

- . . . . . . . . . . . . . . .

CA-45 $\quad 6.450 E-06$

CD-109 2.000E-08

FE-59 3.845E-05

$\mathrm{H}-3 \quad 3.885 \mathrm{E}-05$

NI-63 1.542E-0S

P-32 7.200E-07

TC-99 $5.436 E-05$

$1.230 E-01 \quad 2.637 E-03 \quad 2.669 E-03 \quad 1.595 E-03$

AU-195 1.166E-05

BE -7 3.300E-10

$C=14 \quad 5.629 E-05$

CA-45 1.600E-05

CD-109 2.300E-08

CE-144 7.700E-07

CO-58 4.400E-07

CO- $60 \quad 8.573 E-06$

EU- $152 \quad 1.786 E-05$

EU-154 3.075E-05

EU-155 $1.162 E-06$

FE- $55 \quad 1.600 E-08$

FE-59 6.600E-06

$\mathrm{H}-3 \quad 5.705 \mathrm{E}-02$

I-129 $1.280 \mathrm{E}-12$

MN-54 2.050E-08

NA-22 1.110E-05

HB- 94 2.930E- 15

NI -60 B. BO0E-09

NI -63 2.340E-09

P-32 1.468E-04

PM- $147 \quad 4.746 E-06$

PO-210 5.800E-09

S-35 1.201E-05

SE-79 1.900E-11

$\mathrm{IC}-99 \quad 1.343 \mathrm{E}-05$

2N-65 2.700E-10

ZR-95 2.910E-06

$3.235 E-03$

$\mathrm{SE}-7$

C -14

CA- 45

CD -109

CE -144

CO- 60

C5- 134

Cs. 135

EU- 152

EU-154

EU- 155

FE- 55

FE- 59

H-3

$1-129$

NB -94

$\mathrm{NI}-60$

N1-63

P. 32

PD $-10 \%$

$P M=147$

PO-210

$\mathrm{s}-35$

SB- 125

$2.848 E-02$

4. $900 \mathrm{E}-09$

$1.651 E-04$

2.500E- 04

3. $400 \mathrm{E}-07$

4. $703 E-03$

4. 659E-05

$1.690 \mathrm{E}-06$

5.610E-13

2.020E-07

3. $430 \mathrm{E}-06$

$2.446 \mathrm{E}-06$

2.500E-07

9.800E-05

2. $018 E-02$

9. $283 \mathrm{E}-07$

5. $560 E-15$

$6.592 E-06$

1. $127 \mathrm{E}-06$

4. $900 \mathrm{E}-05$

$1.130 \mathrm{E}-13$

$2.450 \mathrm{E}-03$

$2.000 E-09$

2.300E-09

$1.840 E-08$ 
SWIR328B - (Page 24 of 26)

RUNDATE : 03/15/96

Solid Waste Burial Ground Areas Waste Volumes Disposed and Stored and Nondecayed Curie Content in the 200 Areas from Startup to December 31, 1995 , Including segregated Transuranic Waste.

BURIAL GROUND FACILITIES

fLAMMABLE STDRAGE MODULE 14

flammaBle storage module is

flammable storage module 16

\begin{tabular}{|c|c|c|c|}
\hline $\begin{array}{l}\text { TOTAL } \\
\text { (CURIES) }\end{array}$ & $\begin{array}{c}\text { SR-90 } \\
\text { (CURIES) }\end{array}$ & $\begin{array}{c}\text { RU- } 106 \\
\text { (CURIES) }\end{array}$ & $\begin{array}{c}\text { CS }-137 \\
\text { (CURIES) }\end{array}$ \\
\hline (i) & 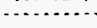 & (c) & \\
\hline
\end{tabular}

FLAMMABLE STORAGE MOOULE 17

\begin{tabular}{ll}
02 & \multicolumn{1}{c}{$6.906 E+00$} \\
C-14 & $1.538 E-03$ \\
CA-45 & $3.783 E-04$ \\
CE-146 & $1.471 E-04$ \\
C0-56 & $1.800 E-06$ \\
CO-57 & $1.511 E-02$ \\
CO-5B & $2.189 E-01$ \\
CO-60 & $2.346 E-01$ \\
CR-51 & $1.002 E-02$ \\
CS-134 & $3.903 E-04$ \\
EU-152 & $7.700 E-06$ \\
EU-156 & $1.780 E-05$ \\
EU-155 & $1.350 E-05$ \\
FE-55 & $3.007 E+00$ \\
FE-59 & $2.150 E-05$ \\
H-3 & $7.206 E-01$ \\
HF- 181 & $4.100 E-04$ \\
$1-129$ & $1.000 E-02$ \\
MN-54 & $1.433 E+00$ \\
MO-93 & $2.703 E-01$ \\
NB-91 & $4.200 E-08$ \\
NB-93M & $6.202 E-02$ \\
NB-94 & $6.500 E-07$ \\
&
\end{tabular}

$2.366 E-03$

$2.354 E-05$
1. 489E- 02

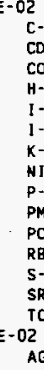

CE- 14

CE -144

$\mathrm{CS}-134$

CS -134
EU- 15?

EU-15i

EU- $15 \%$

$\mathrm{H}-3$

$H-3$
$I-129$
$M N-54$

MN -54
PM -147

PM -147
SB -125

SM- 151

TC -99
$2 R-95$
6. $177 \mathrm{E} \cdot 02$
$7.060 E+00 \quad 1.406 E-02 \quad 7.265 E-04 \quad 6.177 E$

$7.060 E+00 \quad 1.406 E-02 \quad 7.265 E-04 \quad 6.177 E$

$3.670 E-11$

5.000E-04

2. $497 \mathrm{E}-05$

$1.860 \mathrm{E}-08$

3.930E-12

1.330E-02

1.413E-07

6.000E- 07

7.070E-08

5. $705 E-03$

$7.077 \mathrm{E}-03$

$9.900 E-10$

4. $400 \mathrm{E}-08$

2.300E-07

2. $000 \mathrm{E}-06$

$1.355 \mathrm{E}-05$

$6.050 E-07$

2.160E-04

9.400E-07

2. $800 \mathrm{E} \cdot 04$

3.445E-06

$1.966 \mathrm{E}-02$

2. $700 \mathrm{E}-06$

7.210E-04

$1.032 \mathrm{E}-02$

$6.722 \mathrm{E}-06$

$6.513 E-05$

$2.481 E-06$

$2.951 \mathrm{E}-05$

1. $261 \mathrm{E}-05$

2. $590 \mathrm{E}-03$

3. $866 \mathrm{E}-10$

2. $957 \mathrm{E}-07$

4. 800E-03

7.569E- 06

1. 100E-03

3. $002 \mathrm{E}-10$

5.360E-07

$6.906 \mathrm{E}+00$ 
SHIR328B - (Page 25 of 26)

RUNDATE : 03/15/96

Solid Waste Burial Ground Areas Waste Volumes Disposed and Stored and Nondecayed Curie content in the 200 Areas from startup to December 31, 1995, Including Segregated Transuranic Waste.

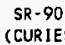

RU- 106 (CURIES)

CS -137
(CURIES)

OTHER RADIOACTIVITY (CIJRIES)

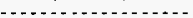

\section{BURIAL GROUND FACILITIES}

FLAMMABLE STORAGE MOOULE 18

FLAMMABLE STORAGE MOOULE 19

FLAMMABLE STORAGE MOOULE 20
FLAMMABLE STORAGE MOOULE 17
$6.648 E-02 \quad 3.780 E-03 \quad 3.775 E-03$

$3.243 \mathrm{E}-02$

4. $132 E-03$

1.210E-03

$1.149 \mathrm{E}-02$
NB-95 2.000E-05

NI-60 3.570E-03

NI-63 8.761E-02

P-32 1.288E-03

PM- 147 3.330E-09

PO- 210 4.500E-06

SB- 125 1.000E-OS

SC-46 $\quad 6.400 E-04$

SI-31 $1.130 \mathrm{E}-06$

TA- $182 \quad 1.700 E-03$

TC.99 $\quad 1.307 E-02$

TI $-51 \quad 4.420 E-07$

$V-49 \quad 4.680 E-02$

W-185 7.661E-01

ZN-65

ZR-95 4.140E-05

03

C- 14

$1.540 \mathrm{E}-02$

6.356E-04

PM-147 7.200E-03

SM-151 2.000E-03

TC-99 8.600E-05

4. $629 \mathrm{E}-03$

EU-15

$\mathrm{H}-3 \quad 1.260 \mathrm{E}-07$

I-129 1.610E-09

PM-147 2.440E-07

TC-99 1.240E-09

$3.751 E-03$

C- $14 \quad 3.810 E-16$ $1.688 E-04$

CE-144 5.600E-08

CO-57 5.997E-08

CO-60 $\quad 1.371 \mathrm{E}-05$

CS-134 2.040E-05

EU-154 2.420E-07

EU-155 5.017E-07

$\mathrm{H}-3$ 2.016E-05

I-129 2.584E-07

$\begin{array}{ll}\mathrm{K}-40 & 4.000 \mathrm{E}-07\end{array}$

MN-54 1.275E-05

NB- $94 \quad 2.140 E-16$

N1-60 $\quad 1.311 E-05$

PM- $147 \quad 3.928 E-05$

SE- $79 \quad 1.410 E-12$

TC-99 4.790E-05

$8.679 E+01 \quad 2.050 E+01 \quad 1.973 E-05 \quad 2.353 E+01$

MIXED WASTE STAGING PAD AT THE CWC

SN- 120
TC -99

$1.450 E-04$

3. $140 \mathrm{E}-03$

$2.290 E+06$

$4.970 E+06$

TOTAL 200 AREAS

$5.286 E+06 \quad 3.914 E+05 \quad 5.388 E+04 \quad 5.827 E+05$

$1.299 E+07$

$1.436 E+06$

$7.922 E+04$

1. $806 E+06$

$4.970 E+06$

SPECIAL CAISSON/TRENCH BREAKOUT FOR FACILITY 218W4B

CAISSON - ALPHA 1

CAISSON - ALPHA 2 $\begin{array}{ll}3.627 E+04 & 7.501 E+02 \\ 2.055 E+04 & 2.472 E+03\end{array}$

$1.550 E+03$

$7.990 \mathrm{E}+02$

$2.648 E+03$
$.000 E+00$ $.000 E+00$ 
SWIR3288 - (Page 26 of 26)

RUNDATE : 03/15/96

Solid Waste Burial Ground Areas Waste Volumes Disposed and stored and Nondecayed Curie Content in the 200 Areas from startup to December 31,1995, Including segregated Transuranic waste.

\begin{tabular}{llllll} 
BURIAL GROUND FACILITIES & $\begin{array}{c}\text { TOTAL } \\
\text { (CURIES) }\end{array}$ & $\begin{array}{c}\text { SR-90 } \\
\text { (CURIES) }\end{array}$ & $\begin{array}{c}\text { RU-106 } \\
\text { (CURIES) }\end{array}$ & $\begin{array}{c}\text { CS-137 } \\
\text { (CURIES) }\end{array}$ & $\begin{array}{c}\text { OTHER RADIOACIIVIIY } \\
\text { (CURIES) }\end{array}$ \\
\hline
\end{tabular}

SPECIAL CAISSON/TRENCH BREAKOUT FOR FACILITY 218W4B

\begin{abstract}
CAISSON - ALPHA 3
CAISSON - ALPHA 4

CAISSON - NO. 1

CAISSON - NO. 2

CAISSON - NO. 3

CAISSON - NO. 4

CAISSON - NO. 5

CAISSON - MO. 6
\end{abstract}

CAISSON - UNI NO. 1

TRENCHES

218 W4B Totals

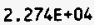

$1.985 \mathrm{E}+04$

4. $336 E+03$

3. $878 E+03$

1. $490 \mathrm{E}+04$

$4,748 E+02$

$2.400 E+02$

CE -144

$\mathrm{H}-3$ PM- 147

2. $000 E+01$

3. $978 E-01$

1. $169 \mathrm{E}+02$

8. $566 \mathrm{E}-01$

4. 268E- 01

$6.345 E+03$

$2.517 \mathrm{E}+02$

$1.338 E+02$

$5.811 E+04$

1. $564 E+03$

1. $191 \mathrm{E}+03$

$2.015 E+02$

$1.492 E+03$

$2.492 E+03$

$3.031 \mathrm{E}+0$

$2.469 \mathrm{E}+02$

1. $272 E+03$

$3.662 E+02$

1. $760 \mathrm{E}+03$

Co- 60

CR-51

CS- 13.4

EU- 15.4

FE- 59

$M N-54$.

SB- 12.5

ZR-95;

$1.250 E+02$

$.000 E+00$

$.000 E+00$

$1.288 E+00$

$2.975 E+05$

$7.469 E+03$

$1.246 E+03$

$8.063 E+03$

CE $-14,4$

$\mathrm{H}-3$

I -120

PM- 147

BA 137

C -14

co- 58

Co. 60

c\$137

EU152

EU154

EU155

FE-59

MN -54

$2.412 E+04$
$.000 E+00$ $1.701 E+03$

$1.694 \mathrm{E}+03$

$7.000 E+00$

$3.099 E+03$

1. 000 E - 02

$2.796 E+03$

$3.030 E+02$

$.000 E+00$

$3.672 \mathrm{E}+02$

$8.418 E+01$

2. $830 E+02$ $.000 \mathrm{E}+00$ $.000 E+00$ $1.928 E+04$

$1.925 E+04$

$1.000 E-01$

3.000E-01

2.200E-01

$2.046 E+00$

$3.623 E+00$

$2.000 \mathrm{E}-02$

$3.000 E+01$ $.000 E+00$

$1.213 E+00$

$1.250 \mathrm{E}+00$

CO-58 $\quad 1.250 E+00$

CO-60 8.375E+01

$\quad 1.288 \mathrm{E}+00$

$1.250 E+00$

$1.063 E+01$

$1.063 E+01$

5. $000 \mathrm{E}+00$

8. $750 E+00$ $2.373 E+05$

6. $000 E-03$

$2.283 \mathrm{E}+05$

$5.000 \mathrm{E}-01$

$9.000 E+03$

$9.881 E+00$

$1.019 E+01$

$1.019 \mathrm{E}+01$

$6.825 E+02$

$1.049 E+01$

$1.019 E+01$

$8.659 E+01$

$8.659 E+01$

$4.075 E+01$

7. $131 \mathrm{E}+01$

$2.617 \mathrm{E}+05$ 
SWIR328C - (Page 1 of 26)

RUNDATE: $03 / 15 / 96$

Solid Waste Burial Ground Areas Decayed Curie Content in the 200 Areas from Startup to December 31, 1995, Including Segregated Transuranic Waste.

BURIAL GROUND FACILITIES

2O0E AREA

BURIAL GROUND $218 E 1$

BURIAL GROUND 218ETO
TOTAL
(CURIES)

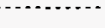

$2.714 E+00$

$2.975 E+06$
SR-90

(CURIES)

RU- 106

(CURIES)

CS-137

(CURIES)

OTHER RADIOACTIVITY (CURIES)
BURIAL GROUND 218E12A
BURIAL GROUND 218E 128
$3.376 E+01$

$1.951 \mathrm{E}+06$

$8.148 E+00$

$2.222 E+04$
3.783E-08

$7.513 E+00$
7.292E-01
$8.267 E+05$

2.273E-01
Co- 60

(CURiEs)

$-60$

$B E-10$

$\mathrm{BE}-7$

C. 14

CE-141

CE- 144

$\mathrm{CL}-36$

CO- 58

CO-60

CR-51

CS -134

FE -55

FE -59

$\mathrm{H}-3$

HF- 181

I -129

$K-40$

MN- 54

MO- 93

NB-93M.

NB -94

NB-95

NI -59

NI -63

PM- 147

$S B-125$

SE -79

$S M-151$

$S N=119$

iA -18 ?

TC- 99

IE- 12 ; 5

$2 \mathrm{~N}-65$

$2 R-93$

ZR-95 
SWIR328C - (Page

RUNDATE: 03/15/96

2 of 26 )

Solid waste Burial Ground Areas Decayed curie Content in the 200 Areas from
startup to December 31, 1995. Including Segregated Transuranic: Waste.

BURIAL GROUND FACILITIES

PUREX TUNNEL (\#2) 218E 15

BUR IAL GROUND 218E2

BURIAL GROUMD 218E 4

BURIAL GROUND 218ES

BURIAL GROUND 218E5A

BURIAL GROUND 218E8

BURIAL GROUND 218EC9

222B VAULTS

Iotal 200E

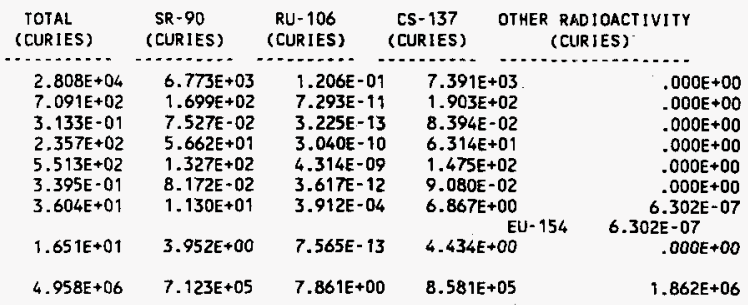


SWIR328C - (Page 3 of 26 )
RUNDATE: $03 / 15 / 96$

Solid Waste Burial Ground Areas Decayed Curie Content in the 200 Areas from Startup to December 31, 1995, Including Segregated Transuranic: Waste.

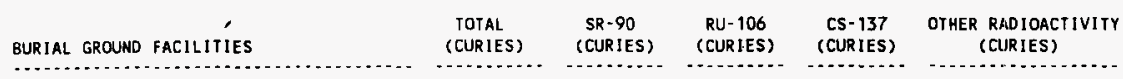

\section{W AREA}

212N BUILDING

214T FACILITY/TPLANT

BURIAL GROUND 218W1 BUR IAL GROUND 218W11 BURIAL GROUND 218W1A BURIAL GROUND 218W2 BURIAL GROUND 218W2A

BURIAL GROUND $218 \mathrm{~W} 3$

BURIAL GROUND 218W3A

$\begin{array}{lll}.000 E+00 & .000 E+00 & .000 E+00 \\ 2.360 E-03 & 7.786 E-07 & 7.215 E-07 \\ & & \\ & & \\ 5.437 E+00 & 1.301 E+00 & 2.694 E-13 \\ 3.516 E-03 & 8.472 E-04 & 9.625 E-14 \\ 1.357 E+03 & 3.251 E+02 & 1.599 E-10 \\ 1.559 E+01 & 3.745 E+00 & 1.752 E-11 \\ 9.228 E+03 & 2.222 E+03 & 8.790 E-05 \\ 3.056 E+01 & 7.356 E+00 & 4.042 E-10 \\ 8.950 E+05 & 9.847 E+04 & 2.383 E+03\end{array}$

$.000 E+00$
$8.326 E-07$

C. 14 EU-152 $\mathrm{H}-3$ NA-22

1. $461 \mathrm{E}+0 \mathrm{O}$

9.398E-04

3. $642 \mathrm{E}+02$

4. $177 \mathrm{E}+00$

$2.466 E+03$

Co- 60

8.173E +00

2. $702 E+05$

AG- 108

$A G-110$

$A G-110$

BA- 133

BE -10

BE -7

BI -207

BI- 214

C- 14

CA -45

CD- 109

CD- 113

CE- 141

CE - 144

$\mathrm{CL}-36$

Co. 56

co. 57

co- 58

Co- 60

CR-51

CS- 134

CS-13!

EU-15?

EU-15\%

EU-15!

FE- 55

FE. 59

GD- 15:

GD - 15

GE -68

H. 3

$1-125$

I - 129

I -131

K -40

$K R-85$

MN- 54

NA-22

NB- $93 \mathrm{M}$

NB- 94

N! -59

Ni -60

N I -63

P-32

PM- 147

PR -144

$$
\begin{array}{r}
.000 E+00 \\
2.349 E-03
\end{array}
$$

8.998E-06

$9.182 E-08$

2. 339E-03

$6.485 \mathrm{E}-07$

$.000 E+00$ $.000 E+00$ $.000 E+00$ $.000 E+00$ $1.691 \mathrm{E}-01$ 1.691E-01 $.000 E+00$ $1.415 E+05$ $.000 \mathrm{E}+00$

5. 105E-05

$.000 E+00$

$1.325 \mathrm{E}-04$

6. $920 E-10$

5.646E-05

2. $494 E-03$

$.000 \mathrm{E}+00$

$1.738 E+00$

$1.062 E-08$

$4.657 \mathrm{E}-05$

3.007E-05

2. $521 E-39$

7.224E-02

2. $003 E-04$

$1.046 E-06$

$1.481 E-03$

$1.004 \mathrm{E}-06$

5. $127 \mathrm{E}+03$

4.612E-15

$2.841 E+02$

$1.102 E-06$

$1.002 \mathrm{E}+00$

4.517E-01

$1.865 \mathrm{E}+00$

$1.695 \mathrm{E}-01$

$1.834 \mathrm{E}-10$

$3.390 \mathrm{E}-03$

$.000 E+00$

5.534E-04

1.349E +05

$1.986 \mathrm{E}-13$

$1.639 \mathrm{E}-02$

$.000 E+00$

1.245E-04

$9.200 E+02$

$9.214 \mathrm{E}-03$

$1.227 E+00$

6. $105 E-05$

2. $128 E-04$

8. 783E-04

$.000 E+00$

$3.608 E+00$

$1.594 \mathrm{E}-46$

$1.343 E+02$

$.000 E+00$ 
SWIR328C - (Page 4 of 26 )

RUNDATE: 03/15/96

Solid Waste Burial Ground Areas Decayed Curie Content in the 200 Areas from Startup to December 31, 1995, Including segregated Transuranic Waste.

BURIAL GROUND 218W3AE

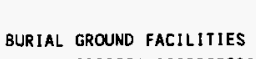

BURIAL GROUND 218W3A

\begin{abstract}
TOTAL
(CURIES)
\end{abstract}

SR-90 (CURIES)

RU- 106

(CURIES)

Cs- 137

(CURIES)

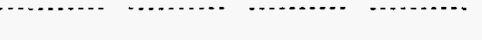
.

IES

.

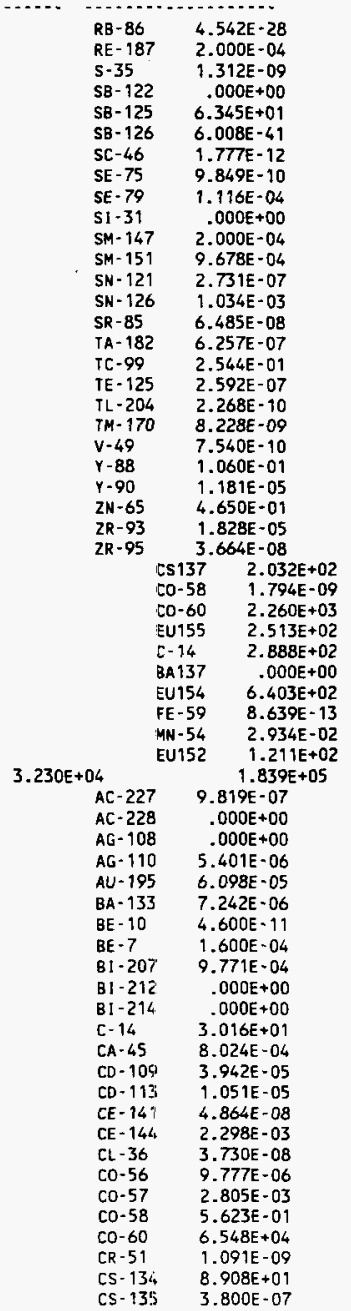


SW1R328C - (Page 5 of 26)

RUNDATE: 03/15/96

Solid Waste Burial Ground Areas Decayed Curie Content in the 200 Areas from Startup to December 31, 1995, Including Segregated Transuranic Waste.

BURIAL GROUND FACILITIES $\begin{array}{lllll}\text { TOTAL } & \text { SR-90 } & \text { RU-106 } & \text { CS-137 OTHER RADIOACTIVITY }\end{array}$

BUR IAL GROUND 21 BUBAE $\begin{array}{lccc}\text { (CURIES) } & \text { (CURIES) } & \text { (CURIES) } & \text { (CURIES) }\end{array}$ (CURIES)

(CUR

.......................

$\begin{array}{ll}\text { ES }-254 & 1.746 E-09 \\ \text { EU-152 } & 1.082 E+03\end{array}$

EU- $153 \quad .000 E+00$

EU-154 $1.511 E+03$

EU-155 $2.891 E+02$

FE-55 $1.146 E+04$

FE-59 $1.750 E+00$

GE-68 3.293E-04

$\mathrm{H}-3 \quad 3.312 \mathrm{E}+04$

HF- $175 \quad 6.480 E-06$

HF- $181 \quad 3.356 E-08$

HG-203 1.224E-07

$\mathrm{I}-125 \quad 9.628 \mathrm{E}-05$

1-129 2.346E-06

I-131 $4.878 E-16$

K-40 5.500E-04

KR-85 $\quad 7.218 E+00$

$M N-54 \quad 6.019 E+03$

MO- 93 5.598E-02

NA-22 1.903E-01

NB-93M 1.225E-03

NB-94 $1.064 E+00$

NB-95 1.099E-02

$\mathrm{NI}-59 \quad 5.200 E+02$

NI-60 $\quad .000 E+00$

N1 $-63 \quad 6.096 E+04$

P-32 2.206E-06

PA-231 1.729E-04

PB-210 9.819E-0B

PB-212 1.329E-41

$\mathrm{PB}-214 \quad .000 \mathrm{E}+00$

PM-147 7.013E-01

PO-21C $1.369 \mathrm{E}-03$

RA-22E; 7.537E-04

$R U-103 \quad 1.279 E-10$

S-35 3.968E-04

SB-125 $\quad 3.108 E+03$

SB-12t, 7.970E-42

SC-46 7.918E-04

SE-75 2.071E-10

SE-79 4.819E-05

SM-151 3.409E-04

$S N-113 \quad 8.506 E-07$

$\mathrm{SN}-119 \quad 1.878 \mathrm{E}+02$

$\mathrm{SN}-121 \quad 1.570 \mathrm{E}+01$

$S N-123 \quad .000 E+00$

SN-126 2.715E-04

SR-85 1.210E-04

TA- $182 \quad 4.738 E-02$

TB-162 $\quad .000 E+00$

TC- $99 \quad 6.508 E-02$

TE- $125 \quad 2.403 E-05$

$\mathrm{TL}-208 \quad .000 \mathrm{E}+00$

TM- $170 \quad 4.522 E-07$

$V-49 \quad 1.385 E-09$

Y-88 1.589E-06

$2 \mathrm{~N}-65 \quad 2.262 \mathrm{E}-02$

$2 R-93 \quad 2.000 E+00$

$Z R-95 \quad 2.431 E+00$

CS137 $9.103 E+00$

CO-58 7.005E-08

CO-60 $\quad 1.896 \mathrm{E}+02$ 
SWIR328C - (Page 6 of 26 )
RUNDATE: $03 / 15 / 96$

Solid waste Burial Ground Areas Decayed Curie Content in the 200 Areas from Startup to December 31, 1995, Including Segregated Transuranic Waste.

BURIAL GROUND FAGILITIE

BURIAL GROUND 218W3AE

BURIAL GROUND 218W4A

BURIAL GROUND 218W4B

BURIAL GROUND $218 W 4 \mathrm{C}$
TOTAL SR-90 RU-106 CS-137 OTHER RADIOACTIVITY (CURIES) (CURIES) (CURIES) (CURIES)
(CIJRIES)

$\begin{array}{ll}1.321 E+02 & 3.182 E+01 \\ 1.222 E+05 & 1.476 E+04\end{array}$

$2.608 \mathrm{E}-07$

8.209E-01

$3.511 E+01$

$1.626 \mathrm{E}+04$

PM- 147

CE- 144

CO-60

CR-51

CS- 134

EU- 154

FE- 59

$\mathrm{H}-3$

I- 129

$M N-54$

PH. 147

PO- 210

SB- 125

ZR-95 ...........

EU155 $2.206 E+01$

$=-14 \quad 1.125 \mathrm{E}+01$

उA $137 \quad .000 E \div 00$

EU154 4.058E+01

FE-59 1.529E-07

HN-54 $1.776 \mathrm{E}-02$

EU152 $6.476 E+00$

3.180E-01

6. $086 \mathrm{E}+04$

$1.598 E-08$

$8.514 E+03$

$1.625 E-39$

4.215E-02

1.354E-01

$3.976 \mathrm{E}-15$

5. $233 E+04$

5. $000 E-01$

$1.941 E-02$

$9.665 \mathrm{E}+00$

$1.051 \mathrm{E}-12$

4. $260 E-03$

$2.554 \mathrm{E}-13$

$\operatorname{cs137} 6.809 E+00$

CO-58 1.021E-24

CO-60 3.754E+01

EU155 $3.984 E+00$

C- $14 \quad 1.140 E+01$

BA $137 \quad .000 \mathrm{E}+00$

EU154 $1.443 E+01$

FE- $59 \quad 2.783 E-38$

MN-54 3.203E-05

EU152 3.306E +00

$5.252 E+05 \quad 7.018 E+04 \quad 3.738 E+01 \quad 1.162 E+05$

AC -228

$A G-110$

$A G-110$

$B A-133$

$\mathrm{BE}-10$

$\mathrm{BE}-7$

$\mathrm{BI}-207$

$\mathrm{BI}-212$

BI-21;

$C-14$

$C A-45$

CD- 107

CD -113

CE- 1414

CL- 36

CO-56

CO- 57

$\mathrm{Co}-58$

CO- 60

CR- 51

CS. 134

CS -135

EU- 152

EU- 154

EU- 155

FE $=55$

FE- 59

GA- 68

$1.573 \mathrm{E}+05$

$.000 E+00$

$3.638 E-07$

$.000 E+00$

$4.951 E-04$

5. $700 \mathrm{E}-11$

3.663E-05

2.107E-04

$.000 E+00$

$.000 E+00$

$8.593 E+00$

$1.237 \mathrm{E}-07$

$1.187 \mathrm{E}-04$

$1.324 \mathrm{E}-05$

$7.622 \mathrm{E}+00$

1.007E-05

1. $137 E-05$

2.854E-05

1. $140 E-03$

1. $210 \mathrm{E}+05$

4. $471 E-06$

$1.245 E+02$

4.800E-07

$1.527 E+01$

$2.059 E+02$

2.314E-01

2. $128 \mathrm{E}+03$

1. $225 \mathrm{E}-06$

$-000 E+00$ 
SWIR328C - (Page 7 of 26)

RUMDATE: $03 / 15 / 96$

Solid Waste Burial Ground Areas Decayed Curie Content in the 200 Areas from Startup to December 31, 1995, Including Segregated TransuraniE Waste.

BURIAL GROUND FACILITIES

BURIAL GROUND 218W4C
CS- 137 (CURIES)
OTHER RADIOACTIVITY (CIJRIES)

GE-68 $1.402 E-12$

$\mathrm{H}=3 \quad 2.426 \mathrm{E}+04$

$1-129$ 1.009E-03

$1-131 \quad 1.117 E-16$

K-40 $1.885 \mathrm{E}-04$

KR-85 2.442E+01

LA-140 .000E+00

MN-54 $1.861 E+02$

MO-93 1.254E-03

NA- $22 \quad 3.672 E-03$

NB-93M 2.751E-05

NB- $94 \quad 3.489 E-01$

NB-95 8.414E-07

NI-59 $\quad 7.622 E+01$

NI $-60 \quad .000 E+00$

NI $-63 \quad 9.275 E+03$

P-32 2.930E-05

PA-231 4.450E-03

PB-210 1.098E-04

PB-212 .000E +00

$\mathrm{PB}-214 \quad .000 \mathrm{E}+00$

PM- $147 \quad 1.241 E-01$

PO- 210 7.775E-05

RA-228 $3.928 E-06$

RU- $103 \quad 2.284 E-09$

S-35 1.369E-03

SB-125 4.020E-01

SB-126 $\quad 1.363 E-39$

SC-46 $2.765 \mathrm{E}-04$

SE-75 1.119E-08

SE-79 2.036E-06

SM-151 4.179E-04

SN-113; $\quad 1.920 \mathrm{E}-07$

SN-121 1.195E-07

SN-126 $\quad 6.400 E-07$

SR-85 $1.021 \mathrm{E}-05$

TA-182 8.033E-02

TA-183 $9.216 E-29$

IC-99 6.173E-02

IE-125) 8.185E-11

$\mathrm{TL}-208 \quad .000 \mathrm{E}+00$

V-49 7.329E-02

W-185 2.427E-10

$Y-88 \quad 9.582 E-08$

ZN-65 $\quad 1.010 E-04$

ZR-93 8.256E-06

$2 R-95$

9.519E-07

$\operatorname{cs} 137 \quad 2.087 \mathrm{E}+00$

CO-58 $1.643 \mathrm{E}-09$

C0-60 $\quad 3.634 E+01$

EU155 $4.180 E+00$

$\mathrm{C}-14 \quad 2.693 \mathrm{E}+00$

BA137 $\quad .000 E+00$

EU154 8.400E +00

FE-59 5.817E-09

MN-54 3.761E-03

EU152 $1.408 E+00$

AC- 227

AC -228

$A C=228$

$1.343 E+04$

$7.942 E-05$

$.000 E+00$

$.000 E+00$

1. $.087 \mathrm{E}-06$ 
WHC-EP-0125-8

SWIR328C - (Page 8 of 26)

RUNDATE: $03 / 15 / 96$

Solid Waste Burial Ground Areas Decayed Curie Content in the 200 Areas from Startup to December 31, 1995, Including Segregated Transuranic Waste.

BURIAL GROUND FACILITIES

TOTAL SR-90 RU-106 CS-137 OTHER RADIOACTIVITY

BURIAL GROUND 218W5

(CURIES) (CURIES) (CURIES)
(CURIES)
(CIJRIES)

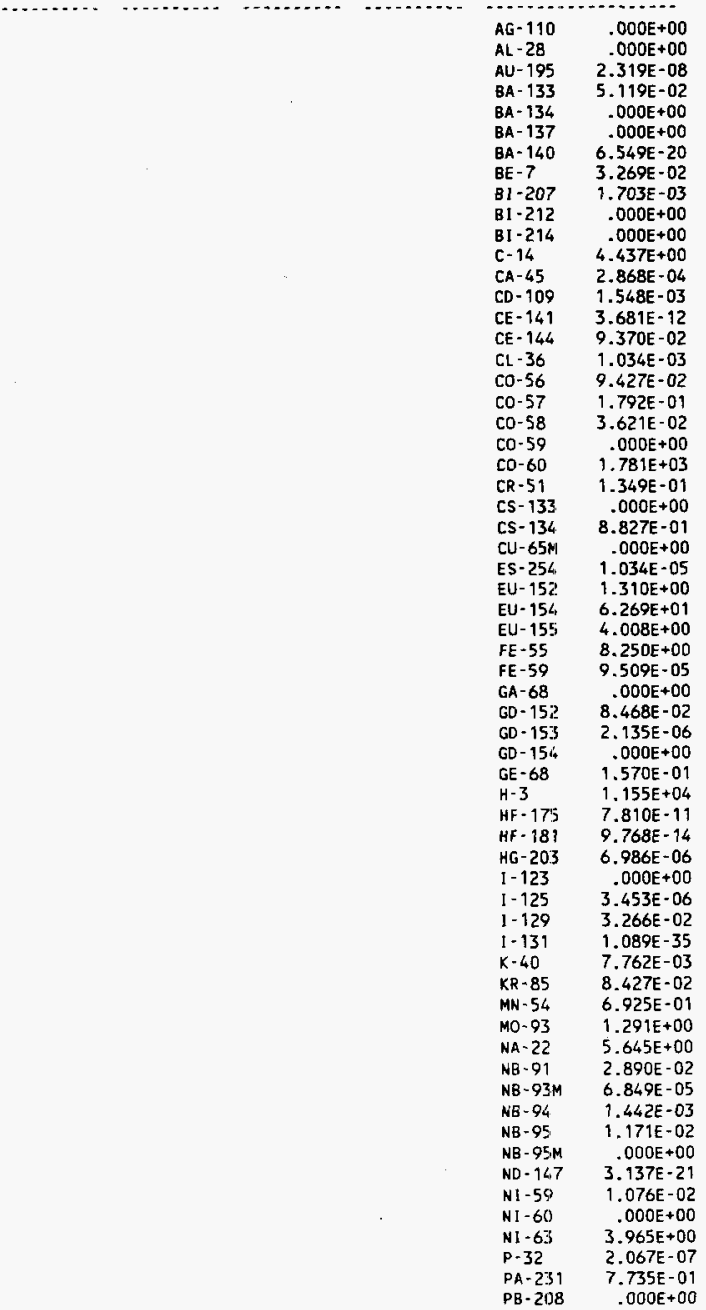


SWIR328C - CPage

RUNDATE: $03 / 15 / 96$

9 of 26 )

Solid Waste Burial Ground Areas Decayed Curie Content in the 200 Areas from Startup to December 31, 1995, Including Segregated Transuranic: Waste.

\begin{tabular}{|c|c|c|c|c|}
\hline BURIAL GROUNO FACILITIES & $\begin{array}{l}\text { TOTAL } \\
\text { (CURJES) }\end{array}$ & $\begin{array}{l}\text { SR-90 } \\
\text { (CURIES) }\end{array}$ & $\begin{array}{l}\text { RU- } 106 \\
\text { (CURIES) }\end{array}$ & $\begin{array}{c}\text { CS-137 } \\
\text { (CURIES) }\end{array}$ \\
\hline 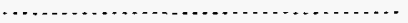 & $\ldots \ldots . .$. & $\cdots \cdots$ & $\cdots+\cdots$ & $\cdots$ \\
\hline
\end{tabular}

BURIAL GROUND 218W5

BUR IAL GROUND $218 W 9$ $221 T$ T PLANT
$3.070 \mathrm{E}-03$

2. $194 \mathrm{E}-04$
7. $371 E-04$

$9.651 \mathrm{E}-10$
1. $766 E-15$

$2.688 E-14$
8. $228 E-04$ $6.483 E-10$

CO- 60

EU-154, EU- 155

FE -59

MN -54 (CURIES)

PB-210

$.000 E+00$

PB-214 .000E+00

PM-147 9.625E-01

PT-195 .000E+00

RA-228 6.166E-04

RB-85M .000E +00

RB-86 1.136E-13

RE- 187 3.000E - 06

RU-103 6.746E-03

5-35 5.664E-04

SB-124 1.081E-07

SB-125 $\quad 1.857 E-01$

SB-126 $\quad .000 E+00$

SC-46 5.317E-04

SE-79 4.978E-03

Si-31 . .000E +00

SM-151 7.696E-01

SN-113 1.257E-05

SN-121 3.208E-07

SN-123 $\quad-000 E+00$

SN-126 $\quad 1.930 E-08$

SR-85 $\quad 4.330 E-04$

SR-89 $1.132 E-13$

TA-182 2.714E-05

C $-99 \quad 1.268 \mathrm{E}-01$

TE- $121 \quad 7.301 E-40$

IE-125 8.013E-09

TE-127 .000E+00

IE-129 3.873E-27

$\mathrm{TL}-204 \quad 2.500 \mathrm{E}-04$

$\mathrm{IL}-208 \quad .000 \mathrm{E}+00$

$\begin{array}{ll}V-49 & 5.690 \mathrm{E}-06\end{array}$

W- $183 \mathrm{M} \quad .000 \mathrm{E}+00$

W-185 2.686E-10

$X E-131 \quad 1.156 E-21$

$Y-87 \quad .000 E+00$

$Y-88 \quad 2.524 E-07$

$Y-90 \quad .000 E+00$

ZN-65 5.004E-01

ZR-95 8.236E-05

CS137 9.287E-01

CO-58 5.377E-06

CO-60 2.399E+01

EU155 $2.838 E+00$

C- $14 \quad 1.097 \mathrm{E}+00$

BA137 .000E +00

EU154 4.649E+00

FE-59 1.205E-05

MN-54 1.121E-02

EU152 7.005E-01 $.000 E+00$

2.194E-04

2. $194 \mathrm{E}-04$

$1.622 E-09$

5.894E- 10

$8.262 E-22$

$4.222 E-08$ 
SWIR328C - (Page 10 of 26 )

RUNDATE : 03/15/96

Solid Waste Burial Ground Areas Decayed Curie Content in the 200 Areas from Startup to December 31, 1995, Including Segregated Transuranic Waste.

\section{BURIAL GROUND FACILIIIIES}

TOTAL
(CURIES)

2211 I PLANT

2225 VAULTS

2221 VAULTS

TRANSURANIC WASTE STORAGE \& ASSAY

FACILITY(TRUSAF)

\begin{abstract}
CENTRAL WASTE COMPLEX $2401 \mathrm{~W}$ BUILDING
\end{abstract}
CENTRAL WASTE COMPLEX $2402 W$ BUILDING

$1.308 E+02$
$2.129 E+01$
$1.630 E+02$

$S R-90$ (CURIES)

RU- 106

(CURIES)

CS- 137

(CURIES)

OTHER RADIOACTIVITY

$1.630 E+02$

3. $147 \mathrm{E}+01$ $5.098 E+00$

$6.818 E+01$

$7.048 \mathrm{E}-10$

1. $101 \mathrm{E}-12$

8.600E-03

(C........

(CURIES)

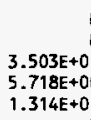

NI -60

HI -63

$.718 E+00$

$1.314 \mathrm{E}+01$

BI -214

C. 14

CE- 144

CO-60

CS- 134

EU-152

EU-154

EU- 155

$\mathrm{H}-3$

K-40

MN -54

P-32

$\$-35$

SB- 125

TE- 125

ZR-95

\section{co-} 3. $642 E-09$ 2. $2.523 \mathrm{E}-03$

EU155 3.118E-04

C- $14 \quad 5.708 E-05$

BA $137 \quad .000 E+00$

EU154 $3.748 E-04$

FE-59 1.469E-10

MN-54 3.232E-05

$1.493 E+05 \quad 7.467 E+04 \quad 4.566 E-06 \quad 1.605 E-03$

EU152 4.834E-05

CO- 60
$E U-154$
$K-40$

$1.344 \mathrm{E}-04$

2. $454 \mathrm{E}-05$

$2.450 \mathrm{E}-07$

CS137 2.908E-08

CO-58 1.610E-17

CO-60 9.879E-07

EU155 1.193E-07

C-14 3.238E-08

BA $137 \quad .000 E+00$

EU154 1.690E-07

FE-59 2.160E-22

MN-54 1.764E-09

EU152 2.366E-08

5.527E-01 7.427E-02 3.404E-04 9.333E-02

C- 14

CE-144

CO- 60

CS- 134,

EU-152?

EU-154

$\mathrm{H} \cdot 3$

PM- 14?

$S M-151$

TC- 99 $1.828 \mathrm{E}-01$

1. $409 \mathrm{E}-12$

$6.720 E-05$

7. $197 \mathrm{E}-07$

5. $025 E-06$

$3.593 \mathrm{E}-03$

$1.050 E-03$

$4.256 E-06$

$7.378 E-04$

$6.724 E-04$

$1.767 \mathrm{E}-01$

CS137 $1.169 E-04$

CO-58 $\quad 1.988 \mathrm{E}-05$

CO-60 $6.909 E-03$

EU155 8.708E-04

[-14 $1.162 \mathrm{E}-04$

BA $137 \quad .000 E+00$ 
WHC-EP-0125-8

SWIR328C - (Page 11 of 26)

RUNDATE: $03 / 15 / 96$

Solid Waste Burial Ground Areas Decayed Curie content in the 200 Areas from Startup to December 31, 1995, Including Segregated Transuranic Waste.

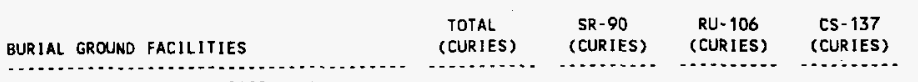

CENIRAL WASTE COMPLEX $2402 W$ BUILDING

$\begin{array}{llll}\text { (CURIES) } & \text { (CURIES) } & \text { (CURIES) } & \text { (CURIES) }\end{array}$

OTHER RADIOACTIVITY (CUIRIES)

CENTRAL WASTE COMPLEX 2402W BUILDING

CENTRAL WASTE COMPLEX 2402WB BUILDING

$3.506 E+00$

$7.876 \mathrm{E}-01$

$3.021 E-03$

$8.480 E-01$

EU154

134E-04

FE-59 3.074E-05

MIN-S4 4.922E-04

EU152 1.103E-04

AC- $228 \quad .000 \mathrm{E}+00$

BA-133 2.222E-06

$B 1-212 \quad .000 E+00$

B $1-214 \quad .000 E+00$

C-14 1.102E-05

CE-144 1.570E-04

CO-60 2.363E-03

CS-134 7.966E-09

EU-152 4.050E-05

EU-154 7.365E-05

EU-155 2.374E-05

FE-59 7.995E-20

$\mathrm{H}-3 \quad 1.331 \mathrm{E}-05$

MN-54 $4.998 E-07$

NA-22 1.694E-05

NB $-95 \mathrm{M} \quad .000 \mathrm{E}+00$

N $1-60 \quad-000 E+00$

PA-231 4.010E-16

PB- $212 \quad .000 E+00$

$P B-214 \quad .000 E+00$

PM- 147 2.072E-03

RA-228 5.072E-04

SM-151 2.009E-03

IC $-99 \quad 1.524 \mathrm{E}-06$

$\mathrm{TL}-208 \quad .000 \mathrm{E}+00$

2R-95 9.687E-08

C\$137 2.908E-07

CO-58 7.206E-17

CO-60 9.637E-06

EU155 1.161E-06

C-14 3.254E-07

BA $137 \quad .000 \mathrm{E}+00$

EU154 1.667E-06

FE-59 6.012E-22

HN-54 1.475E-08

CENTRAL WASTE COMPLEX 240ZWC BUILDING

$2.677 E+00$

6. 193E-01

$4.944 E-04$

4. $458 \mathrm{E}-01$

EU152 2.349E-07

$A G-108 \quad 5.100 E-01$

$.000 E+00$

BE-7 $\quad 6.563 \mathrm{E}-11$

C- $14 \quad 1.045 E-05$

CE-141 5.728E-18

CE-144, $\quad 9.899 E-05$

CO-57 1.980E-11

CO-58 $\quad 1.206 \mathrm{E}-08$

CO-60 1.278E-01

CR-51 6.279E-14

CS-134 8.901E-06

EU-152 4.154E-04

EU-154 1.620E-01

EU-15' 2.122E-04

FE-55 1.463E-01

FE-59 $3.092 \mathrm{E}-19$

$\mathrm{H}-3 \quad 2.732 \mathrm{E}-03$

HF-181 1.946E-07

I. $129 \quad 6.001 E-06$

$M N-54 \quad 5.033 E-04$ 
SWIR328C - (Page 12 of 26 )

RUNDATE: 03/15/96

Solid Waste Burial Ground Areas Decayed Curie Content in the 200 Areas from Startup to December 31, 1995, Including Segregated Transuranic Waste.

$\begin{array}{lllll}\text { BURIAL GROUND FACILITIES } & \begin{array}{c}\text { TOIAL } \\ \text { (CURIES) }\end{array} & \begin{array}{c}\text { SR }-90 \\ \text { (CURIES) }\end{array} & \begin{array}{c}\text { RU-106 } \\ \text { (CURIES) }\end{array} & \begin{array}{c}\text { CS-137 } \\ \text { (CURIES) }\end{array}\end{array}$

CENTRAL WASTE COMPLEX 2402WC BUILDING

CA- 45

CE -144

CL -36

Co- 57

Co- 60

cs- 134

Cs-135.

EU-152:

EU. 154

EU-155

FE- 55

FE- 59

$\mathrm{H}-3$

I -129

$\mathrm{K}-40$

MN -54

NA -22

NB-93M

NB -94

Ni -60

NI -63

NI -63

P. 32

$P B-210$

PD -107

$P M-147$

$P O-210$

RADIOACTIVITY CURIES)

9.943E-08

$1.315 E-04$

2, $088 \mathrm{E}-08$

3.000E-05

$1.314 \mathrm{E}-10$

$.000 E+00$

$.000 E+00$

$5.934 E-02$

$5.268 \mathrm{E}-09$

$7.445 E-03$

$4.365 \mathrm{E}-10$

$1.468 \mathrm{E}-10$

4. $401 E-05$

5.097E-15

$6.000 \mathrm{E}-06$

$2.290 E-03$

$8.017 E-10$

$1.249 E-06$

6. $371 \mathrm{E}-04$

$1.188 \mathrm{E}-08$

$1.538 E-10$

$1.434 \mathrm{E}-08$

CS137 4.555E-08

C0-58 $6.692 \mathrm{E}-16$

C0.60 1.710E-06

EU155 2.081E-07

C-14 4.965E-08

BA $137 \quad .000 E+00$

EU154 2.794E-07

FE-59 6.256E-20

MN-54 5.714E-09

EU152 3.807E-08

$9.268 \mathrm{E}-10$

$5.726 \mathrm{E}-02$

$9.568 \mathrm{E}-09$

$1.077 \mathrm{E}-05$

3.560E-05

8.372E-08

$5.302 E-04$

$2.779 \mathrm{E}-05$

$5.650 E-13$

$4.383 \mathrm{E}-08$

$6.506 E-07$

$9.067 \mathrm{E}-08$

$5.836 \mathrm{E}-07$

8. $247 \mathrm{E}-19$

5.870E-02

7.429E-07

$1.150 E-07$

$3.176 E-09$

4. $842 E-04$

$1.118 \mathrm{E}-10$

$1.520 E-12$

$.000 E+00$

$6.467 \mathrm{E}-06$

$8.166 \mathrm{E}-11$

$5.154 \mathrm{E}-07$

9.551E-06

1. $140 \mathrm{E}-13$

$2.354 E-04$

$4.173 E-09$ 
SWIR328C - (Page 13 of 26)

RUNDATE: $03 / 15 / 96$

Solid Waste Burial Ground Areas Decayed Curie content in the 200 Areas from

startup to December 3.1, 1995, Including Segregated Transuranic Waste.

BURIAL GROUND FACILITIES

CENTRAL WASTE COMPLEX 2402WD BUILDING $\begin{array}{lllll}\text { TOTAL } & \text { SR-90 RU-106 CS-137 OTHER RADIOACIIVITY }\end{array}$

(CURIES)
(CURIES)

(CUR!ES)
(CUR IES)
(CURIES)

C...............

RA-228 $1.931 \mathrm{E}-10$

RU-103 1.779E- 14

$\mathrm{S}-35 \quad 2.692 \mathrm{E}-08$

SE-75 3.729E-12

SE-79 1.585E-06

SM-151 3.821E-04

TC-99 2.618E-04

2N-65 1.659E-09

$2 R-93 \quad 3.960 E-12$

ZR-95 3.689E-10

ES137 9.604E-15

CO-58 4.774E-21

50-60 4.015E-13

EU155 $4.925 E-14$

$=-14 \quad 1.023 E-14$

BA $137 \quad .000 E+00$

EU154 6.243E-14

FE-59 3.593E-24

MN-54 2.630E-15

EU152 8.267E-15

$4.294 \mathrm{E}+00 \quad 6.370 \mathrm{E}-01 \quad 4.905 \mathrm{E}-03 \quad 1.364 \mathrm{E}+00 \quad 1.219 \mathrm{E}-01$

$B A-133 \quad 2.521 E-03$

C-14 7.256E-04

CE-144 2.280E-03

CO-60 2.558E-02

CS-134 $1.140 E-03$

EU-154 1.007E-03

EU- $155 \quad 9.857 \mathrm{E}-06$

FE-59 5.016E-19

$\mathrm{H}-3 \quad 5.065 \mathrm{E}-0.4$

$[-131 \quad .000 E+00$

$K-40 \quad 5.200 E-09$

$M N-54 \quad 2.036 \mathrm{E}-05$

NI $-60 \quad .000 E+00$

N1-63 6.899E-05

PM-14? 3.816E-02

SB-125 $3.484 E-05$

SE-75 2.785E-09

SM- 151 4.827E-02

TA-18Z: $\quad 4.812 \mathrm{E}-09$

TC-99 1.570E-03

TE-125. 3.138E-14

CS137 $1.498 \mathrm{E}-06$

CD.58 $1.406 \mathrm{E}-15$

CO-60 4.976E-05

EU155 $5.997 \mathrm{E}-06$

C- $14 \quad 1.676 \mathrm{E}-06$

BA137 $\quad .000 E+00$

EU154 8.599E-06

FE-59 2.227E-19

MN-54 7.859E-08

EU152 1.211E-06 $4.981 \mathrm{E}-02$

C0-60 4.969E-02

EU-15i $2.550 E-09$

EU-155 8.519E-10

FE-59 2.367E-18

$M N=54 \quad 1.207 E \cdot 04$

NI $-60 \quad .000 E+00$

NI-63 8.517E-08

C\$137 2.240E-0?

Co.58 $2.499 \mathrm{E}-16$ 
WHC-EP-0125-8

SWIR328C - (Page 14 of 26 )

RUMDATE : 03/15/96

Solid Waste Burial Ground Areas Decayed Curie Content in the 200 Areas from Startup to December 31, 1995, Including Segregated Transuranic Waste.

BURIAL GROUND FACILITIES

CENTRAL HASTE COMPLEX 2402WF BUILDING

CENTRAL WASTE COMPLEX 2402WG BUILDING

$\begin{array}{llcc}\begin{array}{l}\text { TOTAL } \\ \text { (CURIES) }\end{array} & \text { SR }-90 & \text { RU-106 } & \text { CS- } 137 \\ \text { (CURIES) } & \text { (CURIES) } & \text { (CURIES) }\end{array}$

(CURIES)

(CURIES)

(CURIES)

OTHER RADIOACTIVITY (CURIES)

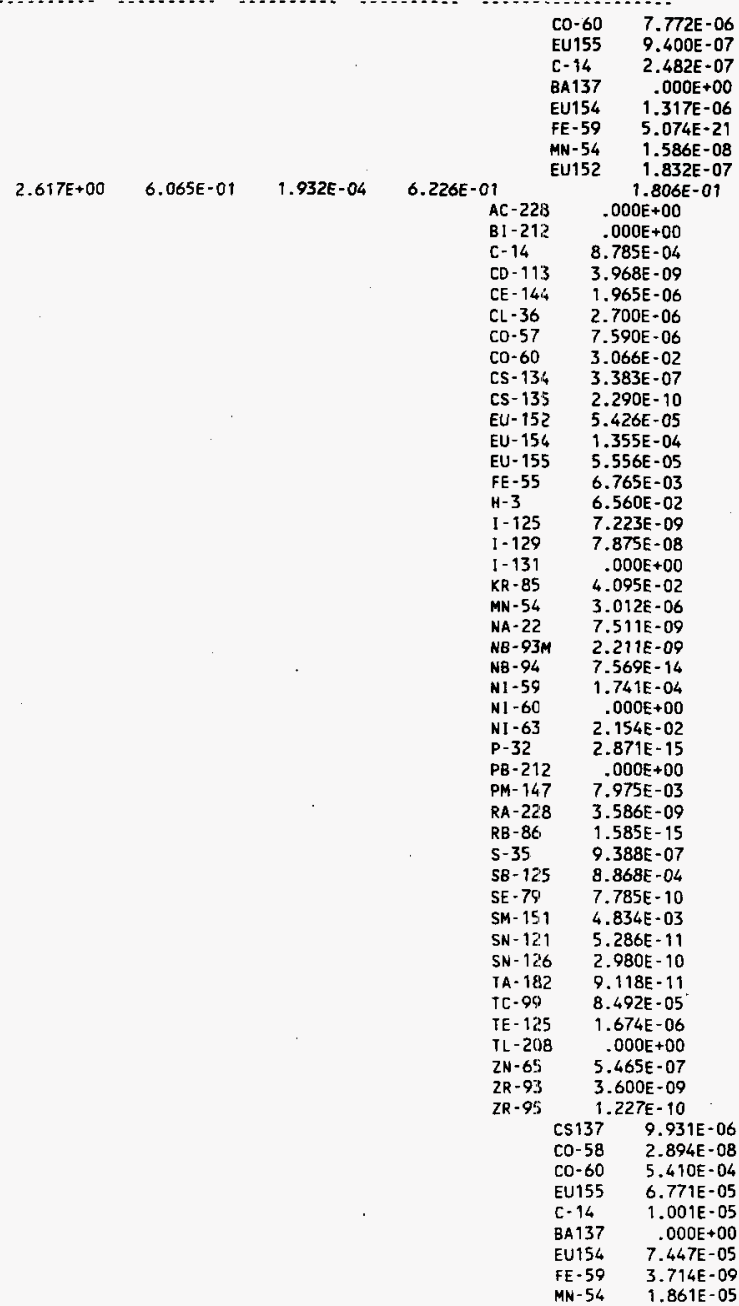


SWIR328C - (Page 15 of 26)

RUNDATE : $03 / 15 / 96$

Solid Waste Burial Ground Areas Decayed Curie Content in the 200 Areas from Startup to December 31, 1995, Including Segregated Transuranic Waste.

\section{BURIAL GROUND FACILITIES}

CENTRAL WASTE COMPLEX 2402WG BUILDING CENTRAL WASTE COMPLEX 2402 WH BUILDING

CENTRAL WASTE COMPLEX 2402WI BUILOING

CENTRAL WASTE COMPLEX $2402 \mathrm{WJ}$ BUILDING

\begin{abstract}
CENTRAL WASTE COMPLEX 2402WH BUILDING
\end{abstract}
CENTRAL WASTE CONPLEX 2402WI BUILOING

\begin{tabular}{cccc}
$\begin{array}{c}\text { TOTAL } \\
\text { (CURIES) }\end{array}$ & $\begin{array}{c}\text { SR-90 } \\
\text { (CURIES) }\end{array}$ & $\begin{array}{c}\text { RU- } 106 \\
\text { (CURIES) }\end{array}$ & $\begin{array}{c}\text { CS-137 } \\
\text { (CURIES) }\end{array}$ \\
\hline & &
\end{tabular}

OTHER RAOIOACTIVITY (CUR!ES)

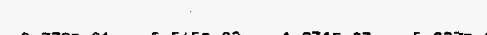

$9.779 E \cdot 01$

$2.910 \mathrm{E}-01 \quad 5.353 \mathrm{E}-03 \quad 1.435 \mathrm{E}-05 \quad 4.944 \mathrm{E}-03$

3.080E-01 2.044E-02 $1.014 E-04 \quad 4.933 E-02$
EU152 $9.187 \mathrm{E}-06$

$5.827 \mathrm{E}-02$

BI -214

C-14 4.192E-08

CE- $144 \quad 1.472 E-04$

CD-60 4.065E-04

CS-135 1.000E-04

EU-152 5.764E-05

EU- $154 \quad 9.437 E-05$

EU-155 2.596E-04

FE-59 1.187E-19

$\mathrm{H}-3 \quad 7.230 \mathrm{E}-01$

1-129 1.000E-04

I-131 $1.107 \mathrm{E}-24$

MN-54 7.793E-07

NA-22 7.655E-06

NB- $94 \quad 3.044 E-14$

NI-59 2.500E-10

$\mathrm{N1}-60 \quad .000 \mathrm{E}+00$

N] $-63 \quad 2.484 \mathrm{E}-08$

PB-214 .000E+00

PD-107 1.000E-04

PM- $147 \quad 1.468 \mathrm{E}-03$

SE-79 3.730E-11

SM-151 9.541E-04

$\mathrm{SN}-123 \quad .000 \mathrm{E}+00$

TC. $99 \quad 1.012 \mathrm{E}-04$

$2 \mathrm{~N}-65 \quad 2.512 \mathrm{E}-11$

ZR- $90 \quad .000 E+00$

ZR-93 1.696E-13

ZR-95 6.573E-09

C. 14

CE -144

CO-60

$\mathrm{H}-3$

1. 129

NB- 94

PM- 147

PO-210

SE -79

SR- 85

TC- 99

C. 14

CE. 144

$\mathrm{CO}-60$

CS. 134

EU- 152

EU-154

$\mathrm{H}-3$

PM- 147

SM- 15

TC- 99

$9.160 E-11$
2.690E-01

$9.992 E-07$

$4.450 \mathrm{E}-08$

$6.665 \mathrm{E}-04$

$6.471 \mathrm{E}-06$

1. $864 \mathrm{E}-12$

$4.270 E-15$

$2.639 E-06$

2.820E-11

$2.057 E-13$

2.683E-01 1.627E-01

7. $384 \mathrm{E}-13$

$1.158 \mathrm{E}-05$

7. $240 \mathrm{E}-06$

1. $174 \mathrm{E}-03$

$1.665 \mathrm{E}-04$

$9.159 \mathrm{E}-04$

2.080E-06

2. $853 \mathrm{E}-04$

$3.805 E-04$

$1.597 \mathrm{E}-01$

CS137 1.909E-12

CO-58 $\quad 1.377 \mathrm{E}-17$

CO-60 8.658E-11

EU155 $1.069 \mathrm{E}-11$

C-14 $1.999 \mathrm{E}-12$

BA137 .000E+00

EU154 1.297E-11

FE-59 $5.053 E-20$ 
SWIR328C - (Page 16 of 26)

RUNDAIE : 03/15/96

Solid Waste Burial Ground Areas Decayed Curie content in the 200 Areas from Startup to December 31, 1995, Including Segregated Transuranic Waste.

BURIAL GROUND FACILITIES

TOTAL (CURIES)

CENTRAL WASTE COMPLEX 2402WJ BUILDING

CENIRAL WASTE COMPLEX 2402WK BUILDING

CENTRAL WASTE COMPLEX 2403WA BUILDING

$\begin{array}{lc}\text { SR }-90 & \text { RU-106 } \\ \text { (CURIES) } & \text { (CUR!ES) }\end{array}$

(CURIES)

(CUR!ES)

CS- 137 (CURIES)

$8.402 E-01 \quad 1.686 E-02$

3.497E-04

$2.115 E+01 \quad 4.358 E+00$

$1.562 E-02$

$4.802 E+00$

1.876E-02

OTHER RADIOACTIVITY (CURIES)

(CIJRIES)

MN-S4 9.458E-13

EU152 $1.680 \mathrm{E}-\{2$

4. $214 E-04$

EU-155 7.585E-05

$\mathrm{H}-3 \quad 5.062 \mathrm{E}-01$

PM-147 8.632E-03

SM-151 1.287E-02

SN-123 .000E+00

TC-99 2.394E-01

$A C-228$

$A C=228$
$A G=110$

$A G=110$

BE -7

BI- 212

B1-214

C. 14

CA- 45

CE- 144

CO-56

CO- 57

co. 58

CO- 60

CR-51

CS- 134

CS- 135

EU- 152

EU-154

EU- 155

FE -55

FE -59

GD - 153

GD- 154 .

$$
\mathrm{H}-3
$$

HF -175

$\mathrm{HG}-203$

$1-125$

I -129

$K-40$

KR-85

$M N-54$

MO- 93

NA- 22

NB-9314

NB- 94

NB -9514

NI-59

$N I-60$

NI -63

$\mathrm{P}-32$

P8- 212

$P B-216$

$P D-107$

PH- 147

PT- 195

5. 35

SB- 125

SC- 46

SE- 79

SM. 151
$2.770 \mathrm{E}+00$

$.000 E+00$

$1.674 E-08$

$.000 \mathrm{E}+00$

2. $467 \mathrm{E}-08$

$.000 E+00$

$.000 E+00$

$3.632 E-05$

1. $398 \mathrm{E}-02$

$6.584 \mathrm{E}-10$

$6.599 E-06$

1. $148 E-09$

2. 105E-01

5. $040 \mathrm{E}-14$

1.371E-03

$6.620 \mathrm{E}-13$

$1.518 \mathrm{E}-03$

2.618E-03

4.623E-04

$1.918 E-01$

$1.170 \mathrm{E}-10$

$3.260 \mathrm{E}-05$

$.000 E+00$

$1.995 E-02$

$1.765 E-08$

$1.136 \mathrm{E}-10$

1.003E-09

$2.425 \mathrm{E}-04$

$1.403 E-02$

2. $119 E-03$

$1.688 \mathrm{E}-02$

2.399E-07

3. $007 E-04$

2.032E-07

2. $003 \mathrm{E}-08$

$.000 E+00$

$.000 E+00$

$9.399 \mathrm{E}-04$

$1.313 E-21$

$.000 E+00$

$.000 \mathrm{E}+00$

$1.336 \mathrm{E}-13$

$1.245 \mathrm{E}-01$

$.000 E+00$

4. $910 \mathrm{E}-06$

$1.095 \mathrm{E}-02$

2. $155 \mathrm{E}-11$

$5.146 \mathrm{E}-08$

9. $846 \mathrm{E}-02$
2. $389 E-03$

$7.650 \mathrm{E}-06$ 
SHIR328C - (Page 17 of 26)

RUNDATE : 03/15/96

$$
\begin{aligned}
& \text { Solid Waste Burial Ground Areas Decayed Curie Content in the } 200 \text { Areas from } \\
& \text { Startup to December 31, 1995, Including Segregated Transuranil Waste. }
\end{aligned}
$$

BURIAL GROUND FACILITIES

CENTRAL WASTE COMPLEX 2403WA BUILDING

CENTRAL WASTE COMPLEX 2403 WB BUILOING

\begin{tabular}{cccc}
$\begin{array}{c}\text { TOTAL } \\
\text { (CURIES) }\end{array}$ & $\begin{array}{c}\text { SR-90 } \\
\text { (CURIES) }\end{array}$ & $\begin{array}{c}\text { RU-106 } \\
\text { (CURIES) }\end{array}$ & $\begin{array}{c}\text { CS-137 } \\
\text { (CUR!ES) }\end{array}$ \\
\hline & &
\end{tabular}

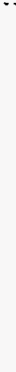

$2.407 E+02 \quad 6.331 E+01 \quad 1.171 E-01$
OTHER RADIOACTIVITY (CURIES)

TA-182 5 .

TC-99 $2.057 \mathrm{E}+00$

TE-125 8.972E-07

TL- $208 \quad .000 E+00$

$\begin{array}{ll}\mathrm{V}-49 & 2.112 \mathrm{E}-05\end{array}$

$X E-131 \quad 5.699 E-36$

2N-65 3.823E-07

ZR-93 4.630E-12

ZR-95 7.036E-09

$\operatorname{Cs137} 9.967 \mathrm{E}-09$

Co-58 7.361E-17

CO-60 3.605E-07

EU155 4.373E-08

C- $14 \quad 1.095 \mathrm{E}-08$

BA $137 \quad .000 E+00$

EU154 5.991E -08

FE-59 1.098E-19

MN-54 $9.544 E-10$

EU152 8.247E-09

5.156E+01

B) -214

C- 14

CD-105)

CE-164,

Co- 60

CS-134,

EU-15\%

EU-154,

FE -55

FE -59

$\mathrm{H}-3$

I -129

K -40

MN -54

NA- 22

NB-93M

NB-94

NB -95

NI -59

N1-60

NI -63

$\mathrm{P}-32$

$P B-210$

PM- 147

PO- 210

$R A=228$

SB- 125

SE -79

$S R-85$

$S R-89$

TC- 99

TE- 125

ZN- 65

$2 R-95$
4.681E-05 . DOOE +00

3. $049 \mathrm{E}-09$

$7.443 E-06$

$5.743 E-03$

1. $380 \mathrm{E}+00$

6. 199E-02

$9.962 E-02$

2. $819 E-01$

2.225E-08

2.064E-16

5.297E-01

3.695E-05

3.113E-05

1.771E-02

$9.333 E-07$

$8.383 E-11$

$6.230 \mathrm{E}-04$

$2.079 \mathrm{E}-04$

$2.900 E-12$

$.000 E+00$

1. $126 \mathrm{E}+00$

5.154E-08

5. $403 E-07$

2.382E-02

$9.507 \mathrm{E}-09$

2.853E- 07

8.342E-02

$7.332 E-12$

$3.110 \mathrm{E}-07$

1. $610 \mathrm{E}-08$

$1.390 E-01$

$3.339 E-10$

$4.412 \mathrm{E}-02$

2.710E-04

$\begin{array}{ll}\text { CS137 } & 3.237 E-04 \\ \text { CO-58 } & 6.066 E-05 \\ \text { CO-60 } & 1.996 E-02 \\ \text { EU155 } & 2.522 E-03 \\ \text { C. } 14 & 3.179 E-04 \\ \text { BA137 } & .000 E+00 \\ \text { EU154 } & 2.595 E-03\end{array}$

$3.794 \mathrm{E}+00$ 
SWIR328C - (Page 18 of 26)

RUNDATE: 03/15/96 Solid Waste Burial Ground Areas Decayed curie content in the 200 Areas from
Startup to December 31,1995 , Including Segregated Transuranic Waste.

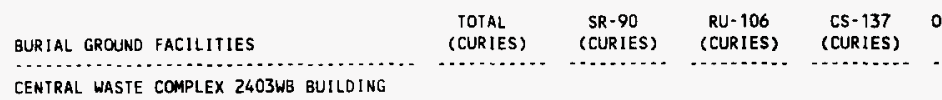

CENTRAL WASTE COMPLEX 2403WC BUILDING

$2.065 E+02 \quad 5.587 E+01 \quad 1.165 E-01$

4. $133 \mathrm{E}+01$

AC -227

AC -228

$A G-110$

AG -110

AU- 195

$B A-133$

$\mathrm{BE}-7$

BI -207

BI -212

$\mathrm{BI}-214$

C- 14

$C A-45$

CD- 109

CD -113

CE - 141

CE -144

$\mathrm{CL}-36$

Co-56

C0.57

CO- 58

CO- 60

CR-51

CS -134

EU- 152

EU- 154

EU- 155

FE- 55

FE -59

GA-68

GD- 154

GE- 68

$\mathrm{H}-3$

HF- 175

HG- 203

I. 125

I +129

-13 ]
$k-40$

$k=40$

KR- 85

MN -54

MO- 93

NA -22

NB-93N

NB- 94

NB- 95

NB-95N

N $1-59$

NI -60

N: -63

N1. 63

P. 32

PA- 231

PB- 210

PB-212

PB-214

PM- 147

PO- 210

RA $-22 B$ RIES)

FE-59 9.595E-05

$M N-54 \quad 1.501 \mathrm{E}-03$

$3.097 E-04$ $2.856 E+00$ $1,110 E-02$ $.000 E+00$

$4.999 E-05$

$.000 E+00$

$1.169 \mathrm{E}-07$

$9.555 E-04$

$2.478 E-05$

$1.304 \mathrm{E}-06$

$.000 E+00$

$.000 E+00$

$4.079 E-03$

$8.232 E-04$

$1.1705-01$

8.593E-01

2. $138 \mathrm{E}-18$

$3.975 E-03$

$1.900 E-05$

$6.275 \mathrm{E}-08$

$1.444 \mathrm{E}-04$

$1.626 \mathrm{E}-05$

$1.813 \mathrm{E}-01$

2.075E-09

$9.240 \mathrm{E}-03$

1.010E-01

2.327E-01

$1.207 \mathrm{E}-01$

1.694E-02

$1.287 \mathrm{E}-14$ $.000 \mathrm{E}+00$ $.000 \mathrm{E}+00$

$1.466 \mathrm{E}-06$

$6.726 \mathrm{E}-01$

$2.996 E-06$

3. $712 E-07$

$5.994 E-05$

$1.440 \mathrm{E}-03$

8.107E-25

3. $799 \mathrm{E}-02$

4. 172E-09

$2.894 E-03$

3.988E-08

$3.015 E-03$

1. $433 E-06$

$1.343 E-05$

$1.494 \mathrm{E}-19$

$.000 E+00$

$2.630 \mathrm{E}-06$

$.000 E+00$

$4.750 E-03$

2. $041 E-10$

$5.945 E-13$

5.949E-05

3.706E-01

$.000 E+00$

$.000 E+00$

$4.987 \mathrm{E}-02$

$4.540 \mathrm{E}-03$

$1.009 E-06$ 
SW1R328C - (Page 19 of 26)

RUNDATE: 03/15/96

Solid waste Burial Ground Areas Decayed Curie Content in the 200 Areas from
startup to December 31,1995 , Including Segregated Transuranic: Waste.

BURIAL GROUND FACILITIES

CENTRAL WASTE COMPLEX 2403 WC BUILDING
TOTAL SR-90 RU-106 CS-137 OTHER RADIOACIIVIIY

(CURIES) (CURIES) (CURIES) (CURIES)

(CURIES)

\begin{tabular}{|c|c|c|}
\hline$s-35$ & \multicolumn{2}{|c|}{ 1. $370 E-04$} \\
\hline S8- 124 & \multicolumn{2}{|c|}{$3.380 E-05$} \\
\hline$S B-125$ & \multicolumn{2}{|c|}{$7.370 E-04$} \\
\hline$S C-46$ & \multicolumn{2}{|c|}{$1.355 E-07$} \\
\hline SC -47 & \multicolumn{2}{|c|}{$1.576 E-38$} \\
\hline SE- 75 & \multicolumn{2}{|c|}{$5.386 E-08$} \\
\hline SE- 79 & \multirow{2}{*}{\multicolumn{2}{|c|}{$\begin{array}{l}8.248 E-08 \\
1.930 E-02\end{array}$}} \\
\hline SH- 151 & & \\
\hline$S N-113$ & \multicolumn{2}{|c|}{$\begin{array}{l}1.052 E-07 \\
3.697 E-11\end{array}$} \\
\hline $5 R-82$ & \multirow{2}{*}{\multicolumn{2}{|c|}{$\begin{array}{l}3.697 \mathrm{E}-11 \\
1.320 E-05\end{array}$}} \\
\hline TA- 18 & & \\
\hline$T A-183$ & \multicolumn{2}{|c|}{$3.074 \mathrm{E}-22$} \\
\hline TC-99 & \multicolumn{2}{|c|}{$2.817 \mathrm{E}-02$} \\
\hline TE - 125 & \multirow{2}{*}{\multicolumn{2}{|c|}{$\begin{array}{r}1.201 E-09 \\
.000 E+00\end{array}$}} \\
\hline$T L-208$ & & \\
\hline$T M-170$ & \multicolumn{2}{|c|}{$3.304 E-08$} \\
\hline$v-49$ & \multirow{2}{*}{\multicolumn{2}{|c|}{$2.260 E-09$}} \\
\hline$w-185$ & & \\
\hline $2 N-65$ & \multicolumn{2}{|c|}{$\begin{array}{l}4.795 E-08 \\
6.632 E-04\end{array}$} \\
\hline $2 R-90$ & \multicolumn{2}{|c|}{$.000 E+00$} \\
\hline $2 R-95$ & \multicolumn{2}{|c|}{$4.587 \mathrm{E}-06$} \\
\hline & CS137 & 4. $151 E-06$ \\
\hline & CO- 58 & $1.053 E-12$ \\
\hline & $c 0-60$ & $1.673 E-04$ \\
\hline & EU155 & $2.046 E-05$ \\
\hline & $c-14$ & $4.458 E-06$ \\
\hline & BA137 & $.000 E+00$ \\
\hline & EU154 & $2.645 E-05$ \\
\hline & $F E-59$ & $1.962 E-15$ \\
\hline & MN -54 & 8. $780 \mathrm{E}-07$ \\
\hline & EU152 & $3.537 E-06$ \\
\hline
\end{tabular}

$1.515 E+04 \quad 7.491 E+00 \quad 1.217 E-01 \quad 2.351 E+01$

BE -10

B] -212

C- 14

CE- 144

Co. 60

EU-152?

EU- 154

EU-155;

FE- 55

$\mathrm{H}-3$

$1-129$

$\mathrm{K}-40$

MN -54

MO- 93

NB-93N

NB -94

NI -59

NI -60

NI -63

PB-212

PM- 147

SE -79

SH- 151

$S N-123$

TC -99

ZN- 65

2R-93
1. $060 \mathrm{E}-05$

$.000 E+00$

5.060E-01

$9.366 E-04$

$4.333 E+03$

7.234E-03

1.818E-01

$1.464 E-03$

7. $132 \mathrm{E}+02$

$1.572 E+00$

$1.642 \mathrm{E}-03$

3. $600 \mathrm{E}-05$

$4.478 E-01$

$3.949 \mathrm{E}-02$

$1.920 E-04$

2.610E-01

$8.639 E+09$

$.000 \mathrm{E}+00$

$9.955 \mathrm{E}+03$

$.000 \mathrm{E}+00$

$1.327 E-02$

1.873E- 05

$1.302 E-02$

$.000 E+00$

$6.353 \mathrm{E}-01$

$1.140 \mathrm{E}-05$

C\$137 4.907E-06

CO-58 2.739E-09

CO-60 2.542E-04
2.564E-11 
WHC-EP-0125-8

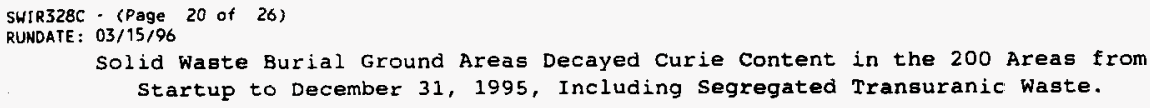

ALKaL INE METAL WASTE STORAGE UNIT 1

ALKALINE METAL WASTE STORAGE UNIT 2

ALKALINE METAL WASTE STORAGE UNII 3

ALKALINE METAL WASIE STORAGE UNIT 4

INVENTORY NOT YET VERIFIED BY SWO

FLAMMABLE STORAGE MOOULE 1

FLAMMABLE STORAGE MODULE 2

flammable storage module 3

$\begin{array}{lll}1.375 E-04 & 8.153 E-07 & 2.629 E-09 \\ 1.077 E-01 & 1.005 E-04 & 4.095 E-07 \\ 2.233 E-03 & 4.088 E-06 & 1.423 E-08 \\ 6.167 E-02 & 4.262 E-06 & 2.232 E-08 \\ 1.206 E-06 & 5.462 E-06 & 4.491 E-12 \\ 2.980 E-03 & 2.686 E-04 & 1.045 E-06\end{array}$

$2.980 E \cdot 03$

$2.686 \mathrm{E}-0.6$

2.898E-0

$C-1,4$

CE- 144

CO-58

Co-60

CR -51

FE -59

MN -54

NA-22

NB -95

$\mathrm{SC}-47$

TA- 182

TA- 183

$2 N-65$

$6.029 E-04$

$3.527 E-07$

8.397E-05

C. 14

CE -144

CS -134

EU- 152

EU- 154

EU. 155

$\mathrm{H}=3$

$I-129$

k-40

$M N-54$

NB -94

PM- 147

SE -79

TA- 18Z':

TC- 99

$2.980 \mathrm{E}+00$

$8.471 E-01$

$2.021 E-04$

$6.478 E-0$

BE -7

C. 14

CA- 45

CD- 10 ?

Co- 60

EU-15?

EU- 154

FE- 55

FE- 59

$\mathrm{H}+3$

NI -63
EU155 3.170E-05

C- $14 \quad 4.999 \mathrm{E}-06$

BA137 .000E +00

EU154 3.581E-05

FE-59 1.320E-10

MN-54 6.377E-06

EU152 4.478E-06 $.000 E+00$

$.000 E+00$ $.000 E+00$

1.131E-02

$1.087 \mathrm{E}-03$

$1.022 \mathrm{E}-02$ $1.544 \mathrm{E}-03$

1.544E-03 $.000 E+00$

$2.406 \mathrm{E}-02$

2.406E-02

1.000 E- 04

1.000E-06 $1.767 E-03$

$9.415 E-04$

$4.595 \mathrm{E}-06$

$4.219 \mathrm{E}-10$

3.817E-04

2.994E-21

$1.429 E-21$

3.824E-OS

3. $921 E-04$

8. $943 \mathrm{E}-19$

$.000 E+00$

$5.989 E-06$

$.000 E+00$

$2.752 E-06$ $1.987 \mathrm{E}-04$

$4.300 E-05$

5.081E-07

1. $786 \mathrm{E}-07$

$2.561 E-05$

4.317E- 05

$5.282 E-06$

$6.468 E-05$

$1.097 \mathrm{E}-07$

$7.850 E-08$

$3.277 \mathrm{E}-10$

$7.419 E-15$

$1.306 \mathrm{E}-05$

$4.900 \mathrm{E}-11$

4. $842 \mathrm{E}-11$

3. $017 \mathrm{E}-06$

$1.328 \mathrm{E}-02$

$4.215 E-11$

$6.016 E-03$

$8.615 E-05$

$6.743 \mathrm{E}-07$

$6.058 \mathrm{E}-04$

3. $989 \mathrm{E}-07$

8.307E-08

3. $719 E-07$

2.914E- 07

6.554E-03

2.814E-06 
WHC-EP-0125-8

SWIR328C - (Page 21 of
RUNDATE: $03 / 15 / 96$
SOlid Waste
startup to
BURIAL GROUND FACILITIES

flamMaBle STORAGE MOOULE 3

FLAMMABLE SIORAGE MODULE 4

FLAMMABLE STORAGE MOOULE 5

FLAMmable Storage MODULE 6

26)

rial Grounci Areas Decayed Curie Content in the 200 Areas from

$\begin{array}{lllll}\text { TOTAL } & \text { SR-90 } & \text { RU-106 } & \text { (S-137 OTHER RADIOACTIVITY }\end{array}$ (CURIES) (CURIES) (CURIES) (CURIES) (CURIES)

$\begin{array}{lll}\text { P-32 } & 1.446 E-13 \\ \text { PM- } 147 & 3.693 E-07 \\ \text { PO-210 } & 1.448 E-07 \\ \text { S-35 } & 6.779 E-08 \\ \text { IC-99 } & 1.260 E-05 \\ & \text { CS137 } & 4.367 E-06 \\ & \text { CO-58 } & 6.484 E-17 \\ & \text { CO-60 } & 1.328 E-04 \\ & \text { EU155 } & 1.590 E-05 \\ & \text { C-14 } & 4.976 E-06 \\ & \text { EA137 } & .000 E+00 \\ & \text { EU154 } & 2.391 E-05 \\ & \text { FE-59 } & 1.020 E-22 \\ & \text { MN-54 } & 1.187 E-07 \\ & \text { EU152 } & 3.447 E-06 \\ 03 & & 2.529 E-02\end{array}$

$\begin{array}{llll}7.467 E-02 & 1.675 E-02 \quad 1.238 E-06 \quad 8.148 E-03\end{array}$

$\mathrm{BE}-7$

$\mathrm{C}-14$

co-56

CO-57

Co-58

$\mathrm{CO}-60$

CR -51

CS- 134

EU- 154

EU- 155

H-3

1 -129

MN -54

NA-22

P-32

$P B-210$

$P M-147$

S-35

SC- 46

TC -99

$2 \mathrm{~N}-65$

$2.222 E+00$

8.818E-06 $\quad 1.855 E-06$

$6.400 E-06$

C- 14

co. 58

CO- 60

EU-154,

EU-155

FE -55

$\mathrm{H} \cdot 3$

1. 129

MN-54

NI -60

NI -63

PM- 147

IC -99

2.552E- 02

8.358E-04 1.044E-03
8. $530 \mathrm{E} \cdot 04$

BE -7

C- 14

CA -45

CD -109

Co- 60

EU- 152

FE -55

FE -59

$\mathrm{H}-3$

N] -63
2.698E- 07

5. $049 \mathrm{E}-04$

$7.357 E-05$

2.844E-03

$1.341 \mathrm{E}-04$

$1.552 \mathrm{E}-04$

i. $766 \mathrm{E}-08$

$1.184 \mathrm{E}-05$

2. $122 \mathrm{E}-05$

2.150E-05

$1.542 \mathrm{E}-02$

$1.210 E-10$

1.032E-03

$1.134 \mathrm{E}-06$

$6.686 \mathrm{E}-12$

$3.242 \mathrm{E}-05$

$2.742 \mathrm{E}-08$

1.906E-08

1.837E-06

$9.370 E-11$

5.038E-03 $2.222 \mathrm{E}+00$

4.827E-02

3.219E- 11

$5.988 E-08$

$1.289 E-07$

$2.438 E-08$

5. $894 \mathrm{E}-07$

$2.174 \mathrm{E}+00$

$5.094 E-11$

6.754E-08

$.000 E+00$

$1.374 E-07$

$3.802 \mathrm{E}-09$

6. $124 E-08$ 1. $120 \mathrm{E}-02$

2. $107 \mathrm{E}-11$

1. $373 \mathrm{E}-06$

4. 308E-05

$7.902 E-07$

3. $440 \mathrm{E}-05$

2. $165 E-08$

$1.859 E-07$

$1.449 E-0$ ?

$1.109 E-02$

$2.788 \mathrm{E}-07$ 
SWIR328C - (Page 22 of
RUNDATE: $03 / 15 / 96$
Solid Waste
Startup t
BURIAL GROUND FACILITIES

FLAMMABLE STORAGE MODULE 6

flammaBle storage mOOULE 7

flamMaBLE STORAGE MOOULE 8

FLAMMABLE STORAGE MODULE 10

fLAMmable StORAGE MOOULE 12

FLAMMABLE STORAGE MOOULE 13 urial Ground Areas Decayed Curie Content in the 200 Areas from o December 31, 1995. Including Segregated Transuranic Waste.

TOTAL SR-90 RU-106 CS-137 OTHER RADIOACTIVITY

(CURIES) (CURIES) (CURIES) (CURIES) (CURIES)

(CURIES) (CURIES) (CURIES) (CURIES)

(.....................

P-32 $7.232 \mathrm{E}-14$

PM- $147 \quad 1.996 \mathrm{E}-05$

PO-210 7.827E-08

$5-35 \quad 3.642 E-08$

TC-99 $9.150 \mathrm{E}-06$

$\operatorname{cs} 137 \quad 1.429 \mathrm{E}-10$

CO-58 $4.942 \mathrm{E}-11$

CO-60 8.903E-09

EU155 1.126E-09

C- $14 \quad 1.400 E-10$

B.A $137 \quad .000 E+00$

EUU154 1.152E-09

FE-59 1.318E-10

MN-54 7.250E-10

EU152 1.371E-10

$1.197 E+00 \quad 6.578 E-04 \quad 2.238 E-06 \quad 6.180 E-04$

C- 14

$1.194 E+00$

8.213E-03

CE- $144 \quad 4.106 E-06$

CO-60 1.096E-06

EU-154 $6.112 E-09$

EU-155 2.715E-09

$\mathrm{H}-3 \quad 1.186 \mathrm{E}+00$

$1-129 \quad 1.815 E-08$

MN-54 3.766E-10

NI $-60 \quad .000 E+00$

NI-63 9.733E-10

PM-147 6.832E-05

$\mathrm{TC}-99 \quad 4.802 \mathrm{E}-07$

$\begin{array}{llll}1.100 E-02 & 8.096 E-05 & 3.698 E-07 & 8.715 E-05\end{array}$

[- 14

$\mathrm{H}-3$

$\begin{array}{llll}8.249 E-02 & 1.213 E-04 & 1.019 E-04 & 1.253 E-04\end{array}$

C. 14

Co- 60

$\mathrm{H}-3$

P-32

PB -210

PO. 210

$1.319 E-04 \quad .000 E+00 \quad .000 E+00 \quad .000 E+00$

$s-35$

C- 14

CA- 45

CD- 109

FE- 59

$\mathrm{H}-3$

NI -63

P. 32

$4.802 E-07$
$1.400 E-10$

$1.063 E-02$

5.959E-03

4. $675 \mathrm{E}-03$

B. $095 \mathrm{E}-02$

$1.780 E-04$

$1.956 \mathrm{E}-06$

8.020E-02

$1.634 \mathrm{E}-10$

4. $858 \mathrm{E}-09$

$1.630 \mathrm{E}-06$

$5.616 \mathrm{E}-04$

$2.586 \mathrm{E}-05$

$6.605 \mathrm{E}-07$

8.716E-09

B. 266 E-09

3.574E-05

$1.526 E-05$

2.588E-18

TC-90 5.436E-05

$\begin{array}{cccc}7.429 E-02 & 2.552 E-03 & 1.208 E-03 \quad 1.548 E-03\end{array}$

5.360

BE-7 $1.419 E-12$

C. $14 \quad 5.626 E-05$

CA-45 2.757E-06

CD $-109 \quad 1.212 E-08$

CE-164 3.054E-08

CO- $58 \quad 6.142 \mathrm{E}-13$

CO-60 7.219E-06

EU-152 1.645E-05

EU-154 2.710E-05

EU-155 9.334E-07

FE- $55 \quad 1.190 \mathrm{E}-08$ 
SHIR328C - (Page 23 of 26 )

RUNOATE: 03/15/96

Solid waste Burial Ground Areas Decayed Curie Content in the 200 Areas from Startup to December 31, 1995, Including Segregated Transuranic Waste.

BURIAL GROUND FACILIIIIES

FLAMMABLE STORAGE MOOULE 13

FLAMMABLE STORAGE MOOULE 16

FLAMMABLE STORAGE MODULE 15 $\begin{array}{cccc}\text { TOTAL } & \text { SR-90 } & \text { RU-106 } & \text { CS-137 } \\ \text { (CURIES) } & \text { (CURIES) } & \text { (CURIES) } & \text { (CURIES) }\end{array}$ (CURIES)

(CUR IES)

(CURIES)

(CURIES)
(.........

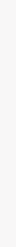

OTHER RADIOACTIVITY (CURIES)

(CURIES)

FE-59 9.761E-0

$\mathrm{H}-3$ 5.346E-02

I-129 $1.280 \mathrm{E}-12$

MN-54 1.153E-09

NA-22 7.076E-06

NB-94 2.930E-15

NI $-60 \quad .000 E+00$

NI.63 2.320E.09

P-32 4.914E-15

PM- 147 1.826E-06

PO- $210 \quad 7.117 E-10$

S-35 $1.083 E-07$

SE-79 $1.900 E-11$

TC-99 1.343E-05

$2 \mathrm{~N}-65 \quad 5.279 \mathrm{E}-11$

2R-95 5.867E-09

BE-7 2.107E-11

C- 14 १.650E-04

CA- $45 \quad 4.308 E-05$

CD-109 1.791E-07

CE-144 8.230E-05

CO-60 3.856E-05

CS- $134 \quad 5.209 \mathrm{E}-07$

CS-135 5.610E-13

EU- 152 1.757E-07

EU-154 2.578E-06

EU-155 1.500E-06

FE-55 1.859E-07

FE-59 1.449E-07

$\mathrm{H}-3$ 1.891E-02

1-129 9.283E-07

NB-94 $\quad 5.559 E-15$

NI $-60 \quad .000 E+00$

NI $-63 \quad 1.115 E-06$

P-32 7.232E-14

PD- 107 1.130E-13

PM- $147 \quad 7.517 E-04$

PO-210 2.454E-10

S-35 8.294E-11

SB-125 9.252E-09

SE-79 3.670E-11

SM-151 4.822E-04

IC- $99 \quad 2.497 \mathrm{E}-05$

TE-125 $1.476 E-13$

2R-Q3 $3.930 E-12$

5.234E-03

[-14 1.413E-07

CD-105, 3.161E-07

CO-60 $\quad 5.476 \mathrm{E}-08$

$\mathrm{H}-3 \quad 5.184 \mathrm{E}-03$

I- $125 \quad 3.524 \mathrm{E}-05$

$1.129 \quad 9.900 E-10$

K-40 4.400E - 08

N1. $63 \quad 2.282 \mathrm{E}-07$

$\mathrm{P} \cdot 32 \quad 3.786 \mathrm{E}-22$

PM- $147 \quad 8.434 \mathrm{E}-06$

PO- $210 \quad 7.423 \mathrm{E}-08$

RB-86 $9.167 \mathrm{E}-12$

S-35 3.390E-08

SR-85 2.031E-06

TC-99 $3.445 E-06$ 
WHC-EP-0125-8

SWIR328C - (Page 24 of 26 )

RUNDATE : 03/15/96

Solid Waste Burial Ground Areas Decayed Curie Content in the 200 Areas from Startup to December 31, 1995. Including Segregated Transuranic Waste.

\begin{tabular}{|c|c|c|c|c|c|}
\hline $1 \mathrm{con}$ & $\begin{array}{l}\text { TOTAL } \\
\text { (CUR!ES) }\end{array}$ & $\begin{array}{l}\text { SR }-90 \\
\text { (CURIES) }\end{array}$ & $\begin{array}{c}\text { RU-106 } \\
\text { (CURIES) }\end{array}$ & $\begin{array}{l}\text { CS }-137 \\
\text { (CURIES) }\end{array}$ & $\begin{array}{l}\text { OTHER RADIOACT IVITY } \\
\text { (CURIES) }\end{array}$ \\
\hline & $\cdots+\cdots$ & $\ldots \ldots$ & 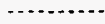 & - & \\
\hline
\end{tabular}

flamkable storage mOOULE 16

$3,500 E-02$

$1.916 \mathrm{E}-03$

4.950E-05

$1.337 E-02$

$A G-110$

C. 14

$4.856 \mathrm{E}-03$

$.000 E+00$

CE-60 $6.327 \mathrm{E}-05$

CS-134 $1.242 E-05$

EU-152 $1.912 \mathrm{E}-06$

EU-154 1.972E-05

EU-155 6.327E-06

$\mathrm{H}-3 \quad 1.924 \mathrm{E}-03$

I-129 3.866E-10

MN-54 5.296E-09

PM- $147 \quad 1.051 E-03$

SB-125 2.147E-06

SM-151 $\quad 1.051 \mathrm{E}-03$

IC- $99 \quad 3.002 E-10$

FLAMMABLE STORAGE MODULE 17

$2.798 E+00 \quad 1.233 E-02 \quad 1.549 E-05$

5.427E-02

ZR-95 $\quad 1.605 E-15$

C- $14 \quad 1.537 \mathrm{E}-03$

CA-45 1.830E-05

CE-164 $1.530 E-06$

C0-56 6.700E-08

CO-57 1.652E-03

CO-58 1.919E-04

CO-60 1.726E-01

$\mathrm{CR}+51 \quad 5.407 \mathrm{E}-11$

CS-134 5.847E-05

EU. $152 \quad 5.986 E-06$

EU-154 1.201E-05

EU. $155 \quad 6.787 E-06$

FE $-55 \quad 1.361 E+00$

FE-59 1.825E-09

$\mathrm{H}-3 \quad 5.651 \mathrm{E}-01$

HF-18, $3.793 E-09$

I-129 $\quad 1.000$ E-02

MN-54 1.251E-01

MO-93 2.702E-01

NB-91 4.192E-08

NB-93M 5.617E-02

NB-94 6.499E-07

NB-95 1.551E-11

$\mathrm{NI}-60 \quad .000 \mathrm{E}+00$

NI $-63 \quad 8.596 E-02$

P-32 1.688E-11

PM- 147 1.350E-09

PO-210 8.537E-07

SB-125: 2.679E-06

SC $-46 \quad 1.804 E-06$

SI $-31 \quad .000 E+00$

TA-18? 2.356E-05

TC-99 $1.307 \mathrm{E}-02$

$\begin{array}{lll}11-51 & .000 \mathrm{E}+00\end{array}$

$V-49 \quad 3.549 E-03$

H- $185 \quad 8.465 E-04$

$2 N-65 \quad 3.773 E-05$

$2 R-95 \quad 1.478 E-10$

FLAMMABLE STORAGE MODULE 18

$2.054 E-02 \quad 3.346 E-03 \quad 1.535 E-04$

$3.722 \mathrm{E}-03$

C- 14

CE -144

$\mathrm{H}-3$

PM+ 147

SM- 151 $5.753 E-03$

8.716E-04

$2.633 E-04$

4. $959 E-04$

2.111E-03

$1.925 \mathrm{E}-03$ 
SWIR328C - (Page 25 of 26)

RUNDATE: $03 / 15 / 96$

Solid Waste Burial Ground Areas Decayed Curie content in the 200 Areas from Startup to December 31, 1995, Including Segregated Transuranic Waste.

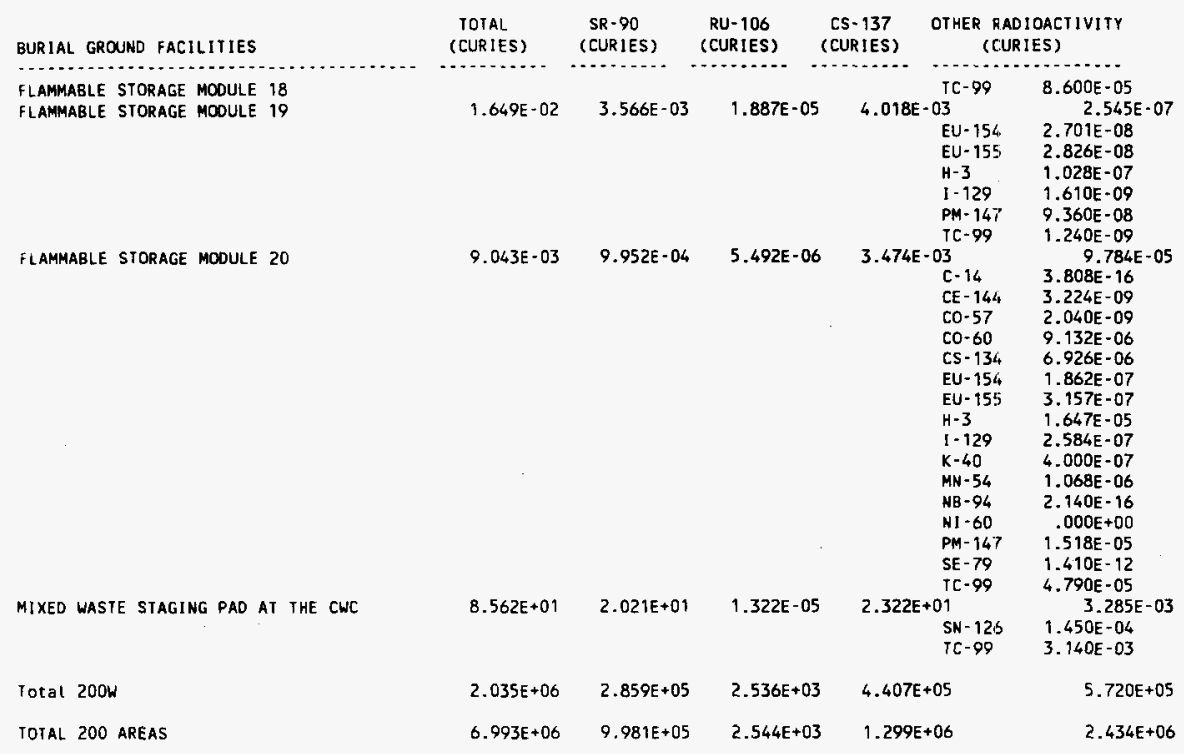

TOTAL 200 AREAS

SPECIAL CAIISSON/TRENCH BREAKOUT FOR FACILITY 218W4B

CAISSON - ALPHA
CAISSON - ALPHA
CAISSON - ALPHA 3
CAISSON - ALPHA 4
CAISSON - NO. 1
CAISSON - NO. 2
CAISSON - NO. 3
CAISSON - NO. 4
CAISSON - NO. 5
CAISSON - NO. 6

$\begin{array}{ll}1.695 E+03 & 4.103 E+02 \\ 6.181 E+03 & 1.495 E+03 \\ 1.159 E+04 & 2.803 E+03 \\ 1.132 E+04 & 2.662 E+03 \\ & \\ 1.337 E+03 & 2.476 E+02 \\ & \\ 8.658 E-01 & 2.094 E-01 \\ 2.666 E+02 & 6.205 E+01 \\ & \\ 2.656 E+03 & 6.422 E+02 \\ 7.013 E+02 & 1.250 E+02 \\ 1.323 E+04 & 1.123 E+03\end{array}$

$5.149 E-05$

$3.636 E-04$

$6.817 \mathrm{E}-04$

5.514E- 03

$1.638 E+03$

$3.072 E+03$

2.917E+03

$3.000 E-06$

$1.279 E+02$

CO. 60 PO. 210

CE - 14.4

$\mathrm{H}+3$

$8.456 \mathrm{E}-09$

2.302E-01

$3.350 E-06$

$7.321 \mathrm{E}+01$

CE $-14,4$

4. 980 E- 05

$3.039 E-05$

$8.130 \mathrm{E}-01$
7.052E+02

2. $318 \mathrm{E}+02$

Co- 60

$\mathrm{CR}+51$

CS- 134

EU- 154

FE - 59

$M N-5$ is

SB- 125

ZR- 95
$.000 E+00$ $.000 E+00$ $.000 E+00$ $3.118 E+02$

$3.118 E+02$

$1.051 \mathrm{E}-12$

$5.938 \mathrm{E}+02$

$1.822 \mathrm{E}-13$

$5.936 E+02$

2.385E-01 $.000 \mathrm{E}+00$ 2. $562 \mathrm{E}-01$

$1.598 \mathrm{E}-08$

2.562E-01 $.000 E+00$ $.000 E+00$ 8. $203 E+03$ 8. $203 \mathrm{E}+03$

1. $625 E-39$

4.215E-02

1.354E-01

$3.976 E-15$

$1.941 \mathrm{E}-02$

4.260E-03

$2.554 E-13$ 
SWIR328C - (Page 26 of 26 )

RUNDATE: 03/15/96

Solid Waste Burial Ground Areas Decayed Curie Content in the 200 Areas from Startup to December 31, 1995, Including Segregated Transuranic Waste.

$\begin{array}{lcccc} & \text { IOIAL } & \text { SR-90 } & \text { RU-106 } & \text { CS-137 OTHER RADIOACTIVITY }\end{array}$

BURIAL GROUND FACILITIES

(CURIES) (CURIES) (CURIES) (CURIES) (CURIES)

SPECIAL CAISSON/TRENCH BREAKOUT FOR FACILITY 218 $18 \mathrm{~B}$

CAISSON - UNI NO. 1

IRENCHES

218w4B Totals
$1.852 E+01$

$.000 E+00$

$.000 E+00$

$7.325 E+04$

$5.185 E+03$

$1.207 E-03$

8.209E-01
8.994E-01

$\begin{array}{ll}\text { BA } 137 & .000 E+00 \\ C-14 & .000 E+00 \\ \text { CO-58 } & 1.248 E+00 \\ \text { CO-60 } & 1.021 E-24 \\ \text { CS137 } & 1.079 E+01 \\ \text { EU152 } & 8.994 E-01 \\ \text { EU154 } & 5.508 E-01 \\ \text { EU155 } & 2.978 E+00 \\ \text { FE-59 } & 1.203 E+00 \\ \text { MN-54 } & 2.783 E-38 \\ & 2.963 E-05 \\ & 5.175 E+04\end{array}$

$5.689 E+03$ $\mathrm{CE}-14$
$\mathrm{H}-3$
$\mathrm{I}-129$

$5.491 \mathrm{E}+13$

$5.174 E+04$

5. $000 E-01$

PM- 147

$9.171 E+00$

BA137 .000E+00

I: $-14 \quad 1.016 \mathrm{E}+01$

150-58 2.679E-28

$100-60 \quad 2.675 E+01$

1.5137 $5.909 \mathrm{E}+00$

EU152 2.755E+00

EU154 1.145E+01

EU155 2.781E+00

FE-59 $1.232 E-43$

IMN-54 2.398E-06

$6.086 E+04$ 


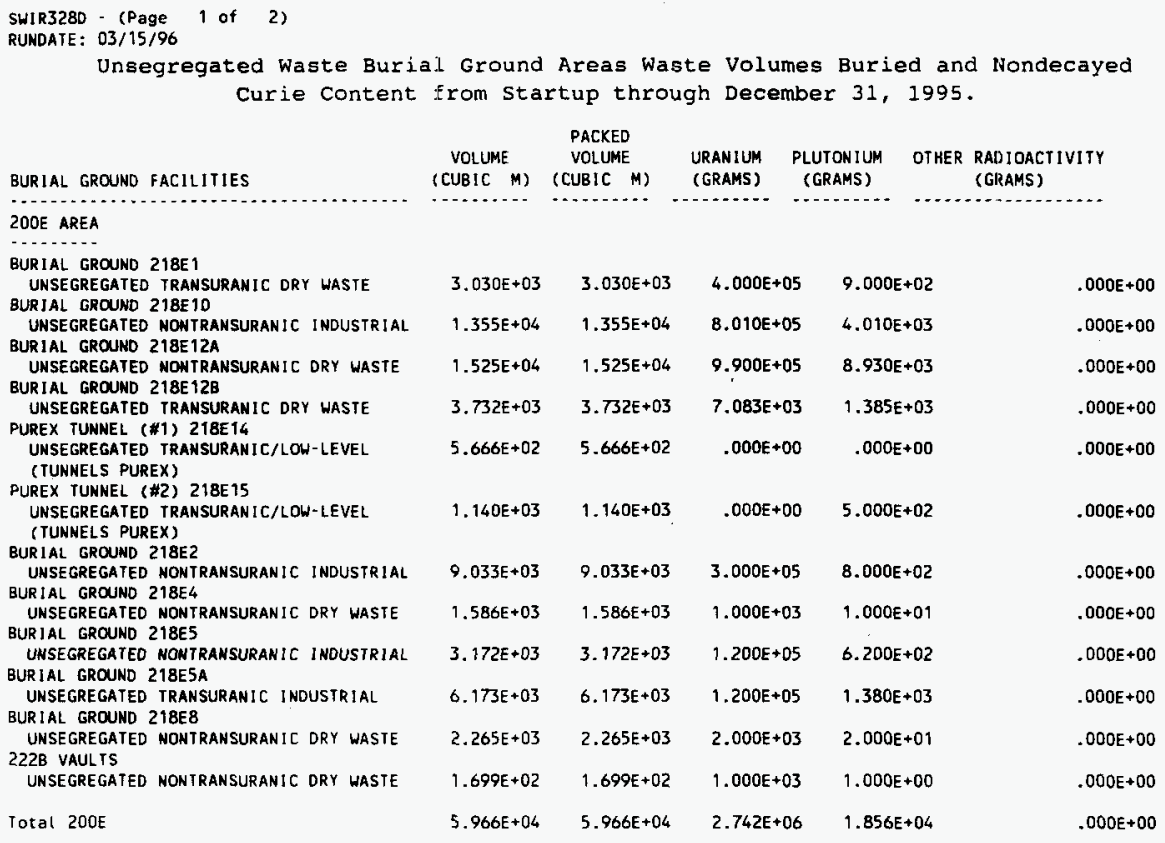


WHC-EP-0125-8

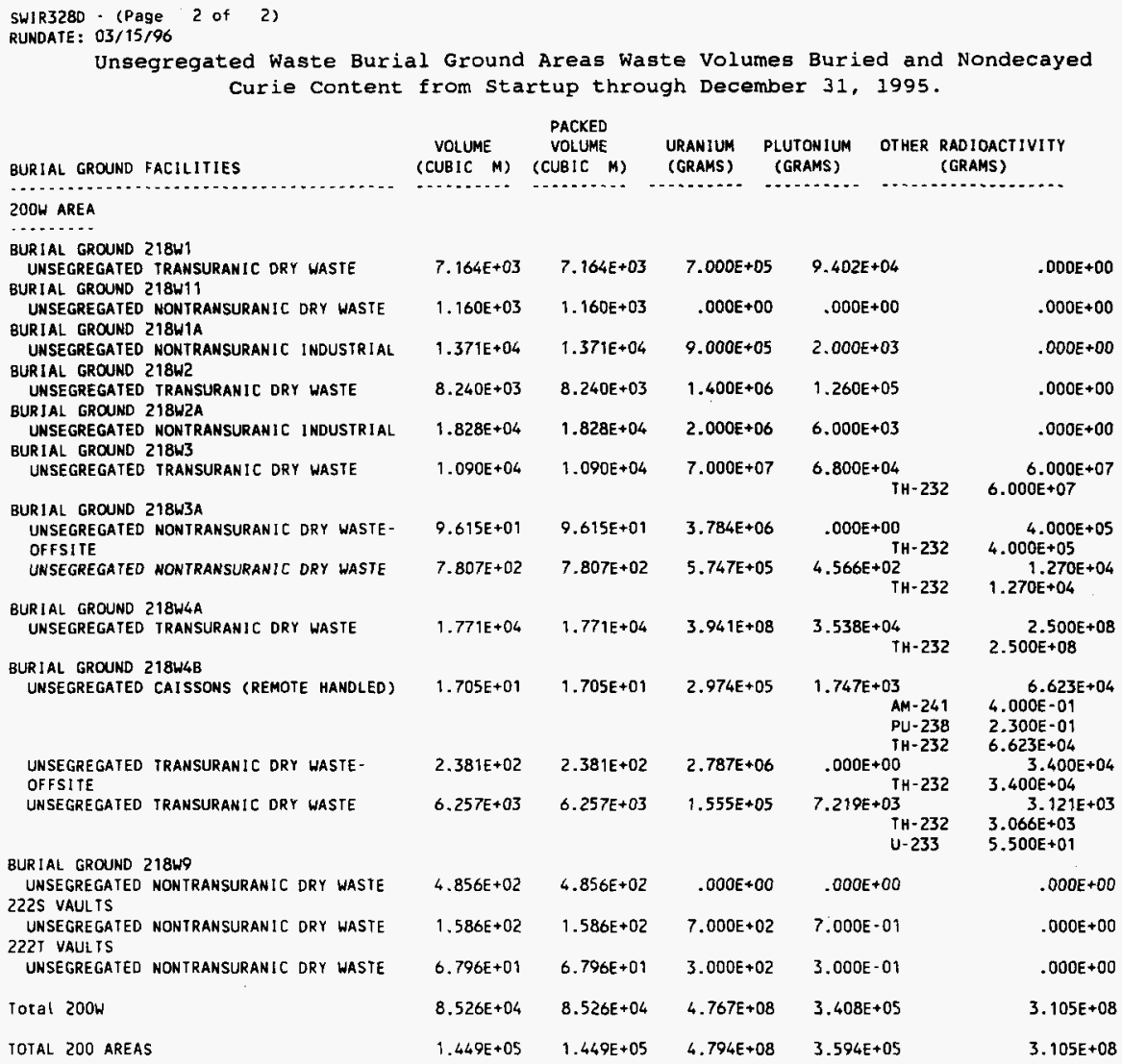




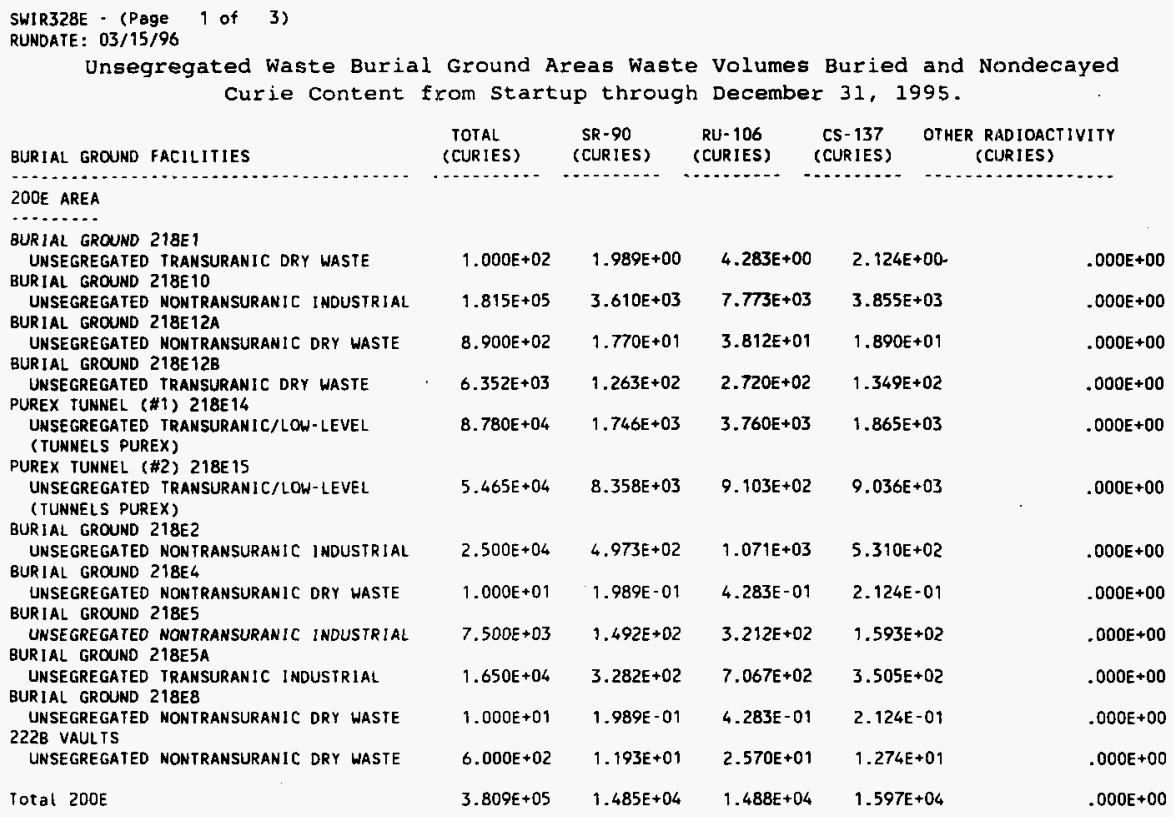




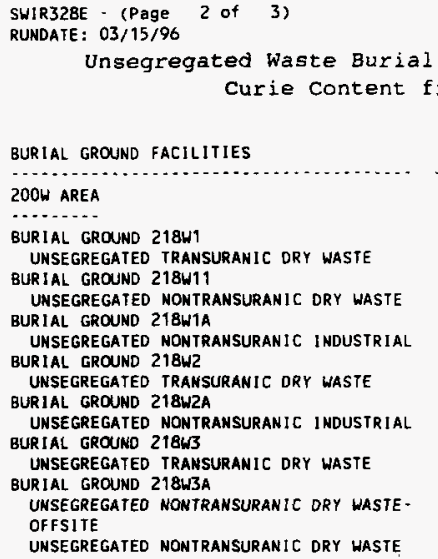

$\begin{array}{lll}3.815 E+03 & 6.594 E+01 & 1.420 E+02 \\ 7.937 E+04 & 1.783 E+03 & 3.229 E+03 \\ 3.393 E+04 & 7.120 E-03 & 1.533 E-02 \\ 2.297 E+05 & 5.358 E+02 & 1.154 E+03\end{array}$

$\begin{array}{lllll}1.000 E-01 & 1.989 E-03 & 4.283 E-03 & 2.124 E-03 & .000 E+00 \\ 4.030 E+03 & 8.016 E+01 & 1.726 E+02 & 8.560 E+01 & .000 E+00 \\ 7.700 E+02 & 1.532 E+01 & 3.298 E+01 & 1.635 E+01 & .000 E+00\end{array}$


WHC-EP-0125-8

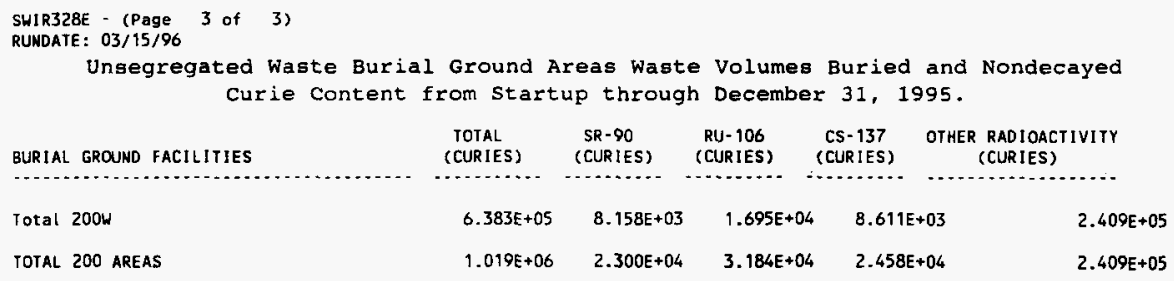




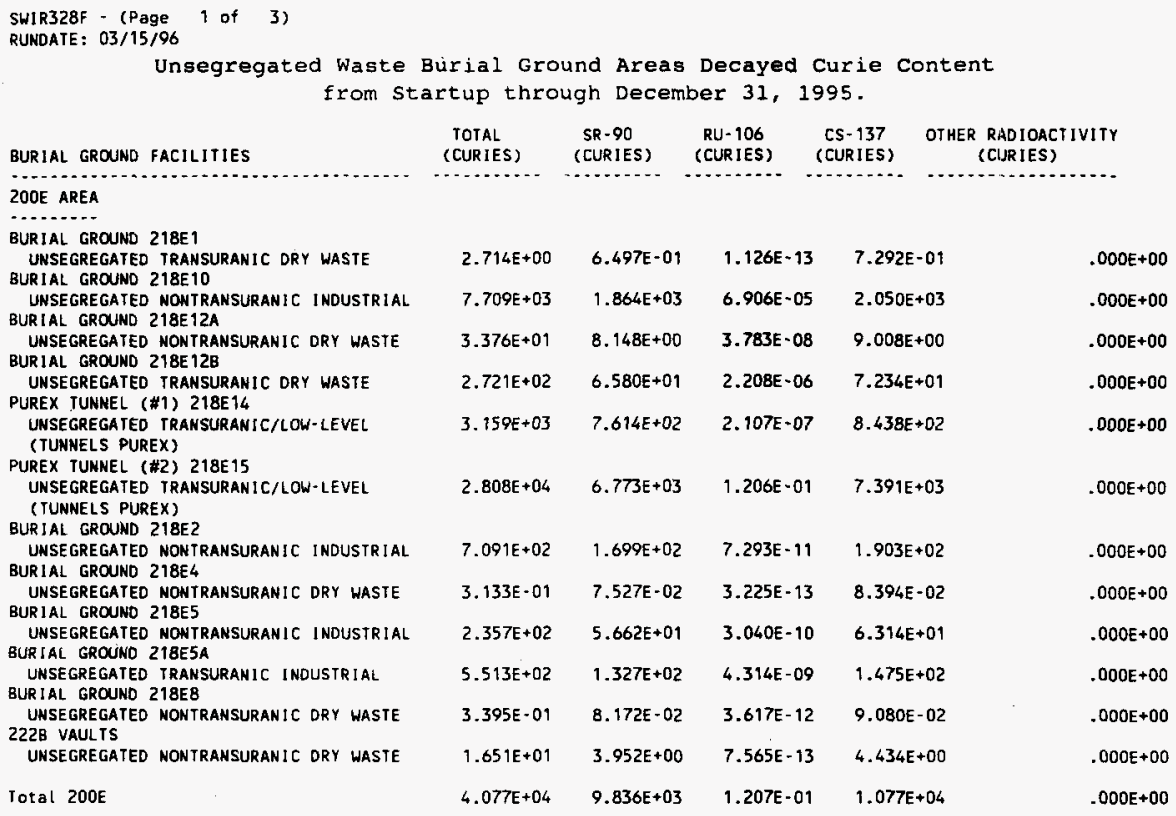


SWIR328F - (Page 2 of 3)

RUNDATE: $03 / 15 / 96$

Unsegregated Waste Burial Ground Areas Decayed Curie Content

from startup through December 31, 1995.

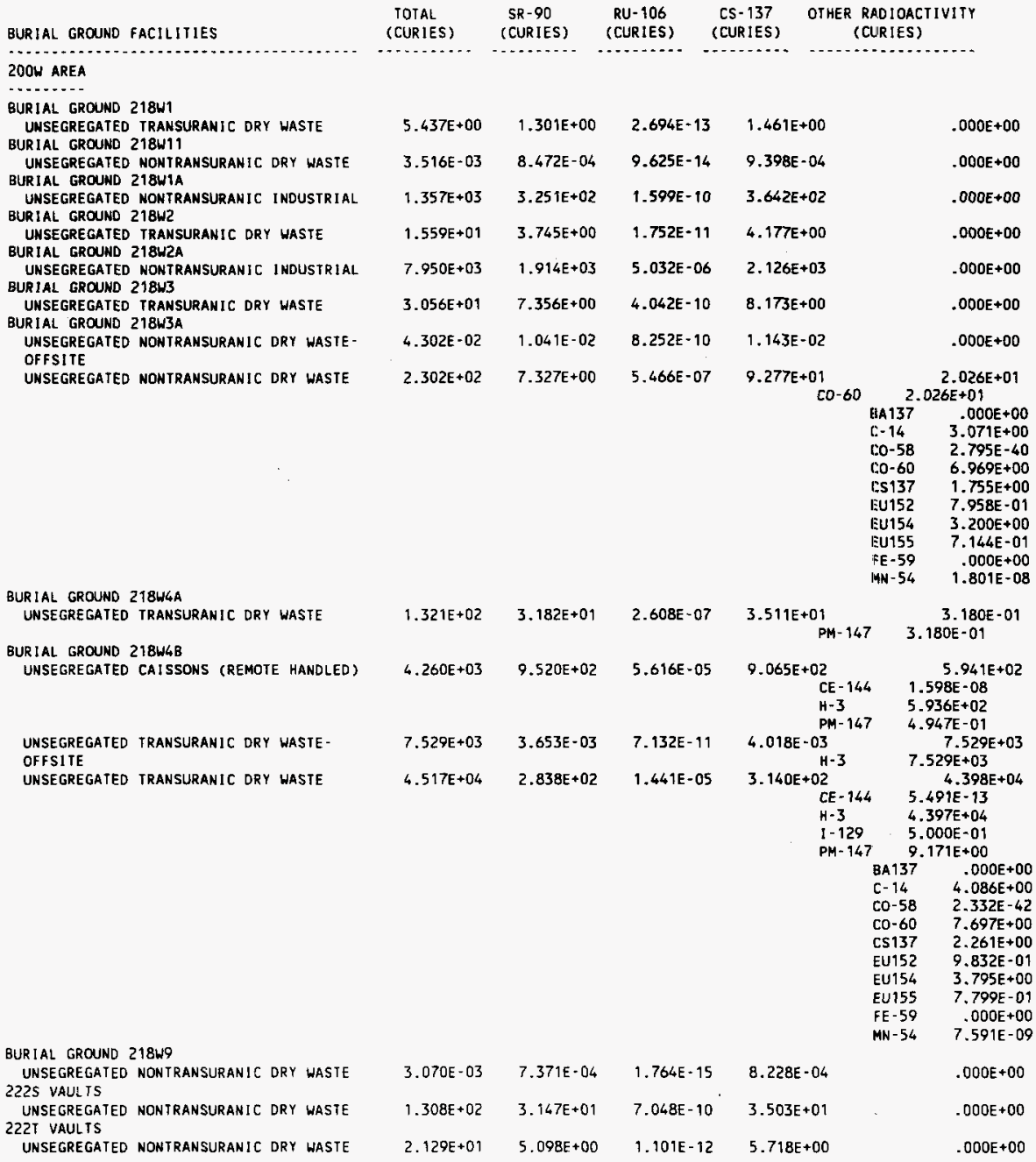


WHC-EP-O125-8

SWIR328F - (Page 3 of 3)

RUNDATE: 03/15/96

Unsegregated Waste Burial Ground Areas Decayed Curie Content from startup through December 31, 1995.

BURIAL GROUND FACILJTIES TOTAL SR-90 RU-106 CS-137 OIHER RADIOACTIVITY

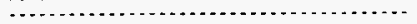
(CURIES) (CURIES) (CURIES) (CURIES) (CURIES)

Total 200w

$6.683 E+04 \quad 3.563 E+03 \quad 7.641 E-05 \quad 3.893 E+03$

$5.212 E+04$

TOTAL 200 AREAS

$1.076 \mathrm{E}+05$

$1.340 E+04$

$1.207 E-01$

$1.467 E+04$

$5.212 E+04$ 


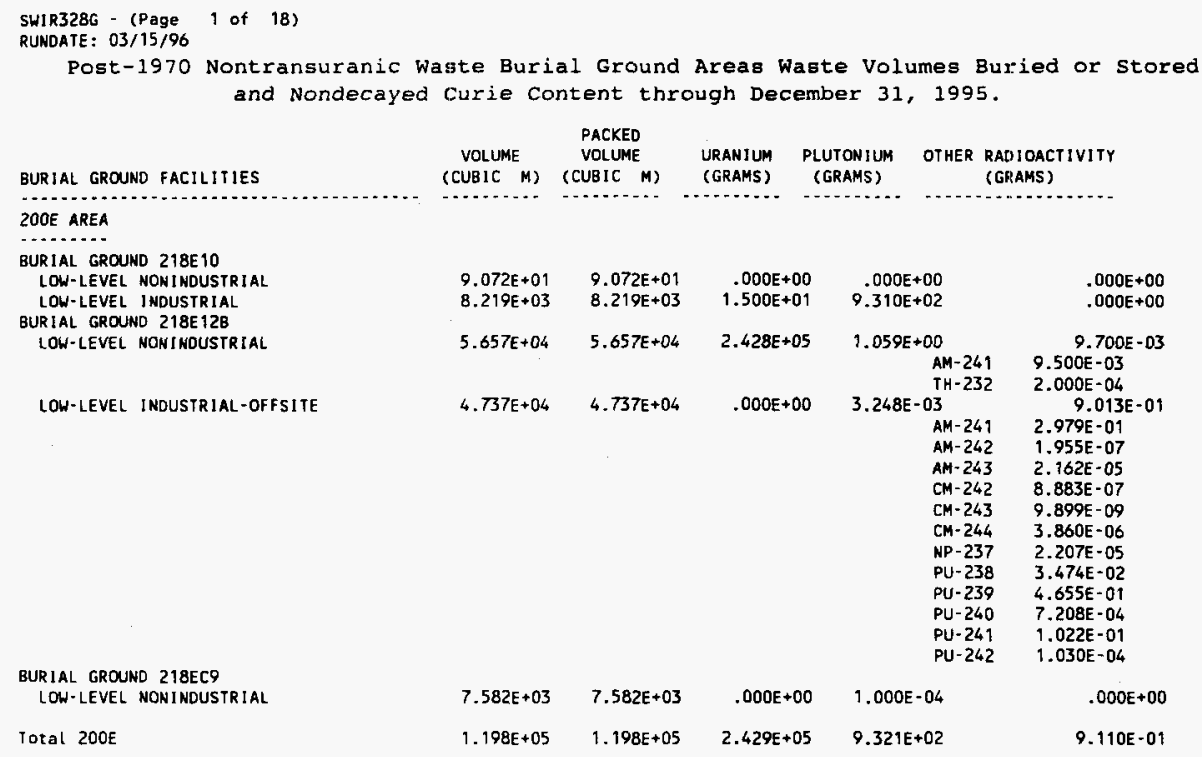




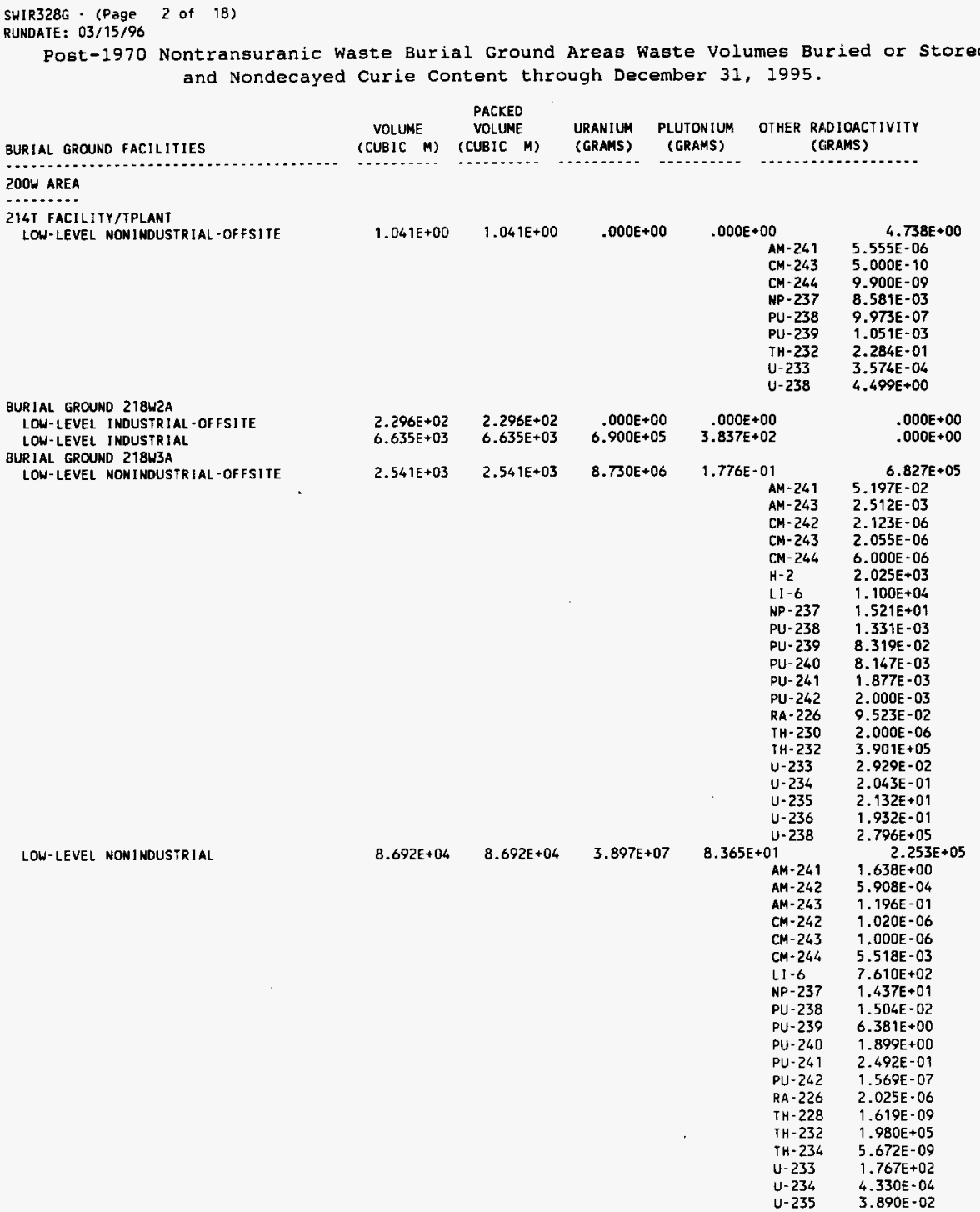




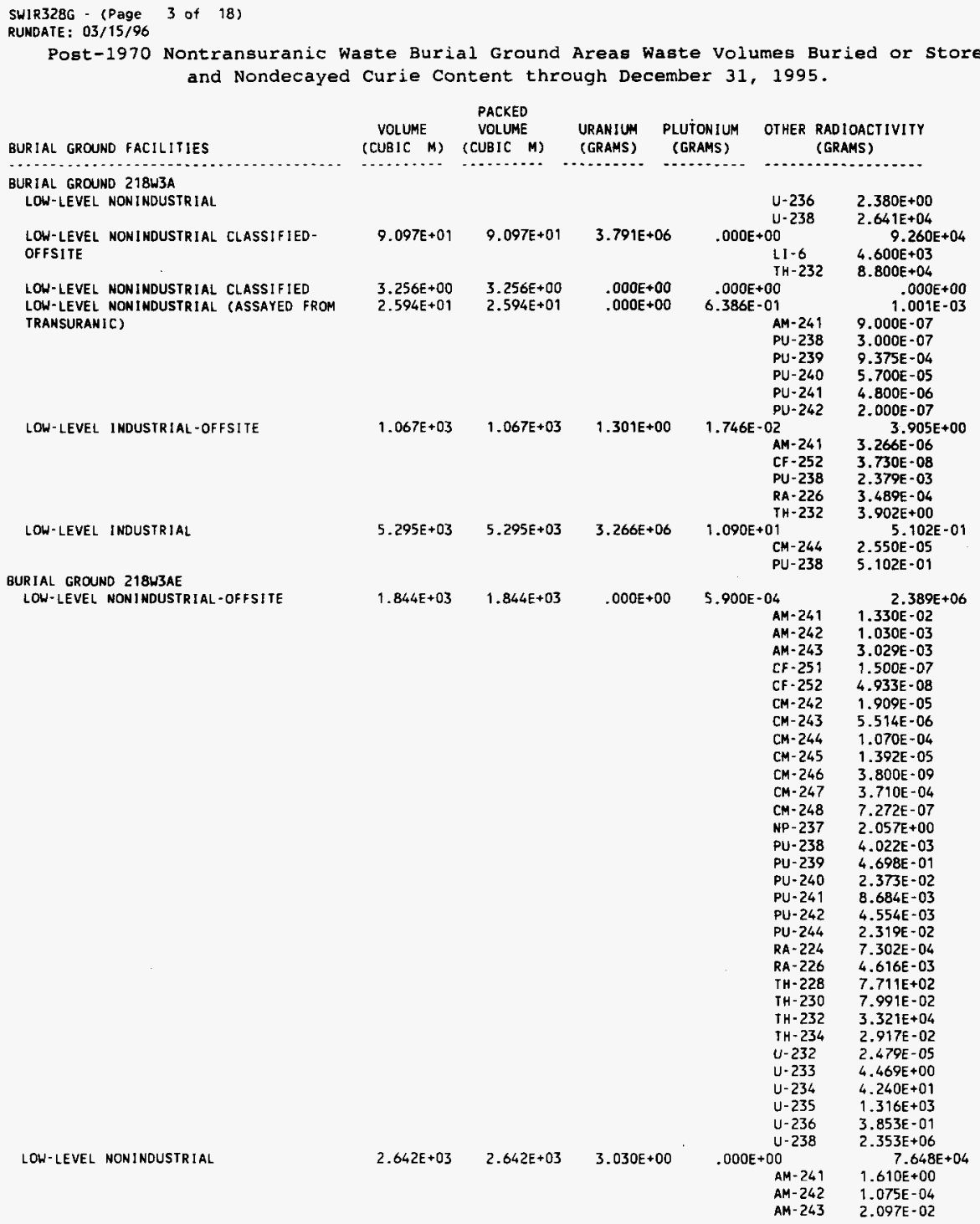




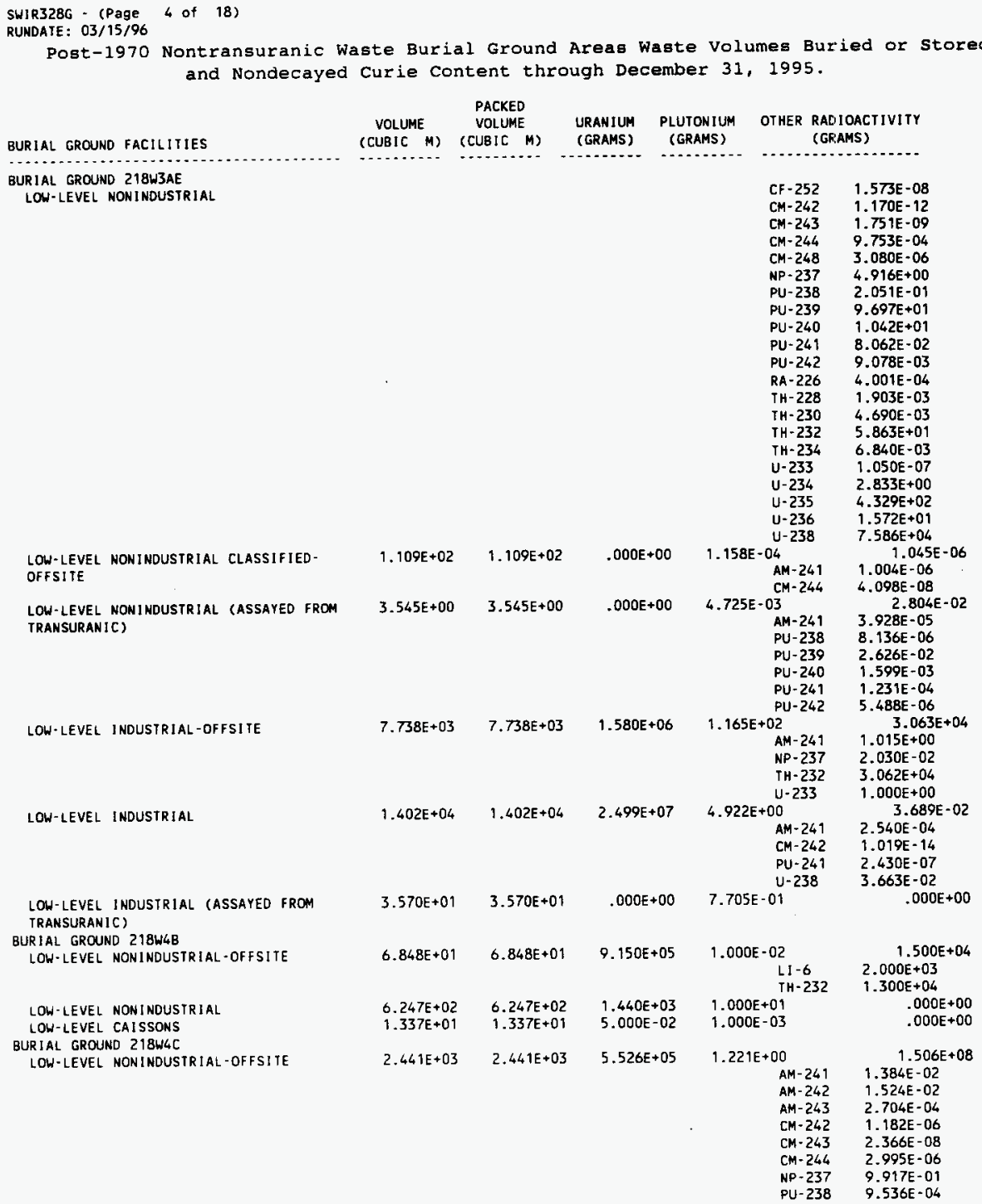




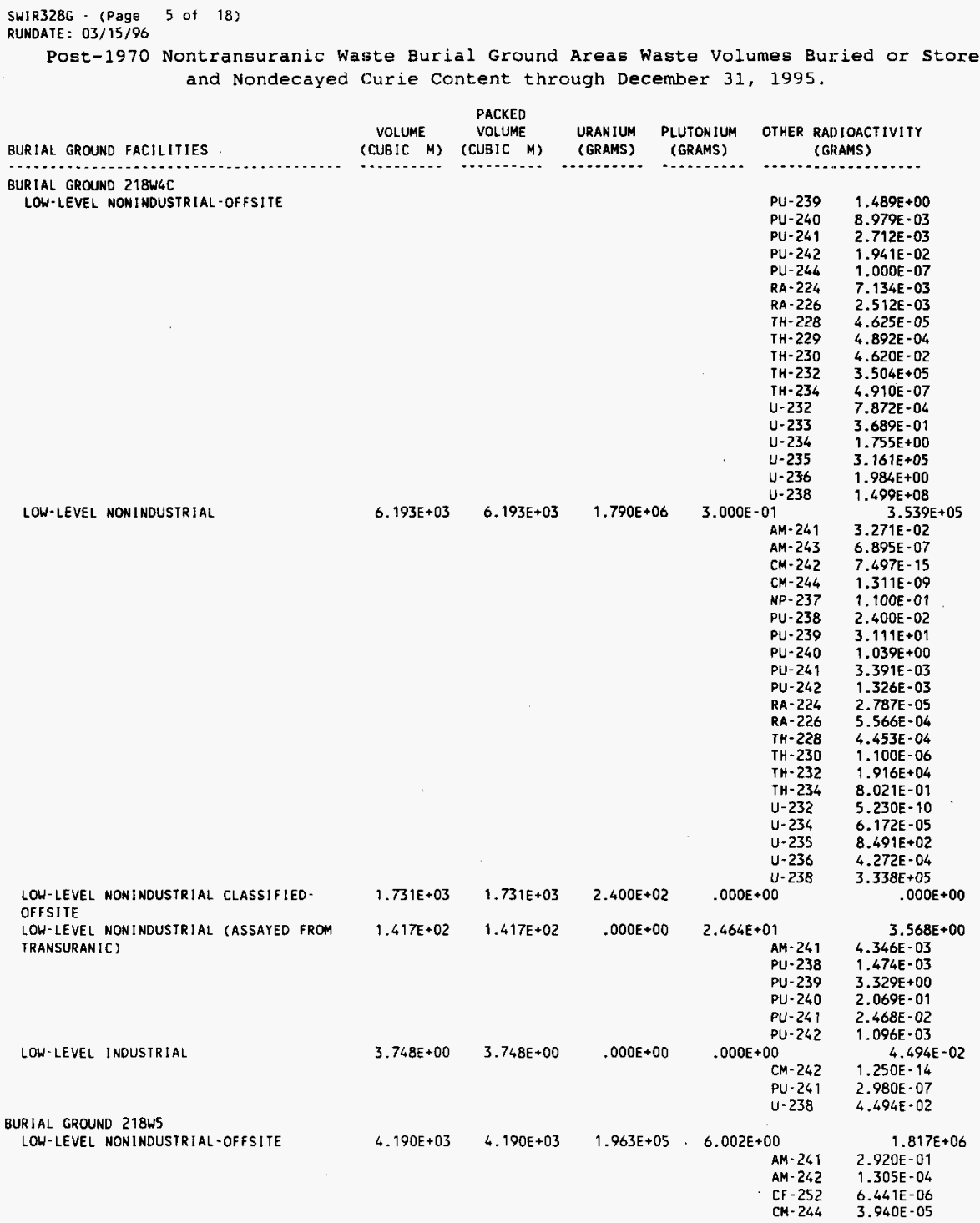




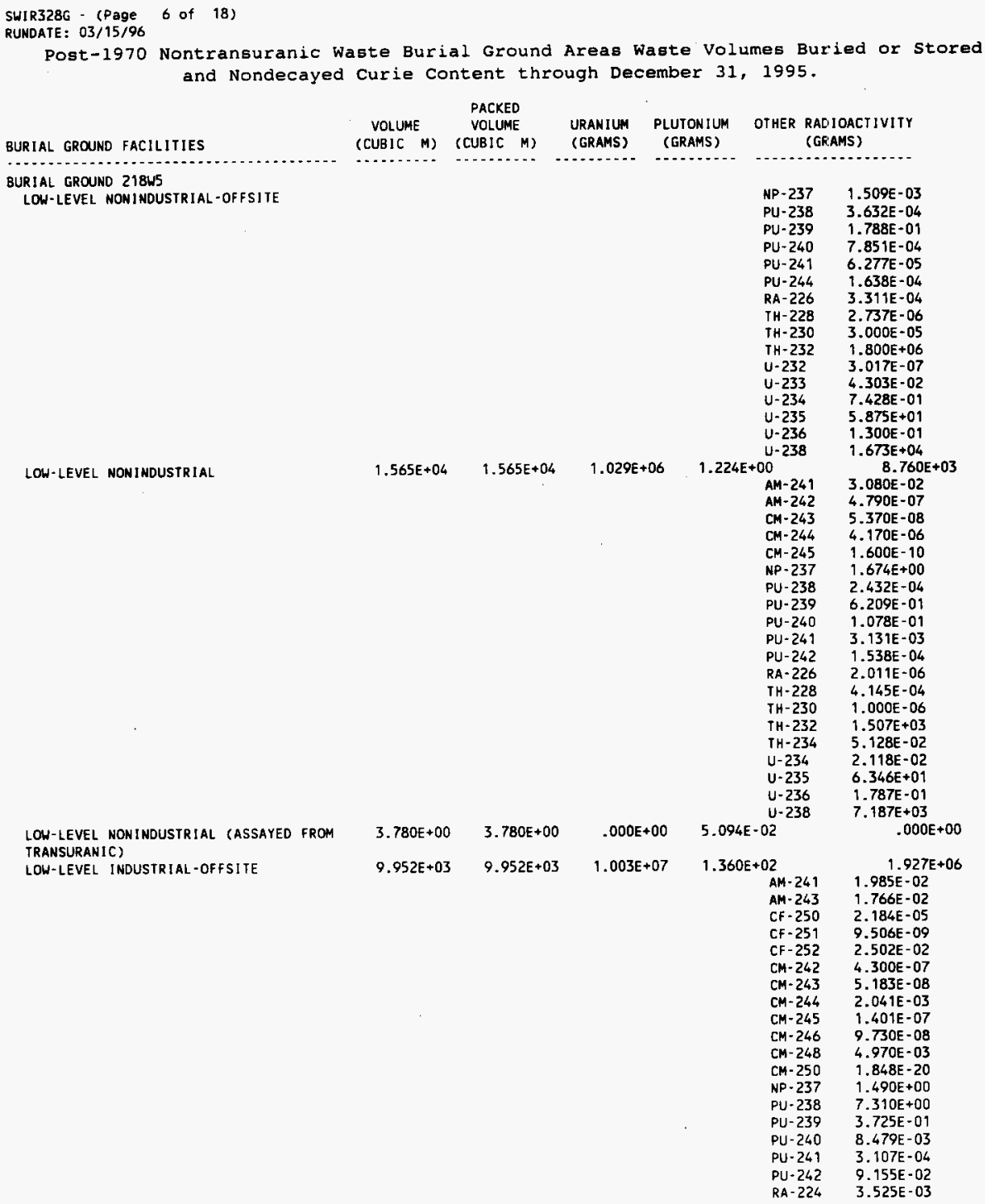

AP -237

PU- 238

PU. 239

PU-240

PU-241

PU -244

RA- 226

IH -228

TH -230

TH-232

$\mathrm{u}-232$

$\mathrm{u}-233$

$U-234$

$\mathrm{U}-235$

U-236

U- 238

ION-LEVEL NONINDUSTRIAL

TRANSURANIC)

LOW-LEVEL INDUSTRIAL-OFFSITE

(1.565e+04

3. $780 E+00$

3. $780 E+00$

$.000 \mathrm{E}+00$

$9.952 E+03$

$9.952 E+03$

AM- 241

AM- 242

CM- 243

CM- 244

CH- 245

NP. 237

PU- 238

PU- 239

PU -240

PU- 241

PU-242

RA- 226

TH- 228

TH- 230

TH-232

TH-234

$\mathrm{U}-234$

U-235

U-236

u-238

$1.003 E+07$

5. $094 \mathrm{E}-02$

$1.360 \mathrm{E}+02$

AM- 241

AM- 243

CF -250

CF -251

CF -252

CM- 242

CM- 243

CM- 244

CM- 245

CM- 246

CM- 248

CM-250

NP. 237

PU- 238

PU- 239

PU -240

PU- 241

PU -242

RA-224

1.509E-03

3. $632 E-04$

1.788E-01

$7.851 \mathrm{E}-04$

$6.277 \mathrm{E}-05$

$1.638 \mathrm{E}-04$

3.311E-04

2.737E-06

3.000E-05

$1.800 E+06$

3. $017 \mathrm{E}-07$

4.303E-02

7.428E- 01

$5.875 \mathrm{E}+01$

$1.300 E-01$

$1.673 E+04$ $8.760 E+03$

3. $080 \mathrm{E}-02$

4. $790 \mathrm{E}-07$

5. 370E-OB

4. $170 \mathrm{E}-06$

1. $600 \mathrm{E}-10$

$1.674 \mathrm{E}+00$

2.432E-04

6.209E-01

1.078E-01

3. $131 E-03$

$1.53 B E-04$

2. $011 \mathrm{E}-06$

4. 145 E- 04

1. $000 \mathrm{E}-06$

$1.507 \mathrm{E}+03$

$5.128 E-02$

2.118E-02

$6.346 E+01$

1. $787 \mathrm{E}-01$

$7.187 E+03$

$.000 E+00$

$1.927 E+06$

1.985E-02

$1.766 \mathrm{E}-02$

2. 184 E- 05

9.506E-09

2.502E-02

4. 300E-07

5. $183 E-08$

2. $041 \mathrm{E}-03$

1. $401 \mathrm{E}-07$

$9.730 E-08$

$4.970 \mathrm{E}-03$

1. $848 \mathrm{E}-20$

$1.490 E+00$

7.310E+00

3. $725 \mathrm{E}-01$

8.479E-03

3. $107 E-04$

$9.155 \mathrm{E}-02$

3. $525 E-03$ 


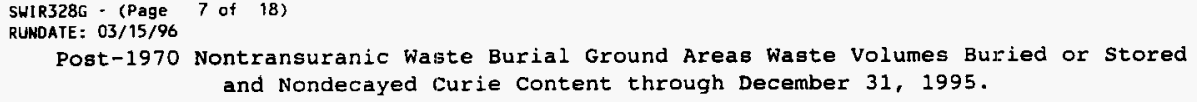

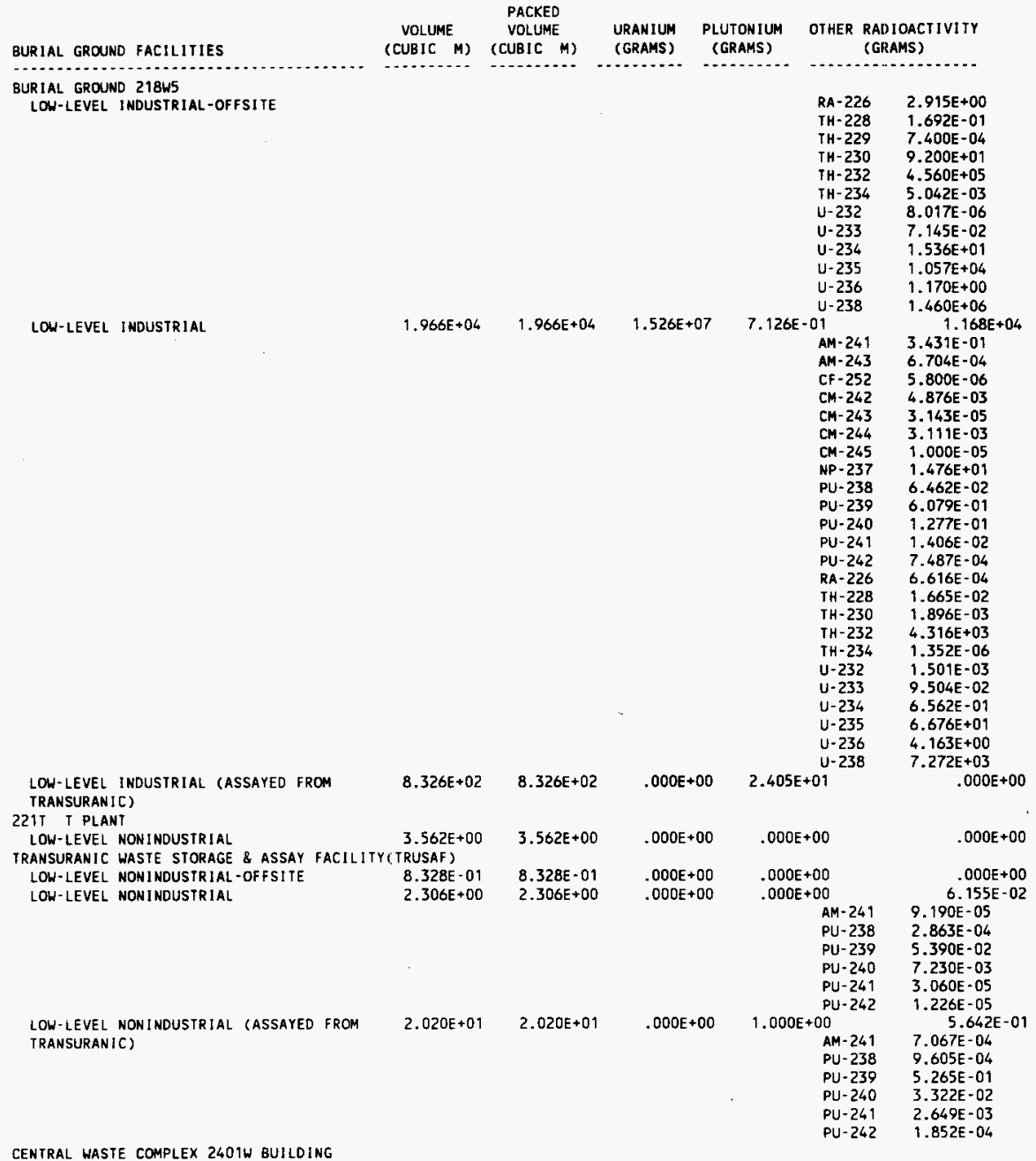




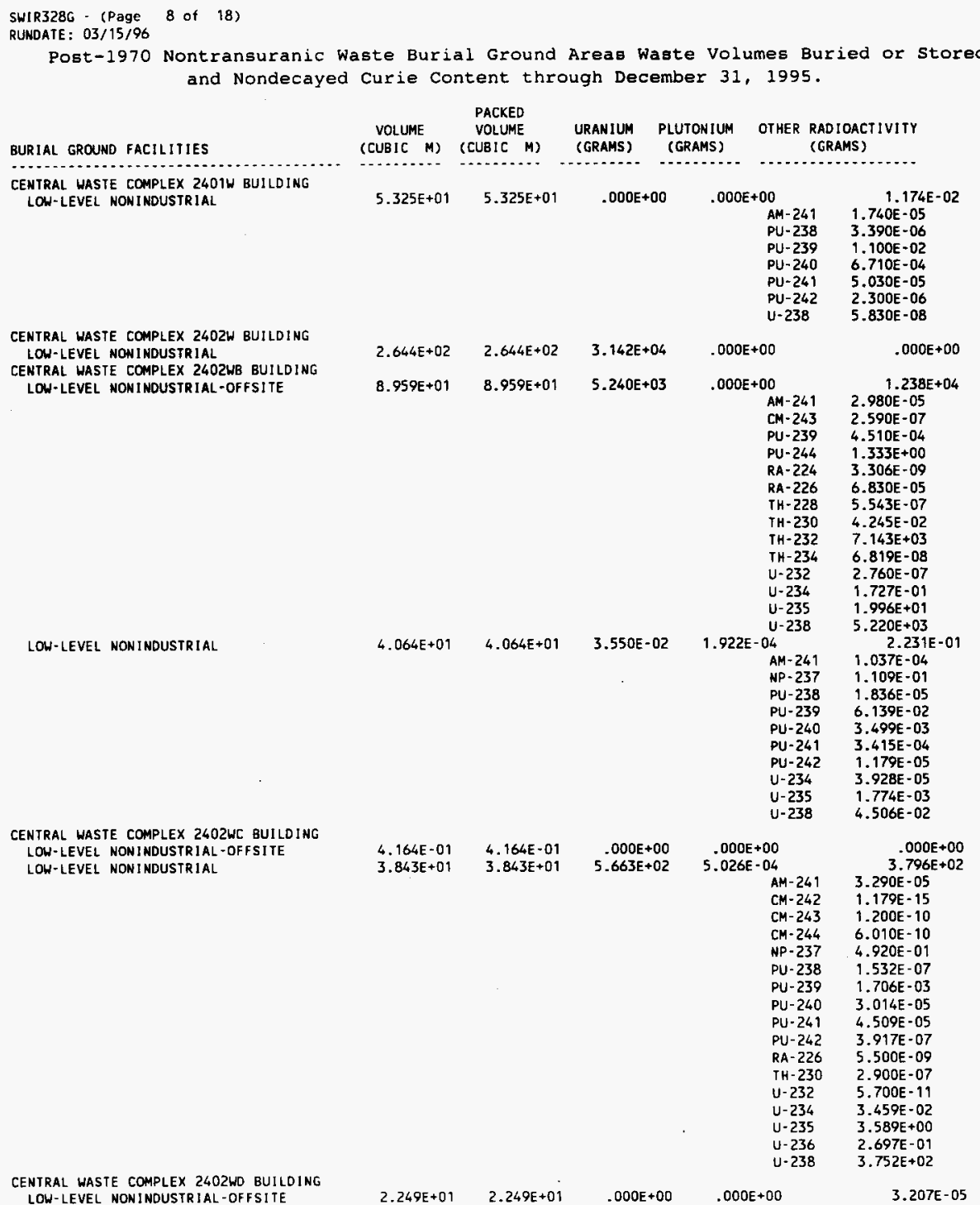




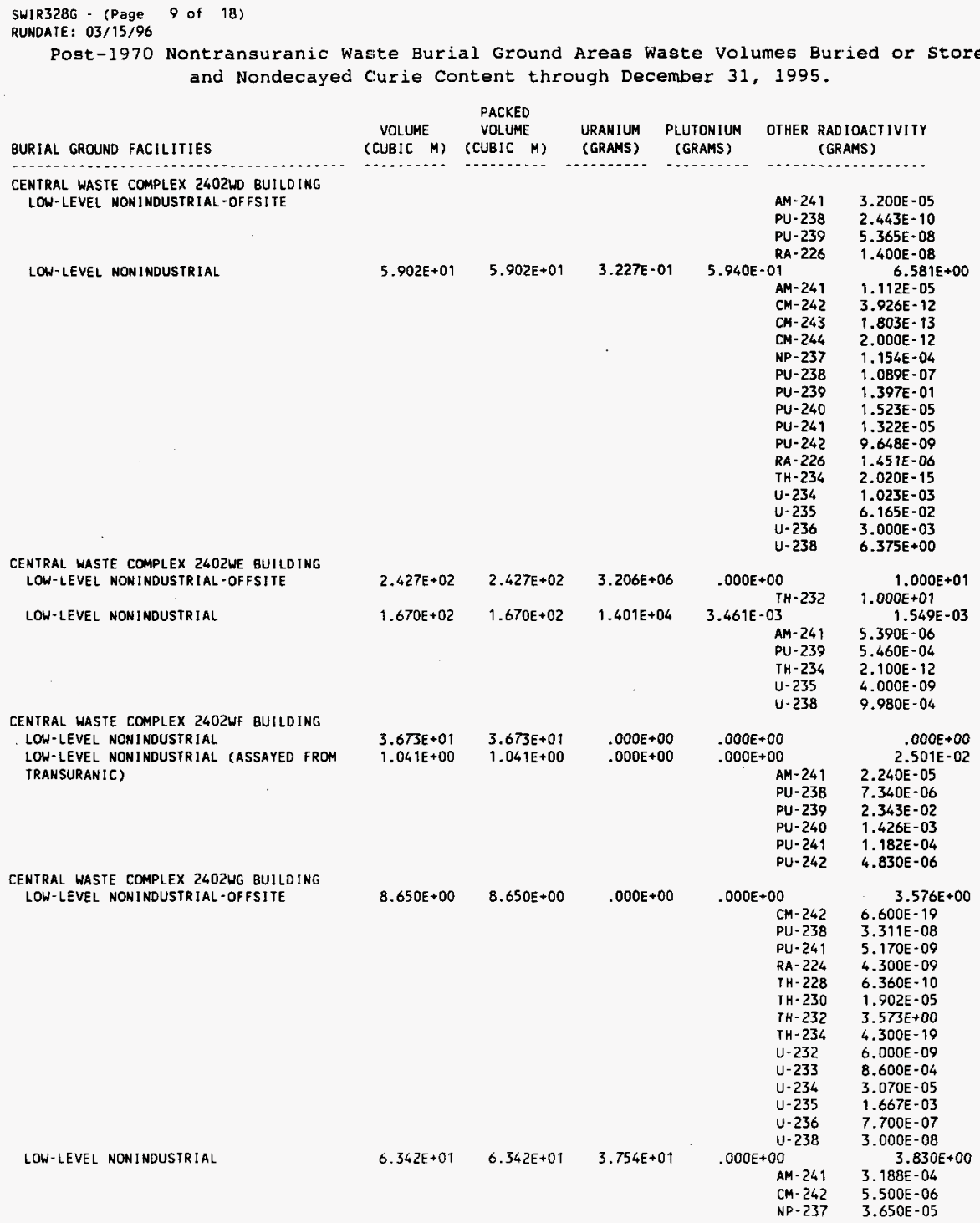




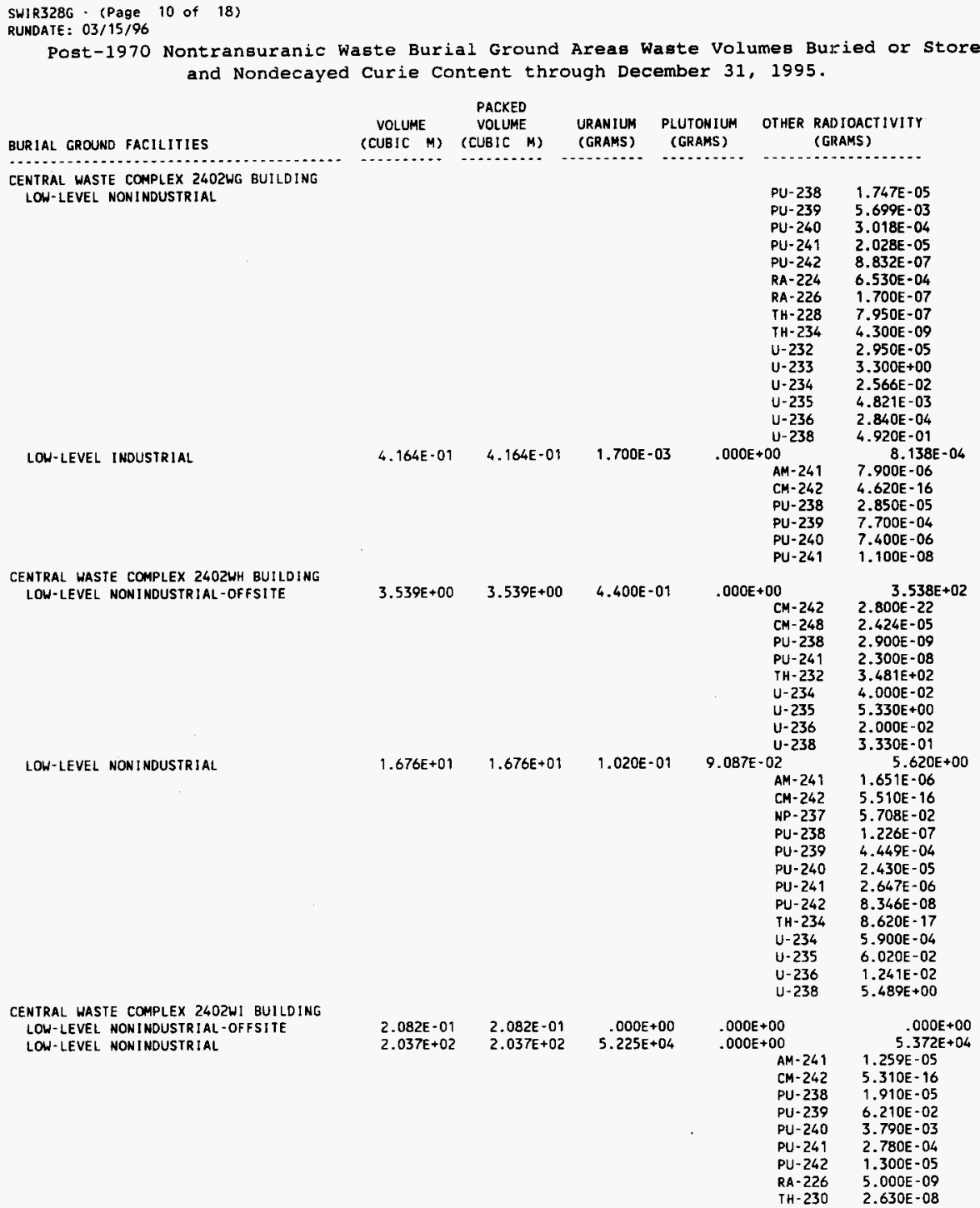




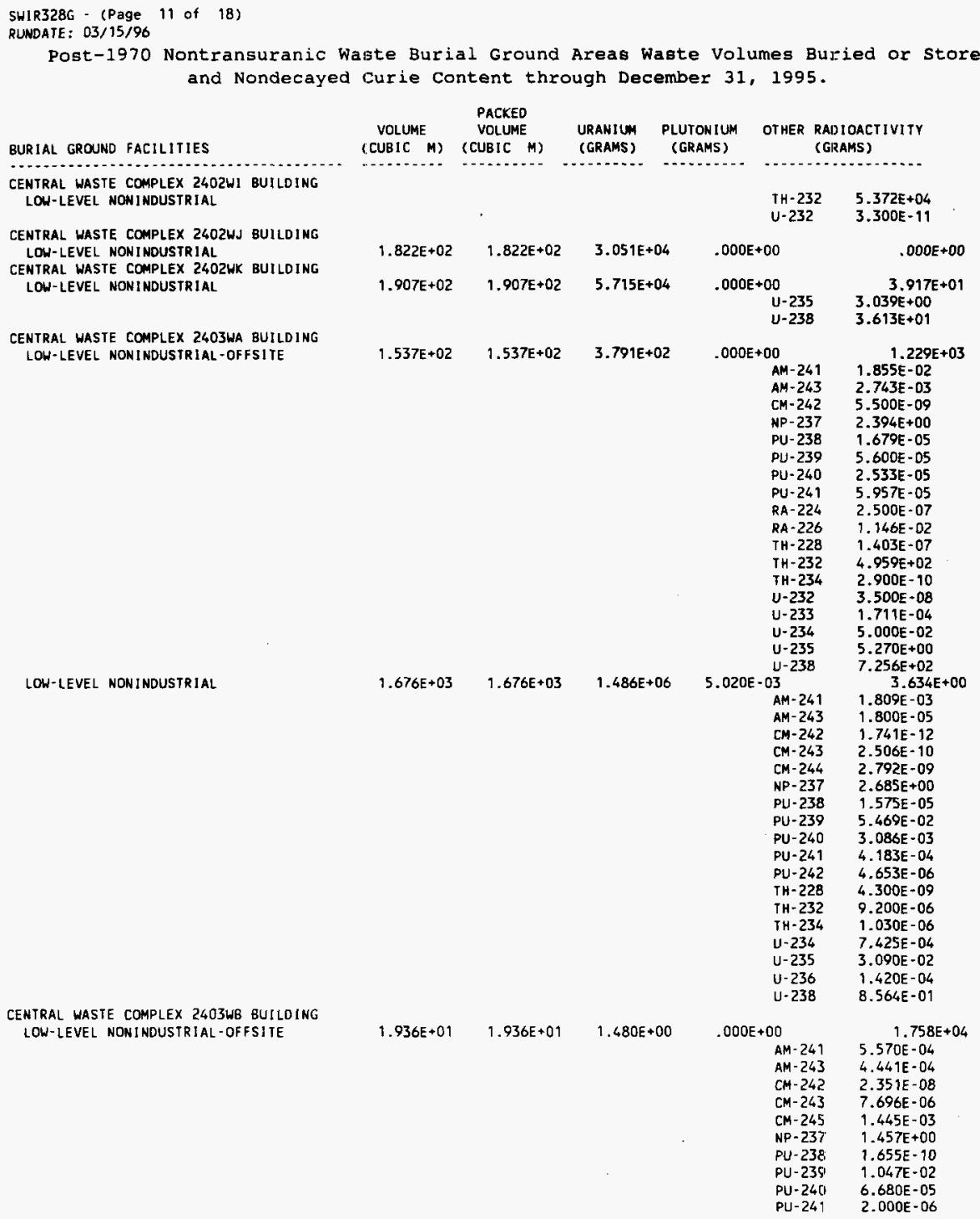




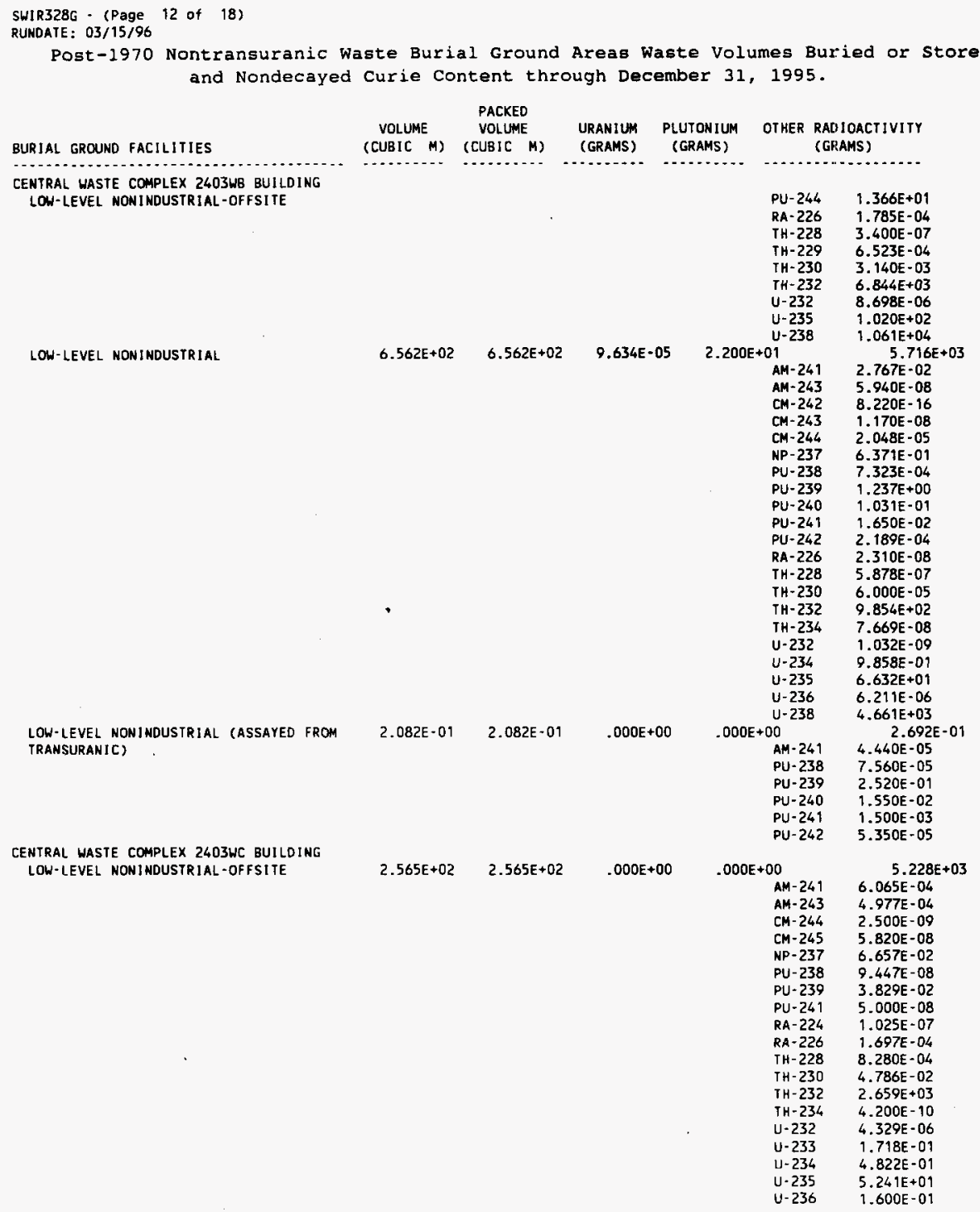




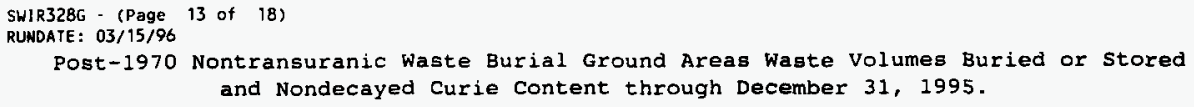

$U-238 \quad 2.516 E+03$

LOW-LEVEL NONINOUSTRIAL

$2.442 E+04$

2.718E-02

$7.900 \mathrm{E}-04$

1. 687E-11

$1.242 E-08$

$1.031 \mathrm{E}-07$

9. $701 E+00$

$5.120 E-04$

7.075E-01

3.500E-02

$4.937 \mathrm{E}-03$

6.789E-05

$9.000 E-04$

8. $039 \mathrm{E}-04$

5.013E-04

6.235E-05

$1.571 \mathrm{E}+04$

$1.580 \mathrm{E}-10$

$4.670 \mathrm{E}-12$

2. $100 \mathrm{E}-02$

$9.774 \mathrm{E}-01$

$4.442 \mathrm{E}+01$

$2.235 \mathrm{E}+00$

$8.659 E+03$ $.000 E+00$$$
\text { 2.087E-01 }
$$

2.920E-06

4.070E-09

2. 050 E -07

8. $700 E-12$

$1.400 E-09$

$9.060 \mathrm{E}-08$

$1.390 E-03$

4. $270 \mathrm{E}-05$

4.970E-0.

$9.900 \mathrm{E}-10$

1. $200 E-12$

8. $900 \mathrm{E}-04$

1.200E-06

6. $000 E-07$

1. $380 E-03$

$1.850 E-05$

2. $050 E-01$ $4.826 E+04$

2.252E-02

$2.115 E-09$

3. $120 \mathrm{E}-07$

3. $485 E-06$

$1.676 E+01$

6. $471 E-04$

$5.914 E+00$

$2.349 \mathrm{E}-01$

$4.835 E-01$

2.808E-03

$2.231 \mathrm{E}+04$

$2.960 \mathrm{E}-08$ 


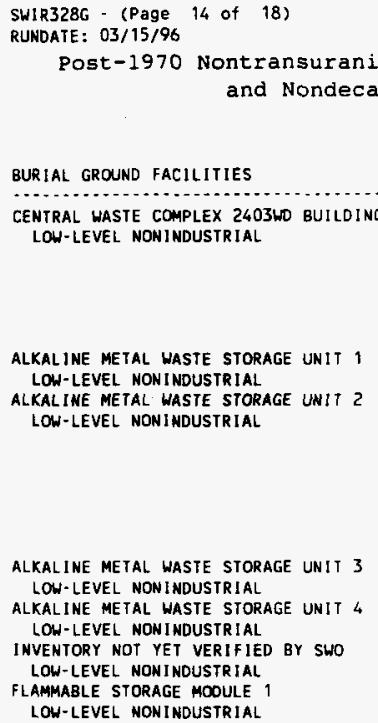

ALKALINE METAL WASTE STORAGE UNIT 3 LOW-LEVEL NONINDUSTRIAL

ALKALINE METAL WASIE STORAGE UNIT 4 LOW-LEVEL NONINDUSIRIAL

INVENTORY NOT YET VERIFIED BY SWO LOW-LEVEL NONINOUSTRIAL

FLAMMABLE STORAGE MOOULE 1 LOW-LEVEL NONINDUSTRIAL

FLAMMABLE STORAGE MOOULE 2

LOW-LEVEL NONINDUSTRIAL-OFFSITE

LOW-LEVEL NONINDUSTRIAL

FLAMMABLE STORAGE MODULE 3

LOW-LEVEL NONINDUSTRIAL-OFFSITE

AM-241 $9.211 \mathrm{E}-10$

CM-242 $9.240 \mathrm{E}-16$

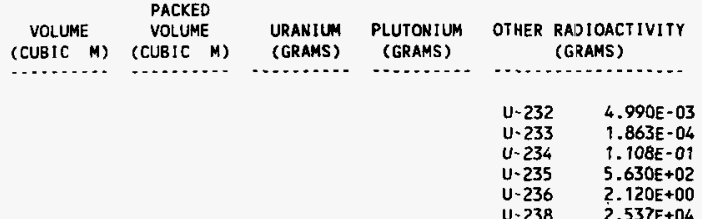

$\begin{array}{rrrrr}7.469 E+00 & 7.469 E+00 & .000 E+00 & .000 E+00 & .000 E+00 \\ 3.331 E+00 & 3.331 E+00 & .000 E+00 & .000 E+00 & 8.968 E-06 \\ & & & \text { AM-241 } & 1.010 E-08 \\ & & P U-238 & 2.620 E-09 \\ & & P U-239 & 8.400 E-06 \\ & & P U-240 & 5.120 E-07 \\ & & P U-241 & 4.160 E-08 \\ & & P U-242 & 1.760 E-09\end{array}$

$\begin{array}{rrrrr}6.371 E+00 & 6.371 E+00 & .000 E+00 & .000 E+00 & .000 E+00 \\ 5.129 E+00 & 5.129 E+00 & .000 E+00 & .000 E+00 & -000 E+00 \\ 4.100 E-01 & 4.100 E-01 & 2.400 E+01 & .000 E+00 & .000 E+00 \\ 3.358 E+00 & 3.358 E+00 & 2.115 E-04 & .000 E+00 & 9.448 E-08 \\ & & & A M-241 & 1.640 E-09 \\ & & & P U-238 & 4.110 E-11 \\ & & & P U-239 & 9.280 E-08\end{array}$

$1.874 E+00 \quad 1.874 E+00 \quad-000 E+00 \quad-000 E+00$

$1.914 E-08$

PU-238 3.043E-10

PU-239 2.248E-09

PU-241 5.160E-10

RA-226 $\quad 1.515 E-08$

$1.851 \mathrm{E}+00 \quad 1.851 \mathrm{E}+00 \quad 1.572 \mathrm{E}-01 \quad .000 \mathrm{E}+00$

$A M-241$

$1.095 \mathrm{E}-01$

PU-238 1.994E-08

PU-239 5.819E-05

PU-241 5.090E-08

TH-234 2.210E-06

U-234 $\quad 1.600 E-04$

U-235 $9.910 \mathrm{E}-02$

$U-238 \quad 1.020 E-02$

$2.290 E+00 \quad 2.290 E+00 \quad .000 E+00 \quad .000 E+00$

AM- 241

$A M-243$

GM- 244

CM-245

CM- 246

NP -237

PU. 238

PU 239

PU-240

PU-242

TH-230

U. 232

U-233

$1.472 E-02$

8. $370 E-06$

$5.800 E-06$

$5.800 E-09$

$6.800 \mathrm{E}-06$

3.800E-06

$9.885 E-03$

1. 580E-06

2. $738 \mathrm{E}-04$

6. $700 E-06$

$6.500 \mathrm{E}-07$

$2.400 E-05$

$5.400 E-0 B$

$3.400 E-03$ 
SWIR32BG - (Page 15 of 18)

RUNDATE: $03 / 15 / 96$

Post-1970 Nontransuranic Waste Burial Ground Areas Waste Volumes Buried or stored and Nondecayed Curie Content through December 31, 1995.

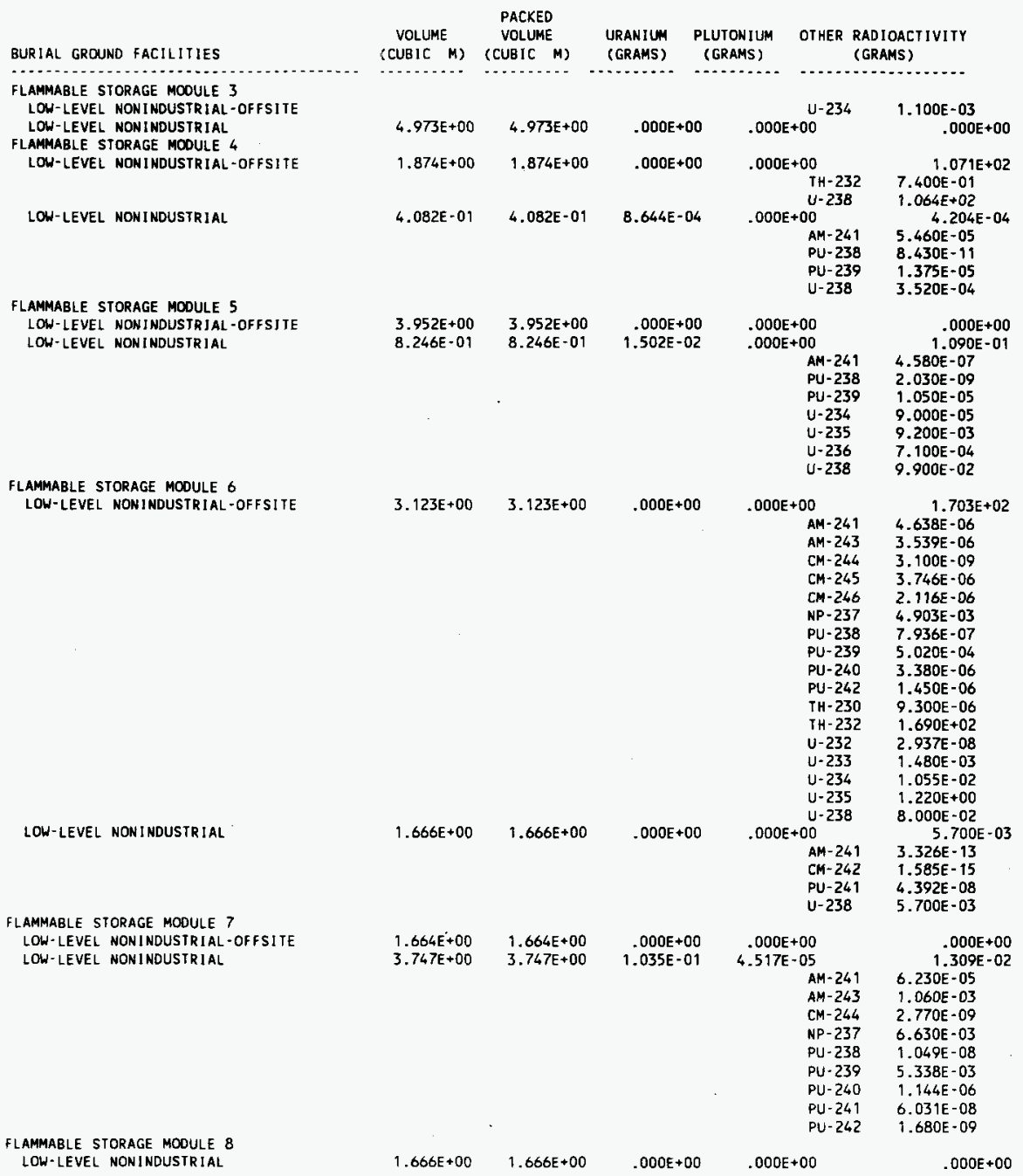




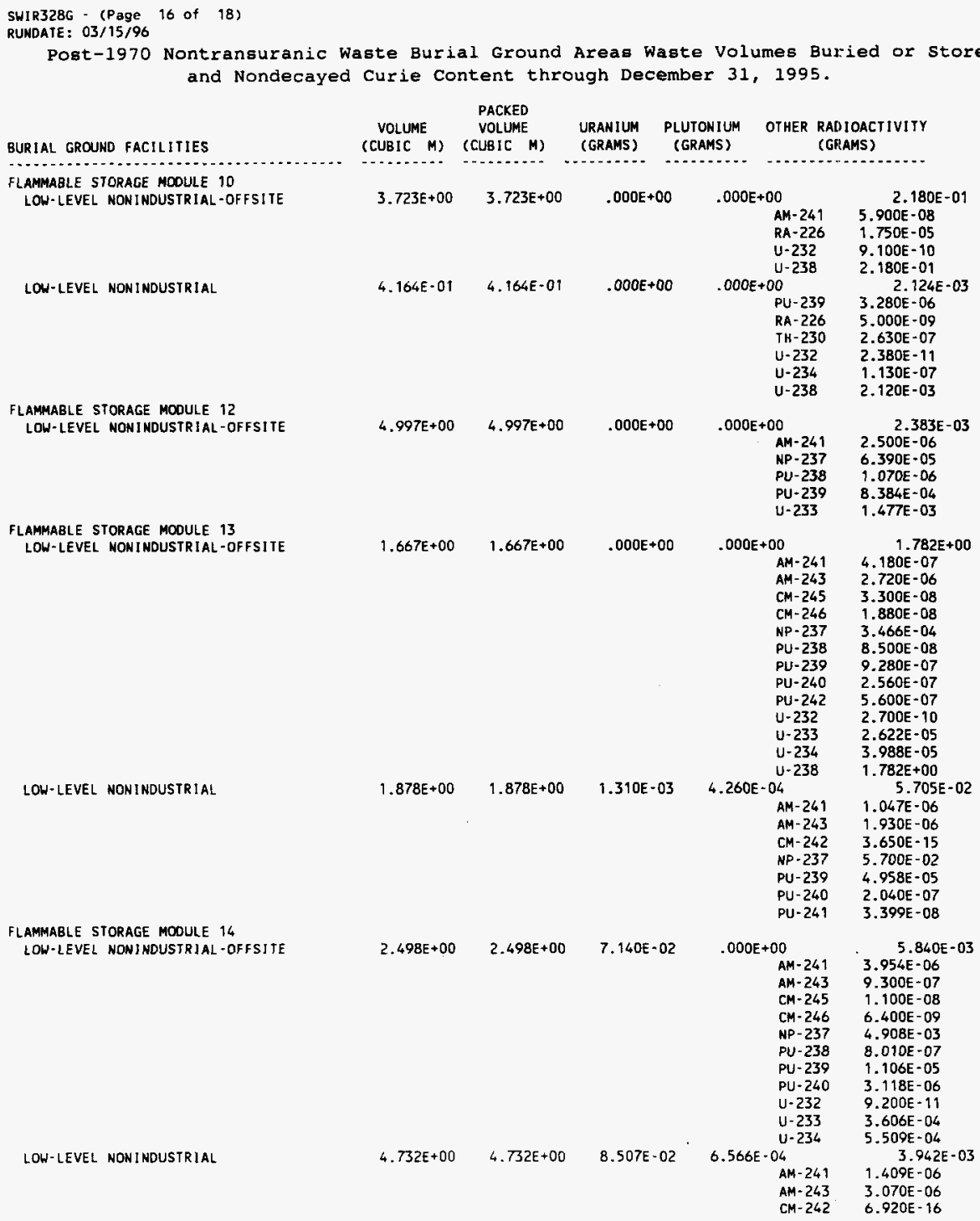




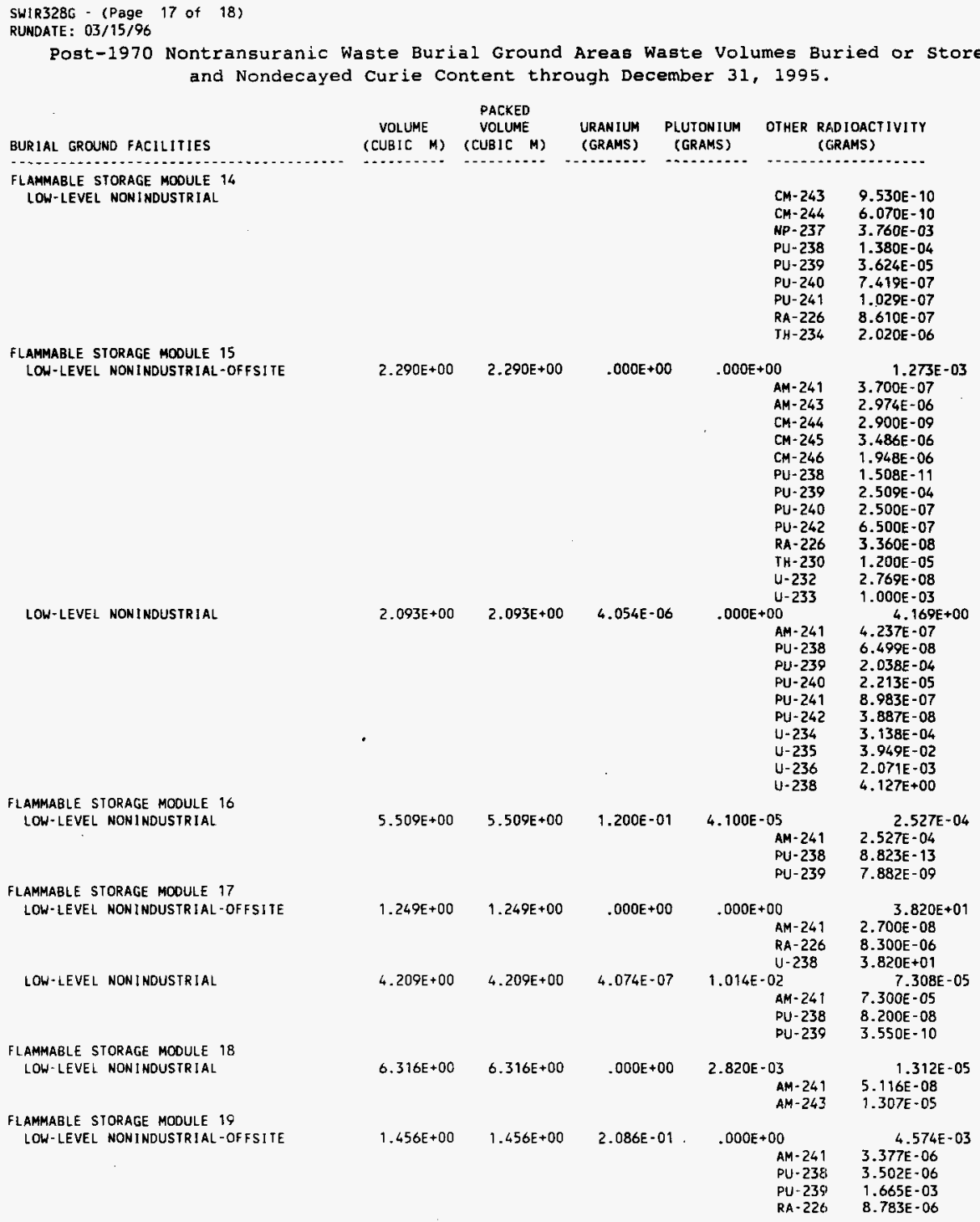




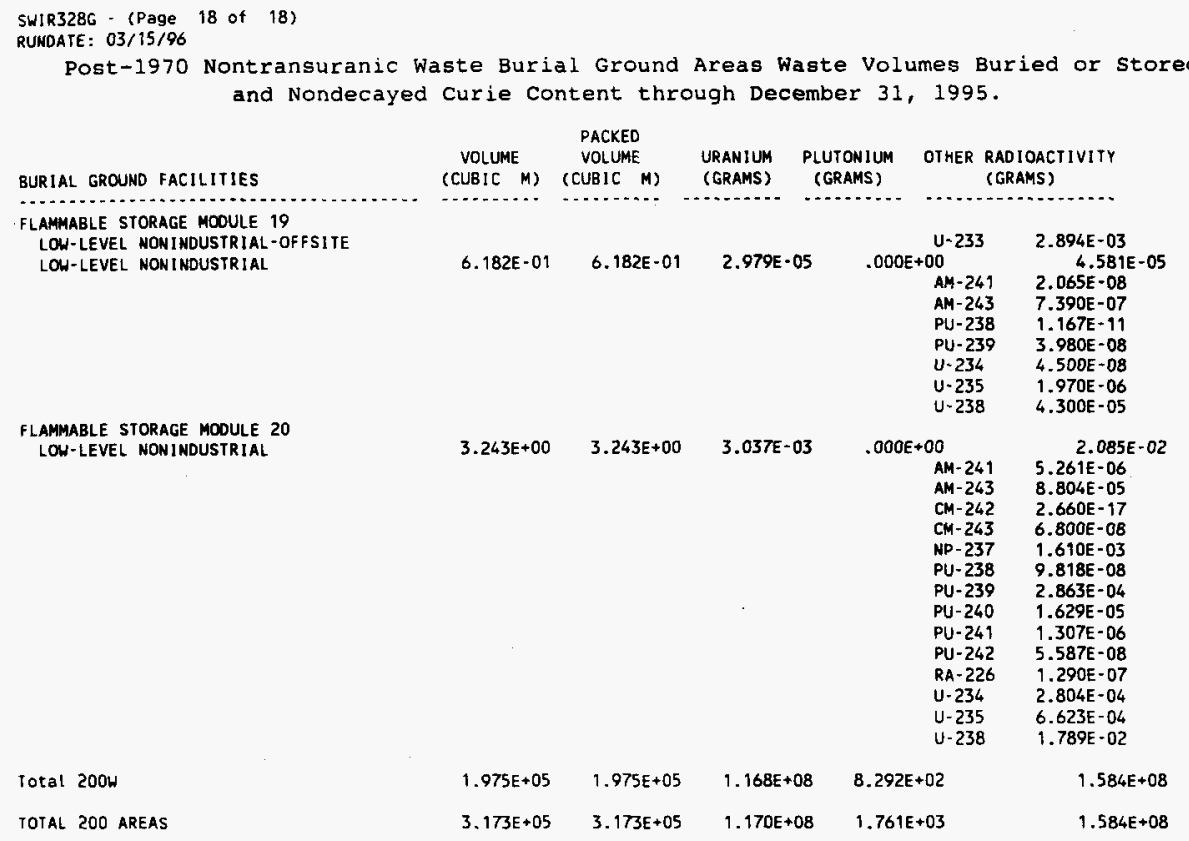


SWIR328H - (Page 1 of 37)

RUNDATE: $03 / 15 / 96$

Post-1970 Nontransuranic Waste Burial Ground Areas Waste Volumes Buried or Stored and Nondecayed Curie Content through DECEMBER 31, 1995.

\begin{tabular}{|c|c|c|c|c|c|}
\hline URIAL GROUND FACILITIES & $\begin{array}{l}\text { TOTAL } \\
\text { (CURIES) }\end{array}$ & $\begin{array}{l}\text { SR }-90 \\
\text { (CUR!ES) }\end{array}$ & $\begin{array}{l}\text { RU-106 } \\
\text { (CURIES) }\end{array}$ & $\begin{array}{l}\text { CS-137 } \\
\text { (CURIES) }\end{array}$ & $\begin{array}{l}\text { OTHER RADIOACIIVITY } \\
\text { (CURIES) }\end{array}$ \\
\hline
\end{tabular}

\section{E AREA}

BURIAL GROUND 218E 10

LOW-LEVEL NONINDUSTRIAL

LOW-LEVEL INDUSTRIAL

\begin{tabular}{|c|c|c|c|}
\hline $\begin{array}{l}1.912 E+03 \\
4.515 E+06\end{array}$ & $\begin{array}{l}5.870 E+02 \\
9.982 E+05\end{array}$ & $\begin{array}{l}5.223 E-03 \\
9.944 E+03\end{array}$ & $\begin{array}{l}3.793 E+02 \\
1.176 E+06 \\
\text { Co-60 }\end{array}$ \\
\hline
\end{tabular}

Co- 60

$\begin{array}{cc} & .000 E+00 \\ 3.800 E+03 & 3.800 E+03 \\ 137 & 4.423 E+01 \\ 14 & 4.560 E+01 \\ -58 & 4.560 E+01 \\ -60 & 3.055 E+03 \\ 137 & 4.696 E+01 \\ 152 & 4.560 E+01 \\ 154 & 3.876 E+02 \\ 155 & 3.876 E+02 \\ -59 & 1.824 E+02 \\ -54 & 3.192 E+02\end{array}$

BURIAL GROUND 218E12B

LOW-LEVEL NONINDUSTRIAL

$3.059 E+04$

$4.817 E+02$

$3.086 E+04$

4. $805 \mathrm{E}+04$

BE - 7

C- 14

$1.300 E-09$

CE-141 4.000E-01

CE-144 3.477E+01

CO-60 4.801E+04

CS-134 $9.923 \mathrm{E}-02$

$\mathrm{H}-3 \quad 2.443 \mathrm{E}-03$

$1-129 \quad 1.980 \mathrm{E}-02$

$\mathrm{K}-40 \quad 9.054 \mathrm{E}-03$

$P M-147 \quad 3.004 E+00$

SB-125 1.100E-06

SM- $151 \quad 4.241 E-01$

ZR-95 1.100E-05

BA $137 \quad 1.166 E-02$

C-14 1.202E-02

CO-58 $1.202 E-02$

CO-60 8.054E-01

CS $137 \quad 1.238 E-02$

EU152 1.202E-02

EU154 1.022E-01

EU155 1.022E-01

FE-59 4.808E-02

LOW-LEVEL INDUSTRIAL-OFFSITE

$2.628 E+06 \quad 1.175 E+01 \quad 1.690 E+01 \quad 2.002 E+01$

MN-54 8.415E-02

$B E-10$

$2.628 E+06$

$1.278 \mathrm{E}-06$

C. $14 \quad 1.253 E+02$

$\mathrm{CL}-36 \quad 5.556 \mathrm{E}-03$

CO-58 $\quad 1.323 E+04$

CO-60 $9.946 E+05$

CR-51 3.614E+02

CS-134, 7.003E-03

FE $-55 \quad 7.322 E+05$

FE-59 7.144E+01

$\mathrm{H}-3 \quad 1.124 \mathrm{E}+03$

$H F-181 \quad 2.320 E+00$

$1.129 \quad 2.941 \mathrm{E}-03$

$M N-54 \quad 1.117 E+04$

MO-93 6.900E-02

NB-93M 5.611E-01

$N B=94 \quad 1.282 E+01$

NB. $95 \quad 1.076 E+01$

NI.59 $5.119 E+03$

NI $-63 \quad 8.699 E+05$ 


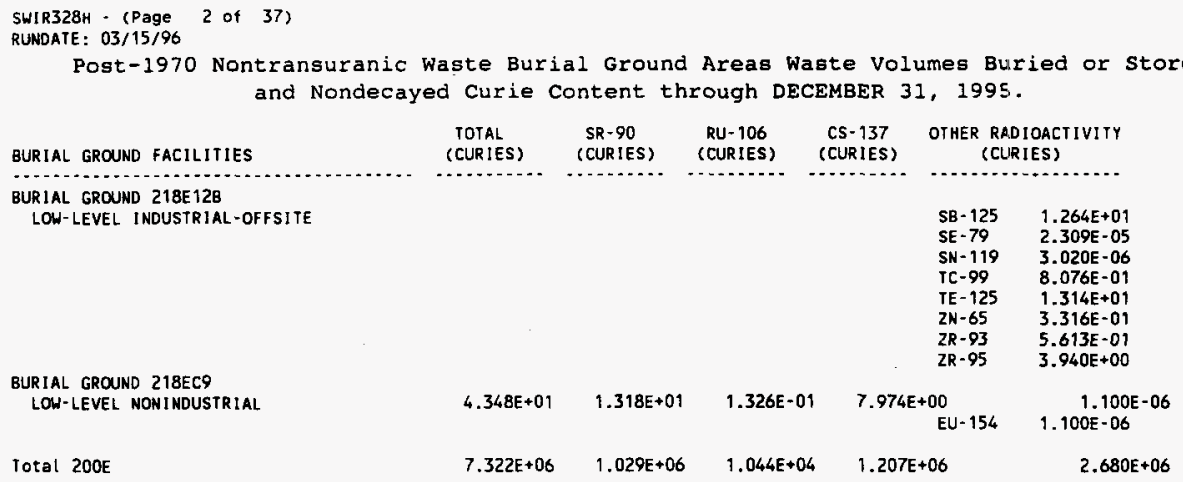


WHC-EP-0125-8

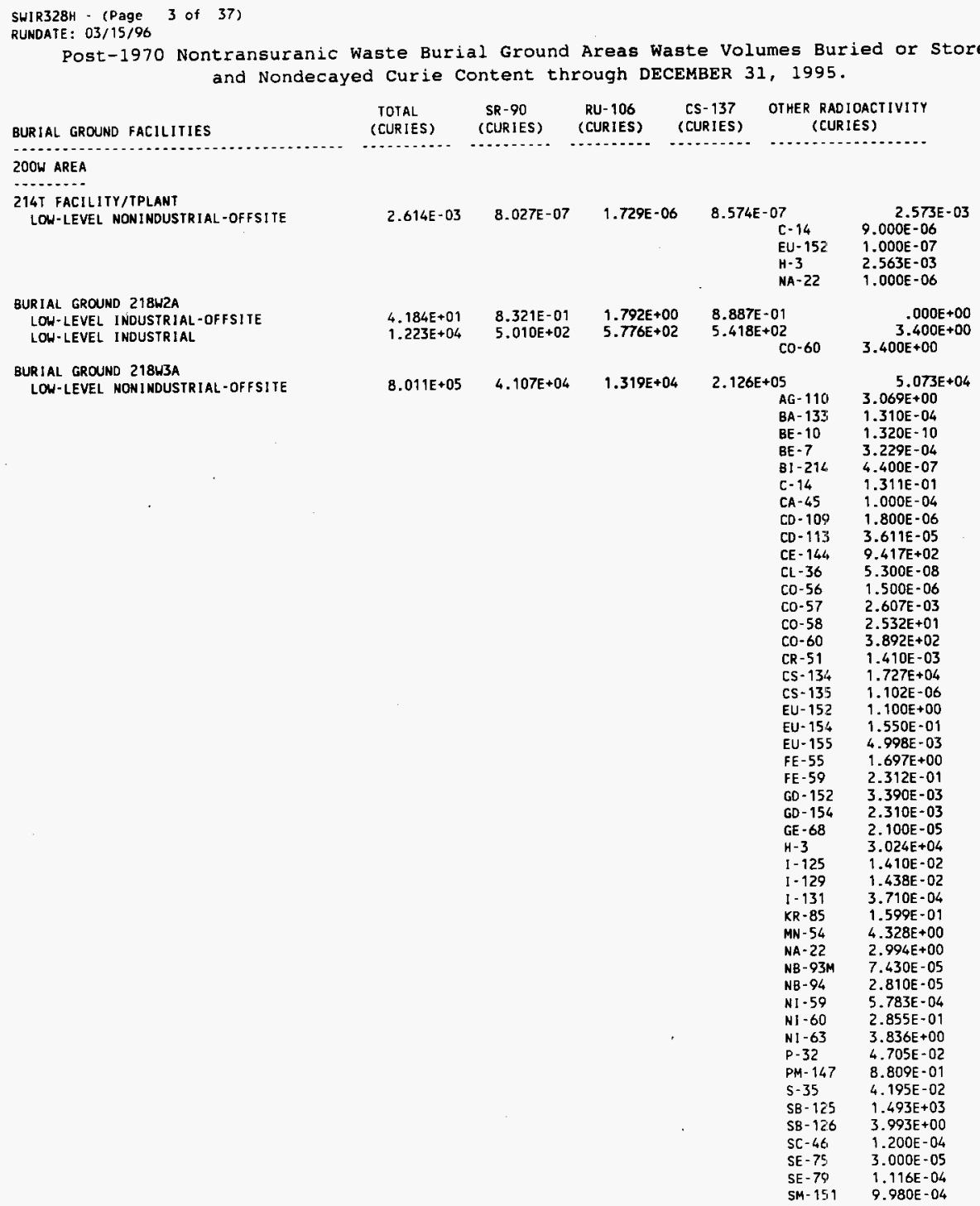


SWIR328H - (Page 4 of 37 )

RUNDATE: $03 / 15 / 96$

Post-1970 Nontransuranic Waste Burial Ground Areas Waste Volumes Buried or stored and Nondecayed Curie Content through DECEMBER 31, 1995.

BURIAL GROUND FACILITIES

BURIAL GROUND 218W3A

LOW-LEVEL NONINDUSTRIAL-OFFSITE
TOTAL SR-90 RU-106 CS-137 OTHER RADIOACTIVITY

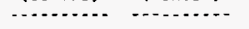

(CURIES) (CURIES) (CURIES) (CURIES)

(CURIES)

.

LOW-LEVEL NONINDUSTRIAL

$7.876 E+05 \quad 4.600 E+04 \quad 1.383 E+03$

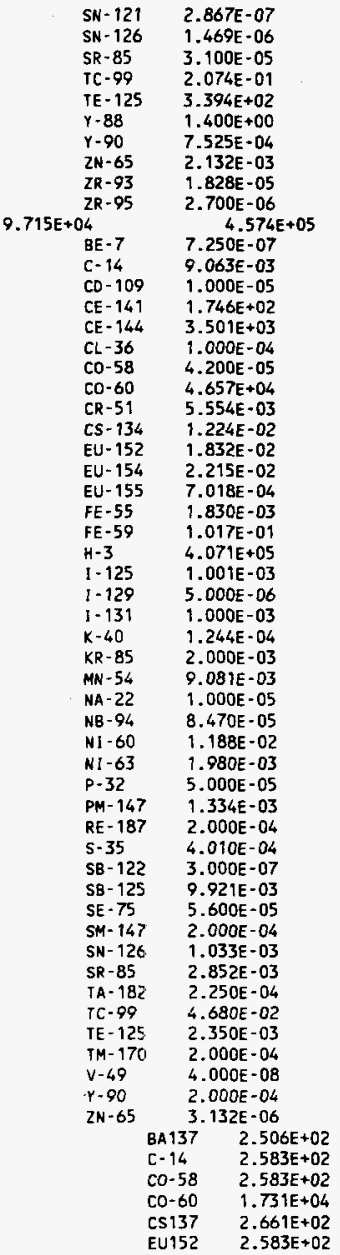




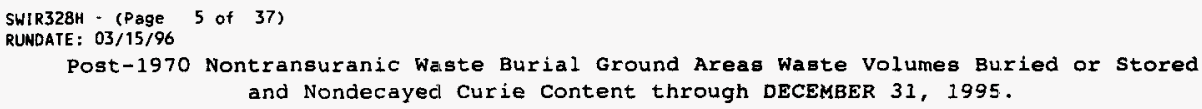

$5.000 E+00 \quad .000 E+00 \quad .000 E+00$
EU154

EU155

FE-59

MN -54

LOW-LEVEL NOWINOUSTRIAL CLASSIFIED
2. $196 \mathrm{E}+03$

2. $196 \mathrm{E}+03$

$1.033 \mathrm{E}+03$

$1.808 E+03$

$.000 E+00$

$.000 E+00$

8A137 $4.850 E-02$

C- $14 \quad 5.000 E-02$

C. $0-58 \quad 5.000 E-02$

C. $0-60$ 3. $350 \mathrm{E}+00$

CS137 $5.150 E-02$

EU152 $5.000 E-02$

EU154 4.250E-01

EU155 4.250E-01

FE-59 2.000E-01

MN $-54 \quad 3.500 E-01$

$\begin{array}{llllll}6.101 E+00 & 8.05 .7 E-01 & 2.294 E-01 & 8.636 E-01 & M N-54 & \begin{array}{l}3.500 E-01 \\ .000 E+00\end{array}\end{array}$
TRANSURANIC)

LOW-LEVEL INDUSTRIAL-OFFSITE
$4.868 E+00$

$5.798 \mathrm{E}-01$
$3.048 E+02$

9.100E-06

3.400E-06

2.745E-05

5. $600 \mathrm{E}-10$

$1.745 \mathrm{E}-01$

2.696E-03

3.710E-04

$4.980 \mathrm{E}-04$

$7.880 \mathrm{E}-01$

$1.002 \mathrm{E}-04$

2.190E-04

$3.017 \mathrm{E}+00$

3. $000 \mathrm{E}-03$

$1.461 E+00$

3.964E-01

2.809E-01

2.090E-04

$7.568 \mathrm{E}-03$

8.801E-06

$2.397 E-02$

2. $526 \mathrm{E}+02$

4. $000 \mathrm{E}-07$

$5.200 E-07$

7. $490 \mathrm{E}-08$

3. $580 \mathrm{E}-01$

1.944E-02

$3.586 E+00$

1. $000 E-04$

$3.000 E-04$

5. $000 E-02$

2. $134 E+00$

$1.240 \mathrm{E}-05$

5.017E-03

3. $000 \mathrm{E}-08$

1. $500 \mathrm{E}-05$

$1.040 E-03$

2. $400 \mathrm{E}-04$

$5.000 \mathrm{E}-10$

1. DOOE- 06

$3.982 \mathrm{E}+01$ 
SWIR328H - (Page 6 of 37)

RLNDATE : 03/15/96

Post-1970 Nontransuranic Waste Burial Ground Areas Waste Volumes Buried or Stored

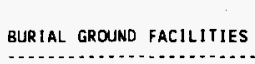

BURIAL GROUND 218W3A

LOH-LEVEL INDUSTRIAL and Nondecayed Curie Content through DECEMBER 31, 1995.

TOTAL

(CURIES)

SR-90

(CURIES)

RU- 106

$\operatorname{cs}-137$

(CURIES)

OTHER RADIOACTIVITY

(CURIES) (CURIES) (CURIES)

\section{$6.906 E+03$}

$6.759 \mathrm{E}+02$

$5.047 E+00$

$\rightarrow+0$

$1.000 \mathrm{E}-12$

$4.332 \mathrm{E}+00$

4. $000 \mathrm{E}-02$

8.230E-02

$1.370 E-01$

1.300E-09

$1.890 \mathrm{E}+00$

$1.300 \mathrm{E}-09$

5.000E- 07

$6.000 E-13$

$3.350 \mathrm{E}-02$

$2.000 \mathrm{E}-13$

7.310E-03

$\begin{array}{ll}01 & 6.532 E+04 \\ A C-227 & 1.000 E-06 \\ A C-228 & 7.801 E-04 \\ A G-108 & 2.700 E-05 \\ A G-110 & 9.850 E-06 \\ A U-195 & 9.250 E-05 \\ B A-133 & 7.656 E-06 \\ B E-10 & 4.600 E-11 \\ B E-7 & 1.101 E-03 \\ B I-207 & 1.000 E-03 \\ B I-212 & 2.975 E-04 \\ B I-214 & 2.790 E-05 \\ C-14 & 1.004 E+01 \\ C A-45 & 3.120 E-03 \\ C D-109 & 6.594 E-05 \\ C D-113 & 1.260 E-05 \\ C E-141 & 1.000 E-06 \\ C E-144 & 3.576 E-02 \\ C L-36 & 1.840 E-08 \\ C O-56 & 9.630 E-05 \\ C O-57 & 3.560 E-03 \\ C O-58 & 1.316 E+02 \\ C O-60 & 2.751 E+04 \\ C R-51 & 7.664 E-03 \\ C S-134 & 1.820 E+00 \\ C S-135 & 3.800 E-07 \\ E S-254 & 6.300 E-09 \\ E U-152 & 2.118 E-02 \\ E U-153 & 1.000 E-09 \\ E U-154 & 5.905 E-01 \\ E U-155 & 1.192 E-01 \\ \text { EE }-55 & 7.415 E+03 \\ F E-59 & 3.249 E-02 \\ & \end{array}$

$$
\begin{aligned}
& 1.000 E-06 \\
& 7.801 E-06 \\
& 2.700 E-05 \\
& 9.850 E-06 \\
& 9.250 E-05 \\
& 7.656 E-06 \\
& 4.600 E-11 \\
& 1.101 E-03 \\
& 1.000 E-03 \\
& 2.975 E-04 \\
& 2.790 E-05 \\
& 1.004 E+01 \\
& 3.120 E-03 \\
& 6.594 E-05 \\
& 1.260 E-05 \\
& 1.000 E-06 \\
& 3.576 E-02 \\
& 1.840 E-08 \\
& 9.630 E-05 \\
& 3.560 E-03 \\
& 1.316 E+02 \\
& 2.751 E+04 \\
& 7.664 E-03 \\
& 1.820 E+00 \\
& 3.800 E-07 \\
& 6.300 E-09 \\
& 2.118 E-02 \\
& 1.000 E-09 \\
& 5.905 E-01 \\
& 1.192 E-01 \\
& 7.415 E+03 \\
& 3.249 E-02
\end{aligned}
$$

$\begin{aligned} & 6.759 E+02 \quad 5.047 E+00 \quad 1.452 E+ 03 \\ & C-14 \\ & C E-144 \\ & C O-60 \\ & C S-134 \\ & E U-152 \\ & E U-154 \\ & F E-55 \\ & H-3 \\ & M N-54 \\ & N A-22 \\ & N I=60 \\ & P M=147 \\ & S B-125 \\ & S M-151 \\ & T E-125\end{aligned}$

\section{GURIAL GROUND 218WBAE}

LOW-LEVEL NONINDUSTRIAL-OFFSITE

\section{$6.593 E+04 \quad 6.403 E+01 \quad 2.807 E+00 \quad 5.588 E+01$}

$3.0005-23 E+0$

3. $000 E-22$

$4.570 \mathrm{E}-04$

E1A $137 \quad 2.405 \mathrm{E}+01$

$\mathrm{C}-14 \quad 2.480 \mathrm{E}+01$

Co-58 $2.480 \mathrm{E}+01$

C. $0.60 \quad 1.661 E+03$

CS137 2.554E+01

EU152 $2.480 E+01$

EU154 2.108E +02

EU155 2.108E+02

PE-59 9.919E+01

$M N-54 \quad 1.736 E+02$ 


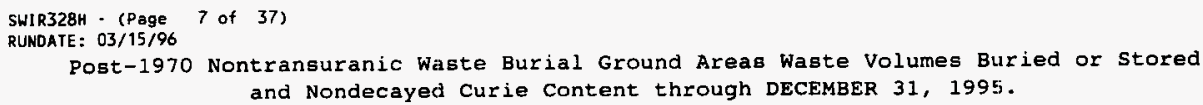
and Nondecayed Curie Content through DECEMBER 31, 1995.

BURIAL GROUND FACILITIES

BURIAL GROUND 218W3AE

LOW-LEVEL MONINDUSTRIAL-OFFSITE $\begin{array}{llll}\text { TOTAL } & \text { SR-90 } 90 & \text { CS }-137\end{array}$ (CURIES)
(CUR IES)

OTHER RADIOACTIVITY (CURIES)

. . . . (CURIES)

(CURJES)


WHC-EP-0125-8

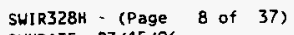

LOW-LEVEL NONINDUSTRIAL

LOW-LEVEL NONINDUSTRIAL CLASSIFIEDOFFSITE
$1.375 E+05 \quad 1.618 E-01 \quad 2.524 E-01$

$1.258 \mathrm{E}-01$

C- 14

$c-14$
Co- 58

Co. 60

$C R=51$

FE- 55

FE- 59

$\mathrm{H}-3$

HF- 181

]. 129

MN -54

NB -94

NB -95
$1.843 E+02$

$1.316 E+03$

$2.032 E+03$

$4.860 \mathrm{E}+02$

$1.145 \mathrm{E}+03$

$1.138 \mathrm{E}+02$

$4.463 E+03$

$1.000 E-09$

5.506E-07

3.374E-04

1.000E-09

8. $375 E+03$

2.090E-10

5.859E-04

$1.829 \mathrm{E}-07$

$9.202 E-05$

5.495E-04

$2.317 E-06$

$1.798 E+03$

$3.453 \mathrm{E}+02$

1. $497 \mathrm{E}-05$

$3.256 E-05$

$4.418 \mathrm{E}-03$

2. $000 \mathrm{E}-09$

$1.441 E+00$

$1.026 \mathrm{E}-08$

2. $710 E-04$

$1.003 E-06$

8. $431 E-02$

2. $130 \mathrm{E}-02$

4.000E-04

8.039E-06

2.001E-06

3. $831 E-09$

2. $100 \mathrm{E}-10$

$9.750 E-04$

$7.410 E+00$

BA $137 \quad 9.196 \mathrm{E}-08$

C-14 9.480E-08

CO-58 $\quad 9.480 \mathrm{E}-08$

CO-60 6.352E-06

CS137 9.764E-08

EU152 9.480E-08

EU154 8.058E-07

EU155 8.058E-07

FE-59 3.792E-07

MN-54 6.636E-07 $1.369 \mathrm{E}+05$

$1.982 E+01$

2. $600 E+02$

$4.600 E+04$

$4.680 E-07$

$1.600 \mathrm{E}+04$

$7.680 \mathrm{E}-05$

9.400E-01

$1.984 \mathrm{E}-0 \mathrm{~S}$

$6.160 \mathrm{E}-08$

$1.660 \mathrm{E}+02$

$5.603 E-01$

$1.420 E+03$ 


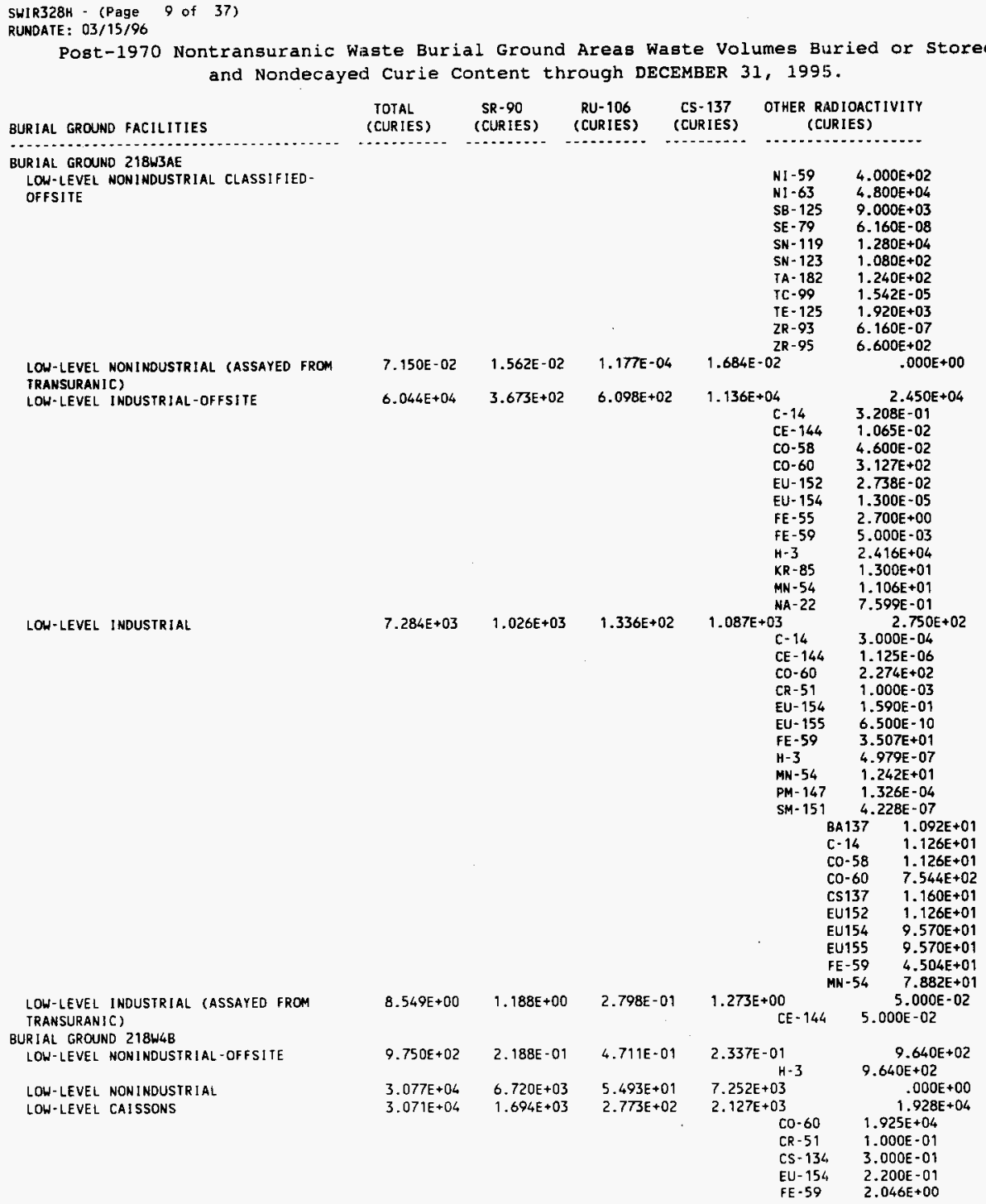


SWIR328H - (Page 10 of 37)

RUMDATE: $03 / 15 / 96$

Post-1970 Nontransuranic Waste Burial Ground Areas Waste Volumes Buried or Stored and Nondecayed Curie Content through DECEMBER 31, 1995.

\begin{tabular}{lllll} 
BURIAL GROUND FACILITIES & $\begin{array}{c}\text { TOTAL } \\
\text { (CURIES) }\end{array}$ & $\begin{array}{c}\text { SR-90 } \\
\text { (CURIES) }\end{array}$ & $\begin{array}{c}\text { RU }-106 \\
\text { (CURIES) }\end{array}$ (CURIES) \\
\hline
\end{tabular}

OTHER RADIOACTIVITY

BURIAL GROUND 218 4B

LOW-LEVEL CAISSONS

- D.................

BURIAL GROUNO $218 W 4 \mathrm{C}$

LOW-LEVEL NONINDUSTRIAL-OFFSITE

$6.531 E+04 \quad 2.761 E+01 \quad 4.646 E+00 \quad 2.737 E+01$

\begin{tabular}{|c|c|c|}
\hline $\begin{array}{l}M N=54 \\
S B-125 \\
Z R-95\end{array}$ & & $\begin{array}{l}3 E+00 \\
0 E-02 \\
D E+01\end{array}$ \\
\hline & BiA 137 & $1.213 \mathrm{E}+00$ \\
\hline & $c-14$ & 1. $.250 E+00$ \\
\hline & $c 0-58$ & $1.250 \mathrm{E}+00$ \\
\hline & c.0-60 & $8.375 E+01$ \\
\hline & $\begin{array}{l}\text { CS137 } \\
\text { E.U152 }\end{array}$ & $\begin{array}{l}1.288 E+00 \\
1.250 E+00\end{array}$ \\
\hline & E:U154 & $1.063 E+01$ \\
\hline & EU155 & $1.063 E+01$ \\
\hline & FE-5S & $5.000 E+00$ \\
\hline & $M N-54$ & $8.750 E+00$ \\
\hline
\end{tabular}

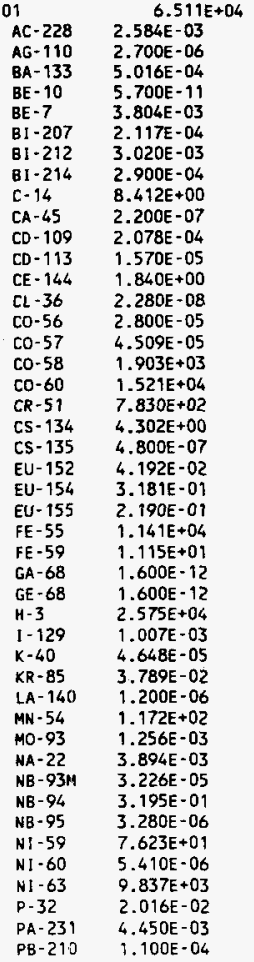


SWIR328H - (Page 11 of 37 )

RUNDATE : $03 / 15 / 96$

Post-1970 Nontransuranic Waste Burial Ground Areas Waste Volumes Buried or stored and Nondecayed Curie Content through DECEMBER $31,1995$.

BURIAL GROUND FACILITIES

BURIAL GROUND 218 W4C

LOW-LEVEL NONINDUSTRIAL-OFFSITE

LOW-LEVEL NONINOUSTRIAL
$8.224 E+04 \quad 5.610 E+03 \quad 4.052 E+02 \quad 4.542 E+03$

\begin{tabular}{cccc}
$\begin{array}{c}\text { IOTAL } \\
\text { (CURIES) }\end{array}$ & $\begin{array}{c}\text { SR-90 } \\
\text { (CURIES) }\end{array}$ & $\begin{array}{c}\text { RU-106 } \\
\text { (CURIES) }\end{array}$ & $\begin{array}{c}\text { CS-137 } \\
\text { (CURIES) }\end{array}$ \\
\hline & (CUR
\end{tabular}

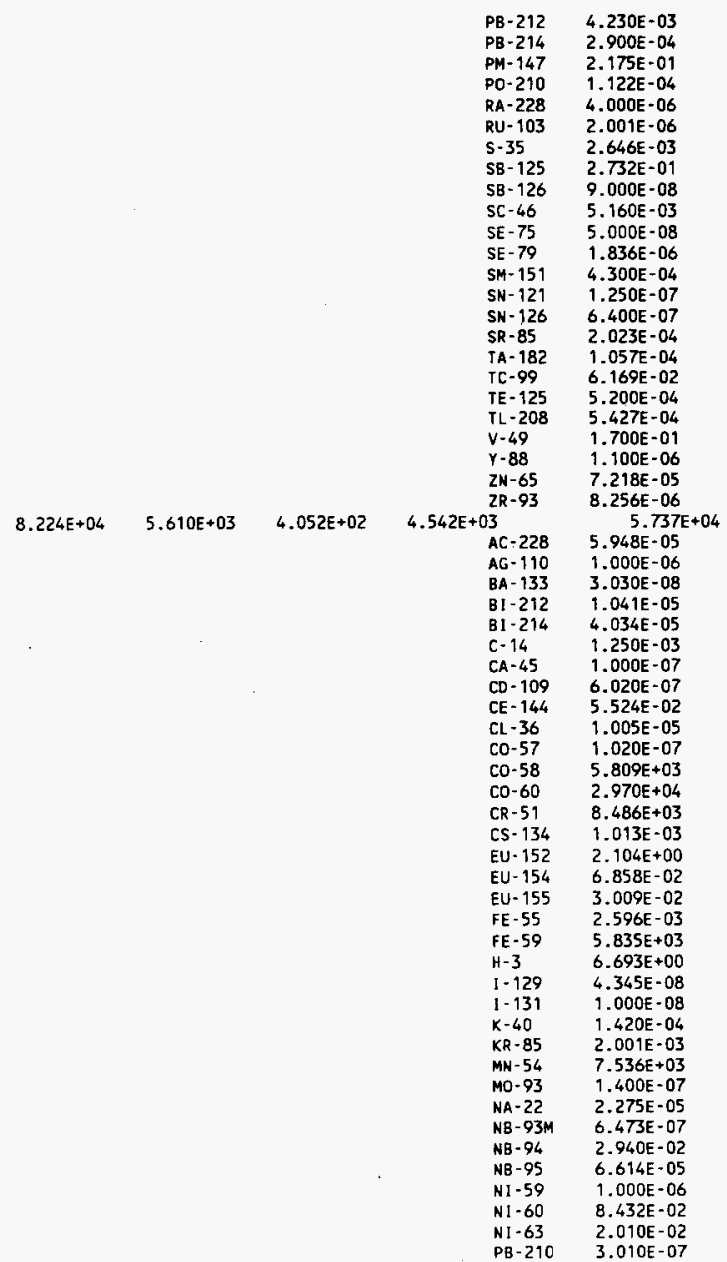

OTHER RADIOACTIVITY (CURIES)

(CURIES)

PO- 210

RA- 228

$5-35$

SE -75

N- 12

$\mathrm{TL}-208$

$A G=110$

BI -212

CA- 45

$\mathrm{CO}-5$

CS. 13

$k-40$

$M N-54$

PB- 210
4. 230E-03

$122 \mathrm{E}-04$

$4.000 E-06$

$5.160 \mathrm{E}-03$

000E - 08

4. 300E-04

OE- 07

$6.400 E-07$

.200E- 0

. 427E-04

6E-06

$.948 E-05$

.041E-05

$4.034 \mathrm{E}-05$

.524E-02

.005E-05

$2.970 E+04$

$8.486 E+03$

$1.420 E-04$

1E-03

$6.614 \mathrm{E}-05$

$8.432 E-02$

3.010E-07 


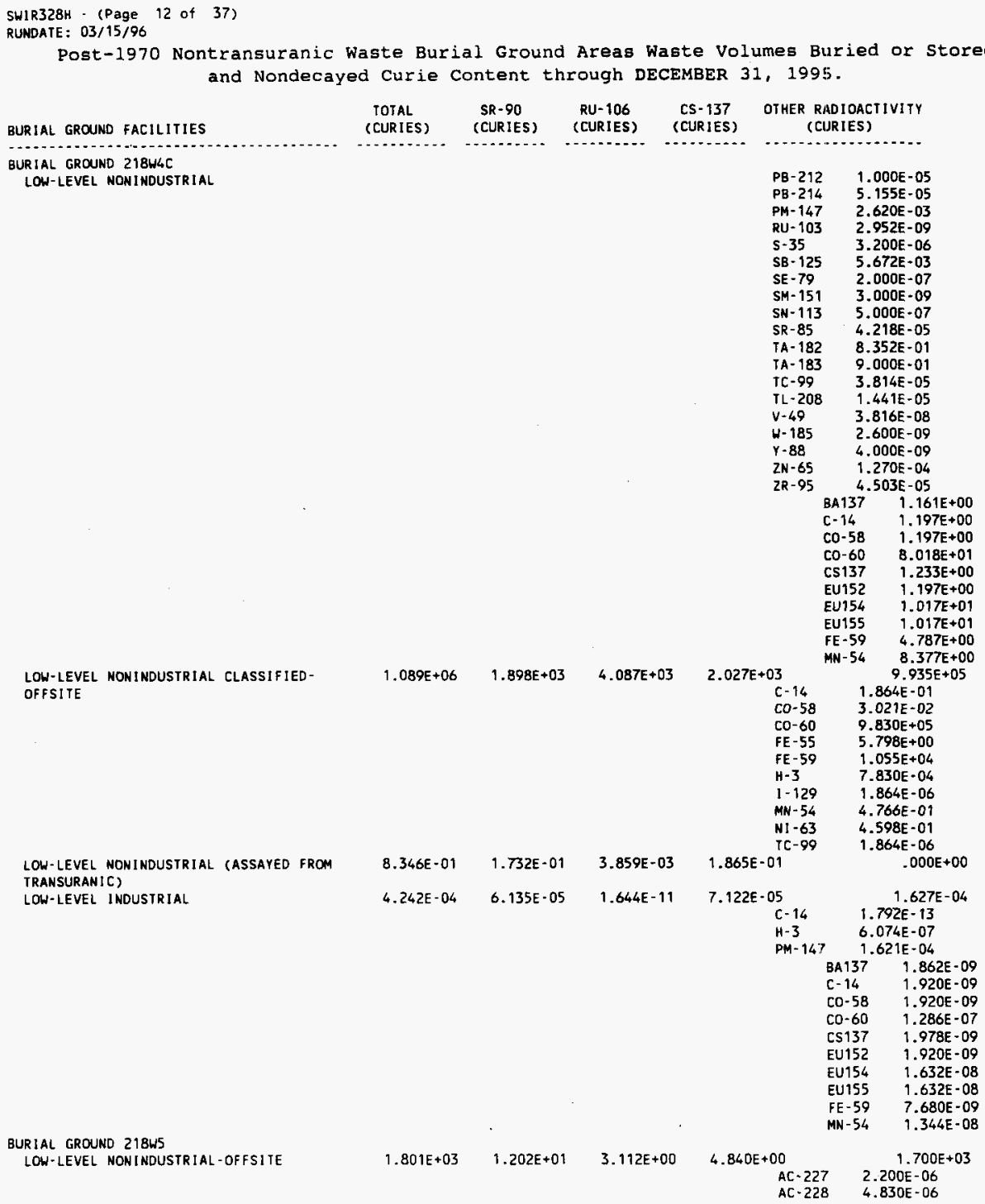


WHC-EP-0125-8

SWIR32BH - (Page 13 of 37)

RUMDATE: $03 / 15 / 96$

Post-1970 Nontransuranic Waste Burial Ground Areas Waste Volumes Buried or Stored and Nondecayed Curie Content through DECEMBER 31, 1995.

\begin{tabular}{|c|c|c|c|c|c|}
\hline 3URIAL GROUND FACILITIES & $\begin{array}{l}\text { TOTAL } \\
\text { (CURIES) }\end{array}$ & $\begin{array}{c}\text { SR-90 } \\
\text { (CURIES) }\end{array}$ & $\begin{array}{c}\text { RU-106 } \\
\text { (CUR IES) }\end{array}$ & $\begin{array}{c}\text { CS-137 } \\
\text { (CURIES) }\end{array}$ & $\begin{array}{l}\text { OTHER RADIOACIIVITY } \\
\text { (CURIES) }\end{array}$ \\
\hline 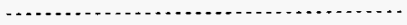 & $\ldots \ldots$ & $-\ldots \ldots . . . .$. & 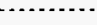 & 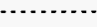 & - \\
\hline
\end{tabular}

BURIAL GRONND $218 W 5$

LOW-LEVEL NONINDUSTRIAL-OFFSITE

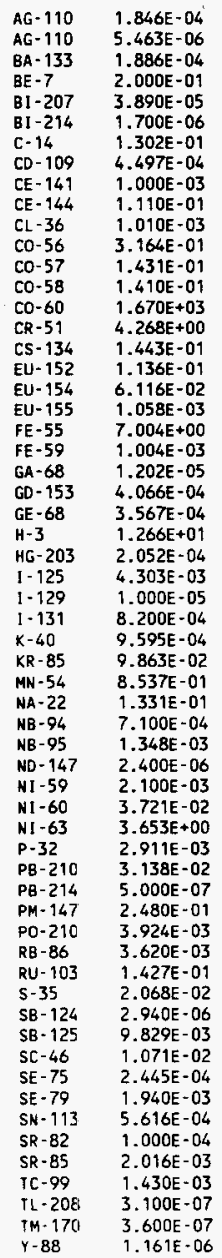


SWIR328H - (Page 14 of 37)

RUNDATE: $03 / 15 / 96$

Post-1970 Nontransuranic Waste Burial Ground Areas Waste Volumes Buried or stored and Nondecayed Curie Content through DECEMBER 31, 199.

\begin{tabular}{|c|c|c|c|c|c|}
\hline BURIAL GROUND FACILITIES & $\begin{array}{l}\text { TOTAL } \\
\text { (CURIES) }\end{array}$ & $\begin{array}{l}\text { SR }-90 \\
\text { (CURIES) }\end{array}$ & $\begin{array}{l}\text { RU }-106 \\
\text { (CURIES) }\end{array}$ & $\begin{array}{l}\text { CS- } 137 \\
\text { (CURIES) }\end{array}$ & $\begin{array}{c}\text { OTHER R.AD IOACT IVITY } \\
\text { (CUJRIES) }\end{array}$ \\
\hline SETL & $\ldots \ldots$ & - & & & \\
\hline
\end{tabular}

BURIAL GROUND $218 W 5$

LOW-LEVEL NONINDUSTRIAL-OFFSITE

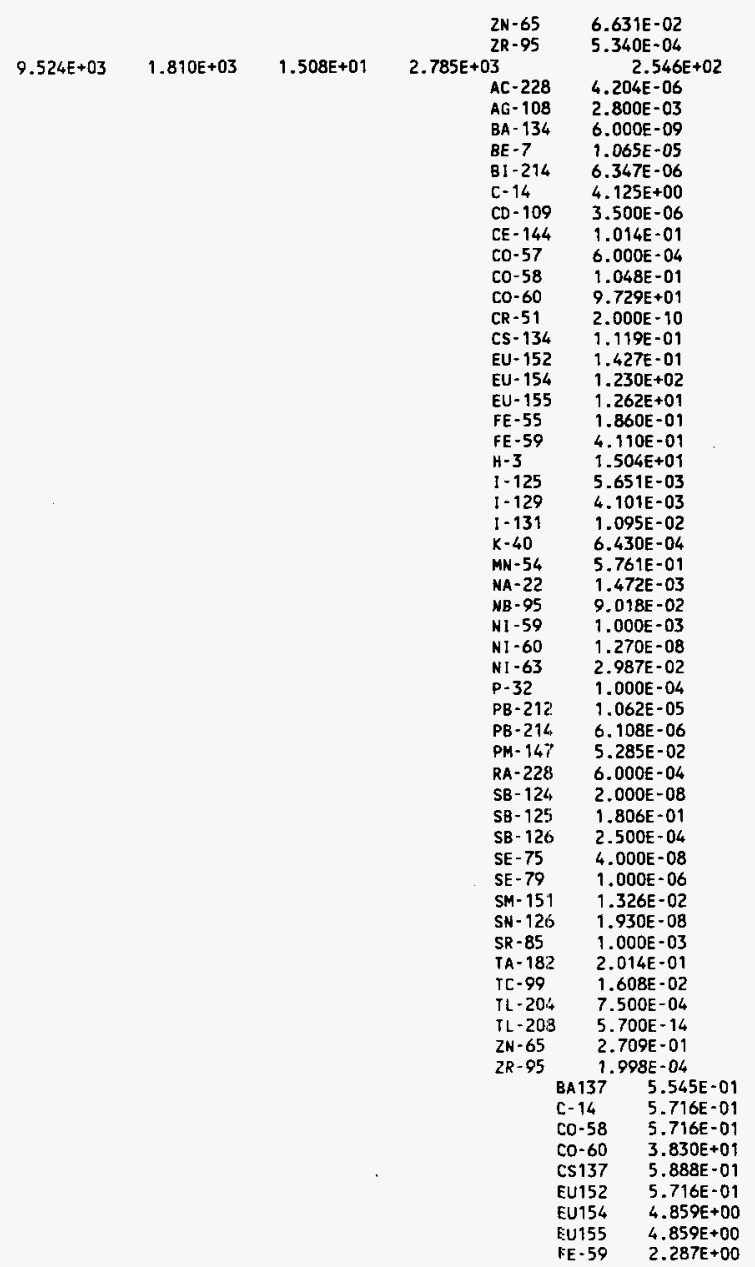


SWIR328H - (Page 15 of 37 )

RUNDATE: 03/15/96

Post-1970 Nontransuranic Waste Burial Ground Areas Waste Volumes Buried or Stored and Nondecayed Curie Content through DECEMBER 31, 1995.

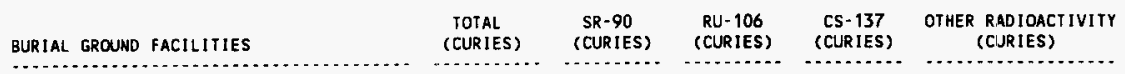

BURIAL GROUND 218W5

LON-LEVEL NONINDUSTRIAL

LDH-LEVEL NONINDUSTRIAL (ASSAYED FROM

TRANSURANIC)

LOH-LEVEL INDUSTRIAL-OFFSITE

$\begin{array}{lll}1.800 E-02 & 1.797 E-03 & 9.832 E-04 \\ 1.937 E+04 & 2.002 E+02 & 1.666 E+01\end{array}$

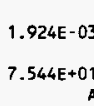
NN-54 $4.002 E+00$ $.000 E+00$

AC -227

AC -228

$A G-108$

AG- 110

$A G=110$

AL -28

AU- 195

BA- 133

BA- 134

$B A-137$

BA- 140

BE - 7

B 1-207

B 1 - 212

BI -214

C. 14

CA-45

CD- 109

CE-141

CE- 146

CL-36

Co-56

$\mathrm{CO}-57$

Co- 58

Co- 59

CO- 60

CR-51

CS-134

CU $-65 \mathrm{M}$

ES- 254

EU- 152

EU-154

EU- 155

FE- 55

FE- 59

GA- 68

GD- 152

GD -153

GO- 154

GE -68

$\mathrm{H}-3$

HF - 175

HG. 203

1- 123

$1-125$

1- 129

] -131

$K-40$

KR-85

MN - 54

MO-93

NA- 22

NB- 91

NB -9314

NB -94

NB- 95

$1.847 E+04$
$2.205 E-05$
$1.373 E-04$
$4.282 E-05$
$5.720 E-05$
$2.412 E-04$
$3.400 E-05$
$4.780 E-05$
$6.323 E-02$
$1.270 E-07$
$2.000 E-07$
$2.800 E-06$
$1.066 E+00$
$1.748 E-03$
$7.034 E-03$
$3.109 E-04$
$1.092 E-01$
$1.692 E-02$
$1.799 E-03$
$1.706 E-04$
$2.363 E-04$
$1.162 E-05$
$2.100 E-01$
$4.218 E-01$
$2.792 E-01$
$4.000 E-06$
$2.741 E+03$
$2.117 E-01$
$1.393 E+00$
$1.983 E-05$
$1.152 E-04$
$1.083 E+00$
$1.264 E-01$
$7.685 E-04$
$1.374 E+01$
$3.847 E-02$
$6.208 E-02$
$8.468 E-02$
$1.811 E-04$
$4.420 E-02$
$6.182 E-01$
$1.569 E+04$
$1.002 E-06$
$1.200 E-05$
$5.610 E-04$
$6.836 E-02$
$6.062 E-05$
$2.021 E-05$
$8.871 E-04$
$8.074 E-04$
$1.935 E+00$
$1.292 E+00$
$1.336 E+01$
$1.020 E-03$
$9.000 E-05$
$7.513 E-05$
$1.111 E-02$


SWIR328H - (Page 16 of 37)

RUNDATE : 03/15/96

Post-1970 Nontransuranic Waste Burial Ground Areas Waste Volumes Buried or Stored and Nondecayed Curie Content through DECEMBER 31, 1995.

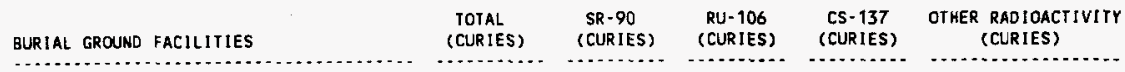

BURIAL GROUND 218W5

LOW-LEVEL INDUSTRIAL-OFFSITE

LOW-LEVEL INDUSTRIAL

B. $076 E+02 \quad 7.026 E+01$

$2.192 E+01$

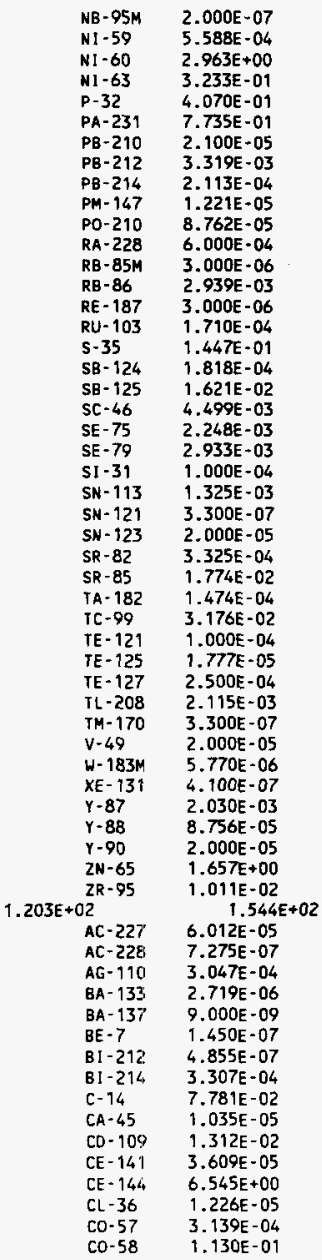


WHC-EP-0125-8

SHIR328H - (Page 17 of 37 )

RUNDATE : 03/15/96

Post-1970 Nontransuranic Waste Burial Ground Areas Waste Volumes Buried or Stored and Nondecayed Curie Content through DECEMBER 31, 1995.

$\begin{array}{lllll}\text { BURIAL GROUND FACILITIES } & \begin{array}{c}\text { IOIAL } \\ \text { (CURIES) }\end{array} & \begin{array}{c}\text { SR-90 } \\ \text { (CURIES) }\end{array} & \begin{array}{c}\text { RU-106 } \\ \text { (CURIES) }\end{array} & \begin{array}{c}\text { CS-137 } \\ \text { (CURIES) }\end{array} \text { OTHER RADIOACTIVITY } \\ \text { (CIJRIES) }\end{array}$

BURIAL GROUND 218W5

LOW-LEVEL INDUSTRIAL

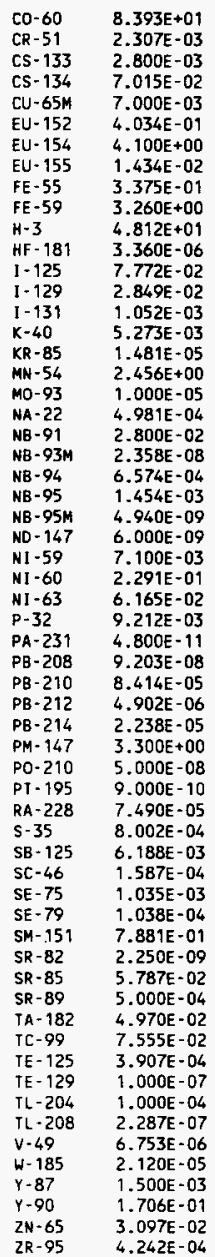




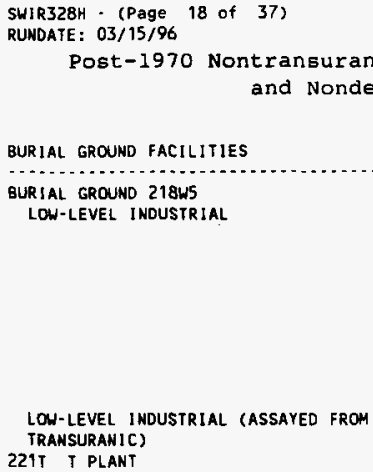

$2.049 \mathrm{E}+05$ $6.561 E-03$

$1.025 E+05$

$1.364 \mathrm{E}-03$
$.000 E+00$

$2.496 \mathrm{E}-05$ (CIRIES) 
SWIR328H - (Page 19 of 37 )

RUNDATE : 03/15/96

Post-1970 Nontransuranic Waste Burial Ground Areas Waste Volumes Buried or stored and Nondecayed Curie Content through DECEMBER 31, 1995.

BURIAL GROUND FACILITIES
CENIRAL WASTE COMPLEX $2402 \mathrm{~W}$ BUILDING
LOH-LEVEL NONINDUSTRIAL

\section{CENTRAL WASTE COMPLEX 2402WB BUILDING} LOW-LEVEL NONINDUSTRIAL-OFFSITE

LOW-LEVEL NOWIMDUSTR IAL

CENTRAL WASIE COMPLEX 2402WC BUILDING LOW-LEVEL NONINDUSTRIAL-OFFSITE

LOW-LEVEL NONINDUSTRIAL

\begin{abstract}
$\begin{array}{lcccc}\begin{array}{l}\text { TOTAL } \\ \text { (CURIES) }\end{array} & \text { SR-90 } & \text { RU-106 } & \text { CS-137 } & \text { OTHER RADIOACTIVITY } \\ \text { (CURIES) } & \text { (CURIES) } & \text { (CURIES) } & \text { (CURIES) }\end{array}$
\end{abstract}

(CURIES)

(CUR

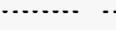


SWIR328H - (Page 20 of 37 )

RUNDATE : 03/15/96

Post-1970 Nontransuranic Waste Burial Ground Areas Waste Volumes Buried or Stored and Nondecayed Curie Content through DECEMBER 31, 1995. $\begin{array}{lcccc}\begin{array}{c}\text { TOIAL } \\ \text { (CURIES) }\end{array} & \begin{array}{c}\text { SR } 90 \\ \text { (CURIES) }\end{array} & \begin{array}{c}\text { RU-106 } \\ \text { (CURIES) }\end{array} & \begin{array}{c}\text { CS-137 } \\ \text { (CURIES) }\end{array} & \text { OTHER RIDIOACTIVITY } \\ \text { (CURIES) }\end{array}$

(CURIES) (CURIES) (CURIES)
BURIAL GROUND FACILITIES
.

CENTRAL WASTE COMPLEX 2402WC BUILDING LOW-LEVEL NONINDUSTRIAL

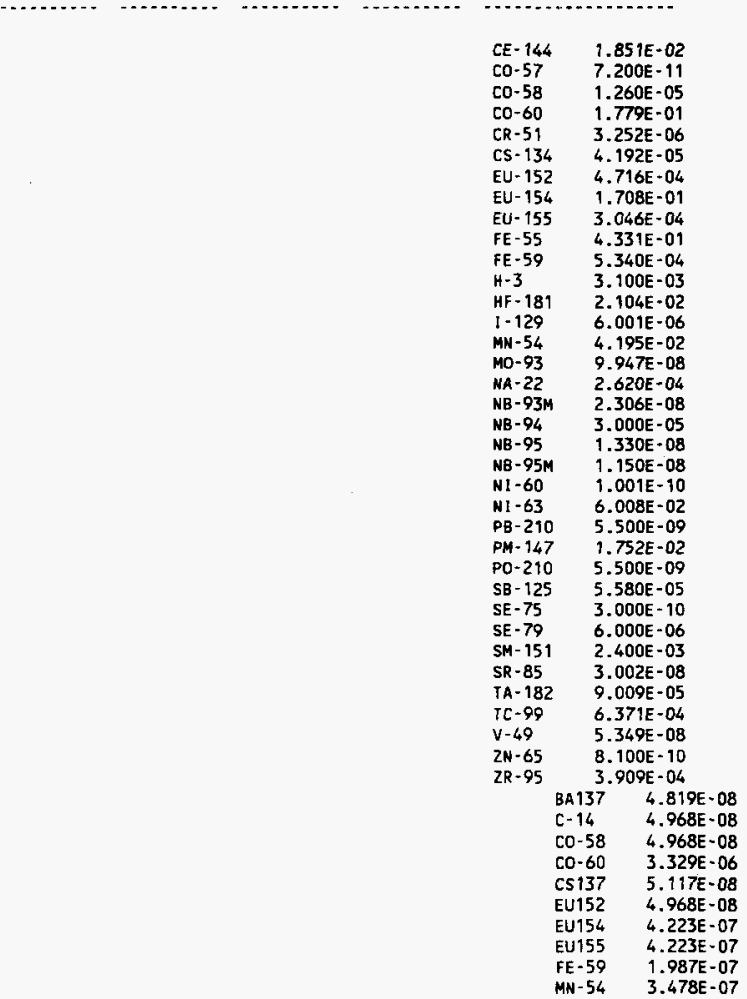

CENTRAL WASTE COMPLEX 2402WD BUILDING LOW-LEVEL NONINDUSTRIAL-OFFSITE

\begin{abstract}
$6.286 \mathrm{E}-02 \quad 1.101 \mathrm{E}-05 \quad 4.959 \mathrm{E}-06$
\end{abstract}
3.959
$6.272 E-02$

$1.200 \mathrm{E}-07$

4. $210 E-04$

6. $000 E-08$

$4.830 E-06$

3.500E-07

8. 300E-07

$6.223 E-02$

$1.150 E-07$

2.800E- 09

2. $790 \mathrm{E}-06$

$9.000 \mathrm{E}-08$

9. $860 E-06$ 


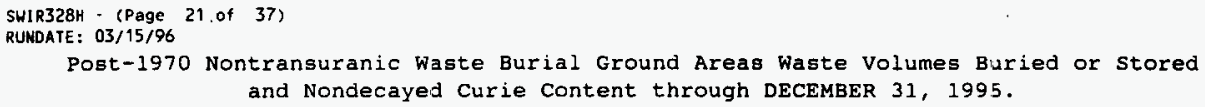


SWIR328H - (Page 22 of 37)

RUNDATE : 03/15/96

Post-1970 Nontransuranic Waste Burial Ground Areas Waste Volumes Blaried or Stored and Nondecayed Curie Content through DECEMBER 31, 1995.

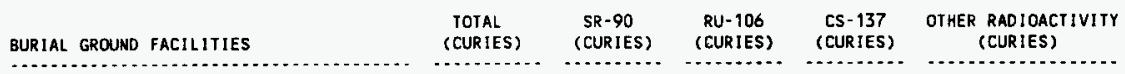

CENTRAL WASTE COMPLEX 2402WE BUILDING LOW-LEVEL NONINOUSTRIAL

CENTRAL WASTE COMPLEX 2402WF BUILDING LOW-LEVEL NONINDUSTRIAL
$1.526 E-01 \quad 3.600 E-04 \quad 8.512 E-13 \quad 4.605 E-04$

CO- 60

EU- 154

EU- 155

FE- 59

$\mathrm{MN}-54$

N1-60

N1 -63

B -1

$$
\begin{aligned}
& \mathrm{C}-14 \\
& \mathrm{CO}-58
\end{aligned}
$$

co-58

CS137

EU152

EU154

EU155

FE-59

MN- 54
$6.806 E-04$

1.600E-02

5. 200E-09

$2.964 \mathrm{E}-03$

$1.200 \mathrm{E}-07$

7.130E -05

1.769E-01

$1.162 E-04$

$1.410 E-03$

$5.060 \mathrm{E}-02$

$4.570 E-03$

1.570E-03

2. $642 \mathrm{E}-05$

BA137 $1.627 E-06$

C- $14 \quad 1.677 \mathrm{E}-06$

CO-58 $\quad 1.677 \mathrm{E}-06$

C0-60 1.124E-04

CS137 1.727E-06

EU152 1.677E-06

EU154 1.425E-05

EU155 1.425E-05

FE-59 6.708E-06

1.510E-01

1. $150 \mathrm{E}-01$

3. $640 E-09$

$1.568 \mathrm{E}-09$

1. $440 \mathrm{E}-02$

2. 160E-02

$1.258 E-06$

8.674E-08

$\begin{array}{ll}8.674 E-08 \\ 137 & 2.409 E-07\end{array}$

$2.409 E-07$
$2.484 E-07$

2. $484 \mathrm{E}-07$

1.664E-05

2.559E-07

2.484E-07

2. 111E-06

2.111E-06

9.936E-07

$\begin{array}{llll}1.150 E-02 & 2.441 E-03 \quad 2.842 E-05 & 2.629 E-03\end{array}$

1. $.739 \mathrm{E}-06$
$.000 E+00$ $.000 E+00$

LOW-LEVEL NONI
TRANSURANIC)

CENTRAL WASTE COMPLEX $2402 W G$ BUILDING

LOW-LEVEL NONINDUSTRIAL-OFFSITE $\begin{array}{llll}7.500 E-02 & 1.686 E-04 & 2.186 E-04 & 1.756 E-04\end{array}$

AC- 228

BI -212

C. 14

$\mathrm{CD}-113$

CO- 60

c5- 134

CS- 135

EU-15z̈:

EU- 154

FE -55

$\mathrm{H}-3$

$1 \cdot 125$

$1-129$

KR- 85
$6.963 E-02$

4.300E-09

4. 300E-09

3. $138 E-04$

4.300E-09

$1.630 E-06$

8. $600 \mathrm{E}-10$

$2.290 E-10$

3.382E-06

3. $381 E-06$

8. $900 \mathrm{E}-10$

$6.918 E-02$

$6.700 E-06$

2. $130 E-11$

3. $600 \mathrm{E}-06$ 
SWIR328H - (Page 23 of 37)

RUNDATE: 03/15/96

Post-1970 Nontransuranic Waste Burial Ground Areas Waste Volumes Buried or stored and Nondecayed Curie Content through DECEMBER 31, 1995.

\begin{tabular}{|c|c|c|c|c|c|}
\hline BURIAL GROUND FAD & $\begin{array}{l}\text { TOTAL } \\
\text { (CURIES) }\end{array}$ & $\begin{array}{l}\text { SR-90 } \\
\text { (CURIES) }\end{array}$ & $\begin{array}{l}\text { RU-106 } \\
\text { (CURIES) }\end{array}$ & $\begin{array}{l}\text { CS- } 137 \\
\text { (CURIES) }\end{array}$ & $\begin{array}{l}\text { OTHER RADIOACTIVITY } \\
\text { (CURIES) }\end{array}$ \\
\hline
\end{tabular}

CENTRAL WASIE COMPLEX 2402WG BUILDING

LOW-LEVEL NONINOUSTRIAL-OFFSIIE

LOW-LEVEL NONINDUSTRIAL

\section{...}




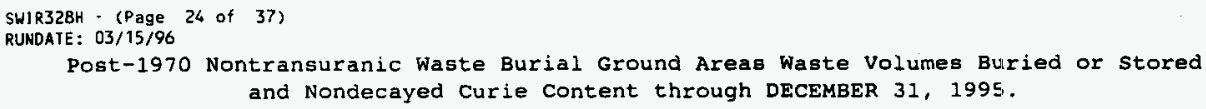

3.135E-0.

$4.532 E-06$

8.610E-07

$5.490 \mathrm{E}-06$

C. 14

$7.953 E-06$

H.3 2.240E-OB

$1-129 \quad 1.530 \mathrm{E}-12$

NB-94 3.710E-15

PM-147 5.980E-06

SE-79 2.450E- 11

TC $-99 \quad 9.060 \mathrm{E}-10$

CENTRAL WASTE COMPLEX 2402WH BUILDING LOW-LEVEL NONINDUSTRIAL-OFFSITE

LOW-LEVEL NONINDUSTRIAL

CENTRAL WASTE COMPLEX 2402WI BUILDING LOW-LEVEL NONINDUSTRIAL-OFFSITE

LOW-LEVEL NONINDUSTRIAL
$6.502 E-02$

(2)

$8.392 E-03$

5.020E-03

7.063E-03

$1.384 E-03$

C- 14

CE-146

CO- 60

$\mathrm{H} \cdot 3$

$1-129$

1- 131

NB-94

H1 $1-59$

NI -63

SE -79

TC-99

ZR -95

Bi -214

C- 14

CE -144

CO- 60

CS- 135

EU-152

EU-154

EU- 155

FE-59

$\mathrm{H}=3$

I -129

MN-54

NA -22

$\mathrm{NB}-94$

NI -60

$N !-63$

PB-214

PD- 107

PM- 147

SE - 79

SM- 151

SN- 123

TC- 99

$2 \mathrm{~N}-65$

$2 \mathrm{R}-90$

$2 R-93$

2R-95

$\begin{array}{rr}6.750 E-06 & .000 E+00 \\ 2.919 E-01 & 5.452 E-03\end{array}$

$.000 E+00$

$.000 E+00$

2.837E-05

5.030E-03
$\mathrm{H}-3$

C- 14 CE -146 CO- 60

$\mathrm{H} \cdot 3$

I- 129
2. $445 E-06$

3.550E-08

3. $000 \mathrm{E}-07$

$5.800 \mathrm{E}-07$

$1.104 \mathrm{E}-06$

$9.200 \mathrm{E}-13$

3.000E-07

$2.600 E-14$

$2.500 \mathrm{E}-10$

2.500E-08

$1.800 E-11$

$5.000 \mathrm{E}-10$

$1.000 E-07$ $1.021 E+00$

1. $140 \mathrm{E}-06$

$6.424 \mathrm{E}-09$

1. 160 E-O2

$9.092 E-04$

1.000 E -04

6.694E-05

$1.191 \mathrm{E}-04$

4.816E-04

$6.000 E-05$

$1.000 E+00$

$1.000 \mathrm{E}-04$

$9.500 \mathrm{E}-05$

$1.162 \mathrm{E}-05$

4.440E-15

$9.146 E-07$

$2.700 \mathrm{E}-10$

1. $140 \mathrm{E}-06$

$1.000 E-04$

5.837E-03

$1.930 E-11$

$1.000 E-03$

$6.000 E-05$

$1.012 E-04$

1. $350 \mathrm{E}-10$

1.000E-04

$1.696 \mathrm{E}-13$

2.915E-06

$6.750 E-06$

2.691E-01

$1.000 E-06$

1. $122 \mathrm{E}-06$

$7.609 \mathrm{E}-04$

2. $580 \mathrm{E}-08$

$1.864 E-12$ 


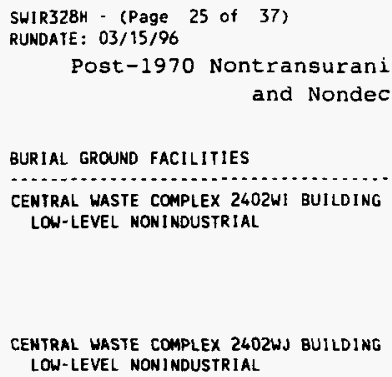

CENTRAL WASTE COMPLEX 2602WJ BUILDING LOW-LEVEL NON] NDUSTRIAL

CENTRAL WASTE COMPLEX 2402WK BUILDING LOW-LEVEL NONINDUSTRIAL

CENTRAL WASTE COMPLEX 2403WA BUILDING LOW-LEVEL NONI HDUSTRIAL-OFFSITE

\begin{tabular}{llccc}
$\begin{array}{l}\text { TOTAL } \\
\text { (CUR!ES) }\end{array}$ & $\begin{array}{c}\text { SR-90 } \\
\text { (CURIES) }\end{array}$ & $\begin{array}{c}\text { RU-106 } \\
\text { (CURIES) }\end{array}$ & $\begin{array}{c}\text { CS-137 } \\
\text { (CURIES) }\end{array}$ & $\begin{array}{c}\text { OTHER FADIOACTIVITY } \\
\text { (CURIES) }\end{array}$ \\
\hline
\end{tabular}

\begin{tabular}{|c|c|c|c|c|}
\hline & & & $\begin{array}{l}\text { NB-94 } \\
\text { PM- } 147 \\
\text { PO-210 } \\
\text { SE-79 } \\
\text { SR }-85 \\
\text { TC }-99\end{array}$ & $\begin{array}{l}4.270 E-15 \\
6.880 E-06 \\
5.000 E-10 \\
2.820 E-11 \\
1.050 E-11 \\
2.683 E-01\end{array}$ \\
\hline $1.717 E-01$ & $5.228 E-04$ & $8.066 E-04$ & $\begin{array}{r}6.244 E-04 \\
\text { C- } 14 \\
\text { CE }-144 \\
H-3 \\
\text { PM- } 147 \\
\text { SM-151 } \\
\text { TC- } 99\end{array}$ & $\begin{array}{l}1.647 E-01 \\
7.390 E-13 \\
3.100 E-03 \\
2.960 E-06 \\
1.500 E-03 \\
4.000 E-04 \\
1.597 E-01\end{array}$ \\
\hline $1.312 E+00$ & $1.954 \mathrm{E}-02$ & $2.280 E-02$ & $\begin{array}{l}2.160 E-02 \\
\text { C- } 14 \\
\text { CE-144 } \\
\text { EU-155 } \\
H-3 \\
\text { PH-147 } \\
\text { SM-151 } \\
\text { SN-123 } \\
\text { IC }-99\end{array}$ & $\begin{array}{l}1.085 E+00 \\
2.127 E-11 \\
8.970 E-02 \\
1.700 E-04 \\
7.001 E-01 \\
4.246 E-02 \\
1.350 E-02 \\
4.000 E-05 \\
2.394 E-01\end{array}$ \\
\hline
\end{tabular}

$7.885 E-01 \quad 6.328 E-02 \quad 2.452 E-05 \quad 1.987 E-01$
$A G=110$

$A G-110$

$\mathrm{BE}-7$

BI- 212

BI-214

C- 14

CA- 45

CE- 144

Co-56

CO- 57

CO- 58

Co- 60

CR-51

CS- 134

EU- 152

EU- 154

EU. 155

FE-55

FE- 59

H-3

HF- 175

HG- 203

] -125

$1-129$

K- 40

KR- 85

$M N=54$

NA- 22

NB- 94

NI -59

NI -60

Ni -63

P- 32

PB-212
2.739E-01

2.235E-07

$8.526 \mathrm{E}-08$

5.224E-03

2.500E-07

1. $288 \mathrm{E}-05$

$2.389 \mathrm{E}-03$

$1.173 \mathrm{EE}-03$

8. 500 E-04

2.642E-06

$7.318 E-05$

1.174E-05

1.073E-01

5.000E-05

$1.271 E-03$

5.182E-04

3.581E-04

4.870E-06

2.521E-04

3.310E-05

2. $055 E-02$

3. $200 E-04$

2.133E-04

2.050E-05

3. $500 \mathrm{E}-06$

$1.396 \mathrm{E}-02$

$2.486 \mathrm{E}-03$

$1.211 E-04$

$1.319 E-04$

$1.793 \mathrm{E}-08$

$7.644 \mathrm{E}-06$

$6.892 E-02$

$9.516 \mathrm{E}-04$

$9.330 \mathrm{E}-04$

$2.500 E-07$ 
SWIR328H - (Page 26 of 37 )

RUNDATE : $03 / 15 / 96$

Post-1970 Nontransuranic Waste Burial Ground Areas waste Volumes Buried or Stored and Nondecayed Curie content through DECEMBER 31, 1995 .

\begin{tabular}{llllll} 
BURIAL GROUND FACILITIES & $\begin{array}{c}\text { TOIAL } \\
\text { (CURIES) }\end{array}$ & $\begin{array}{c}\text { SR-90 } \\
\text { (CURIES) }\end{array}$ & $\begin{array}{c}\text { RU-106 } \\
\text { (CURIES) }\end{array}$ & $\begin{array}{c}\text { CS-137 } \\
\text { (CURIES) }\end{array}$ \\
\hline
\end{tabular}

CENTRAL WASTE COMPLEX 2403WA BUILDING

LOH-LEVEL NONINDUSTRIAL-OFFSITE

OTHER RADIOACTIVITY (CURIES)

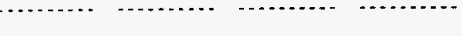

LOH-LEVEL NONINDUSTRIAL-OFFSITE

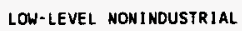

$1.20060-01$

$9.715 E-01 \quad 4.844 E-01$

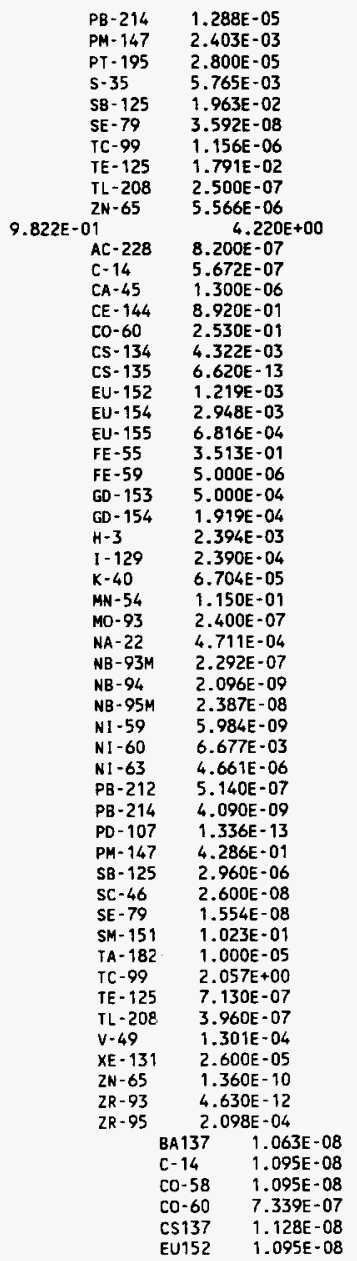


WHC-EP-0125-8

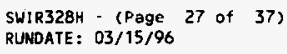

\begin{tabular}{|c|c|c|c|c|c|c|c|}
\hline BURIAL GROUND FACILITIES & $\begin{array}{l}\text { TOTAL } \\
\text { (CURIES) }\end{array}$ & $\begin{array}{l}\text { SR }-90 \\
\text { (CURIES) }\end{array}$ & $\begin{array}{l}\text { RU- } 106 \\
\text { (CURIES) }\end{array}$ & $\begin{array}{l}\text { CS-137 } \\
\text { (CURIES) }\end{array}$ & OTHER & $\begin{array}{l}\text { RADIOAC } \\
\text { (CIJRIES) }\end{array}$ & VITY \\
\hline $\begin{array}{l}\text { CENTRAL WASTE COMPLEX 2403WA BUILDING } \\
\text { LOW-LEVEL NONINDUSTRIAL }\end{array}$ & & & & & & $\begin{array}{l}\text { EU154 } \\
\text { EU155 } \\
\text { FE-59 } \\
\text { IMN-54 }\end{array}$ & $\begin{array}{l}9.311 E-08 \\
9.311 E-08 \\
4.382 E-08 \\
7.668 E-08\end{array}$ \\
\hline
\end{tabular}

CENTRAL WASTE COMPLEX 2403WB BUILDING LOW-LEVEL NONINOUSTR IAL-OFFSITE
$1.313 E+00$

$2.755 E-03$
3.470E-03

\subsection{E-02}

BI -214

\section{14}

CD- 109

CO- 60

CS -134

EU- 154

FE -55

FE-59

$\mathrm{H}-3$

I -129

k-40

NA -22

NB- 94

NI -60

NI -63

PO- 210

$\mathrm{SB}-125$

SE -79

IC- 99

TE- 125

LOW-LEVEL NON I NDUSTRIAL
B. $649 \mathrm{E}+01$

$2.907 E+01$ $1.227 E+01$

3. $142 \mathrm{E}-02$
BA -133

$C=14$

CE -144

CO- 60

CS- 134

EU- 152

EU- 154

$\mathrm{H}-3$

I- 129

K -40

MN -54

NB -93 H

NB- 94

NB -95

N1 -59

NI -63

$\mathrm{P}-32$

$P B=210$

PM- 147

RA- 228

SB- 125

SE. 79

SR-85

$S R-89$

IC. 99

$\mathrm{ZN}-65$
$1.132 E+00$

1.200E-08

$4.600 \mathrm{E}-12$

$1.000 E-05$

$1.282 \mathrm{E}-03$

$3.255 E-04$

3. $331 E-05$

$4.400 \mathrm{E}-08$

$6.800 \mathrm{E}-10$

8. $705 \mathrm{E}-05$

4. $400 E-08$

$4.870 E-06$

$1.900 E-06$

$4.400 \mathrm{E}-18$

2. $700 \mathrm{E}-04$

1. $130 E+00$

$2.861 \mathrm{E}-08$

$5.899 \mathrm{E}-05$

8. $200 E-14$

1.659E-05

3. $825 E-05$ $2.309 E+00$

4.715E-05

$3.044 \mathrm{E}-09$

$1.059 \mathrm{E}-02$

$1.347 E+00$

$4.066 \mathrm{E}-04$

$2.880 \mathrm{E}-09$

4. $505 \mathrm{E}-02$

5.381E-01

3.691E-05

2. $626 \mathrm{E}-05$

$2.000 E-02$

8.670E-11

$6.230 E-04$

3.001E-02

2. $900 \mathrm{E}-12$

$5.247 \mathrm{E}-08$

$1.000 \mathrm{E}-04$

5.500E-07

2. $738 \mathrm{E}-02$

$3.042 E-07$

$6.060 E-02$

$7.250 \mathrm{E}-12$

$8.520 \mathrm{E}-07$

$5.670 \mathrm{E}-08$

$1.390 \mathrm{E}-01$

9. $000 \mathrm{E}-02$

BA $137 \quad 2.744 E-04$ E-14 2.829E-04 C0-58 2.829E-04 100-60 1.895E-02 C\$137 2.914E-04 EU152 2.829E-04 EU154 $2.405 E-03$ 
SWIR328H - (Page 28 of 37 )

RUNDATE : 03/15/96

Post-1970 Nontransuranic Waste Burial Ground Areas Waste Volumes Buried or stored and Nondecayed Curie Content through DECEMBER 31, $1995_{1}$.

\begin{tabular}{|c|c|c|c|c|c|}
\hline BURIAL GROUND FACILITIES & $\begin{array}{l}\text { TOIAL } \\
\text { (CURIES) }\end{array}$ & $\begin{array}{l}\text { SR-90 } \\
\text { (CURIES) }\end{array}$ & $\begin{array}{l}\text { RU- } 106 \\
\text { (CURIES) }\end{array}$ & $\begin{array}{l}\text { CS- } 137 \\
\text { (CURIES) }\end{array}$ & $\begin{array}{c}\text { OTHER RADIOACTIVITY } \\
\text { (CUIRIES) }\end{array}$ \\
\hline
\end{tabular}

CENTRAL WASTE COMPLEX 2403 WB BUILDING LOW-LEVEL NONINDUSTRIAL

LOW-LEVEL NONINDUSTRIAL (ASSAYED FROM TRANSURANIC)

CENTRAL WASTE COMPLEX 2403WC BUILDING LOW-LEVEL NONINDUSTRIAL-OFFSITE

\begin{tabular}{|c|c|c|c|c|c|}
\hline & & & & $\begin{array}{l}\text { EU155 } \\
\text { FE-59 } \\
\text { MNN-54 }\end{array}$ & $\begin{array}{l}2.405 E-03 \\
1.132 E-03 \\
1.980 E-03\end{array}$ \\
\hline $1.830 E-01$ & $8.893 E-02$ & $6.722 E-07$ & $\begin{array}{l}2.536 E-03 \\
\text { CO-60 } \\
\text { EU- } 154\end{array}$ & & $\begin{array}{l}1.570 E-04 \\
\text { OOE-05 } \\
\text { OOE }-04\end{array}$ \\
\hline
\end{tabular}

$1.238 E+01 \quad 3.749 E-02$

4. $487 \mathrm{E}-02$

$1.207 E+01$
AC- 228

$A G-110$

AG- 110

AU- 195

BE- 7

BI -207

BI -212

BI - 214

C -14

CA -45

CD- 109

CD- 113

CE- 144

Co-56

CO-57

Co-58

Co-60

CR-51

CS-134

EU-152

EU-154

EU-155

FE -55

GA- 68

GE -68

$\mathrm{H}-3$

HF- 175

HG- 203

I- 125

I -129

I- 131

$\mathrm{K}-40$

KR- 85

$M N-54$

NA- 22

NI-60

N1 -63

$\mathrm{P}-32$

PA- 231

PB- 212

PB- 214

PM- 147

PO- 210

RA-228

S- 35

SB- 124

SB- 125

SC -46

SC. 47

SE- 75

SH- 151

$\mathrm{SN}-113$
9.710E-08

1.068E-06

$4.809 E-05$

4.270E-07

2.116E-03

1. $340 E-06$

1. $025 \mathrm{E}-07$

$6.369 E-05$

3.476E-03

7. DOOE-03

1. $747 E-01$

8.891E-01

6. $211 E-06$

1. 272E-06

5. 128E-04

1. $760 \mathrm{E}-03$

1.606E-01

2.400E-05

8.513E-04

2.979E-05

8. $092 E-04$

$9.506 E-04$

2.738E-02

7.800E-0?

$5.000 E-06$

6.809E-01

B.850E-05

5. 900E-05

$2.115 E-02$

$9.545 E-06$

$1.000 E-05$

$3.756 \mathrm{E}-02$

$4.600 E-09$

B.692E-03

$1.773 \mathrm{E}-03$

8.910 E-05

4.500E-03

$3.296 \mathrm{E}-02$

$5.925 E-05$

$1.025 \mathrm{E}-07$

$6.210 \mathrm{E}-05$

2. $830 \mathrm{E}-08$

$7.450 E-05$

$9.710 \mathrm{E}-08$

$7.101 E-03$

$6.952 E-04$

$1.475 E-04$

$3.430 E-06$

1.510E-05

4. $800 \mathrm{E}-07$

$4.700 E-10$

$1.000 E-06$ 
WHC-EP-0125-8

SWIR328H - (Page 29 of 37 )

RUNDATE : 03/15/96

Post-1970 Nontransuranic Waste Burial Ground Areas Waste Volumes Buried or Stored and Nondecayed Curie Content through DECEMBER 31, 1995.

$\begin{array}{lllll}\text { BURIAL GROUND FACILITIES } & \begin{array}{c}\text { TOTAL } \\ \text { (CURIES) }\end{array} & \begin{array}{c}\text { SR-90 } \\ \text { (CURIES) }\end{array} & \begin{array}{c}\text { RU-106 } \\ \text { (CURIES) }\end{array} & \begin{array}{c}\text { CS-137 } \\ \text { (CURIES) }\end{array} \\ \text { (CUHER RADIOACTIVITY } \\ \text { (CURIES) }\end{array}$

CENTRAL WASTE COMPLEX 2403WC BUILDING

LOW-LEVEL NONINDUSTRIAL-OFFSITE

LOW-LEVEL NONINDUSTR IAL

(CUR...... - -

(1)

$6.040 E+01 \quad 2.341 E+01$

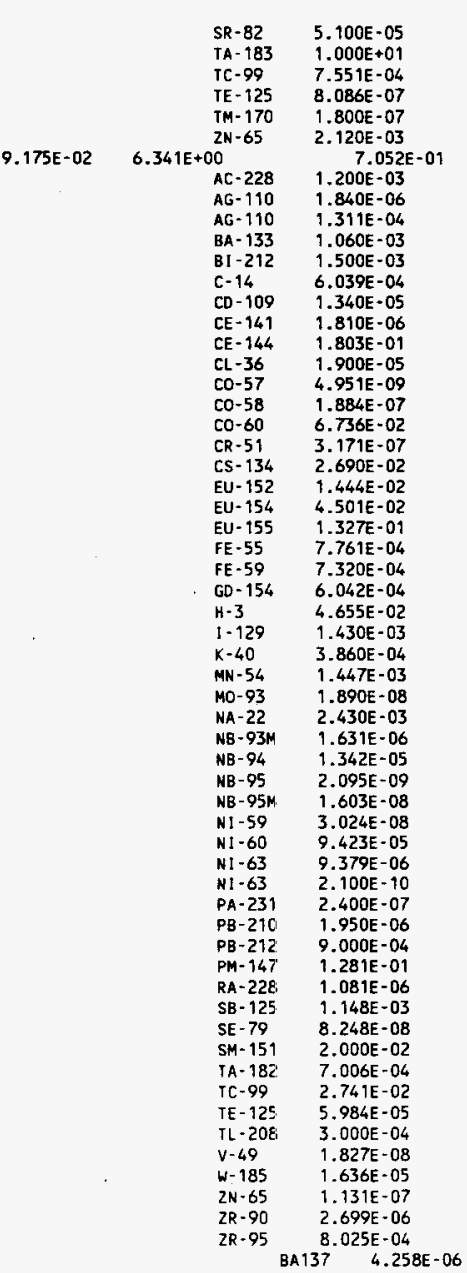




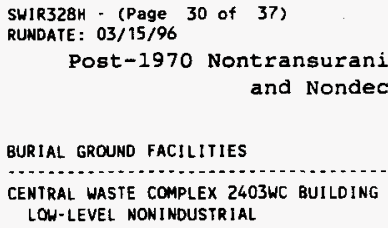

LOW-LEVEL NONINDUSTRIAL (ASSAYED FROM TRANSURANIC)

CENTRAL HASTE COMPLEX 2403WD BUILDING LOW-LEVEL NONIMDUSTRIAL-OFFSITE

LOW-LEVEL NONINDUSTRIAL

ALKALINE METAL WASTE STORAGE UNIT LOW-LEVEL NONINDUSTRIAL

ALKALINE METAL WASIE STORAGE UNIT 2 LOW-LEVEL NONINDUSTRIAL

$\begin{array}{lllll}1.000 E-03 \quad 1.310 E-04 \quad 3.818 E-05 \quad 1.404 E-04 & \text { MN-54 } & \begin{array}{l}3.073 E-05 \\ .000 E+00\end{array}\end{array}$

$1.343 E+04 \quad 1.270 E-01 \quad 2.728 E-01 \quad 1.356 E-0$

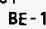

B1-212

C. 14

Co- 60

EU-154

FE -55

$\mathrm{H}-3$

$1 \cdot 129$

MN-54

MO- 93

NB- 94

N1 -59

N1 -63

PB- 212

SE -79

SM-15?

TC- 99

$3.191 E+03 \quad 7.097 E+00 \quad 3.520 E-02$

$2.429 \mathrm{E}+01$

2R-93

C- 14

CE- 144

CD- 60

EU-152

EU- 154

EU- 155

H-3

1. 129

K. 40

$M N-54$

NB-93M

NB -94

N! -59

N1-60

NI -63

PM- 147

SE - 79

SM- 151

$S N-123$

TC. 99

ZN-65

$1.400 \mathrm{E}-04$

8.305E-07

$4.438 E-0$

$7.090 \mathrm{E}-05$

$$
\text { BA- } 133
$$$$
\text { NA }-22
$$

$1.342 E+04$

$1.060 \mathrm{E}-05$

$9.900 E-10$

5.060E-01

$2.440 \mathrm{E}+03$

4. $480 E-06$

$9.760 E+02$

3.670E-01

$1.010 E-06$

$1.200 \mathrm{E}+00$

3.950E-02

2.610E-01

$8.530 E+01$

$9.920 E+03$

$9.900 \mathrm{E}-10$

8. 250 E-08

5. 660 E-06

1. $070 \mathrm{E}-02$

1. 140E-OS 3. $129 \mathrm{E}+03$

5.3BOE-05

$1.085 E-01$

3. $005 \mathrm{E}+03$

8.121E-03

2.294E-01

2.177E-03

1. $700 \mathrm{E}+00$

1. $641 \mathrm{TE}-03$

3.600E-05

4. $050 \mathrm{E}-10$

2.230E-04

4. $100 \mathrm{E}-06$

$1.092 E+00$

$2.410 E-05$

$1.200 E+02$

$5.452 E-02$

1. $865 E-05$

$1.360 \mathrm{E}-02$

$1.000 E-04$

$6.243 \mathrm{E} \cdot 01$

$1.350 E-10$

$1.134 E-01 \quad 1.064 E-04$

5.062E-02
$.000 E+00$

$.000 E+00$ $.000 E+00$

$1,460 \mathrm{E}-02$ 1. $162 E-03$
$2.117 E-06$ 
SHIR328H - (Page 31 of 37 )

RUNDATE: $03 / 15 / 96$

Post-1970 Nontransuranic Waste Burial Ground Areas Waste Volumes Buried or Stored and Nondecayed Curie Content through DECEMBER 31, 1995.

\begin{tabular}{llllll} 
BURIAL GROUND FACILITIES & $\begin{array}{l}\text { TOTAL } \\
\text { (CURIES) }\end{array}$ & $\begin{array}{c}\text { SR-90 } \\
\text { (CURIES) }\end{array}$ & $\begin{array}{c}\text { RU-106 } \\
\text { (CURIES) }\end{array}$ & $\begin{array}{c}\text { CS-137 } \\
\text { (CURIES) }\end{array}$ \\
\hline
\end{tabular}

\section{ALKALINE METAL WASTE SIORAGE UNIT 2} LOW-LEVEL NONINDUSTRIAL

ALKALINE METAL WASTE STORAGE UNIT 3 LOW-LEVEL NONINDUSTRIAL

ALKALINE METAL. WASTE STORAGE UNIT 4 LOW-LEVEL NONINDUSTRIAL

INVENTORY NOT YET VERIFIED BY SWO LOW-LEVEL NONINDUSTRIAL

flamagle storage moOUle 1 LOW-LEVEL NONINDUSTRIAL

\begin{tabular}{|c|c|c|c|c|}
\hline & & & NA -22 & $1.344 E-02$ \\
\hline $2.310 E-03$ & $4.152 E-06$ & $2.219 E-08$ & $\begin{aligned} 3.545 E-04 \\
\text { BA }-133 \\
\text { NA }-22\end{aligned}$ & $\begin{array}{c}1.610 E-03 \\
1.610 E-03 \\
.000 E+00\end{array}$ \\
\hline $7.016 \mathrm{E}-02$ & $4.368 E-06$ & $4.545 E-08$ & $\begin{array}{l}1.978 E-02 \\
\text { NA }-22\end{array}$ & $\begin{array}{l}3.165 E-02 \\
3.165 E-02\end{array}$ \\
\hline $1.227 E-06$ & $6.021 \mathrm{E}-06$ & $7.403 E-11$ & $\begin{array}{l}5.471 E-06 \\
\text { TC }-99\end{array}$ & $\begin{array}{c}1.000 E-04 \\
1.000 E-04\end{array}$ \\
\hline $1.348 \mathrm{E}-01$ & $2.990 E-04$ & $2.300 E-05$ & $\begin{array}{c}3.211 E-04 \\
C-14 \\
C E-144 \\
C O-58 \\
C O-60 \\
C R-51 \\
\text { FE }-59 \\
\text { MN-54 } \\
\text { NA- } 22 \\
\text { NB- } 95 \\
\text { SC }-47 \\
\text { TA }-182 \\
\text { TA }-183 \\
2 N-65\end{array}$ & $\begin{array}{l}1.329 E-01 \\
9.420 E-04 \\
2.465 E-04 \\
6.980 E-03 \\
7.671 E-04 \\
3.042 E-03 \\
1.000 E-04 \\
2.974 E-03 \\
1.385 E-03 \\
1.054 E-04 \\
4.900 E-05 \\
1.156 E-01 \\
4.390 E-04 \\
2.860 E-04\end{array}$ \\
\hline $1.229 E-04$ & $9.515 E-06$ & $1.053 E-08$ & $\begin{aligned} 5.221 E-09 \\
C-14 \\
H-3 \\
K=40 \\
M N-54\end{aligned}$ & $\begin{array}{l}1.037 E-04 \\
4.300 E-05 \\
6.060 E-05 \\
7.850 E-08 \\
7.000 E-10\end{array}$ \\
\hline $5.656 E-04$ & $1.189 E-04$ & $1.945 E-06$ & $\begin{array}{l}9.057 E-05 \\
\text { C- } 14 \\
\text { CE }-144 \\
\text { CS }-134 \\
\text { EU- } 152 \\
\text { EU- } 154 \\
\text { EU }-155 \\
H-3 \\
1-129 \\
N B-94 \\
\text { PH }-147 \\
\text { SE }-79 \\
\text { TA }-182 \\
\text { TC }-99\end{array}$ & $\begin{array}{l}1.427 E-04 \\
1.322 E-14 \\
5.010 E-06 \\
5.813 E-07 \\
3.080 E-05 \\
5.727 E-05 \\
8.620 E-06 \\
8.601 E-06 \\
1.097 E-07 \\
7.420 E-15 \\
2.857 E-05 \\
4.900 E-11 \\
1.110 E-07 \\
3.017 E-06\end{array}$ \\
\hline $1.232 E-02$ & $1.796 E-04$ & $2.144 E-04$ & $\begin{array}{l}1.063 E-04 \\
\text { BE }-7 \\
C-14 \\
C A-45 \\
C D-109 \\
C O-60 \\
F E-55 \\
F E-59 \\
H-3 \\
N 1-63 \\
P=32 \\
P M-147 \\
P O-210\end{array}$ & $\begin{array}{l}7.149 E-03 \\
9.800 E-09 \\
1.035 E-05 \\
5.000 E-04 \\
1.280 E-06 \\
8.000 E-05 \\
5.000 E-07 \\
2.030 E-04 \\
6.240 E-03 \\
5.340 E-07 \\
9.800 E-05 \\
5.000 E-07 \\
1.180 E-06\end{array}$ \\
\hline
\end{tabular}

Flammable storage mODULE 2

LOW-LEVEL NONINDUSTRIAL - OFFSITE

LOW-LEVEL NONINOUSTRIAL

FLAMMABLE STORAGE MODULE 3

LOW-LEVEL NONINDUSTRIAL-OFFSITE

. 
SWIR328H - (Page 32 of 37)

RUNDATE: $03 / 15 / 96$

Post-1970 Nontransuranic Waste Burial Ground Areas Waste Volumes Buried or Stored and Nondecayed Curie Content through DECEMBER 31, 1995.

\section{BURIAL GROUND FACILITIES}

FlamMABLE STORAGE MODULE 3

LON-LEVEL NONINDUSTRIAL-OFFSITE

LOW-LEVEL NOWINDUSTRIAL

flammable storage moDULE 4

LOW-LEVEL NONINDUSTRIAL-OFFSITE

LOW-LEVEL MONINDUSTRIAL

\section{FLAmMable storage module 5}

LOW-LEVEL NONINDUSTRIAL-OFFSITE

LOW-LEVEL NONINDUSTRIAL

\begin{tabular}{ccccc}
$\begin{array}{c}\text { TOTAL } \\
\text { (CURIES) }\end{array}$ & $\begin{array}{c}\text { SR-90 } \\
\text { (CURIES) }\end{array}$ & $\begin{array}{c}\text { RU-106 } \\
\text { (CURIES) }\end{array}$ & $\begin{array}{c}\text { CS-137 } \\
\text { (CURIES) }\end{array}$ & $\begin{array}{c}\text { OTHER RADIOACTIVITY } \\
\text { (CURIES) }\end{array}$ \\
\hline
\end{tabular}

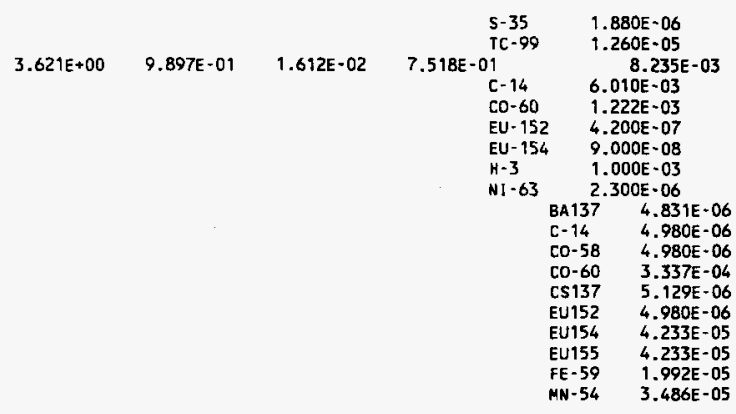

5.041E-02 1.166E-06 2.510E-06 5.312E-04

BE -7

C -14

CO- 56

Co- 57

Co. 58

CO-60

CR -51

$\mathrm{H}-3$

$M N=54$

NA- 22

P. 32

$\mathrm{S}-35$

$\mathrm{SC}-46$

$2 \mathrm{~N}-65$

5.059E-02 1.750E-02 1.999E-09 7.954E-03

CS- 134

EU- 154

EU- 155

$\mathrm{H}-3$

I -129

PB - 210

PM- 147

$4.932 \mathrm{E}-02$

$3.493 E-05$

5. $050 \mathrm{E}-06$

1.977E-03

7. 384E-03

$5.219 E-03$

1. $776 \mathrm{E}-04$

$2.045 E-04$

$1.633 \mathrm{E}-02$

2.367E-03

$1.490 \mathrm{E}-06$

5. 100E-04

3. $700 E-07$

4. 050 E - 05

$1.457 E-02$

1.085E-04

2. 180E-05

$2.460 E-05$

2.770E-05

$9.480 E-09$

1.210E-10

3. $430 E-05$

5.780E-08

$9.370 E-11$

\begin{tabular}{|c|c|c|c|c|}
\hline $2.602 E+00$ & $6.225 E-06$ & $1.341 E-05$ & $\begin{aligned} 6.648 E-06 \\
\text { C }-14 \\
H-3\end{aligned}$ & $\begin{array}{l}\quad 2.601 E+00 \\
4.828 E-02 \\
2.553 E+00\end{array}$ \\
\hline $9.795 E-06$ & $3.207 E-06$ & $1.667 E-12$ & $\begin{array}{l}1.856 E=07 \\
C O-58 \\
C O-60 \\
\text { EU-154 } \\
\text { EU- } 155 \\
F E-55 \\
H-3 \\
I-129 \\
M N-54 \\
N I-60 \\
N I-63 \\
P M-147\end{array}$ & $\begin{array}{l}3.020 E-06 \\
6.100 E-07 \\
8.600 E-08 \\
1.600 E-07 \\
3.530 E-08 \\
1.200 E-06 \\
3.991 E-09 \\
5.094 E-11 \\
6.300 E-07 \\
8.600 E-08 \\
1.400 E-07 \\
7.720 E-09\end{array}$ \\
\hline
\end{tabular}




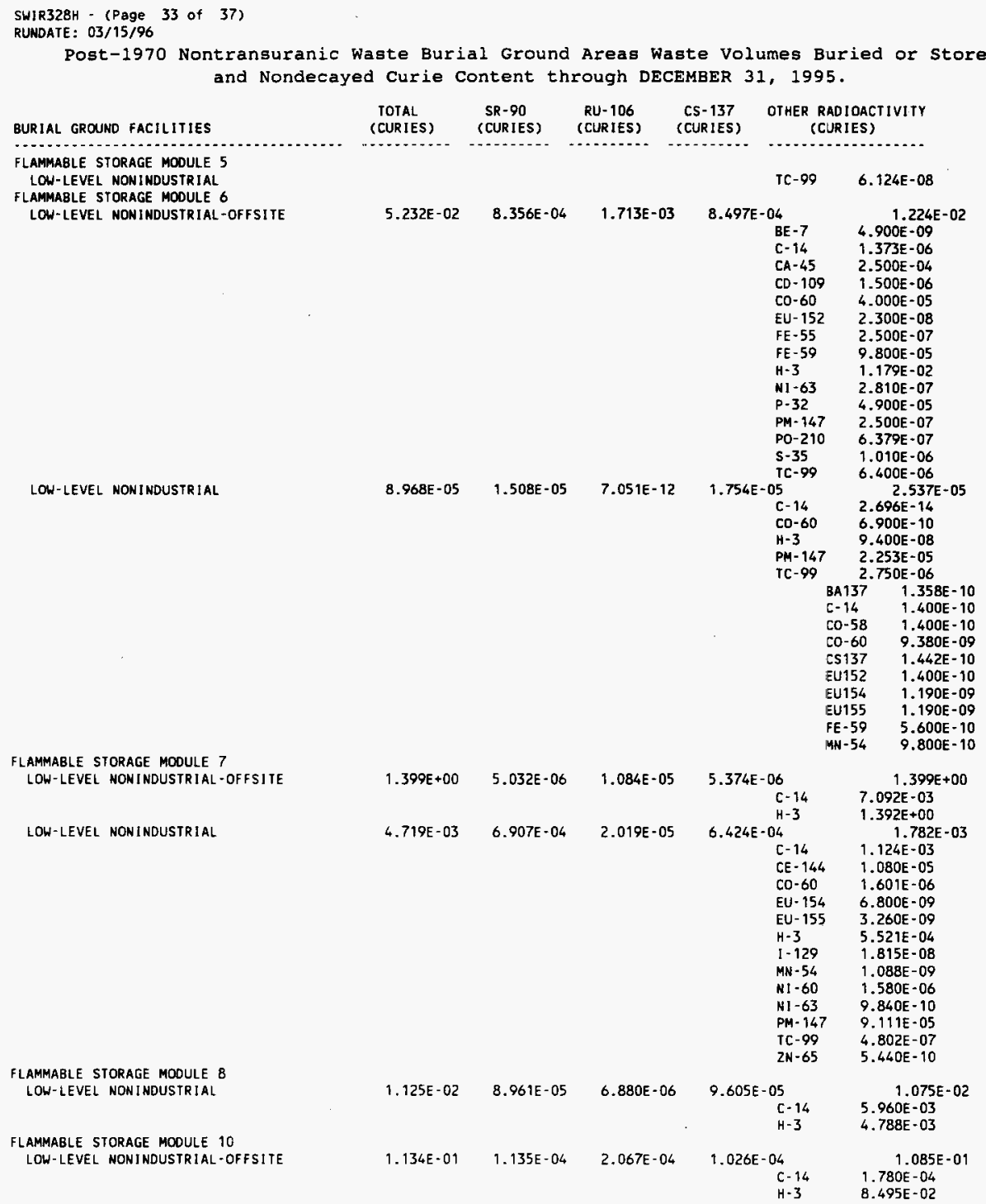




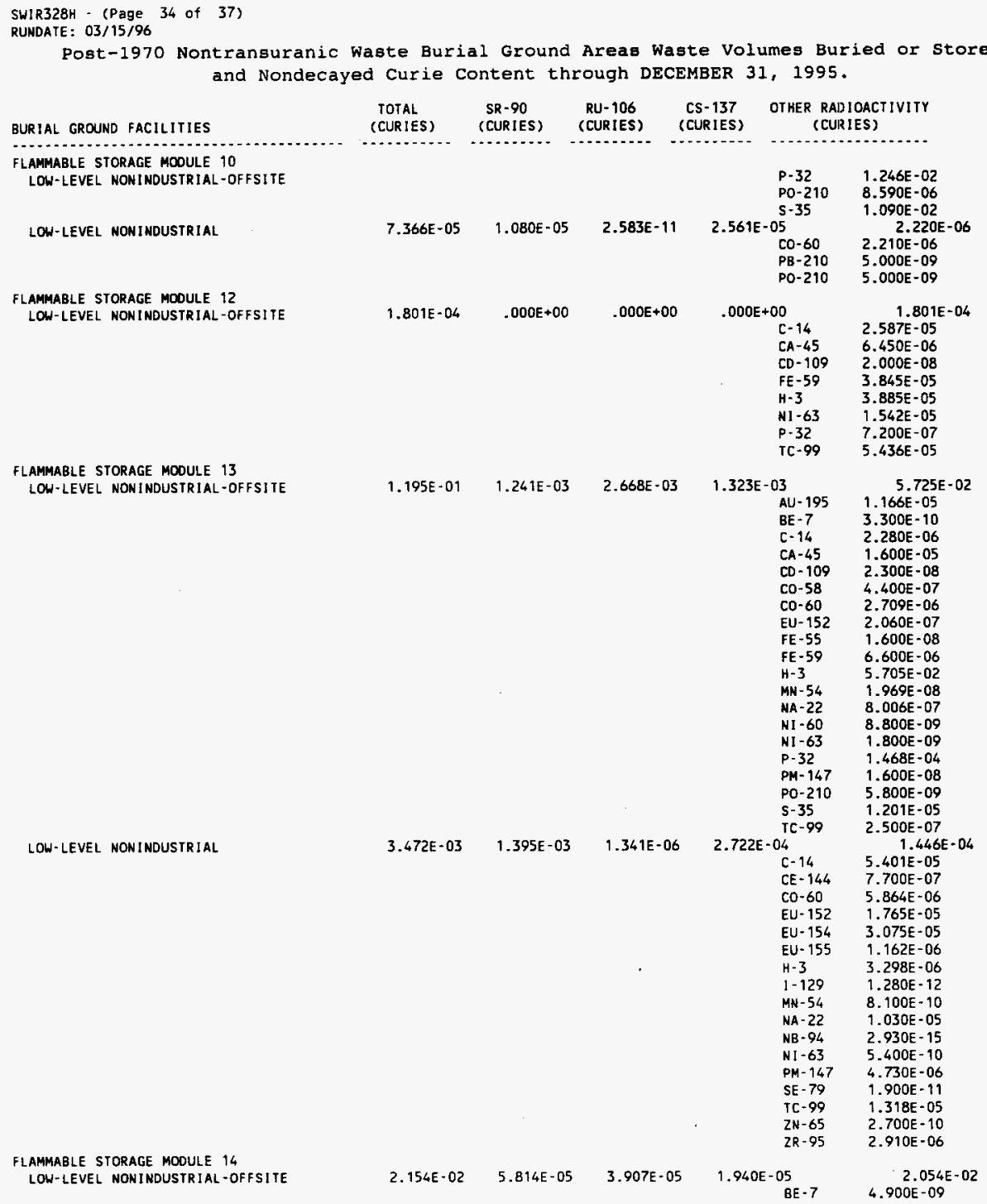


SWIR328H - (Page 35 of 37 )

RUNDATE : $03 / 15 / 96$

Post-1970 Nontransuranic Waste Burial Ground Areas Waste Volumes Buried or Stored and Nondecayed Curie Content through DECEMBER 31, 1995.

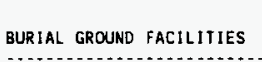

FLAMMABLE STORAGE MODULE 14

LOW-LEVEL NONINDUSTRIAL-OFFSITE

LOW-LEVEL NON INDUSTRIAL

\begin{abstract}
FLAMMABLE STORAGE MODULE 15
LOW-LEVEL NONINDUSTRIAL-OFFSITE
\end{abstract}

LOW-LEVEL NONINDUSTRIAL

FLAMMABLE STORAGE MODULE 16 LOW-LEVEL NONINDUSTRIAL

$\begin{array}{lcccc}\text { TOTAL } & \text { SR }-90 & \text { RU }-106 & \text { CS-137 } & \text { OTHER RADIOACTIVITY } \\ \text { (CURIES) } & \text { (CURIES) } & \text { (CURIES) } & \text { (CURIES) } & \text { (CURIES) }\end{array}$

(CURIES) (CURIES) (CURIES) (CURIES)

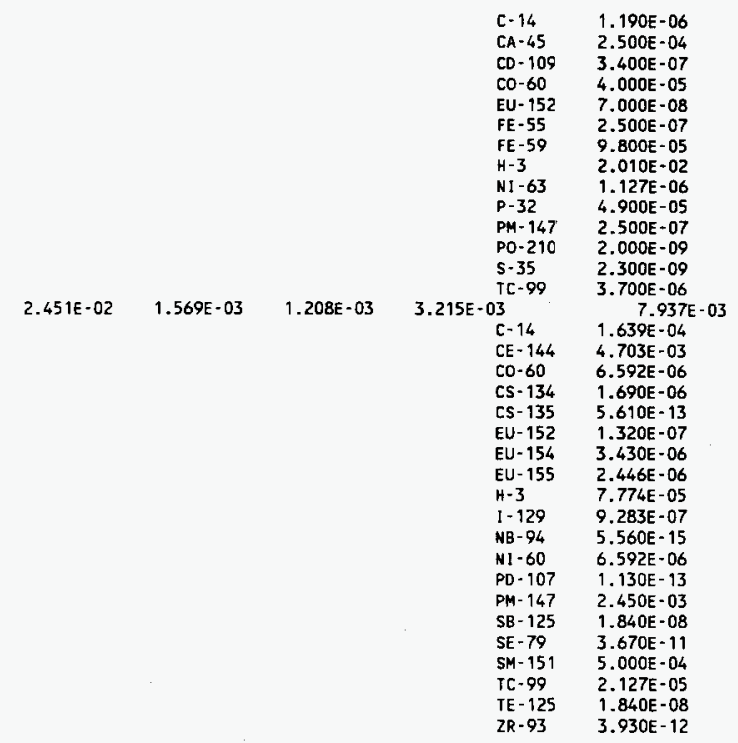

$1.331 E-02 \quad 1.250 E-05 \quad 2.238 E-07 \quad 1.110 E-07$

C- 14 1.300E-07

CD-105' 6.000E-07

$\mathrm{H}-3 \quad 5.704 \mathrm{E}-03$

I-125 7.077E-03

$\mathrm{K}-40 \quad 4.400 \mathrm{E}-08$

NI-63 2.300E-07

P-32 2.000E-06

PO-210 6.050E-0?

RB-B6 2.160E-04

S-35 9.400E-07

$S R-85 \quad 2.800 E-04$

TC. $99 \quad 2.600 \mathrm{E}-06$

$3.569 E-02 \quad 2.354 E-03 \quad 2.332 E-05 \quad 1.489 E-02$

C- 14

$1.592 \mathrm{E}-05$

1.130E-08

CO-60 7.070E-08

$\mathrm{H}-3 \quad 1.447 \mathrm{E}-06$

$\mathrm{I}-129 \quad 9.900 \mathrm{E}-10$

PM- $147 \quad 1.355 E-05$

IC-99 8.450E-07

$6.922 E-02 \quad 2.195 E-03 \quad 2.564 E-03 \quad 1.501 E-02$

AG- $1101 \quad 2.700 E-06$

C-14 $\quad 7.210 E-04$ 


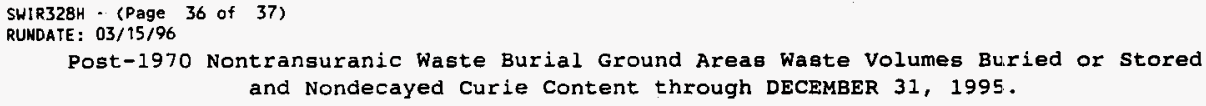

FLAMMABLE STORAGE MOOULE 17 LOW-LEVEL NONINOUSTRIAL-OFFSITE

LOW-LEVEL NONIMDUSTR IAL

\begin{tabular}{|c|c|c|c|c|}
\hline & & & $\begin{array}{l}C E-144 \\
C O-60 \\
C S-134 \\
E U-152 \\
E U-154 \\
E U-155 \\
H=3 \\
1-129 \\
M N-54 \\
P M-147 \\
S B-125 \\
S M-151 \\
I C-99 \\
Z R-95\end{array}$ & $\begin{array}{l}1.032 E-02 \\
6.722 E-06 \\
6.513 E-05 \\
2.481 E-06 \\
2.951 E-05 \\
1.261 E-05 \\
2.590 E-03 \\
3.866 E-10 \\
2.957 E-07 \\
4.800 E-03 \\
7.569 E-06 \\
1.100 E-03 \\
3.002 E-10 \\
5.360 E-07\end{array}$ \\
\hline $6.613 E-03$ & $8.732 \mathrm{E}-06$ & $6.954 \mathrm{E}-08$ & $\begin{aligned} 5.37 B E-08 \\
C-14 \\
C O-56 \\
C O-57 \\
C 0-58 \\
F E-59 \\
H-3 \\
M N-54 \\
P-32 \\
P O-210 \\
Z N-65\end{aligned}$ & $\begin{array}{l}6.594 E-03 \\
1.025 E-03 \\
1.800 E-06 \\
1.401 E-05 \\
1.650 E-05 \\
5.000 E-07 \\
4.230 E-03 \\
1.000 E-06 \\
1.288 E-03 \\
4.500 E-06 \\
1.290 E-05\end{array}$ \\
\hline $7.053 E+00$ & $1.405 E-02$ & $7.264 E-04$ & 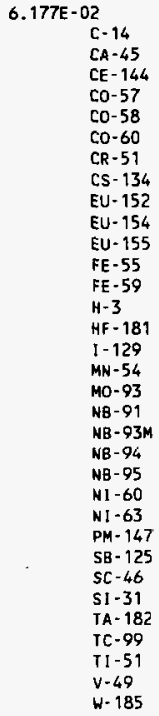 & $\begin{array}{l}6.899 E+00 \\
5.126 E-04 \\
3.783 E-04 \\
1.471 E-04 \\
1.510 E-02 \\
2.189 E-01 \\
2.346 E-01 \\
1.002 E-02 \\
3.903 E-04 \\
7.700 E-06 \\
1.780 E-05 \\
1.350 E-05 \\
3.007 E+00 \\
2.100 E-05 \\
7.164 E-01 \\
4.100 E-04 \\
1.000 E-02 \\
1.433 E+00 \\
2.703 E-01 \\
4.200 E-08 \\
6.202 E-02 \\
6.500 E-07 \\
2.000 E-05 \\
3.570 E-03 \\
8.761 E-02 \\
3.330 E-09 \\
1.000 E-05 \\
6.400 E-04 \\
1.130 E-06 \\
1.700 E-03 \\
1.307 E-02 \\
4.420 E-07 \\
4.680 E-02 \\
7.661 E-01\end{array}$ \\
\hline
\end{tabular}




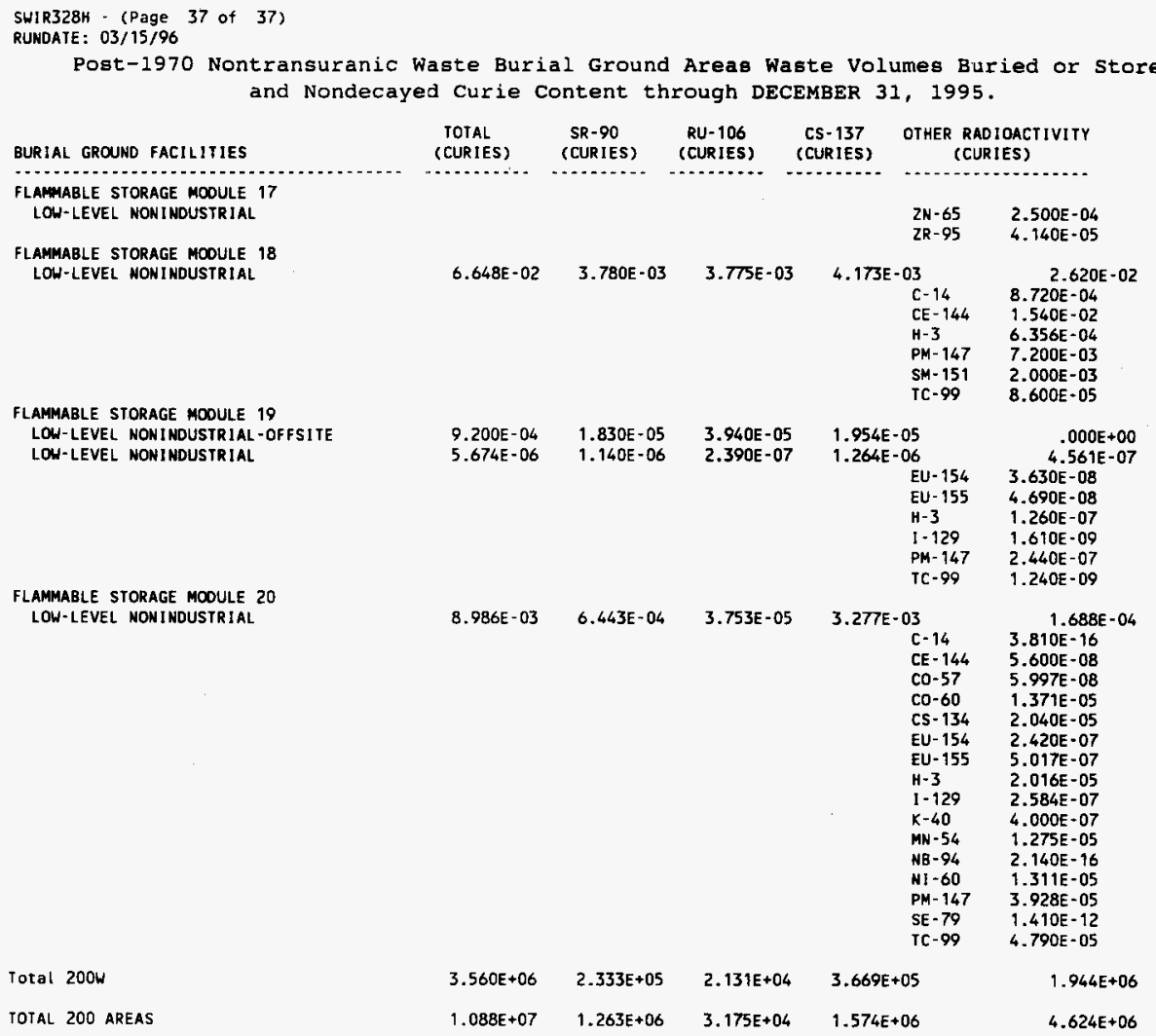


SHIR3281 - (Page 1 of 37 )

RUNDATE: $03 / 15 / 96$

Post-1970 Nontransuranic Waste Burial Ground Areas Decayed Curie Content through DECEMBER 31, 1995.

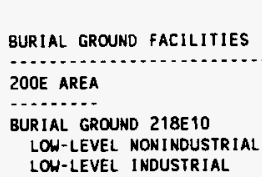

LOW-LEVEL INDUSTRIAL

BURIAL GROUND 218E12B LOW-LEVEL NONINDUSTRIAL

LOW-LEVEL INOUSIRIAL-OFFSITE

$\begin{array}{lccc}\text { TOTAL } & \text { SR-90 } & \text { RU- } 106 & \text { CS-137 } \\ \text { (CURIES) } & \text { (CURIES) } & \text { (CURIES) } & \text { (CURIES) }\end{array}$

OTHER RADIOACTIVITY (CLIRIES)

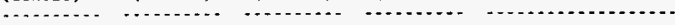

$1.811 E+03$

$2.965 E+06$

$5.555 \mathrm{E}+02$

$6.797 \mathrm{E}+05$

$1.276 \mathrm{E}-03$

$2.259 \mathrm{E}-01$

$3.599 E+02$

CO-60

137

EA 137
$C=14$

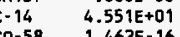

CO-58 1.462E-16

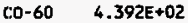

CS137 3.311E+01

EU152 2.065E+01

EU154 1.144E+02

EU155 4.955E+01

FE-59 1.995E-25

MIN-54 8.833E-03

$9.523 E+04 \quad 2.214 E+04 \quad 2.703 E-01 \quad 2.265 E+04$

BE-7 1.745E-19

$6.791 E+03$

C-14 3.498E-10

CE-141 3.846E-35

CE-144 3.541E-02

CO-60 6.790E+03

CS-134 $\quad 1.223 \mathrm{E}-02$

$\mathrm{H}-3 \quad 1.725 \mathrm{E}-03$

$1-129 \quad 1.980 E-02$

K-40 $9.054 \mathrm{E}-03$

PM- $147 \quad 5.426 E-01$

SB-125 1.896E-07

SM-151 4.035E-01

ZR-95 1.696E-17

$13 \mathrm{~A} 137 \quad .000 E+00$

E-14 1.201E-02

CO-58 4.174E-18

CO-60 2.177E-01

CS137 9.851E-03

EU152 7.127E-03

EU154 4.539E-02

EU155 2.543E-02

FE-59 1.346E-26

MN-54 2.629E-05 $1.855 \mathrm{E}+06$

BE- 10

C- 14

$\mathrm{CL}-36$

$\mathrm{CO}-58$

CO-60

CR-51

CS -134

FE -55

FE- 59

$\mathrm{H}-3$

HF -181

I -129

$M N \cdot 54$

MO- 93

NB-93M

NB- 94

NB- 95

$\mathrm{Nl}-59$

NI -63

$1.278 E-06$

$1.252 E+02$

5.556E-03

$1.604 \mathrm{E}+03$

$6.429 E+05$

5. $127 \mathrm{E}-03$

1. $218 \mathrm{E}-03$

$3.537 E+05$

4. $791 \mathrm{E}-02$

$8.492 E+02$

1. 197E-03

2.941E-03

$1.611 \mathrm{E}+03$

$6.893 E-02$

5. $225 \mathrm{E}-01$

$1.282 E+01$

$9.758 \mathrm{E}-04$

$5.119 \mathrm{E}+03$

$8.492 E+05$ 


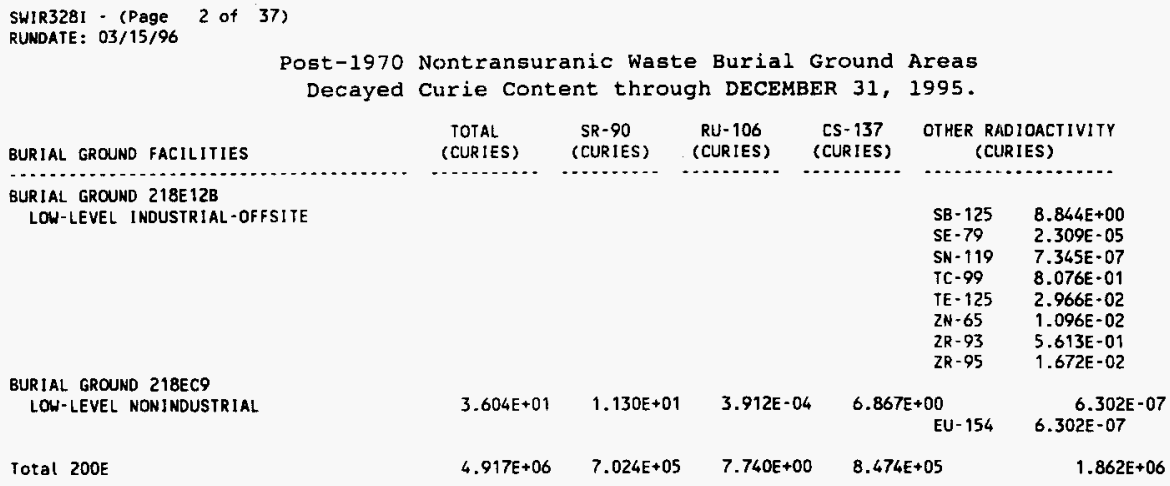

$3.604 E+01$

$1.130 E+01$

$4.917 E+06$

$7.024 E+05$

$7.740 E+00$

$8.474 E+05$

(.....................

$\begin{array}{ll}\text { SB-125 } & 8.844 E+00 \\ \text { SE-79 } & 2.309 E-05 \\ \text { SN-119 } & 7.365 E-07 \\ \text { TC-99 } & 8.076 E-01 \\ \text { TE-125 } & 2.966 E-02 \\ \text { ZN-65 } & 1.096 E-02 \\ 2 R-93 & 5.613 E-01 \\ \text { ZR-95 } & 1.672 E-02\end{array}$

00

EU- 154

$6.302 E-07$

$1.862 \mathrm{E}+06$ 
SWIR328I - (Page 3 of 37 )
RUNDATE: $03 / 15 / 96$

Post-1970 Nontransuranic Waste Burial Ground Areas

Decayed Curie Content through DECEMBER 31, 1995.

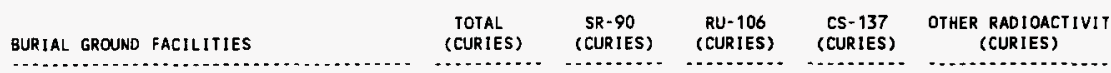

2ODW AREA

294 T FACILITY/TPLANT

LOW-LEVEL NONINDUSTRIAL-OFFSITE

BURIAL GROUND 218W2A

LOW-LEVEL INDUSTRIAL-OFFSITE

LOW-LEVEL INOUSTRIAL

BURIAL GROUND 218W3A

LON-LEVEL NONI NDUSTRIAL-OFFS!TE
2.360E-03 7.786E-07 7.215E-07 8.326E-07

C- 14 EU -152

H-3

NA -22

$\begin{array}{rrrrr}2.278 E+00 & 5.468 E-01 & 1.033 E-05 & 5.950 E-01 & .000 E+00 \\ 1.276 E+03 & 3.074 E+02 & 7.253 E-05 & 3.397 E+02 & 1.691 E-01 \\ & & & 00-60 & 1.691 E-01\end{array}$

$4.251 E+05 \quad 3.173 E+04 \quad 2.378 E+03 \quad 1.612 E+05$

AG -110

$B A-133$.

BE -10

BE -7

BI-216

C- 14

CA-45

CD -109

CD- 113

CE-144

$\mathrm{CL}-36$

Co- 56

Co- 57

CO- 58

co- 60

CR-51

CS-13i:

CS- 135

EU-15:

EU-15\%

EU-15!5

FE- 55

FE -59

GD - 15?

GD $=15 i^{\prime}$

GE -68

H-3

$1-125$

1- 129

1-131

KR-85

MN -54

NA- 22

NB- $93 M$

NB -94

NI -59

NI -60

N I -63

P-32

PM- 147

S-35

SB- 125

SB- 126

SC $-4 \theta$

SE -75

SE -79

SM-151

\subsection{E-03}

8.996E-06

9. $1 \mathrm{B2E}-08$

2.339E-03

$6.485 E-07$

$1.691 E-01$

$164 E+04$

5.105E-05

$1.117 \mathrm{E}-04$

$1.320 \mathrm{E}-10$

$5.646 \mathrm{E}-05$

$.000 E+00$

1. 309E-01

$1.062 E-08$

$9.806 \mathrm{E}-07$

3.007E-05

5.967E-02

5.300E-08

$1.046 \mathrm{E}-06$

$1.477 \mathrm{E}-03$

1.004E-06

$6.794 E+01$

2. $905 E-27$

$2.838 E+02$

1. 102E-06

6.090E-01

1.328E-01

$3.021 E-03$

1.665E-01

$1.834 E-10$

3.390E-03

$.000 \mathrm{E}+00$

$1.107 \mathrm{E}-07$

$2.122 E+04$

$1.817 \mathrm{E}-13$

1. $438 \mathrm{E}-02$

$.000 E+00$

1. $413 \mathrm{E}-01$

8. $461 E-03$

8.207E-02

$6.105 E-05$

2.810E-05

5.783E-04

$.000 E+00$

$3.558 E+00$

$.000 E+00$

$5.373 \mathrm{E}-01$

1. $303 E-09$

$6.343 E+01$

6. $008 E-41$

1. $777 \mathrm{E}-12$

8.718E- 11

$1.116 E-04$

$9.678 E-04$ 
SWIR3281 - (Page 4 of 37)

RUNDATE : $03 / 15 / 96$

Post-1970 Nontransuranic Waste Burial Ground Areas Decayed Curie Content through DECEMBER 31, 1995.

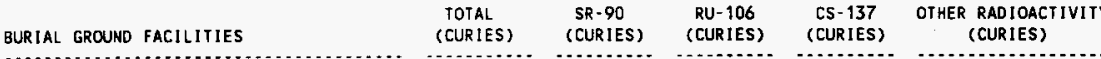

BURIAL GROUND 218W3A

LOW-LEVEL NOWINDUSTRIAL-OFFSI TE

LOW-LEVEL NONINDUSTRIAL

$3.324 E+05 \quad 3.404 E+04 \quad 4.664 E+00$

$7.305 E+04$

\section{BE -7}

C- 14

CD-109 5.688E-07

CE-141 2.521E-39

CE-144 7.127E-06

$\mathrm{CL}-36 \quad 1.000 \mathrm{E}-04$

CO-58 4.206E- 13

CO-60 $4.906 \mathrm{E}+03$

CR-51 4.612E-15

CS- $134 \quad 3.223 \mathrm{E}-03$

EU- $152 \quad 1.365 E-02$

EU-154 1.697E-02

EU-155 2.223E-04

$\mathrm{FE}=55 \quad 4.868 \mathrm{E}-04$

FE-59 1.294E-18

$\mathrm{H}-3 \quad 1.135 \mathrm{E}+05$

$\mathrm{I}-125 \quad 1.087 \mathrm{E}-16$

I-129 5.000E-06

$\begin{array}{ll}1-131 & .000 E+00\end{array}$

K-40 1.244E-04

KR-85 1.355E-03

MN-54 1.581E-04

NA-22 2.177E-06

NB-94 8.469E-05

N I $-60 \quad .000 E+00$

N $1-63 \quad 1.899 E-03$

P.32 $\quad 1.594 E-44$

PM-147 8.162E-04

RE-187 2.000E-04

S-35 $9.329 \mathrm{E}-12$

SB-122 $\quad .000 E+00$

SB-125 $\quad 5.720 E-03$

SE-75 $8.977 \mathrm{E}-10$

SM-147 2.000E-04

SN-12t, $\quad 1.033 \mathrm{E}-03$

SR-85 1.200E-12

TA-18:: $\quad 5.428 E-07$

TC-99 4.680E-02

TE- $125, \quad 1.961 E-07$

TH- $170 \quad 8.228 \mathrm{E}-09$

$V-49 \quad 7.540 E-10$

$Y-90 \quad .000 E+00$

ZN-65 8.419E-08

BA137 .000E +00

C- $14 \quad 2.578 E+02$

CO-58 $3.123 E-15$

CO-60 2.046E+03

CS137 $1.818 E+02$

EU152 1.087E+02 
SWIR3281 - (Page 5 of 37)

RUNDATE: 03/15/96

Post-1970 Nontransuranic Waste Burial Ground Areas

Decayed Curie Content through DECEMBER 31, 1995.

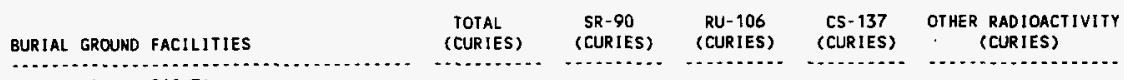

BURIAL GROUND 218W3A

LOW-LEVEL NONINDUSTRIAL

LOU-LEVEL NONIMDUSTRIAL CLASSIFIEDOFFSITE

LOW-LEVEL NONINDUSTRIAL CLASSIFIED

LOW-LEVEL MONINDUSTRIAL (ASSAYED FROM TRANSURANIC)

LOW-LEVEL I NDUSTR IAL-OFFSITE

\begin{tabular}{|c|c|c|c|c|c|c|}
\hline $7.868 E-01$ & $1.903 \mathrm{E}-01$ & $4.786 E-07$ & $2.083 E-01$ & \multicolumn{2}{|c|}{$\begin{array}{l}\text { EU154 } \\
\text { EU155 } \\
\text { FE-59 } \\
\text { MN-54 }\end{array}$} & $\begin{array}{l}5.766 E+02 \\
2.277 E+02 \\
1.681 E-20 \\
2.706 E-02 \\
.000 E+00\end{array}$ \\
\hline $2.723 E+00$ & 6.367E-01 & $2.635 E-04$ & $6.893 E-01$ & & & $\begin{array}{c}.000 E+00 \\
.000 E+00 \\
4.903 E-02 \\
5.745 E-20 \\
7.340 E-01 \\
3.950 E-02 \\
2.727 E-02 \\
1.658 E-01 \\
8.466 E-02 \\
6.386 E-30 \\
2.998 E-05 \\
.000 E+00\end{array}$ \\
\hline $2.213 E+02$ & $4.297 E+00$ & $1.901 E-03$ & $\begin{array}{r}4.641 \mathrm{E}+00 \\
\mathrm{C} \\
\mathrm{B} \\
\mathrm{B} \\
\mathrm{C} \\
\mathrm{C} \\
\mathrm{C} \\
\mathrm{C} \\
\mathrm{C} \\
\mathrm{C} \\
\mathrm{C} \\
\mathrm{C} \\
\mathrm{E} \\
\mathrm{E} \\
\mathrm{E} \\
\mathrm{F} \\
\mathrm{F} \\
\mathrm{G} \\
\mathrm{H} \\
\mathrm{I} \\
\mathrm{I} \\
\mathrm{K} \\
\mathrm{K} \\
\mathrm{M} \\
\mathrm{N} \\
\mathrm{N} \\
\mathrm{N} \\
\mathrm{N} \\
\mathrm{P} \\
\mathrm{P} \\
\mathrm{R} \\
\mathrm{S} \\
\mathrm{S} \\
\mathrm{T} \\
\mathrm{T} \\
\mathrm{T} \\
\mathrm{Y} \\
\mathrm{Z}\end{array}$ & & $\begin{array}{l}. \\
2 . \\
5 . \\
2 . \\
3 . \\
4 . \\
1 . \\
1 . \\
4 . \\
1 . \\
2 . \\
3 . \\
3 . \\
1 . \\
1 . \\
2 . \\
2 . \\
5 . \\
1 . \\
6 . \\
5 . \\
7 . \\
2 . \\
5 . \\
1 . \\
9 . \\
3 . \\
4 . \\
6 . \\
4 . \\
1 . \\
8 . \\
2 . \\
3 . \\
4 .\end{array}$ & $\begin{array}{l}2.036 E+02 \\
0 E+00 \\
0 E+00 \\
7 E-05 \\
0 E-10 \\
0 E-10 \\
44 E-03 \\
8 E E-04 \\
12 E-05 \\
2 E-02 \\
2 E-04 \\
2 E-06 \\
36 E+00 \\
25 E-20 \\
23 E-01 \\
99 E-01 \\
17 E-01 \\
6 E-04 \\
93 E-03 \\
58 E-16 \\
33 E-04 \\
36 E+02 \\
16 E-15 \\
20 E-07 \\
90 E-08 \\
20 E-01 \\
49 E-04 \\
65 E+00 \\
98 E-05 \\
00 E-04 \\
21 E-02 \\
33 E-01 \\
00 E+00 \\
62 E-28 \\
57 E-13 \\
00 E+00 \\
90 E-08 \\
00 E-04 \\
68 E-10 \\
90 E-11 \\
48 E-01 \\
\end{array}$ \\
\hline
\end{tabular}


SWIR3281 - (Page 6 of 37 )
RUNDATE: $03 / 15 / 96$

Post-1970 Nontransuranic Waste Burial Ground Areas

Decayed Curie content through DECEMBER 31, 1995.

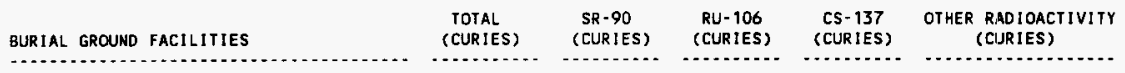

BURIAL GROUND 218W3A

LOW-LEVEL INDUSTRIAL

$3.622 \mathrm{E}+03$

$4.971 \mathrm{E}+02$

$2.079 E-04$

1. $195 E+03$

$2.452 E+00$

C-14 2.998E-22

CE-144 1.358E-14

C0-60 6.239E-01

CS-134 7.824E-03

EU-152 7.153E-02

EU-154 1.102E-01

FE-55 4.329E-10

$H-3 \quad 1.622 E+00$

MN-54 4.111E-11

NA-22 $1.723 E-07$

NI $-60 \quad .000 E+00$

PM-147 1.674E-13

SB-125 1.691E-02

SM-151 1.924E-13

IE-125 6.300E-08

BA137 .000E+00

$E-14 \quad 2.475 E+01$

100-58 $1.794 \mathrm{E}-09$

$150-60 \quad 1.888 E+02$

CS137 $1.746 E+01$

EU152 1.040E+01

EU154 $5.467 \mathrm{E}+01$

EUt55 2.090E $\div 01$

FE-59 8.639E-13

BURIAL GROUND 218W3AE

LOW-LEVEL NONINDUSTRIAL-OFFSITE

$5.831 E+04 \quad 6.333 E+01 \quad 1.266 E+00 \quad 5.532 E+01$

$\begin{array}{lr}A C-227 & 9.819 E-07 \\ A C-228 & .000 E+00 \\ A G-108 & .000 E+00 \\ A G-110 & 5.401 E-06 \\ A U-195 & 6.098 E-05 \\ B A-133 & 7.211 E-06 \\ B E-10 & 4.600 E-11 \\ B E-7 & 1.600 E-04 \\ B I-207 & 9.771 E-04 \\ B I-212 & .000 E+00 \\ B 1-214 & .000 E+00 \\ C-14 & 1.003 E+01 \\ C A-45 & 8.023 E-04 \\ C D-109 & 3.941 E-05 \\ C D-113 & 1.051 E-05 \\ C E-141 & 4.864 E-08 \\ C E-144 & 1.503 E-03 \\ C L-36 & 1.860 E-08 \\ C O-56 & 9.777 E-06 \\ C O-57 & 2.625 E-03 \\ C O-58 & 5.622 E-01 \\ C O-60 & 2.456 E+04 \\ C R-51 & 1.091 E-09 \\ C S-134 & 1.578 E+00 \\ C S-135 & 3.800 E-07 \\ \text { ES-254 } & 1.746 E-09 \\ E U-152 & 1.792 E-02 \\ E U-153 & .000 E+00 \\ E U-154 & 5.691 E-01 \\ E U-155 & 1.123 E-01 \\ F E-55 & 5.008 E+03 \\ F E-59 & 2.883 E-05 \\ & \end{array}$


WHC-EP-0125-8

SWIR328I - (Page 7 of 37)

RUMDATE : $03 / 15 / 96$

Post-1970 Nontransuranic Waste Burial Ground Areas

Decayed Curie Content through DECEMBER 31, 1995.

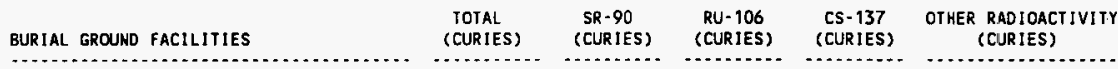

BURIAL GROUND 218W3AE

LOW-LEVEL NONINDUSTRIAL-OFFSITE

LOW-LEVEL NONINDUSTR IAL

$1.166 E+05$

$2.199 E+04$

$1.119 E+02$

$2.252 E+04$

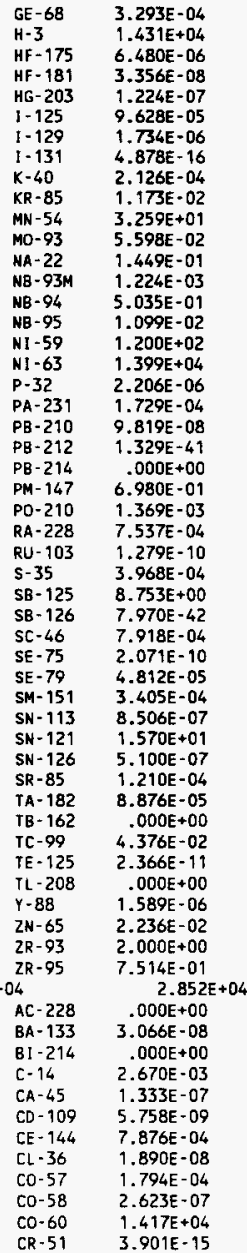


SWIR328I - (Page 8 of 37 )
RUNDATE: $03 / 15 / 96$

Post-1970 Nontransuranic Waste Burial Ground Areas Decayed Curie Content through DECEMBER 31, 1995.

\begin{tabular}{|c|c|c|c|c|c|}
\hline BURIAL GROUND FACILITIES & $\begin{array}{l}\text { TOTAL } \\
\text { (CURIES) }\end{array}$ & $\begin{array}{l}\text { SR-90 } \\
\text { (CURIES) }\end{array}$ & $\begin{array}{l}\text { RU- } 106 \\
\text { (CURIES) }\end{array}$ & $\begin{array}{l}\text { CS.- } 137 \\
\text { (CURIES) }\end{array}$ & $\begin{array}{l}\text { OIHER RADIOACTIVITY } \\
\text { (CURIES) }\end{array}$ \\
\hline
\end{tabular}

BURIAL GROUND 218W3AE

LOW-LEVEL NONIHDUSTRIAL
LOH-LEVEL NONINDUSTRIAL CLASSIFIEDOFFSITE
8.239E $+04 \quad 1.463 E-01 \quad 1.417 E-02 \quad 1.143 E-01$

C -14

Co- 58

CO-60

CR-51

$\mathrm{FE}-55$

FE-59

$\mathrm{H}=3$

$\mathrm{HF}=181$

] -129

$M N-54$

NB- 94

NB-95
$8.751 E+01$

$1.082 E+03$

$1.511 E+03$

$2.890 E+02$

$9.929 E+02$

$1.750 \mathrm{E}+00$

$4.064 E+03$

4. $181 \mathrm{E}-11$

5.506E-07

$3.374 E-04$

$9.522 \mathrm{E}-10$

$5.981 E+03$

$2.089 \mathrm{E}-10$

$4.143 \mathrm{E}-04$

1.759E-07

9.202E-05

$3.668 E-07$

2.317E-06

$.000 E+00$

$3.403 E+02$

$.000 E+00$

$.000 E+00$

3. 135E-03

4. $446 E-11$

8.873E-01

$1.026 \mathrm{E}-08$

$2.710 \mathrm{E}-04$

$5.984 E-09$

$3.448 \mathrm{E}-02$

2. $130 E-02$

2.506E-08

$.000 E+00$

$4.522 \mathrm{E}-07$

$1.385 \mathrm{E}-09$

$9.914 \mathrm{E}-12$

2.580E-04

$1.680 \mathrm{E}+00$

BA $137 \quad .000 E+00$

C- $14 \quad 9.480 E-08$

CO-58 3.229E-08

C0-60 $6.105 \mathrm{E}-06$

CS137 9.697E-08

EU152 $9.331 \mathrm{E}-08$

EU154 $\quad 7.862 \mathrm{E}-07$

EU155 7.726E-07

FE-59 6.852E-0B

MN-54 5.199E-07 8. $239 E+04$

$1.981 E+01$

8.690E-05

$2.659 E+04$

$1.326 \mathrm{E}-23$

$5.455 E+03$

$3.970 E-15$

7. $438 \mathrm{E}-01$

$3.161 E-16$

6. $160 E-08$

$5.651 \mathrm{E}+00$

5. $603 E-01$

$1.108 E-10$ 
SWIR3281 - (Page 9 of 37)

RUNDATE : $03 / 15 / 96$

$$
\begin{array}{r}
\text { Post-1970 Nontransuranic Waste Burial Ground Areas } \\
\text { Decayed Curie Content through DECEMBER 31, } 1995 .
\end{array}
$$

\begin{tabular}{llllll} 
BURIAL GROUND FACILITIES & $\begin{array}{c}\text { TOTAL } \\
\text { (CURIES) }\end{array}$ & $\begin{array}{c}\text { SR-90 } \\
\text { (CURIES) }\end{array}$ & $\begin{array}{c}\text { RU-106 } \\
\text { (CURIES) }\end{array}$ \\
\hline
\end{tabular}

\section{BURIAL GROUND 218W3AE}

LOW-LEVEL NONINDUSTRIAL CLASSIFIEDOFFSITE

LOW-LEVEL NONINDUSTRIAL (ASSAYED FROM TRANSURANIC)

LOW-LEVEL INOUSIRIAL-OFFSITE

\begin{tabular}{|c|c|c|c|c|}
\hline $6.914 E-02$ & $1.539 \mathrm{E}-02$ & 5.739E-05 & $\begin{array}{l}\text { NI }-59 \\
\text { NI }-63 \\
\text { SB-12! } \\
\text { SE }-79 \\
\text { SN- } 719 \\
\text { SN- } 123 \\
T A-18: \\
T C-99 \\
T E-125 \\
2 R-93 \\
2 R-95 \\
1.660 E-02\end{array}$ & $\begin{array}{c}4.000 E+02 \\
4.663 E+04 \\
3.099 E+03 \\
6.160 E-08 \\
1.878 E+02 \\
.000 E+00 \\
1.281 E-02 \\
1.542 E-05 \\
2.400 E-05 \\
6.160 E-07 \\
4.521 E-05 \\
.000 E+00\end{array}$ \\
\hline $3.278 E+04$ & $2.810 E+02$ & $3.499 E-01$ & $\begin{array}{c}8.897 E+03 \\
C-14 \\
C E-144 \\
C O-58 \\
C O-60 \\
E U-152 \\
E U-154 \\
F E-55 \\
F E-59 \\
H-3 \\
K R-85 \\
M N-54 \\
N A-22\end{array}$ & $\begin{array}{l}1.484 E+04 \\
3.205 E-01 \\
6.424 E-08 \\
3.467 E-14 \\
9.077 E+01 \\
1.790 E-02 \\
6.934 E-06 \\
3.210 E-01 \\
3.015 E-29 \\
1.475 E+04 \\
7.206 E+00 \\
1.548 E-03 \\
4.495 E-02\end{array}$ \\
\hline $3.564 E+03$ & $7.701 E+02$ & $8.851 E-02$ & $\begin{array}{c}8.284 E+02 \\
C-14 \\
C E-144 \\
C O-60 \\
C R-51 \\
E U-154 \\
E U-155 \\
F E-59 \\
H-3 \\
M N-54 \\
P M-147 \\
\text { SM }-151\end{array}$ & $\begin{array}{l}6.448 E+01 \\
2.998 E-04 \\
7.773 E-09 \\
6.436 E+01 \\
4.074 E-34 \\
8.485 E-02 \\
2.972 E-10 \\
3.216 E-17 \\
4.896 E-07 \\
3.289 E-02 \\
1.229 E-04 \\
4.044 E-07\end{array}$ \\
\hline & 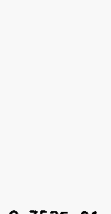 & ? & & $\begin{array}{ll}37 & .000 E+00 \\
14 & 1.125 E+01 \\
58 & 3.776 E-08 \\
60 & 1.896 E+02 \\
37 & 9.103 E+00 \\
52 & 6.476 E+00 \\
54 & 4.058 E+01 \\
55 & 2.206 E+01 \\
59 & 8.442 E-08 \\
54 & 1.776 E-02\end{array}$ \\
\hline $3.983 E+00$ & $9.352 E-01$ & $2.973 \mathrm{E}-04$ & $\begin{array}{l}1.013 E+00 \\
\text { CE }-144\end{array}$ & $\begin{array}{l}8.045 E-06 \\
8.045 E-06\end{array}$ \\
\hline $2.424 E+02$ & $1.210 E-01$ & $1.927 \mathrm{E}-08$ & $\begin{array}{r}1.327 E-01 \\
H-3\end{array}$ & $\begin{array}{c}2.419 E+02 \\
2.419 E+02\end{array}$ \\
\hline $\begin{array}{l}1.970 E+04 \\
1.395 E+04\end{array}$ & $\begin{array}{l}4.766 E+03 \\
1.248 E+03\end{array}$ & $\begin{array}{l}1.159 E-03 \\
8.131 E-01\end{array}$ & $\begin{aligned} 5.223 E+03 \\
1.590 E+03 \\
\text { CO- } 60 \\
\text { CR }-51 \\
\text { CS- } 134 \\
\text { EU }-154 \\
\text { FE }-59\end{aligned}$ & $\begin{array}{l}\quad .000 E+00 \\
8.203 E+03 \\
8.203 E+03 \\
1.625 E-39 \\
4.215 E-02 \\
1.354 E-01 \\
3.976 E-15\end{array}$ \\
\hline
\end{tabular}

LOW-LEVEL INDUSTRIAL
LOW-LEVEL INDUSTRIAL (ASSAYED FROM TRANSURAN IC)

BUR:AL GROUND $218 W 4 \mathrm{~B}$

LOW-LEVEL NONINDUSTRIAL-OFFSITE

LOW-LEVEL NONINDUSTRIAL

LOW-LEVEL CAISSONS

(CUR IES)

(CURIES)

CURIES)

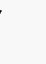


SWIR328: - (Page 10 of 37)

RUNDATE : 03/15/96

Post-1970 Nontransuranic Waste Burial Ground Areas

Decayed Cirie Content through DECEMBER 31, 1995.

BURIAL GROUND FACILITIES

TOTAL

(CURIES)

BURIAL GROUND 218W4B

LOW-LEVEL CAISSONS

BURIAL GROUND $218 W 4 C$

LOW-LEVEL NONINDUSTRIAL-OFFSITE

SR -90

(CURIES)

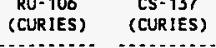

-.......
OTHER RADIOACTIVITY (CURIES)

\begin{tabular}{lrr} 
MN-54 & $1.941 E-02$ \\
SB-125 & \multicolumn{2}{c}{$4.260 E-03$} \\
ZR-95 & $2.554 E-13$ \\
\multicolumn{2}{c}{ BA137 } & $.000 E+00$ \\
C- 14 & $1.24 B E+00$ \\
CO-58 & $1.021 E-24$ \\
CO- 60 & $1.079 E+01$ \\
CS137 & $8.994 E-01$ \\
EU152 & $5.508 E-01$ \\
EU154 & $2.978 E+00$ \\
EU155 & $1.203 E+00$ \\
FE-59 & $2.783 E-38$ \\
MN-54 & $2.963 E-05$
\end{tabular}

\begin{tabular}{|c|c|c|c|}
\hline 4. $156 \mathrm{E}+04$ & $2.683 E+01 \quad 3.893 E-01$ & 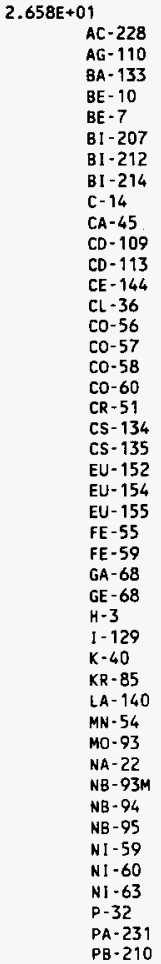 & $\begin{array}{l}4.145 E+04 \\
-000 E+00 \\
.000 E+00 \\
4.951 E-04 \\
5.700 E-11 \\
3.663 E-05 \\
2.107 E-04 \\
.000 E+00 \\
.000 E+00 \\
8.406 E+00 \\
7.243 E-08 \\
1.182 E-04 \\
1.324 E-05 \\
1.085 E+00 \\
2.280 E-08 \\
1.137 E-05 \\
2.847 E-05 \\
2.676 E-04 \\
5.711 E+03 \\
4.471 E-06 \\
3.490 E+00 \\
4.800 E-07 \\
3.715 E-02 \\
2.995 E-01 \\
2.056 E-01 \\
2.126 E+03 \\
1.394 E-08 \\
.000 E+00 \\
1.402 E-12 \\
2.425 E+04 \\
1.007 E-03 \\
4.648 E-05 \\
3.279 E-02 \\
.000 E+00 \\
1.929 E+00 \\
1.254 E-03 \\
3.654 E-03 \\
2.688 E-05 \\
3.195 E-01 \\
7.721 E-07 \\
7.622 E+01 \\
.000 E+00 \\
9.274 E+03 \\
2.930 E-05 \\
4.450 E-03 \\
1.095 E-04 \\
.03\end{array}$ \\
\hline
\end{tabular}


SWIR328I - (Page 19 of 37)

RUNDATE : 03/15/96

Post-1970 Nontransuranic Waste Burial Ground Areas

Decayed Curie Content through DECEMBER 31, 1995.

BURIAL GROUND FACILITIES

TOTAL

(CURIES)

SR -90

(CUR IES)

RU-106

(CURIES)

CS -137
(CURIES)

OTHER RADIOACTIVITY

BURIAL GROUND 218W4C

LOW-LEVEL NON!NDUSTRIAL-OFFSITE

LOW-LEVEL NONINDUSIRIAL

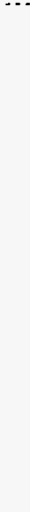

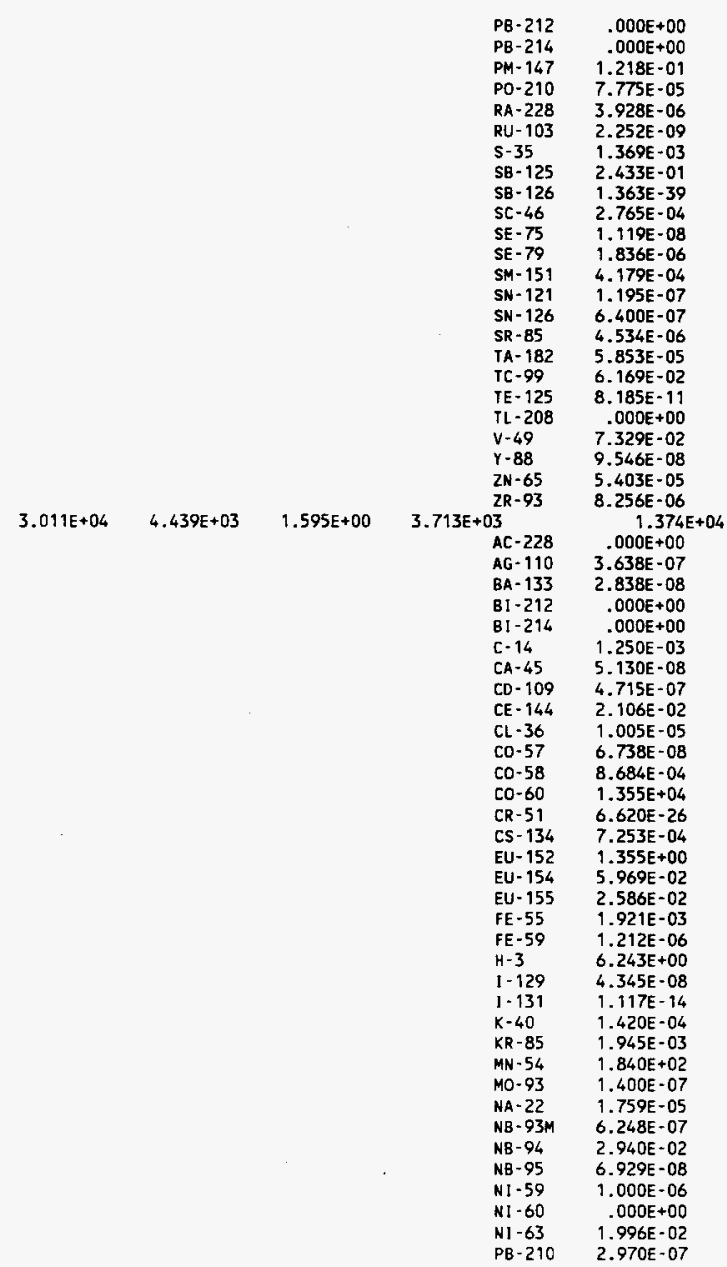



(CURIES)
SWIR328I - (Page 12 of 37 )
RUWDATE: $03 / 15 / 96$

Post-1970 Nentransuranic Waste Burial Ground Areas Decayed Curie Content through DECEMBER 31, 1995.

$\begin{array}{llllll}\text { BURIAL GROUND FACILITIES } & \begin{array}{c}\text { TOTAL } \\ \text { (CURIES) }\end{array} & \begin{array}{c}\text { SR }-90 \\ \text { (CURIES) }\end{array} & \begin{array}{c}\text { RU-106 } \\ \text { (CURIES) }\end{array} & \begin{array}{c}\text { CS-137 } \\ \text { (CURIES) }\end{array}\end{array}$

BURIAL GROUND $218 W 4 C$

LOU-LEVEL HONINDUSTRIAL

LOW-LEVEL NONIHDUSTRIAL CLASSIFIED-

OFFSITE

8.376E-02

$1.398 \mathrm{E}+03$

C- 14

$\mathrm{CO}-58$

CO- 60

FE -55

FE- 59

$\mathrm{H}-3$

$1-129$

MN -54

NI -63

TC- 99

$7.498 E-01$

$1.665 E-01$

$6.702 E-04$

$1.796 \mathrm{E}-0$

$1.589 E-11$

$7.114 \mathrm{E}-05$

$6.128 E-05$

4.218E-04

LOW-LEVEL INDUSTRIAL
$1.069 E+05$

$1.287 E+03$
4.218E-04

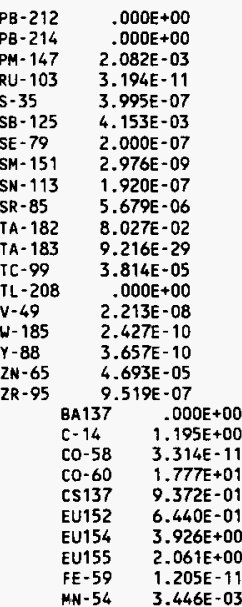

$M-54 \quad \begin{aligned} & 3.446 E-03 \\ & 1.015 E+05\end{aligned}$

$1.863 \mathrm{E}-01$

3.603E-06

$1.015 E+05$

$2.445 \mathrm{E}+00$

7.937E-27

$6.483 E-04$

$1.864 E-06$

$3.499 \mathrm{E}-02$

$4.492 \mathrm{E}-01$

$1.864 \mathrm{E}-06$ $.000 \mathrm{E}+00$

$1.606 \mathrm{E}-04$

C-14 $1.792 \mathrm{E}-13$

$\mathrm{H}-3 \quad 6.057 \mathrm{E}-07$

PM- $147 \quad 1.600 E-04$

IIA $137 \quad .000 E+00$

C. $14 \quad 1.920 E-09$

CO-58 $\quad 1.610 E-09$

प0-60 $1.278 \mathrm{E}-07$

CS137 1.975E-09

EU152 $1.915 E-09$

EU154 $1.625 \mathrm{E}-08$

RU155 $1.621 \mathrm{E}-08$

FE-59 $5.805 E-09$

MN-54 1.291E-08

BUR IAL GROUND $218 W 5$

LOW-LEVEL NONINDUSTRIAL-OFFSITE
$8.762 E+02 \quad 1.035 E+01 \quad 7.504 E-02 \quad 4.219 E+00$

AC -227

$A C-228$
$8.458 \mathrm{E}+02$

2. $157 \mathrm{E}-06$ $.000 E+00$ 
SHIR3281 - (Page 13 of 37 ) RUNDATE: $03 / 15 / 96$

$$
\text { Post-1970 Nontransuranic Waste Burial Ground Areas }
$$
Decayed Curie Content through DECEMBER 31, 1995.

$\begin{array}{lllll}\text { BURIAL GROUND FACILITIES } & \begin{array}{c}\text { TOTAL } \\ \text { (CURIES) }\end{array} & \begin{array}{c}\text { SR-90 } \\ \text { (CURIES) }\end{array} & \begin{array}{c}\text { RU }-106 \\ \text { (CURIES) }\end{array} \text { (CS }-137 \\ \text { (CURIES) }\end{array}$
BURIAL GROUND 218 H5

OTHER RADIOACTIVITY (CIJRIES)

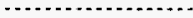

AG- 110

AG -110

BA -133

BE -7

BI - 207

BI- 214

C. -14

CD- 109

CE- 141

CE -144

CL -36

CO- 56

Co- 57

Co- 58

CO-60

CR- 51

CS -134

EU-152

EU- 154

EU- 155

FE - 55

FE- 59

GA- 68

GD -153

GE -68

$\mathrm{H}-3$

HG-203

I- 125

I. 129

I -131

$K-40$

KR-85

MN -54

NA- 22

NB- 94

NB- 95

ND -147

N1 -59

NI -60

NI -63

P- 32

PB- 210

PB- 214

PM- 147

PO- 210

RB-86

RU -103

S- 35

SB- 124

SB- 125

SC -46

SE-75

SE - 79

SN- 113

SR -82

SR -85

TC- 99

TL-208

$T M-170$

$y-88$
9. $865 E-05$ $.000 E+00$

$1.810 E-04$

3. 26BE-02

3. 846E- 05

$.000 E+00$

$1.301 E-01$

2. 866E-04

8. $847 \mathrm{E} \cdot 32$

1.531E-02

1.010E-03

$9.378 E-02$

1.016E-01

3.579E-02

$8.294 E+02$

1.349E-01

3.261E-02

9.531E-02

4. $174 \mathrm{E}-02$

4. 131E-04

$2.485 E+00$

$9.441 \mathrm{E}-05$

$.000 E+00$

$1.475 \mathrm{E}-06$

$1.257 \mathrm{E}-04$

8. $925 \mathrm{E}+00$

$6.986 \mathrm{E}-06$

$7.048 E-13$

$1.000 E-05$

$.000 E+00$

$9.595 \mathrm{E}-04$

8.360E-02

$4.212 E-01$

8.536E-02

7:100E-04

$2.349 E-05$

3. 137E-21

2. $100 E-03$

$.000 E+00$

$3.560 E+00$

2.067E-0?

$3.085 E-02$

$.000 E+00$

B. 196E-02

$6.875 E-04$

$3.802 E-22$

$6.746 \mathrm{E}-03$

3.141E-04

1.015E-07

8. $151 \mathrm{E}-03$

5.295E-04

$1.079 E-05$

$1.940 \mathrm{E}-03$

$1.170 \mathrm{E}-05$

$1.800 \mathrm{E}-11$

$4.328 E-04$

$1.430 \mathrm{E}-03$

$.000 E+00$

$1.030 \mathrm{E}-07$

2. $875 \mathrm{E}-08$ 
WHC-EP-0125-8

SWIR3281 - (Page 16 of 37)

RUNDATE: $03 / 15 / 96$

Post-1970 Nentransuranic Waste Burial Ground Areas

Decayed Curie Content through DECEMBER 31, 1995.

$\begin{array}{llcccc}\text { BURIAL GROUND FACILITIES } & \begin{array}{c}\text { TOTAL } \\ \text { (CURIES) }\end{array} & \begin{array}{c}\text { SR-90 } \\ \text { (CURIES) }\end{array} & \begin{array}{c}\text { RU-106 } \\ \text { (CURIES) }\end{array} \text { (CURIES) } & \text { OTHER RADIOACTIVITY } \\ \text { (CURIES) }\end{array}$

BUR IAL GROUND 218WS

LOW-LEVEL NONINDUSTRIAL-OFFSITE

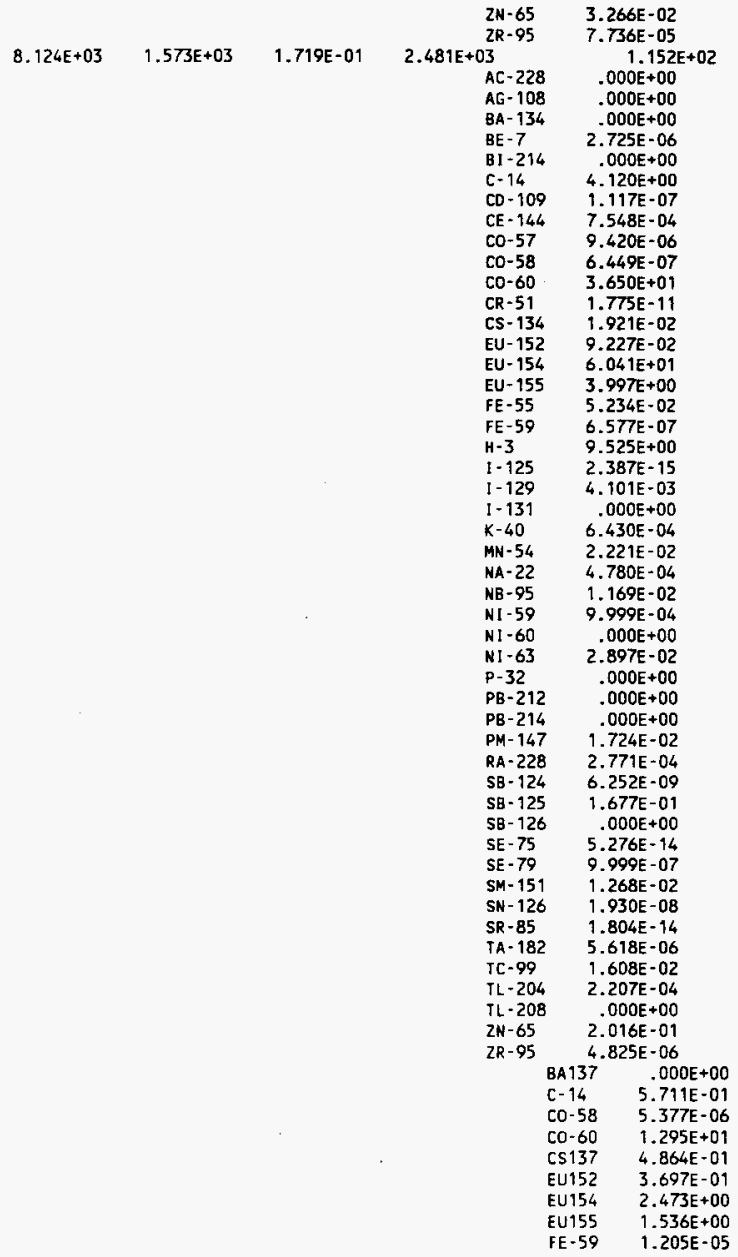


SHIR3281 - (Page 15 of 37 ) RUNDATE: $03 / 15 / 96$

Post-1970 Nontransuranic Waste Burial Ground Areas Decayed Curie Content through DECEMBER 31, 1995.

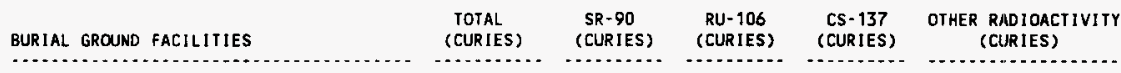

BURIAL GROUND 218W5

LOW-LEVEL NONINDUSTRIAL

LOW-LEVEL NONINDUSTRIAL (ASSAYEO FROM

$\begin{array}{rrrr}6.712 E-03 & 1.498 E-03 & 5.162 E-06 & 1.616 E-03 \\ 1.287 E+04 & 1.717 E+02 & 3.689 E-01 & 6.517 E+01\end{array}$

HIN-54 8.274E-03

TRANSURANIC)

LOW-LEVEL INDUSTRIAL-OFFSI TE

\section{AC-}

AC- 228

AG- 108

$A G-110$

AG -110

AL -28

AU -195

BA- 133

BA- 134

BA- 137

BA- 140

$\mathrm{BE}-7$

BI -207

B I -212

B: -214

C- 14

CA- 45

CD- 109

CE- 141

CE-144

$\mathrm{CL}-36$

Co-56

co- 57

co- 58

co- 59

Co-60

CR-51

CS-134

CU-65M

ES- 254

EU- 152

EU-154

EU- 155

FE- 55

FE- 59

GA- 68

GD- 152

GD- 153

GD- 154

GE -68

$\mathrm{H}-3$

HF -175

HG -203

I -123

I -125

I -129

I -131

$K=40$

KR-85

$M N-54$

MO- 93

NA -22

NB-91

NB-93M

NB -94

NB-95 $.000 E+00$

$1.239 \mathrm{E}+04$

2.058E-05 $.000 E+00$ $.000 \mathrm{E}+00$

1. $010 \mathrm{E}-05$ $.000 \mathrm{E}+00$ $.000 E+00$

$2.319 E-08$

5. 100E-02

$.000 E+00$ $.000 \mathrm{E}+00$ $6.549 \mathrm{E}-20$

$1.280 \mathrm{E}-05$

$1.664 E-03$ $.000 E+00$ $.000 E+00$ $1.092 \mathrm{E}-01$

2.868E-04 4.228E- 04

$3.509 E-12$

2.091E-05

1. 142E-05

4. $883 E-04$

$7.756 \mathrm{E}-02$

$4.082 E-04$

$.000 E+00$

B. $817 E+02$

$5.553 \mathrm{E}-11$

8. 157E-01

$.000 \mathrm{E}+00$

1.034E-05

8.585E-01

8.898E-02

5.624E-04

$5.598 \mathrm{E}+00$

$2.486 \mathrm{E}-08$

$.000 E+00$

$8.468 \mathrm{E}-02$

$6.599 E-07$

$.000 E+00$

$1.568 \mathrm{E}-01$

1. $149 E+04$

7.810E- 11

$1.556 \mathrm{E}-12$

$.000 \mathrm{E}+00$

$3.338 \mathrm{E}-06$

$6.062 \mathrm{E}-05$

1. $089 E-35$

8. B71E-04

$6.527 E-04$

2. 330E-01

$1.291 E+00$

$5.559 E+00$

$1.018 \mathrm{E}-03$

6.847E-05

7.512E-05

$6.387 \mathrm{E}-12$ 
WHC-EP-0125-8

SWIR3281 - (Page 16 of 37 )

RUNDATE : $03 / 15 / 96$

Post-1970 Nontransuranic Waste Burial Ground Areas

Decayed Curie Content through DECEMBER 31, 1995.

$\begin{array}{llllll}\text { BURIAL GROUND FACILITIES } & \begin{array}{c}\text { TOTAL } \\ \text { (CURIES) }\end{array} & \begin{array}{c}\text { SR }-90 \\ \text { (CURIES) }\end{array} & \begin{array}{c}\text { RU-106 } \\ \text { (CURIES) }\end{array} & \begin{array}{c}\text { CS-137 } \\ \text { (CURIES) }\end{array} & \begin{array}{c}\text { OTHER RADIOACTIVITY } \\ \text { (CURIES) }\end{array}\end{array}$

BURIAL GROUND 218 W5

LOW-LEVEL I HOUSTRIAL-OFFSITE

LOW-LEVEL INOUSIRIAL

$4.463 \mathrm{E}+02$

$6.205 E+01$

$2.577 \mathrm{E}-01$

$1.076 \mathrm{E}+02$

\begin{tabular}{|c|c|}
\hline $\begin{array}{l}\text { NB- } 95 M \\
N I-59\end{array}$ & $\begin{array}{r}.000 E+00 \\
5.588 E-04\end{array}$ \\
\hline $\begin{array}{l}N 1-59 \\
N I-60\end{array}$ & $\begin{array}{r}3.300 \mathrm{E}-04 \\
.000 E+00\end{array}$ \\
\hline NI -63 & 3.158E-01 \\
\hline P-32 & $1.389 E-14$ \\
\hline PA-23! & $7.735 E-01$ \\
\hline PB-210 & $1.909 E-05$ \\
\hline PB-212 & $.000 E+00$ \\
\hline PB-214 & $.000 E+00$ \\
\hline PM- 147 & $6.155 E-06$ \\
\hline$P 0-210$ & $3.188 E-06$ \\
\hline RA-228 & $2.783 E-04$ \\
\hline$R B-85 M$ & $.000 E+00$ \\
\hline RB-86 & $1.136 E-13$ \\
\hline RE- 187 & $3.000 E-06$ \\
\hline RU- 103 & $1.777 \mathrm{E}-19$ \\
\hline s.35 & $2.523 E-04$ \\
\hline SB- 124 & $2.671 E-10$ \\
\hline SB -125 & $8.198 \mathrm{E}-03$ \\
\hline$S C-46$ & $2.184 E-06$ \\
\hline SE-75 & $7.520 \mathrm{E}-05$ \\
\hline SE-79 & $2.933 E-03$ \\
\hline $5 I-31$ & $.000 E+00$ \\
\hline SN- 113 & $8.737 E-07$ \\
\hline SN-121 & $3.208 E-07$ \\
\hline SN-123 & $.000 E+00$ \\
\hline SR-82 & $9.788 E-15$ \\
\hline SR - 85 & 1.524E-07 \\
\hline $\begin{array}{l}\text { TA- } 182 \\
\text { TC- } 99\end{array}$ & $\begin{array}{l}3.732 E-06 \\
3\end{array}$ \\
\hline $\begin{array}{l}T C \cdot 99 \\
T E=121\end{array}$ & $\begin{array}{l}3.176 E-02 \\
7.301 E-40\end{array}$ \\
\hline$T E-125$ & $9.539 E-10$ \\
\hline TE - 127 & $.000 E+00$ \\
\hline$T L-208$ & $.000 E+00$ \\
\hline$T M-170$ & $6.824 E-10$ \\
\hline$v-49$ & $5.130 E-06$ \\
\hline$W-183 M$ & $.000 E+00$ \\
\hline$X E-131$ & $1.156 E-21$ \\
\hline$\gamma \cdot 87$ & $.000 E+00$ \\
\hline$\gamma-88$ & $2.236 E-07$ \\
\hline$y-90$ & $.000 E+00$ \\
\hline $2 N-65$ & $2.625 \mathrm{E}-01$ \\
\hline $2 R \cdot 95$ & $3.671 E-08$ \\
\hline 02 & $7.779 E+01$ \\
\hline$A C-227$ & $5.668 E-05$ \\
\hline$A C-228$ & $.000 E+00$ \\
\hline$A G-110$ & $.000 E+00$ \\
\hline$B A \cdot 133$ & $2.170 E-06$ \\
\hline BA- 137 & $.000 E+00$ \\
\hline$B E-7$ & $1.084 E-10$ \\
\hline BI-212 & $.000 E+00$ \\
\hline BI -214 & $.000 E+00$ \\
\hline$c-14$ & $7.776 E-02$ \\
\hline$C A-45$ & $2.526 E-08$ \\
\hline$C 0-109$ & $8.388 E-04$ \\
\hline$C E-141$ & $1.726 E-13$ \\
\hline$C E=144$ & $7.762 E-02$ \\
\hline $\mathrm{CL}-36$ & $1.226 E-05$ \\
\hline $\begin{array}{l}C 0-57 \\
C 0.58\end{array}$ & $\begin{array}{l}4.547 \mathrm{E}-06 \\
2.997 \mathrm{E}-06\end{array}$ \\
\hline
\end{tabular}


SWIR3281 - (Page 17 of 37 )

RUNDATE: 03/15/96

Post-1970 Nontransuranic Waste Burial Ground Areas

Decayed Curie Content through DECEMBER 31, 1995.

$\begin{array}{llllll}\text { BURIAL GROUND FACILITIES } & \begin{array}{c}\text { TOTAL } \\ \text { (CURIES) }\end{array} & \begin{array}{c}\text { SR }-90 \\ \text { (CURIES) }\end{array} & \begin{array}{c}\text { RU-106 } \\ \text { (CURIES) }\end{array} & \begin{array}{c}\text { CS-137 } \\ \text { (CURIES) }\end{array} & \begin{array}{c}\text { OTHER RADIOACTIVITY } \\ \text { (CIIRIES) }\end{array}\end{array}$

BURIAL GROUND 218 W5

LOW-LEVEL INDUSTRIAL

\begin{tabular}{|c|c|}
\hline co- 60 & $3.387 E+01$ \\
\hline$C R-51$ & $5.281 E-14$ \\
\hline Cs. 133 & $.000 E+00$ \\
\hline Cs-134 & $1.523 \mathrm{E}-02$ \\
\hline $\mathrm{CU}-65 \mathrm{M}$ & $.000 E+00$ \\
\hline EU- 152 & $2.640 \mathrm{E}-01$ \\
\hline EU-154 & $2.141 E+\infty 0$ \\
\hline$E U-155$ & $1.000 E-02$ \\
\hline FE - 55 & $1.144 E-01$ \\
\hline FE -59 & $2.113 E-10$ \\
\hline $\mathrm{H}-3$ & $3.937 E+01$ \\
\hline HF- 181 & $9.768 E-14$ \\
\hline $\mathrm{I}-125$ & $1.141 \mathrm{E}-07$ \\
\hline $1-129$ & $2.849 E-02$ \\
\hline $1-131$ & $5.904 E-46$ \\
\hline$k-40$ & $5.273 E-03$ \\
\hline$K R-85$ & $1.338 E-05$ \\
\hline MN -54 & $1.612 \mathrm{E}-02$ \\
\hline MO-93 & $9.993 E-06$ \\
\hline NA-22 & $2.425 E-04$ \\
\hline NB +91 & 2. $788 E-02$ \\
\hline NB-93M & $2.132 E \cdot 08$ \\
\hline NB - 94 & $6.573 E-04$ \\
\hline NB-95 & $1.256 \mathrm{E}-12$ \\
\hline NB $-95 M$ & $.000 E+00$ \\
\hline ND .147 & $.000 E+00$ \\
\hline N1 159 & $7.100 E-03$ \\
\hline N $1-60$ & $.000 E+00$ \\
\hline N1 -63 & $6.040 E-02$ \\
\hline P-32 & $1.326 E-20$ \\
\hline$P A-231$ & $4.800 E-11$ \\
\hline PB - 208 & $.000 E+00$ \\
\hline PB- 210 & $7.740 E-05$ \\
\hline PB-212 & $.000 E+00$ \\
\hline PB-214 & $.000 E+00$ \\
\hline PM- 147 & $8.633 E-01$ \\
\hline PO- 210 & $2.965 E-13$ \\
\hline PT - 195 & $.000 E+00$ \\
\hline$R A-228$ & $6.126 \mathrm{E}-05$ \\
\hline$s-35$ & $1.902 E-09$ \\
\hline SB- 125 & $1.706 E-03$ \\
\hline SC- -46 & $8.139 E-10$ \\
\hline$S E-75$ & $9.189 E-08$ \\
\hline SE -79 & $1.038 E-04$ \\
\hline$S M=151$ & $7.569 \mathrm{E}-01$ \\
\hline SR - 82 & $4.374 E-24$ \\
\hline SR-85 & $1.027 E-08$ \\
\hline SR -89 & 1. $132 E-13$ \\
\hline TA- 182 & $1.779 E-05$ \\
\hline TC-99 & $7.555 \mathrm{E}-02$ \\
\hline TE- 125 & $7.060 \mathrm{E}-09$ \\
\hline$T E-129$ & $3.873 E-27$ \\
\hline $\mathrm{Tl}-204$ & $2.934 E-05$ \\
\hline$T L-208$ & $.000 E+00$ \\
\hline$v \cdot 49$ & $5.601 E-07$ \\
\hline W- 185 & $2.686 E-10$ \\
\hline$Y-87$ & $.000 E+00$ \\
\hline$Y-90$ & $.000 E+00$ \\
\hline $2 N-65$ & $3.690 \mathrm{E}-03$ \\
\hline $2 R-95$ & $1.319 E-07$ \\
\hline
\end{tabular}


SWIR3281 - (Page 18 of 37 )
RUNOATE: $03 / 15 / 96$

Post-1970 Nontransuranic Waste Burial Ground Areas Decayed Curie content through DECEMBER 31, 1995.

BURIAL GROUND FACILITIES

TOTAL SR-90 RU-106 CS-137 OTHER RADIOACTIVIIY

BURIAL GROUND 218 W5

LOH-LEVEL INDUSTRIAL

$\begin{array}{lccc}\text { (CURIES) } & \text { (CURIES) } & \text { (CURIES) } & \text { (CURIES) } \\ \ldots & & \end{array}$

\begin{tabular}{|c|c|c|c|c|c|}
\hline $7.091 E+01$ & $1.679 E+01$ & $1.054 E-02$ & $1.827 E+01$ & $\begin{array}{l}\text { B.A } 137 \\
\text { C- } 14 \\
\text { CD-58 } \\
\text { CD-60 } \\
\text { CS } 137 \\
\text { EU152 } \\
\text { EU154 } \\
\text { EU155 } \\
\text { FE-59 } \\
M N-54\end{array}$ & $\begin{array}{l}.000 E+00 \\
5.256 E-01 \\
9.892 E-12 \\
1.104 E+01 \\
4.424 E-01 \\
3.308 E-01 \\
2.176 E+00 \\
1.302 E+00 \\
1.572 E-13 \\
2.933 E-03 \\
.000 E+00\end{array}$ \\
\hline $2.194 E-04$ & $9.651 E-10$ & $2.688 E-14$ & $\begin{array}{r}6.483 E-10 \\
\text { CO- } 60 \\
\text { EU-154 } \\
\text { EU-155 } \\
\text { FE }-59 \\
M N-54 \\
N I-60 \\
N I-63\end{array}$ & $\begin{array}{l}1 \\
5 \\
8 \\
4\end{array}$ & $\begin{array}{l}2.194 E-04 \\
94 E-04 \\
22 E-09 \\
94 E-10 \\
62 E-22 \\
22 E-08 \\
00 E+00 \\
83 E-09\end{array}$ \\
\hline
\end{tabular}

TRAHSURANIC WASTE STORAGE \& ASSAY FACILITYY(TRUSAF) LOW-LEVEL NONINDUSTRIAL-OFFSITE

3. $473 E-06$

$.000 E+00$

LOW-LEVEL NONI NDUSTRIAL

$7.483 E-03$

$1.699 E-03$

$6.611 E-06$

$1.730 \mathrm{E}-03$

$.000 E+00$

$-000 E+00$

C- 14

$\mathrm{H}-3$

p- 32

$5-35$

LOW-LEVEL NONIMDUSTRIAL

LOW-LEVEL NOWINDUSTRIAL (ASSAYED FROM TRANSURANIC)

CENTRAL WASTE COMPLEX $2401 \mathrm{~W}$ BUILDING

LOW-LEVEL NONINDUSTRIAL-OFFSITE
$3.473 E-06$

$1.699 E-06$

$1.496 \mathrm{E}-06$

$6.481 E-22$

2. $770 E-07$

9.148E-05

8.945E-05

9.561E-07

4.124E- 07

6.614E-07 $.000 E+00$

$\begin{array}{cccc}1.286 E-01 & 2.858 E-02 & 1.136 E-04 & 3.083 E-02 \\ & & & M N-54 \\ 1.493 E+05 & 7.467 E+04 & .000 E+00 & .000 E+00 \\ 5.848 E-03 & 1.307 E-03 & 3.646 E-06 & 1.363 E-03 \\ & & & C D-60 \\ & & & E U-154 \\ K-40\end{array}$

CO- 60

EU- 152

EU -154

$\mathrm{MN}-54$

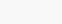

LOW-LEVEL NONINDUSTRIAL

$1.592 \mathrm{E}-04$

$1.344 \mathrm{E}-04$

$2.454 E-05$

2.450E-07

EA $137 \quad .000 E+00$

c: $-16 \quad 3.238 \mathrm{E}-08$

C. $0-58 \quad 1.610 \mathrm{E}-17$

Co-60 $9.879 \mathrm{E}-07$

CS137 2.908E-08

EU152 2.366E-08

EU154 1.690E-07

EU155 $1.193 E-07$

FE-59 2.160E-22

MN-54 $1.764 E-09$

CENTRAL WASTE COMPLEX $2402 \mathrm{~W}$ BUILDING LOW-LEVEL NONINDUSTRIAL

\begin{tabular}{|c|c|c|c|c|}
\hline \multirow[t]{2}{*}{$1.836 \mathrm{E}-01$} & $1.075 \mathrm{E}-03$ & $4.356 \mathrm{E}-05$ & $\begin{aligned} 1.291 E-03 \\
C-14 \\
C E-144 \\
H-3 \\
P M-147 \\
5 M-151 \\
T C-99\end{aligned}$ & $\begin{array}{l}1.782 E-01 \\
1.409 E-12 \\
6.720 E-05 \\
4.256 E-06 \\
7.378 E-04 \\
6.724 E-04 \\
1.767 E-01\end{array}$ \\
\hline & & & & $\begin{array}{ll}37 & .000 E+00 \\
4 & 1.119 E-05\end{array}$ \\
\hline
\end{tabular}


SWIR3281 - (Page 19 of 37 )
RUNDATE: $03 / 15 / 96$

Post-1970 Nontransuranic Waste Burial Ground Areas

Decayed Curie Content through DECEMBER 31, 1995.

\begin{tabular}{|c|c|c|c|c|c|c|}
\hline BURIAL GROUND FACILITIES & $\begin{array}{l}\text { TOTAL } \\
\text { (CURIES) }\end{array}$ & $\begin{array}{l}\text { SR-90 } \\
\text { (CUR!ES) }\end{array}$ & $\begin{array}{l}\text { RU-106 } \\
\text { (CURIES) }\end{array}$ & $\begin{array}{l}\text { CS-137 } \\
\text { (CURIES) }\end{array}$ & $\begin{array}{l}\text { OTHER RADIOAC } \\
\text { (CIJRIES) }\end{array}$ & VITY \\
\hline \multirow{2}{*}{$\begin{array}{l}\text { CENTRAL WASTE COMPLEX } 2402 W \text { BUILDING } \\
\text { LOW-LEVEL NONINDUSTRIAL }\end{array}$} & n............ & 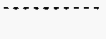 & 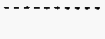 & . & & \\
\hline & & & & & $\begin{array}{l}\text { CO-58 } \\
\text { CO-60 } \\
\text { ES137 } \\
\text { EU152 } \\
\text { EU154 } \\
\text { EU155 } \\
\text { VE-59 } \\
\text { MN-54 }\end{array}$ & $\begin{array}{l}5.458 E-15 \\
3.413 E-04 \\
1.005 E-05 \\
8.175 E-06 \\
5.840 E-05 \\
4.120 E-05 \\
7.240 E-20 \\
6.069 E-07\end{array}$ \\
\hline
\end{tabular}

CENTRAL WASTE COMPLEX 2402WB BUILDING LOW-LEVEL NONINDUSTRIAL-OFFSITE

LOW-LEVEL NONINDUSTRIAL

CENTRAL WASTE COMPLEX 2402WC BUILDING LOW-LEVEL NONINDUSTRIAL-OFFSITE

LOW-LEVEL NON INDUSTRIAL
$9.288 E-03 \quad 2.597 E-04 \quad 4.047 E-04 \quad 1.784 E-03$

$A C-228$

$\mathrm{BA}-133$

$81-212$

B1-214

CO-60

EU-154

PA- 231

PB- 212

PB-214

$\mathrm{RA}-22 \mathrm{~B}$

$T L-208$

$2.661 E+00 \quad 5.923 E-01 \quad 2.412 E-03 \quad 6.346 E-01$

C- 14

CE- 144

Co- 60

cs-134

EU- 152

EU- 154

EU- 155

FE -59

$\mathrm{H}-3$

MN- 54

NA-22

NB - $95 \mathrm{~N}$

N] -60

PM- 147

SM- 151

TC-99

ZR-95

BA137

c:-14

co-58

100-60

CS137

EU152

lEU154

EU155

FE- 59

IAN- 54

$2.471 E-07 \quad .000 E+00 \quad .000 E+00 \quad .000 E+00$

$\mathrm{BE}-7$

C- 14

EU- 154

$\mathrm{SB} \cdot 124$

$1.227 E+00 \quad 2.810 E-01$

8.786E-05.

$7.869 E-02$

AG -108

$A G-110$

C- 14

$6.219 E-04$

$.000 E+00$

2. $222 E-06$

$.000 E+00$

$.000 E+00$

$1.056 E-04$

$6.800 E-06$

$4.010 E-16$

. OOOE +00

$.000 E+00$

5.072E-04

OOOE +00

6.670E-03

$1.102 \mathrm{E}-05$

$1.570 E-04$

2.257E-03

$7.966 \mathrm{E}-09$

$4.050 E-05$

$6.685 \mathrm{E}-05$

$2.374 \mathrm{E}-05$

$7.995 \mathrm{E}-20$

$1.331 \mathrm{E}-05$

$4.998 \mathrm{E}+07$

$1.694 \mathrm{E}-05$

$.000 E+00$

$.000 \mathrm{E}+00$

2.072E-03

$2.009 E-03$

$1.524 \mathrm{E}-06$

9.687E-08

$-000 E+00$

3.254E-07

$7.206 \mathrm{E}-17$

9.637E-06

2. $908 E-07$

2.349E-07

1.667E-06

1.161E-06

$6.012 \mathrm{E}-22$

$1.475 E-08$

2. 471 E- 07

$6.563 E-11$

$9.998 \mathrm{E}-08$

$1.469 \mathrm{E}-07$

$1.468 \mathrm{E}-10$ 5. 100E-01 $.000 E+00$ $.000 E+00$

$1.035 \mathrm{E}-05$

CE-14] $5.728 E-18$ 
WHC-EP-0125-8

SWIR3281 - (Page 20 of 37)

RUNDATE : $03 / 15 / 96$

Post-1970 Nontransuranic Waste Burial Ground Areas Decayed Curie Content through DECEMBER 31, 1995.

\begin{tabular}{|c|c|c|c|c|}
\hline BURIAL GROUND FACILITIES & $\begin{array}{l}\text { IOTAL } \\
\text { (CURIES) }\end{array}$ & $\begin{array}{l}\text { SR-90 } \\
\text { (CURIES) }\end{array}$ & $\begin{array}{l}\text { RU-106 } \\
\text { (CURIES) }\end{array}$ & $\begin{array}{c}\text { CS-137 } \\
\text { (CURIES) }\end{array}$ \\
\hline
\end{tabular}

OTHER RADIOACTIVITY

CENTRAL WASTE COMPLEX $2402 W C$ BUILDING

LOW-LEVEL NONINDUSTRIAL

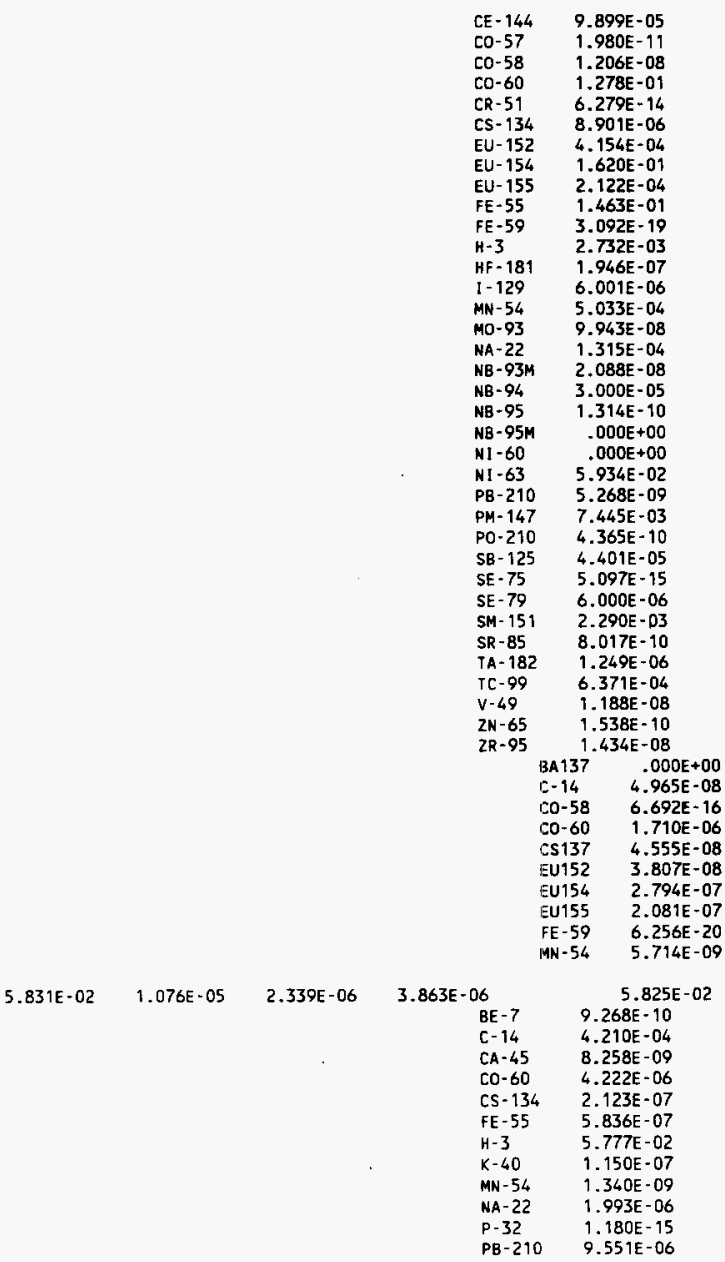

CENIRAL WASTE COMPLEX 2402WD BUILDING LOW-LEVEL NONINDUSTRIAL-OFFSITE

(n)


SWIR3281 - (Page 21 of 37)

RUNDATE: 03/15/96

Post-1970 Nontransuranic Waste Burial Ground Areas

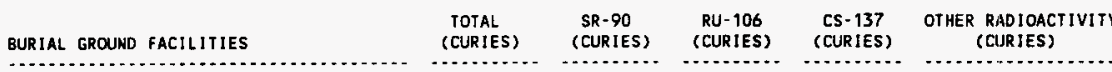

CENTRAL WASTE COMPLEX 2402WO BUILDING LON-LEVEL NONINDUSTRIAL-OFFSITE

LOW-LEVEL NONIHDUSTRIAL
CENTRAL WASTE COMPLEX 2402WE BUILDING LOW-LEVEL NONINDUSTRIAL-OFFSITE

LOH-LEVEL NONINDUSTRIAL

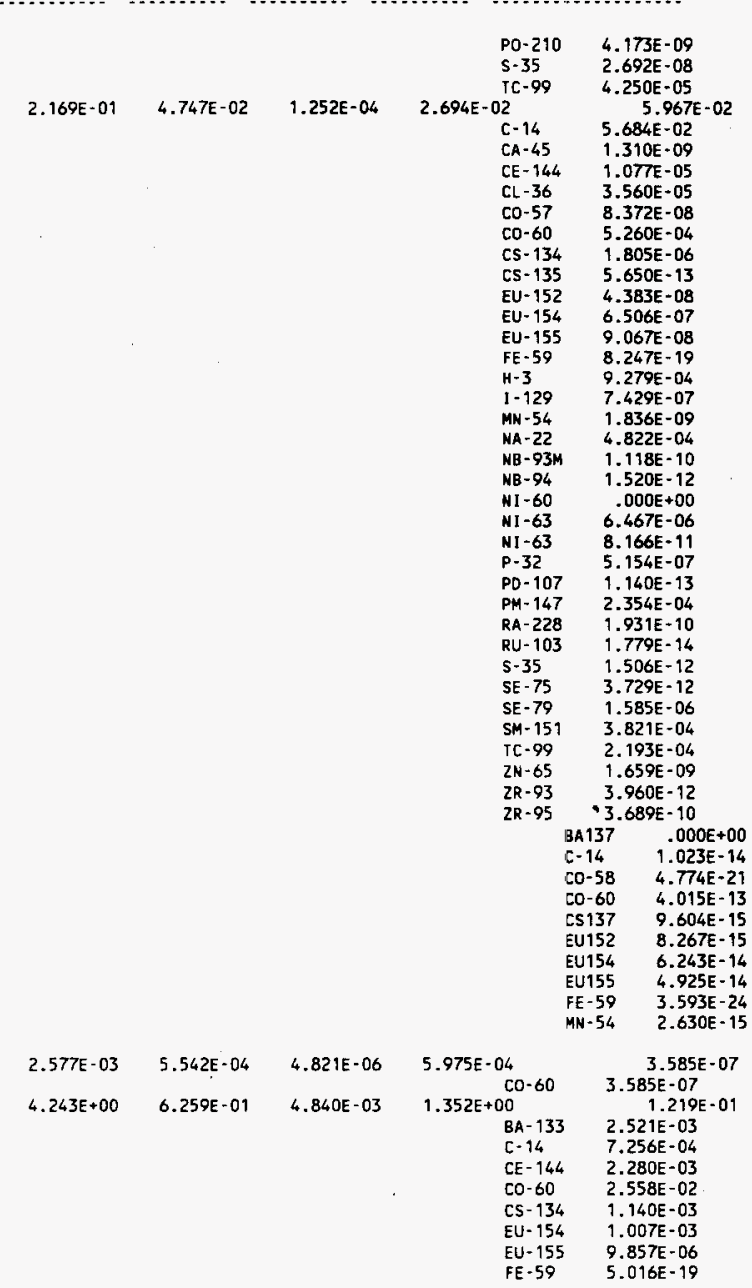


SWIR3281 - (Page 22 of 37)

RUNDATE: $03 / 15 / 96$

$$
\text { Post-1970 Nontransuranic Waste Burial Ground Areas }
$$
Decayed Curie Content through DECEMBER 31, 1995.

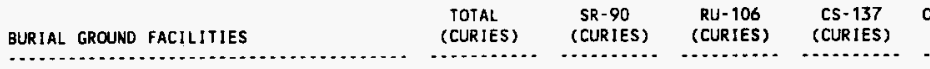

CENTRAL WASTE COMPLEX 2402WE BUILDING LOW-LEVEL NONIMDUSTRIAL
CENTRAL WASTE COMPLEX 2402WF BUILDING LOW-LEVEL NOHINDUSTRIAL
5. 123E-02

$3.132 E-04$
$1.378 E-13$
OTHER RIDIOACTIVITY (CURIES)

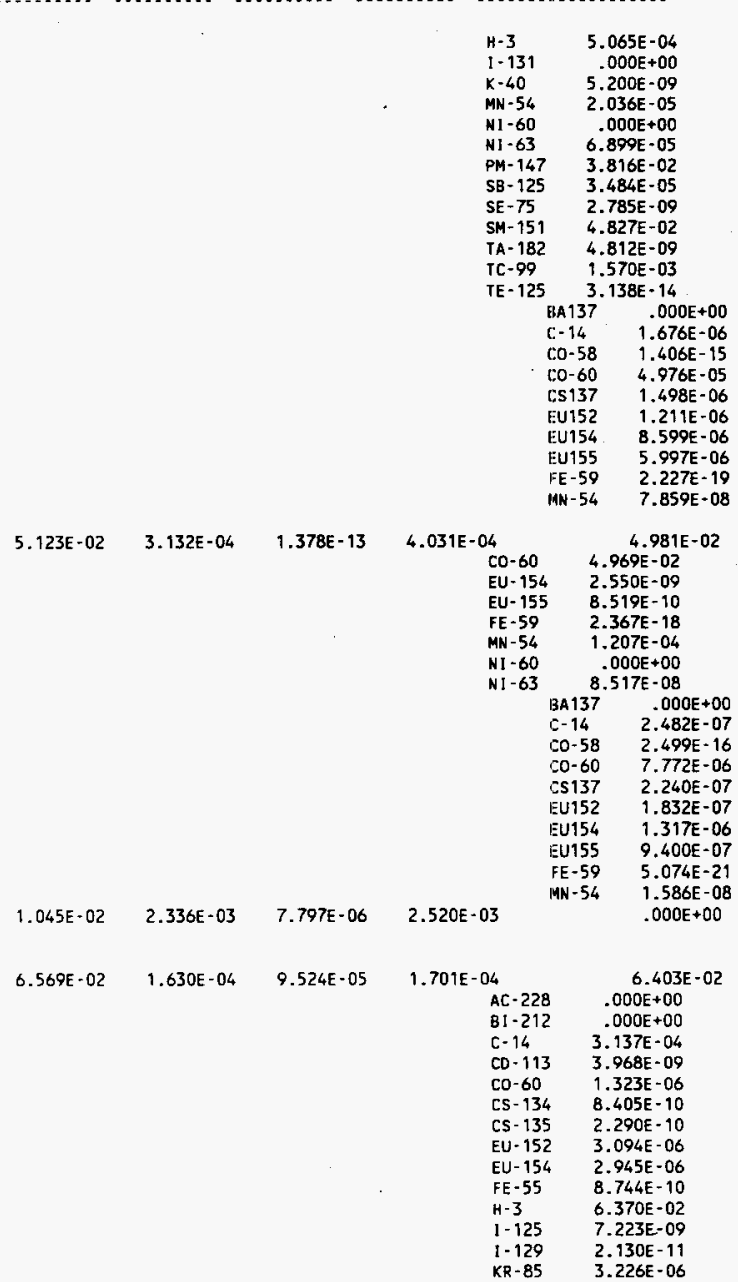

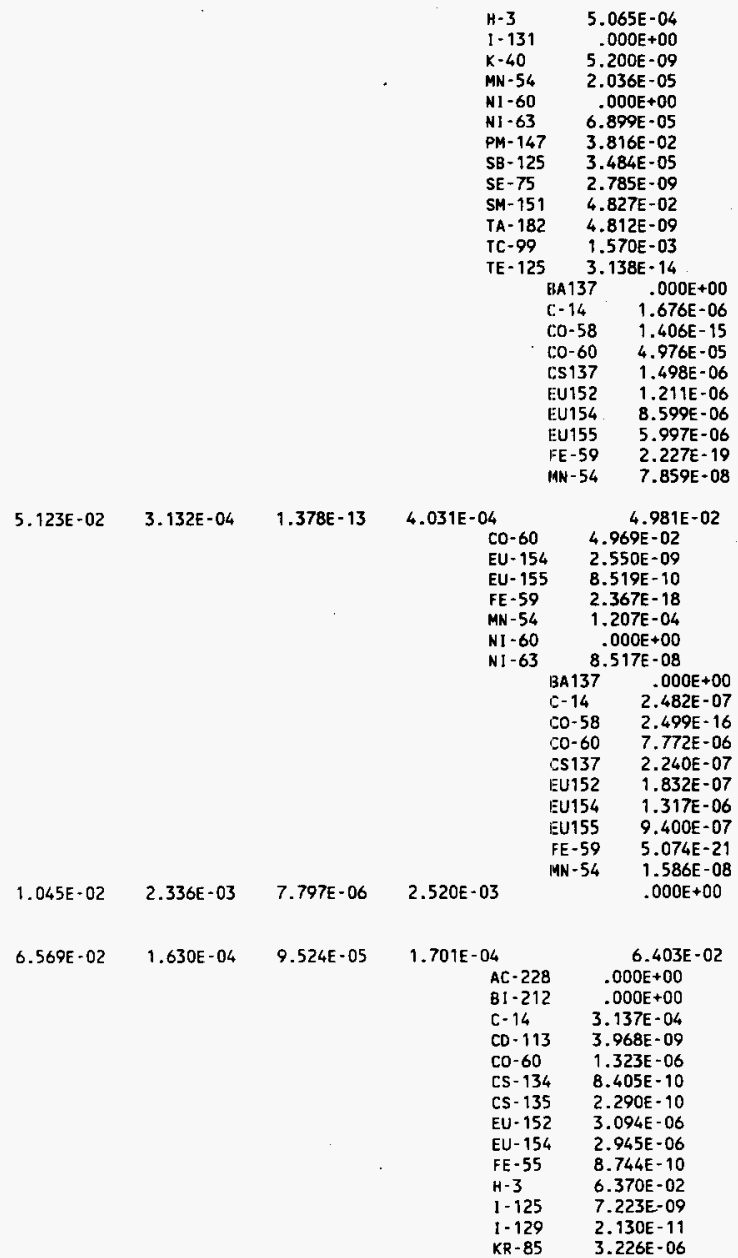

LOH-LEVEL NOHINDUSTRIAL (ASSAYED FROM TRANSURANIC)

CENTRAL WASTE COMPLEX 2402WG BUILDING LOW-LEVEL NONINDUSTRIAL-OFFSITE 
SWIR328I - (Page 23 of 37)

RUNDATE: $03 / 15 / 96$ Post-1970 Nontransuranic Waste Burial Ground Areas
Decayed Curie Content through DECEMBER 31, 1995.

\begin{tabular}{|c|c|c|c|c|c|}
\hline BURIAL GROUND FACILITIES & $\begin{array}{l}\text { TOTAL } \\
\text { (CURIES) }\end{array}$ & $\begin{array}{l}\text { SR-90 } \\
\text { (CURIES) }\end{array}$ & $\begin{array}{c}\text { RU-106 } \\
\text { (CURIES) }\end{array}$ & $\begin{array}{l}\text { CS-137 } \\
\text { (CURIES) }\end{array}$ & $\begin{array}{l}\text { OTHER RADIOACTIVITY } \\
\text { (CURIES) }\end{array}$ \\
\hline
\end{tabular}

CENTRAL WASTE COMPLEX 2402WG BUILDING LOW-LEVEL NONINDUSTRIAL-OFFSITE
LOW-LEVEL NONINDUSTRIAL
$2.307 E+00 \quad 5.481 E-01$

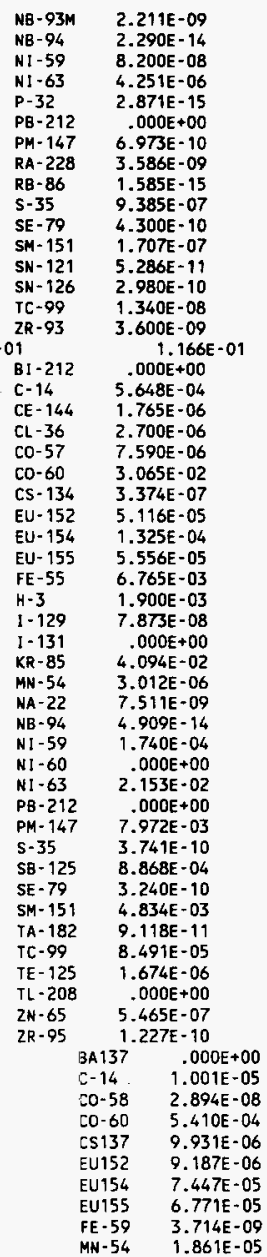




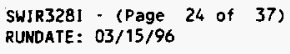

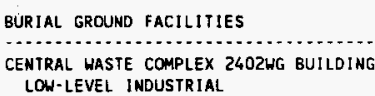

CENTRAL WASTE COMPLEX 2402WH BUILDING LOW-LEVEL NONINDUSTRIAL-OFFSI TE

LON-LEVEL NONINDUSTRIAL

CENTRAL WASTE COMPLEX 2402WI BUILDING LOW-LEVEL NONINDUSTRIAL-OFFSITE

LOW-LEVEL NONINDUSTRIAL

\begin{tabular}{|c|c|c|c|c|}
\hline $\begin{array}{l}\text { TOTAL } \\
\text { (CUR IES) }\end{array}$ & $\begin{array}{l}\text { SR-90 } \\
\text { (CURIES) }\end{array}$ & $\begin{array}{l}\text { RU-106 } \\
\text { (CURIES) }\end{array}$ & $\begin{array}{l}\text { CS-137 } \\
\text { (CURIES) }\end{array}$ & $\begin{array}{l}\text { OTHER RADIOACTIVITY } \\
\text { (CURIES) }\end{array}$ \\
\hline . & n......... & - & . & - \\
\hline
\end{tabular}

$2.235 E \cdot 05$

$1.470 \mathrm{E}-07$

5.178E-06

C- 14

$3.261 E-06$

CE-144 1.999E-07

$\mathrm{H}-3 \quad 1.940 \mathrm{E}-08$

1-129 1.530E-12

NB-94 3.710E-15

PM- $147 \quad 3.041 E-06$

SE-79 2.450E-11

IC $-99 \quad 9.060 \mathrm{E}-10$

2.305E-02 1.274E-03 $1.697 \mathrm{E}-03 \quad 1.361 \mathrm{E}-03$

C- 14

$1.577 \mathrm{E}-06$

$3.549 \mathrm{E}-08$

CE-144 8.859E-08

CO-60 4.197E-07

$\mathrm{H}=3 \quad 1.007 \mathrm{E}-06$

$1-129 \quad 9.200 E-13$

I-131 1.107E-24

NB-94 2.600E-14

NI $-59 \quad 2.500 E-10$

NI $-63 \quad 2.458 E-08$

SE-79 $1.800 E-11$

TC- 99 5. DOOE-10

$2 R-95 \quad 1.606 E-10$

7.564E-01 7.697E-03 1.760E-04 6.403E-03

BI -214

C- 14

CE -144

CO- 60

CS- 135

EU-152

EU-154

EU-155

FE-59

$\mathrm{H}-3$

I -129

MN -54

NA- 22

NB- 94

N1 -60

N1 -63

PB- 214

PD -107

PM- 147

SE-79

SM- 151

SN- 123

TC- 99

$2 \mathrm{~N}-65$

$2 R-90$

ZR-93

$2 R-95$

$.606 \mathrm{E}-10$
$7.268 \mathrm{E}-01$

$.000 E+00$

$6.423 \mathrm{E}-09$

1.471E-04

4.061E-04

$1.000 E-04$

5.764E-05

$9.437 \mathrm{E}-05$

2. $596 \mathrm{E}-04$

$1.187 \mathrm{E}-19$

7.230E-01

$1.000 E-04$

7.793E-07

7. $655 E-06$

$4.440 E-15$

$.000 E+00$

$2.670 E-10$

$.000 E+00$

$1.000 E-04$

$1.468 E-03$

$1.930 E-11$

9.541E-04

$.000 E+00$

1.012E-04

2.512E-11

.000E +00

$1.696 \mathrm{E}-13$

$6.412 E-09$

\begin{tabular}{|c|c|c|c|c|}
\hline $6.450 E-06$ & $.000 E+00$ & $.000 E+00$ & $.000 E+00_{H-3}$ & $\begin{array}{r}6.450 E-06 \\
6.450 E-06\end{array}$ \\
\hline $2.910 \mathrm{E}-01$ & $5.353 E-03$ & $1.435 E-05$ & $\begin{aligned} 4.944 E-03 \\
\text { C- } 14 \\
\text { CE }-144 \\
\text { CO-60 } \\
H-3 \\
1-129\end{aligned}$ & $\begin{array}{l}2.690 \mathrm{E}-01 \\
9.992 \mathrm{E}-07 \\
4.450 \mathrm{E}-08 \\
6.665 \mathrm{E}-04 \\
2.105 \mathrm{E}-08 \\
1.864 \mathrm{E}-12\end{array}$ \\
\hline
\end{tabular}


WHC-EP-O125-8

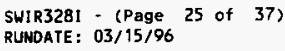

CENTRAL WASTE COMPLEX 2402WJ BUILDING LOH-LEVEL NONINDUSTRIAL

$1.624 E-01 \quad 4.495 E-04 \quad 1.052 E-05 \quad 5.405 E-04$

NB-94 4.270E- 15

PH- $147 \quad 2.639 E-06$

PO- 210 9.160E-11

SE - 79 2.820E-11

SR-8S 2.057E-13

TC $-99 \quad 2.683 \mathrm{E}-01$

CENTRAL WASTE COMPLEX 2402WK BUILDIMG LOW-LEVEL NON INDUSTRIAL

8.402E-01 1.686E-02 3.497E-04 1.876E-02

C- 14

CE-144

$\mathrm{H}-3$

PM- 147

$S M-151$

TC- 99

EU- 155

$\mathrm{H}-3$

PH- 147

SM- 151

SN-123:

TC-99

CENTRAL WASTE COMPLEX 2403WA BUILDING LOW-LEVEI NONINDUSTRIAL-OFFSITE

6.037E-01 5.982E-02 3.269E-06 1.882E-0

AG -110

$A G-110$

BE -7

BI-212:

BI -214 .

C- 14

CA -45

CE- 144

CO-56

Co-57

Co- 58

CO-60

CR -51

CS -134

EU. 152

EU-154,

EU-155;

$\mathrm{FE}-55$

FE- 59

$\mathrm{H}-3$

$\mathrm{HF}-175$

HG-203

$1-125$

$1-129$

K-40

$\mathrm{KR}-85$

$\mathrm{MN}-54$

$\mathrm{NA}-22$

NB- 94

NI -59

N1 -60

N I -63

P-32

PB- 212

1.604E-01

$7.384 E-13$

$1.158 \mathrm{E}-05$

$2.080 E-06$

2. $853 \mathrm{E}-06$

3. 805E-04

1.597E-01

7.676E-0

$2.125 \mathrm{E}-11$

4.214E-04

$7.585 E-05$

5.062E-01

8.632E-03

$1.287 E-02$

$.000 E+00$

2.394E-01

1.177E-01

$1.674 E-08$

$.000 \mathrm{E}+00$

$2.467 E-08$

$.000 E+00$

$.000 E+00$

$2.388 E-03$

3.629E-05

$9.512 E-05$

$6.584 \mathrm{E}-10$

$6.599 E-06$

1. $148 \mathrm{E}-09$

$6.636 E-02$

5. $040 \mathrm{E}-14$

$5.557 E-04$

$4.547 E-04$

$2.920 \mathrm{E}-04$

$3.460 \mathrm{E}-06$

$1.335 \mathrm{E}-04$

$1.170 \mathrm{E}-10$

$1.802 \mathrm{E}-02$

$1.765 \mathrm{E}-08$

$1.136 E-10$

$1.003 E-09$

3.500E-06

1.396E-02

2.119E-03

$1.493 E-05$

$6.602 \mathrm{E}-05$

$1.793 \mathrm{E}-08$

7.644E-06

$.000 E+00$

$9.354 \mathrm{E}-04$

$1.313 E-21$

$.000 E+00$ 
SWIR328I - (Page 26 of 37 )
RUNDATE: $03 / 15 / 96$

Post-1970 Nontransuranic Waste Burial Ground Areas Decayed Curie content through DECEMBER 31, 1995.

\begin{tabular}{llllll} 
BURIAL GROUND FACILITIES & $\begin{array}{c}\text { TOIAL } \\
\text { (CURIES) }\end{array}$ & $\begin{array}{c}\text { SR-90 } \\
\text { (CURIES) }\end{array}$ & $\begin{array}{c}\text { RU-106 } \\
\text { (CURIES) }\end{array}$ & $\begin{array}{c}\text { CS-137 } \\
\text { (CURIES) }\end{array}$ & $\begin{array}{c}\text { OTHER RADIOACTIVITY } \\
\text { (CURIES) }\end{array}$ \\
\hline
\end{tabular}

CENTRAL WASTE COMPLEX 2403WA BUILDING LOW-LEVEL NONINDUSTRIAL-OFFSITE

LOW-LEVEL NONINDUSTRIAL

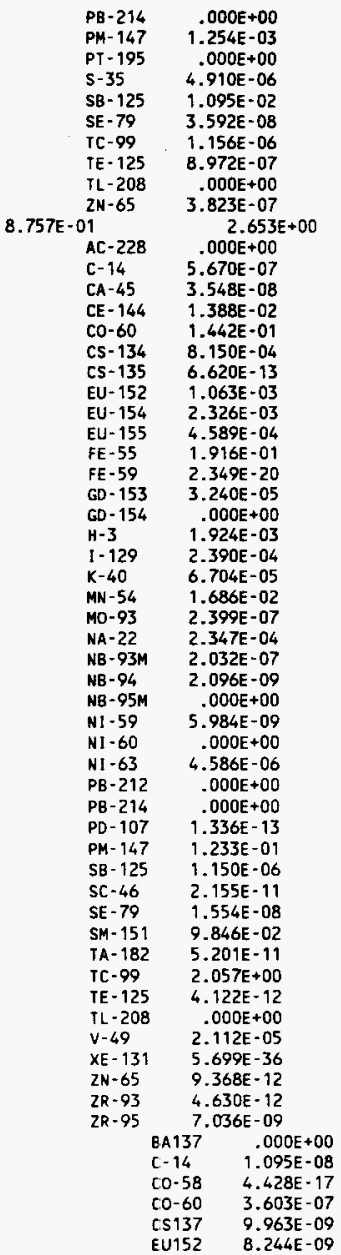


SWIR3281 - (Page 27 of 37 ) RUNDATE: $03 / 15 / 96$

Post-1970 Nontransuranic Waste Burial Ground Areas Decayed Curie Content through DECEMBER 31, 1995.

\begin{tabular}{|c|c|c|c|c|c|c|c|}
\hline BURIAL GROUND FACILITIES & $\begin{array}{l}\text { TOTAL } \\
\text { (CURIES) }\end{array}$ & $\begin{array}{c}\text { SR-90 } \\
\text { (CURIES) }\end{array}$ & $\begin{array}{c}\text { RU-106 } \\
\text { (CURIES) }\end{array}$ & $\begin{array}{c}\text { CS }-137 \\
\text { (CURIES) }\end{array}$ & OTHER & $\begin{array}{l}\text { RADIOAC } \\
\text { (CURIES) }\end{array}$ & IVITY \\
\hline $\begin{array}{l}\text { CENTRAL WASTE COMPLEX } 2403 \text { WA BUILOING } \\
\text { LOW-LEVEL NONINDUSTRIAL }\end{array}$ & & & & & & $\begin{array}{l}\text { EU154 } \\
\text { EU155 } \\
\text { FE-59 } \\
\text { MN-54 }\end{array}$ & $\begin{array}{l}5.989 E-08 \\
4.371 E-08 \\
2.203 E-21 \\
9.524 E-10\end{array}$ \\
\hline
\end{tabular}

CENTRAL WASTE COMPLEX 2403WB BUILDING LOW-LEVEL NONINDUSTRIAL-OFFSITE
$1.258 E+00$

2.718E-03

$2.401 E-03$

5. 102E-02
B1 -214

C- 14

CD- 109

CO-60

CS- 134

EU- 154

FE- 55

FE- 59

H-3

$1-129$

$\mathrm{K}-40$

NA-22

NB- 94

N] -60

NI -63

PO- 210

$\mathrm{SB} \cdot 125$

SE -79

IC -99

LOW-LEVEL NONINDUSTRIAL

\begin{abstract}
8. 307E+01
\end{abstract}
$2.864 E+01$

$1.952 E-02$

$1.210 E+0$

TE-125;

$B A-133$

C- 14

CE -144

CO-60

CS-13:

EU-15:?

EU-15i;

$\mathrm{H}-3$

I -129

$\mathrm{K}-40$

MN- 54

NB- 93 M

NB- 94

NB- 95

NI-59

N1 -63

P-32

PB-21D

PM- 147

RA- 2213

SB- 125

SE -79

SR-85

SR-89

TC- 99

ZN-65

\section{BA 137}

C- 14

CO-58

Co- 60

CS 137

EU152

EU154

$1.127 \mathrm{E}+00$

.000E+00

4.599E-12

7. $443 E-06$

$1.125 E-03$

2.726E-04

3.176E-05

2. 225E-08

2. $064 \mathrm{E}-16$

8.416E-05

4. $400 \mathrm{E}-08$

4.870E-06

9.333E-07

4. $400 E-18$

$.000 E+00$

$1.126 \mathrm{E}+00$

9.507E-09

$3.701 E-05$

8. $200 \mathrm{E}-14$

$1.659 \mathrm{E}-05$

3. $339 E-10$

$4.681 \mathrm{E}-05$

3. 044E-09

5.743E-03

1. $244 E+00$

3.564E-04

2.812E-09

4. $277 \mathrm{E}-02$

$5.296 \mathrm{E}-01$

3.691E-05

2.626E-05

1. 146E-02

8.383E- 11

6. 230E-04

2.079E-04

2.900E-12

5. 226E-08

5. $154 E-08$

5. $403 E-07$

2.382E-02

2. $853 E-07$

5. 086E-02

7. $250 E-12$

3. $110 \mathrm{E}-07$

1.610E-08

$1.390 E-01$

$4.412 E-02$ $.000 E+00$ $2.829 E-04$ $5.963 E-05$ $1.790 E-02$ 2. 885E-04 2.765E-04 2.321E-03 
SWIR328I - (Page 28 of 37 )

RUNDATE : 03/15/96

Post-1970 Nontransuranic Waste Burial Ground Areas Decayed Curie Content through DECEMBER 31, 1995.

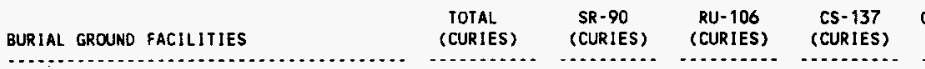

OTHER RADIOACTIVITY

CENTRAL WASTE COMPLEX $2403 \mathrm{WB}$ BUILDING LOW-LEVEL NONINDUSTRIAL

LOW-LEVEL NONINDUSTRIAL (ASSAYED FROM

$1.789 \mathrm{E}-01$

$8.696 \mathrm{E}-02$

$3.542 E-07$

2.482E- 03 (CLRIES) IRANSURANIC

$2.021 E+00$

3.65 IE-02

$3.994 E-03$

4.399E-02

AC -228

AG -110

AG- 110

AU- 195

BE- 7

BI -207

BI -212

B I - 214

C- 14

CA- 45

CD- 109

CD -113

CE- 144

Co-56

CO-57

CO- 58

CO- 60

CR-51

CS- 134

EU- 152

EU- 154

EU- 155

FE -55

GA- $6 B$

GE - 68

$\mathrm{H}-3$

HF- 175

HG -203

I- 125

I - 129

I- $\{31$

$k-40$

KR-85

MN -54

NA -22

NI -60

NI -63

P. 32

PA- 231

PB-212

PB- 214

PM- 147

PO-210

RA- 228

S- 35

SB- 124

SB- 125

$\mathrm{SC}-46$

SC. 47

SE - 75

SM- 151

SN- 113

$\begin{array}{ll}\text { EU155 } & 2.263 E-03 \\ \text { FE-59 } & 9.543 E-05\end{array}$

$M N-54 \quad 1.391 E-03$ $1.435 E-04$

4.337E-05

1.001E-04

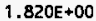

$1.820 \mathrm{~F}+00$

$.000 \mathrm{E}+00$

6. $995 \mathrm{E}-05$

$.000 \mathrm{E}+00$

1. $169 \mathrm{E}-07$

2.47BE-05

$1.304 E-06$

$.000 E+00$

$.000 \mathrm{E}+00$

$3.475 \mathrm{E}-03$

8.232E-04

1. $170 \mathrm{E}-01$

8.593E-01

1.793E-06

$6.275 E-08$

$1.444 \mathrm{E}-04$

$1.610 E-05$

1.397E-01

2. $073 \mathrm{E}-09$

$6.678 \mathrm{E}-04$

$2.856 \mathrm{E}-05$

$7.379 \mathrm{E}-04$

8. $238 \mathrm{E}-04$

$1.513 \mathrm{E}-02$ $.000 E+00$

$1.466 \mathrm{E}-06$

6.343E-01

$2.996 \mathrm{E}-06$

3.712E-07

$5.994 \mathrm{E}-05$

$9.545 \mathrm{E}-06$

$8.107 E-25$

$3.756 E-02$

4. $172 E-09$

2.698E-03

1. $287 E-03$

$.000 E+00$

$4.456 \mathrm{E}-03$

$5.945 \mathrm{E}-13$

$5.925 E-05$

$.000 E+00$

$.000 E+00$

1.900E-08

1.169E-05

8. $100 E-08$

$1.370 E-04$

$3.380 E-05$

$1.212 \mathrm{E}-04$

$1.355 \mathrm{E}-07$

$1.576 E-38$

$5.386 \mathrm{E}-08$

4. $644 E-10$

1. $052 E-07$ 
SHIR328I - (Page 29 of 37)

RUNDATE : $03 / 15 / 96$

Post-1970 Nontransuranic Waste Burial Ground Areas Decayed Curie content through DECEMBER 31, 1995.

\begin{tabular}{llllll} 
BURIAL GROUND FACILITIES & & $\begin{array}{c}\text { TOTAL } \\
\text { (CURIES) }\end{array}$ & $\begin{array}{c}\text { SR-90 } \\
\text { (CURIES) }\end{array}$ & $\begin{array}{c}\text { RU-106 } \\
\text { (CURIES) }\end{array}$ & $\begin{array}{c}\text { CS-137 } \\
\text { (CURIES) }\end{array}$ \\
\hline
\end{tabular}
CENTRAL WASTE COMPLEX 2403WC BUILDING LOW-LEVEL NONIMDUSTRIAL-OFFSITE

LON-LEVEL NONINDUSIRIAL

$5.492 E+01 \quad 2.156 E+01 \quad 4.356 E-03$

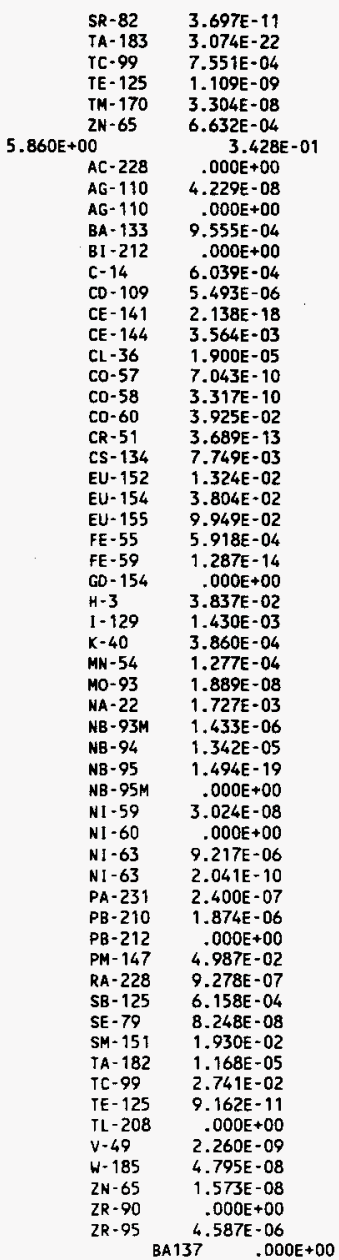




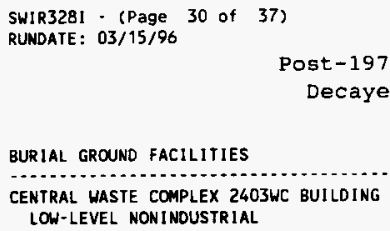

LOW-LEVEL MONIMOUSTRIAL (ASSAYED FROM TRANSURANIC)

CENTRAL HASTE COMPLEX 2403WD BUILDING LOW-LEVEL NONINDUSTRIAL-OFFSITE

LOH-LEVEL NON ] MDUSTRIAL

ALKaL IME METAL WASTE STORAGE UNIT 1 LOW-LEVEL NONINDUSTRIAL

ALKALINE METAL WASTE STORAGE UNIT 2 LOW-LEVEL NONINDUSTRIAL
$4.936 E-04 \quad 1.108 E-04$
3.110E-07
$1.196 \mathrm{E}-04$

$\begin{array}{ll}\text { C. }-14 & 4.388 E-06 \\ \text { CO-58 } & 5.734 E-13 \\ \text { CO-60 } & 1.643 E-04 \\ \text { CS137 } & 4.084 E-06 \\ \text { EU152 } & 3.479 E-06 \\ \text { EU154 } & 2.600 E-05 \\ \text { EU155 } & 2.009 E-05 \\ \text { FE }-59 & 2.039 E-16 \\ \text { MN-54 } & 8.451 E-07 \\ & .000 E+00\end{array}$

$1.272 E+04 \quad 1.231 E-01 \quad 1.168 E-01 \quad 1.317 E-01$ $\mathrm{BE}-10$

$1.272 E+04$

BI $-212 \quad .000 E+00$

C. $14 \quad 5.059 E-01$

CO-60 $2.080 E+03$

EU-154 4.058E-06

FE-55 7.132E+02

$\mathrm{H}-3 \quad 3.428 \mathrm{E}-01$

1-129 $9.010 E-06$

MN-54 4.478E-01

MO-93 $3.949 E-02$

NB-94 2.610E-01

N1-59 8.530E+01

N1 $-63 \quad 9.837 \mathrm{~F}+03$

PB-212 . O00E +00

SE-79 8.250E-08

SM-151 5.606E-06

TC-99 $1.070 \mathrm{E}-02$

ZR-93 1.140E-05

2.375E+03

C. 14

CE -144

$9.366 E-04$

2. $253 \mathrm{E}+03$

EU-152 7.234E-03

EU-154 1.818E-01

EU-155 $1.464 \mathrm{E}-03$

$\mathrm{H}-3 \quad 1.229 \mathrm{E}+0 \mathrm{O}$

$1.129 \quad 1.641 E-03$

K-40 3.600E-05

MN-54 1.105E-10

NB-93M 1:920E-04

MB-94 4.100E-06

NI $-59 \quad 1.092 E+00$

NI $-60 \quad .000 \mathrm{E}+00$

N $-63 \quad 1.182 E+02$

PM-147 1.327E-02

SE-79 1.865E-05

SM-151 1.302E-02

$\mathrm{SN}-123 \quad .000 \mathrm{E}+00$

IC $-99 \quad 6.243 \mathrm{E}-01$

ZN-65 2.564E-11

$\begin{array}{ccccc}1.375 E-04 & 8.153 E-07 & 2.629 E-09 & \begin{array}{r}6.967 E-05 \\ B A-133 \\ \text { NA- } 22\end{array} & \begin{array}{l}.000 E+00 \\ .000 E+00 \\ .000 E+00\end{array} \\ & & & & \\ 1.077 E-01 & 1.005 E-04 & 4.095 E-07 & 4.943 E-02 & 1.131 E-02 \\ B A-133 & 1.087 E-03\end{array}$


SWIR3281 - (Page 31 of 37 )
RUNDATE: $03 / 15 / 96$

Post-1970 Nontransuranic Waste Burial Ground Areas

Decayed Curie Content through DECEMBER 31, 1995.

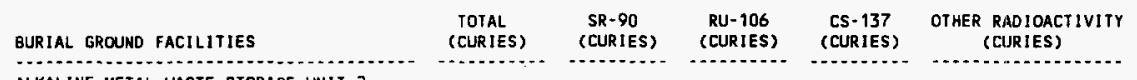

ALKALINE METAL WASTE STORAGE UNIT 2

LOW-LEVEL NONIMDUSTRIAL

ALKALINE METAL WASTE STORAGE UNIT 3

LOW-LEVEL NONINDUSTRIAL

\begin{tabular}{|c|c|c|c|c|}
\hline & & & $N A-22$ & $1.022 E-02$ \\
\hline $2.233 E-03$ & $4.088 E-06$ & $1.423 E-08$ & $\begin{array}{r}3.493 E-04 \\
\quad 8 A-133 \\
\text { NA- } 22\end{array}$ & $\begin{array}{l}1.544 E-03 \\
1.544 E-03 \\
.000 E+00\end{array}$ \\
\hline $6.167 \mathrm{E}-02$ & $4.262 E-06$ & $2.232 E-08$ & $\frac{1.932 E-02}{\text { NA }-22}$ & $\begin{array}{l}2.406 E-02 \\
2.406 E-02\end{array}$ \\
\hline $1.206 \mathrm{E}-04$ & $5.462 E-06$ & $4.491 E-12$ & $\begin{array}{l}4.984 E-06 \\
T C-99\end{array}$ & $1.000 E-04$ \\
\hline
\end{tabular}

FLAMMABLE STORAGE MODULE 1

LOW-LEVEL NOWIMDUSTRIAL

2.980E-03

2.686E-04 1.045E-06

2.898E-04

$1.767 \mathrm{E}-03$

C. $14 \quad 9.415 E-04$

CE-144 4.595E-06

CO-58 4.219E-10

CO-60 3.817E-04

CR-51 2.994E-21

FE-59 1.429E-21

MN-54 3.824E-05

NA-22 $3.921 E-04$

NB-95 8.943E-19

SC $-47 \quad .000 E+00$

IA- $182 \quad 5.989 E-06$

TA $-183 \quad .000 E+00$

FLAMMABLE STORAGE MODULE 2

LOW-LEVEL NONINDUSTRIAL-OFFSITE

$1.193 E-04 \quad 9.309 E-06 \quad 5.617 E-09 \quad 5.113 E-09$

2.752E-06

LOW-LEVEL NONINDUSTRIAL

4.835E-04 1.104E-04 3.471E-07 8.397E-05

09

$1.007 E-04$

C-14 $4.300 E-05$

$\mathrm{H}-3 \quad 5.758 \mathrm{E}-05$

K-40 7.850E-08

MN-54 $3.277 E-10$

C-14 $1.322 \mathrm{E}-14$

CE-144 5.081E-07

CS-134 1.786E-07

EU-152 2.561E-05

EU- $154 \quad 4.317 E-05$

EU-155 5.282E-06

$\mathrm{H}-3 \quad 7.099 \mathrm{E}-06$

$1.129 \quad 1.097 \mathrm{E}-07$

NB-94 7.419E-15

PM- 147 1.306E-05

SE-79 4. $900 \mathrm{E}-11$

TA- $182 \quad 4.842 E-11$

TC- $99 \quad 3.017 E-06$

FLAMMABLE STORAGE MODULE 3

LOW-LEVEL NONINDUSTRIAL-OFFSITE

$\begin{array}{llll}7.383 E-03 \quad 1.739 E-04 & 7.682 E-05 \quad 1.028 E-04\end{array}$

BE -7

6.030E-03

C-14 1.035E-05

CA-45 8.615E-05

CD $-109 \quad 6.743 \mathrm{E}-07$

CO-60 6.881E-05

FE-55 3.719E-07

FE-59 2.914E-07

$\mathrm{H}-3 \quad 5.850 \mathrm{E}-03$

NI- 63 5.297E-07

P-32 $1.446 E-13$

PM- $147 \quad 3.693 \mathrm{E}-07$

PO- $210 \quad 1.448 E-07$ 


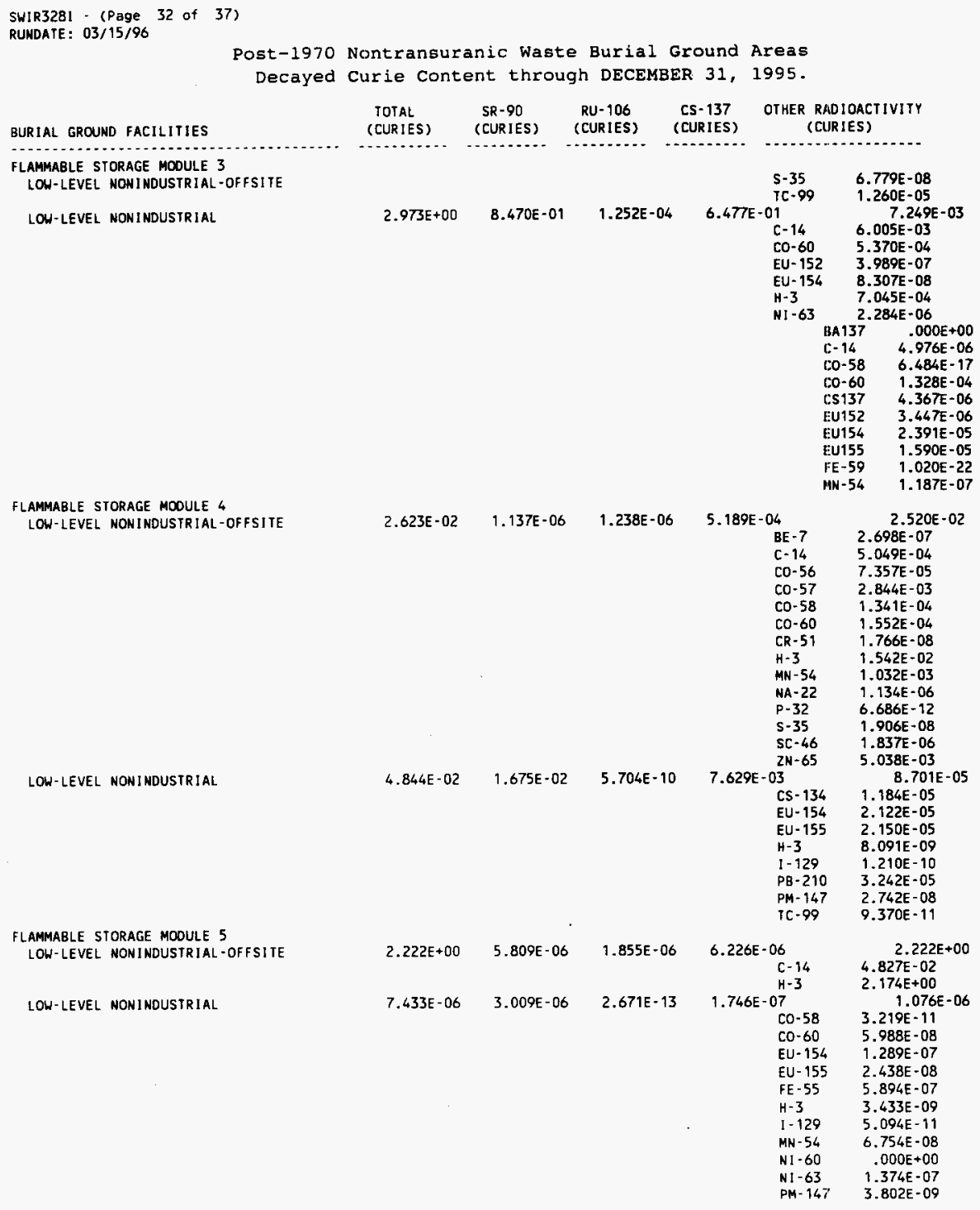


SWIR3281 - (Page 33 of 37)

RUNDATE : $03 / 15 / 96$

Post-1970 Nontransuranic Waste Burial Ground Areas

Decayed Curie Content through DECEMBER 31, 1995.

BURIAL GROUND FACILITIES

FLAMMABLE STORAGE MODULE 5

LOW-LEVEL NONINDUSTRIAL

flamMABLE STORAGE MODULE 6

LOW-LEVEL MONINDUSTRIAL-OFFSI TE

LOW-LEVEL NOW I MOUSTRIAL

FLAMMABLE STORAGE MOOULE 7

LOW-LEVEL NONINDUSTRIAL-OFFSITE

LOW-LEVEL NONINDUSTRIAL

FLAMMABLE STORAGE MODULE 8 LOW-LEVEL NONINDUSTRIAL

Flammable stoRAGE MODULE 10 LOW-LEVEL NONINDUSIRIAL-OFFSITE

TOTAL SR-90 RU-106 CS-137 OTHER R.AOIOACIIVITY

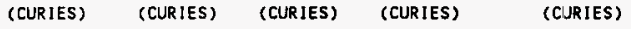

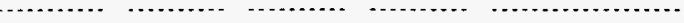

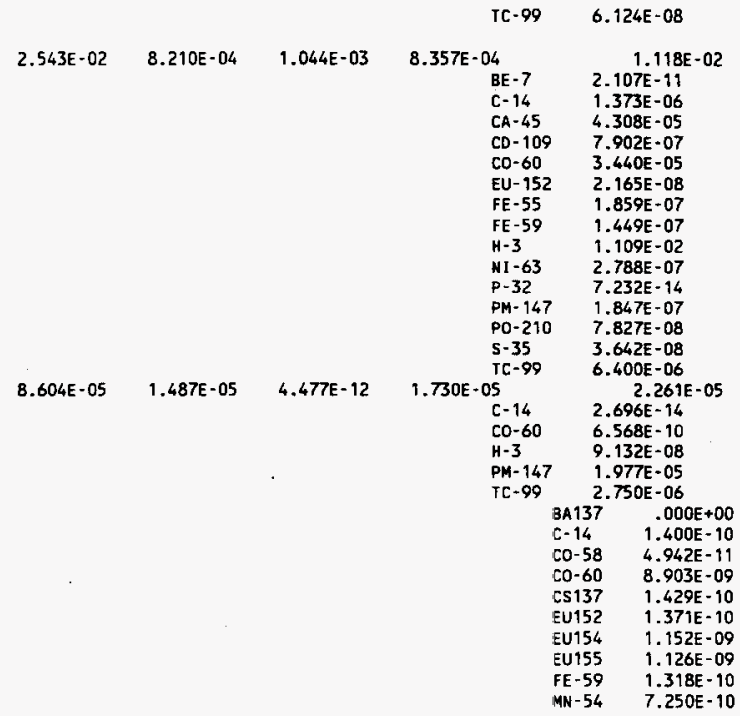

\begin{tabular}{|c|c|c|c|c|}
\hline $1.192 \mathrm{E}+00$ & $4.696 E-06$ & $1.499 E-06$ & $\begin{array}{r}5.032 E-06 \\
C-14 \\
H-3\end{array}$ & $\begin{array}{l}1.192 \mathrm{E}+00 \\
7.090 \mathrm{E}-03 \\
1.185 \mathrm{E}+00\end{array}$ \\
\hline $4.201 E-03$ & $6.531 E-04$ & $7.390 E-0 ?$ & $\begin{aligned} 6.130 E-04 \\
C-14 \\
C E-144 \\
C O-60 \\
E U-154 \\
\text { EU }-155 \\
H-3 \\
I-129 \\
M N-54 \\
N I-60 \\
N I-63 \\
P M-147 \\
I C-99 \\
2 N-65\end{aligned}$ & $\begin{array}{l}1.630 E-03 \\
1.123 E-03 \\
4.106 E-06 \\
1.096 E-06 \\
6.112 E-09 \\
2.715 E-09 \\
4.322 E-04 \\
1.815 E-08 \\
3.766 E-10 \\
.000 E+00 \\
9.733 E-10 \\
6.832 E-05 \\
4.802 E-07 \\
1.400 E-10\end{array}$ \\
\hline $1.100 E-02$ & 8.096E-05 & $3.698 E-07$ & $\begin{array}{r}8.715 E-05 \\
C-14 \\
H-3\end{array}$ & $\begin{array}{l}\quad 1.063 \mathrm{E}-02 \\
5.959 \mathrm{E}-03 \\
4.675 \mathrm{E}-03\end{array}$ \\
\hline $8.242 \mathrm{E}-02$ & $1.108 E-04$ & $1.019 E-04$ & $\begin{aligned} 1.002 E-04 \\
c-14 \\
H-3\end{aligned}$ & $\begin{array}{l}\quad 8.094 \mathrm{E}-02 \\
1.780 \mathrm{E}-04 \\
8.020 \mathrm{E}-02\end{array}$ \\
\hline
\end{tabular}


SWIR328I - (Page 34 of 37 )
RUNDATE: $03 / 15 / 96$

Post-1970 Nontransuranic Waste Burial Ground Areas Decayed Curie Content through DECEMBER 31, 1995.

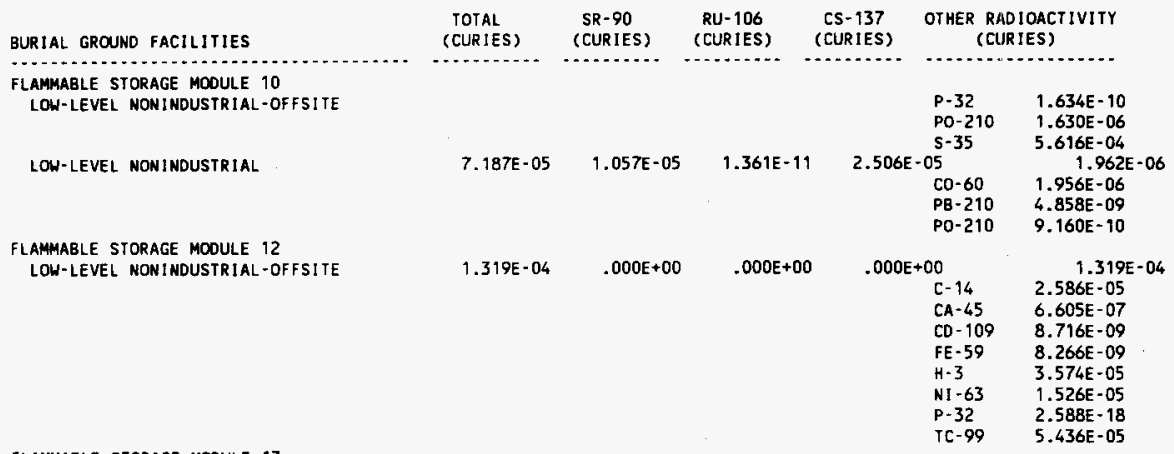

flammable storage mOOULE 13 LOW-LEVEL MONIMDUSTRIAL-OFFSITE

7.096E-02 $1.208 E-03 \quad 1.208 E-03 \quad 1.289$ E-03

BE $-7 \quad 1.419 E-12$

C- $14 \quad 2.279 \mathrm{E}-06$

CA-45 2.757E-06

CD-109 1.212E-08

CO-58 6.142E-13

CO-60 2.328E-06

EU-152 $1.939 E-07$

FE-55 1.190E-08

FE-59 $\quad 9.761 E-09$

$\mathrm{H}-3 \quad 5.346 \mathrm{E}-02$

MN-54 9.264E-10

NA-22 $\quad 2.933 E-07$

NI $-60 \quad .000 E+00$

NI-63 1.786E-09

P-32 4.914E-15

PM- $147 \quad 1.182 E-08$

PO-210 7.117E-10

S-35 $\quad 1.083 \mathrm{E}-07$

LOW-LEVEL NONINOUSIRIAL

3.322E-03 1.344E-03 5.269E-07 2.595E-04

IC $-99 \quad 2.500 \mathrm{E}-07$

C- $14 \quad 5.398 E-05$

CE-144 3.054E-08

CO-60 4.891E-06

EU-152 $1.625 E-05$

EU-154 2.710E-05

EU-155 9.334E-07

$\mathrm{H}-3 \quad 3.057 \mathrm{E}-06$

$\mathrm{I}-129 \quad 1.280 \mathrm{E}-12$

MN-54 2.261E-10

NA-22 $6.783 E-06$

NB-94 2.930E-15

N1-63 5.341E-10

PM- $147 \quad 1.814 \mathrm{E}-06$

SE $-79 \quad 1.900 E-11$

TC-99 1.318E-05

$2 \mathrm{~N}-65 \quad 5.279 \mathrm{E}-11$

$2 R-95 \quad 5.867 E-09$

FLAMMABLE STORAGE MOOULE 14

LOW-LEVEL NONINOUSIRIAL-OFFSIIE

$1.916 \mathrm{E}-02 \quad 5.602 \mathrm{E}-05 \quad 7.279 \mathrm{E}-06 \quad 1.833 \mathrm{E}-05$

$1.893 \mathrm{E}-02$

$\mathrm{BE}-?$ 
SWIR328I - (Page 35 of 37 )

RUNDATE: 03/15/96

Post-1970 Nontransuranic Waste Burial Ground Areas Decayed Curie Content through DECEMBER 31, 1995.

\begin{tabular}{|c|c|c|c|c|c|}
\hline BURIAL GROUND FACILITIES & $\begin{array}{l}\text { TOTAL } \\
\text { (CURIES) }\end{array}$ & $\begin{array}{l}\text { SR-90 } \\
\text { (CURIES) }\end{array}$ & $\begin{array}{l}\text { RU-106 } \\
\text { (CURIES) }\end{array}$ & $\begin{array}{l}\text { CS-137 } \\
\text { (CURIES) }\end{array}$ & $\begin{array}{l}\text { OTHER RADIOACTIVITY } \\
\text { (CURIES) }\end{array}$ \\
\hline$\ldots \ldots \ldots$ & $\ldots \ldots$ & $\ldots \ldots$ & $\ldots+\ldots$ & .......... & \\
\hline
\end{tabular}

FLAMMABLE STORAGE MOOULE 14

LOU-LEVEL MONINDUSTRIAL-OFFSITE

LOW-LEVEL NONINOUSTRIAL

FLAMMABLE STORAGE MOOULE 15

LOW-LEVEL NONINDUSTRIAL-OFFSITE

LOW-LEVEL NONINDUSTRIAL

FLAMMABLE STORAGE MODULE 16 LOW-LEVEL NONINDUSTRIAL

\begin{tabular}{|c|c|c|c|c|}
\hline & & - & $\begin{array}{l}C-14 \\
C A-45 \\
C D-109 \\
C O-60 \\
E U-152 \\
F E-5 S \\
F E-59 \\
H-3 \\
N I-63 \\
P-32 \\
P M-147 \\
P O-210 \\
S-35 \\
T C-99\end{array}$ & $\begin{array}{l}1.190 \mathrm{E}-06 \\
4.308 \mathrm{E}-05 \\
1.791 \mathrm{E}-07 \\
3.440 \mathrm{E}-05 \\
6.591 \mathrm{E}-08 \\
1.859 \mathrm{E}-07 \\
1.449 \mathrm{E}-07 \\
1.885 \mathrm{E}-02 \\
1.115 \mathrm{E}-06 \\
7.232 \mathrm{E}-14 \\
1.847 \mathrm{E}-07 \\
2.454 \mathrm{E}-10 \\
8.294 \mathrm{E}-11 \\
3.700 \mathrm{E}-06\end{array}$ \\
\hline $1.036 \mathrm{E}-02$ & $1.422 E-03$ & $5.220 E-05$ & 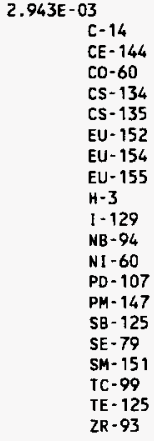 & $\begin{array}{l}1.575 E-03 \\
1.638 E-04 \\
8.230 E-05 \\
4.161 E-06 \\
5.209 E-07 \\
5.610 E-13 \\
1.098 E-07 \\
2.578 E-06 \\
1.500 E-06 \\
6.376 E-05 \\
9.283 E-07 \\
5.559 E-15 \\
.000 E+00 \\
1.130 E-13 \\
7.516 E-04 \\
9.252 E-09 \\
3.670 E-11 \\
4.822 E-04 \\
2.127 E-05 \\
1.476 E-13 \\
3.930 E-12\end{array}$ \\
\hline $5.249 E-03$ & 1.223E-05 & $9.473 E-08$ & $\begin{aligned} 1.079 E-07 \\
C-14 \\
C D-109 \\
H-3 \\
I-125 \\
K-40 \\
N I-63 \\
P-32 \\
P O-210 \\
R B-86 \\
S-35 \\
\text { SR }-85 \\
\text { IC }-99\end{aligned}$ & $\begin{array}{l}5.223 E-03 \\
1.300 E-07 \\
3.161 E-07 \\
5.182 E-03 \\
3.524 E-05 \\
4.400 E-08 \\
2.282 E-07 \\
3.786 E-22 \\
7.423 E-08 \\
9.167 E-12 \\
3.390 E-08 \\
2.031 E-06 \\
2.600 E-06\end{array}$ \\
\hline $3.306 \mathrm{E}-02$ & $2.247 \mathrm{E}-03$ & $6.107 E-06$ & $\begin{array}{c}1.428 E-02 \\
\text { C- } 14 \\
\text { CO- } 60 \\
H-3 \\
1-129 \\
\text { PM- } 147 \\
\text { TC }-99\end{array}$ & $\begin{array}{l}1.065 E-05 \\
1.130 E-08 \\
5.476 E-08 \\
1.303 E-06 \\
9.900 E-10 \\
8.434 E-06 \\
8.450 E-07\end{array}$ \\
\hline $\mathrm{OE}-02$ & $1.916 \mathrm{E}-03$ & $4.950 E-05$ & $\begin{array}{r}1.337 E-02 \\
\text { AG }-110 \\
C-16\end{array}$ & $\begin{array}{l}4.856 \mathrm{E}-03 \\
.000 \mathrm{E}+00 \\
7.206 \mathrm{E}-04\end{array}$ \\
\hline
\end{tabular}


WHC-EP-0125-8

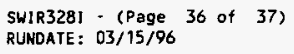

FLAMMABLE STORAGE MOOULE 17

LOW-LEVEL MONIMDUSTRIAL-OFFSITE

LOW-LEVEL NONINDUSTRIAL

$\begin{array}{llll}\text { (CURIES) } & \text { (CURIES) } & \text { (CURIES) } & \text { (CURIES) }\end{array}$
(CURIES)

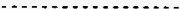

\begin{tabular}{|c|c|c|c|c|}
\hline & & & $\begin{array}{l}\text { CE }-144 \\
C O-60 \\
C S-134 \\
E U-152 \\
E U-154 \\
E U-155 \\
H-3 \\
I-129 \\
M N-54 \\
P M-147 \\
S B-125 \\
S M-151 \\
T C-99 \\
Z R-95\end{array}$ & $\begin{array}{l}6.327 \mathrm{E}-05 \\
3.514 \mathrm{E}-06 \\
1.242 \mathrm{E}-05 \\
1.912 \mathrm{E}-06 \\
1.972 \mathrm{E}-05 \\
6.327 \mathrm{E}-06 \\
1.924 \mathrm{E}-03 \\
3.866 \mathrm{E}-10 \\
5.296 \mathrm{E}-09 \\
1.051 \mathrm{E}-03 \\
2.147 \mathrm{E}-06 \\
1.051 \mathrm{E}-03 \\
3.002 \mathrm{E}-10 \\
1.605 \mathrm{E}-15\end{array}$ \\
\hline $5.048 E-03$ & 8.544E-06 & $3.432 E-08$ & $\begin{array}{c}5.259 E-08 \\
\mathrm{C}-14 \\
\mathrm{CO}-56 \\
\mathrm{CO}-57 \\
\mathrm{CO}-58 \\
\mathrm{FE}-59 \\
\mathrm{H}-3 \\
\mathrm{MN}-54 \\
\mathrm{P}-32 \\
\mathrm{PO}-210 \\
\mathrm{ZN}-65\end{array}$ & $\begin{array}{l}5.031 E-03 \\
1.025 E-03 \\
6.700 E-08 \\
5.395 E-06 \\
4.239 E-07 \\
1.489 E-09 \\
3.994 E-03 \\
4.360 E-07 \\
1.688 E-11 \\
8.537 E-07 \\
4.460 E-06\end{array}$ \\
\hline $2.793 E+00$ & $1.232 E-02$ & $1.565 E-05$ & 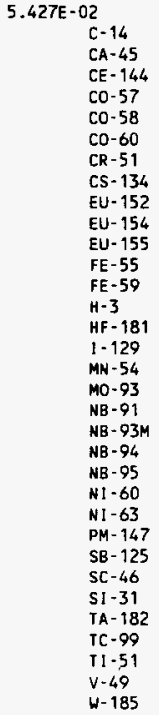 & $\begin{array}{l}2.662 E+00 \\
5.122 E-04 \\
1.830 E-05 \\
1.530 E-06 \\
1.647 E-03 \\
1.914 E-04 \\
1.726 E-01 \\
5.407 E-11 \\
5.847 E-05 \\
5.986 E-06 \\
1.201 E-05 \\
6.787 E-06 \\
1.361 E+00 \\
3.364 E-10 \\
5.611 E-01 \\
3.793 E-09 \\
1.000 E-02 \\
1.251 E-01 \\
2.702 E-01 \\
4.192 E-08 \\
5.617 E-02 \\
6.499 E-07 \\
1.551 E-11 \\
.000 E+00 \\
8.596 E-02 \\
1.350 E-09 \\
2.679 E-06 \\
1.804 E-06 \\
-000 E+00 \\
2.356 E-05 \\
1.307 E-02 \\
.000 E+00 \\
3.549 E-03 \\
8.465 E-04\end{array}$ \\
\hline
\end{tabular}




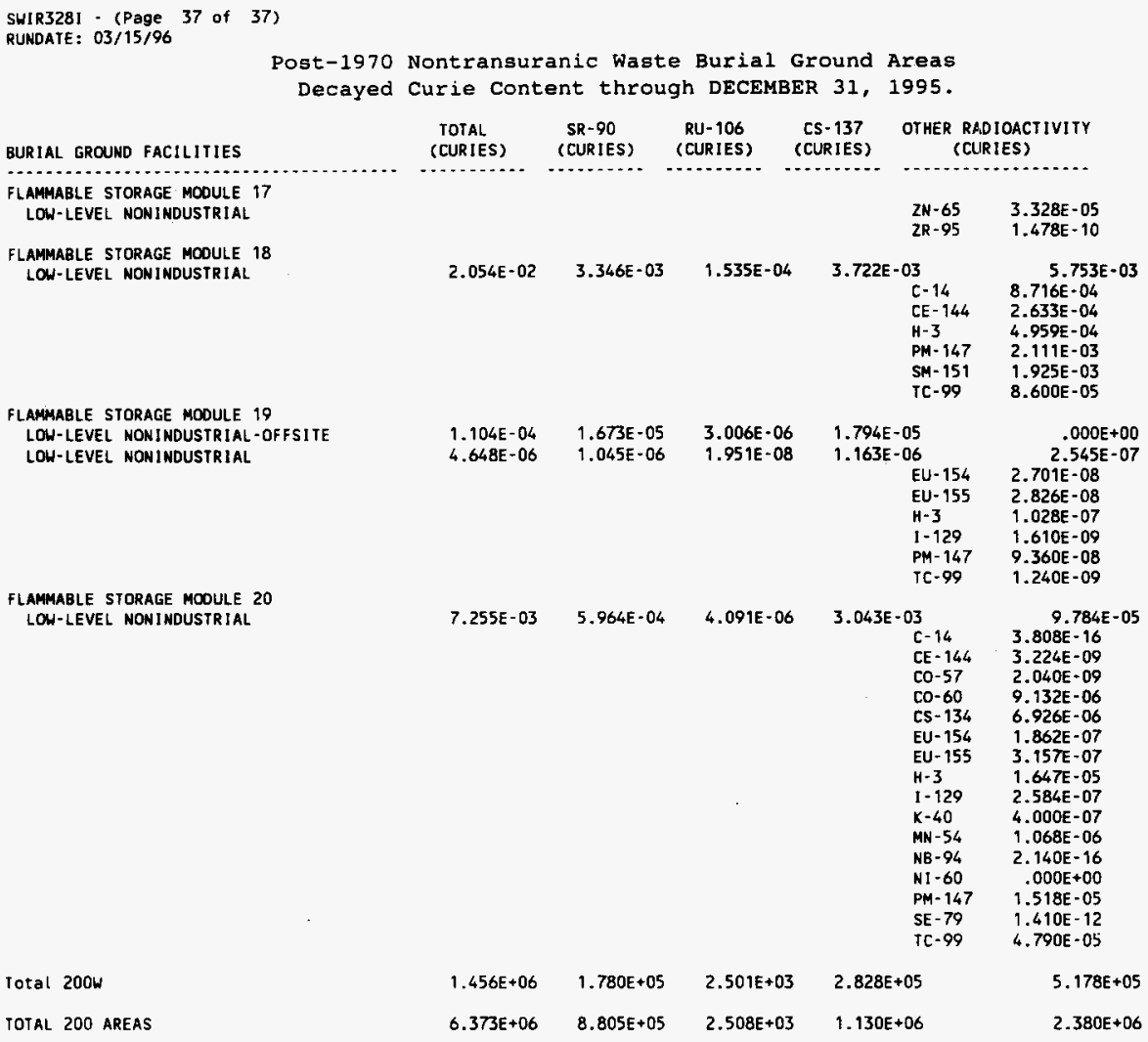




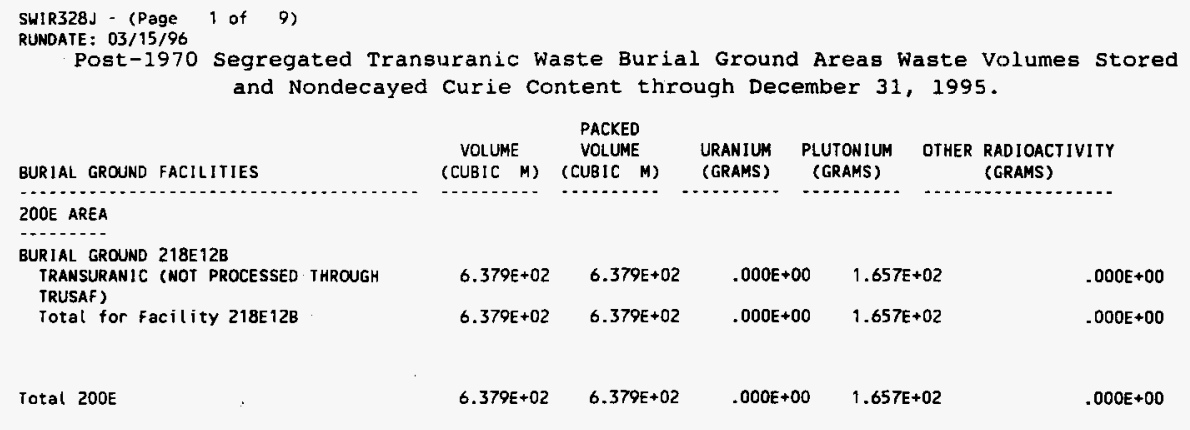




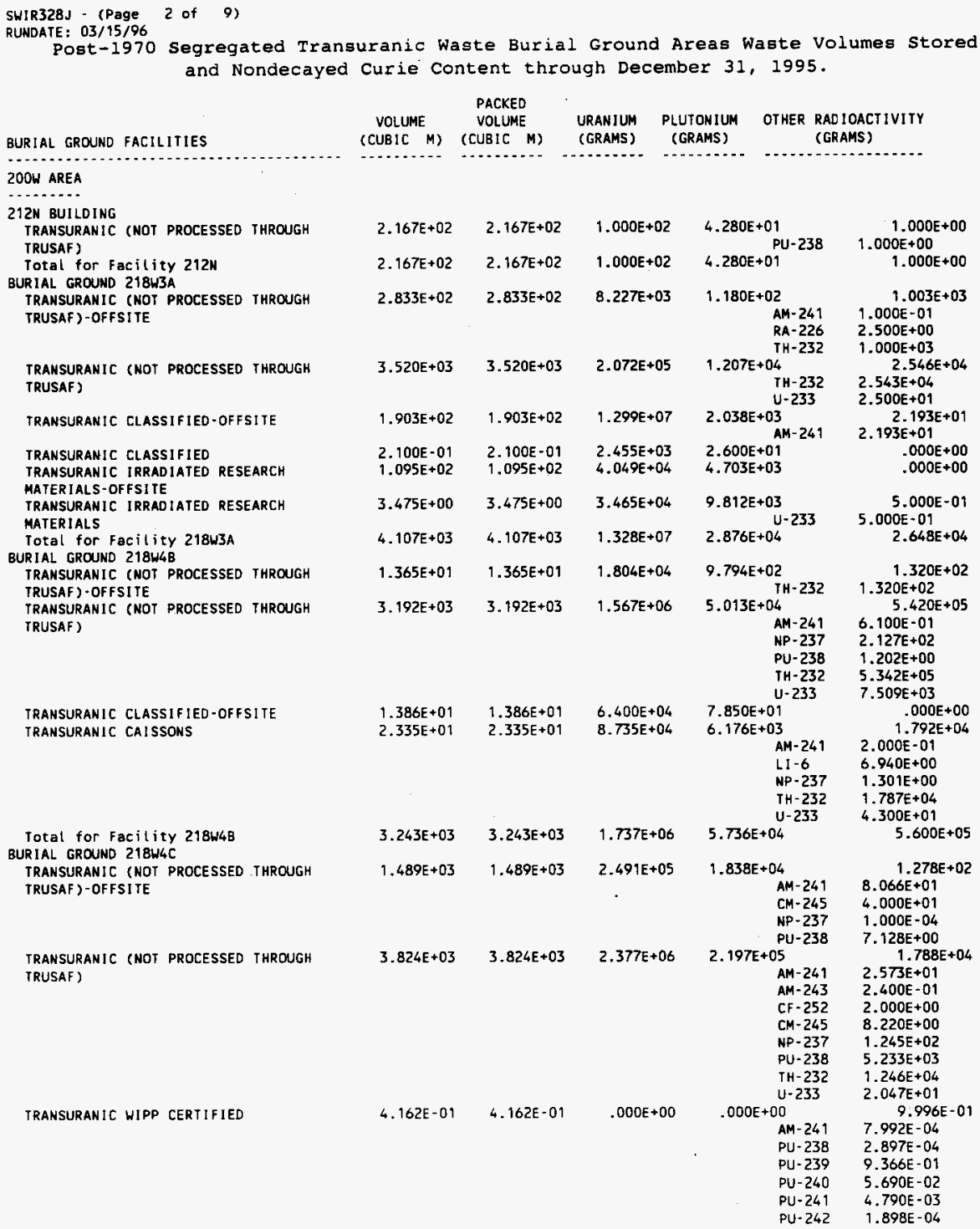


Segregated Transuranic Waste Burial Ground Areas Waste Volumes stored and Nondecayed Curie Content through December 31, 1995.

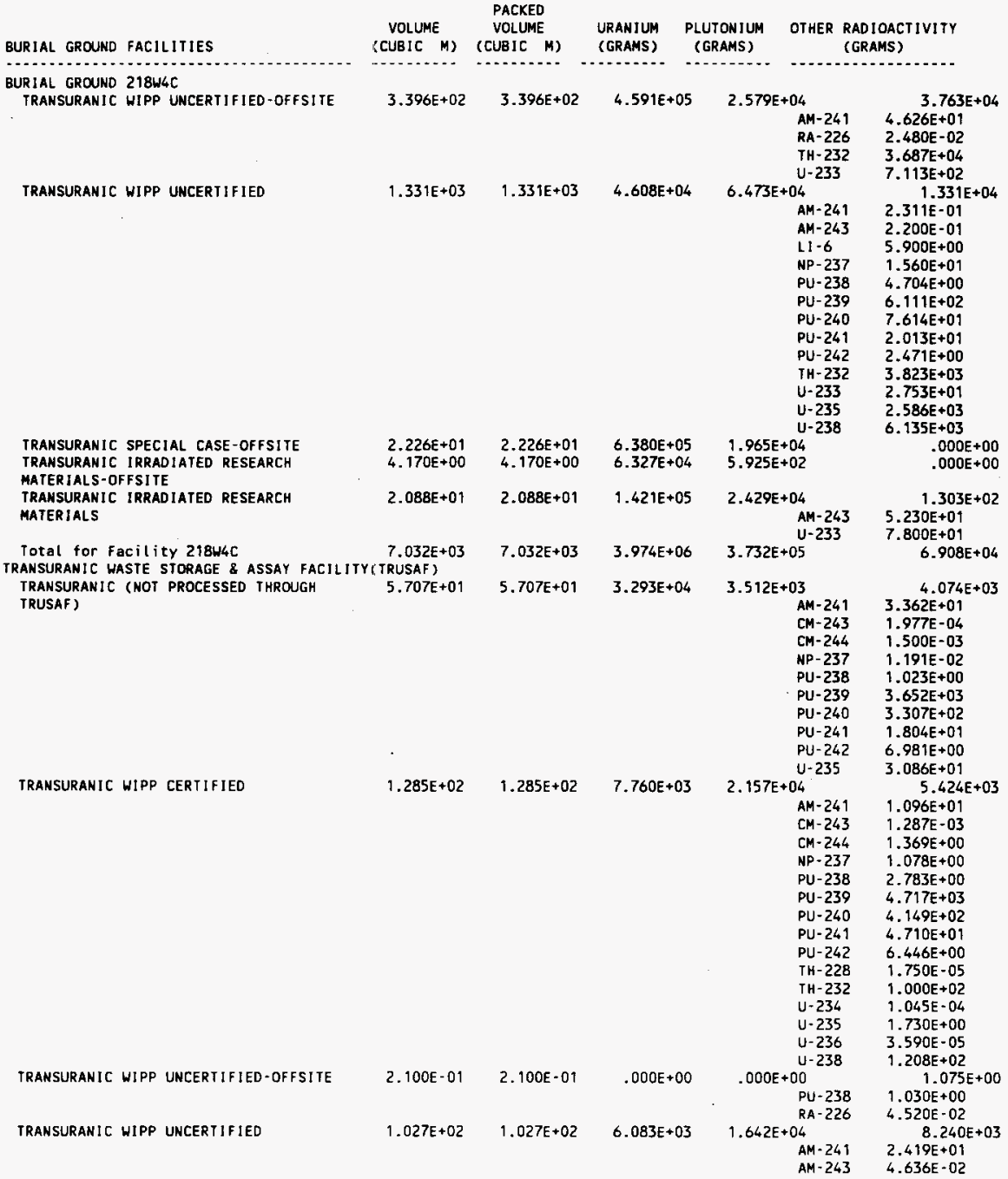




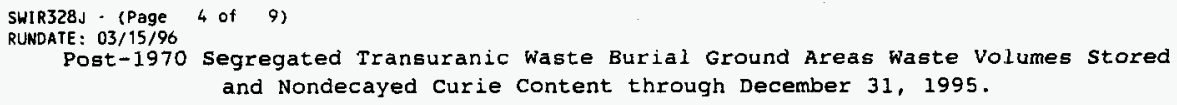

$.000 E+00$

$.000 E+00$

AM- 241

AM- 243

$\mathrm{CM}-243$

CM- 244

NP -237

PU-238

PU-239

$\mathrm{PU}-240$

PU-241

PU -242

TH -228

$\mathrm{U}-235$

U-238

IRANSURANIC HIPP UNCERTIFIED

$4.794 E+00 \quad 4.794 E+00 \quad .000 E+00 \quad .000 E+00$

$9.599 E-02$

$5.140 E-08$

$5.640 \mathrm{E}-05$

$9.915 \mathrm{E}-03$

$2.785 E+01$

$1.824 \mathrm{E}+00$

$1.500 \mathrm{E}-01$

$9.233 \mathrm{E}-03$

$1.230 \mathrm{E}+00$

$2.439 \mathrm{E}+02$

8.017E-01

4.950E-05

$7.160 E-05$

1. $568 \mathrm{E}-03$

2. $128 \mathrm{E}-01$

6. $778 \mathrm{E}-02$

$2.200 E+02$

$1.385 \mathrm{E}+01$

$1.472 \mathrm{E}+00$

$3.826 \mathrm{E}-01$

2.881E-07

$1.820 E+00$

$5.230 E+00$ $2.013 E+02$

2.035E-01

4. $870 E-03$

5. $441 E-04$

$1.443 \mathrm{E}-03$

2.081E-02

$7.886 \mathrm{E}-03$

$2.846 \mathrm{E}+01$

$1.790 \mathrm{E}+00$

$1.754 \mathrm{E}-01$

$8.338 \mathrm{E}-03$

4.014E-05

$6.730 E+01$

U. 235

$1.300 E+00$ 


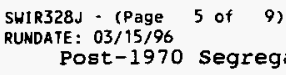

TRANSURANIC WIPP CERTIFIED

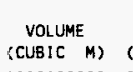

PACKEO

(CUBIC $M$ )

...........

$1.444 E+01$

Total for Facility 2402WB

CENTRAL WASTE COMPLEX 2402WC BUILDING TRANSURANIC (NOT PROCESSED THROUGH TRUSAF ?

IRANSURANIC WIPP CERTIFIED

TRANSURANIC WIPP UNCERTIFIED

Iotal for Facility $2402 W C$

CENTRAL WASTE COMPLEX 2402WD BUILDING IRANSURANIC (NOT PROCESSED THROUGH TRUSAF)

TRANSURANIC WIPP UNCERIIFIED

AM- 241

$3.248 E+01$

$3.248 E+01$

$6.288 \mathrm{E}+00$

$6.288 \mathrm{E}+00$

$1.444 E+01$

$.000 E+00$

$2.090 \mathrm{E}+02$

AM- 24

PU-238

PU-239

PU-240

PU-241
1. $680 \mathrm{E}+00$

1. $680 E+00$

$2.241 E+01$

$1.040 E+00$

DOOE +00

$.000 E+00$

1. $102 \mathrm{E}+00$

PU-242

2.197E+02

5.000E-01

AM- 24

PU -238

PU- 239

PU -240

PU-241

PU -242

$2.908 E+01$

$2.908 E+01$

$.000 E+00$

$5.686 \mathrm{E}+00$

$5.686 E+00$

$.000 E+00$

$2.040 E+01$

AM- 241

PU- 238

PU-239

PU-240

PU- 241

$\mathrm{PU}-242$

AM- 24

CM-244

PU -238

PU -239

PU-240

PU- 241

PU-242

$3.581 E+01$

$3.581 E+01$

2.082E-01

2. $082 E-01$

$.000 E+00$

$2.530 E+02$

$.000 E+00$

$.000 E+00$

AM- 241

PU- 238

PU-239

PU- 241

2. $100 E+00$

2. $100 E+00$

$.000 E+00$

2.676E+00

AM- 241

PU -238

PU- 239

PU-240

PU- 241

PU- 242

$2.308 E+00$

2. 308E +00

$.000 E+00$

$2.676 \mathrm{E}+00$

$1.456 \mathrm{E}+00$

$.000 E+00$

$1.000 E+00$

$1.456 E+00$
$1.020 E+02$ $4.763 E+02$

\section{$3.069 E+00$}

$2.519 E-03$

8.778E-04

$2.838 E+00$

2.122E-01

$1.462 E-02$

5.751E-04

$5.346 E+00$

3.817E-03

2.297E-03

$4.768 E+00$

5. $226 \mathrm{E}-01$

$4.526 \mathrm{E}-02$

4. $328 \mathrm{E}-03$ $.000 E+00$

$8.415 E+00$

3.372E-01

$2.696 \mathrm{E}-04$

$9.780 \mathrm{E}-05$

3. 159E-01

$1.921 \mathrm{E}-02$

$1.618 \mathrm{E}-03$

$6.410 E-05$

$$
4.058 E+01
$$

2. $225 \mathrm{E}-02$

2.829E-02

$3.626 E+01$

$3.693 E+00$

5.090E-01

$6.060 E-02$ $9.234 E+00$

$1.336 E-01$

7. BBOE-D9

2.563E-03

$8.482 E+00$

5.502E-01

$6.323 E-02$

$2.983 E-03$

$5.015 E+01$

7. 100E-04

2.000E-05

$1.000 \mathrm{E}-05$

2.000E-05

6. $600 \mathrm{E}-04$ $1.000 E+00$

9.000E-05

2. BO0E- 04

9.364E-01

5. $750 \mathrm{E}-02$

$5.600 E-03$

2.000E-04

$1.001 E+00$

.0OOE+OO TRANSURANIC (NOT PROCESSEO THROUGH TRUSAF ) 


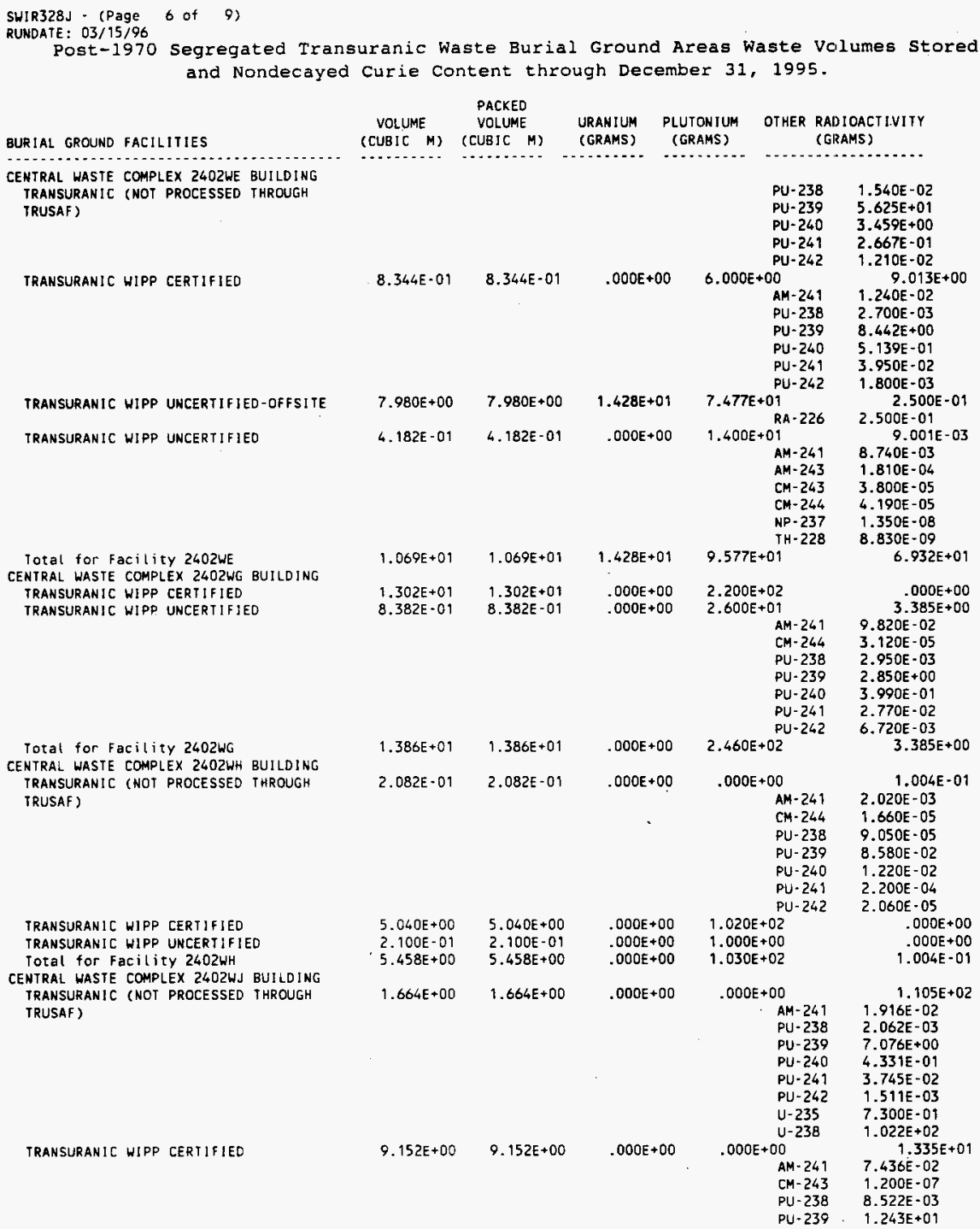




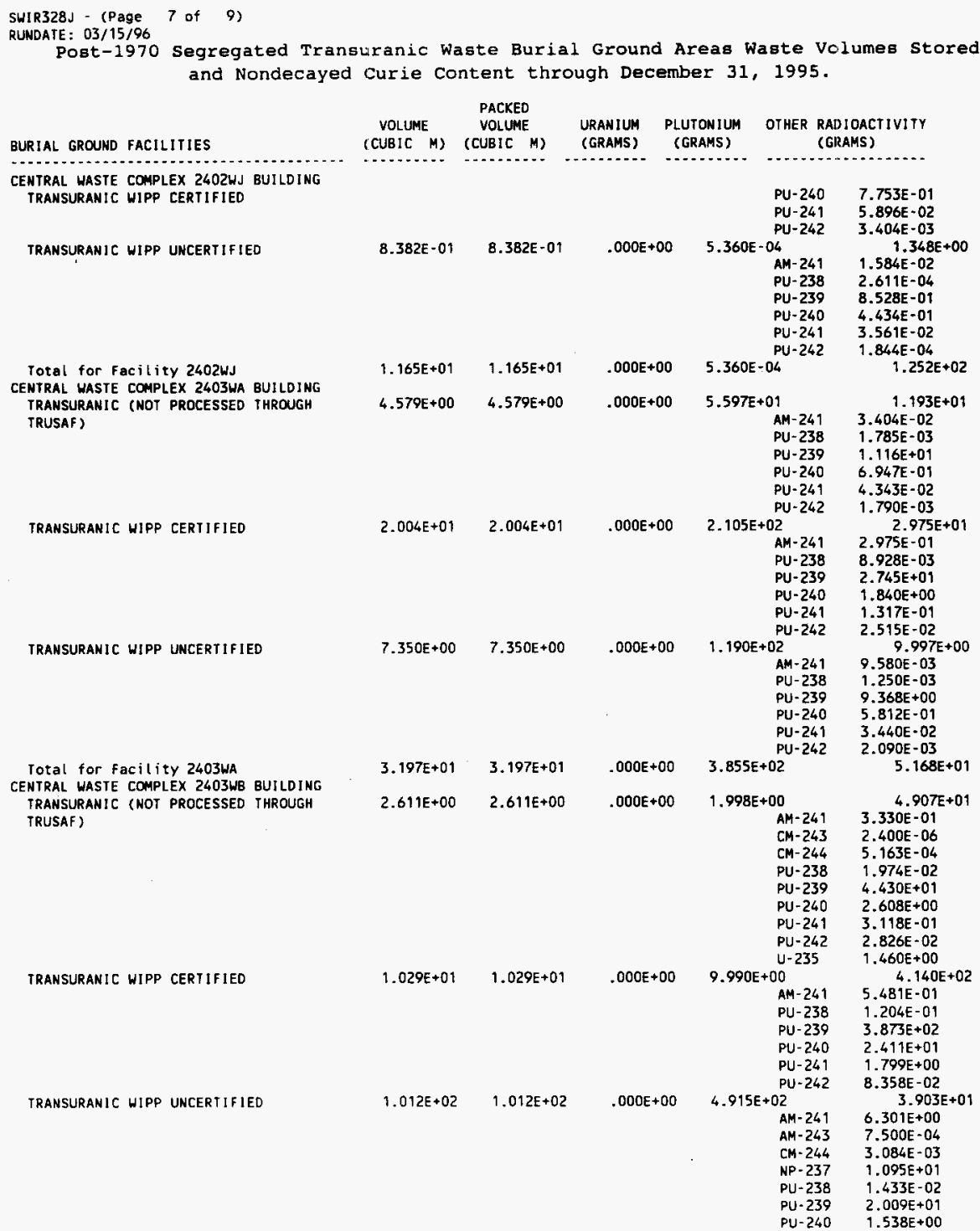


SHIR328J - (Page 8 of 9)

RUNDATE : $03 / 15 / 96$

Post-1970 segregated Transuranic Waste Burial Ground Areas Waste Volumes stored and Nondecayed Curie Content through December 31, 1995.

\begin{abstract}
BURIAL GROUND FACILITIES
CENTRAL WASTE COMPLEX 2403 WB BUILDING

TRANSURANIC WIPP UNCERTIFIED

Total for Facility $2403 \mathrm{WB}$

CENTRAL WASTE COMPLEX 2403WC BUILDING

TRANSURANIC (NOT PROCESSED THROUGH

TRUSAF)-OFFSITE

TRANSURANIC (NOT PROCESSED THROUGH

(RUSAF)
\end{abstract}

TRANSURANIC WIPP CERTIFIED

TRANSURANIC WIPP UNCERTIFIED

Total for Facility $2403 W C$

CENIRAL WASIE COMPLEX 2403 WO BUILDING TRANSURANIC (NOT PROCESSED THROUGH TRUSAF)

TRANSURANIC WIPP CERTIFIED

TRANSURANIC WIPP UNCERTIFIED

Total for Facility 240360

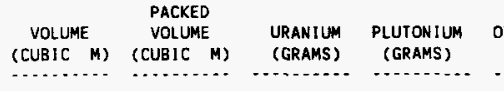

$$
1 .
$$

$1.141 \mathrm{E}+02$

1. $141 E+02$

$.000 E+00$

$5.035 \mathrm{E}+02$

PU- 241 (GRAMS)

2.082E-01

2.082E-01 1.616E+05

$3.060 E+01$

3. $060 E+09$

$.000 E+00$

2.898E+02

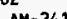

AM -243

CM- 243

CM- 244

NP -237

PU -238

PU-239

PU -240

PU-241

PU-242

$4.993 \mathrm{E}+0$

$4.993 E+01$

$9.250 \mathrm{E}+02$

4.442E+02

TH- 228

AM- 241

CM-243

NP -237

PU -238

PU-239

PU-240

$\mathrm{PU}-241$

PU-242

$1.383 \mathrm{E}+02$

$1.383 E+02$

$4.000 E+00$

9.957E+02

$\mathrm{TH}-228$

AM- 241

NP -237

PU-238

PU -239

PU- 240

PU-241

2. $190 \mathrm{E}+02$

2. $190 E+02$

$1.626 E+05$

$1.730 E+03$

PU- 242

$1.156 \mathrm{E}+00$

1. $154 \mathrm{E}+00$

$5.454 E+01$

$.000 E+00$

AM- 241

PU- 238

PU-239

PU-240

PU-241

PU- 242

$6.454 E+00$

$6.454 E+00$

$.000 E+00$

$.000 E+00$

AM- 241

PU- 238

PU-239

PU. 240

PU-241

PU-242

$6.246 E-01 \quad 6.246 E-01 \quad .000 E+00 \quad .000 E+00$

AM- 241

PU- 238

PU-239

PU- 240

PU. 241

$\mathrm{PU}-242$

$.000 E+00$
1.283E-01

3. 357 E- 04

$5.021 E+02$

4.000E-01

4. $000 \mathrm{E}-01$

$1.394 E+02$

4. $119 \mathrm{E}+00$

2. $770 \mathrm{E}-04$

$5.950 E+07$

8.608E-04

1. 087E-01

$1.141 \mathrm{E}-01$

$1.155 E+02$

$1.703 E+01$

$1.811 E+00$

$6.380 \mathrm{E}-01$

3.012E-09 $1.041 E+02$

7.718E-01

$7.853 \mathrm{E}-05$

8.223E-01

3.710E-02

$9.391 \mathrm{E}+01$

$7.349 E+00$

$1.121 E+00$

8.459E-02

$3.960 E-08$

4. $085 E+02$

$1.385 E+00$

2. $000 \mathrm{E}+00$

2. $047 \mathrm{E}-01$

$3.796 E+02$

2. $332 E+01$

$1.784 \mathrm{E}+00$

2. 106E- 01

$6.523 \mathrm{E}+02$

$7.008 E+00$

8. $100 E-03$

2.000E-03

$6.565 E+00$

3.997E-01

3.220E-02

$1.400 E-03$ $1.665 E+02$

7.053E-01

2. 299E-01

$1.538 E+02$

$1.099 E+01$

6. $148 E-01$

7.921E-02

$1.242 \mathrm{E}+02$

1. $727 \mathrm{E}-01$

3. 602 E-02

$1.163 E+02$

$7.081 E+00$

$5.424 \mathrm{E}-01$

$2.427 E-02$

$2.976 E+02$ 


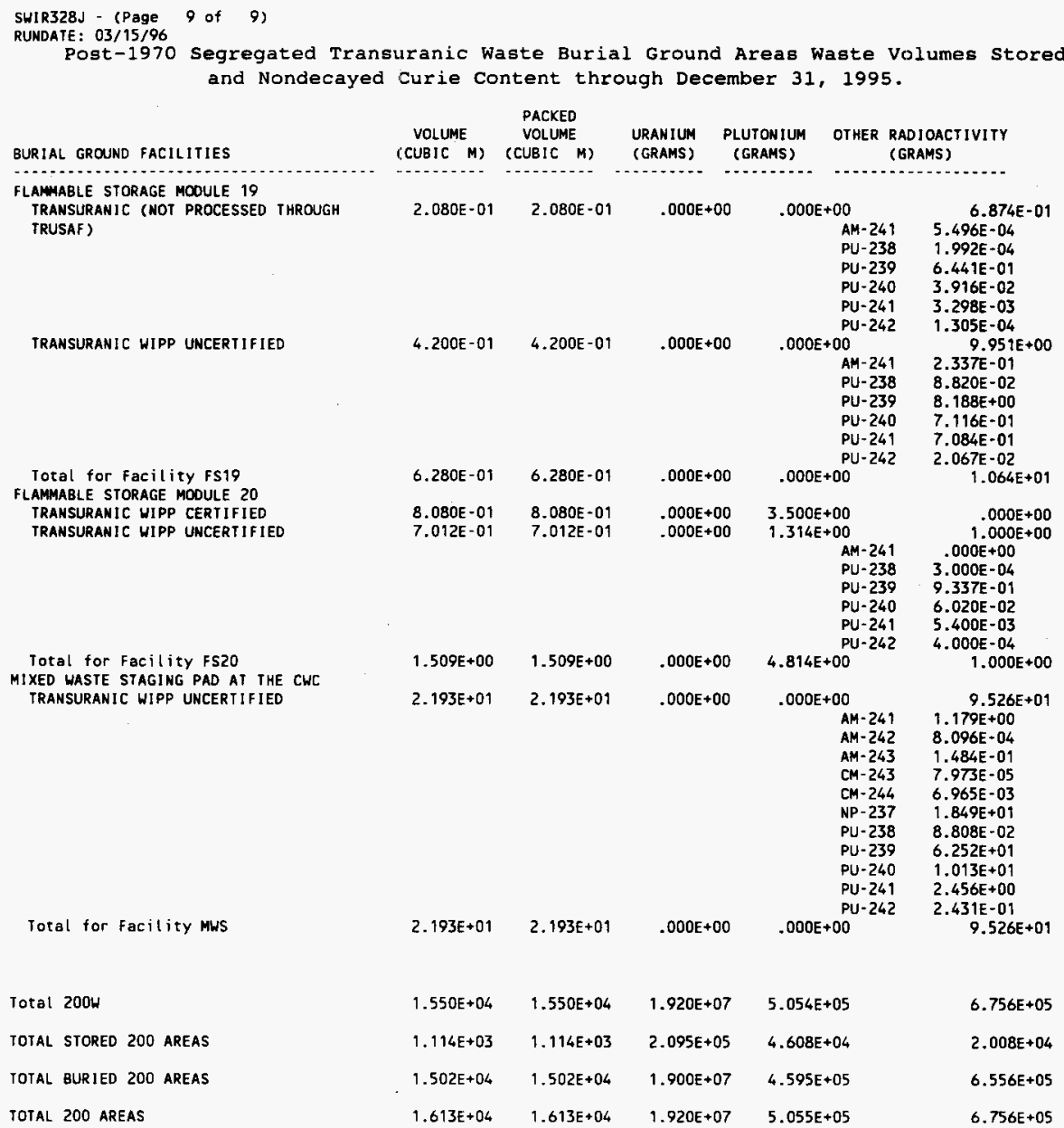


WHC-EP-0125-8

SWIR328K - (Page 1 of 8 )

RUNDATE: 03/15/96

Post-1970 Segregated Transuranic Waste Burial Ground Areas Waste Vo:Lumes Stored and Nondecayed Curie Content through December 31, 1995.

BURIAL GROUND FACILITIES

$\begin{array}{lllll}\text { TOTAL } & \text { RR-90 } 106 & \text { CS-137 OTHER RADIOACTIVITY }\end{array}$

ZOOE AREA

BURIAL GROUND 218E12B

TRANSURANIC (NOT PROCESSED THROUGH

TRUSAF )

(CURIES)

(CUR IES)

(CURIES)

(CUR!ES)

(CUIRIES)

Total for Facility 218E12B

$3.070 E+02$

6. $107 E+00$

$1.315 E+01$

$6.521 E+00$

CE-14

$3.070 E+02$

$6.107 E+00$

$1.315 E+01$

6.521E+00

TA- 182

$1.000 E-03$

OOOE -03

2.000E-03

Total $200 E$

$3.070 E+02$

6. $107 E+00$

$1.315 E+01$

$6.521 E+00$

2.000E-03 
WHC-EP-0125-8

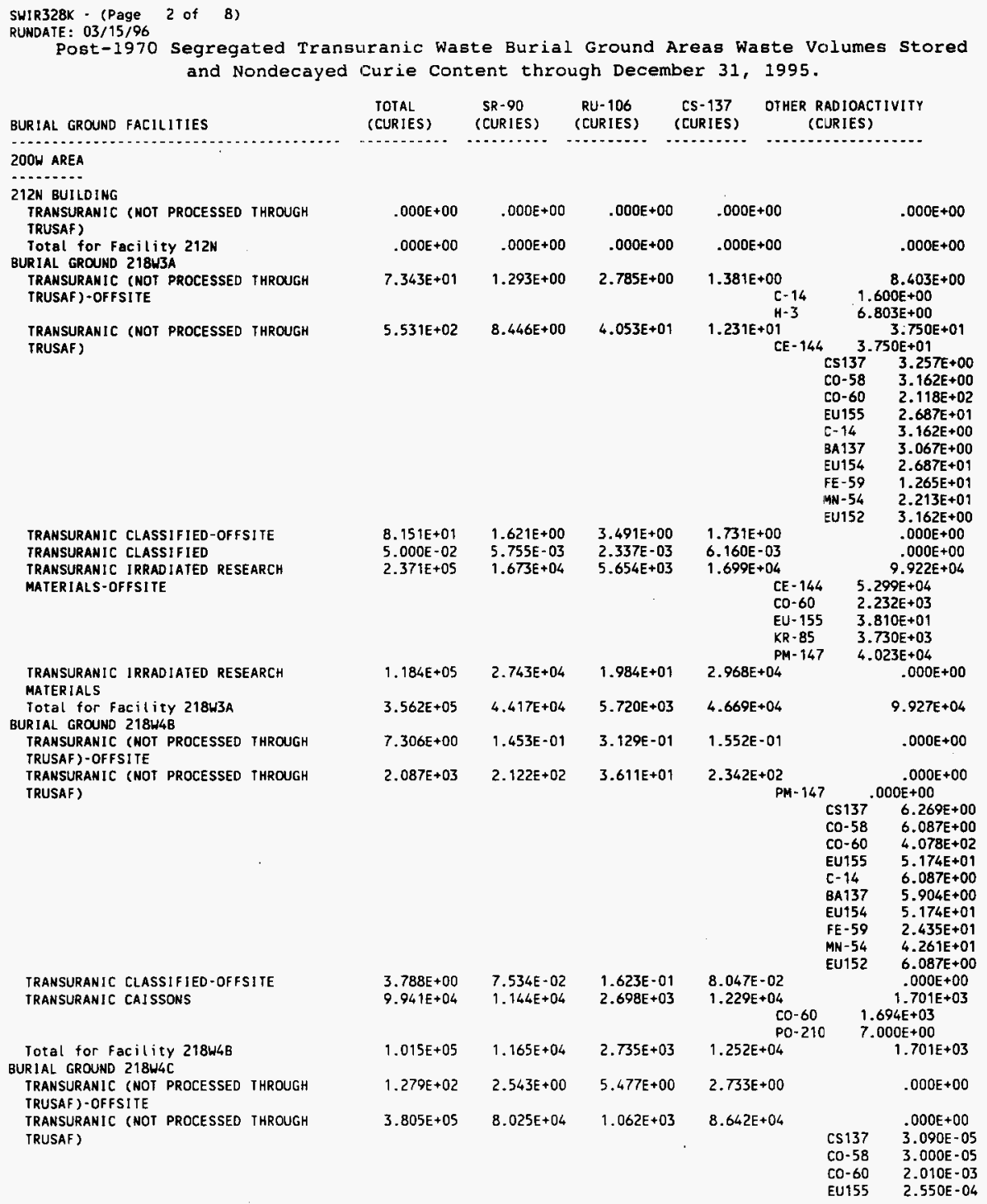




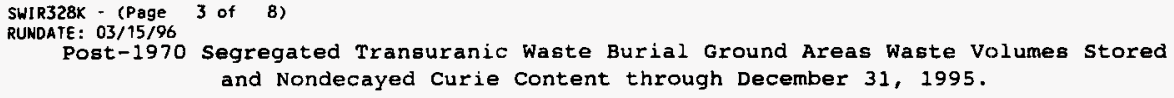

BURIAL GROUND FACILITIES
BURIAL GROUND 218W4C
TRANSURANIC (NOT PROCESSED THROUGH
TRUSAF)

TRANSURANIC WIPP CERTIFIED TRANSURANIC WIPP UNCERTIFIED-OFFSITE TRANSURANIC WIPP UNCERIIFIED

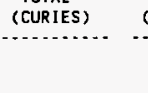

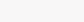

TRANSURANIC SPECIAL CASE-OFFSITE TRANSURANIC IRRADIATED RESEARCH MATERIALS-OFFSITE

TRANSURANIC IRRADIATED RESEARCH MATERIALS

$\begin{array}{llll}5.300 E+00 & 1.054 E-01 & 2.270 E-01 & 1.126 E-01 \\ 5.997 E+04 & 1.193 E+03 & 2.569 E+03 & 1.274 E+03 \\ & & & \\ 1.462 E+05 & 1.059 E+04 & 1.970 E+03 & 5.737 E+04\end{array}$

$1.462 \mathrm{E}+05$
$5.000 E-04$
$3.398 E+04$
$7.873 E+03$

9.700E-05

$6.758 E+02$

$1.249 \mathrm{E}+03$
$3.824 \mathrm{E}-06$
$1.455 \mathrm{E}+03$
$9.330 \mathrm{E}+01$

$1.970 E+03$
$1.043 E-04$
$7.218 E+02$

$1.978 \mathrm{E}+03$
C-14 3.000E-05

BA $137 \quad 2.910 E-05$

EU154 2.550E-04

FE $-59 \quad 1.200 E-04$

MN-54 2.100E-04

EU152 3.000E-05

$.000 E+00$

$.000 E+00$

$7.235 E+01$

$1.470 E+01$

$9.580 E+00$

$\begin{array}{ll}C S-134 & 9.580 E+00 \\ E U-154 & 2.284 E+01\end{array}$

KR-85 $2.480 E+01$

TL-208 4.320E-01

CS137 5.150E-06

CO-58 5.000E-06

CO-60 3.350E-04

EU155 4.250E-05

C- 14 5.000E-06

BA137 4.850E-06

EU154 4.250E-05

FE-59 2.000E-05

MN-54 3.500E-05

EU152 5.000E-06 $.000 E+00$ $.000 E+00$

$3.675 E+03$

$$
\begin{array}{ll}
\text { CE }-144 & 1.282 E+03 \\
C O-58 & 5.452 E+01 \\
C O-60 & 4.478 E+02 \\
\text { CR-51 } & 1.116 E+02 \\
\text { CS-134 } & 8.591 E+02 \\
E U-152 & 1.880 E+01 \\
E U-154 & 3.026 E+02 \\
\text { FE }-59 & 5.713 E+02 \\
M N-54 & 2.603 E+01 \\
S B-125 & 8.350 E-01
\end{array}
$$

CS137 $1.545 E+00$

CO-58 $1.500 E+00$

CO- 60 1.005E+02

EU155 1.275E+01

C- $14 \quad 1.500 E+00$

BA $137 \quad 1.455 \mathrm{E}+00$

EU154 $1.275 \mathrm{E}+01$

FE $-59 \quad 6.000 E+00$

MN-54 1.050E+01

EU152 $1.500 \mathrm{E}+00$

Iotal for Facility $218 \mathrm{WC}$

$6.287 E+05$

$9.396 \mathrm{E}+04$

7. $156 \mathrm{E}+03$

$1.478 E+05$

$5.480 E+01$

2. 135E-02

$3.436 E+00$

3. $747 \mathrm{E}+03$

IRANSURANIC WASTE STORAGE \& ASSAY FACIL
TRANSURANIC (NOT PROCESSED THROUGH

$1.171 \mathrm{E}+02$

TRUSAF )

$1.171 \mathrm{E}+02$

$$
\begin{aligned}
& +00 \\
& \text { CO }-60 \\
& \text { CS }-13 \\
& \text { EU }-15 \\
& K-40 \\
& S B-125
\end{aligned}
$$

$8.484 E-04$

4.573E-06

2.215E-04

5.105E-04

1. $120 \mathrm{E}-06$

1.107E-04

CS $137 \quad 7.210 E-06$

CO-58 7.000E-06

CO-60 4.690E-04

EU155 $5.950 E-05$ 
WHC-EP-0125-8

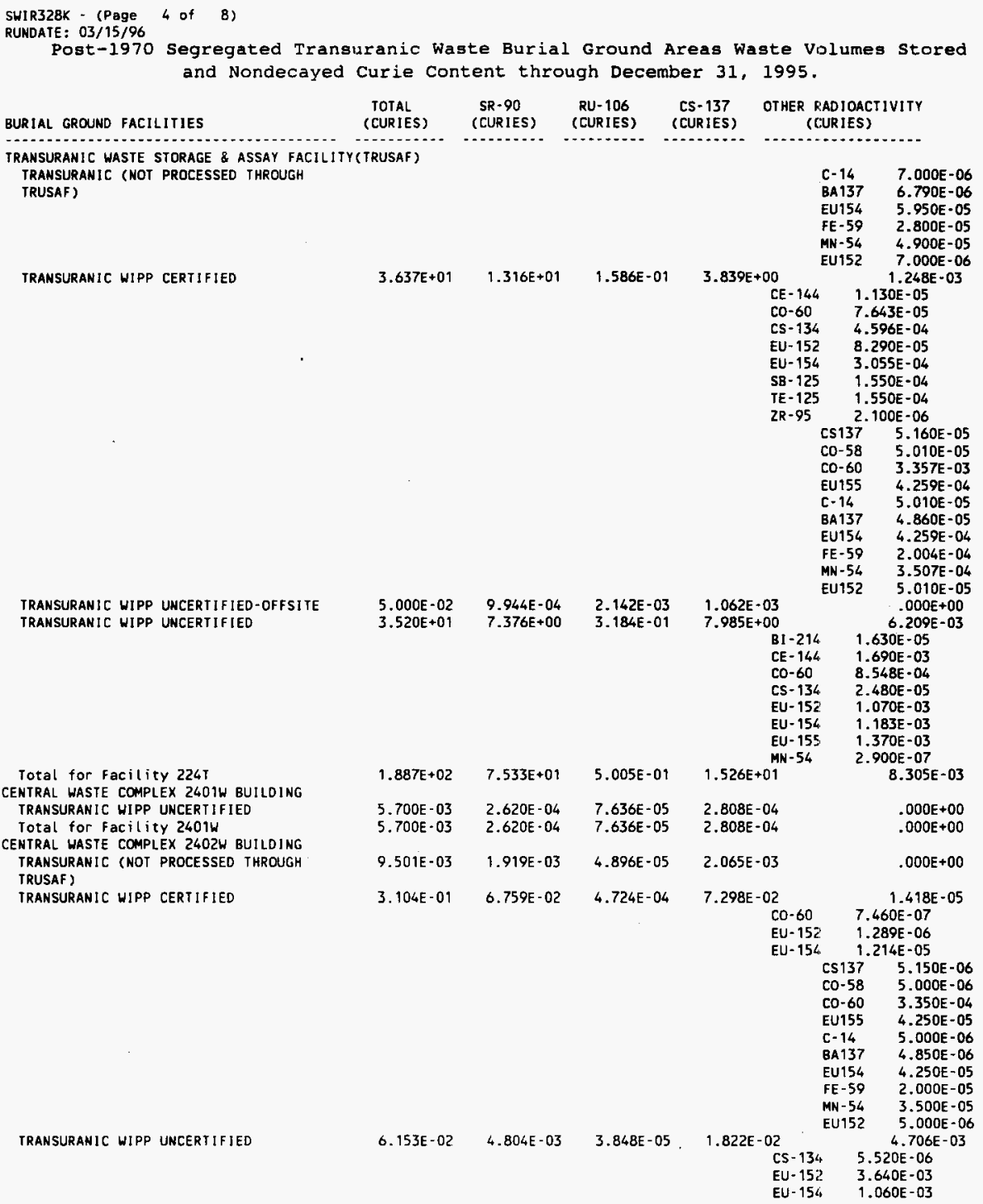


WHC-EP-0125-8

SWIR328K - (Page 5 of 8 )

RUNDATE: $03 / 15 / 96$

Post-1970 Segregated Transuranic Waste Burial Ground Areas Waste Volumes Stored and Nondecayed Curie Content through December 31, 1995.

\begin{tabular}{|c|c|c|c|c|c|}
\hline BURIAL GROUND FACILITIES & $\begin{array}{l}\text { TOTAL } \\
\text { (CURIES) }\end{array}$ & $\begin{array}{l}\text { SR-90 } \\
\text { (CUR!ES) }\end{array}$ & $\begin{array}{c}\text { RU- } 106 \\
\text { (CURIES) }\end{array}$ & $\begin{array}{c}\text { CS }-137 \\
\text { (CURIES) }\end{array}$ & $\begin{array}{l}\text { OTHER RMDIOACTIVITY } \\
\text { (CURIES) }\end{array}$ \\
\hline 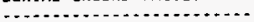 & $-\ldots \ldots . .$. & $\ldots \ldots \ldots$ & 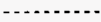 & $\cdots-\cdots \cdots$ & $\cdots$ \\
\hline
\end{tabular}

CENTRAL WASTE COMPLEX 2402W BUILDING

TRANSURANIC WIPP UNCERTIF IED

Total for Facility $2402 W$

CENTRAL HASTE COMPLEX 2402WB BUILDING

TRANSURANIC (NOT PROCESSED THROUGH

TRUSAF )

TRANSURANIC WIPP CERTIFIED

TRANSURANIC WIPP UNCERTIFIEO

Total for Facility 2402WB

CENTRAL WASTE COMPLEX 2402WC BUILOING IRANSURANIC (NOT PROCESSED THROUGH

TRUSAF)

TRANSURANIC UIPP CERTIFIED

TRANSURANIC WIPP UNCERTIFIED

Total for Facility $2402 W C$

CENTRAL WASTE COMPLEX 2402WD BUILDING

TRANSURANIC (NOI PROCESSED THROUGH

TRUSAF)

TRANSURANIC WIPP UNCERTIFIED

Total for Facility 240210

CENIRAL WASTE COMPLEX 2402WE BUILDING IRANSURANIC (NOT PROCESSED THROUGH TRUSAF ?

TRANSURANIC WIPP CERTIFIED

TRANSURANIC WIPP UNCERTIFIED-OFFSITE

TRANSURANIC WIPP UNCERTIFIED

Total for Facility 2402WE

CENTRAL WASTE COMPLEX 2402WG BUILDING

TRANSURANIC WIPP CERTIFIED

IRANSURANIC WIPP UNCERTIFIED

Total for Facility 2402WG

CENTRAL WASTE CONPLEX 2402WH BUILDING IRANSURANIC (NOT PROCESSED THROUGH

TRUSAF )

TRANSURANIC WIPP CERTIFIED

TRANSURANIC WIPP UNCERTIFIED

Total for Facility $2402 W H$

CENTRAL WASTE COMPLEX $2402 W J$ BUILDING TRANSURANIC (NOT PROCESSED THROUGH TRUSAF )

TRANSURAN IC WIPP CERTIFIED

\begin{tabular}{|c|c|c|c|}
\hline $3.814 E-01$ & $7.431 E-02$ & $5.598 E-04$ & $9.327 \mathrm{E}-02$ \\
\hline $1.068 E+00$ & $1.373 E-01$ & $3.171 E-02$ & $1.473 \mathrm{E}-01$ \\
\hline $\begin{array}{l}1.117 E+00 \\
4.580 E-02 \\
2.230 E+00\end{array}$ & $\begin{array}{l}1.057 \mathrm{E}-01 \\
2.134 \mathrm{E}-03 \\
2.451 \mathrm{E}-01\end{array}$ & $\begin{array}{l}3.279 E-02 \\
5.914 E-04 \\
6.509 E-02\end{array}$ & $\begin{array}{l}1.137 \mathrm{E}-01 \\
2.287 \mathrm{E}-03 \\
2.632 \mathrm{E}-01\end{array}$ \\
\hline $2.500 E-03$ & $3.880 E-04$ & $1.529 \mathrm{E}-05$ & $4.170 E-04$ \\
\hline $\begin{array}{l}3.367 E+00 \\
5.700 E-02 \\
3.427 E+00\end{array}$ & $\begin{array}{l}4.077 E-01 \\
6.112 E-03 \\
4.142 E-0 .\end{array}$ & $\begin{array}{l}9.283 E-02 \\
2.897 E-03 \\
9.575 E-02\end{array}$ & $\begin{array}{l}4.386 E-01 \\
6.546 E-03 \\
4.455 E-01\end{array}$ \\
\hline $4.723 E-03$ & $2.330 E-03$ & $3.986 E-09$ & $\begin{array}{r}2.118 \mathrm{E}-07 \\
\text { CD }-60 \\
\text { CS }-134 \\
\text { N }]-60\end{array}$ \\
\hline $\begin{array}{l}1.000 E-02 \\
1.472 E-02\end{array}$ & $\begin{array}{l}5.880 E-04 \\
2.918 E-03\end{array}$ & $\begin{array}{l}3.335 E-04 \\
3.335 E-04\end{array}$ & $\begin{array}{l}6.294 E-04 \\
6.296 E-04\end{array}$ \\
\hline $8.000 E-03$ & $1.607 \mathrm{E}-03$ & $4.370 E-05$ & $1.729 \mathrm{E}-03$ \\
\hline $\begin{array}{l}5.150 E-02 \\
2.805 E-01 \\
1.100 E-03 \\
3.411 E-01\end{array}$ & $\begin{array}{l}5.206 \mathrm{E}-03 \\
5.578 \mathrm{E}-03 \\
1.686 \mathrm{E}-04 \\
1.256 \mathrm{E}-02\end{array}$ & $\begin{array}{l}2.788 E-03 \\
1.201 E-02 \\
3.397 \mathrm{E}-05 \\
1.488 \mathrm{E}-02\end{array}$ & $\begin{array}{l}5.574 \mathrm{E}-03 \\
5.958 \mathrm{E}-03 \\
1.813 \mathrm{E}-04 \\
1.344 \mathrm{E}-02\end{array}$ \\
\hline $\begin{array}{l}2.072 E+00 \\
2.050 E-03 \\
2.074 E+00\end{array}$ & $\begin{array}{l}7.069 \mathrm{E}-02 \\
3.688 \mathrm{E}-04 \\
7.105 \mathrm{E}-02\end{array}$ & $\begin{array}{l}6.091 E-03 \\
2.758 E-05 \\
6.119 E-03\end{array}$ & $\begin{array}{l}7.636 \mathrm{E}-02 \\
3.960 \mathrm{E}-04 \\
7.675 \mathrm{E}-02\end{array}$ \\
\hline $5.000 E-03$ & $1.067 \mathrm{E}-03$ & $1.110 \mathrm{E}-05$ & $1.149 E-03$ \\
\hline $\begin{array}{l}5.630 E-01 \\
5.000 E-04 \\
5.685 E-01\end{array}$ & $\begin{array}{l}5.519 E-02 \\
6.550 E-05 \\
5.624 E-02\end{array}$ & $\begin{array}{l}1.141 E-02 \\
1.909 E-05 \\
1.144 E-02\end{array}$ & $\begin{array}{l}5.939 E-02 \\
7.020 E-05 \\
6.061 E-02\end{array}$ \\
\hline $5.923 E-02$ & $6.893 E-04$ & $2.459 E-05$ & $\begin{array}{r}2.814 E-02 \\
\text { CO-60 } \\
\text { CS }-134 \\
\text { EU- } 152 \\
\text { EU- } 154 \\
2.086 E-02\end{array}$ \\
\hline
\end{tabular}

8.901E-02 $\quad 1.935 \mathrm{E}-02 \quad 1.394 \mathrm{E}-04 \quad 2.086 \mathrm{E}-02$

$\begin{array}{ll}\text { CS137 } & 1.030 E-04 \\ \text { CO-58 } & 1.000 E-04 \\ \text { CO-60 } & 6.700 E-03 \\ \text { EU155 } & 8.500 E-04 \\ \text { C-14 } & 1.000 E-04 \\ \text { BA137 } & 9.700 E-05 \\ \text { lE } 154 & 8.500 E-04 \\ \text { FE }-59 & 4.000 E-04 \\ \text { IAN-54 } & 7.000 E-04 \\ \text { EU152 } & 1.000 E-04 \\ & 4.720 E-03 \\ & .000 E+00 \\ & .000 E+00 \\ & .000 E+00 \\ & .000 E+00 \\ & .000 E+00 \\ & .000 E+00 \\ .000 E+00 \\ .000 E+00 \\ 6.204 E-05\end{array}$

2. $000 E-08$

$6.200 E-05$

$2.000 E-08$

$.000 E+00$

$6.204 E-05$

$.000 \mathrm{E}+00$

$.000 E+00$

$.000 E+00$

$.000 E+00$

$.000 E+00$

$.000 E+00$

$.000 E+00$

$.000 \mathrm{E}+00$

$.000 E+00$

$.000 E+00$

$.000 E+00$

$.000 E+00$

2. $404 \mathrm{E}-03$

$7.510 E-06$

$1.290 E-03$

$1.690 E-06$

$9.370 \mathrm{E}-04$ $.000 E+00$

CS137 2.060E-12

CO-58 2.00OE-12

Co-60 1.340E-10

EU155 1.700E-11

C. $14 \quad 2.000 E-12$

BA $137 \quad 1.940 E-12$ 


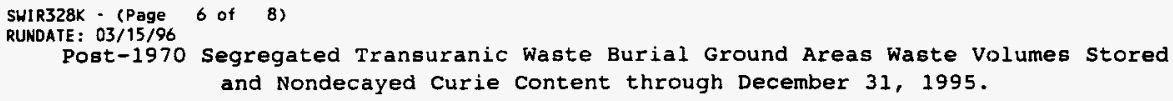

TRANSURANIC WIPP UNCERT IFIED

Total for Facility 2402WJ

CENTRAL WASTE COMPLEX 2403WA BUILDING TRAMSURAHIC (NOT PROCESSED THROUGH

TRUSAF)

TRANSURAMIC WIPP CERTIF IED

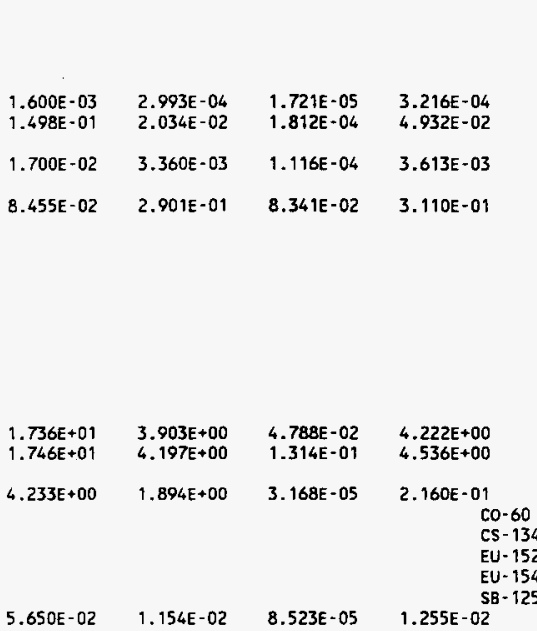

IRANSURANIC WIPP CERTIFIED

TRANSURANIC WIPP UNCERTIFIED

Total for Facility 2403WB

CENTRAL WASTE COMPLEX 2403 WC BUILDING TRANSURANIC (NOT PROCESSED THROUGH TRUSAF)-OFFSITE

TRANSURANIC WIPP UNCERTIFIED

CENTRAL WASTE COMPLEX 2403WB BUILDING TRANSURANIC (NOT PROCESSED THROUGH IRUSAF)

$5.650 E-02$

(.650 -02

$1.154 E-02$

$1.255 E-02$

EU154

FE- 59

MN -54

EU152

1. $700 \mathrm{E}-11$

8. $000 E-12$

8.
$1.4000 E-11$

2. $000 \mathrm{E}-12$

$.000 E+00$

2.404E-03

$.000 E+00$

Cs 137

Co-58

CO-60

EU155

C. 14

BA137

EU154

FE-59

MN- 54

EU152

$.000 E+00$

4. $388 \mathrm{E}-12$

4. $260 \mathrm{E}-12$

2. $854 E-10$

3.621E-11

4. 260E-12

4. $132 E+12$

$3.621 \mathrm{E}-11$

1.704E-11

2. $982 E-11$

4.260E-12

$.000 E+00$

$.000 E+00$

2. $196 \mathrm{E}-02$

8.543E-04

4. $755 \mathrm{E}-03$

7.910E-07

$1.154 \mathrm{E}-02$

$4.810 E-03$

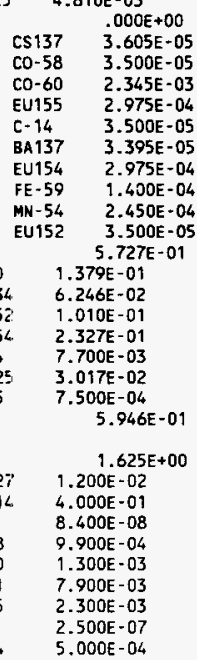


WHC-EP-0125-8

SWIR328K - (Page 7 of 8 )

RUNOATE: 03/15/96

Post-1970 Segregated Transuranic Waste Burial Ground Areas Waste Volumes Stored and Nondecayed Curie Content through December 31, 1995.

BURIAL GROUND FACILITIES
CENTRAL WASTE COMPLEX 2403 WC BUILDING
TRANSURANIC (NOT PROCESSED THROUGH TRUSAF) - OFFSITE

TRANSURANIC (NOT PROCESSED THROUGH IRUSAF)

TRANSURANIC WIPP CERTIFIED
TOTAL SR-90 RU-106 CS-137 OTHER RADIOACTIVITY (CURIES) (CURIES) (CURIES) (CURIES) (CIJRIES)

\begin{tabular}{|c|c|c|c|c|c|}
\hline & & & \multicolumn{2}{|l|}{$\begin{array}{l}M O-93 \\
N B-94 \\
N 1-59 \\
N 1-63 \\
P B-210 \\
P B-214 \\
P 0-210 \\
T C-99\end{array}$} & $\begin{array}{l}2.100 E-08 \\
9.900 E-09 \\
2.600 E-06 \\
2.900 E-04 \\
4.000 E-01 \\
4.000 E-01 \\
4.000 E-01 \\
4.500 E-09\end{array}$ \\
\hline $4.337 E+00$ & $1.755 E+00$ & $9.605 E-04$ & $\begin{aligned} 2.260 E-01 \\
C E-144 \\
C O-60 \\
C S-134 \\
E U-152 \\
E U-154 \\
E U-155 \\
K=40 \\
T A-182\end{aligned}$ & $\begin{array}{l}3 . \\
1 . \\
1 . \\
1 . \\
2 . \\
2 . \\
4 . \\
3 .\end{array}$ & $\begin{array}{l}3.708 E-01 \\
.800 E-03 \\
.647 E-03 \\
.900 E-03 \\
.000 E-01 \\
.340 E-01 \\
.900 E-02 \\
.513 E-05 \\
.800 E-04\end{array}$ \\
\hline $7.195 E+00$ & $1.491 E+00$ & $1.035 E-01$ & $\begin{array}{r}1.627 E+00 \\
\text { CE }-144 \\
\text { CD- } 60 \\
\text { CS-134 } \\
\text { EU }-152 \\
\text { EU- } 154\end{array}$ & $\begin{array}{l}2 . \\
6 . \\
1 \\
1 . \\
2 .\end{array}$ & $\begin{array}{l}3.771 E-04 \\
.600 E-05 \\
.804 E-06 \\
.621 E-05 \\
.072 E-04 \\
.208 E-04\end{array}$ \\
\hline & & & & $\begin{array}{l}\text { CS } 137 \\
\text { CO-58 } \\
\text { CO-60 } \\
\text { EU155 } \\
\text { C- } 14 \\
\text { BA } 137 \\
\text { EU154 } \\
\text { FE-59 } \\
\text { IMN }-54 \\
\text { EU152 }\end{array}$ & $\begin{array}{l}7.169 \mathrm{E}-08 \\
6.960 \mathrm{E}-08 \\
4.663 \mathrm{E}-06 \\
5.916 \mathrm{E}-07 \\
6.960 \mathrm{E}-08 \\
6.751 \mathrm{E}-08 \\
5.916 \mathrm{E}-07 \\
2.786 \mathrm{E}-07 \\
4.872 \mathrm{E}-07 \\
6.960 \mathrm{E}-08\end{array}$ \\
\hline $\begin{array}{l}1.632 E+02 \\
1.764 E+02\end{array}$ & $\begin{array}{l}3.379 E+01 \\
3.704 E+01\end{array}$ & $\begin{array}{l}6.534 E-01 \\
7.579 E-01\end{array}$ & $\begin{array}{l}3.639 E+01 \\
3.824 E+01\end{array}$ & & $\begin{array}{l}.000 E+00 \\
1.996 E+00\end{array}$ \\
\hline $3.001 E+00$ & $5.820 E-01$ & $2.294 E-02$ & $\begin{array}{r}6.255 E-01 \\
T C-99\end{array}$ & & $\begin{array}{c}2.800 E=04 \\
800 E-04\end{array}$ \\
\hline & & & & $\begin{array}{l}\text { CS137 } \\
\text { CO-58 } \\
\text { CO-60 } \\
\text { EU155 } \\
\text { C- } 14 \\
\text { BA } 137 \\
\text { EU154 } \\
\text { FE-59 } \\
\text { MN }-54 \\
\text { EU152 }\end{array}$ & $\begin{array}{l}5.150 E-06 \\
5.000 E-06 \\
3.350 E-04 \\
4.250 E-05 \\
5.000 E-06 \\
4.850 E-06 \\
4.250 E-05 \\
2.000 E-05 \\
3.500 E-05 \\
5.000 E-06\end{array}$ \\
\hline $\begin{array}{l}3.800 E-02 \\
1.500 E-02 \\
3.054 E+00\end{array}$ & $\begin{array}{l}8.105 E-03 \\
3.200 E-03 \\
5.933 E-01\end{array}$ & $\begin{array}{l}8.432 E-05 \\
3.329 E-05 \\
2.306 E-02\end{array}$ & $\begin{array}{l}8.732 E-03 \\
3.447 E-03 \\
6.377 E-01\end{array}$ & & $\begin{array}{r}.000 E+00 \\
.000 E+00 \\
2.800 E-04\end{array}$ \\
\hline $5.000 E-04$ & $1.025 E-04$ & $2.076 \mathrm{E}-06$ & $1.103 E-04$ & & $.000 E+00$ \\
\hline $\begin{array}{l}3.100 \mathrm{E}-02 \\
3.150 \mathrm{E}-02\end{array}$ & $\begin{array}{l}4.010 E-03 \\
4.113 E-03\end{array}$ & $\begin{array}{l}1.169 \mathrm{E}-03 \\
1.171 \mathrm{E}-03\end{array}$ & $\begin{array}{l}4.498 E-03 \\
4.608 E-03\end{array}$ & & $\begin{array}{l}.000 E+00 \\
.000 E+00\end{array}$ \\
\hline $\begin{array}{l}1.000 E-03 \\
1.500 E-03 \\
2.500 E-03\end{array}$ & $\begin{array}{l}1.939 E-04 \\
2.472 E-04 \\
4.611 E-04\end{array}$ & $\begin{array}{l}7.659 E-06 \\
3.302 E-05 \\
4.068 E-05\end{array}$ & $\begin{array}{l}2.085 E-04 \\
2.655 E-04 \\
4.740 E-04\end{array}$ & & $\begin{array}{l}.000 E+00 \\
.000 E+00 \\
.000 E+00\end{array}$ \\
\hline
\end{tabular}

\section{$2.500 \mathrm{E}-03$ \\ $1.500 E-02$}

$4.411 \mathrm{E}-04$
4. $740 \mathrm{E}-04$
TRANSURANIC WIPP UNCERTIFIED

Total for Facility $2403 W C$

CENTRAL WASTE COMPLEX 2603WD BUILDING TRANSURANIC (NOT PROCESSED THROUGH TRUSAF)
IRANSURANIC HIPP CERTIFIED

IRANSURANIC WIPP UNCERTIFIED

Total for Facility 2403WO

FLAMMABLE STORAGE MOOULE 19 IRANSURANIC (NOT PROCESSED THROUGH TRUSAF)

IRANSURANIC WIPP UNCERTIFIED

Total for facility FS19

FLAMMABLE STORAGE MODULE 20

IRANSURANIC WIPP CERTIFIED

TRANSURANIC WIPP UNCERTIFIED

Iotal for facility FS2O 
WHC-EP-0125-8

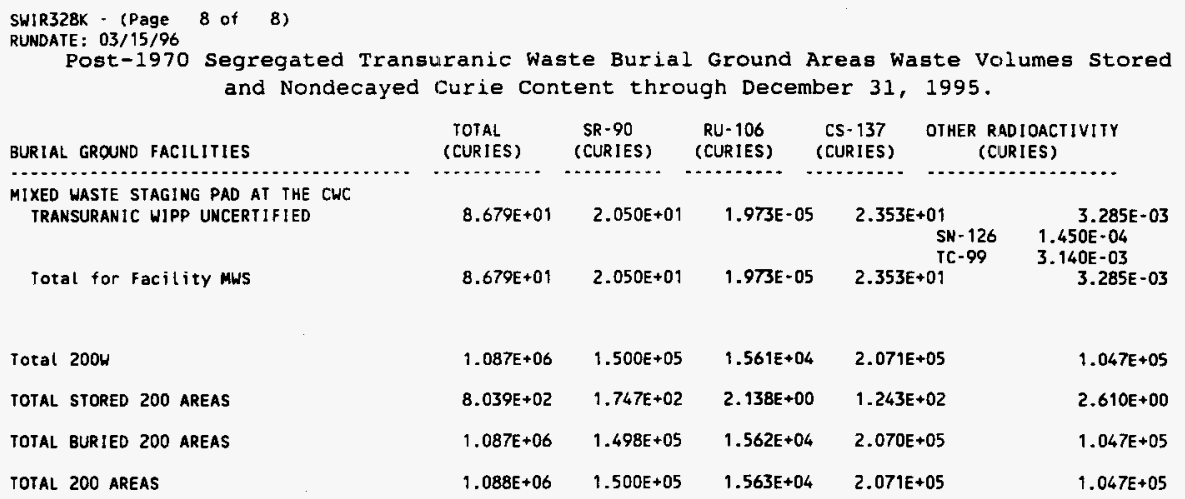


SWJR328L - (Page 1 of 8 )

RUNDATE : 03/15/96

Post-1970 Segregated Transuranic Waste Burial Ground Areas Decayed

Curie Content through December 31, 1995.

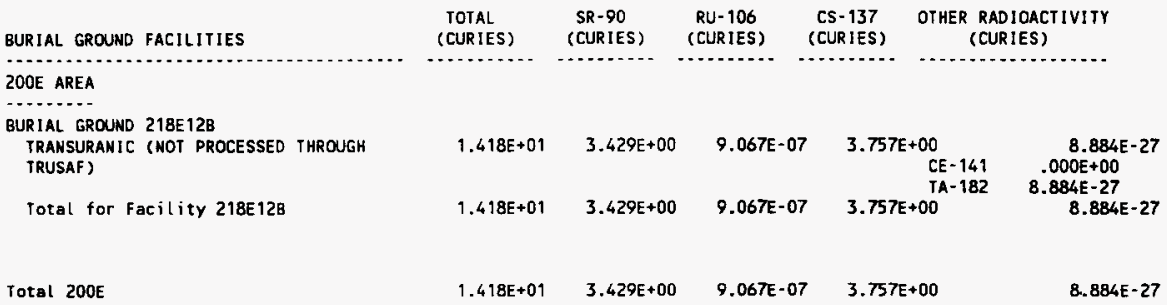




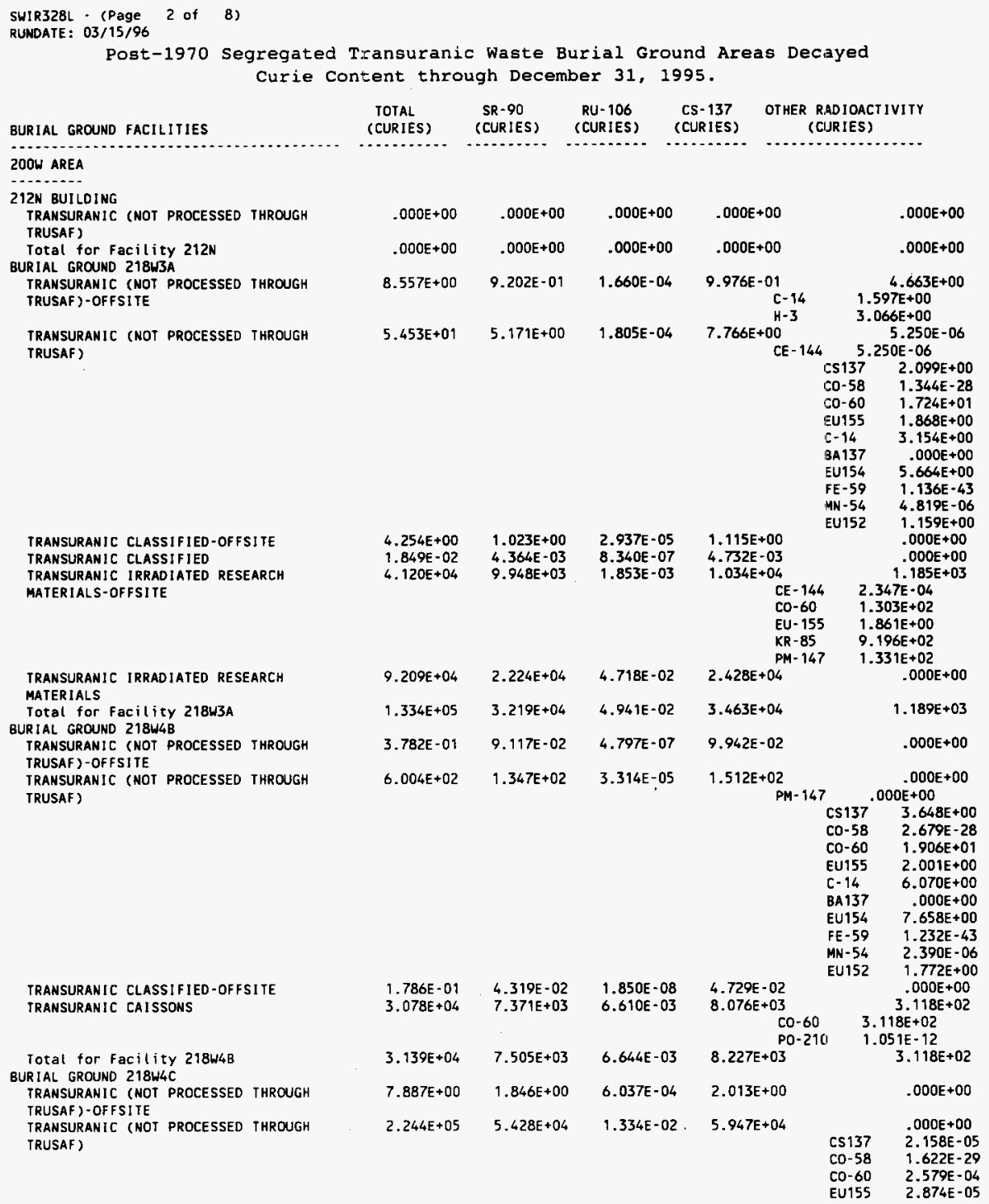


SWIR328L - (Page 3 of 8 )

RUNDATE: 03/15/96

Post-1970 Segregated Transuranic Waste Burial Ground Areas Decayed

Curie Content through December 31, 1995.

BURIAL GROUND FACILITIES

BURIAL GROUND 218 W4C

TRANSURANIC (NOT PROCESSED THROUGH TRUSAF)

IRANSURANIC WIPP CERTIFIED TRANSURANIC WIPP UNCERTIFIED-OFFSITE TRANSURANIC WIPP UNCERTIFIED
TOTAL SR-90 RU-106 CS-137 OTHER RADIOACTIVITY (CURIES) (CURIES) (CURIES) (CURIES) (CURIES)

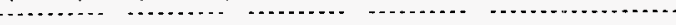

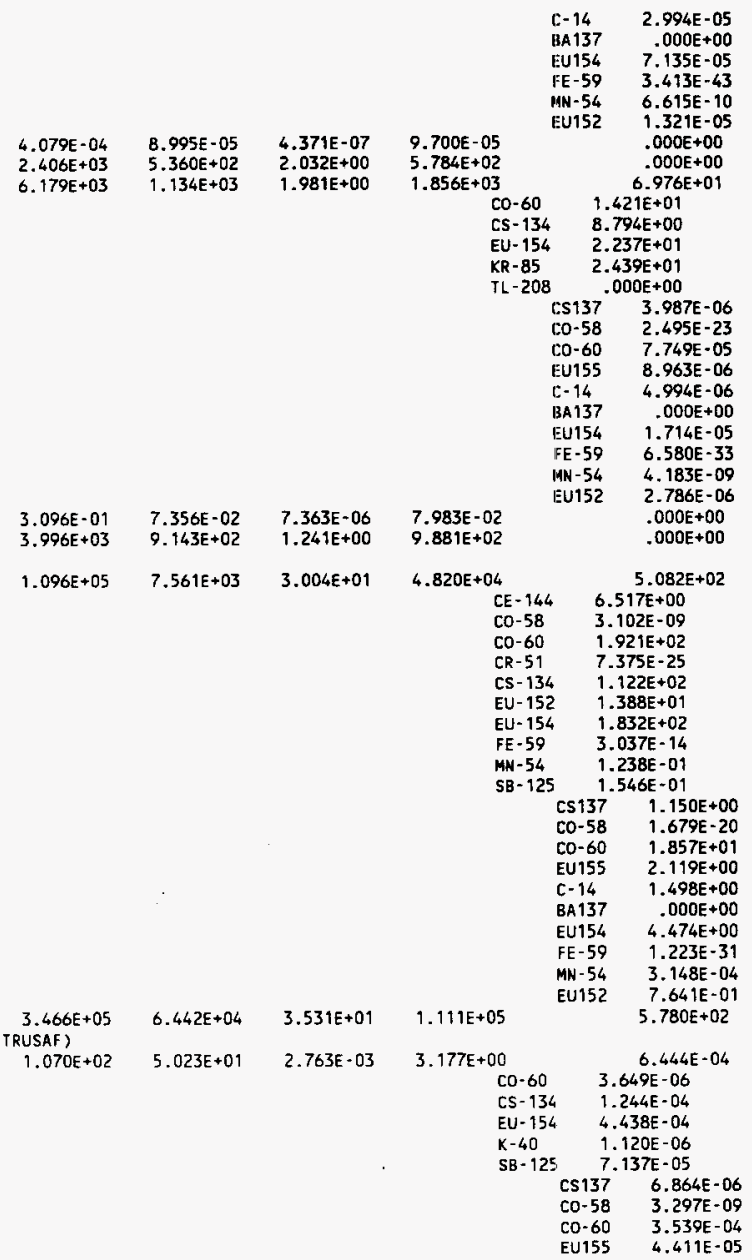

TRANSURANIC SPECIAL CASE-OFFSITE TRANSURANIC IRRADIATED RESEARCH MATERIALS - OFFSITE

IRANSURANIC IRRADIATED RESEARCH MATERIALS
Total for Facility 218W4C

IRANSURANIC WASTE STORAGE \&

TRANSURANIC (NOT PROCESSEO THROUGH

TRUSAF)

TRRAOATED RESEARCH

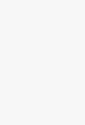




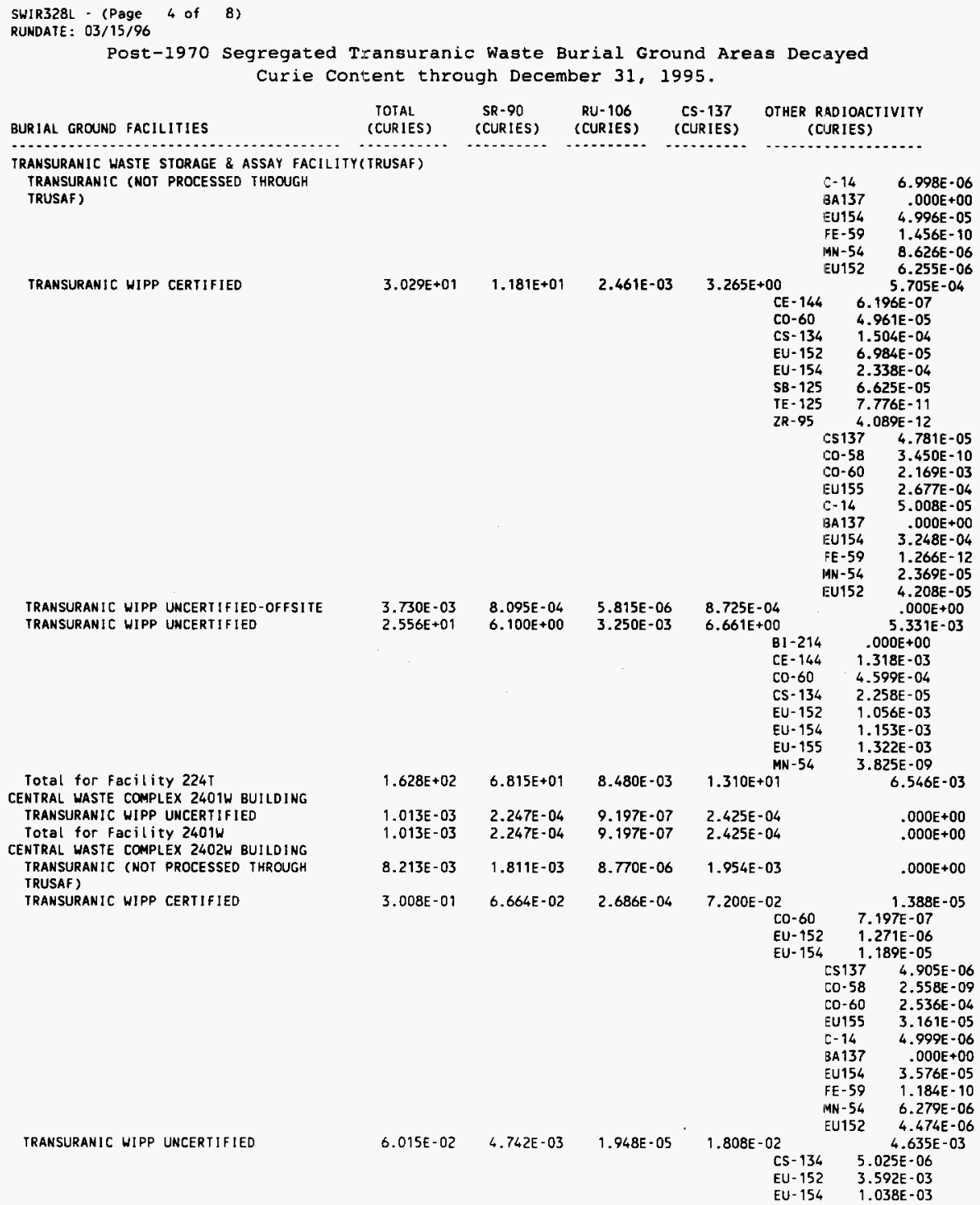




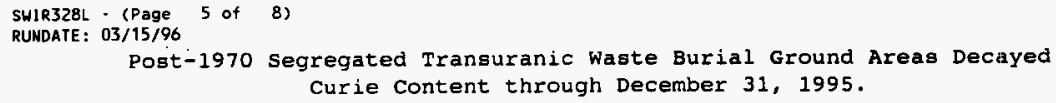

Total for Facility $2402 \mathrm{~W}$

CENTRAL WASTE COMPLEX 2402WB BUILDING TRANSURANIC (NOI PROCESSED IHROUGH TRUSAF ?

TRANSURANIC WIPP CERTIFIED TRANSURANIC WIPP UNCERTIFIED Total for Facility 2402WB

CENTRAL WASTE COMPLEX 2402WC BUILDING TRANSURANIC (NOI PROCESSED IHROUGH TRUSAF ?

TRANSURANIC HIPP CERTIFIED

TRANSURANIC WIPP UNCERTIFIED

Total for Facility $2402 W C$

CENTRAL WASIE COMPLEX $2402 W 0$ BUILDING TRANSURANIC (NOT PROCESSED THROUGH TRUSAF)

TRANSURANIC WIPP UNCERTI I IED Total for Facility $2402 \mathrm{WD}$ CENIRAL WASTE COMPLEX 2402WE BUILDING TRANSURANIC (NOT PROCESSED IHROUGH TRUSAF)

TRANSURANIC WIPP CERTIFIED

TRANSURANIC WIPP UNCERTIFIED-OFFSITE TRANSURANIC WIPP UNCERTIFIED

Total for Facility 2402WE

CENTRAL WASTE COMPLEX $2402 W G$ BUILDING

TRANSURANIC WIPP CERTIFIED

TRANSURANIC WIPP UNCERTIFIED

Iotal for Facility $2402 W G$

CENTRAL WASTE COMPLEX 2402WH BUILDING TRANSURANIC (NOT PROCESSEO THROUGH TRUSAF )

TRANSURANIC WIPP CERTIFIED

IRANSURANIC WIPP UNCERTIFIED

Total for Facility $2402 \mathrm{WH}$

CENTRAL WASTE COMPLEX $2402 \mathrm{WJ}$ BUILDING TRANSURANIC (NOT PROCESSED THROUGH TRUSAF)

IRANSURANIC WIPP CERIJFIED

$\begin{array}{llll}3.691 E-01 & 7.320 E-02 & 2.968 E-04 & 9.204 E-02 \\ 4.421 E-01 & 1.047 E-01 & 1.899 E-05 & 1.138 E-01 \\ 3.850 E-01 & 8.843 E-02 & 1.784 E-04 & 9.581 E-02 \\ 8.220 E-03 & 1.830 E-03 & 6.832 E-06 & 1.974 E-03 \\ 8.354 E-01 & 1.950 E-01 & 2.042 E-04 & 2.116 E-01 \\ 1.620 E-03 & 3.585 E-04 & 1.606 E-06 & 3.867 E-04 \\ 1.426 E+00 & 3.327 E-01 & 3.848 E-04 & 3.611 E-01 \\ 2.330 E-02 & 5.178 E-03 & 2.014 E-05 & 5.587 E-03 \\ 1.450 E+00 & 3.383 E-01 & 4.065 E-04 & 3.671 E-01 \\ 4.403 E-03 & 2.188 E-03 & 6.563 E-10 & 1.994 E-07\end{array}$

$2.257 E-03$

$4.964 \mathrm{E}-04$ $2.685 E-03$

2.577E-06 2.578E-06

$1.514 \mathrm{E}-03$

7.517E-06

$4.345 \mathrm{E}-03$

$1.325 E-05$

$1.940 \mathrm{E}-02$

$2.122 \mathrm{E}-02$

4. $570 \mathrm{E}-03$

$1.577 \mathrm{E}-04$

$4.820 \mathrm{E}-02$

1. $059 \mathrm{E}-02$

$3.925 \mathrm{E}-05$

$6.303 \mathrm{E}-07$

$6.064 \mathrm{E} \cdot 05$

2.429E-01

5. $784 \mathrm{E}-02$

$3.339 E-04$

3. 622E-05

$1.510 E-03$

$5.818 \mathrm{E}-02$

$1.501 \mathrm{E}-06$

3. 772E-05

4.616E- 03

$1.028 \mathrm{E}-03$

3.805E-06

$4.539 E-02$

$1.936 \mathrm{E}-0$

$2.576 \mathrm{E}-04$

$5.665 \mathrm{E}-05$

5.421E-05

$1.984 \mathrm{E}-01$

$4.647 \mathrm{E}-02$

$2.936 \mathrm{E}-07$

$5,812 \mathrm{E}-02$

$6.396 \mathrm{E}-04$

$2.623 \mathrm{E}-06$

8.626E-02 1.907E-02

$8.699 E \cdot 05$
2.057E-02

$\begin{array}{lr}\text { CS137 } & 1.019 E-04 \\ \text { CO-58 } & 1.988 E-05 \\ \text { CO-60 } & 6.314 E-03 \\ \text { EU155 } & 7.980 E-04 \\ \text { C-14 } & 9.999 E-05 \\ \text { BA137 } & .000 E+00 \\ \text { EU154 } & 8.192 E-04 \\ \text { BE-59 } & 3.074 E-05 \\ \text { HW-54 } & 4.853 E-04 \\ \text { EU152 } & 9.766 E-05 \\ & 4.649 E-03 \\ & .000 E+00 \\ & .000 E+00 \\ & .000 E+00 \\ & .000 E+00 \\ & .000 E+00 \\ & .000 E+00 \\ & .000 E+00 \\ & .000 E+00 \\ & \\ & \\ & .579 E+05\end{array}$

Co- 60

CS- 134 NI -60

$1.419 E-08$

$2.577 \mathrm{E}-05$

5.354E-06

5.356E-04

1.633E-03

$.000 E+00$

$.000 E+00$

2.579E-05

$.000 E+00$

4.690E -03

$4.923 \mathrm{E}-03$

$1.702 \mathrm{E}-04$

1.142E-02

$.000 E+00$

$.000 E+00$

$.000 E+00$

$.000 E+00$

$6.303 \mathrm{E}-02$

3.602E-04

$6.339 E-02$

1. 109E-03

$4.934 E-02$

$6.110 E-05$

5.051E-02

2.791E-02

Co- 60

CS- 134

EU-15iे

EU-154

$.000 E+00$

$.000 E+00$

$.000 E+00$

$.000 E+00$

$.000 E+00$

$.000 E+00$

$.000 E+00$

$2.264 \mathrm{E}-03$

7.240E-06

1.174E-03

$1.665 \mathrm{E}-04$

$9.159 E-04$

CS137 $\quad .000 \mathrm{E}+00$

$1.909 \mathrm{E}-12$

CO-60 8.658E-11

EU155 1.069E-11

C- 14 1.999E- 12

BA137 . DOOE+00 


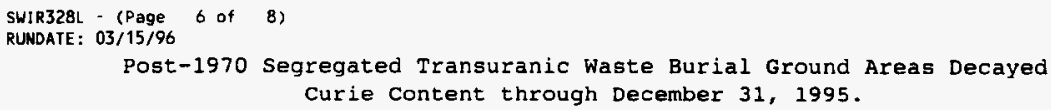

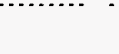

TRANSURANIC WIPP UNCERT IFIED

Total for Facility 2403WA

CENTRAL WASTE COMPLEX 2403 WB BUILDING TRANSURANIC (NOT PROCESSED THROUGH TRUSAF )

TRANSURANIC HIPP CERTIFIEO

IRANSURANIC WIPP UNCERIIFIED

Total for Facility $2403 \mathrm{WB}$

CENTRAL WASTE COMPLEX 2403 WC BUILOING IRANSURANIC (NOT PROCESSED THROUGH TRUSAF ) -OFFSITE

$1.562 E+02$

$\begin{array}{llll}1.246 E-03 & 2.751 E-04 & 1.286 E-06 & 2.967 E-04 \\ 1.456 E-01 & 1.999 E-02 & 9.090 E-05 & 4.879 E-02 \\ 1.411 E-02 & 3.127 E-03 & 1.326 E-05 & 3.373 E-03 \\ 9.972 E-01 & 2.318 E-01 & 1.467 E-04 & 2.509 E-01\end{array}$

1. $325 E+01$

$3.193 \mathrm{E}+00$

$9.441 E-05$

$3.428 E+00$

$2.544 E+04$

4. $184 E+00$

$1.874 E+00$

1. 020E-05

$2.136 \mathrm{E}-01$

Co- 60

CS- 134

EU- 152

EU- 154

$5.395 E-02$

$1.132 E-02$

4. 155E-05

1.232E-02

SB- 125
EU154

FE- 59

NN-54

EU152

$\begin{array}{cc} & .000 E+00 \\ & .000 E+00 \\ \text { CS137 } & 4.065 E-12 \\ \text { Co-58 } & 2.933 E-17 \\ \text { CO-60 } & 1.844 E-10 \\ \text { EU155 } & 2.276 E-11 \\ \text { C-14 } & 4.258 E-12 \\ \text { BA137 } & .000 E+00 \\ \text { BU154 } & 2.762 E-11 \\ \text { FE-59 } & 1.076 E-19 \\ \text { IAN-54 } & 2.015 E-12 \\ \text { BU152 } & 3.578 E-12 \\ & .000 E+00 \\ & .000 E+00 \\ & \\ & 2.028 E-02\end{array}$

8.041E-04

4.077E-03

7. 147E-07

1. 112E-02

4. $279 E-03$

$\begin{array}{ll}\text { CS137 } & -000 E+00 \\ \text { CO-58 } & 3.524 E-05 \\ \text { CO-60 } & 2.031 E-06 \\ \text { EU155 } & 2.060 E-03 \\ \text { C-14 } & 3.502 E-04 \\ \text { BA137 } & .000 E-05 \\ \text { EU154 } & 2.745 E-06 \\ \text { FE-59 } & 5.184 E-07 \\ \text { MN-54 } & 1.102 E-04 \\ \text { EU152 } & 3.324 E-05\end{array}$

$1.520 E+02 \quad 3.269 E+09 \quad 9.507 E-02 \quad 3.917 E+01$

Co-60

CS- 134

EU-152

EU- 154

MN -54

SB-125

2R-95

$3.458 \mathrm{E}+01$

$9.512 E-02$

$3.940 \mathrm{E}+01$

$5.654 E-06$

$1.440 \mathrm{E}-05$

AC -227

BI -214

$\mathrm{C}-14$

Co- 58

C0-60

CR- 51

FE -55

$\mathrm{H}-3$

MN -54
$1.333 E-01$

$5.728 \mathrm{E}-02$

$9.962 \mathrm{E}-02$

2. $279 \mathrm{E}-01$

$6.250 \mathrm{E}-03$

$2.710 E-04$

$5.731 \mathrm{E}-01$

3.888E-01

1. $110 \mathrm{E}-02$

$.000 E+00$

8.397E-08

$1.548 E-07$

$9.421 E-04$

$1.490 \mathrm{E}-12$

$1.222 \mathrm{E}-03$

2. $179 \mathrm{E}-07$

$6.860 E \cdot 05$
2.824E-02 


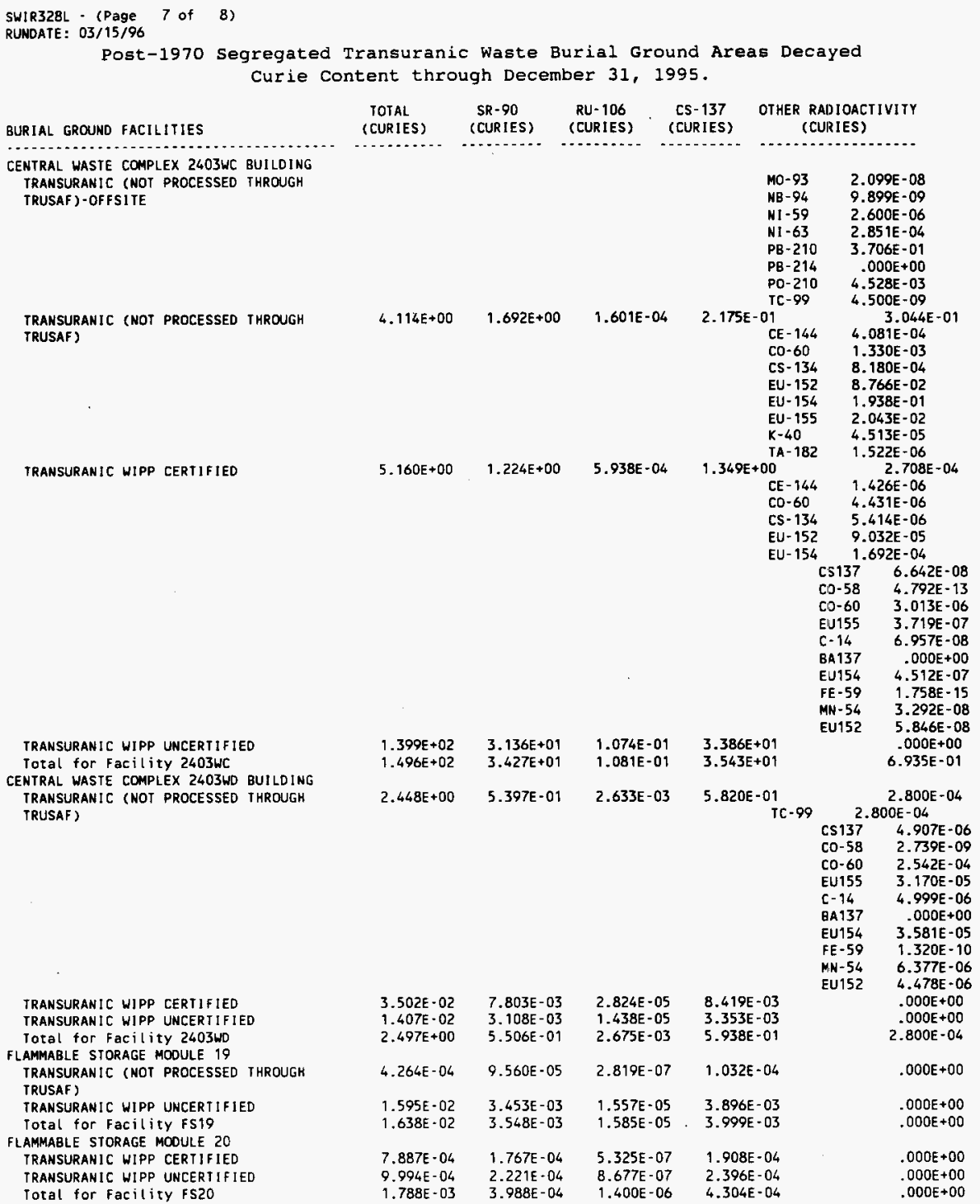




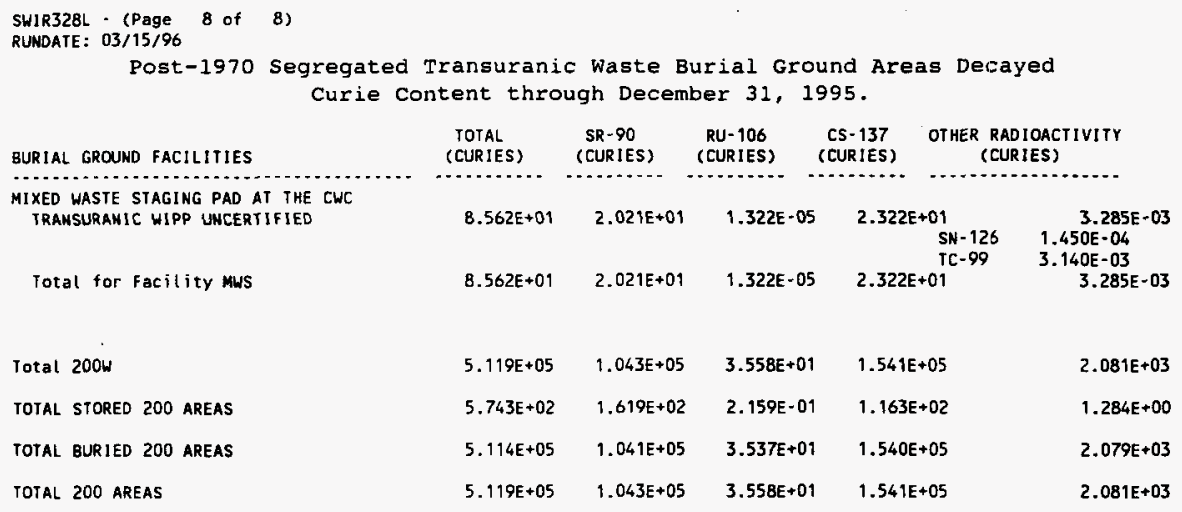




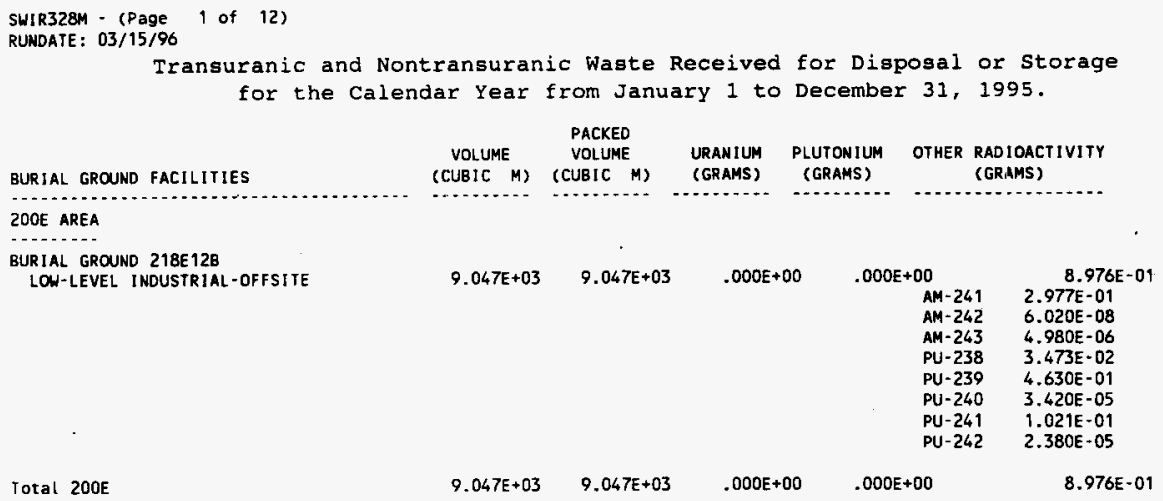


SWIR328M - (Page 2 of 12)

RUNDATE: 03/15/96

Transuranic and Nontransuranic Waste Received for Disposal or Storage for the Calendar Year from January 1 to December $31,1995$.

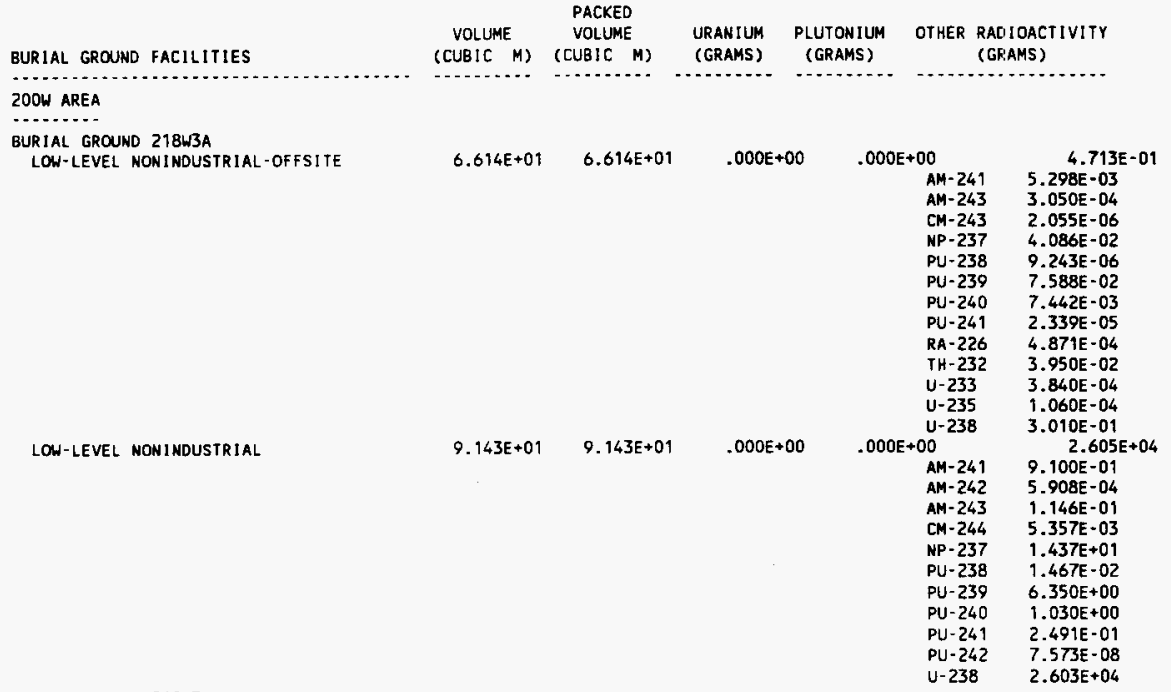

BURIAL GROUND 218W3AE

$1.087 E+03 \quad 1.087 E+03 \quad .000 E+00 \quad .000 E+00$

AM-24

$2.377 \mathrm{E}+06$

AM- 24

AM -243

CF -251

CF -252

$\mathrm{CM}-242$

$\mathrm{CM} \cdot 243$

CM-244

CM- 245

$\mathrm{CM}-247$

NP- 237

PU- 238

PU- 239

PU -240

PU-241

PU- 242

PU- 244

RA- 224

RA- 226

TH-228

TH- 230

TH- 232

TH- 234

$\mathrm{U}-232$

U. 233

U-234

$\mathrm{U}-235$

U. 236

9.422E-03

$1.021 E-03$

2. $416 E-03$

1.500E-07

$9.322 \mathrm{E}+10$

3. $020 \mathrm{E}-12$

3. $606 E-06$

5.719E-06

8. 100E-06

3. $710 E-04$

$1.596 \mathrm{E}+00$

4. $1922 \mathrm{E}-04$

4. $289 \mathrm{E}-01$

1. $709 \mathrm{E}-02$

8.342E-05

$1.000 E-04$

2.319E-02

$7.302 \mathrm{E}-04$

4. $360 \mathrm{E}-03$

$7.709 \mathrm{E}+02$

7.490 E -02

$3.041 E+04$

8.619E- 07

2. $128 E-05$

$4.429 E+D 0$

$1.678 \mathrm{E}+00$

1. $276 E+03$

$3.433 E-01$

U- 238

2. $345 E+06$ 
WHC-EP-0125-8

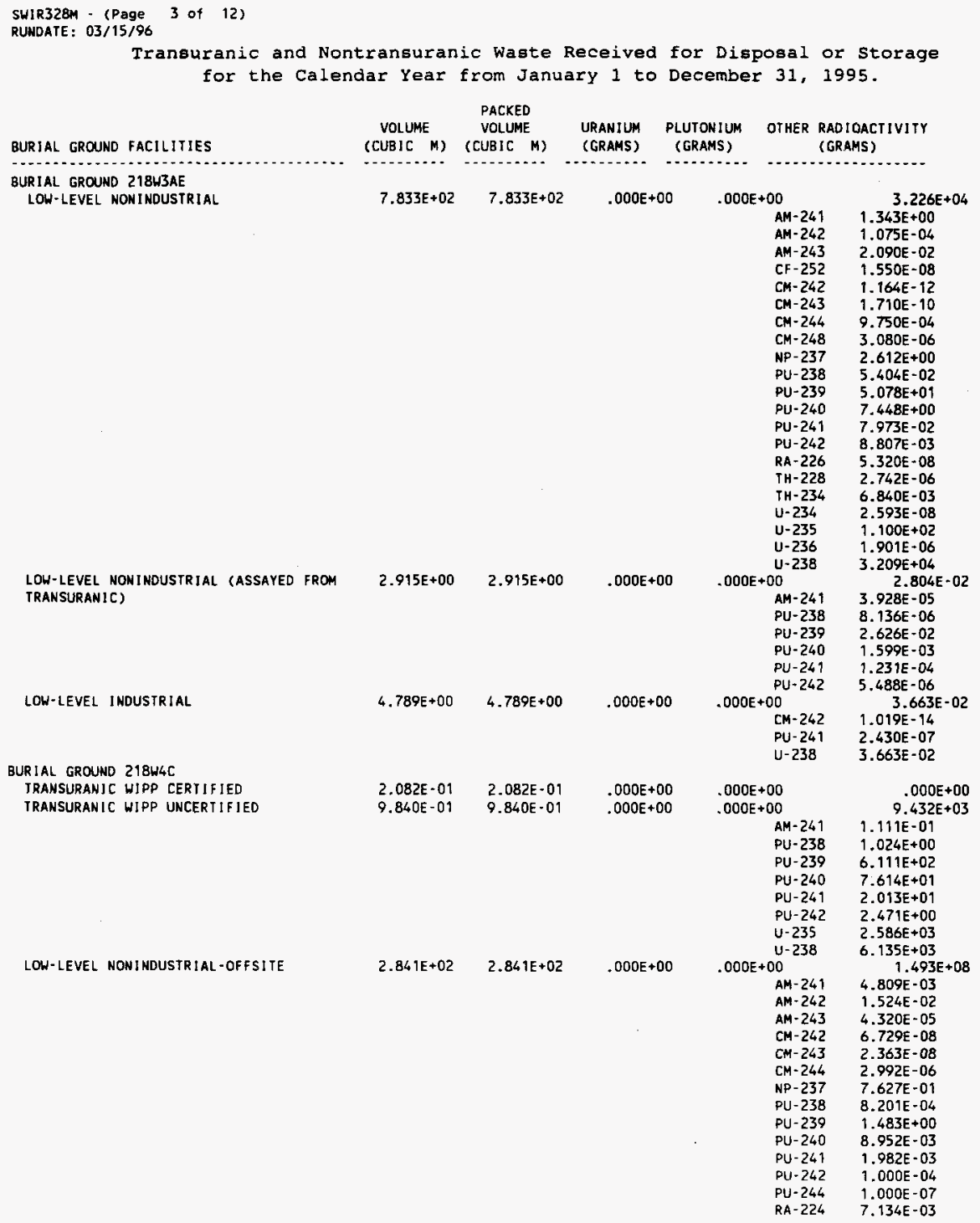


SHIR328M - (Page 4 of 12)

RUNDATE: 03/15/96

Transuranic and Nontransuranic Wagte Received for Disposal or Storage

for the Calendar Year from January 1 to December 31, 1995.

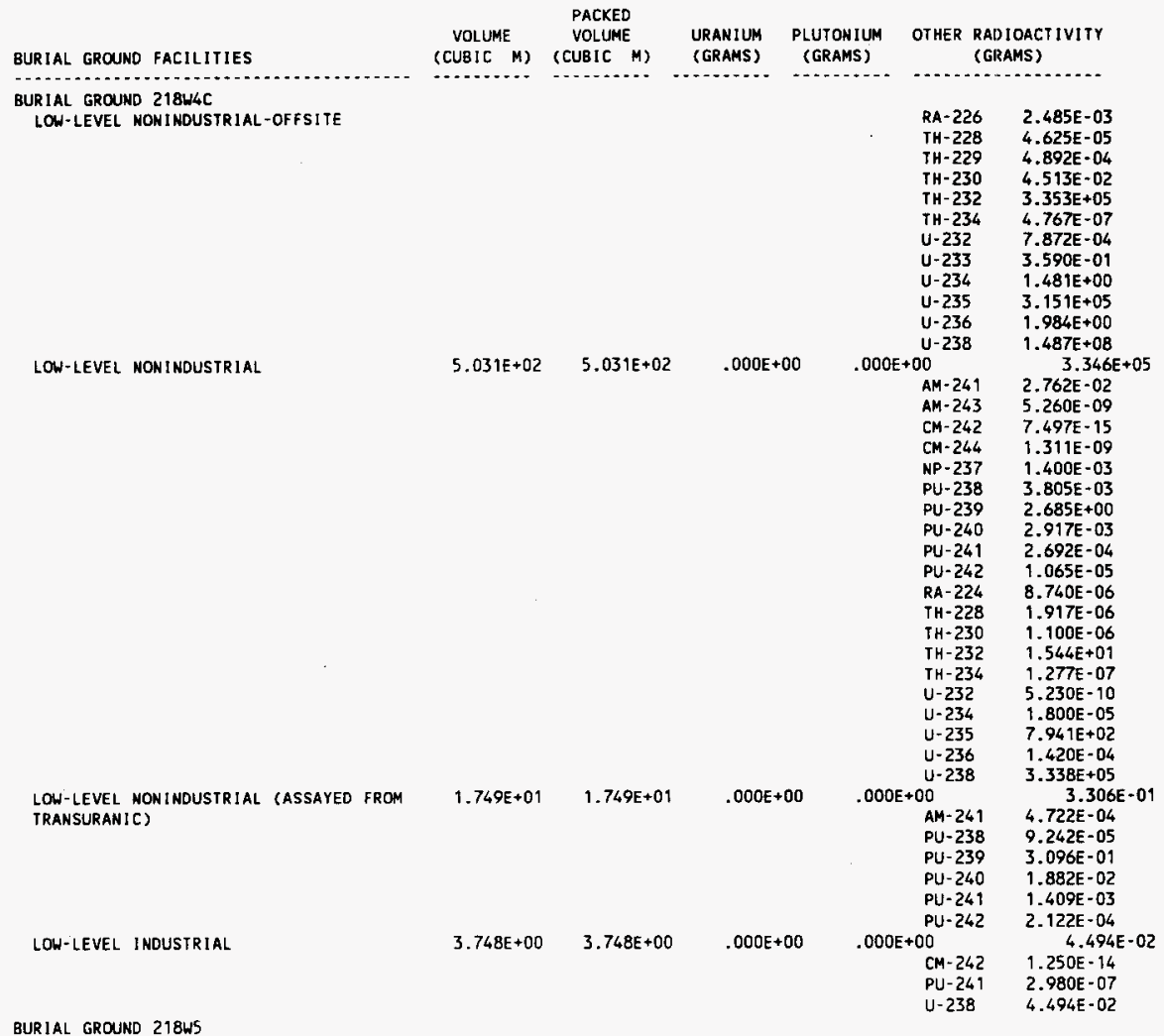

LOW-LEVEL NONINDUSTRIAL-OFFSITE

$5.085 E+02 \quad 5.085 E+02 \quad .000 E+00 \quad .000 E+00$

$\begin{array}{ll}\text { AM-241 } & 2.912 E-01 \\ A M-242 & 1.305 E-04 \\ C M-244 & 3.940 E-05 \\ P U-238 & 1.295 E-04 \\ P U-239 & 3.036 E-02 \\ P U-240 & 3.400 E-04 \\ P U-241 & 4.360 E-05 \\ P U-244 & 1.638 E-04 \\ R A-226 & 8.865 E-05 \\ T H-228 & 1.801 E-07 \\ T H-232 & 5.572 E+03 \\ U-232 & 7.170 E-08 \\ U-233 & 3.297 E-02\end{array}$


SWIR328M - (Page 5 of 12)

RUNDATE: 03/15/96

Transuranic and Nontransuranic Waste Received for Disposal or Storage for the Calendar Year from January 1 to December 31, 1995 .

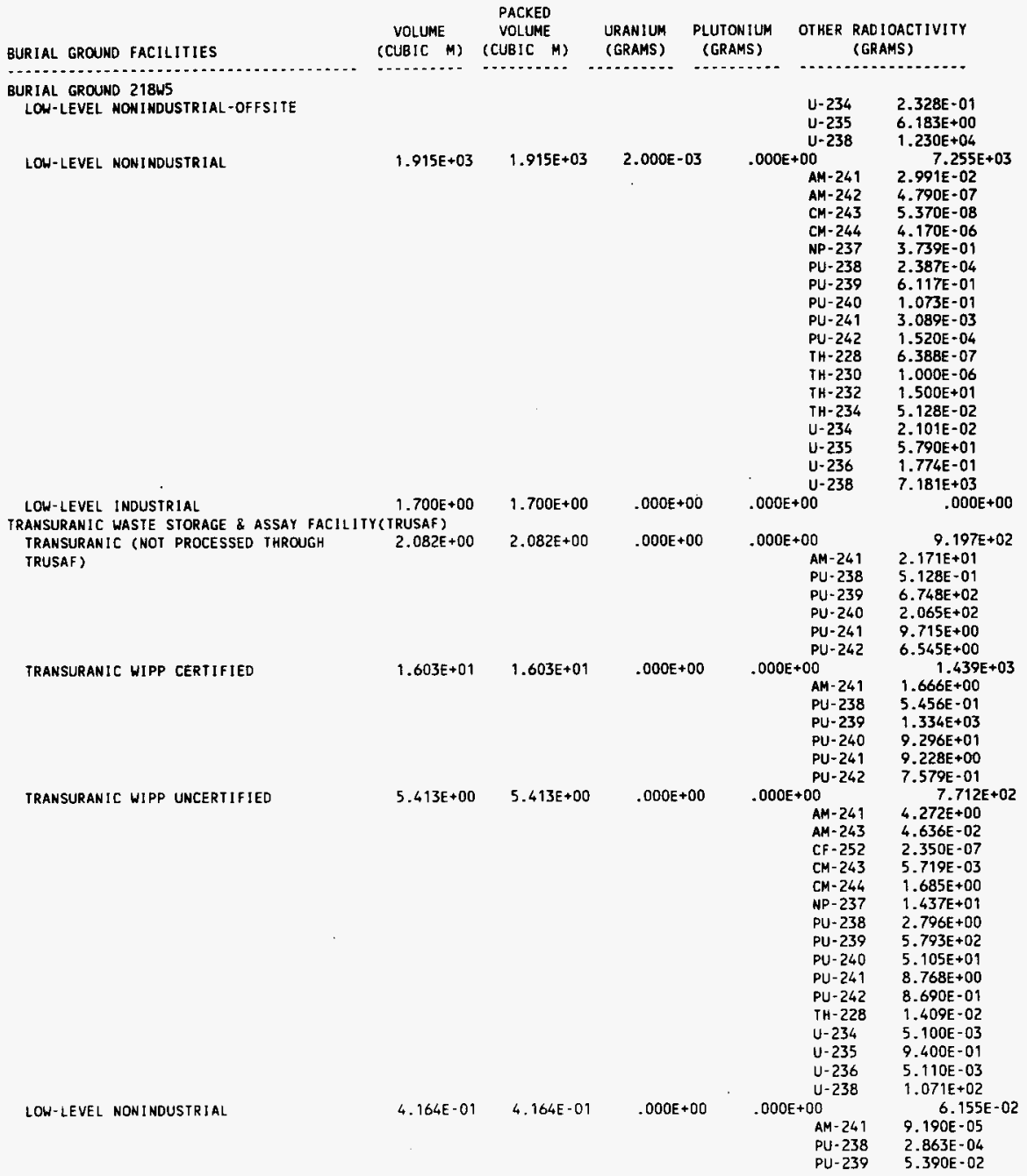




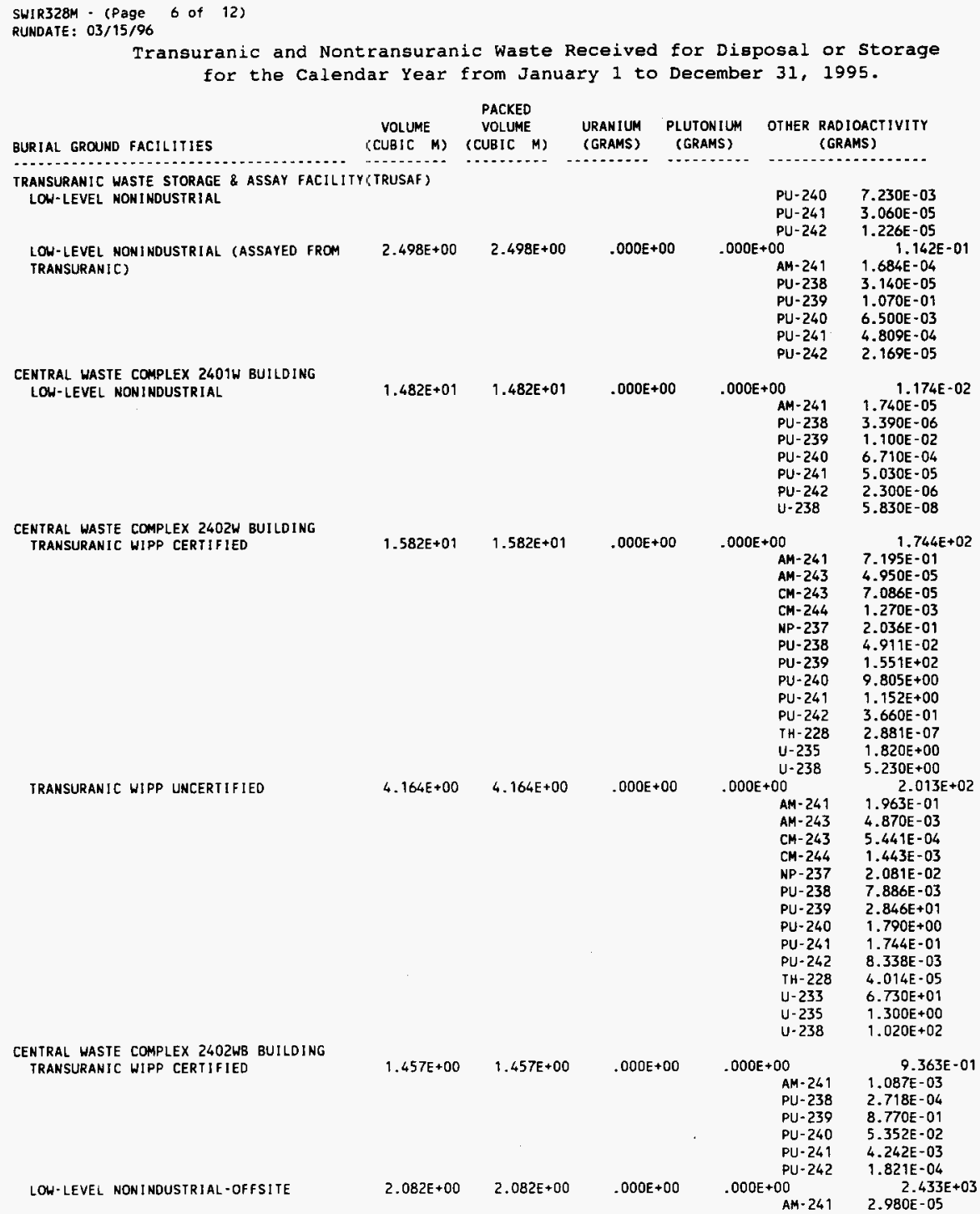




\begin{abstract}
SWIR328M - (Page 7 of 12) RUNDATE: 03/15/96

CENTRAL WASTE COMPLEX 2402WC BUILDING TRANSURANIC WIPP CERTIFIED

LON-LEVEL NONINDUSTRIAL
\end{abstract}

Transuranic and Nontransuranic Waste Received for Disposal or storage for the Calendar Year from January 1 to December $31,1995$.

IRANSURANIC WIPP UNCERTIFIEO

LOW-LEVEL NONINOUSTRIAL

CENTRAL WASTE COMPLEX 2402WD BUILDING LOH-LEVEL NONINDUSIRIAL-OFFSITE

LOW-LEVEL NONINDUSTRIAL

CENTRAL NASIE COMPLEX 2402WE BUILDING TRANSURANIC WIPP UNCERTIFIED

\begin{tabular}{|c|c|c|c|c|c|}
\hline & PACKED & & & & \\
\hline (CUBIC M) & (CUBIC M) & $\begin{array}{l}\text { URAAIIUM } \\
\text { (GRAMS) }\end{array}$ & $\begin{array}{l}\text { PLUTONIUA } \\
\text { (GRAMS) }\end{array}$ & OTHER & $\begin{array}{l}\text { RAOIOACIVITY } \\
\text { (GRAMS) }\end{array}$ \\
\hline
\end{tabular}

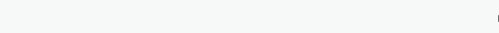

CM- 243

PU -230

PU -244

RA -226

TH-232

$\mathrm{U}-232$

$\mathrm{U}-238$

$4.372 E+00$

$4.372 E+00$

$.000 E+00$

4. $164 \mathrm{E}-01$

4. 164E- 01

$1.249 E+00 \quad 1.249 E+00 \quad .000 E+00$

4.

$.000 E+00 \quad .000 E+00$

AM- 241

NP- 237

PU-23B

PU-239

PU -240

PU -241

PU-242

$.000 E+00$

AM- 241

PU- 238

PU-239

PU 240 .

PU-241

PU-242

AM- 24

CM- 244

PU -238

PU- 239

$\mathrm{PU}-240$

PU- 241

$\mathrm{PU}-242$

$7.192 E+00 \quad 7.192 E+00 \quad .000 E+00 \quad .000 E+00$

AM -241

PU -238

PU -239

$\mathrm{PU}-240$

PU -241

PU -242

$\mathrm{U}-234$

$u-235$

$\mathrm{U}-236$

u- 238

$1.666 E+00 \quad 1.666 E+00 \quad .000 E+00 \quad .000 E+00$

AM- 241

$\mathrm{PU}-238$

PU- 239

$R A-226$

$1.249 E+00 \quad 1.249 E+00 \quad .000 E+00 \quad .000 E+00$

AM- 241

NP -237

PU. 239

$2.082 E-01 \quad 2.082 E-01 \quad .000 E+00 \quad .000 E+00$

AM- 241

AM- 243

$\mathrm{CM}-243$

$\mathrm{CM}-244$

NP -237

$1.333 E+00$
$2.590 E-07$

$4.510 E-04$

$6.270 E-05$

$9.329 E+02$

2. $760 E-07$

$1.499 E+03$

$9.268 E-02$

8.786E-05

$3.636 \mathrm{E}-02$

$1.625 \mathrm{E}-05$

$5.276 \mathrm{E}-02$

$3.214 \mathrm{E}-03$

2.390E-04

1. $103 \mathrm{E}-05$

$3.141 E+00$

$4.983 \mathrm{E}-03$

$9.399 E-04$

$2.942 E+00$

1.791E-01

$1.306 E-02$

$6.260 E-04$ $1.806 E+00$

$1.335 \mathrm{E}-01$

$7.880 E-09$

3.630E-04

$1.546 E+00$

$1.027 \mathrm{E}-01$

2. $293 \mathrm{E}-02$

2.830E-OL

$3.668 E-01$

$3.913 E-06$

6. $019 \mathrm{E}-08$

2. $022 \mathrm{E}-04$

$1.202 E-05$

$9.448 E-07$

$3.011 E \cdot 07$

9. $000 E-05$

$1.068 E-01$

7. 100 E -04

2.590E-01

\section{$6.872 E-08$}

$8.271 E-10$

$2.443 \mathrm{E}-10$

$5.365 \mathrm{E}-08$

$1.400 E-08$ $1.098 E-04$

3. $022 \mathrm{E}-09$

$1.097 \mathrm{E}-04$

$1.272 \mathrm{E}-07$

\section{$9.001 \mathrm{E}-03$}

8. $740 \mathrm{E}-03$

$1.810 \mathrm{E}-04$

3. 800E-05

4. $190 \mathrm{E}-05$

$1.350 \mathrm{E}-08$ 


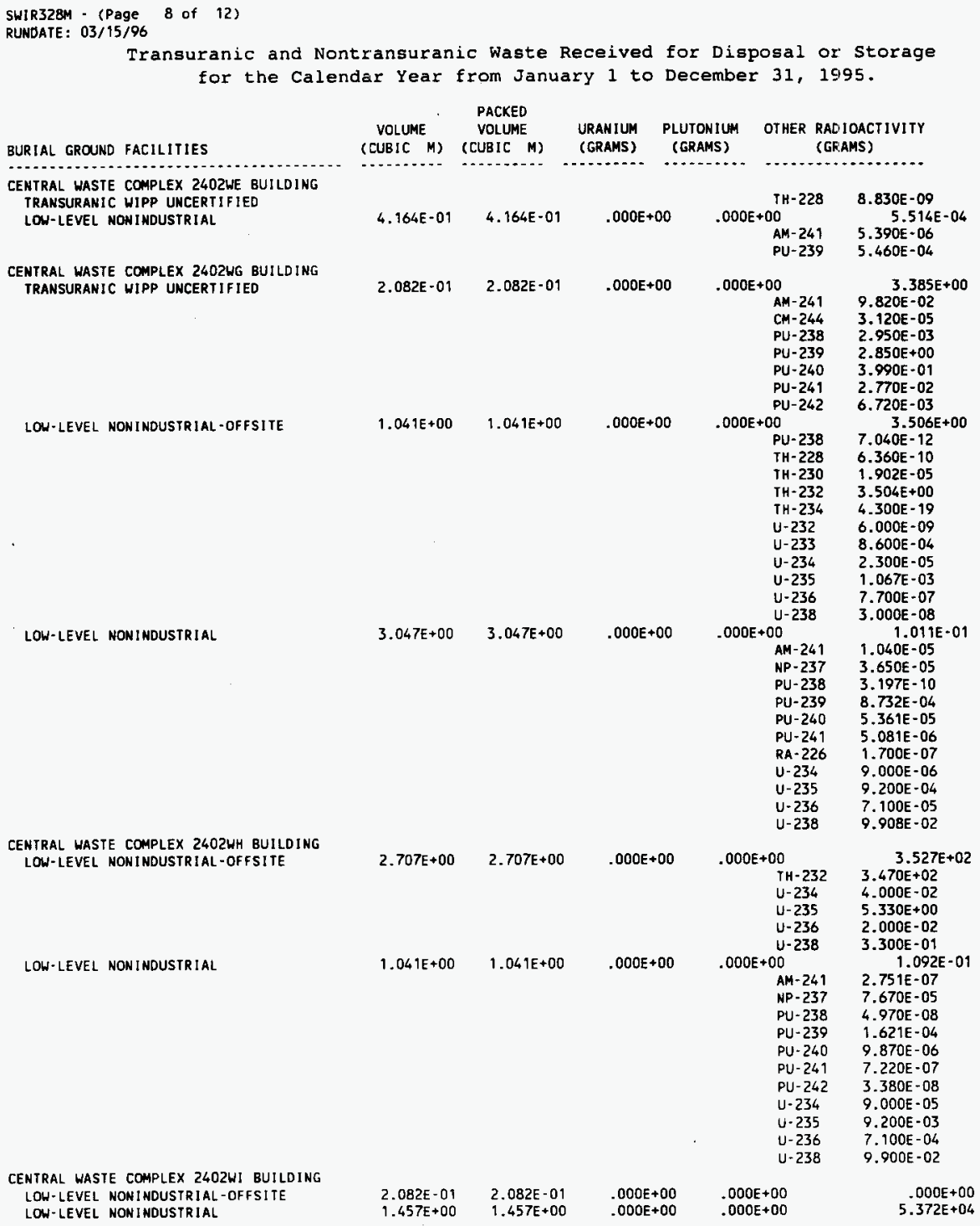




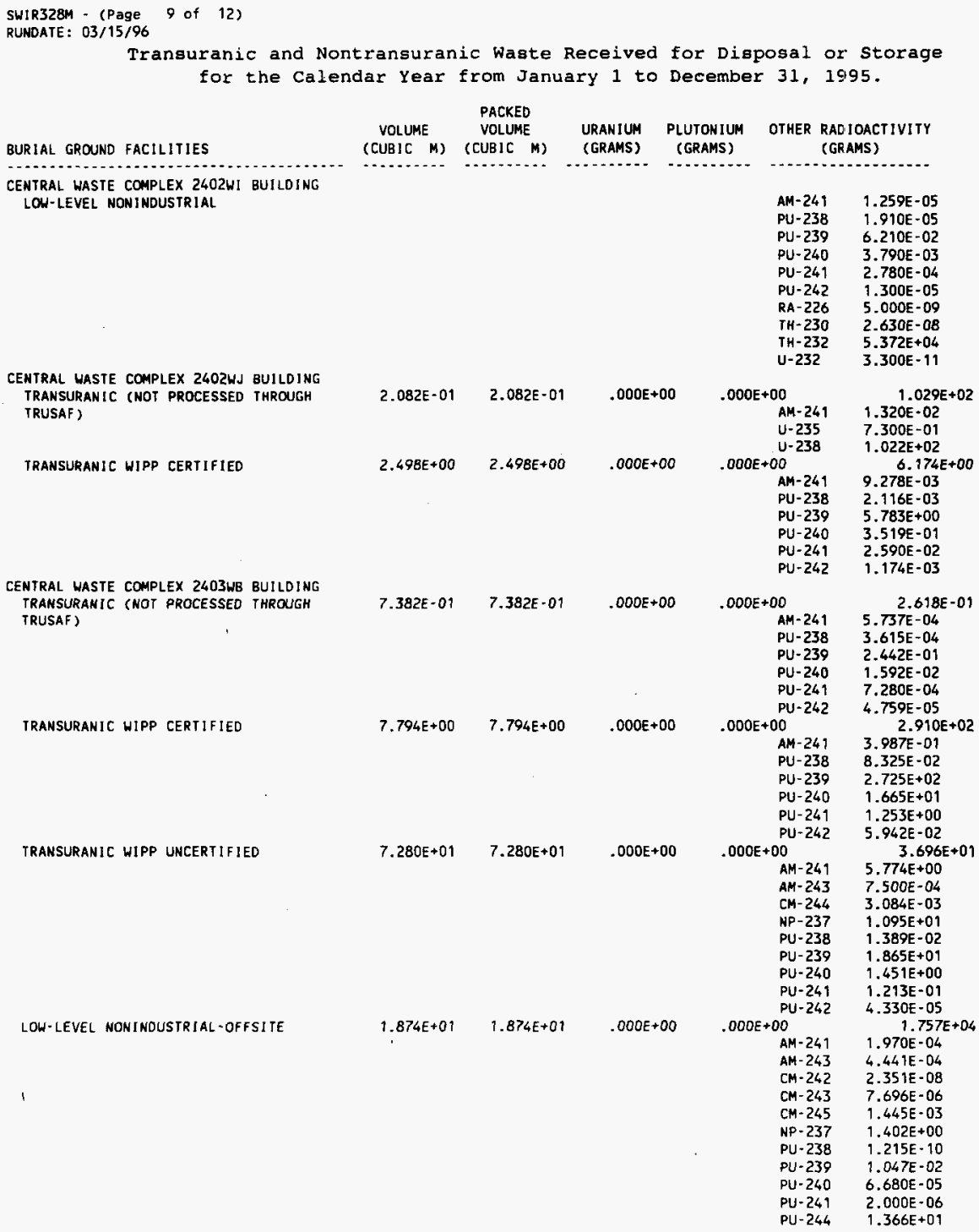




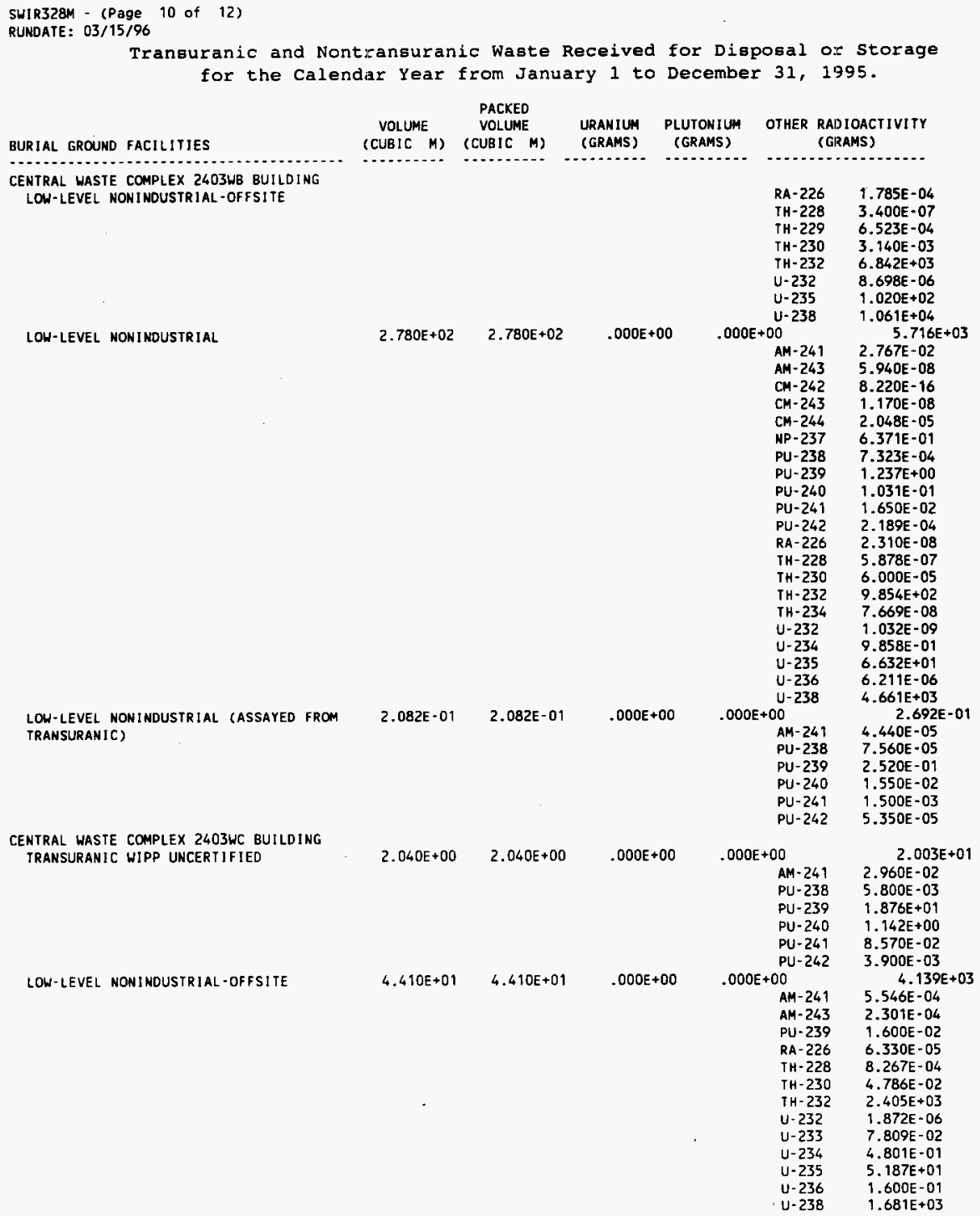




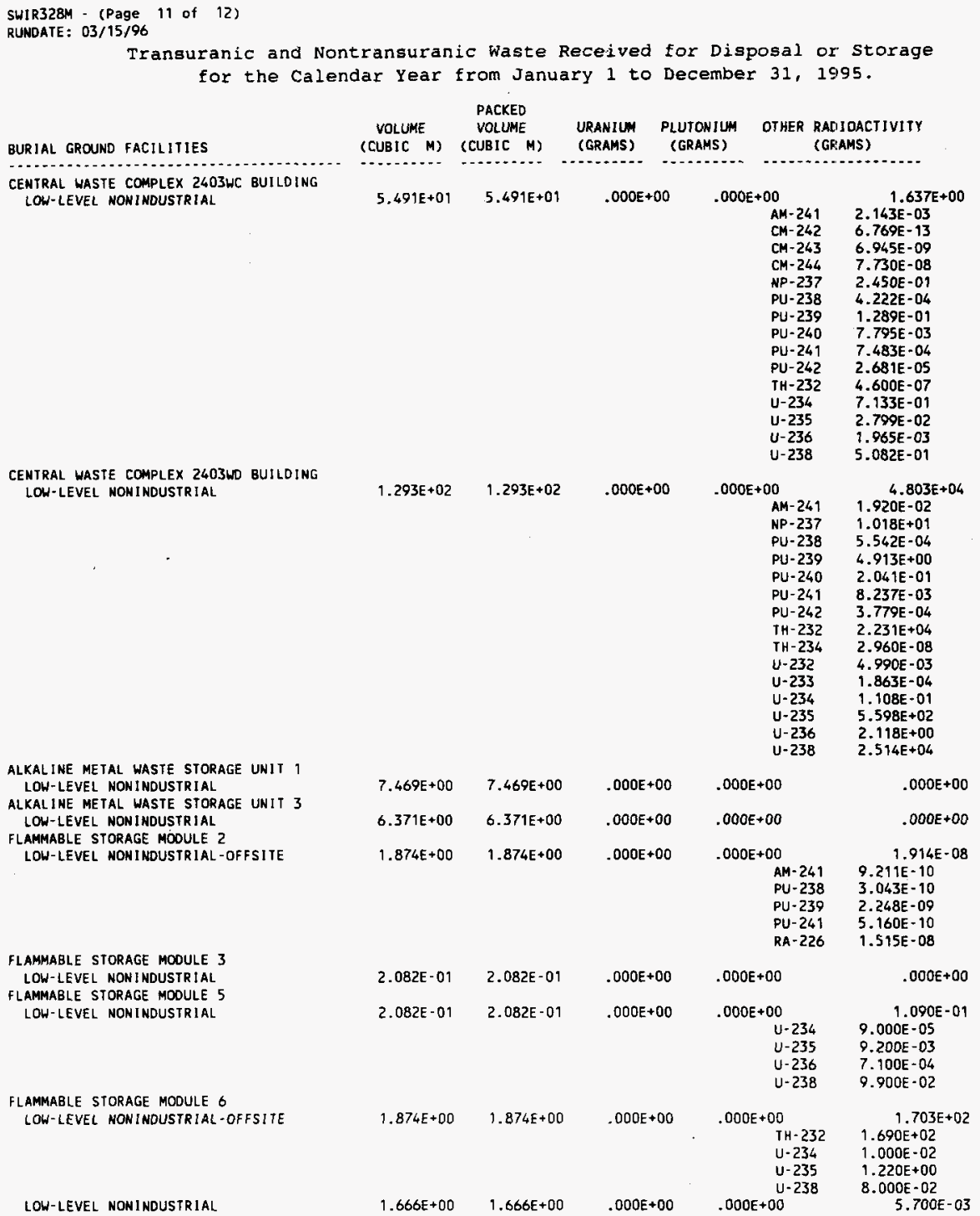




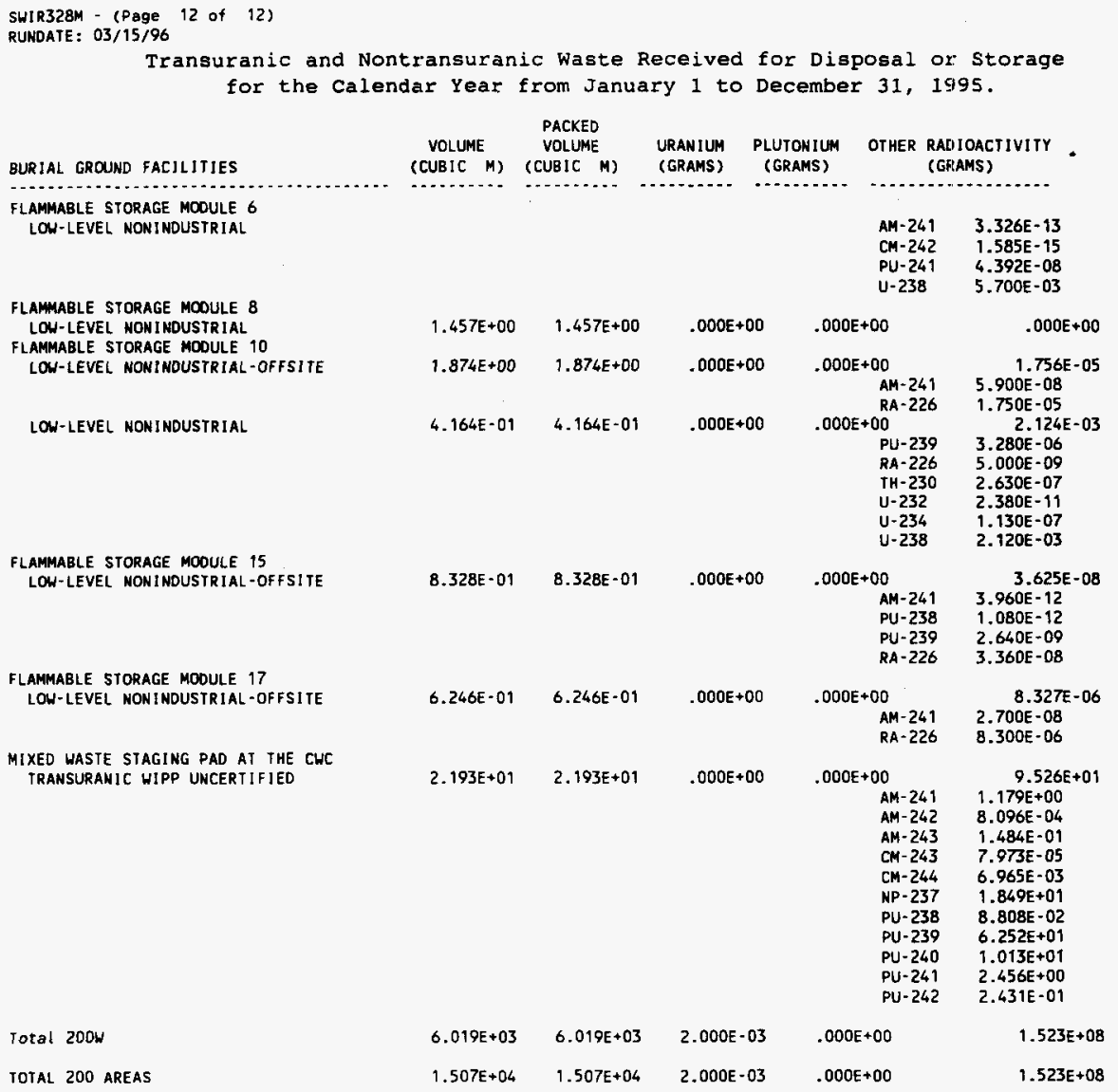


WHC-EP-0125-8

SWIR328N - (Page 1 of 14)

RUNDATE : $03 / 15 / 96$

Tranguranic and Nontransuranic Waste Received for Disposal or Storage for the Calendar Year from January 1 to December 31, 1995.

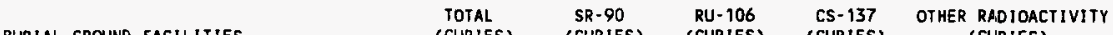

BURIAL GROUND FACILIIIES

(CURIES) (CURIES) (CURIES) (CURIES) (CURIES)

\section{E AREA}

BURIAL GROUND 218E12B

LOW-LEVEL INDUSTRIAL-OFFSITE

$2.561 E+05 \quad 7.354 E+00$

8.007E +00

$1.535 \mathrm{E}+01$

c- 14

CO- 58

co-60

FE -55

NB - 94

N! -63 (CURIES)

Total 200E

$2.561 E+05$

$7.354 E+00$

8.007E+00

$1.535 E+01$

$\begin{aligned} & 2.559 E+05 \\ & 2.784 E+01 \\ & 3.072 E+03 \\ & 6.195 E+04 \\ & 7.480 E+04 \\ & 1.079 E+01 \\ & 1.161 E+05 \\ & 2.559 E+05\end{aligned}$


WHC-EP-0125-8

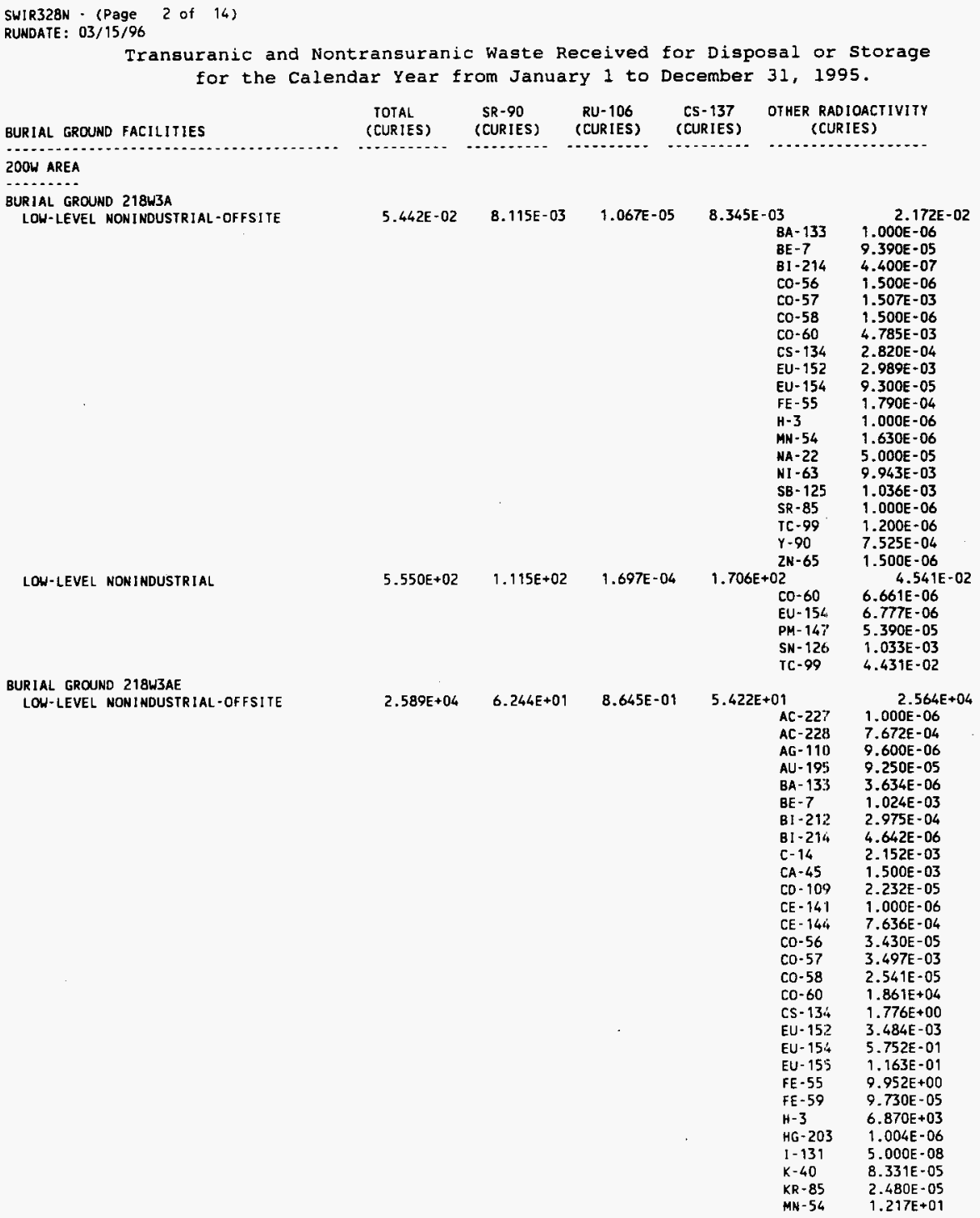


SWIR328N - (Page 3 of 16 )
RUMDATE: $03 / 15 / 96$

Transuranic and Nontransuranic Waste Received for Disposal or storage for the Calendar Year from January 1 to December 31, 1995.

\begin{tabular}{|c|c|c|c|c|c|}
\hline BURIAL GROUND FACILITIES & $\begin{array}{l}\text { TOTAL } \\
\text { (CURIES) }\end{array}$ & $\begin{array}{l}\text { SR-90 } \\
\text { (CURIES) }\end{array}$ & $\begin{array}{l}\text { RU-106 } \\
\text { (CURIES) }\end{array}$ & $\begin{array}{l}\text { CS-137 } \\
\text { (CURIES) }\end{array}$ & $\begin{array}{l}\text { OTHER RADIOACTIVITY } \\
\text { (CIJRIES) }\end{array}$ \\
\hline
\end{tabular}

BURIAL GROUND 21BW3AE

LOH-LEVEL NONINDUSTRIAL-OFFSITE

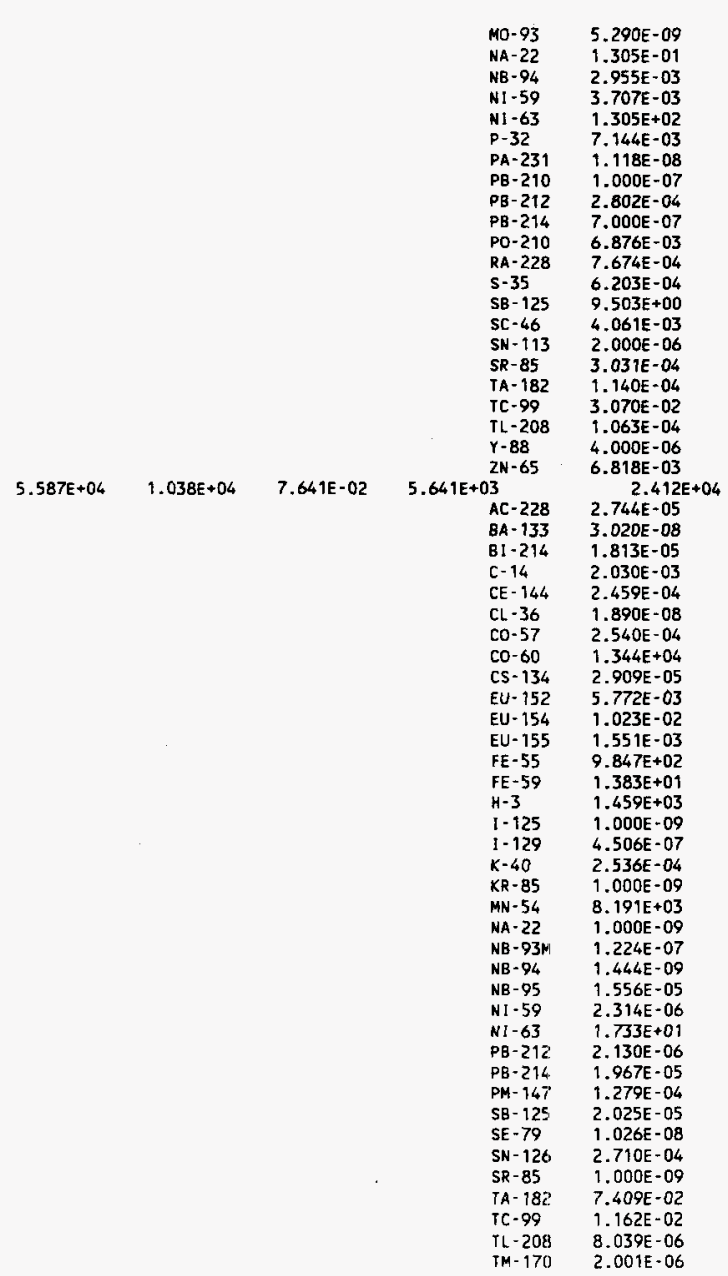




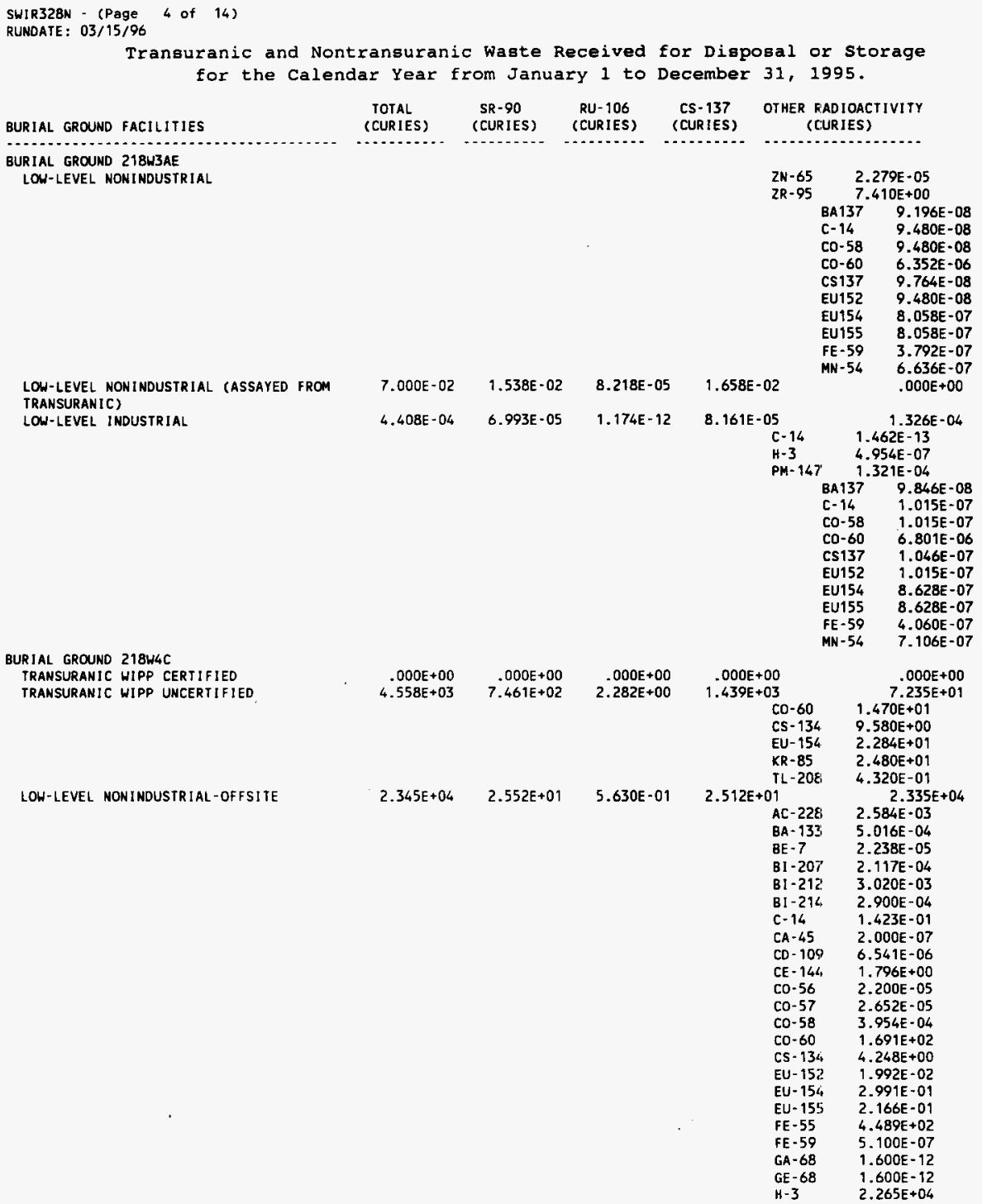


WHC-EP-0125-8

SWIR328N - (Page 5 of 14 )
RUNDATE: $03 / 15 / 96$

Transuranic and Nontransuranic Waste Received for Disposal or Storage for the Calendar Year from January 1 to December 31, 1995.

\begin{tabular}{|c|c|c|c|c|c|}
\hline URIAL GROUMD FACILITIES & $\begin{array}{l}\text { TOTAL } \\
\text { (CURIES) }\end{array}$ & $\begin{array}{l}\text { SR-90 } \\
\text { (CURIES) }\end{array}$ & $\begin{array}{c}\text { RU-106 } \\
\text { (CURIES) }\end{array}$ & $\begin{array}{l}\text { CS-137 } \\
\text { (CURIES) }\end{array}$ & $\begin{array}{l}\text { OTHER RADIOACTIVITY } \\
\text { (CURIES) }\end{array}$ \\
\hline & & 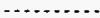 & - & & \\
\hline
\end{tabular}

BURIAL GROUMD 218W4C

LON-LEVEL NONIHDUSTRIAL-OFFSITE

LOW-LEVEL NONINDUSTRIAL

$2.117 \mathrm{E}+03$

$2.339 E+00$

$2.878 E-03$

2. $166 \mathrm{E}+00$

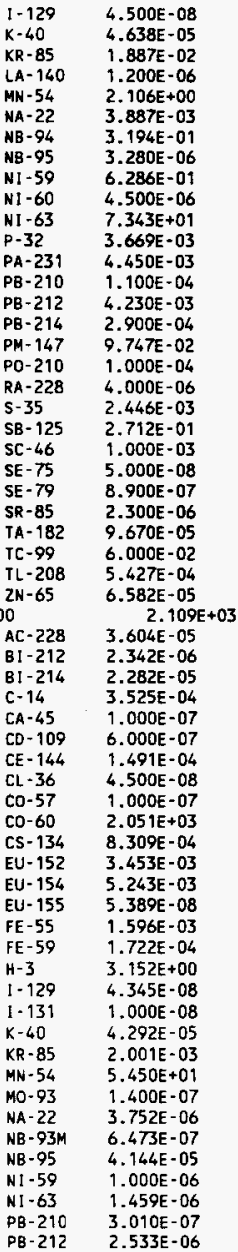


SWIR328N - (Page 6 of 14 )
RUNDATE: $03 / 15 / 96$

Transuranic and Nontransuranic Waste Received for Disposal or storage for the Calendar Year from January 1 to December 31, 1995.

BURIAL GROUND FACILITIES

BURIAL GROUND $218 W 6 C$

LOW-LEVEL. NONINDUSTRIAL
(CURIES)
OTHER RADIOACIIVITY (CUR IES)

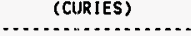

LOW-LEVEL NONINDUSTRIAL (ASSAYED FRON TRANSURANIC)

LOW-LEVEL INDUSTRIAL
$3.795 \mathrm{E}-01$

$4.242 E-04$

$6.135 E-05$

$4.455 E-04$

$1.644 \mathrm{E}-11$

$8.990 E-02$

7. 122E-05
8.338E -02

$\begin{array}{ll}\text { PB- } 214 & 2.682 E-05 \\ \text { PM-147 } & 1.905 E-03 \\ \text { RU-103 } & 2.952 E-09 \\ \text { S-35 } & 3.000 E-06 \\ \text { SB-125 } & 9.324 E-04 \\ \text { SN-113 } & 5.000 E-07 \\ \text { SR }-85 & 1.603 E-05 \\ \text { TA-182 } & 2.200 E-02 \\ \text { IC-99 } & 3.694 E-06 \\ \text { IL-208 } & 1.035 E-05 \\ V-49 & 3.816 E-08 \\ W-185 & 2.600 E-09 \\ Z N-65 & 8.254 E-05 \\ Z R-95 & 2.485 E-05\end{array}$

BA137 7.915E-10

C $-14 \quad 8.160 E-10$

CO-58 8. 160 E- 10

CO-60 5.467E-0B

CS $1378.405 \mathrm{E}-10$

FU152 8.160E-10

EU154 6.936E-09

HU155 6.936E-09

IE-59 3.264E-09

MN $-54 \quad 5.712 E-09$ $.000 E+00$

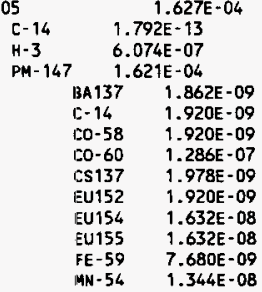

BURIAL GROUND 218W5

LOW-LEVEL NONINDUSTRIAL-OFFSITE
$1.033 E+01 \quad 1.264 E-01 \quad 6.673 E-02 \quad 1.687 E-01$

AC -227

AG -110

BA -133

$\mathrm{BE}=7$

B ! -207

C -14

CD- 109

CE -144

$\mathrm{CL}-36$

CO. 56

Co- 57

Co- 58

Co- 60

CR- 51

C\$- 134

EU- 152

EU- 154

EU- 155
8. $158 E+00$

$2.200 E-06$ $1.846 \mathrm{E}-04$

$1.876 \mathrm{E}-04$

$1.990 \mathrm{E}-01$

$3.890 \mathrm{E}-05$

5.165E-05

$3.363 E-04$

$2.000 \mathrm{E}-02$

$1.000 \mathrm{E}-05$

3. 163E-01

$1.420 \mathrm{E}-01$

$1.384 \mathrm{E}-01$

$7.715 \mathrm{E}-02$

$4.260 E+00$

$2.603 E-03$

$3.158 \mathrm{E}-02$

$3.441 \mathrm{E}-03$

$6.790 E-05$ 
SHIR328N - (Page 7 of 14)

RUNDATE: 03/15/96

Transuranic and Nontransuranic Waste Received for Disposal or Storage for the Calendar Year from January 1 to December 31, 1995.

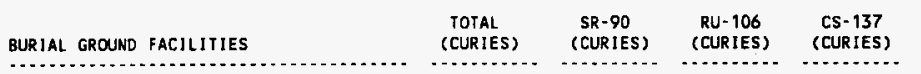
BURIAL GROUND 218W5

LOW-LEVEL NONINDUSTRIAL-OFFSIIE

\begin{tabular}{|c|c|c|c|c|}
\hline & & & 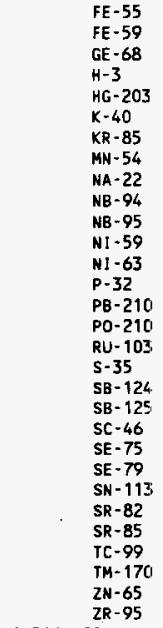 & $\begin{array}{l}3.614 E-01 \\
8.156 E-04 \\
1.037 E-04 \\
1.072 E+00 \\
2.050 E-04 \\
9.259 E-04 \\
2.350 E-06 \\
5.303 E-01 \\
5.802 E-02 \\
7.100 E-04 \\
7.240 E-04 \\
2.100 E-03 \\
6.892 E-01 \\
5.797 E-04 \\
3.136 E-02 \\
3.923 E-03 \\
1.420 E-01 \\
1.692 E-03 \\
1.360 E-06 \\
9.818 E-03 \\
1.483 E-03 \\
3.370 E-05 \\
1.940 E-03 \\
4.520 E-05 \\
1.000 E-08 \\
2.001 E-03 \\
4.269 E-05 \\
3.500 E-07 \\
5.469 E-02 \\
5.040 E-04\end{array}$ \\
\hline $7.651 E+00$ & $1.160 E+00$ & $5.373 E-03$ & 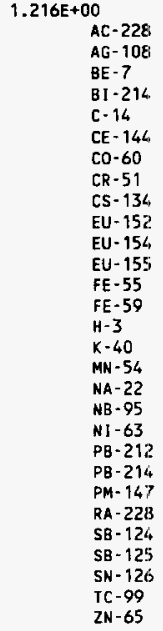 & $\begin{array}{l}2.508 E+00 \\
4.204 E-06 \\
2.800 E-03 \\
1.040 E-05 \\
6.347 E-06 \\
1.563 E-06 \\
4.597 E-05 \\
1.932 E+00 \\
2.000 E-10 \\
2.111 E-04 \\
7.591 E-04 \\
1.133 E-03 \\
1.046 E-04 \\
2.200 E-09 \\
5.359 E-05 \\
1.567 E-05 \\
4.360 E-04 \\
2.254 E-02 \\
1.496 E-04 \\
9.001 E-02 \\
4.947 \mathrm{E}-07 \\
1.062 E-05 \\
6.108 E-06 \\
5.547 E-03 \\
2.300 E-10 \\
2.000 E-08 \\
1.800 E-01 \\
1.930 E-08 \\
1.568 E-03 \\
2.700 E-01\end{array}$ \\
\hline
\end{tabular}


SWIR328N - (Page 8 of 14)

RUNDATE: $03 / 15 / 96$

Transuranic and Nontransuranic Waste Received for Disposal or storage for the Calendiar Year from January 1 to December 31, 1995.

\begin{tabular}{|c|c|c|c|c|c|}
\hline BURIAL -GROUND FACILITIES & $\begin{array}{l}\text { TOTAL } \\
\text { (CURIES) }\end{array}$ & $\begin{array}{l}\text { SR-90 } \\
\text { (CURIES) }\end{array}$ & $\begin{array}{l}\text { RU-106 } \\
\text { (CURIES) }\end{array}$ & $\begin{array}{l}\text { CS }-137 \\
\text { (CURIES) }\end{array}$ & $\begin{array}{l}\text { OTHER RADIOACTIVITY } \\
\text { (CURIES) }\end{array}$ \\
\hline
\end{tabular}

BURIAL GROUND 218 L5

LOW-LEVEL NONINDUSTRIAL
LOW-LEVEL INDUSTRIAL

TRANSURANIC WASIE STORAGE \& ASSAY FACILITY(TRUSAF)

TRANSURANIC (NOT PROCESSED THROUGH

IRUSAF)

IRANSURANIC WIPP CERIIFIED

TRANSURANIC WIPP UNCERTIFIED

\section{5. $000 \mathrm{E}-03$}

$3.964 \mathrm{E}-01$

8. $107 E-02$
$6.591 E-11$

$1.099 E-03$

8. $709 E-02$

$1.156 \mathrm{E}-02$
$3.522 E-13$

$5.870 E-06$

4.654E-04

$6.177 \mathrm{E}-05$
2.355E-06

2R-95

$\begin{array}{ll}\text { BA } 137 & 1.399 E-05 \\ \text { C- } 14 & 1.442 E-05 \\ \text { CO-58 } & 1.442 E-05 \\ \text { Co-60 } & 9.661 E-04 \\ \text { CS137 } & 1.485 E-05 \\ \text { EU152 } & 1.442 E-05 \\ \text { EU154 } & 1.226 E-04 \\ \text { EU155 } & 1.226 E-04 \\ \text { FE-59 } & 5.768 E-05 \\ \text { IMN-54 } & 1.009 E-04 \\ & 4.197 E-06 \\ & 4.197 E-06\end{array}$

Co- 60

$1.185 E-03$

9.391E-02

2.341E-02

B! -214

CE- 144

Co-60

C5- 134

EU-152

$$
\text { EU- } 154
$$

$.000 E+00$

EU- 155

$1.390 \mathrm{E}-06$

$.000 E+00$

$.000 \mathrm{E}+00$

EU- 152

EU- 154

5.550E-02

$1.219 E-02$

$6.516 E-05$

1.315E-02

5.870E-06

1. 185E-03

K-40

$2.874 E-01$

$6.310 E-02$

$3.372 E-04$

6.813E-02

CO- 60

EU- 152 EU- 154

$6.053 E-02$

4. $626 E-03$

$.000 E+00$

$.000 E+00$ $5,493 \mathrm{E}-03$

$1.630 \mathrm{E}-05$

$1.690 E-03$

$1.597 \mathrm{E}-04$

$2.480 E-05$

$1.070 E-03$

$1.162 \mathrm{E}-03$

1.370E-03 1.390E- 06

9.690E-07

4. $210 E-07$ $.000 E+00$

\subsection{E-07} 2. $450 \mathrm{E}-07$

$1.418 E-05$

7.460E-07

1.289E-06

$1.214 E-05$ 4.706E-03
$1.803 E-02$ CS -134
EU- 152
EU- 154

5.520E-06

$3.640 E-03$

$1.060 \mathrm{E}-03$

BA137 9.700E-05

C. $14 \quad 1.000 E-04$

CO-58 1.000E-04

CO-60 6.700E-03

CS137 $1.030 \mathrm{E}-04$

EU152 1.000E-04

EU154 8.500E-04

EU155 8.500E-04

FE-59 4.000E-06

MN-54 7.000E-04

CENTRAL WASTE COMPLEX 2402 WB BUILOING TRANSURANIC WIPP CERTIFIED LOW-LEVEL NONINDUSTRIAL-OFFS!TE

$3.500 E-02 \quad 7.690 E-03$

4. $109 E-05$

8. 292E-03

1. $567 \mathrm{E}-02$

$2.511 E-04$

S. 407E-04

$1.778 \mathrm{E}-03$

BA- 13:3

CO- 60

EU-15i;

LON-LEVEL NONINDUSTRIAL

8.061E-02 1.852E-02

8.870E-05

$1.848 \mathrm{E}-02$

$.000 E+00$ $1.074 \mathrm{E}-04$

2.300E-06

9.800E-05

$7.100 E-06$

8.787E-05

$8.787 E$
$5.729 E-09$ 
SHIR32BN - (Page 9 of 14 )

RUNDATE: $03 / 15 / 96$

Transuranic and Nontransuranic Waste Received for Disposal or storage for the Calendar Year from January 1 to December $31,1995$.

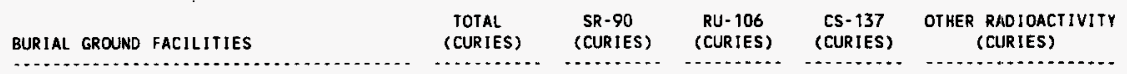

CENTRAL WASTE COMPLEX 2402WB BUILDING LOW-LEVEL NONINDUSTRIAL

CENTRAL WASTE COMPLEX 2402WC BUILDING TRANSURANIC WIPP CERTIFIED TRANSURANIC WIPP UNCERTIFIED LOW-LEVEL NONINDUSTRIAL

CENTRAL WASTE COMPLEX 2402WD BUILOING LOW-LEVEL NOWINDUSTRIAL-OFFS!TE

LOW-LEVEL NONINDUSTRIAL

CENTRAL WASTE COMPLEX 2402WE BUILDING IRANSURANIC WIPP UNCERTIFIED LOW-LEVEL NONINDUSTRIAL

CENTRAL WASTE COMPLEX 2402WG BUILDING TRANSURANIC WIPP UNCERTIFIED LOW-LEVEL NONINDUSTRIAL-OFFSITE

\begin{tabular}{|c|c|c|c|c|}
\hline & & & $\begin{array}{l}C O-60 \\
E U-152 \\
E U-154 \\
E U-155 \\
N A-22 \\
P H-147 \\
T C-99 \\
Z R-95\end{array}$ & $\begin{array}{l}2.856 E-06 \\
1.513 E-05 \\
2.591 E-05 \\
1.036 E-05 \\
9.192 E-06 \\
2.167 E-05 \\
1.567 E-07 \\
2.594 E-06\end{array}$ \\
\hline $\begin{array}{l}3.000 E-02 \\
1.000 E-04 \\
8.737 E-01\end{array}$ & $\begin{array}{l}6.591 E-03 \\
2.197 E-05 \\
2.374 E-01\end{array}$ & $\begin{array}{l}3.522 E-05 \\
1.174 E-07 \\
6.981 E-06\end{array}$ & $\begin{array}{l}7.107 E-03 \\
2.369 E-05 \\
5.600 E-02 \\
\text { AG- } 108 \\
\text { CO-60 } \\
\text { CS-134 } \\
\text { EU-152 } \\
\text { EU-154 } \\
\text { EU-155 } \\
H-3 \\
\text { MN-54 } \\
\text { NB-94 } \\
\text { NB-95 } \\
\text { NI }-63 \\
\text { SB-125 } \\
\text { SR-85 } \\
\text { TC }-99 \\
\text { ZR- } 95\end{array}$ & $\begin{array}{r}-000 E+00 \\
.000 E+00 \\
2.894 E-01 \\
3.340 E-02 \\
7.624 E-02 \\
1.247 \mathrm{E}-06 \\
3.829 \mathrm{E}-05 \\
1.701 \mathrm{E}-01 \\
4.567 \mathrm{E}-06 \\
1.100 \mathrm{E}-08 \\
1.030 \mathrm{E}-04 \\
3.000 \mathrm{E}-05 \\
1.330 \mathrm{E}-08 \\
9.420 \mathrm{E}-03 \\
5.580 \mathrm{E}-05 \\
3.000 \mathrm{E}-08 \\
6.120 \mathrm{E}-08 \\
2.200 \mathrm{E}-08\end{array}$ \\
\hline $3.116 \mathrm{E}-05$ & $7.610 \mathrm{E}-06$ & $5.782 E-10$ & $\begin{aligned} 2.868 E-10 \\
C-14 \\
H-3 \\
K-40 \\
M N-54 \\
P O-210\end{aligned}$ & $\begin{array}{l}1.592 E-05 \\
4.836 E-07 \\
1.530 E-05 \\
1.150 E-07 \\
2.800 E-09 \\
2.200 E-08\end{array}$ \\
\hline $3.620 E-03$ & $5.834 E-06$ & $7.275 E-09$ & $\begin{aligned} 5.435 E-04 \\
C-14 \\
C O-60 \\
E U-152 \\
E U-154 \\
E U-155 \\
H-3 \\
N A-22 \\
P-32 \\
T C-99 \\
Z R-95\end{aligned}$ & $\begin{array}{l}2.550 E-03 \\
5.000 E-04 \\
8.617 \mathrm{E}-09 \\
4.564 \mathrm{E}-08 \\
7.815 \mathrm{E}-08 \\
3.125 \mathrm{E}-08 \\
5.100 \mathrm{E}-04 \\
5.400 \mathrm{E}-04 \\
1.000 \mathrm{E}-03 \\
4.718 \mathrm{E}-10 \\
7.824 \mathrm{E}-09\end{array}$ \\
\hline $\begin{array}{l}5.000 E-04 \\
2.189 E-02\end{array}$ & $\begin{array}{l}1.099 E-06 \\
4.289 E-03\end{array}$ & $\begin{array}{l}5.870 E-07 \\
4.759 E-07\end{array}$ & $\begin{array}{l}1.185 E-04 \\
6.558 E-03 \\
P M-147\end{array}$ & $\begin{array}{l}.000 E+00 \\
5.086 E-04 \\
5.086 E-04\end{array}$ \\
\hline $\begin{array}{l}5.000 E-05 \\
1.008 E-04\end{array}$ & $\begin{array}{l}1.099 E-05 \\
2.092 E-06\end{array}$ & $\begin{array}{l}5.870 E-08 \\
4.283 E-06\end{array}$ & $\begin{array}{l}1.185 E-05 \\
2.326 E-06 \\
\text { CO- } 60 \\
\text { CS }-134 \\
\text { EU- } 152 \\
\text { EU-154 } \\
\text { FE }-55 \\
\text { KR }-85 \\
\text { NI }=63 \\
\text { PM- }-147\end{array}$ & $\begin{array}{r}.000 E+00 \\
1.578 E-07 \\
1.503 E-07 \\
8.600 E-10 \\
1.780 E-09 \\
1.360 E-09 \\
8.900 E-10 \\
2.800 E-10 \\
1.530 E-09 \\
7.100 E-10\end{array}$ \\
\hline
\end{tabular}


SWIR328N - (Page 10 of 14)

RUNDATE : 03/15/96

Transuranic and Nontransuranic Waste Received for Disposal or Storage for the Calendar Year from January 1 to December 31, 1995.

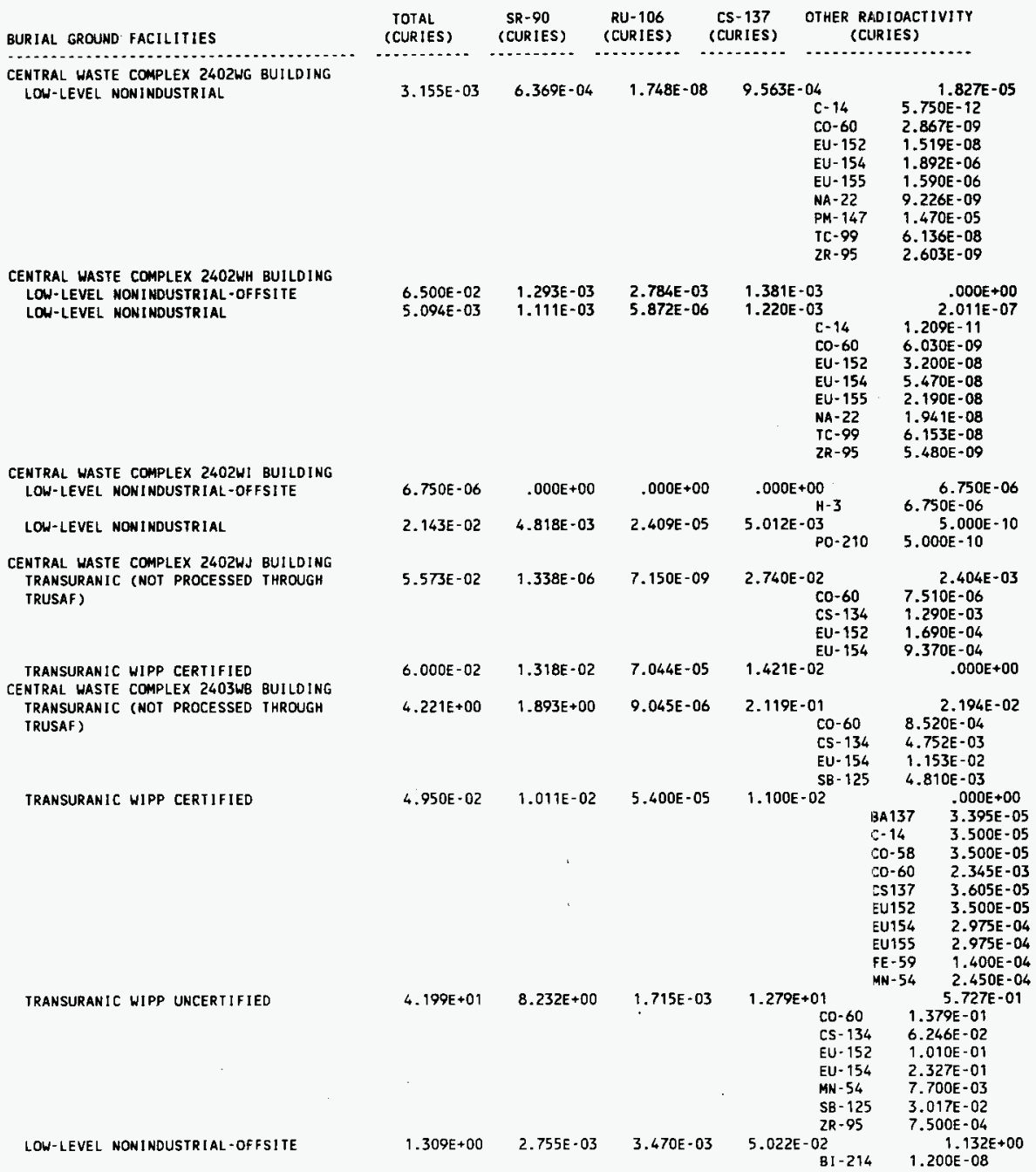




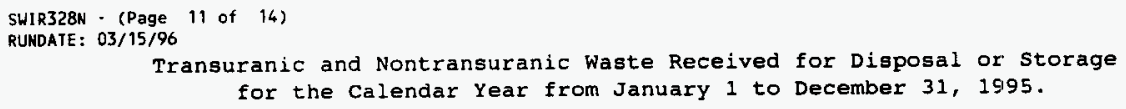

LOW-LEVEL NONINDUSTRIAL

$8.449 E+01 \quad 2.907 E+01 \quad 3.130 E-02 \quad 1.227 E+01$

RADIOACTIVITY
(CURIES)

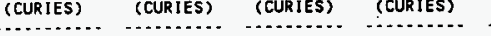

(......................

LOW-LEVEL NONINOUSTRIAL

CD -109

1.000E-05

CO-60 1.012E-03

CS-134 3.255E-04

EU-154 3.331E-05

$\mathrm{H}-3 \quad 8.705 \mathrm{E}-0 \mathrm{~S}$

$\mathrm{K}-40 \quad 4.870 E-06$

NI $-63 \quad 1.130 E+00$

PO-210 2.861E-08

SB-125 $1.989 E-05$

TC- $99 \quad 1.659 \mathrm{E}-05$

BA-133 4.715E-05

C-14 3.044E-09

CE-144 1.059E-02

CO-60 $\quad 1.347 \mathrm{E}+00$

CS-134 4.058E-04

EU-152 2.880E-09

EU-154 4.505E-02

$\mathrm{H}-3 \quad 5.381 \mathrm{E} \cdot 01$

$1-129 \quad 3.690 E-05$

K-40 2.626E-05

MN-54 2.000E-02

NB-93M 8.670E-11

NB- $94 \quad 6.230$ E-04

NB-95 3.001E-02

N1-59 2.900E-12

N1-63 5.247E-0B

P-32 $1.000 E-04$

PB-210 5.500E-07

PM- 147 2.737E-02

RA-228 3.042E-07

SB-125 6.060E-02

SE-79 7.250E-12

SR-85 8.520E-07

SR-89 5.670E-08

TC- $99 \quad 1.390 \mathrm{E}-01$

$2 \mathrm{~N}-65 \quad 9.000 \mathrm{E}-02$

IBA $137 \quad 2.744 \mathrm{E}-04$

E- $14 \quad 2.829 \mathrm{E}-04$

CO-58 2.829E-04

1C0-60 1.895E-02

CS137 2.914E-04

EU152 2.829E-04

EU154 2.405E-03

EU155 2.405E-03

$\mathrm{FE}-59 \quad 1.132 \mathrm{C}-03$

MN-54 1.980E-03

LOW-LEVEL NONINDUSIRIAL (ASSAYED FROM TRANSURANIC)

CENTRAL WASTE COMPLEX 2403 WC BUILDING TRANSURANIC WIPP UNCERTIFIEO LOW-LEVEL NONINDUSTRIAL-OFFSITE
1.830E-01

8.893E-02

$6.722 E-07$

2.536E-03 Co- 60 EU-154

$5.000 E-03$

$1.099 E-03$

5. $732 E-03$

$5.870 \mathrm{E}-06$

$4.727 E-03$

1. $185 \mathrm{E}-03$

3.749E-02

AG- 110

AG- 110

AU -195

BE -7

BI-214

CD- 105

$C D-113$
$1.570 E-04$

4.900E-05

1. $080 \mathrm{E}-04$

$\quad .000 E+00$
$1.277 E+00$
$9.900 E-05$
$4.809 E-05$
$4.270 E-07$
$2.116 E-03$
$2.550 E-08$
$1.746 E-01$
$8.891 E-01$




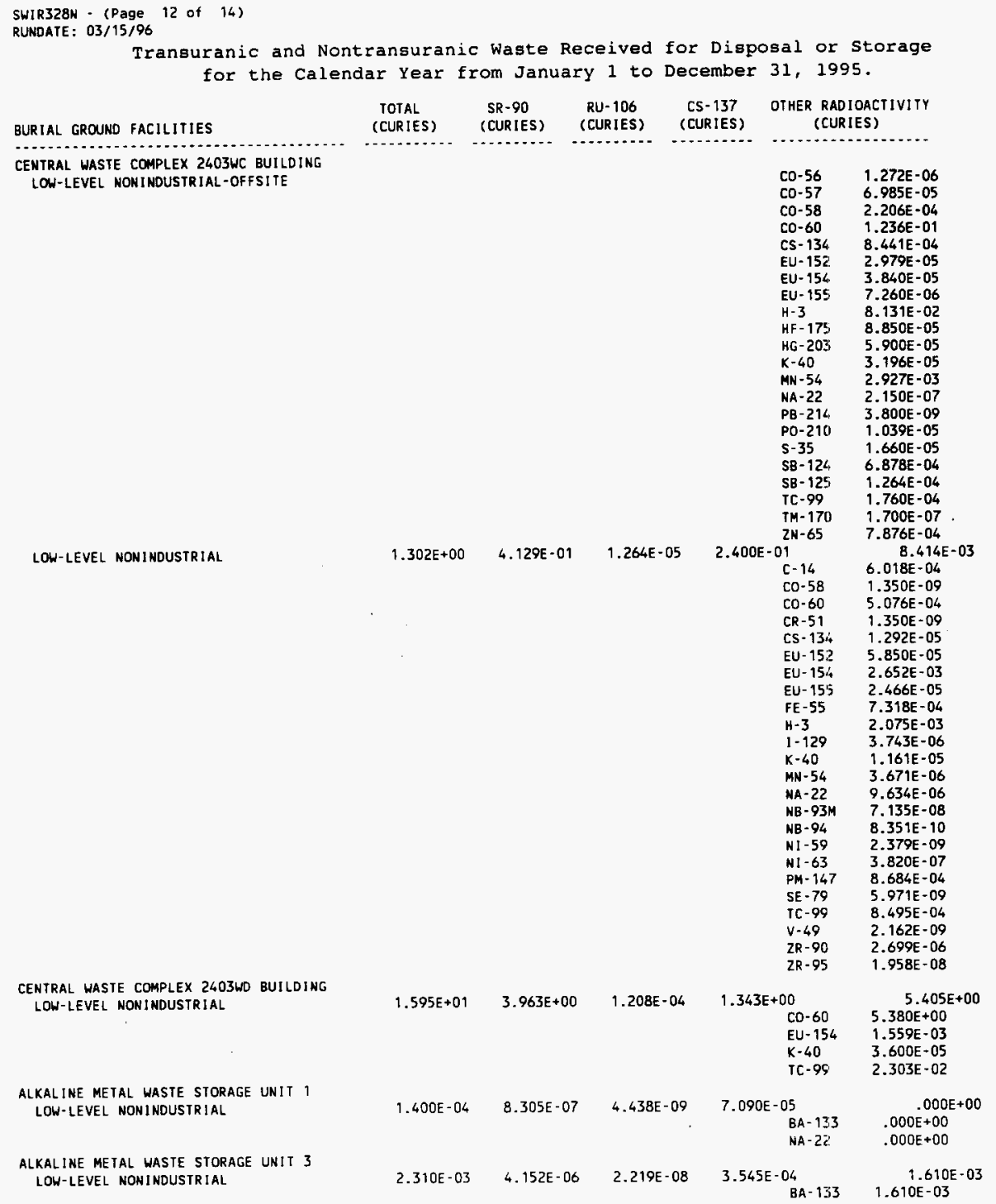




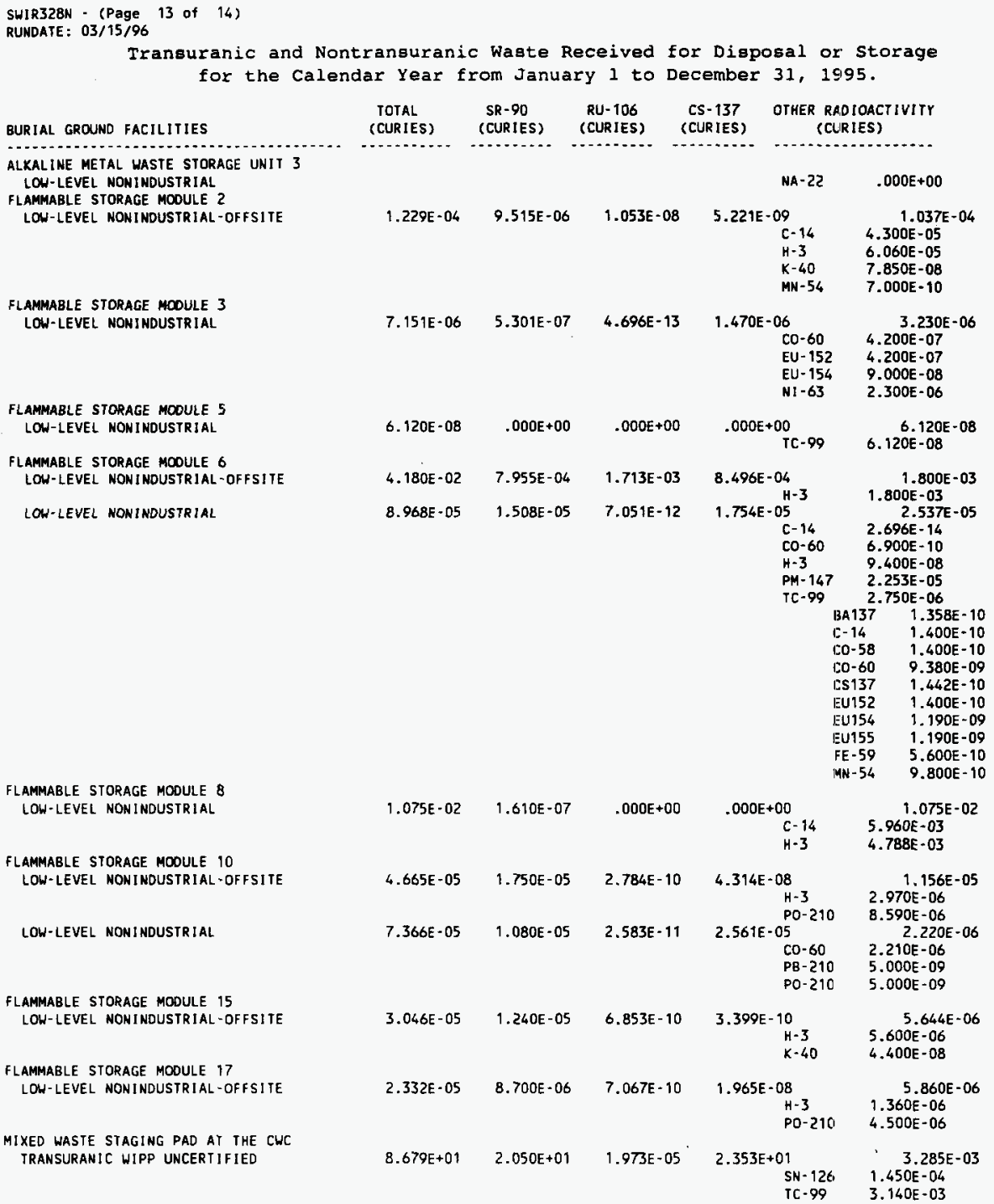


WHC-EP-0125-8

SWIR328N - (Page 14 of 14 )

RUNDATE: 03/15/96

Transuranic and Nontransuranic Waste Received for Disposal or storage for the Calendar Year from January 1 to December 31, 1995.

BURIAL GROUND FACILITIES

Jotal 200W

TOTAL 200 AREAS

\begin{tabular}{lcccc}
$\begin{array}{l}\text { TOTAL } \\
\text { (CURIES) }\end{array}$ & $\begin{array}{c}\text { SR-90 } \\
\text { (CURIES) }\end{array}$ & $\begin{array}{c}\text { RU-106 } \\
\text { (CURIES) }\end{array}$ & $\begin{array}{c}\text { CS-137 } \\
\text { (CURIES) }\end{array}$ & $\begin{array}{c}\text { OTHER RADIOACTIVITY } \\
\text { (CURIES) }\end{array}$ \\
\hline$\ldots \ldots . .$. & $-\ldots \ldots \ldots \ldots$
\end{tabular}

$\begin{array}{lllll}1.127 E+05 & 1.139 E+04 & 3.910 E+00 & 7.384 E+03 & 7.532 E+04 \\ 3.689 E+05 & 1.140 E+04 & 1.192 E+01 & 7.399 E+03 & 3.312 E+05\end{array}$


WHC-EP-0125-8

SWIR3280 - (Page 46 of 107)

RUN DATE: 03/15/96

Land Disposal or Storage of Solid Radioactive Waste from January 1 to Decenber 31 , 1995

TRANSURANIC WASTE STORAGE \& ASSAY FACILITY(TRUSAF), 200 WEST AREA, LOW-LEVEL NONINDUSTRIAI (

( A

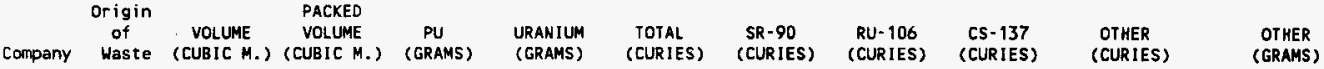

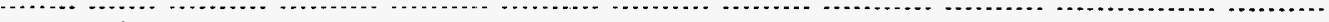

FLOOR LEVEL 1

$\begin{array}{lllllllllll}\text { WHC } & 23452 & 1.457 E+00 & 1.457 E+00 & .000 E+00 & .000 E+00 & 3.500 E-02 & 7.690 E-03 & 4.109 E-05 & 8.292 E-03\end{array}$

FLOOR LEVEL 3

$\begin{array}{lllllllll}\text { WHC } & 23452 & 1.041 E+00 & 1.041 E+00 & .000 E+00 & .000 E+00 & 2.050 E-02 & 4.504 E-03 \quad 2.407 E-05 & 4.856 E-03\end{array}$

TOTALS

$2.498 E+00 \quad 2.498 E+00 \quad .000 E+00 \quad .000 E+00 \quad 5.550 E-02 \quad 1.219 E-02 \quad 6.516 E-05 \quad 1.315 E-0.2$

$\begin{array}{rr}.000 E+00 & 9.714 E-02 \\ A M-241 & 1.652 E-04 \\ P U-238 & 2.645 E-05 \\ P U-239 & 9.101 E-02 \\ P U-240 & 5.529 E-03 \\ P U-241 & 4.079 E-04 \\ P U-242 & 1.844 E-05 \\ & \\ .000 E+00 & 1.702 E \quad 02 \\ A M-241 & 2.320 E-05 \\ P U-238 & 4.950 E-06 \\ P U-239 & 1.595 E-02 \\ P U-240 & 9.706 E-04 \\ P U-241 & 7.300 E-05 \\ P U-242 & 3.250 E-06 \\ .000 E+00 & 1.142 E-01\end{array}$


WHC-EP-0125-8

SW1R3280 - (Page 2 of 107 )

RUN DATE: 03/15/96

Land Disposal or Storage of Solid Radioactive Waste from January 1 to December 31 , 1995

BURIAL GROUND 218 W3A, 200 WEST AREA, LOW-LEVEL NONINDUSTRIAL-OFFSITE.

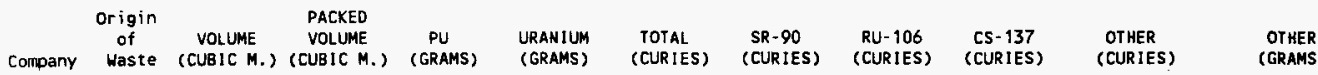

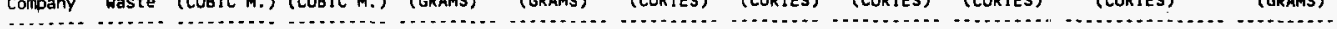

TRENCH 49

ANL ARGON $\quad 5.522 \mathrm{E}+01 \quad 5.522 \mathrm{E}+01 \quad .000 \mathrm{E}+00 \quad .000 \mathrm{E}+00 \quad 5.215 \mathrm{E}-02 \quad 7.355 \mathrm{E}-03 \quad 1.067 \mathrm{E}-05 \quad 8.345 \mathrm{E}-03$

AM- $241 \quad 5.298 \mathrm{E}-03$

AM- $243 \quad 3.050 E-04$

CM-243 2.055E-06

NP-237 4.086E-02

PU-238 9.243E-06

PU-239 7.588E-02

PU-240 7.442E-03

PU-241 2.339E-05

TH-232 3.950E-02

U-233 3.840E-04

U-235 1.060E-04

U-238 3.010E-01

BA-133 1,000E-06

BE-7 $\quad 9.390 E-05$

BI-214 4.400E-0?

CO-56 1.500E-06

CO-57 1.507E-03

CD-58 $\quad 1.500 E-06$

C0.60 4.785E-03

CS-134 2.820E-04

EU-152 2.989E-03

EU-154 9.300E-05

FE-55 $1.790 E-04$

$\mathrm{H}-3 \quad 1.000 \mathrm{E}-06$

MN-56 1.630E-06

NA- $22 \quad 5.000 E-05$

$\mathrm{N} 1-63 \quad 9.943 \mathrm{E}-03$

SB-125 $\quad 1.036 E-03$

SR-85 1.000E-06

TC- $99 \quad 1.200 E-06$

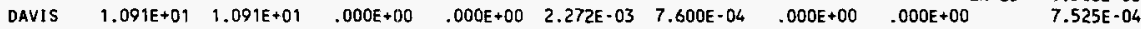

RA-226

$4.871 E-04$

$Y-90 \quad 7.525 \mathrm{E}-04$

4.871E-04 
SWIR3280 - (Page 44 of 107)

RUN DATE: $03 / 15 / 96$

Land Disposal or storage of Solid Radioactive Waste from January 1 to December 31 , 1995

BURIAL GROUND 218W5, 200 WEST AREA, LOW-LEVEL NONINDUSTRIAL.

\begin{tabular}{|c|c|c|c|c|c|c|c|c|c|c|c|}
\hline om & $\begin{array}{c}\text { Origin } \\
\text { of } \\
\text { Waste }\end{array}$ & $\begin{array}{c}\text { VOLUNE } \\
\text { (CUBIC M.) }\end{array}$ & $\begin{array}{l}\text { PACKED } \\
\text { VOLUME } \\
\text { (CUB!C M.) }\end{array}$ & $\begin{array}{c}\text { PU } \\
\text { (GRAMS) }\end{array}$ & $\begin{array}{l}\text { URAN IUM } \\
\text { (GRAMS) }\end{array}$ & $\begin{array}{l}\text { TOTAL } \\
\text { (CUR1ES) }\end{array}$ & $\begin{array}{l}\text { SR-90 } \\
\text { (CURIES) }\end{array}$ & $\begin{array}{l}\text { RU-106 } \\
\text { (CURIES) }\end{array}$ & $\begin{array}{l}\text { CS }-137 \\
\text { (CURIES) }\end{array}$ & $\begin{array}{l}\text { OTHER } \\
\text { (CURIES) }\end{array}$ & $\begin{array}{c}\text { OTHER } \\
\text { (GRAMS) }\end{array}$ \\
\hline
\end{tabular}

TRENCH 29

WHC $2415 X$

$2415 Y$

$3.625 E+00 \quad 3.625 E+00$

$.000 E+00$

$.000 E+00 \quad 5.030 E-06 \quad 1.931 E-06$

$2.818 E-12$

6.006E-07

$241 T x$

$3.625 E+00 \quad 3.625 E+00$

$.000 E+00$

$.000 E+$

$6.870 E-$

$\begin{array}{lll}1.243 E-05 & 1.350 E-10 & 2.253 E-05\end{array}$

2410

$7.250 E+00 \quad 7.250 E+00$

$.000 \mathrm{E}+100$

$.000 \mathrm{E}+00$

$6.024 E-04$

$2.304 E-04$

8.139E-10 7.274E-05

2718

$.000 E+00$

$.000 E+00$

4.216E+01 4.216E+01

$.000 E+100$

$2.447 \mathrm{E}-02$

$1.100 \mathrm{E}-02$

$2.556 \mathrm{E}-03$

5.446E-07 9.923E-03

308
$3.920 E+01 \quad 3,920 E+01$

313

\section{$2.538 E+01 \quad 2.538 E+01$}

$.000 E+00$

$.000 \mathrm{E}+00$

$2.076 \mathrm{E}-05$

$.000 \mathrm{E}+00$

333

$9.788 \mathrm{E}+01 \quad 9.788 \mathrm{E}+01$

$.000 E+00$

$.000 E+00$

$2.994 E-05$

$.000 E+00$

$.000 E+00$

\section{EU- 152}

437

6290

$$
\text { 4.164E-01 4. 164E-01 }
$$

$.000 E+00$

$.000 \mathrm{E}+0$

$9.391 E-06$

$2.541 E-07 \quad 1.890 E-91$

4.564E-06
$.000 E+00 \quad 1.255 E-02 \quad 4.609 E-03$

6

$6.077 E-08 \quad 1.628 E-03$

IC-

IC

Co- 60

EU- 154

MN -54

EU-152 1.080E-06

EU- $154 \quad 1.950 E-07$

$.000 E+00 \quad 7.954 E-08$

AM-241 $1.980 \mathrm{E}-12$

PU-238 2.230E-11

PU-239 7.450E-08

PU-240 4.570E-09

PU-241 4.330E-10

PU-242 1.580E 11

$.000 E+00 \quad 4.047 E-06$

AM-241 4.580E-10

PU-238 1.130E-09

PU-239 3.790E-06

PU-240 2.330E-07

$\begin{array}{ll}P U-241 & 2.200 E-08\end{array}$

PU-242 8.040E-10

$.000 E+00 \quad 1.753 E-05$

AM-241 1.982E-09

PU-238 4.904E-09

PU-239 $1.641 E-05$

PU-240 1.011E-06

PU-241 9.529E-0B

PU-262 3.473E-09

$.000 E+00 \quad .000 E+00$

$9.896 E-04 \quad 3.400 E+03$

AM-241 1.840E-10

PU-240 2.780E-09

TH-228 6.295E-07

$\mathrm{U}-235 \quad 2.248 \mathrm{E}+01$

U-238 3.377E+03

$4.251 E-04$

$5.573 E-04$

4. $453 \mathrm{E}-06$

2.700E-06

2.076E-05

$\mathrm{U}-234$

U-235

u-236

U-238

2.076E-05

2.994E-05

$\mathrm{u}-234$

$\mathrm{U}-235$

$\mathrm{U}-236$

U-238

TC-99 2.994E-05

$4.504 \mathrm{E}-01$

Co-60 4.281E-01

CS-134 1.694E-04

MN-54 2.211E-02

NA-22 $5.700 E-05$

$.000 \mathrm{E}+00$

AM- 241

PU-238

PU -239

PU-240

PU-241

PU-242

$1.617 \mathrm{E}-04$

AM- -241
$1.221 \mathrm{E}+02$

8.195E-03

$1.157 E+00$

7. 206E-02

$1.208 E+02$

$1.761 E+02$

$1.178 \mathrm{E}-02$

$1.667 \mathrm{E}+00$

$1.038 \mathrm{E}-01$

$1.743 \mathrm{E}+02$

$.000 E+00$

2.129E 10

$5.270 \mathrm{E}-10$

1.758E-06

1.081E-07

1.022E-08

$3.740 \mathrm{E}-10$

1.835E-04

$2.077 \mathrm{E}-08$ 
SWIR3280 - (Page 4 of 107)

RUN DATE: 03/15/96

Land Disposal or Storage of Solid Radioactive Waste from January 1 to Decenber 31 , 1995

BURIAL GROUND 218W3A, 200 WEST AREA, LOW-LEVEL NONINDUSTRIAL.

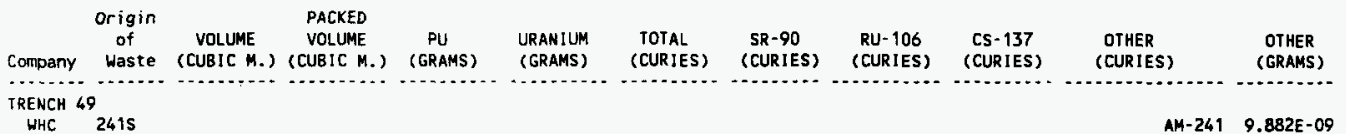

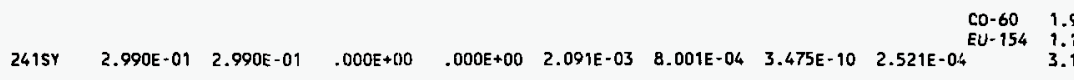

PU-238 $2.117 \mathrm{E}-08$

PU-239 1.014E-04

PU-240 4.355E-06

PU-241 4.114E-07

PU-242 1.368E-08

$.124 E-06$

$120 \mathrm{E}-07$ 3.311E-05

AM-241 3.740E-09

PU-238 9.290E-09

PU- 239 3.100E-05

PU-240 1.910E-06

PU-241 1.800E-07

PU-242 6.620E-09

$2411 \quad 2.990 E-01 \quad 2.990 E-01 \quad 000 E+00 \quad 000 E+00 \quad 2.150 E-04 \quad 8.601 E-05 \quad 4.031 E-11 \quad 2.101 E-05 \quad C 0-60 \quad 3.120 E-07$

$3.410 \mathrm{E}-07 \quad 7.540 \mathrm{E}-06$

AM- $241 \quad 8.540 E-10$

PU-238 $2.190 \mathrm{E} \cdot 09$

PU-239 7.060E-06

PU-240 $4.340 E-07$

PU-241 6.110E-08

PU-242 $1.500 E-09$

$241 \mathrm{TX} \quad 2.990 \mathrm{E}-01 \quad 2.990 \mathrm{E}-01 \quad .000 \mathrm{0}+100 \quad .000 \mathrm{E}+00 \quad 4.910 \mathrm{E}-04 \quad 1.731 E-04 \quad 5.612 \mathrm{E}-10 \quad 7.421 E-05$

$1.540 \mathrm{E}-07$

EU-154 1.870E-07

.000400 4.910E-04 1.731E-04 $5.612 E-10 \quad 7.421 E-05$

$1.870 E-07$
$3.230 E-07$

AM-241 1.380E-09

PU-23B 3.420E-09

PU-239 1.160E-05

PU-260 7.050E-07

PU-24? 6.640E-08

PU-242 2.420E-09

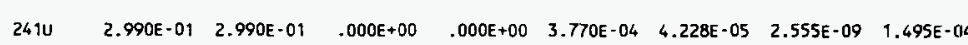

CO-60 1.360E-07

EU-154 1.870E-07

$1.270 \mathrm{E}-06$

AM-241 $1.720 \mathrm{E}-09$

PU $-238 \quad 6.260 E-09$

PU $-239 \quad 1.430 E-05$

PU-240 8.770E-07

PU-241 8.280E-08

PU-242 3.030E-09

$\begin{array}{llllllll}\text { T PLANT } 1.940 E+01 & 1.940 E+01 & .000 E+00 & .000 E+00 & 5.200 E-02 & 1.545 E-02 & 1.371 E-06 & 1.079 E-02\end{array}$

CO.60 $\quad 1.700 E-07$

EU-154 1.100E-06

$.000 \mathrm{E}+00 \quad 3.127 \mathrm{E}-02$

PU-239 2.930E-02

PU-260 1.799E-03

PU-241 1.705E-04 
SWIR3280 - (Page 42 of 107)

RUN DATE: 03/15/96

Land Disposal or Storage of Solid Radioactive Waste from January 1 to December 31 , 1995 BURIAL GROUND 218W5, 200 WEST AREA, LOW-LEVEL NONINDUSTRIAL.

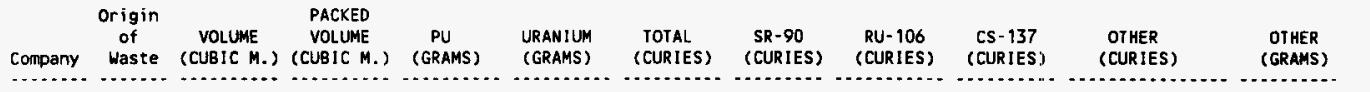

TRENCH 29

PNL 329

331

$1.245 E+01 \quad 1.245 E+01 \quad .000 E+00$

$.000 E+00$

$1.500 E-03 \quad 3.296 E-04$

1. $761 \mathrm{E}-06$

$3.554 E-014$

WHC

$105 \mathrm{KE}$

$7.658 \mathrm{E}+01 \quad 7.658 \mathrm{E}+01$

$.000 E+00$

$9.145 E-06$

1.769E-06 $\quad 1.281 E-12 \quad 2.712 E-016$

$$
016
$$

$105 \mathrm{KW}$

$1.813 E+01 \quad 1.813 E+01$

$0005+00$

$0005+00$

$3.060 E-07$

$07 \quad 5.221 E-08 \quad 5.870 E-13 \quad 9.706 \mathrm{E}-\mathrm{C} \mid 8$ $M N+54$

\section{CO- 60}

MN -54

182KE

$4.078 E+00 \quad 4.078 E+00$

$.000 E+00$

$9.879 \mathrm{E}-08$

$08 \quad 1.740 E-08 \quad 1.057 E-14 \quad 3.176 E-018$

NI -63

$\mathrm{CO}-60$

MN- 54

$202 \mathrm{~A}$

$1.885 E+02 \quad 1.885 E+02$

$.000 E+00$

$.000 E+00 \quad 1.597 E-02 \quad 2.892 E-03$

$3.805 E-0 B \quad 3.391 E-013$

N1-63

(1)

209E

$1.163 E+01 \quad 1.163 E+01$

$.000 E+00$

$.000 E+00$

1.585E-03 1.748E-09 5.846E-04$$
04
$$

2225

$1.269 E+02 \quad 1.269 E+02$

$.000 E+00$

$.000 E+00$

$3.462 E-0$

9.6

$9.623 \mathrm{E} \cdot 02$

6

.490E-06 7.628E-012

Co- 60

C -14

$\mathrm{H}-3$

PM- 147

C- 99

22
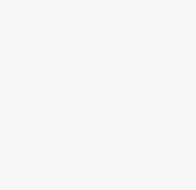

2240

$7.613 E+01 \quad 7.613 E+01$

$.000 E+00$

CS137 1.485E-05

CO-58 1.442E-05

CO-60 $9.661 \mathrm{E}-04$

EU155 1.226E-04

MN-54 1.009E-04

7.613E+01 $7.613 E+01.000+00.000+000$


WHC-EP-0125-8

SWIR3280 - (Page 6 of 107)

RUN DATE: 03/15/96

Land Disposal or storage of solid Radioactive Waste from January 1 to Decenber 31 , 1995

BURIAL GROUND 218 W3AE, 200 WEST AREA, LOW-LEVEL NONINDUSTRIAL (ASSAYED FROM TRANSURANIC).

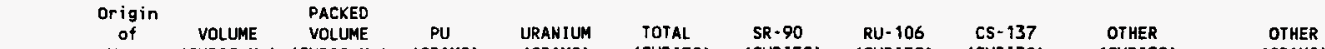

Company Waste (CUBIC M.) (CUBIC M.) (GRAMS) (GRAMS) (CURIES) (CURIES) (CURIES) (CURIES) (CURIES)

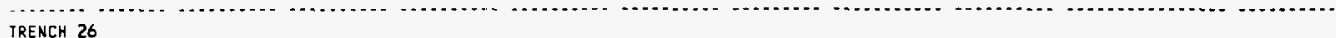

WHC $\quad 23452 \quad 2.915 E+00 \quad 2.915 E+00 \quad .000 E+00 \quad .000 E+00 \quad 7.000 E-02 \quad 1.538 E-02 \quad 8.218 E-05 \quad 1.658 E-02$

$.000 E+00 \quad 2.804 E-02$ AN-241 3.928E-05

PU-238 8.136E-06 PU-239 2.626E-02 PU-240 1.599E-03 PU-241 1.231E-04

$\begin{array}{rr}\text { PU-242 } & 5.488 E-06 \\ .000 E+00 & 2.804 E-02\end{array}$

.


SWIR3280 - (Page 40 of 107 )

RUN DATE : 03/15/96

Land Disposal or storage of Solid Radioactive Waste from January 1 to December 31 , 1995

BURIAL GROUND 218W5, 200 WEST AREA, LOW-LEVEL NONINDUSTRIAL.

\begin{tabular}{|c|c|c|c|c|c|c|c|c|c|c|c|}
\hline & Origin & & PACKED & & & & & & & & \\
\hline$y$ & $\begin{array}{l}\text { of } \\
\text { Waste }\end{array}$ & $\begin{array}{c}\text { VOLUME } \\
\text { (CUBIC M.) }\end{array}$ & $\begin{array}{l}\text { VOLUME } \\
\text { (CUB IC M.) }\end{array}$ & $\begin{array}{c}\text { PU } \\
\text { (GRAMS) }\end{array}$ & $\begin{array}{l}\text { URANIUM } \\
\text { (GRANS) }\end{array}$ & $\begin{array}{l}\text { IOTAL } \\
\text { (CURIES) }\end{array}$ & $\begin{array}{l}\text { SR-90 } \\
\text { (CURIES) }\end{array}$ & $\begin{array}{l}\text { RU- } 106 \\
\text { (CURIES) }\end{array}$ & $\begin{array}{l}\text { CS- } 137 \\
\text { (CURIES) }\end{array}$ & $\begin{array}{l}\text { OTHER } \\
\text { (CURIES) }\end{array}$ & $\begin{array}{c}\text { OTHER } \\
\text { (GRAMS) }\end{array}$ \\
\hline
\end{tabular}

TRENCH 29

BHI $105 \mathrm{~N}$
$109 \mathrm{~N} \quad 2.538 \mathrm{E}+01 \quad 2.538 \mathrm{E}+01 \quad .000 \mathrm{0}+00 \quad .000 \mathrm{O}+00 \quad 2.153 \mathrm{E}-07 \quad 7.703 \mathrm{E}-09 \quad 1.644 \mathrm{E}-14 \quad 2.100 \mathrm{E}-0.08$

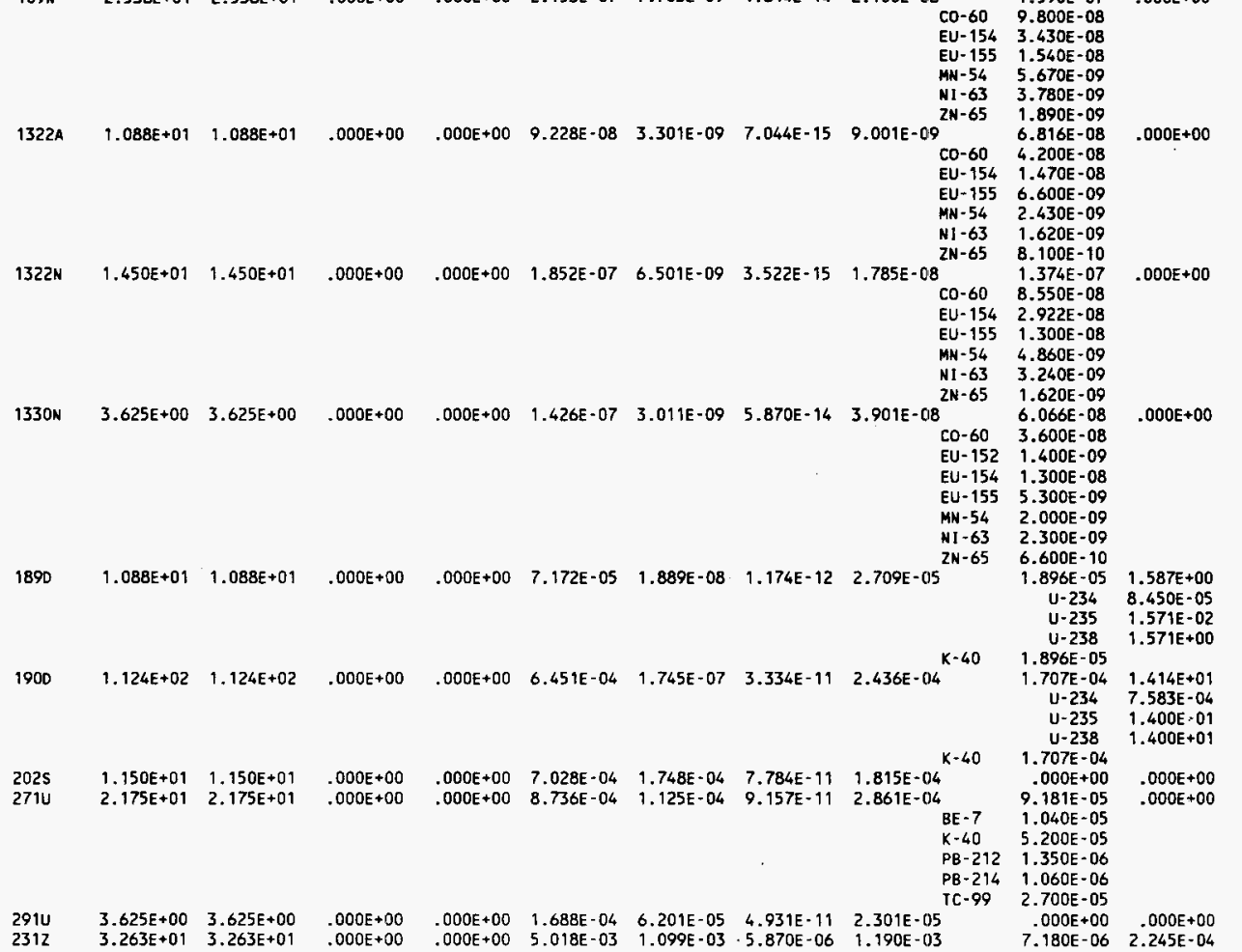

$2.780 E-02$

EU-154 7.414E-04

EU- 155 1.575E-07

MN-54 5.894E-08

NI-63 3.996E-08

SN-126 1.930E-08

TC-99 3.860E-07

ZN-65 1.978E-08

$1.590 E-07$

EU-155

EU-152 1.400E-09

EU-154 1.300E-08

EU-155 5.300E-09

$\mathrm{NI}-63 \quad 2.300 \mathrm{E}-09$

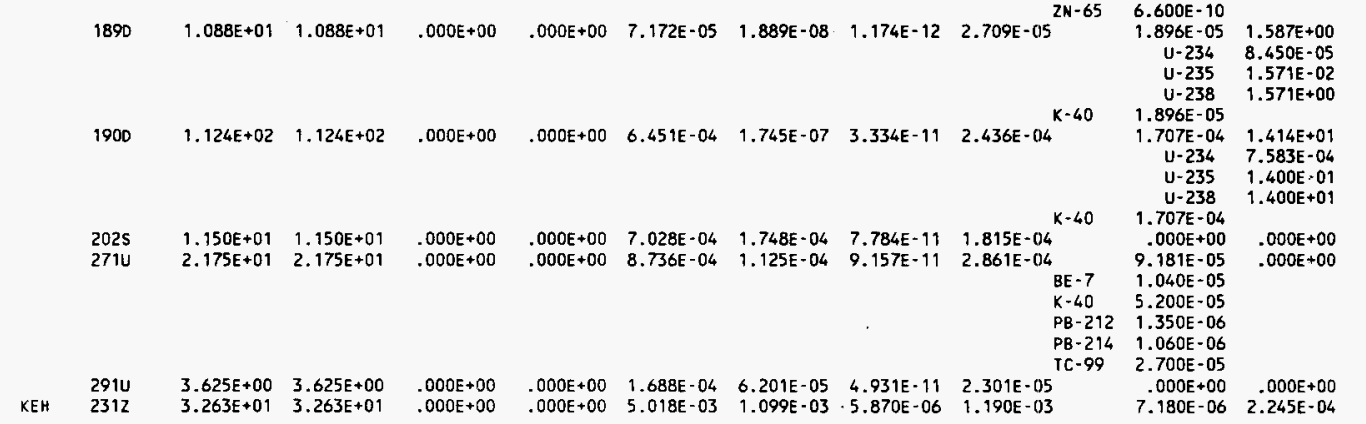


SWIR3280 - (Page 8 of 107 )

RUN DATE: 03/15/96

Land Disposal or Storage of Solid Radioactive Waste from January 1 to Decernber 31 , 1995

BURIAL GROUND 218 W3AE, 200 WEST AREA, LOW-LEVEL NONINDUSTRIAL-OFFSITE.

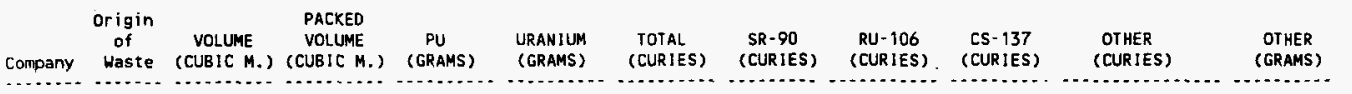

TRENCH 16

ANL ARGON

PPL PRINC

PRINC

$2.340 E+00 \quad 2.340 E+00$

$.000 E+10$

$\begin{array}{lllll}.000 E+00 & 7.500 E+02 & .000 E+00 & .000 E+00 & .000 E+00\end{array}$

TC -99

UCD DAVIS

$5.678 E+00 \quad 5.678 E+00$

$.000 E+00$

$.000 E+00$

2.847E-04 1.421E-04 $1.756 E-08$

8. $709 E-0$ ?

TRENCH 26

AME AMES

AMES

$1.176 E+01 \quad 1.176 E+01$

$.000 E+00$

$.000 E+00 \quad 4.367 E-03$

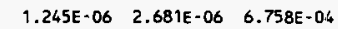

DC-227
$A C-228$
$B I-212$
$B I-214$
$C O-60$
$P A-231$
$P B-212$
RA-228
$T L-208$
02

$.000 E+00$

$3.555 E-04$ 3.555E-04

$2.992 E-03$ TH-228 5.817E 07 $\mathrm{IH}-232 \quad 7.673 \mathrm{E}+\mathrm{O} 3$ TH-234 8.680E-08 U-234 7.111E-04 $U-235 \quad 1.600 E+00$ $U-238 \quad 3.200 E+03$

$3.900 E-10$

$7.672 \mathrm{E}-06$

$2.802 E-04$

3. $720 E-07$

7.961E-04

1.500E-09

2. $802 E-04$

$7.674 \mathrm{E}-04$

$1.001 E-04$

$3.163 \mathrm{E}+00$

$A M-241$

AM- 243

CF- 251

$\mathrm{CF}-252$

CM. 242

$\mathrm{CM}-243$

$\mathrm{CM}-244$

$\mathrm{CM}-245$

NP -237

PU- 238

PU-239

PU-240

PU-24

PU-242

PU- 244

RA- 22

RA- 226

TH -228

$\mathrm{TH}-230$

$\mathrm{TH}-232$

TH-234

$\mathrm{U}-232$

$\mathrm{U}-233$

u-234

$\mathrm{U}-235$

U- 236

$\mathrm{U}-238$

AC -227

$1.000 \mathrm{E}-06$

$A G-110 \quad 9.600 E-06$

$B A=133 \quad 3.634 E-06$

BE-7 $1.022 \mathrm{E}-03$

B1-212 1.730E-05

BI $-214 \quad 3.670 E-06$

C. $14 \quad 5.245 \mathrm{E}-05$

CA-45 $1.500 E-03$

CD-109 2.232E-05
$2.428 E+04$

$1.161 E-03$

2.316E-03

$1.500 E-07$

$9.322 E-10$

$3.020 E-12$

$2.928 E-06$

5.719E-06

8. $100 E-06$

3.975E-01

$1.473 E-05$

3. 158E-01

$1.070 E-02$

3.490E-OS

1. $000 \mathrm{E}-04$

$2.319 E-02$

$5.000 E-14$

$3.531 \mathrm{E}-05$

1. $400 E-09$

$2.856 \mathrm{E}-03$

$2.811 E+00$

2. $950 E-11$

2. $128 \mathrm{E}-05$

7.132E-01

1.270E-01

$1.154 \mathrm{~F}+01$

2.816E-01

$2.426 E+04$ 
SWIR3280 - (Page 38 of 107 )

RUN DATE: $03 / 15 / 96$

Land Disposal or Storage of Solid Radioactive Waste from January 1 to December 31 , 1995

BURIAL GROUND 218W5, 200 WEST AREA, LOW-LEVEL NONINDUSTRIAL-OFFSTTE.

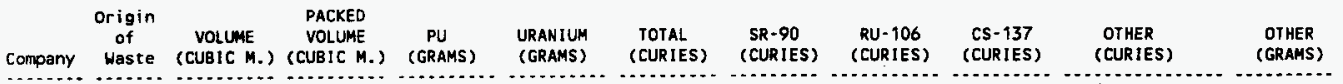

IRENCH 29

BNL BNLAB
HA-22 B.122E-03

NB- $94 \quad 7.100 E-04$

NB-95 7.240E-04

N1-63 4.400E-05

P-32 5.797E-04

PB-210 8.300E-05

PO-210 3.240E-06

RU-103 1.420E-01

S-35 1.692E-03

SB-126 1.360E-06

SB- $125 \quad 7.100 E-06$

SC-46 1.483E-03

SE-75 3.370E-05

SN-113 4.520E-05

SR-82 1.000E-08

SR-85 2.001E-03

TC-99 4.269E-05

TM- 170 3.500E-07

ZN-65 5.209E-02

ZR-95 5.040E-04

RFP RF $\quad 5.565 E+01 \quad 5.565 E+01 \quad .000 E+00 \quad .000 E+00 \quad 1.750 E-01 \quad 3.480 E-03 \quad 7.495 E-03 \quad 3.717 E-03$

RKD ESG $\quad 1.950 E+01 \quad 1.950 E+01 \quad .000 E+00 \quad .000 E+00 \quad 8.349 E-01 \quad 4.398 E-04 \quad 3.505 E-07 \quad 4.134 E-03$

SLA SLACU $\quad 1.675 E+01 \quad 1.675 E+01 \quad .000 E+00 \quad .000 E+00 \quad 1.105 E-01 \quad 3.083 E-04 \quad 6.639 E-04 \quad 3.292 E-04$

$\operatorname{Co} 57$

TOTALS
$.000 E+00$

$.000 E+00$

$1.033 E+0$
$1.264 E-01$

$6.673 \mathrm{E}-0$
$M N-54$

NA- 22

$2 \mathrm{~N}-65$

$.000 E+00$ AM- 241 PU- 239

U-233

U. 235

U-238

8.260E-01 PU-238 $\mathrm{u}-234$

$\mathrm{J}-235$ U-238

CO- 60

CS-134

EU-152

EU-154

FE -55

NI -59

NI-63

$1.088 \mathrm{E}-02$

3. $030 \mathrm{E}-05$

2.004E-02

1.881E-03

3.567E- 01

2. 100E-03

4. 344E-01

9.500E-02

8. 800 - 03

$3.390 E-02$

4. $990 \mathrm{E}-02$

2. $400 E-03$

8. $158 E+00$

$1.788 E+04$ 
SWIR3280 - (Page 10 of 107)

RUN DAIE: 03/15/96

Land Disposal or Storage of Solid Radioactive Waste from January 1 to December 31 , 1995 BURIAL GROUND 218W3AE, 200 WEST AREA, LOW-LEVEL NONINDUSTRIAL-OFFSITE.

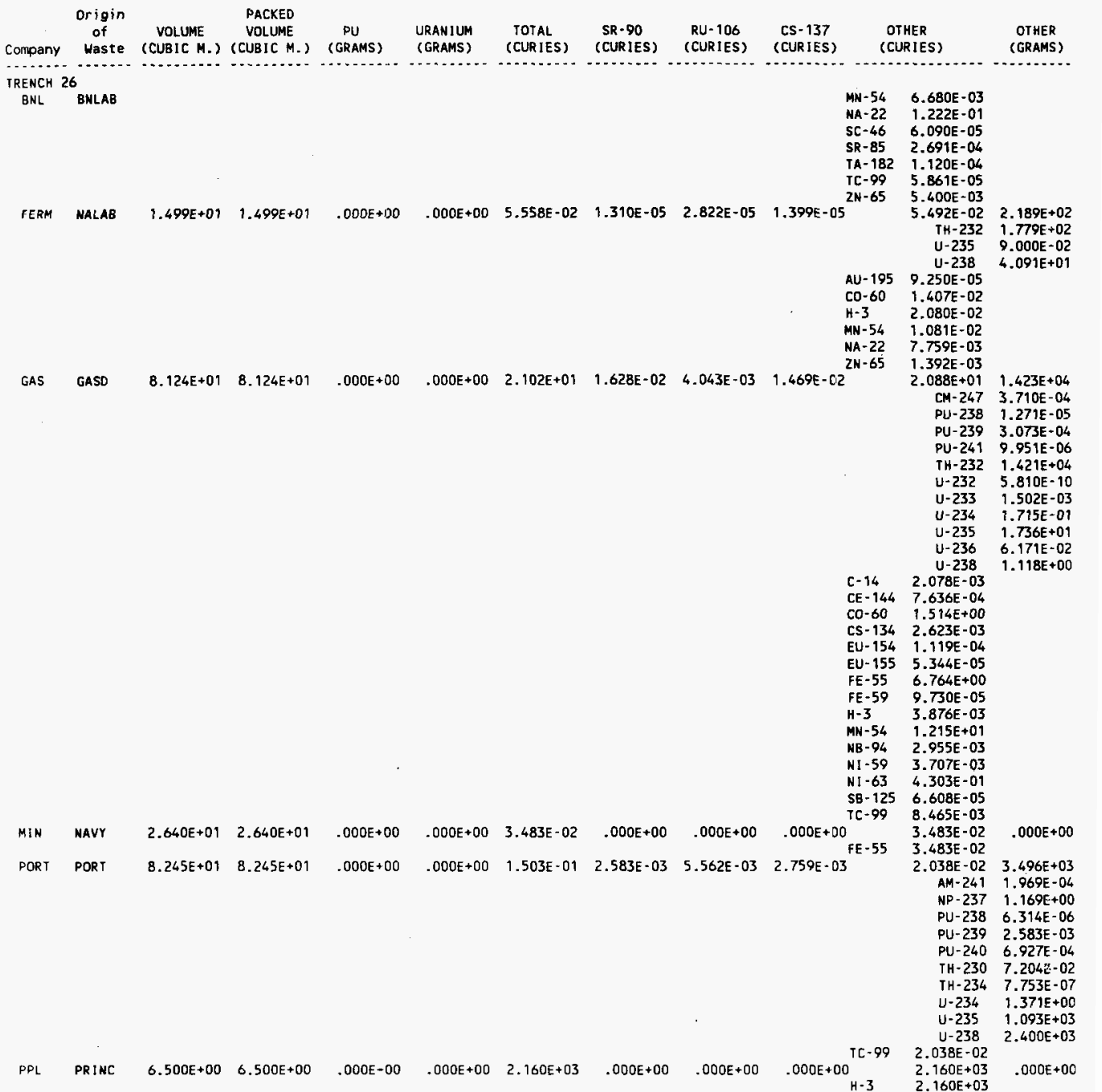


WHC-EP-0125-8

SWIR3280 - (Page 36 of 107)

RUN DATE: $03 / 15 / 96$

Land Disposal or Storage of Solid Radioactive Waste from January 1 to December 31 , 1995 BURIAL GROUND 218W5, 200 WEST AREA, LOW-LEVEL INDUSTRIAL.

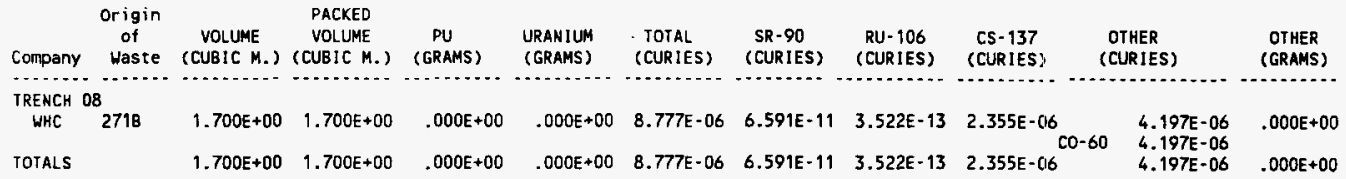


SWIR3280 - (Page 12 of 107)

RUN DATE : 03/15/96

Land Disposal or storage of Solid Radioactive Waste from January 1 to December 31 , 1995

BURIAL GROUND 218W3AE, 200 WEST AREA, LOW-LEVEL NONINDUSTRIAL.

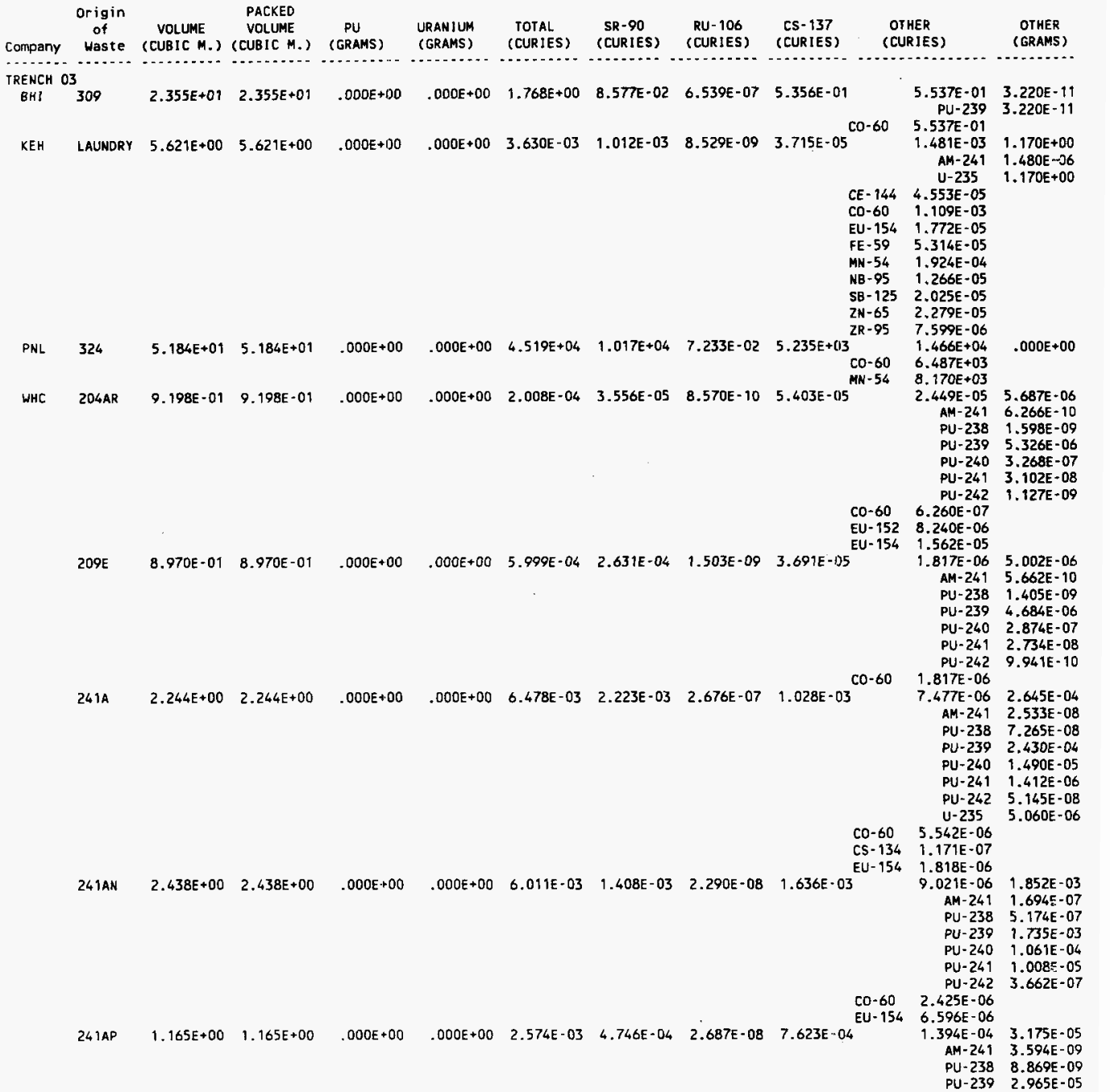




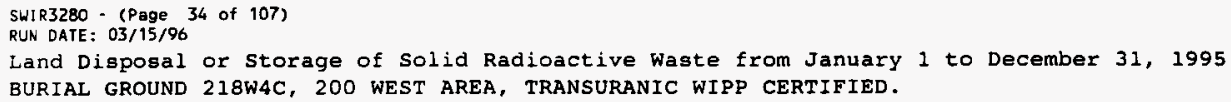

TOTALS

2.082E-01 2.082E-01

2.082E-01 2.082E-01

$\begin{array}{lllll}.000 E+00 & .000 E+00 & .000 E+00 & .000 E+00 & .000 E+00 \\ .000 E+00 & .000 E+00 & .000 E+00 & .000 E+00 & .000 E+00\end{array}$

$-000 E+00$
$-000 E+00$

$.000 E+00$
$.000 E+00$

$.000 E+00$
$.000 E+00$ 
SWIR3280 - (Page 14 of 107)

RUN DATE: 03/15/96

Land Disposal or storage of Solid Radioactive Waste from January 1 to Decenber 31 , 1995 BURIAL GROUND 218W3AE, 200 WEST AREA, LOW-LEVEL NONINDUSTRIAL.

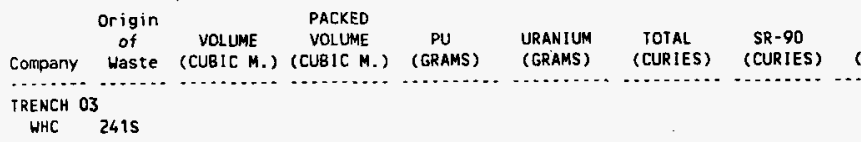

RU-106

(CURIES)

CS- 137 OTHER
(CURIES)

OTHER (GRAMS) RENCH 03

$\begin{array}{lllllllll}2415 X & 8.928 E-01 & 8.928 E-01 & .000 E+C 10 & .000 E+00 & 2.561 E-04 & 9.833 E-05 & 1.749 E-10 & 3.056 E-0.5 \\ 2415 Y \quad 3.874 E+00 & 3.874 E+00 & .000 E+010 & .000 E+00 & 9.018 E-03 & 3.177 E-03 & 2.685 E-08 & 1.365 E-0.3\end{array}$

EU-154

7.076E-06

4.731E-06

$.000 E+00 \quad 4.047 \mathrm{E}-06$

$A M-261 \quad 4.570 E-10$

PU-238 1.136E-09

PU-239 3.790E-06

PU-240 2.324E-07

PU-241 2.195E-08

PU-242 8.020E-10

4.278E-06 3.893E-04

AM-241 3.792E-08

PU-238 1.090E-07

PU-239 3.646E-04

PU-240 2.238E-05

PU-241 2.124E-06

PU-242 7.725E-0B

$241 T \quad 2.415 E+00 \quad 2.415 E+00 \quad .000 E+00 \quad .000 E+00 \quad 1.303 E-03 \quad 3.711 E-04 \quad 4.114 E-09 \quad 2.756 E-04$

CO-60 2.056E-06

CS-134 1.061E-07

EU-154 2.116E-06

2.411E-05

AM- 241

$1.674 E-04$

PU-238 4.658E-08

PU-239 1.569E-04

PU-240 9.578E-06

PU-241 9.041E-07

PU-242 3.295E-08

$2411 \times \quad 1.135 E+00 \quad 1.135 E+00 \quad .000 E+00 \quad .000 E+00 \quad 2.487 E-03 \quad 7.941 E-04 \quad 1.099 E-09 \quad 4.613 E-C 4$

Co-60 1.946E-06

EU- $154 \quad 2.160 E-06$

$\begin{array}{ll}\mathrm{K}-40 & 2.000 \mathrm{E}-05\end{array}$

4.639E-07

AM-24?

PU- 238

$1.143 \mathrm{E}-03$

PU- 239

$1.031 \mathrm{E}-07$

1. OTOE 03

PU-240 $6.595 E-05$

PU-241 6.200E-06

PU-242 2.269E-07

U-235 2.230E-07

\begin{tabular}{|c|c|c|c|c|c|c|c|c|}
\hline & & & & & & & & $\begin{array}{l}\text { CO- } 60 \\
\text { CS-134 } \\
\text { EU- } 154\end{array}$ \\
\hline 410 & $2.358 \mathrm{E}+00 \quad 2.358 \mathrm{E}+00$ & $.000 E+00$ & $.000 E+00$ & $5.267 E-03$ & $1.410 E-03$ & $3.799 E-08$ & $1.252 \mathrm{E}-03$ & \\
\hline
\end{tabular}

$3.187 \mathrm{E}-07$

2.230E-07

$2.358 \mathrm{E}+00 \quad 2.358 \mathrm{E}+$

(n)

9.280E-08

$5.240 E-08$

$6.132 E-06$

AM- 241

PU-23

$1.452 E-04$

PU -239 4.031E-08

PU-240 8.275E-06

PU-241 7.857E-07

PU- $242 \quad 2.860$ - 08

U-235 9.970E-07

$242 A \quad 1.491 E+00 \quad 1.491 E+00 \quad .000 E+00 \quad .000 E+00 \quad 6.455 E-04 \quad 3.073 E-05 \quad 1.971 E-09 \quad 2.997 E-04$

C0-60 1.585E-06

CS-134 8.055E-08

EU-154 4.467E-06
6.727E-07 6.221E-0?
AM-241 5.721E-11
PU-238 $1.746 E-10$
PU- $239 \quad 5.826 \mathrm{E}-07$
PU-240 $3.572 E-08$


SWIR3280 - (Page 32 of 107)

RUN DATE: $03 / 15 / 96$

Land Disposal or Storage of Solid Radioactive Waste from January 1 to December 31 , 1995

BURIAL GROUND 218 W4C, 200 WEST AREA, LOW-LEVEL NONINDUSTRIAL.

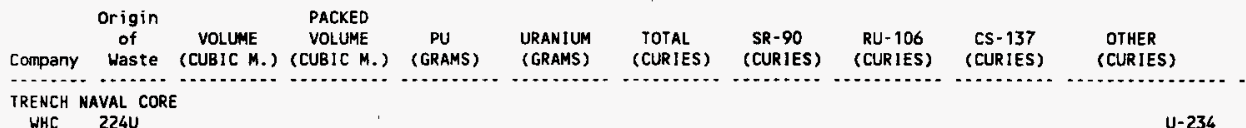

RENCH HAVAL CORE

$\begin{array}{lllllllll}23452 & 2.49 B E+01 & 2.498 E+01 & .000 E+00 & .000 E+00 & 3.120 E-01 & 6.745 E-02 & 3.604 E-04 & 7.273 E-02 \\ 241 A & 2.082 E-01 & 2.082 E-01 & .000 E+00 & .000 E+00 & 2.580 E-06 & 1.101 E-06 & 2.935 E-12 & 1.946 E-07\end{array}$

TC-99 1.224E-07

U. $236 \quad 1.420 \mathrm{E}-04$

U-238 1.982E-01

(2)

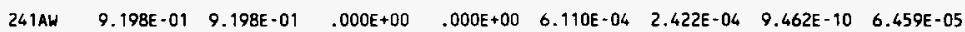

$60-60$

$241 \mathrm{AY}$

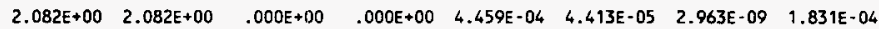

$2418 x$

$2.438 E+00 \quad 2.438 E+00 \quad .000 E+00$

$000 E+00$

$6.914 \mathrm{E}-03$

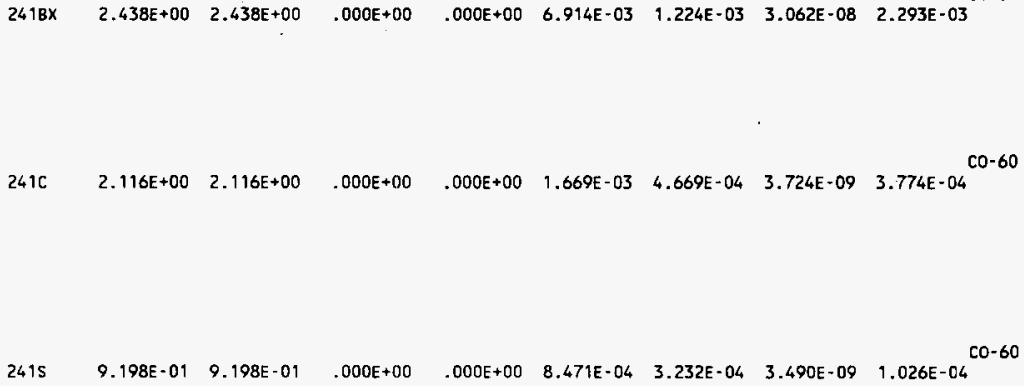

$\mathrm{co}-$

$9.198 \mathrm{E}-01 \quad 9.198 \mathrm{E}-01 \quad .000 \mathrm{E}+00 \quad .000 \mathrm{E}+00$

(1)

(n)


SWIR3280 - (Page 16 of 107 )

RUN DATE: 03/15/96

Land Disposal or Storage of Solid Radioactive Waste from January 1 to December 31 , 1995 BURIAL GROUND 218 WAE, 200 WEST AREA, LOW-LEVEL NONINDUSTRIAL.

\begin{tabular}{|c|c|c|c|c|c|c|c|c|c|c|c|c|}
\hline Company & $\begin{array}{l}\text { Origin } \\
\text { of } \\
\text { Waste }\end{array}$ & $\begin{array}{l}\text { VOLUME } \\
\text { (CUBIC M.) }\end{array}$ & $\begin{array}{c}\text { PACKED } \\
\text { VOLUME } \\
\text { (CUBIC M.) }\end{array}$ & $\begin{array}{c}\text { PU } \\
\text { (GRAMS) }\end{array}$ & $\begin{array}{l}\text { URAN IUH } \\
\text { (GRAMS) }\end{array}$ & $\begin{array}{c}\text { TOTAL } \\
\text { (CURIES) }\end{array}$ & $\begin{array}{l}\text { SR-90 } \\
\text { (CURIES) }\end{array}$ & $\begin{array}{c}R U-106 \\
\text { (CURIES) }\end{array}$ & $\begin{array}{c}\text { CS-137 } \\
\text { (CURIES) }\end{array}$ & \multicolumn{2}{|c|}{$\begin{array}{c}\text { OTHER } \\
\text { (CURIES) }\end{array}$} & $\begin{array}{c}\text { OTHER } \\
\text { (GRAMS) }\end{array}$ \\
\hline TRENCH 1 & 16 & & & & & & & & & & & \\
\hline PNL & $\begin{array}{l}306 \\
324\end{array}$ & $4.320 E+00$ & $4.320 E+00$ & $.000 E+00$ & $.000 E+00$ & $6.878 E+03$ & $3.528 E-01$ & $1.865 \mathrm{E}-03$ & 3.963E-01 & $\begin{array}{l}T C-99 \\
C O-57 \\
C O-60 \\
F E-59 \\
M N-54 \\
\text { NB- } 95 \\
\text { NI }-63 \\
T A-182 \\
\text { TC- } 99 \\
\text { ZR- }-95\end{array}$ & $\begin{array}{l}1.000 E-11 \\
6.869 E+03 \\
2.540 E-04 \\
6.810 E+03 \\
1.383 E+01 \\
2.110 E+01 \\
2.900 E-06 \\
1.660 E+01 \\
7.400 E-02 \\
4.000 E-06 \\
7.410 E+00\end{array}$ & $.000 \mathrm{E}+00$ \\
\hline & 331 & $1.269 E+01$ & $1.269 E+01$ & $.000 E+90$ & $.000 E+00$ & $4.201 E-02$ & $8.789 E-03$ & $4.697 \mathrm{E}-05$ & $9.477 \mathrm{E}-03$ & $\begin{array}{l}C-14 \\
C O-60 \\
H-3 \\
1-125 \\
\text { KR-85 } \\
M A-22 \\
\text { SR-85 } \\
\text { TM-170 }\end{array}$ & $\begin{array}{r}2.002 E-03 \\
P U-238 \\
P U-239 \\
P U-240 \\
2.000 E-03 \\
1.000 E-09 \\
8.002 E-09 \\
1.000 E-09 \\
1.000 E-09 \\
1.000 E-09 \\
1.000 E-09 \\
2.001 E-06\end{array}$ & $\begin{array}{l}3.019 E-09 \\
1.003 E-09 \\
1.956 E-09 \\
6.048 E-11\end{array}$ \\
\hline WHC & 300LEF & $7.360 E+01$ & $7.360 E+01$ & $.000 E+00$ & $.000 E+00$ & $1.457 E-04$ & $1.685 E-05$ & $2.801 E-05$ & $2.843 E-0.8$ & $\mathrm{co}-60$ & $\begin{array}{r}5.595 E-05 \\
A M-241 \\
P U-241 \\
U-238 \\
5.595 E-05\end{array}$ & $\begin{array}{l}5.418 E+02 \\
4.314 E-06 \\
7.256 E-06 \\
5.418 E+02\end{array}$ \\
\hline
\end{tabular}

TRENCH 26

BHI $105 \mathrm{~F}$

\begin{abstract}
$6.070 E+00 \quad 6.070 E+00$
\end{abstract}
$.000 \mathrm{E}+00$

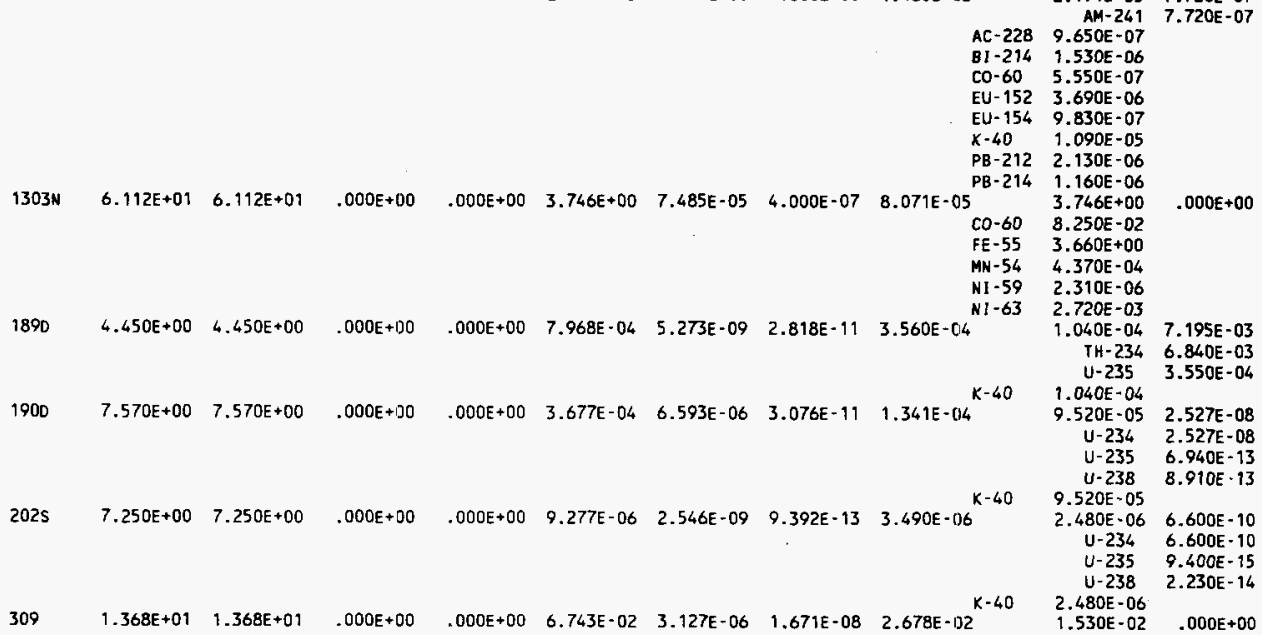


SWIR3280 - (Page 30 of 107)

RUN OATE: 03/15/96

Land Disposal or Storage of Solid Radioactive Waste from January 1 to December 31 , 1995 BURIAL GROUND 218 W4C, 200 WEST AREA, LOW-LEVEL NONINDUSTRIAL.

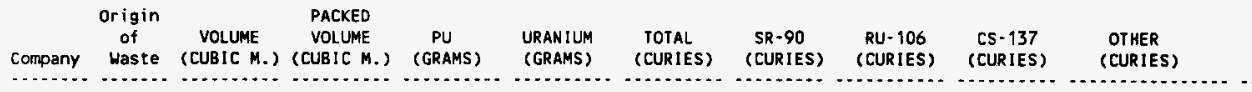

TRENCH 33

HHC 1706KE

2225

$8.744 E+00 \quad 8.744 E+00$

$.000 \mathrm{E}+00$

$.000 E+00$

$4.512 E-02 \quad 1.436 E-02 \quad 1.021 E-06$

$8.308 E-03$

CS-134 1.620E-DS

2345

$\begin{array}{lllllll}4.268 E+01 & 4.268 E+01 \quad .000 E+00 & .000 E+00 & 1.025 E+00 & 2.252 E-01 & 1.203 E-03 & 2.428 E-01\end{array}$

300

$\begin{array}{llllllll}9.785 E+00 & 9.785 E+00 & .000 E+00 & .000 E+00 & 1.833 E-05 & 2.115 E-06 & 3.525 E-06 & .000 E+00\end{array}$

3OOLE

2.165E+01 $2.165 E+01$

$.000 E+00$

$.000 E+00 \quad 4.056 E-05 \quad 4.680 E-06 \quad 7.800 E-06 \quad .000 E+00$

$10-60$

$308 \quad 1.249 E+00 \quad 1.249 E+00 \quad .000 E+00 \quad .000 E+00 \quad 1.500 E-02 \quad 3.296 E-03 \quad 1.761 E-05 \quad 3.554 E-0.3$

$60-60$

$\begin{array}{rlllllllllll}308 & 1.249 E+00 & 1.249 E+00 & .000 E+00 & .000 E+00 & 1.500 E-02 & 3.296 E-03 & 1.761 E-05 & 3.554 E-0.3 \\ 377 & 1.874 E+00 & 1.874 E+00 & .000 E+00 & .000 E+00 & 7.358 E-06 & .000 E+00 & .000 E+00 & .000 E+0\end{array}$

20


SWIR3280 - (Page 18 of 107)

RUN DATE: 03/15/96

Land Disposal or storage of Solid Radioactive Waste from January 1 to December 31 , 1995 BURIAL GROUND 218W3AE, 200 WEST AREA, LOW-LEVEL NONINDUSTRIAL.

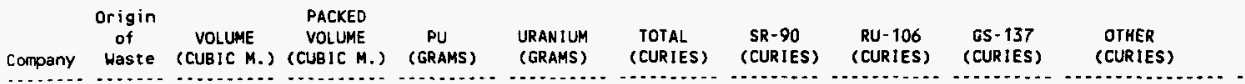
IRENCH 26

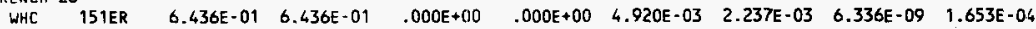

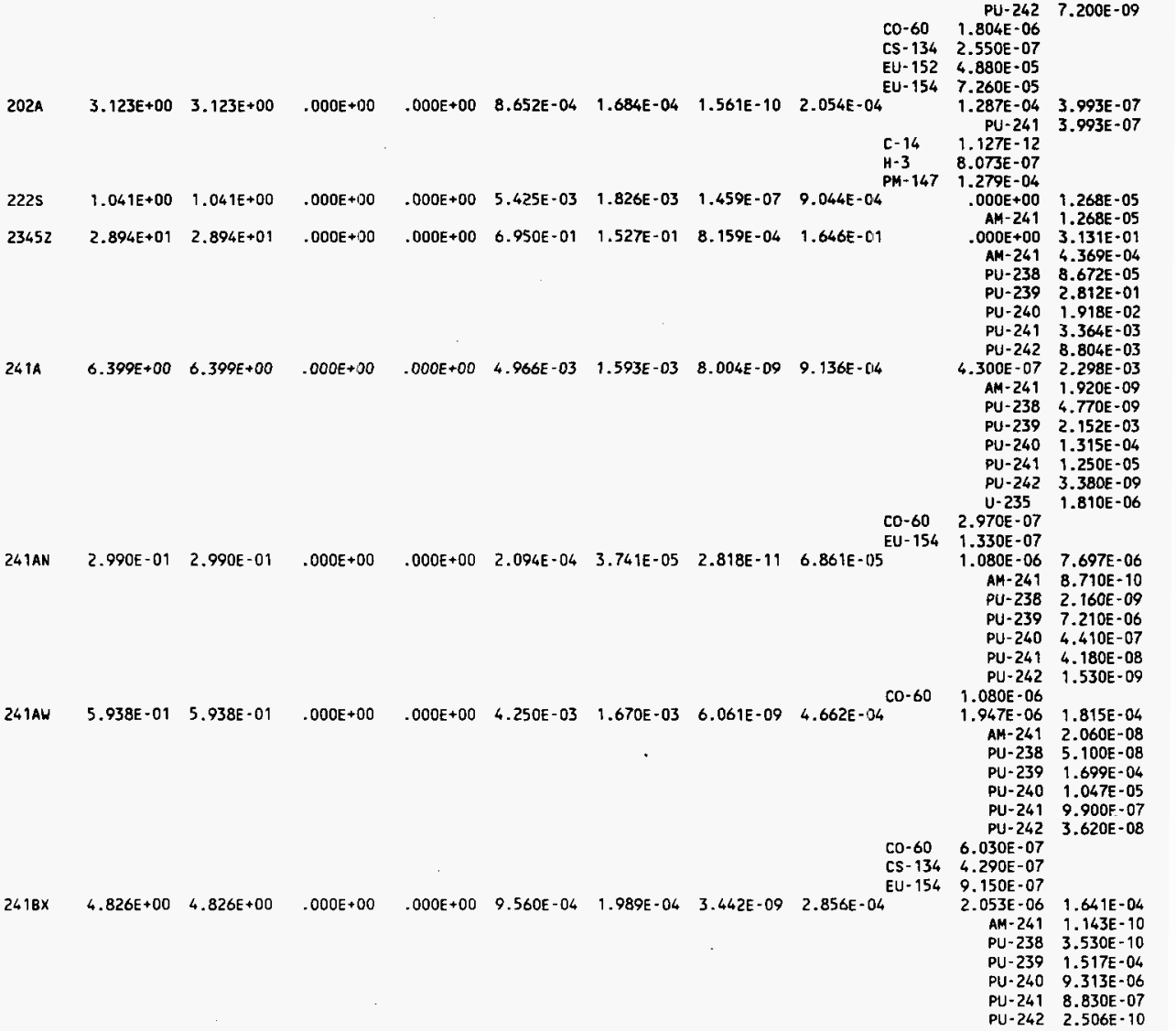


SWIR3280 - (Page 28 of 107 )

RUN DATE: $03 / 15 / 96$

Land Disposal or storage of Solid Radioactive Waste from January 1 to Decenber 31 , 1995 BURIAL GROUND 218W4C, 200 WEST AREA, LOW-LEVEL NONINDUSTRIAL.

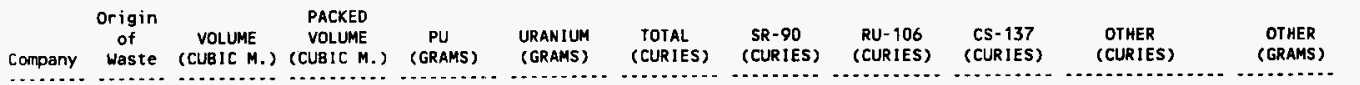

IRENCH 33

BHI 200

$202 S \quad 6.246 E-01 \quad 6.246 E-01$

$300-F F-\quad 5.499 E+00 \quad 5.499 E+00$

KEH 200W

$1.145 E+01 \quad 1.145 E+01$

$.000 E+00 \quad .000 E+00$

$6.460 E-03$

1

788E-03 $1.883 E-08 \quad 6.640 E-05$

$300-$ ARE $\quad 1.416 E+01 \quad 1.416 E+01$

$.000 E+00$

$.000 \mathrm{E}+$

$4.937 E-0$

$2.988 E-09$

1

597E-11 3.222E-09

PNL

324

$4.372 E+00 \quad 4.372 E+00$

$.0006+0$

$.000 \mathrm{E}+$

$1.261 E-02$

3.523E-03 1.335E-08 2.838E-03

325

$9.369 E+00 \quad 9.369 E+00$

$.000 \mathrm{E}+0$
OOOE+OO
$1.024 E+00 \quad 4.847 E-03 \quad 2.449 E-05 \quad 5.110 E-03$
EU-155 1.034E-11

TC $-99 \quad 3.620 \mathrm{E}-09$

$.000 E+00$

$.000 E+00$

u-235

u- 238

2.661E-03

AM-241

U-235

CE-144 8.053E-05

CO-60 1.959E-03

EU-154 3.131E-05

FE-59 9.212E-05

MN-54 3.871E-04

NB- 95 2.237E-05

SB-125 3.577E-05

ZN-65 4.025E-05

$28-95 \quad 1.342 E-05$

$4.935 \mathrm{E}-05$

TH- 228

TH-234

$\mathrm{U}-235$

AC-228 1.877E-05

B1-214 1.176E-05

PB-214 $1.312 E-05$

TL-208 5.698E-06

$3.835 E-05$

CO-60 2.465E-05

MN-54 1.370E-05

$1.002 E+00$

AM- 241

AM- 243

CM-244

NP-23?

PU-238

PU-239

PU-241

PU-242

TH-228

TH-230

$\mathrm{TH}-232$

U -232

$\mathrm{U}-235$

U-238

C- 14

$7.018 E-05$

CA-45 $1.000 E-07$

CD-109 6.000E-07

CL-36 4.500E-08

CO-57 1.000E-07

CO-60 2.522E-04

EU-152 1.200E-06

EU-154 $1.299 E-06$

FE-55 1.000E-06

FE-59 1.000E-12

$\mathrm{H}-3 \quad 1.000 \mathrm{E}+00$

I-129 4.260E-08

$1-131$ 1.000E-08

KR-85 2.000E-03

MN-54 2.105E-06

NA-22 3.752E-06
$.000 E+00$

$1.179 E+03$

$6.397 E+90$

1. $172 \mathrm{E}+03$

$2.071 E+00$

2.624E-06

$2.071 E \div 00$

$6.324 E+01$

$1.047 \mathrm{E}-06$

6. $664 \mathrm{E}-08$

$6.324 E+01$

$.000 E+00$

$1.241 E+02$

$1.632 E-04$

$5.260 E-09$

$6.600 E-10$

$1.400 \mathrm{E}-03$

$1.170 E-08$

$1.384 \mathrm{E}-04$

$3.524 \mathrm{E}-08$

3.016E-07

$1.500 E-11$

$1.100 E-06$

$1.544 E+01$

5. $230 \mathrm{E}-10$

3.073E-02

$1.086 \mathrm{E}+02$ 
SWIR3280 - (Page 20 of 107)

RUN DATE: 03/15/96

Land Disposal or Storage of Solid Radioactive Waste from January 1 to December 31 , 1995

BURIAL GROUND 218W3AE, 200 WEST AREA, LOW-LEVEL NONINDUSTRIAL.

\begin{tabular}{|c|c|c|c|c|c|c|c|c|c|}
\hline Company & $\begin{array}{c}\text { Origin } \\
\text { of } \\
\text { Waste }\end{array}$ & $\begin{array}{c}\text { VOLUME } \\
\text { (CUBIC M.) }\end{array}$ & $\begin{array}{c}\text { PACKED } \\
\text { VOLUME } \\
\text { (CUBIC } M .)\end{array}$ & $\begin{array}{c}\text { PU } \\
\text { (GRAMS) }\end{array}$ & $\begin{array}{l}\text { URANIUM } \\
\text { (GRAMS) }\end{array}$ & $\begin{array}{l}\text { TOTAL } \\
\text { (CURIES) }\end{array}$ & $\begin{array}{l}\text { SR-90 } \\
\text { (CURIES) }\end{array}$ & $\begin{array}{l}\text { RU- } 106 \\
\text { (CURIES) }\end{array}$ & $\begin{array}{l}\text { CS-137 } \\
\text { (CURIES) }\end{array}$ \\
\hline WHC & 410 & & & & & & & & \\
\hline
\end{tabular}

$244 \mathrm{~A} \quad 3.218 \mathrm{~A}-01 \quad 3.218 \mathrm{0}-01 \quad .000 \mathrm{E}+00 \quad .000 \mathrm{E}+00 \quad 3.254 \mathrm{E}-03 \quad 7.263 \mathrm{E}-04 \quad 1.838 \mathrm{E}-09 \quad 9.254 \mathrm{E}-0.04$

CO-60

EU-154

(1)

2718

300

$1.880 E+01 \quad 1.880 E+01$

$1.582 E+09 \quad 9.582 E+01$

$.000 E+$

$.000 E+00$

$000 E+0$

300LEF $1.187 E+01 \quad 1.187 E+01$

$1.741 E+01 \quad 1.741 E+01$

$.000 E+20$

$.000 E+00$

$2.037 E-02 \quad 2.245 E-04$

2.091E-08 $1.270 E-013$

co-60

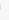

0

\begin{tabular}{|c|c|c|c|c|c|c|c|c|c|}
\hline 308 & $2.082 E-01$ & $2.082 E-01$ & $.000 E+00$ & $.000 E+00$ & $5.000 E-05$ & $1.099 E-05$ & $5.870 E-08$ & $1.185 E-05$ & $5^{c 0-60}$ \\
\hline 09 & 2.082E-01 & $2.082 E-01$ & $.000 E+00$ & $.000 E+00$ & $8.275 E-05$ & $1.241 \mathrm{E}-05$ & $3.405 E-11$ & $5.367 E-08$ & \\
\hline 40 & $1.741 E+01$ & $1.741 E+01$ & $.000 E+D O$ & $.000 E+00$ & $2.037 E-02$ & $2.245 E-04$ & 2.091E-08 & $1.270 E-03$ & $\begin{array}{l}\mathrm{C}-14 \\
\mathrm{CO}-60 \\
\mathrm{H}=3\end{array}$ \\
\hline
\end{tabular}

377

$$
9.400 E+00 \quad 9.400 E+00
$$

$7.833 E+02 \quad 7.833 E+02$ $\begin{array}{lllll}.000 E+00 & 7.820 E-04 & .000 E+00 & .000 E+00 \quad .000 E+00\end{array}$

$.000 E+00 \quad 5.587 E+04 \quad 1.038 E+04 \quad 7.641 E-02 \quad 5.641 E+03$ (CURIES)

OT HER (GRAMS)

PU-239 4.166E-06 PU-240 2.559E-07 PU-24t 2.416E-08 PU-242 8.810E-10 $U-235 \quad 3.790 E-96$

2. $150 E-08$

$1.740 \mathrm{E}-07$

2.850E-06

$3.840 \mathrm{E}-07 \quad 2.788 \mathrm{E}-06$ AM-241 3.160E-10 PU-238 7.830E-10 PU-239 2.610E-06 PU-240 1.610E-07 PU-241 1.520E-08 PU $-242 \quad 5.550 E-10$

3.840E-07 5. $000 E-08$ 5.000E-08

$1.242 E-05 \quad 1.363 E+02$ AM-241 5.372E-07 PU-241 9.044E-0? U-238 $1.363 E+02$

$1.242 \mathrm{E}-05$

8.550E-06 $9.240 E+01$ $A M-241 \quad 7.524 E-13$ PU-241 1.260E-12 $\mathrm{U}-238 \quad 9.240 \mathrm{E}+01$ 8.550E-06 .000E+00 3.096E-06 CF-252 1.550E-08 CH-248 3.080E-06 $5.783 E-05 \quad 4.180 E-11$ PU-239 4.180E-11

3.000E-05

3.040E-08 2.780E-05

$1.725 E-02 \quad 6.148 E-02$ AM-241 1.758E-04 PU-239 3.580E-07 PU-241 5.062E-06 RA-226 5.320E-08 U-238 $6.130 E-02$
$B A-133$

CE- 144

$\mathrm{CL}=36$

CD- 60

EU-152

EU- 154

EU-155

$\mathrm{CO}-60$
3.020E-08

2.004E-04

$1.890 E-08$

$3.860 \mathrm{E}-05$

$5.552 E-03$

9.918E-03

$1.546 \mathrm{E}-03$

$7.820 E-04$

$7.820 \mathrm{E}-04$

$2.412 E+0.4$
$.000 E+00$

$3.226 E+06$ 


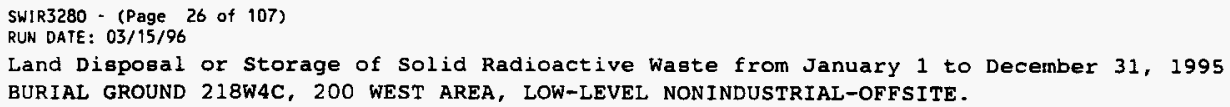

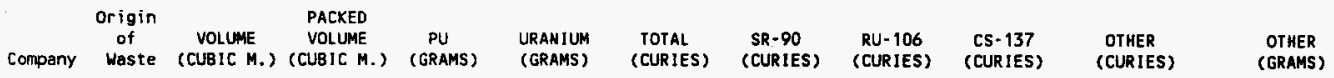

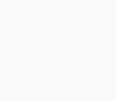

$\begin{array}{ll}\text { LA-140 } & 1.200 E-06 \\ \text { MN-54 } & 3.694 E-04 \\ \text { NA-22 } & 2.200 E-05 \\ \text { NB-95 } & 3.280 E-06 \\ \text { NI-60 } & 4.500 E-06 \\ \text { P-32 } & 3.669 E-03 \\ \text { S-35 } & 2.446 E-03 \\ \text { SB-125 } & 5.596 E-05 \\ \text { SC-46 } & 1.000 E-03 \\ \text { SE-75 } & 5.000 E-08 \\ \text { SR-85 } & 2.300 E-06 \\ \text { TC- }-99 & 3.200 E-06 \\ \text { ZN-65 } & 2.200 E-05\end{array}$


SWIR3280 - (Page 22 of 107)

RUN DATE: $03 / 15 / 96$

Land Disposal or storage of Solid Radioactive Waste from January 1 to December. 31 , 1995

BURIAL GROUND 218 W4C, 200 WEST AREA, LOW-LEVEL NONINDUSTRIAL (ASSAYED FROM TRANSURANIC).

\begin{tabular}{|c|c|c|c|c|c|c|c|c|c|c|}
\hline any & $\begin{array}{c}\text { Origin } \\
\text { of } \\
\text { Waste }\end{array}$ & $\begin{array}{l}\text { VOLUME } \\
\text { (CUBIC M.) }\end{array}$ & $\begin{array}{c}\text { PACKED } \\
\text { VOLUME } \\
\text { (CUBIC M.) }\end{array}$ & $\begin{array}{c}\text { PU } \\
\text { (GRAMS) }\end{array}$ & $\begin{array}{l}\text { URANIUM } \\
\text { (GRAMS) }\end{array}$ & $\begin{array}{l}\text { TOTAL } \\
\text { (CURIES) }\end{array}$ & $\begin{array}{l}\text { SR-90 } \\
\text { (CURIES) }\end{array}$ & $\begin{array}{c}\text { RU- } 106 \\
\text { (CUR IES) }\end{array}$ & $\begin{array}{c}\text { CS-137 } \\
\text { (CURIES) }\end{array}$ & $\begin{array}{l}\text { OTHER } \\
\text { (CURIES) }\end{array}$ \\
\hline
\end{tabular}

TRENCH 20

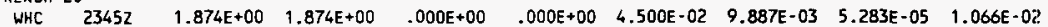

TRENCH 33

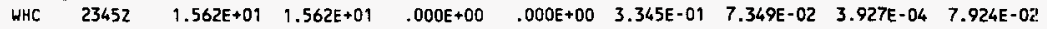

TOTALS

$\begin{array}{lllllll}1.749 E+01 & 1.749 E+01 \quad .000 E+00 \quad .000 E+00 & 3.795 E-01 & 8.338 E-02 & 4.455 E-04 & 8.990 E-02\end{array}$

$\begin{array}{rr}.000 E+00 & 1.804 E-01 \\ A M-241 & 2.700 E-04 \\ P U-238 & 4.884 E-05 \\ P U-239 & 1.689 E-01 \\ P U-240 & 1.026 E-02 \\ P U-241 & 7.560 E-04 \\ P U-242 & 1.830 E-04 \\ & \\ .000 E+00 & 1.502 E-01 \\ A M-241 & 2.022 E-04 \\ P U-238 & 4.358 E-05 \\ P U-239 & 1.407 E-01 \\ P U-240 & 8.557 E-03 \\ P U-241 & 6.533 E-04 \\ P U-242 & 2.925 E-05 \\ 000 E+00 & 3.306 E-01\end{array}$


SWIR3280 - (Page 24 of 107)

RUN DATE: $03 / 15 / 96$

Land Disposal or Storage of Solid Radioactive Waste from January 1 to December 31 , 1995

BURIAL GROUND 218 W4C, 200 WEST AREA, LOW-LEVEL NONINDUSTRIAL-OFFSITE.

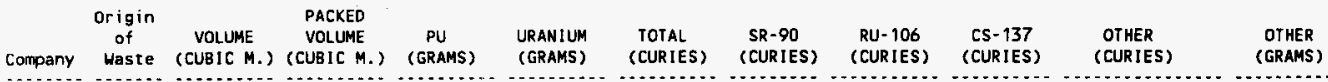

TRENCH 20

ANL ARGON

BNL BNLAB $\quad 4.098 E+01 \quad 4.098 E+01 \quad .000 E+00 \quad .000 E+00 \quad 2.038 E+00 \quad 8.453 E-01 \quad 4.084 E-03 \quad 1.014 E-01$

$\begin{array}{ll}\text { AC-228 } & 2 . \\ B I-207 & 2 \\ B 1-212 & 1 \\ \text { CO-60 } & 4 \\ \text { CS-134 } & 2 \\ \text { EU-154 } & 1 \\ \text { EU-155 } & 9 \\ \text { NA-22 } & 3 \\ \text { PB-212 } & 4 \\ \text { SB-125 } & 8 \\ \text { TL-208 } & 1 \\ & \end{array}$

TRENCH 24

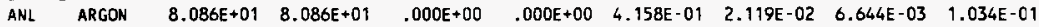

$\mathrm{u}-233$

$\mathrm{U}-238$

$2.580 \mathrm{E}-03$

$2.117 \mathrm{E}-04$

$1.660 \mathrm{E}+03$

$4.299 \mathrm{E}-05$

$2.539 E-02$

$1.781 E-02$

9.522E-02

$3.642 E-04$

4. $230 \mathrm{E}-03$

8.843E-02

$1.937 \mathrm{E}-04$

6.250E-02

AM- 241

AM-24

PU- 238

PU- 239

PU-240

RA- 226

TH-228

TH- 232

U- 234

$\mathrm{u}-235$

U. 238

C. 14

$5.470 \mathrm{E}-06$

CO-57 4.50OE-06

CO-60 2.062E-02

CS-134 1.860E-06

$\mathrm{H}-3 \quad 3.812 \mathrm{E}-02$

NA-22 3.501E-03

TA- 182 9.670E-05

TC-99 $1.114 \mathrm{E}-04$

ZN-65 4.382E-05

$7.042 E-02$ $1.000 E+00$

$4.842 E+02$

8.671E-04

$1.524 \mathrm{E}-02$

$6.133 \mathrm{E}-06$

$1.198 \mathrm{E}-01$

1. $419 E-03$

$9.084 E-04$

$2.333 \mathrm{E} \cdot 07$

$7.994 E+01$

2. $083 \mathrm{E}-02$

$1.138 E+02$

$2.904 E+02$

\subsection{E-02}

AM- 241

NP -237

PU-238

PU-239

PU-240

PU-241

RA- 224

RA- 226

TH- 228

IH-232

U-233

U-234

$\mathrm{u}-235$

$\mathrm{U}-238$

BI - 2

$1.360 E-03$

C- $14 \quad 6.000 E-06$

CO-60 9.359E-03

CS-134 2.002E-03

EU- $154 \quad 2.330$ E- 04

$\mathrm{H}-3 \quad 5.000 \mathrm{E}-05$

K-40 4.510E-05

$P A-231 \quad 4.450 E-03$

SB- $125 \quad 1.180 E-02$

$\mathrm{TL}-208 \quad 3.490 \mathrm{E}-04$
$1.490 E+08$

2. $160 \mathrm{E}-03$

$1.400 E-03$

2.029E-09

$1.336 E+00$

$1.600 \mathrm{E}-06$

$5.000 E-08$

5.730E-03

$1.264 E-03$

6.721E-06

$9.207 E+03$

$9.640 E-04$

$1.800 \mathrm{E}-06$

$3.143 \mathrm{E} \div 05$

$1.487 \mathrm{E}+08$ 
SWIR3280 - (Page 24 of 107 )

RUN DATE: 03/15/96

Land Disposal or Storage of Solid Radioactive Waste from January 1 to December 31 , 1995

BURIAL GROUND 218 W4C, 200 WEST AREA, LOW-LEVEL NONINDUSTRIAL-OFFSITE.

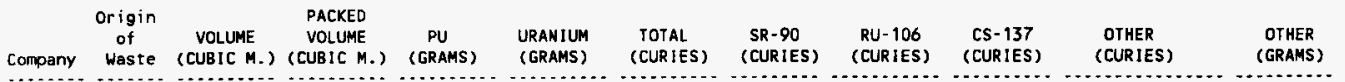

TRENCH 20

ANL ARGON

BNLAB

$4.098 E+01 \quad 4.098 E+01$

$.000 \mathrm{E}+0$

$.000 E+00$

$2.038 \mathrm{E}+00$

$8.453 \mathrm{E}-0$

4.084E-03 1.014E-01

TRENCH 24

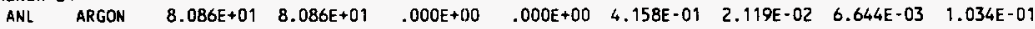

\begin{tabular}{|c|c|c|}
\hline & $\begin{array}{l}U-233 \\
U-238\end{array}$ & $\begin{array}{l}7.042 \mathrm{E}-02 \\
1.000 \mathrm{E}+00\end{array}$ \\
\hline AC- -228 & $2.580 E-03$ & \\
\hline BI -207 & $2.117 E-04$ & \\
\hline B! -212 & $1.660 \mathrm{E}-03$ & \\
\hline$c 0-60$ & $4.299 E-05$ & \\
\hline CS-134 & $2.539 \mathrm{E}-02$ & \\
\hline $\begin{array}{l}E U-154 \\
E U-155\end{array}$ & $1.781 \mathrm{E}-02$ & \\
\hline $\begin{array}{l}\text { EU- } 155 \\
\text { NA-22 }\end{array}$ & $9.522 \mathrm{E}-02$ & \\
\hline $\begin{array}{l}\text { NA-22 } \\
\text { PB-212 }\end{array}$ & $\begin{array}{l}3.642 E-04 \\
4.230 E-03\end{array}$ & \\
\hline $\begin{array}{l}S B-125 \\
T L-208\end{array}$ & $\begin{array}{l}8.843 E-02 \\
1.937 E-04\end{array}$ & \\
\hline 1 & $6.250 \mathrm{E}-02$ & $4.842 E+02$ \\
\hline & $A M-241$ & 8.671E-04 \\
\hline & AM-242 & $1.524 \mathrm{E}-02$ \\
\hline & $\begin{array}{l}P U-238 \\
P U-239\end{array}$ & $\begin{array}{l}6.133 E-06 \\
1.198 E-01\end{array}$ \\
\hline & PU -240 & $1.419 \varepsilon-03$ \\
\hline & $R A-226$ & 9.084E-04 \\
\hline & TH-228 & $2.333 E-07$ \\
\hline & TH-232 & $7.994 E+01$ \\
\hline & $U-234$ & 2.083E-02 \\
\hline & $\begin{array}{l}u-235 \\
u-238\end{array}$ & $\begin{array}{l}1.138 \mathrm{E}+02 \\
2.904 \mathrm{E}+02\end{array}$ \\
\hline$c=14$ & $5.470 E=06$ & \\
\hline CO- 57 & $4.500 E-06$ & \\
\hline$c 0-60$ & $2.062 E-02$ & \\
\hline CS- 134 & $1.860 \mathrm{E}-06$ & \\
\hline $\mathrm{H}-3$ & $3.812 E-02$ & \\
\hline NA- 22 & $3.501 E-03$ & \\
\hline TA-182 & $9.670 E-05$ & \\
\hline $\begin{array}{l}T C-99 \\
2 N-65\end{array}$ & $\begin{array}{l}1.114 E-04 \\
4.382 E-05\end{array}$ & \\
\hline \multirow[t]{14}{*}{01} & $2.9655-02$ & $1.490 E+08$ \\
\hline & $A M-241$ & $2.160 \mathrm{E}-03$ \\
\hline & NP- 237 & $1.400 E-03$ \\
\hline & PU- 238 & $2.029 E-09$ \\
\hline & PU-239 & $1.336 \mathrm{E}+00$ \\
\hline & $P U-240$ & $1.600 \mathrm{E}-06$ \\
\hline & PU-241 & $5.000 \mathrm{E}-08$ \\
\hline & RA-224 & $5.730 E-03$ \\
\hline & RA-226 & $1.264 E-03$ \\
\hline & TH-228 & $6.721 \mathrm{E}-06$ \\
\hline & TH-232 & $9.207 E+03$ \\
\hline & $U-233$ & $9.640 \mathrm{E}-04$ \\
\hline & $U-234$ & $\begin{array}{l}1.800 E-06 \\
3.143 E \div 05\end{array}$ \\
\hline & $\begin{array}{l}u-235 \\
u-238\end{array}$ & $\begin{array}{l}3.143 E \div 05 \\
1.487 E+08\end{array}$ \\
\hline B $!-212$ & $1.360 E-03$ & \\
\hline C- 14 & $6.000 \mathrm{E}-06$ & \\
\hline$C 0-60$ & $9.359 \mathrm{E}-03$ & \\
\hline CS- 134 & $2.002 E-03$ & \\
\hline EU- 154 & $2.330 \mathrm{E}-04$ & \\
\hline $\mathrm{H}-3$ & 5.000 E-05 & \\
\hline$K-40$ & $4.510 E-05$ & \\
\hline$P A-231$ & $4.450 E-03$ & \\
\hline SB-125 & $1.180 E-02$ & \\
\hline TL-208 & $3.490 E-04$ & \\
\hline
\end{tabular}


SHIR3280 - (Page 22 of 107)

RUN DATE : 03/15/96

Land Disposal or Storage of Solid Radioactive Waste from January 1 to December, 31 , 1995

BURIAL GROUND 218 W4C, 200 WEST AREA, LOW-LEVEL NONINDUSTRIAL (ASSAYED FROM TRANSURANIC) .

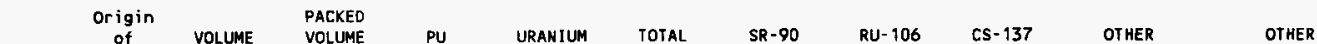

Company Waste (CUBIC M.) (CUBIC M.) (GRAMS) (GRAMS) (CURIES) (CURIES) (CURIES) (CURIES) (CURIES)
(GRAMS)

TRENCH 20

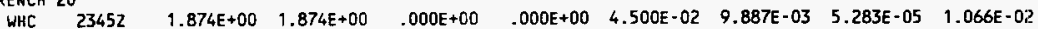

TRENCH 33

WHC

23452

$1.562 E+01 \quad 1.562 E+01$

$.000 E+00 \quad .000 E+00$

$3.345 \mathrm{E}-01$

7.349E-02 3.927E-04 7.924E-02

TOTALS

$1.749 E+01 \quad 1.749 E+01$

$.000 \mathrm{E}+00$

$.000 E+00$

3.795E-0

8.338E-02

4. 455E-04 8.990E-0?

$\begin{array}{rr}.000 E+00 & 1.804 E-01 \\ A M-241 & 2.700 E-04 \\ P U-238 & 4.884 E-05 \\ P U-239 & 1.689 E-01 \\ P U-240 & 1.026 E-02 \\ P U-241 & 7.560 E-04 \\ P U-242 & 1.830 E-04 \\ & \\ .000 E+00 & 1.502 E-01 \\ A M-241 & 2.022 E-04 \\ P U-238 & 4.35 B E-05 \\ P U-239 & 1.407 E-01 \\ P U-240 & 8.557 E-03 \\ P U-241 & 6.533 E-04 \\ P U-242 & 2.925 E-05 \\ .000 E+00 & 3.306 E-01\end{array}$




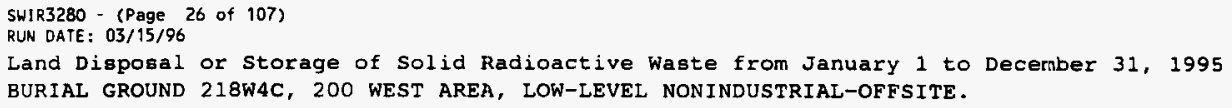

\section{LA- 140 1.200E-06 \\ $M N-54 \quad 3.694 E-04$ \\ NA-22 2.200E-05 \\ NB-95 3.280E-06 \\ NI $-60 \quad 4.500 E-06$ \\ P-32 3.669E-03 \\ S-35 2.446E-03 \\ SB-125 5.596E-05 \\ SC-46 1.000E-03 \\ SE $-75 \quad 5.000 E-08$ \\ SR-85 2.300E-06 \\ TC-99 3.200E-06 \\ ZW-65 2.200E-05}

\section{$2.262 \mathrm{E}+04$} $2.262 \mathrm{E}+04$ 1.263E-02 $A M-241$ NP-237 7.014E-01 PU-238 3.886E-06 PU- $239 \quad 1.592 E-03$ PU-240 4.371E-04 TH-230 4.501E-02 TH-234 4.767E-07 U-236 8.573E-01 $U-235 \quad 6.710 E+02$ U-238 $1.506 \mathrm{E}+03$ 1.263E-02 $2.693 E+01 \quad .000 E+00$ $\mathrm{H}-3 \quad 2.693 \mathrm{E}+01$ 
SWIR3280 - (Page 20 of 107)

RUN DATE: $03 / 15 / 96$

Land Disposal or Storage of Solid Radioactive Waste from January 1 to Decernber 31 , 1995

BURIAL GROUND 218 WAE, 200 WEST AREA, LOW-LEVEL NONINDUSTRIAL.

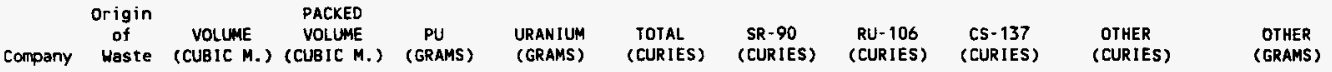

(GRAMS)

TRENCH 26

WHC 2410

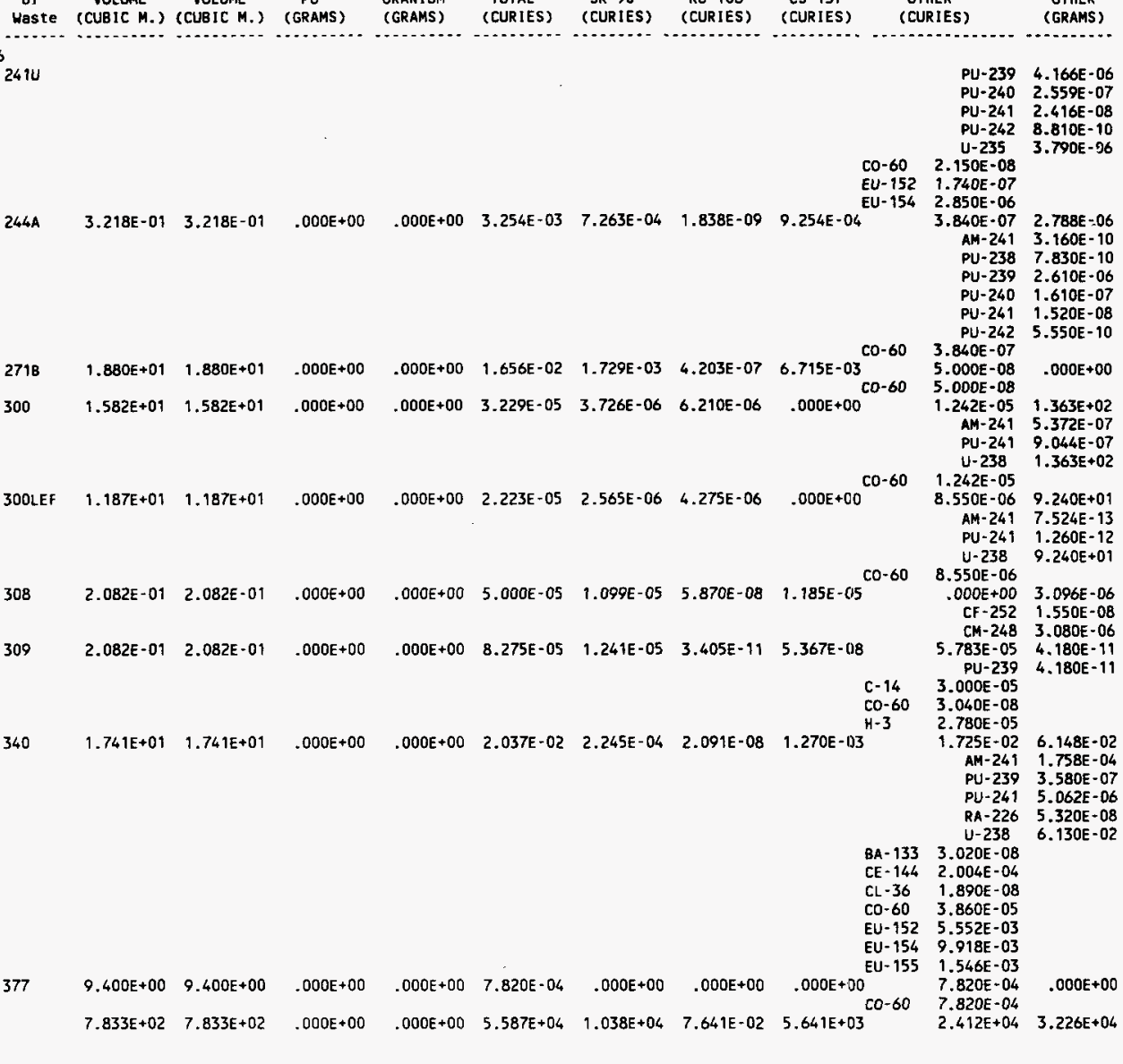

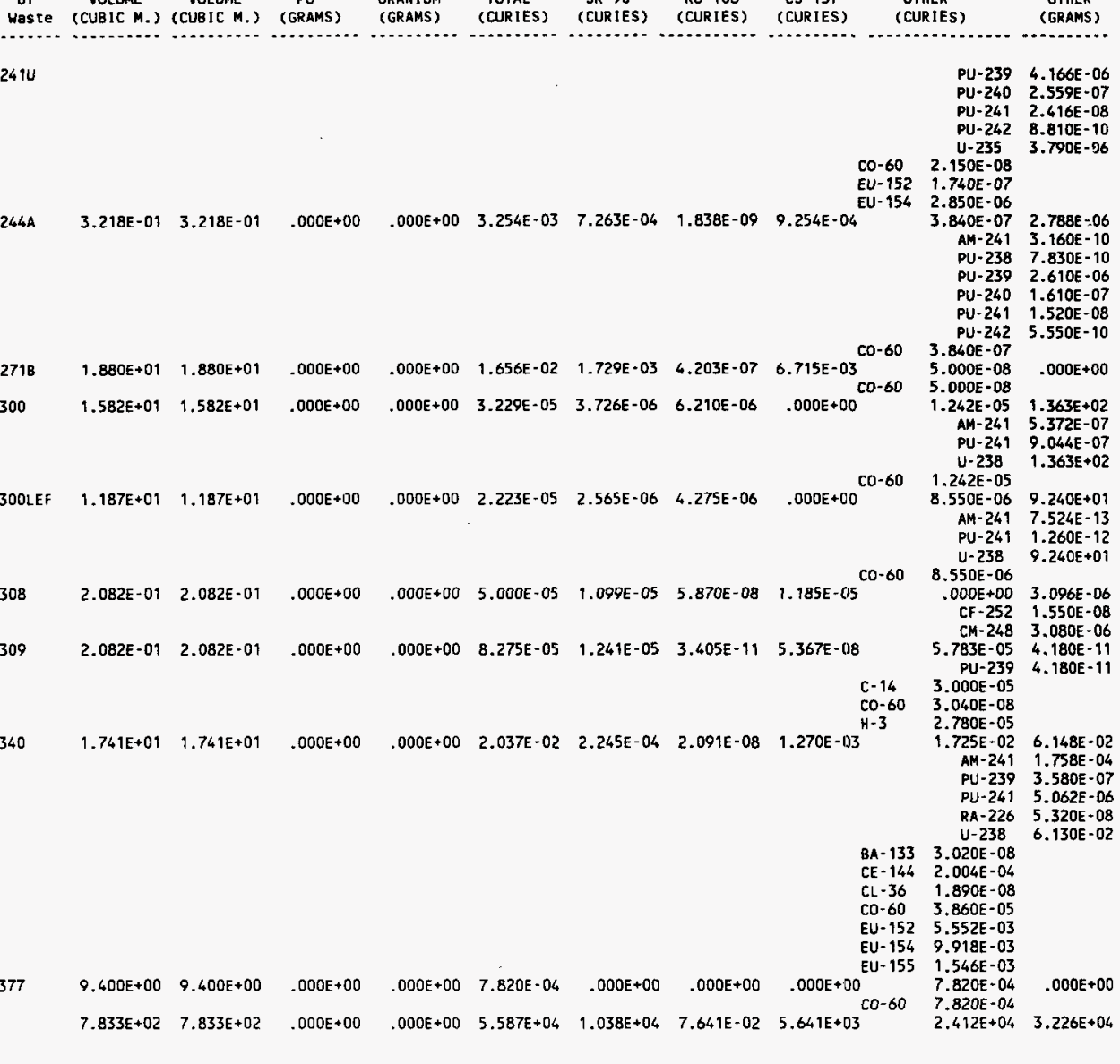

300LEF $\quad 1.187 E+01 \quad 1.187 E+01 \quad .000 E+00 \quad .000 E+00 \quad 2.223 E-05 \quad 2.565 E-06 \quad 4.275 E-06 \quad .000 E+00$

Co-60$$
1.187 \mathrm{E}+01
$$

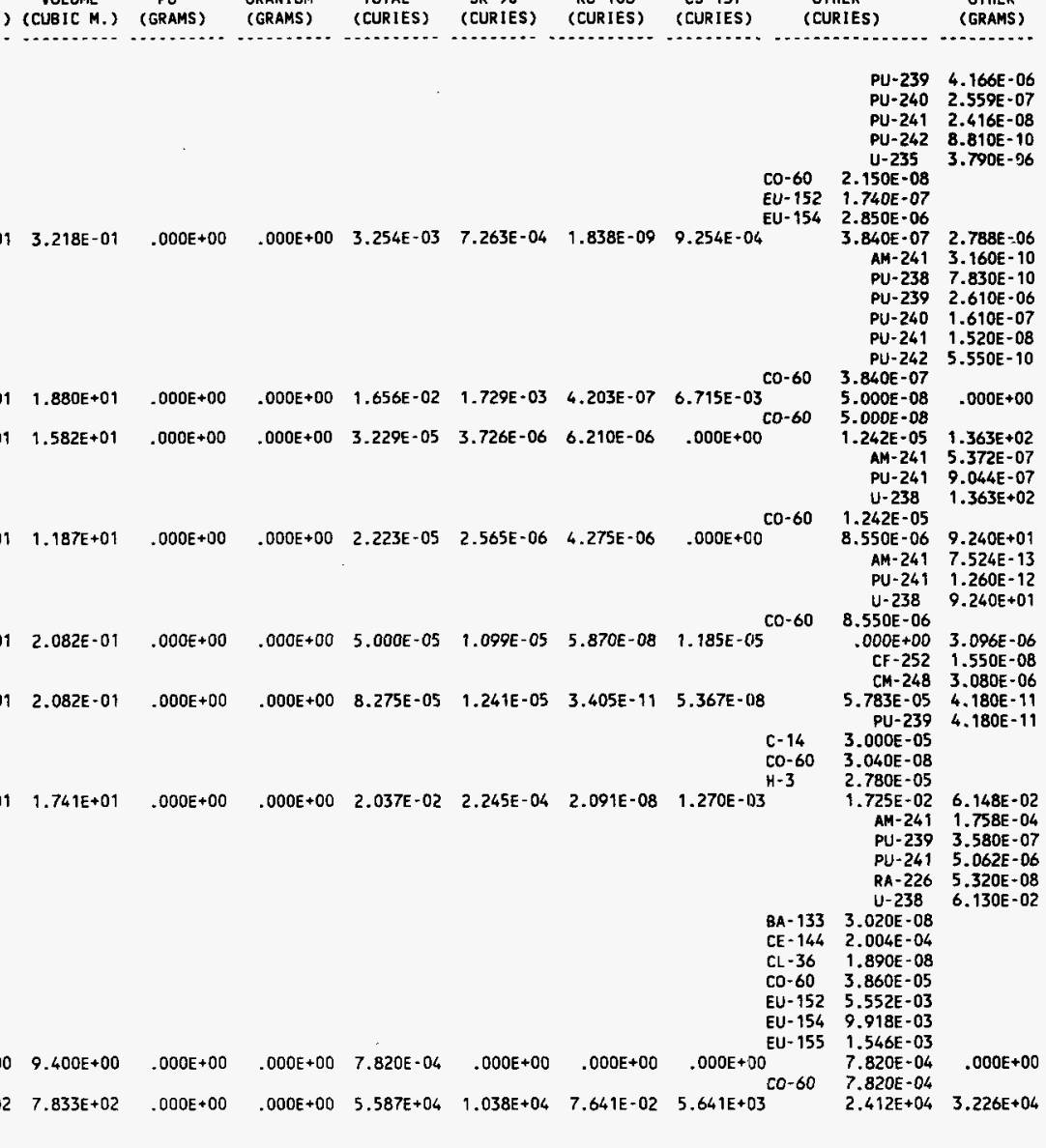

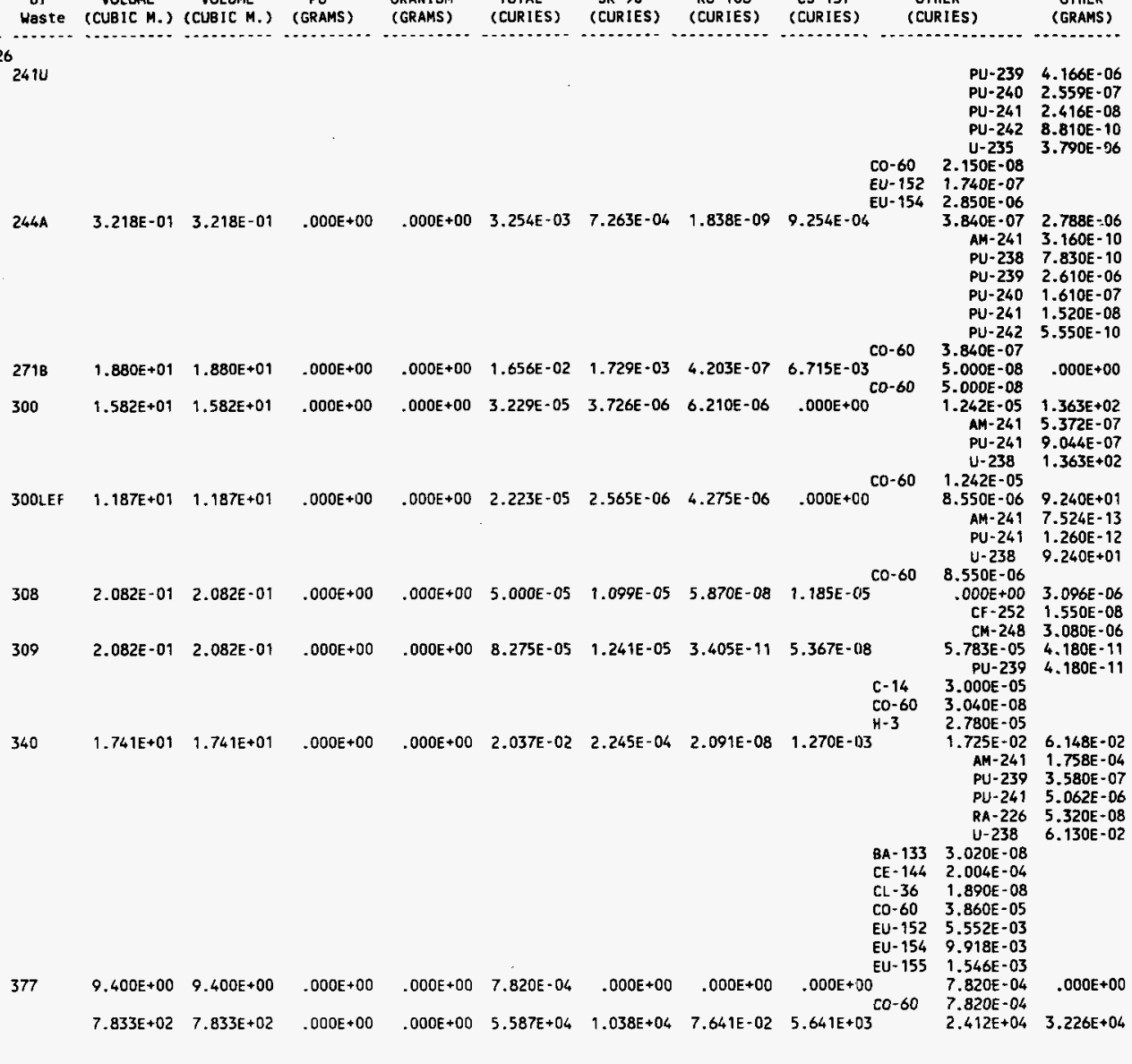

309

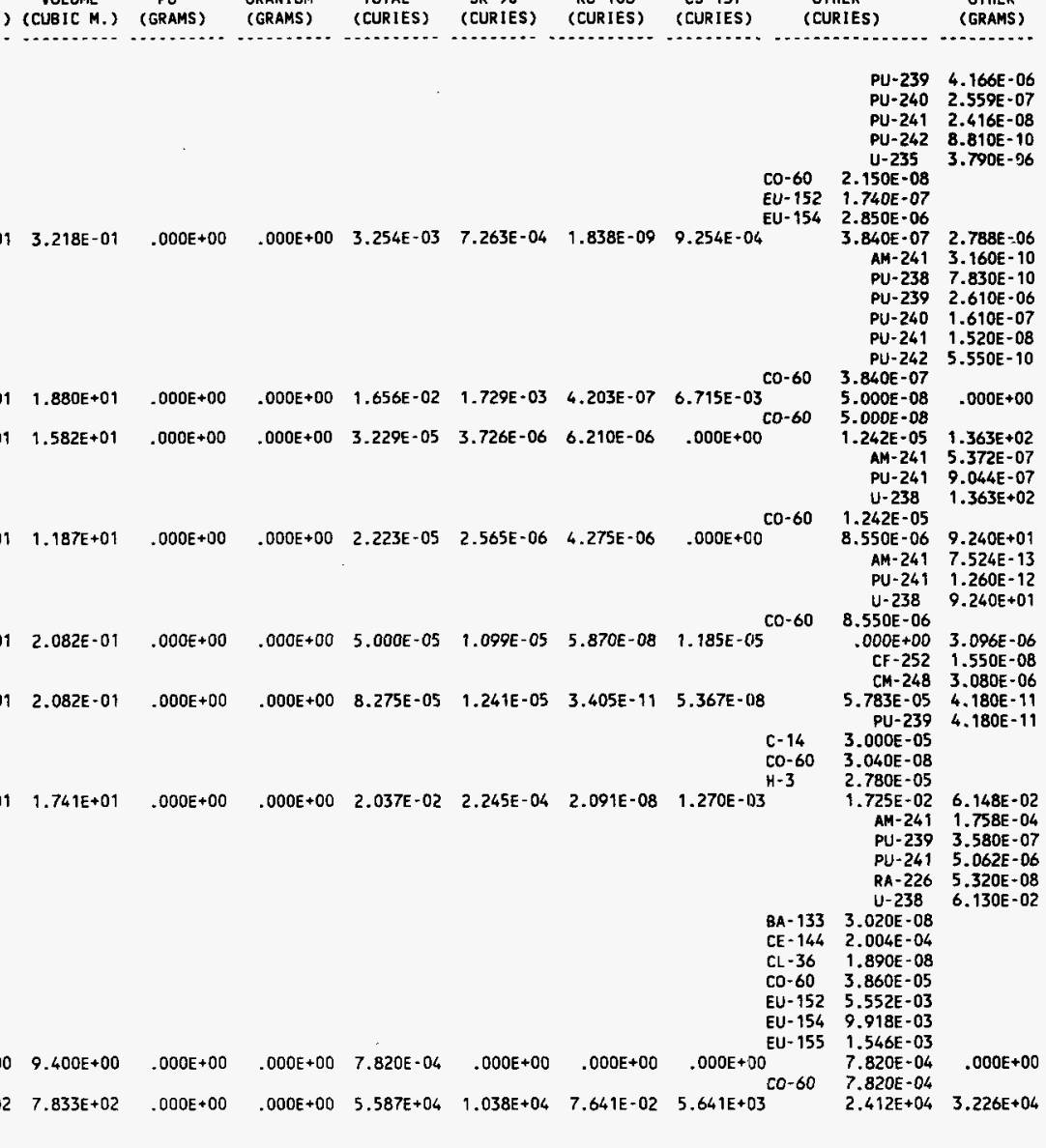

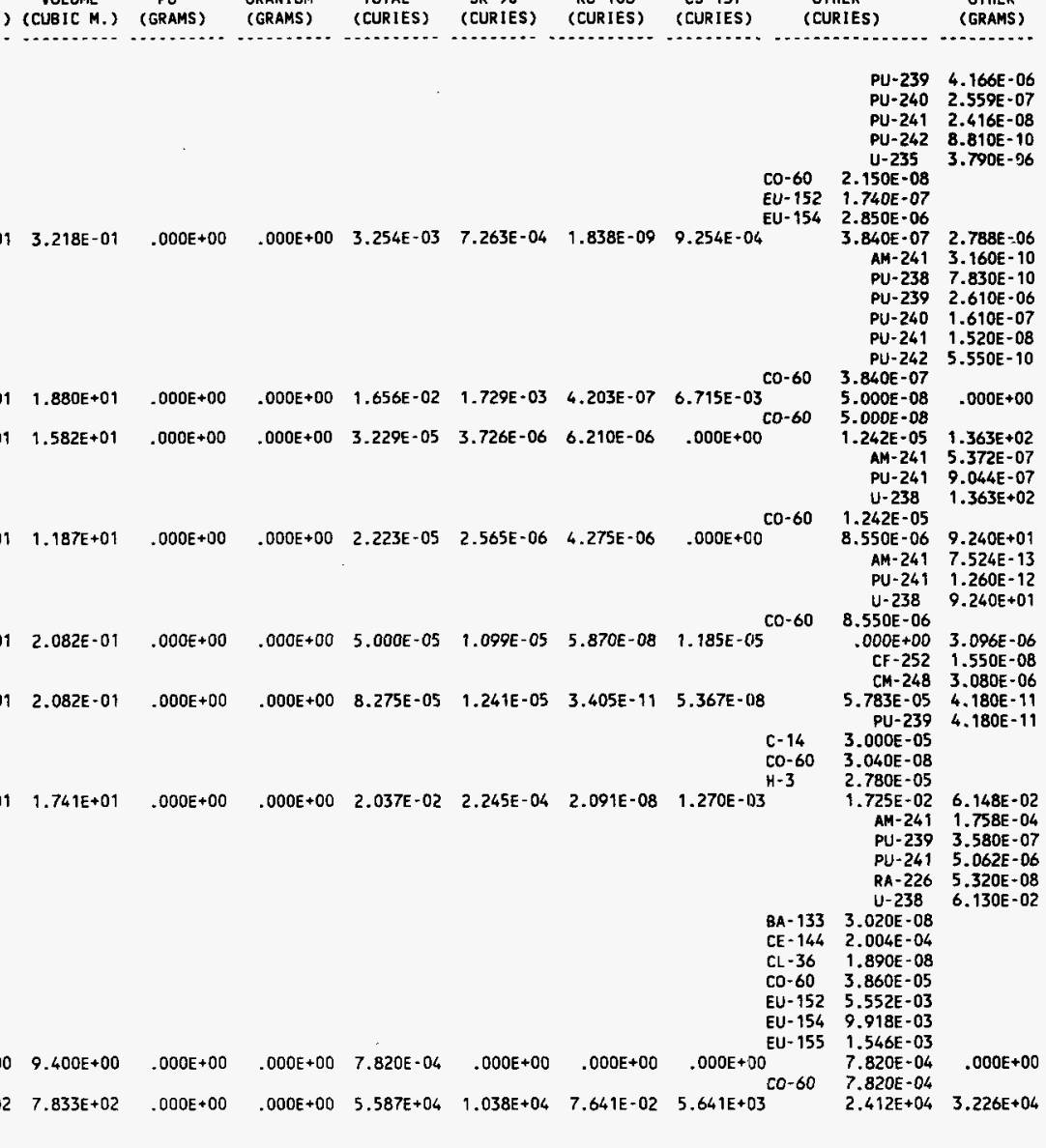

340

$$
1.741 E+01 \quad 1.741 E+01
$$

$.000 E+00$

$.000 E+00$

$2.037 E-02$

$2.245 E-04$

2.091E-08

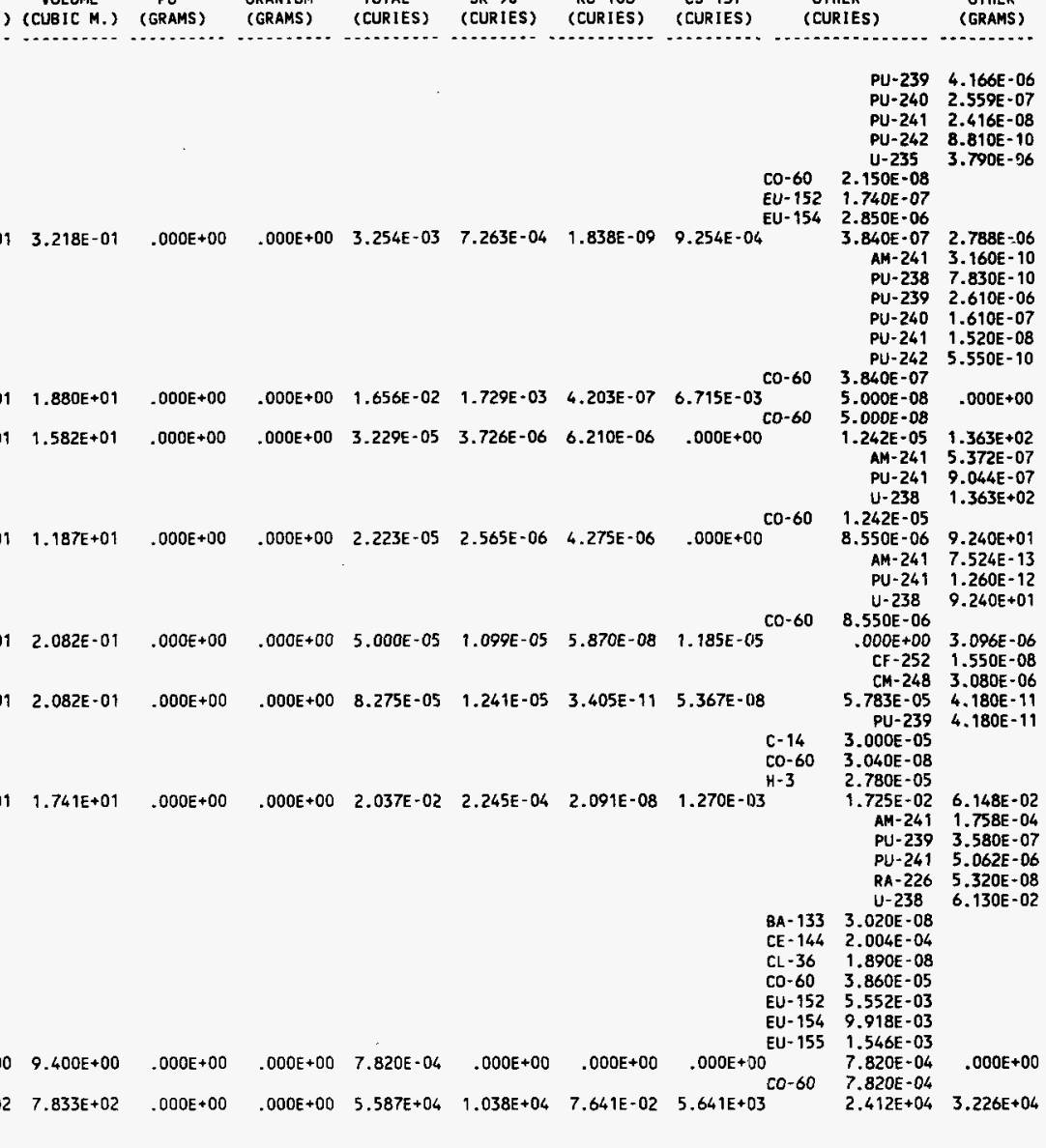

$.000 E+00$

$.000 E+00$

\begin{abstract}
$.000 E+00$
\end{abstract}
$B A-133$
$C E-144$

$\mathrm{CL}-36$

Co. 60

EU- 952

EU- 154

EU- 155

TOTALS
$7.833 E+02$
$7.833 E+0.2$

$7.8336+0$


SWIR3280 - (Page 28 of 107 )

RUN DATE: $03 / 15 / 96$

Land Disposal or Storage of Solid Radioactive Waste from January 1 to Decernber 31 , 1995

BURIAL GROUND 218 W4C, 200 WEST AREA, LOW-LEVEL NONINDUSTRIAL.

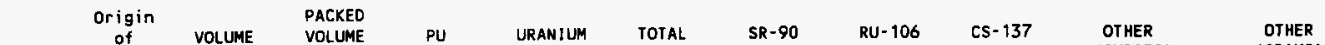
Company Waste (CUBIC M.) (CUBIC M.) (GRAMS) (GRAMS) (CURIES) (CURIES) (CURIES) (CURIES) (CURIES)

TRENCH 33

BH1 200E

$2025 \quad 6.246 E-01 \quad 6.246 E-01$

$.000 E+00$

$.000 \mathrm{E}+00$

$.000 \mathrm{E}+00$

8.220E-08

OOOE-08

3.000E-08 $\quad 1.761 E-14 \quad 1.140 E-08$

EU- $155+1.034 \mathrm{E}-11$

KEH 200W

$1.145 E+01 \quad 1.145 E+01$

$.000 E+C 10$

$.000 E+00$

$6.460 E-03$

$1.788 E-03 \quad 1.883 E-08 \quad 6.640 E-05$

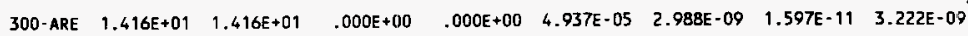

PNL

324

4.

325
$4.372 E+00 \quad 4.372 E+00$

$9.369 E+00 \quad 9.369 E+00$
$.000 E+00$

$.000 E+0$$$
\text { A }
$$

AC -228

BI- 214

PB- 214

TL-208

Co- 60

$\mathrm{MN}-54$

\begin{tabular}{l} 
CE- 144 \\
CO- 60 \\
EU-154 \\
FE-59 \\
MN-54 \\
NB-95 \\
SB- 125 \\
ZN-65 \\
ZR-95 \\
\\
AC- 228 \\
$B I-214$ \\
PB- 214 \\
TL-208 \\
CO- 60 \\
MN-54 \\
\hline
\end{tabular}

C -14

CA -45

CD -109

$C L-36$

co. 57

co- 60

EU- 152

EU-154

FE -55

FE- 59

$\mathrm{H}-3$

]- 129

$\mid-131$

KR -85

MN -54

NA -22
1. $034 E-11$

$.000 E+00 \quad .000 E+00$

$.000 E+00 \quad 1.179 E+03$

$\mathrm{U}-235 \quad 6.397 E+90$

U-238 $1.172 E+03$

2.661E-03 2.071E+00

AM-241 2.624E-06

$\mathrm{U}-235 \quad 2.071 \mathrm{E} \pm 00$

$8.053 E-05$

1.959E-03

3.131E-05

9.212E-05

$3.871 E-04$

$2.237 \mathrm{E}-05$

3.577E-05

4. $025 \mathrm{E}-05$

$1.342 \mathrm{E}-05$

4.935E-05

TH-228

TH-234

$\mathrm{U}-235$

1.877E-05

1. 176E-05

$1.312 E-05$

5.698E-06

$3.835 E-05$

$.465 E-05$

1. 370E-05

$1.002 E+00 \quad 1.241 E+02$

AM-241 1.632E-04

AM-243 5.260E-D9

CM-244 6.600E-10

NP- 237 1.600E-03

PU-238 1.170E-08

PU-239 1.384E-04

PU- 241 3.524E-08

PU-242 3.016E-07

TH-228 1.500E- 11

TH-230 1.100E-06

$\mathrm{TH}-232 \quad 1.544 \mathrm{E}+01$

U-232 5.230E-10

$U-235 \quad 3.073 E-02$

$\mathrm{U}-238 \quad 1.086 \mathrm{E}+02$

$7.018 \mathrm{E}-05$

1. $000 E-07$

6. $000 E-07$

4. $500 E-08$

$1,000 E-07$

$2.522 E-04$

$1.200 E-06$

1. $299 \mathrm{E}-06$

1. $000 \mathrm{E}-06$

1. $000 \mathrm{E}-12$

$1.000 \mathrm{E}+00$

4. $260 \mathrm{E}-08$

1. $000 \mathrm{E}-08$

2. $000 \mathrm{E}-03$

2. $105 E-06$

3. $752 E-06$ 
SHIR3280 - (Page 18 of 107)

RUN DATE: $03 / 15 / 96$

Land Disposal or storage of Solid Radioactive waste from January 1 to December 31 , 1995

BURIAL GROUND 218 WAE, 200 WEST AREA, LOW-LEVEL NONINDUSTRIAL.

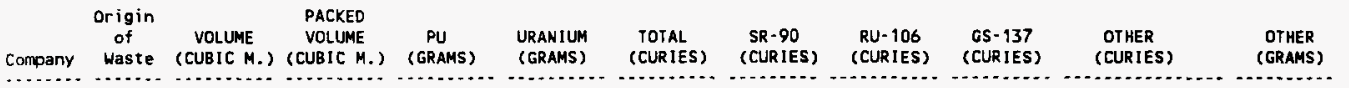

TRENCH 26

$\begin{array}{llllllllll}\text { WHC } & 151 E R & 6.436 E-01 & 6.436 E-01 & .000 E+00 & .000 E+00 & 4.920 E-03 & 2.237 E-03 & 6.336 E-09 & 1.653 E-0.4\end{array}$

$202 A \quad 3.123 E+00 \quad 3.123 E+00 \quad .000 E+00 \quad .000 E+00 \quad 8.652 E-04 \quad 1.684 E-04 \quad 1.561 E-10 \quad 2.054 E-04$

\begin{tabular}{|c|c|c|c|c|c|c|c|c|}
\hline 2225 & $1.041 E+00$ & $1.041 E+00$ & $.000 E+00$ & $.000 E+00$ & $5.425 E-03$ & $1.826 E-03$ & $1.459 \mathrm{E}-07$ & $9.044 E-04$ \\
\hline 3452 & $2.894 E+01$ & $2.894 E+01$ & $.000 E+00$ & $.000 E+00$ & $6.950 E-01$ & $1.527 \mathrm{E}=01$ & $8.159 E-04$ & $1.646 E-01$ \\
\hline
\end{tabular}

$241 \mathrm{~A}$

$6.399 \mathrm{E}+00 \quad 6.399 \mathrm{E}+00$

$.000 E+0$ 
SWIR3280 - (Page 30 of 107)

RUN DATE: $03 / 15 / 96$

Land Disposal or storage of Solid Radioactive Waste from January 1 to Decernber 31 , 1995

BURIAL GROUND 218 W4C, 200 WEST AREA, LOW-LEVEL NONINDUSTRIAL.

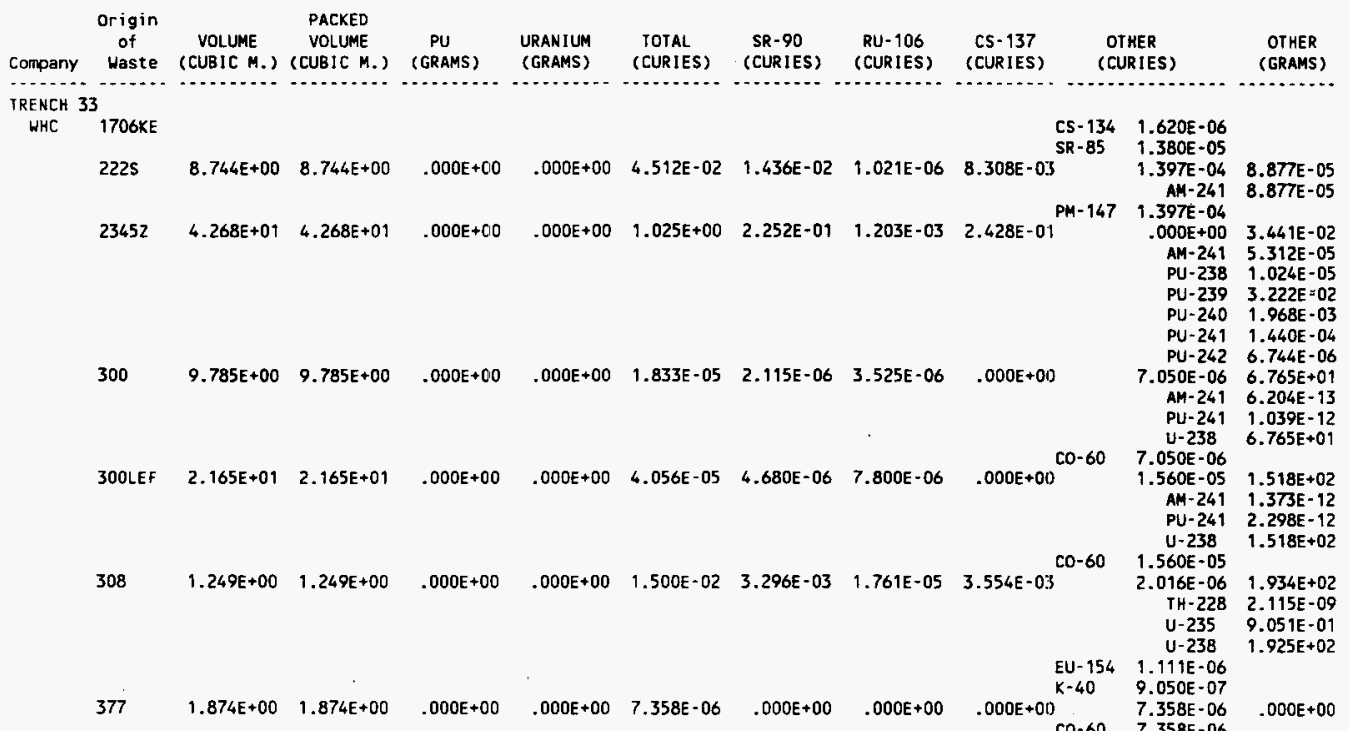

IRENCH 53

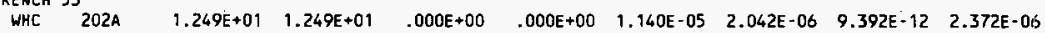

$7.358 E-06$
$7.358 E-06$

TRENCH NAVAL CORE

KEH 200W

$8.953 E+00$

$8.953 E+00$

$.000 E+00$

$.000 E+0$

$4.896 \mathrm{E}-03$

$1.349 E-03$

$9.338 E-09 \quad 4.924 E-0 '$

\section{C.}

AC - 228

BI -214

2.700E-06

4. $950 E-08$

PU-241 4.950E-08

$\begin{array}{ll}-14 & 2.980 \mathrm{E}-15\end{array}$

$\mathrm{H}-3$ 1.010E-08

PM-147 2.690E-06

CE-144 6.074E-05

CO-60 1.478E-03

EU- $154 \quad 2.363 E-05$

FE-59 7.099E-05

MN-54 3.094E-04

AB-95 1.689E-05

PB-214 1.640E-06

SB-125 2.700E-05

2N-65 3.038E-05

$2 \mathrm{R}-95 \quad 1.013 \mathrm{E}-05$

$2724 \mathrm{~W} \quad 8.328 \mathrm{E}-01 \quad 8.328 \mathrm{E}-01 \quad .000 \mathrm{E}+00 \quad .000 \mathrm{E}+00 \quad 6.197 \mathrm{E}-04 \quad 1.728 \mathrm{E}-04 \quad 4.109 \mathrm{E}-11 \quad 6.061 \mathrm{E}-0.5$

$2.533 E-04$

AM- 241

$2.004 E-01$

U- 235

CE-144 7.800E-06

2. $004 E-01$

CO-60 1.896E-04

EU-154 3.030E-06

FE-59 $9.090 E-06$

MN-54 3.294E-05

\section{$1.354 E-04$}

$1.307 E-04$ 4. $642 E-06$ 
WHC-EP-0125-8

SWIR3280 - (Page 16 of 107)

RUN DATE: $03 / 15 / 96$

Land Dispoeal or Storage of Solid Radioactive Waste from January 1 to December 31 , 1995

BURIAL GROUND 218W3AE, 200 WEST AREA, LOW-LEVEL NONINDUSTRIAL.

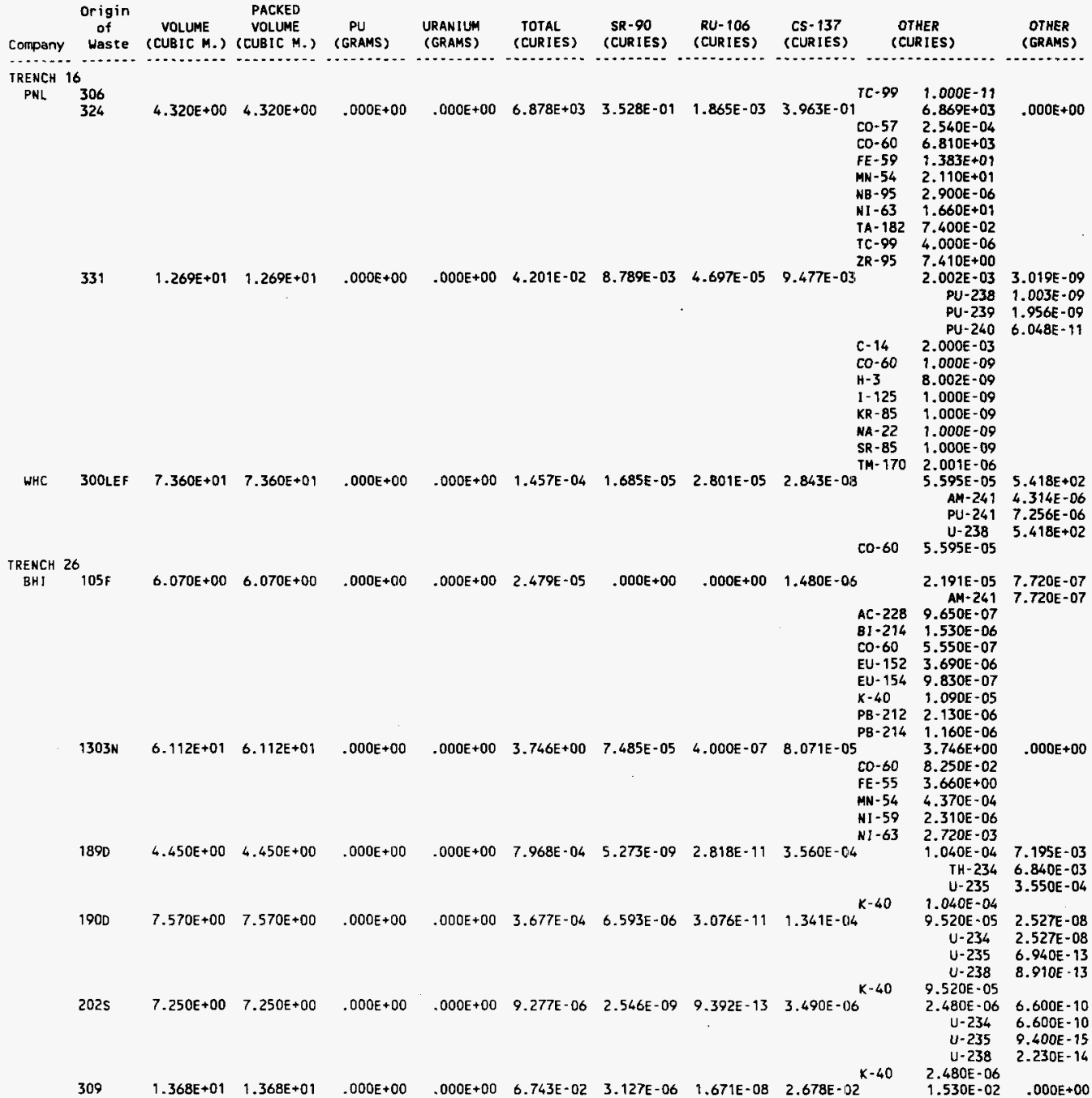


SWIR3280 - (Page 32 of 107)

RUN DATE: $03 / 15 / 96$

Land Disposal or Storage of Solid Radioactive waste from January 1 to Decernber 31 , 1995

BURIAL GROUND $218 W 4 \mathrm{C}$, 200 WEST AREA, LOW-LEVEL NONINDUSTRIAL.

Origin
of VOLUME $\begin{gathered}\text { PACKED } \\ \text { VOLUME }\end{gathered}$

Company Waste (CUBIC M.) (CUBIC M.) (GRAMS)

(GRAMS) (CURIES) (CURIES) (CURIES) (CURIES)

(CURIES)

(GRAMS)

TRENCH MAVAL CORE

WHC $224 \mathrm{U}$

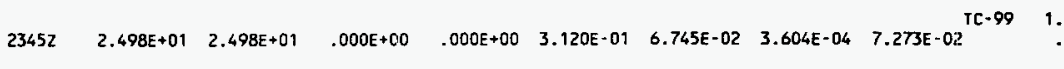

$\mathrm{U}-236 \quad 1.800 \mathrm{E}-05$

U-235 1.840E-03

U-236 1.420E-04

U-238 1.982E-01

.224E-07

$.000 E+00 \quad 1.896 E-02$

AM-241 2.199E-05

PU-23B 3.785E-03

PU-239 $1.410 E-02$

PU-240 9.248E-04

$P U-241 \quad 1.226 E-04$

PU-242 3.582E-06

$241 \mathrm{~A} \quad 2.082 \mathrm{E}-01 \quad 2.082 \mathrm{E}-01 \quad .000 \mathrm{E}+00 \quad, 000 \mathrm{E}+00 \quad 2.580 \mathrm{E}-06 \quad 1.101 \mathrm{E}-06 \quad 2.935 \mathrm{E}-12 \quad 1.946 \mathrm{E}-0.7$

.00E $+00 \quad 2.200 \mathrm{E}-08$

AM-241 $2.490 \mathrm{E}-12$

PJ- 238 6.170E-12

PU-239 2.060E-08

PU-240 1.270E-09

PU-241 1.200E-10

241AW $\quad 9.198 E-01 \quad 9.198 E-01 \quad .000 E+00 \quad .000 E+00 \quad 6.110 E-04 \quad 2.422 E-04 \quad 9.462 E-10 \quad 6.459 E-0 ; 5$

8. $720 \mathrm{E}-07 \quad 2.922 \mathrm{E}-05$

AM-241 3.301E-09

PU-238 8.140E-09

PU-239 2.737E-05

PU $-240 \quad 1.674 \mathrm{E}-06$

PU-241 1.584E-07

PU-242 5.780E-09

$241 \mathrm{AY} \quad 2.082 E+00 \quad 2.082 E+00 \quad .000 E+00 \quad .000 E+00 \quad 4.459 E-04 \quad 4.413 E-05 \quad 2.963 E-09 \quad 1.831 E-0.4$

CO-60 8.720E-07

$1.023 E-06 \quad 5.563 E-06$

AM- $241 \quad 5.022 E-10$

PU-238 1.563E-09

PU $-239 \quad 5.210 \mathrm{E}-06$

PU-240 3.184E-07

PU- 241 3.171E-OB

PU-242 1.101E-09

$241 B X \quad 2.438 E+00 \quad 2.438 E+00 \quad .000 E+C O \quad .000 E+00 \quad 6.914 E-03 \quad 1.224 E-03 \quad 3.062 E-08 \quad 2.293 E-03^{C O}$

C0-60 1.023E-06

$.023 \mathrm{E}-06$

$.034 E-06 \quad 1.408 E-05$

AM-241 $1.272 E-09$

PU- 238 3.951E-09

PU-239 1.319E-05

$\mathrm{PU}-240 \quad 8.094 \mathrm{E}-07$

PU-241 7.736E-08

PU-242 2.801E-09

$241 C \quad 2.116 E+00 \quad 2.116 E+00 \quad .000 E+C D \quad .000 E+00 \quad 1.669 E-03 \quad 4.669 E-04 \quad 3.724 E-09 \quad 3.774 E-0.4$

CO-60 1.034E-06

$5.986 \mathrm{E}-07 \quad 1.238 \mathrm{E}+01$

AM- $241 \quad 1.853 E-09$

PU-238 5.282E -09

PU $-239 \quad 1.763 E-05$

PU-240 1.081E-06

PU-241 1.036E-07

PU- $242 \quad 3.734 E \cdot 09$

$U-238 \quad 1.238 E+01$

5. $986 \mathrm{E}-07$

8.300E-07 5.577E+01

AM-241 1.192E 09

PU-238 3.716E-09

PU-239 1.239E-05

PU-240 7.615E-0?

PU-241 7.343E-08

PU-242 2.635E-09

CO-60 8.300E-07

$U-238$
$300 E-07$ 
SWIR3280 - (Page 14 of 107)

RUN DATE: $03 / 15 / 96$

Land Disposal or storage of Solid Radioactive Waste from January 1 to December 31 , 1995 BURIAL GROUND 218 W3AE, 200 WEST AREA, LOW-LEVEL NONINDUSTRIAL.

\begin{tabular}{|c|c|c|c|c|c|c|c|c|c|}
\hline Company & $\begin{array}{c}\text { Origin } \\
\text { of } \\
\text { Waste }\end{array}$ & $\begin{array}{l}\text { VOLUME } \\
\text { (CUBIC M.) }\end{array}$ & $\begin{array}{l}\text { PACKED } \\
\text { VOLUME } \\
\text { (CUBIC M.) }\end{array}$ & $\begin{array}{c}\text { PU } \\
\text { (GRAMS) }\end{array}$ & $\begin{array}{l}\text { URANIUM } \\
\text { (GRAMS) }\end{array}$ & $\begin{array}{l}\text { TOTAL } \\
\text { (CURIES) }\end{array}$ & $\begin{array}{l}\text { SR-90 } \\
\text { (CURIES) }\end{array}$ & $\begin{array}{c}\text { RU- } 106 \\
\text { (CURIES) }\end{array}$ & $\begin{array}{c}\text { CS- } 137 \\
\text { (CURIES) }\end{array}$ \\
\hline $\begin{array}{l}\text { RENCH } \\
\text { WHE }\end{array}$ & ${ }^{3} 2415$ & & & & & & & & \\
\hline
\end{tabular}

$\begin{array}{lllllllll}8.928 E-01 & 8.928 E-01 & .000 E+00 \quad .000 E+00 & 2.561 E-04 & 9.833 E-05 & 1.749 E-10 & 3.056 E-05\end{array}$

$2415 Y \quad 3.874 E+00 \quad 3.874 E+00 \quad .000 E+00 \quad .000 E+00 \quad 9.018 E-03 \quad 3.177 E-03 \quad 2.685 E-08 \quad 1.365 E-03$
$241 T \quad 2.415 E+00 \quad 2.415 E+00 \quad .000 E+00 \quad .000 E+00 \quad 1.303 E-03 \quad 3.711 E-04 \quad 4.114 E-09 \quad 2.756 E-0$.

$.000 E+00$

\section{.}

CS-134 $1.061 E-07$

CD-60 7.076E-06

EU- $154 \quad 4.731 E-06$

.000E+00 4.047E-06

AM-241 4.570E-10

PU-238 1.136E =09

PU-239 3.790E-06

PU-240 2.324E-07

PU-241 2.195E-08

PU-242 8.020E-10

4.27BE-06 3.893E-04

AM-241 $3.792 E-08$

PU-238 1.090E-07

PU-239 3.646E-04

PU-240 2.238E-05

PU-241 2.124E*06

PU-242 7.725E-08

2.411E-05

AM- 241

PU-238 4.658E-08

PU-239 1.569E-04

PU-240 9.578E-06

PU-241 9.041E-07

$P U-242 \quad 3.295 E-08$

$2411 X \quad 1.135 E+00 \quad 1.135 E+00 \quad .000 E+00 \quad .000 E+00 \quad 2.487 E-03 \quad 7.941 E-04 \quad 1.099 E-09 \quad 4.613 E-04$

Co-60 1.946E-06

EU- 154 2.160E-06

K-40 2.000E-05

2410

$2.358 E+00 \quad 2.358 E+00$

$.000 E+00$

$.000 \mathrm{E}+$

$00 \quad 5.267 E-03 \quad 1.410 E-03 \quad 3.799 E-08 \quad 1.252 E-03$

4
CO- 60
CS- 134
EU- 154
3

4.639E- 07

AN- 241

$P U=238$

PU-239

PU- 240

PU- 241

PU-242

$\mathrm{U}-235$

3. 187E-07

CS- $134 \quad 9.280 \mathrm{E}-08$

9.280E -08
$5.240 E-08$

6. 132E-06

AM- 241

PU -238

PU-239

PU-240

PU-241

PU -242

U- 235

C0.60 1.585E-06

CS-134 B.055E-08

EU-154 4.467E-06

$6.727 \mathrm{E}-07$

AM- 241

PU-238

PU- 239

$6.221 \mathrm{E}-07$

$5.721 E-11$

$1.746 \mathrm{E}-10$

PU-240 $3.572 E-08$ 
SWIR3280 - (Page 34 of 107)

RUN DATE: $03 / 15 / 96$

Land Disposal or storage of Solid Radioactive Waste from January 1 to December 31 , 1995 BURIAL GROUND 218W4C, 200 WEST AREA, TRANSURANIC WIPP CERTIFIED.

\begin{tabular}{|c|c|c|c|c|c|c|c|c|c|c|c|}
\hline Company & $\begin{array}{c}\text { Origin } \\
\text { of } \\
\text { Waste }\end{array}$ & $\begin{array}{c}\text { VOLUME } \\
\text { (CUBIC M.) }\end{array}$ & $\begin{array}{c}\text { PACKED } \\
\text { VOLUME } \\
\text { (CUBIC M.) }\end{array}$ & $\begin{array}{c}\mathrm{PU} \\
\text { (GRAMS) }\end{array}$ & $\begin{array}{l}\text { URANIUM } \\
\text { (GRAMS) }\end{array}$ & $\begin{array}{l}\text { TOIAL } \\
\text { (CURIES) }\end{array}$ & $\begin{array}{l}\text { SR-90 } \\
\text { (CURIES) }\end{array}$ & $\begin{array}{l}\text { RU- } 106 \\
\text { (CURIES) }\end{array}$ & $\begin{array}{c}\text { CS- } 137 \\
\text { (CURIES) }\end{array}$ & $\begin{array}{l}\text { OTHER } \\
\text { (CURIES) }\end{array}$ & $\begin{array}{l}\text { OTHER } \\
\text { (GRAMS) }\end{array}$ \\
\hline RENCH & - & & $\cdots$ & . & & 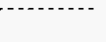 & $\cdots$ & & & & \\
\hline $\begin{array}{c}\text { WHC } \\
\text { TOIALS }\end{array}$ & 23452 & $\begin{array}{l}2.082 E-01 \\
2.082 E-01\end{array}$ & $\begin{array}{l}2.082 E-01 \\
2.082 E-01\end{array}$ & $\begin{array}{l}.000 E+100 \\
.000 E+130\end{array}$ & $\begin{array}{l}.000 E+00 \\
.000 E+00\end{array}$ & $\begin{array}{l}.000 E+00 \\
.000 E+00\end{array}$ & $\begin{array}{l}.000 E+00 \\
.000 E+00\end{array}$ & $\begin{array}{l}.000 E+00 \\
.000 E+00\end{array}$ & $\begin{array}{l}.000 E+00 \\
.000 E+00\end{array}$ & $\begin{array}{l}.000 E+00 \\
.000 E+00\end{array}$ & $\begin{array}{l}.000 E+00 \\
.000 E+00\end{array}$ \\
\hline
\end{tabular}


SWIR3280 - (Page 12 of 107)

RUN DATE: $03 / 15 / 96$

Land Disposal or storage of Solid Radioactive Waste from January 1 to December 31 , 1995

BURIAL GROUND 218 W3AE, 200 WEST AREA, LOW-LEVEL NONINDUSTRIAL.

$\begin{array}{cccccccc}\text { Origin } & \text { PACKED } & \text { POLUME } & \text { VOLUME } & \text { PU } & \text { URANIUM TOTAL } & \text { SR-90 } & \text { RU-106 CS-137 OTHER }\end{array}$

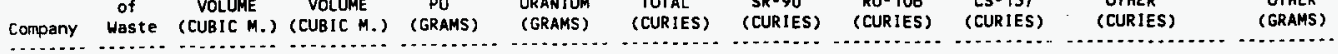

TRENCH 03

TRENCH 03

BHI 309

2.355E+01 $2.355 E+01$

(CURIES)

(CURIES)

(CURIES)

(GRAMS)

KEH LAUNDRY $\quad 5.621 E+00 \quad 5.621 E+00 \quad .000 E+00 \quad .000 E+00 \quad 3.630 E-03 \quad 1.012 E-03 \quad 8.529 E-09 \quad 3.715 E-05$

$5.537 E-01$
$1.481 E-03$

3.220E-11

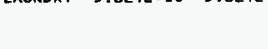

(1)

$\begin{array}{lllllllllll}\text { PNL } & 324 & 5.184 E+01 & 5.184 E+01 & .000 E+00 & .000 E+00 & 4.519 E+04 & 1.017 E+04 & 7.233 E-02 & 5.235 E+03 \\ \text { WHC } \quad 204 A R & 9.198 E-01 & 9.198 E-01 & .000 E+00 & .000 E+00 & 2.008 E-04 & 3.556 E-05 & 8.570 E-10 & 5.403 E-05\end{array}$

$\begin{array}{lcc} & \begin{array}{c}\text { AM-241 } \\ U-235\end{array} & 1.480 E-J 6 \\ \text { CE-144 } & 4.553 E-05 & 1.170 E+00 \\ \text { CO-60 } & 1.109 E-03 & \\ \text { EU-154 } & 1.772 E-05 & \end{array}$

EU- $154 \quad 1.772 E-05$

FE-59 5.314E-05

MN-54 1.924E-04

NB-95 1.266E-05

SB-125 2.025E-05

ZN-65 2.279E-05

ZR-95 ?.599E-06

$\begin{array}{ll}\text { Co-60 } & 1.466 \mathrm{E}+04 \\ 6.487 \mathrm{E}+03\end{array}$

MN-54 8.170E+03

2.449E-05

AM- 241

PU-238

PU-239

PU-240

PU-241

PU-242

CD-60 6.260E-07

EU-152 8.240E-06

EU-154 1.562E-05

209

$\begin{array}{llllllll}8.970 E-01 & 8.970 E-01 \quad .000 E+00 \quad .000 E+00 & 5.999 E-04 & 2.631 E-04 & 1.503 E-09 & 3.691 E-05\end{array}$

$1.562 E-05$
$1.817 E-06$

AM-241

PU-238 $1.405 E-09$

PU-239 4.684E-06

PU-240 2.874E-07

PU-241 2.734E-08

PU-262 $9.961 E-10$

$241 \mathrm{~A} \quad 2.244 \mathrm{E}+00 \quad 2.244 \mathrm{E}+00 \quad .000 E+00 \quad .000 E+00 \quad 6.478 E-03 \quad 2.223 E-03 \quad 2.676 E-07 \quad 1.028 E-03$

CO-60 1.817E-06

7.477E-06 2.645E-04

AM-241 $2.533 E-08$

PU- $238 \quad 7.265 E-08$

PU $-239 \quad 2.430 E-04$

PU-240 1.490E-05

PU-241 1.412E-06

PU-242 5.145E-08

CO-60 $\quad \begin{gathered}\mathrm{U}-235 \\ \mathrm{C}-\mathrm{5} 4 \mathrm{EE}-06\end{gathered}$

CS-134 1.171E-07

241 AN $\quad 2.438 E+00 \quad 2.438 E+00 \quad .000 E+00 \quad .000 E+00 \quad 6.011 E-03 \quad 1.408 E-03 \quad 2.290 E-08 \quad 1.636 E-03$

EU-154 $1.818 E-06$

(n)

3

$9.021 \mathrm{E}-06$

AM- 24 PU- 238

PU-239

PU-240

PU-241

PU-242

$5.0605-06$

$1.852 \mathrm{E} \cdot 03$

1. $.6945-07$

5. 174E-07

$1.735 E-03$

$1.061 \mathrm{E}-04$

$\begin{array}{ll}\text { CO-60 } & 2.425 E-06 \\ \text { EU-154 } & 6.596 E-06\end{array}$

$241 A P \quad 1.165 E+00 \quad 1.165 E+00 \quad .000 E+00 \quad .000 E+00 \quad 2.574 E-03 \quad 4.746 E-04 \quad 2.687 E-08 \quad 7.623 E-04$

$1.394 \mathrm{E}-04$

AM- 241

$\mathrm{PU}-238$

3.175E-05

$3.594 \mathrm{E}-09$

8.869E-09

$2.965 E-05$ 
WHC-EP-0125-8

SHIR3280 - (Page 36 of 107 )

RUN DATE: 03/15/96

Land Disposal or Storage of Solid Radioactive Waste from January 1 to December 31 , 1995 BURIAL GROUND 218W5, 200 WEST AREA, LOW-LEVEL INDUSTRIAL.

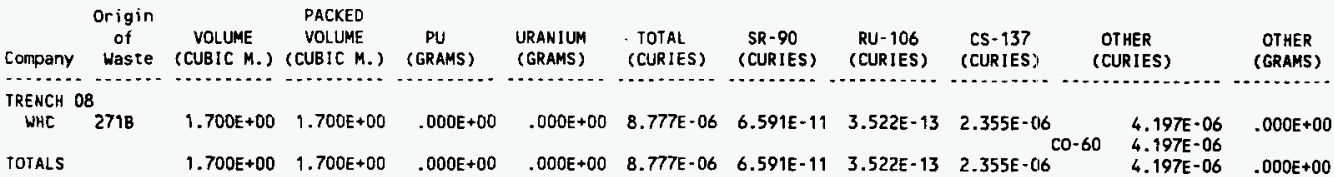


SWIR3280 - (Page 10 of 107 )

RUN DATE: $03 / 15 / 96$

Land Disposal or Storage of Solid Radioactive Waste from January 1 to. Decernber 31 , 1995 BURIAL GROUND 218W3AE, 200 WEST AREA, LOW-LEVEL NONINDUSTRIAL-OFFSITE.

\begin{tabular}{|c|c|c|c|c|c|c|c|c|c|c|c|c|}
\hline Company & $\begin{array}{c}\text { Origin } \\
\text { of } \\
\text { Haste }\end{array}$ & $\begin{array}{l}\text { VOLUME } \\
\text { (CUBIC M.) }\end{array}$ & $\begin{array}{c}\text { PACKED } \\
\text { VOLUME } \\
\text { (CUBIC M.) }\end{array}$ & $\begin{array}{c}\text { PU } \\
\text { (GRAMS) }\end{array}$ & $\begin{array}{l}\text { URANIUM } \\
\text { (GRAMS) }\end{array}$ & $\begin{array}{l}\text { TOTAL } \\
\text { (CURIES) }\end{array}$ & $\begin{array}{l}\text { SR-90 } \\
\text { (CURIES) }\end{array}$ & $\begin{array}{l}\text { RU-106 } \\
\text { (CURIES) }\end{array}$ & $\begin{array}{c}\text { CS- } 137 \\
\text { (CURIES) }\end{array}$ & \multicolumn{2}{|c|}{$\begin{array}{l}\text { OTHER } \\
\text { (CLRIES) }\end{array}$} & $\begin{array}{c}\text { OTHER } \\
\text { (GRAMS) }\end{array}$ \\
\hline \multicolumn{13}{|c|}{ TRENCH 26} \\
\hline BNL & BNLAB & & & & & & & & & $\begin{array}{l}M N-54 \\
N A-22 \\
S C-46 \\
S R-85 \\
\text { IA }-182 \\
\text { IC }-99 \\
2 N-65\end{array}$ & $\begin{array}{l}6.680 E-03 \\
1.222 E-01 \\
6.090 E-05 \\
2.691 E-04 \\
1.120 E-04 \\
5.861 E-05 \\
5.400 E-03\end{array}$ & \\
\hline FERM & MALAB & $1.499 \mathrm{E}+01$ & $1.499 \mathrm{E}+01$ & $.000 E+00$ & $.000 E+00$ & $5.558 E-02$ & $1.310 \mathrm{E}-05$ & $2.822 E-05$ & $1.399 \mathrm{E}-05$ & $\begin{array}{l}A U-195 \\
C O-60 \\
H-3 \\
M N-54 \\
N A-22 \\
Z N-65\end{array}$ & $\begin{array}{c}5.492 E-02 \\
T H-232 \\
U-235 \\
U-238 \\
9.250 E-05 \\
1.407 E-02 \\
2.080 E-02 \\
1.081 E-02 \\
7.759 E-03 \\
1.392 E-03\end{array}$ & $\begin{array}{l}2.189 \mathrm{E}+02 \\
1.779 \mathrm{E}+02 \\
9.000 \mathrm{E}-02 \\
4.091 \mathrm{E}+01\end{array}$ \\
\hline GAS & GASD & 8. $124 E+01$ & $8.124 E+01$ & $.000 \mathrm{E}+00$ & $.000 E+00$ & 2. $102 E+01$ & $1.62 B E-02$ & $4.043 E-03$ & $1.469 E-02$ & & $\begin{array}{c}2.088 E+01 \\
C M-247 \\
P U-238 \\
P U-239 \\
P U-241 \\
T H-232 \\
U-232 \\
U-233 \\
U-234 \\
U-235 \\
U-236 \\
U-238\end{array}$ & $\begin{array}{l}1.423 E+04 \\
3.710 E-04 \\
1.271 E-05 \\
3.073 E-04 \\
9.951 E-06 \\
1.421 E+04 \\
5.810 E-10 \\
1.502 E-03 \\
1.715 E-01 \\
1.736 E+01 \\
6.171 E-02 \\
1.118 E+00\end{array}$ \\
\hline & & & & 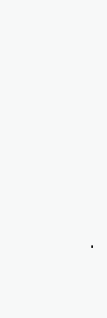 & & & & & & $\begin{array}{l}C-14 \\
C E-144 \\
C O-60 \\
C S-134 \\
E U-154 \\
E U-155 \\
\text { FE }-55 \\
F E-59 \\
H-3 \\
M N-54 \\
N B-94 \\
N I-59 \\
N I-63 \\
\text { SB- } 125 \\
T C-99\end{array}$ & $\begin{array}{l}2.078 E-03 \\
7.636 E-04 \\
1.514 E+00 \\
2.623 E-03 \\
1.119 E-04 \\
5.344 E-05 \\
6.764 E+00 \\
9.730 E-05 \\
3.876 E-03 \\
1.215 E+01 \\
2.955 E-03 \\
3.707 E-03 \\
4.303 E-01 \\
6.608 E-05 \\
8.465 E-03\end{array}$ & \\
\hline$M ! N$ & MAVY & $2.640 E+01$ & $2.640 E+01$ & $.000 E+00$ & $.000 E+00$ & $3.483 E-02$ & $.000 E+00$ & $.000 E+00$ & $.000 E+130$ & FE- 55 & $\begin{array}{l}3.483 E-02 \\
3.483 E-02\end{array}$ & $.000 E+00$ \\
\hline PORT & PORT & $8.245 E+01$ & $8.245 E+01$ & $.000 E+00$ & $.000 E+00$ & $1.503 E-01$ & $2.583 E-03$ & $5.562 E-03$ & $2.759 E-103$ & & $\begin{array}{r}2.038 E-02 \\
A M-241 \\
\text { NP- } 237 \\
P U-238 \\
P U-239 \\
P U-240 \\
T H-230 \\
T H-234 \\
U-234 \\
U-235 \\
U-238\end{array}$ & $\begin{array}{l}3.496 E+03 \\
1.969 E-04 \\
1.169 E+00 \\
6.314 E-06 \\
2.583 E-03 \\
6.927 E-04 \\
7.204 E-02 \\
7.753 E-07 \\
1.371 E+00 \\
1.093 E+03 \\
2.400 E+03\end{array}$ \\
\hline PPL & PRINC & $6.500 \mathrm{E}+00$ & $6.500 E+00$ & $.000 E+00$ & $.000 E+00$ & 2. $160 E+03$ & $.000 E+00$ & $.000 E+00$ & $.000 E+00$ & $\begin{array}{l}{ }_{0}^{T C}-99 \\
H-3\end{array}$ & $\begin{array}{l}2.038 E-02 \\
2.160 E+03 \\
2.160 E+03\end{array}$ & $.000 E+00$ \\
\hline
\end{tabular}


SWIR3280 - (Page 38 of 107 )

RUN DATE : 03/15/96

Land Disposal or storage of solid Radioactive waste from January 1 to December 31 , 1995

BURIAL GROUND 218W5, 200 WEST AREA, LOW-LEVEL NONINDUSTRIAL-OFFSITE.

$\begin{gathered}\text { Origin } \\ \text { of } \\ \text { Company }\end{gathered}$ Uaste
(CULIC M.)

TRENCH 29

BNL BNLAB
NA-22

RFP RF $\quad 5.565 E+01 \quad 5.565 E+01 \quad .000 E+00 \quad .000 E+00 \quad 1.750 E-01 \quad 3.480 E-03 \quad 7.495 E-03 \quad 3.717 E-03$

RKD ESG

$1.950 E+0$

$.000 E+10$

$.000 E+00$

$8.349 E-01$

$4.398 E-04 \quad 3.505 E-07 \quad 4.134 E-03$

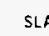

SLACU

$1.675 E+0$

$.000 E+00$

$3.083 E-04$

6

$.639 E-04$

043

SLA SLACU

TOTALS
$5.085 E+02 \quad 5.085 E+02$

$.000 E+00$
$.000 E+00 \quad 1.033 E+01 \quad 1.264 E-01$

$6.673 \mathrm{E}$
NB-94

NB-95

$\mathrm{NI}+63$

P- 32

PB-210

PO- 210

RU- 103

s- 35

5B- 124

$58-125$

SC- 46

SE -75

SN- 113

$S R-82$

SR-B5

TC- 99

TM- 170

ZN-65

$Z R-95$

8. 122E-03

$7.100 E-04$

$7.240 E-04$

$4.400 E+05$

5.797E-04

8.300E-05

$3.240 E-06$

$1.420 E-01$

$1.692 \mathrm{E}-03$

$1.360 \mathrm{E}-06$

$7.100 E-06$

$1.483 E-03$

$3.370 E-05$

4.520E-05

$1.000 E-08$

$2.001 E-03$

4.269E-05

3.SO0E-07

$5.209 \mathrm{E}-02$

5.040E-04 $.000 \mathrm{E}+00$ AM- 241

PU- 239

$\mathrm{U}-233$

u-235

U-238

8.260E-01

PU- 238

U-234

$\mathrm{U}-235$

$\mathrm{U}-238$

co-60

$1.088 E .02$

CS-134 3.030E-05

EU-152 2.004E-02

EU- 154 1.881E-03

FE-55 3.567E-01

NI $-59 \quad 2.100$ E- 03

NI-63 4.344E-01

$9.500 \mathrm{E}-02$

CO-57 8.8OOE-03

MN $-54 \quad 3.390 E-02$

NA-22 $4.990 \mathrm{E}-02$

2N-65 2.400E-03

B. $158 \mathrm{E}+00$

$1.788 E+04$ 
SWIR3280 - (Page 8 of 107)

RUN DATE: $03 / 15 / 96$

Land Disposal or storage of Solid Radioactive Waste from January 1 to December 31 , 1995 BURIAL GROUND 218 W3AE, 200 WEST AREA, LOW-LEVEL NONINDUSTRIAL-OFFSITE.

\begin{tabular}{|c|c|c|c|c|c|c|c|c|c|c|c|}
\hline Company & $\begin{array}{c}\text { Origin } \\
\text { of } \\
\text { Waste }\end{array}$ & $\begin{array}{l}\text { VOLUME } \\
\text { (CUBIC M.) }\end{array}$ & $\begin{array}{l}\text { PACKED } \\
\text { VOLUME } \\
\text { (CUBIC M.) }\end{array}$ & $\begin{array}{c}\text { PU } \\
\text { (GRAMS) }\end{array}$ & $\begin{array}{l}\text { URANIUM } \\
\text { (GRAMS) }\end{array}$ & $\begin{array}{l}\text { TOTAL } \\
\text { (CURIES) }\end{array}$ & $\begin{array}{l}\text { SR-90 } \\
\text { (CURIES) }\end{array}$ & $\begin{array}{l}\text { RU-106 } \\
\text { (CURIES) }\end{array}$ & $\begin{array}{l}\text { CS-137 } \\
\text { (CURIES) }\end{array}$ & $\begin{array}{l}\text { OTHER } \\
\text { (CURIES) }\end{array}$ & $\begin{array}{c}\text { OTHER } \\
\text { (GRAMS) }\end{array}$ \\
\hline & & & & & & & & & & & \\
\hline
\end{tabular}

TRENCH 16

ANL ARGON

PPL PRINC

UCD DAVIS

$2.340 E+00 \quad 2.340 E+00$

$5.678 \mathrm{E}+00 \quad 5.678 \mathrm{E}+00$

$.000 \mathrm{E}+00$

$.000 E+00$

TRENCH 26

AME AMES

$1.176 \mathrm{E}+01 \quad 1.176 \mathrm{E}+01$

$.000 \mathrm{E}+00$

$\begin{array}{lllll}.000 E+00 \quad 4.367 E-03 & 1.245 E-06 & 2.681 E-06 & 6.758 E-04\end{array}$
AC -2

AC- 228

B1 -212

BI-214

CO-60

PA- 231

PB- 212

$\mathrm{RA}-228$

$T L-208$

ANL
$5.054 E+02 \quad 5.054 E+02$

$.000 E+00$
$.000 E+0$

\section{2}$$
\text { AC- } 227 \quad 1.000 \mathrm{E}-06
$$$$
A G-110 \quad 9.600 E-06
$$$$
B A-133 \quad 3.634 E-06
$$$$
\mathrm{BE}-7 \text { 1.022E-03 }
$$$$
\text { B1-212 1.730E-05 }
$$$$
\text { B1-214 3.670E-06 }
$$$$
\text { C. } 14 \quad 5.245 E-05
$$$$
\text { CA-45 } 1.500 \text { E- } 03
$$$$
\text { CD- } 109 \quad 2.232 E-05
$$

$.000 E+00$

3.555E-04

3. $555 E-04$

$1.087 E+04$ $7.302 E-04$

$5.817 E=07$

$7.673 E+03$

8.660E-08

$7.111 \mathrm{E}-04$

$1.600 E+00$

3. $200 E+03$

U- 238
$.900 E-10$

$7.672 E-04$

$3.720 E-07$

$1.900 E-09$

$2.802 E-04$
$7.674 E-04$

$.001 E-04$
$3.163 E+00$

$1.161 \mathrm{E}-03$

AM-243 2.316E-03

CF-251 1.500E-07

3.020E-12

CM-264 $5.719 \mathrm{E}-06$

CM-245 8.100E-06

PU $-238 \quad 1.473 \mathrm{E}-05$

1.070E-02

PU-242 $9.000 E-04$

PU-244 2.319E-02

A-226 3.531E-05

$1.400 E-09$
$2.856 E-03$

$2.811 \mathrm{E}+00$

$2.950 E-11$

$2.128 E-D S$

7. $132 \mathrm{E}-01$

1.270E-01

1. $154 \mathrm{~F}+01$

2.816E-01

$2.426 E+04$ 
SWIR3280 - (Page 40 of 107)

RUN DATE: $03 / 15 / 96$

Land Disposal or storage of Solid Radioactive Waste from January 1 to December 31 , 1995

BURIAL GROUND 218W5, 200 WEST AREA, LOW-LEVEL NONINDUSTRIAL.

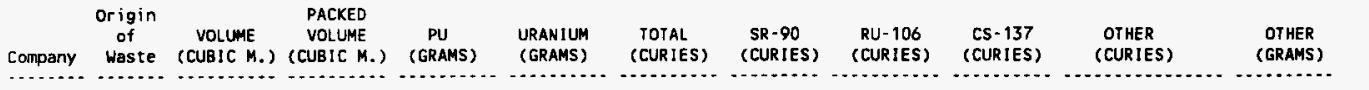

TRENCH 29

BHI $105 \mathrm{~N}$

105N

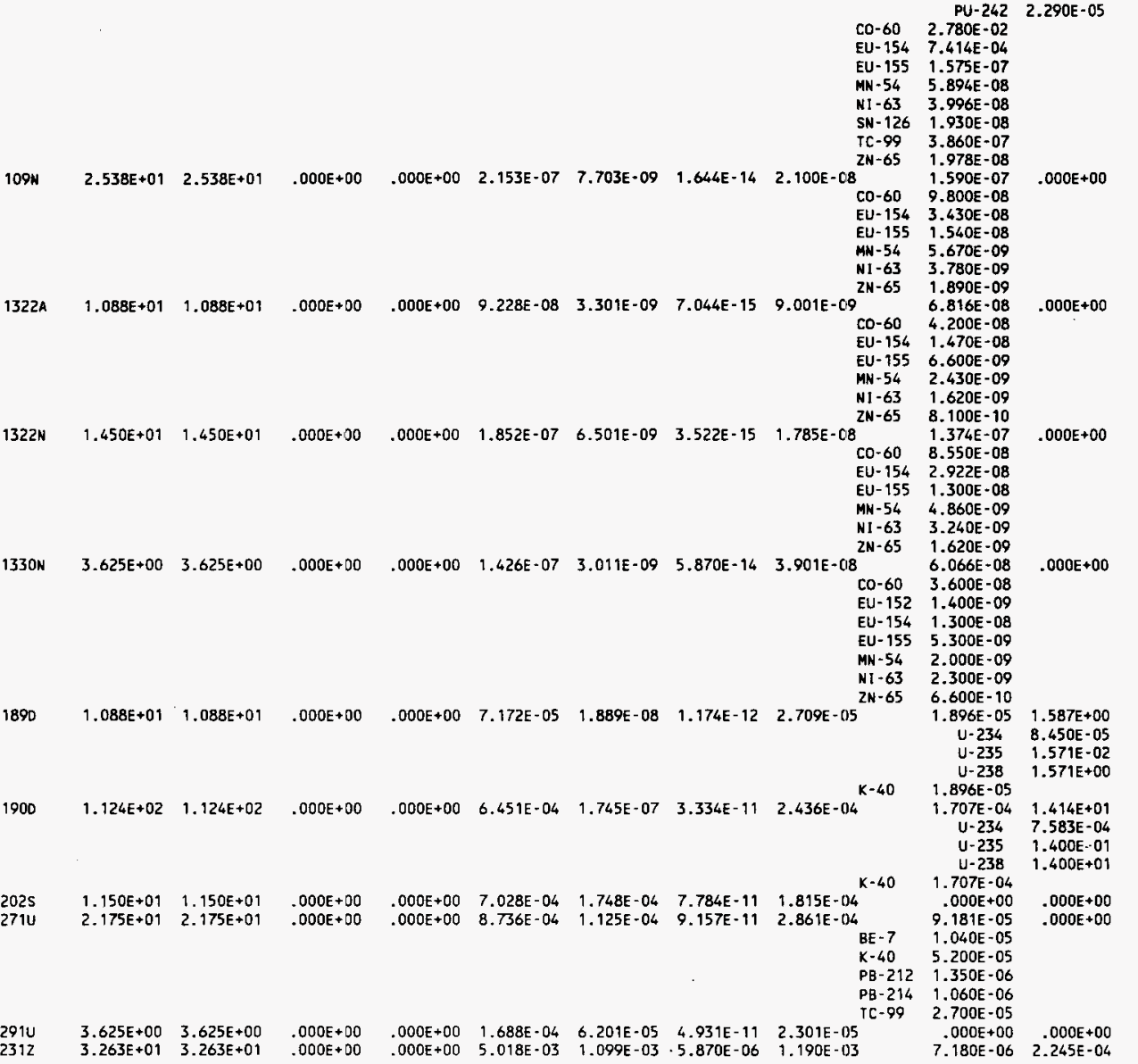


WHC-EP-0125-8

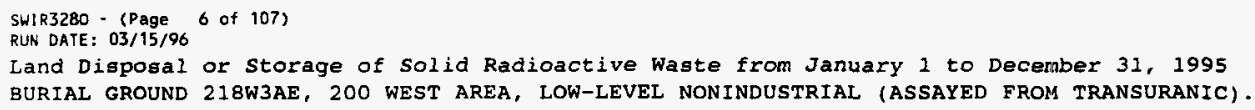


SHIR3280 - (Page 42 of 107)

RUN DATE: $03 / 15 / 96$

Land Disposal or Storage of Solid Radioactive Waste from January 1 to December 31 , 1995

BURIAL GROUND 218W5, 200 WEST AREA, LOW-LEVEL NONINDUSTRIAL.

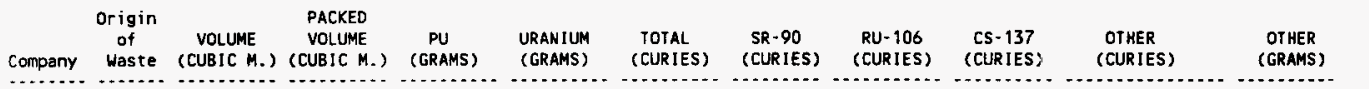

TRENCH 29

PNL 329

331

$\begin{array}{llllllll}1.245 E+01 & 1.245 E+01 & .000 E+00 & -000 E+00 & 1.500 E-03 & 3.296 E-04 & 1.761 E-06 & 3.554 E-C l 4\end{array}$

N: $-63 \quad 4.000 E-08$

PB- $214 \quad 2.400 E-10$

RA-228 $2.300 E-10$

SB-124 2.000E-08

$1.245 E+01$ 1.245e+01

OOOE+00

DOE+OO

then

WHC

$105 K$

$7.658 E+01 \quad 7.658 E+0$

$.000 \mathrm{E}+100$ $.000 E+00 \quad 9.145 E-06 \quad 1.769 E-06 \quad 1.281 E-12 \quad 2.712 E-C 16$

Co- 60

CO- 60

EU- 155

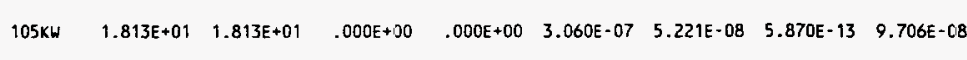

$\begin{array}{lllllllll}182 K E & 4.078 E+00 & 4.078 E+00 \quad .000 E+100 & .000 E+00 & 9.879 E-08 & 1.740 E-08 & 1.057 E-14 & 3.176 E-C B\end{array}$

$202 \mathrm{~A} \quad 1.885 E+02 \quad 1.885 E+02 \quad .000 E+00 \quad .000 E+00 \quad 1.597 E-02 \quad 2.892 E-03 \quad 3.805 E-0 B \quad 3.391 E-013$

202

$209 E \quad 1.163 E+01 \quad 1.163 E+01 \quad .000 E+00 \quad .000 E+00 \quad 4.308 E-03 \quad 1.585 E-03 \quad 1.748 E-09 \quad 5.846 E-04$

$2225 \quad 1.269 E+02 \quad 1.269 E+02 \quad .000 E+100 \quad .000 E+00 \quad 3.462 E-01 \quad 9.623 E-02 \quad 6.490 E-06 \quad 7.628 E-02$

Co-60

CD-60

MN -54

NI -63

8

CO- 60

MN -54
N] -63

$1.885 E+02 \quad 1.885 E+02$

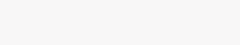

(n)

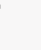

C- 14

$\mathrm{H}-3$

PM -147

TC -99

$1.269 E+02 \quad 1.269 E+02$

$.000 \mathrm{E}+10$

PM- $147 \quad$ AM- 241

CS137 $3.412 E-03$

CO-58 $1.442 E-05$

CO-60 $9.661 E-04$

EU155 1.226E-04

C- 14 1.442E-05

BA $137 \quad 1.399 E-05$

EU154 1.226E-0.4

FE-59 5.768E-05

MN-54 1.009E-04

EU152 $1.442 E-05$

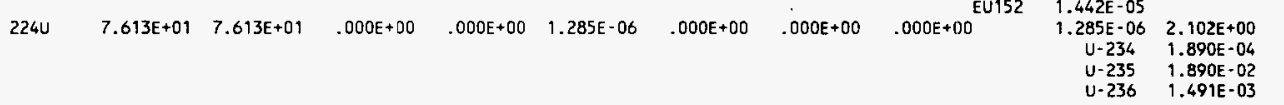


SWIR3280 - (Page 4 of 107)

RUN DATE: $03 / 15 / 96$

Land Disposal or Storage of Solid Radioactive Waste from January 1 to December 31 , 1995

BURIAL GROUND 218W3A, 200 WEST AREA, LOW-LEVEL NONINDUSTRIAL.

\begin{tabular}{|c|c|c|c|c|c|c|c|c|c|}
\hline Company & $\begin{array}{c}\text { Origin } \\
\text { of } \\
\text { Waste }\end{array}$ & $\begin{array}{l}\text { VOLUME } \\
\text { (CUBIC M.) }\end{array}$ & $\begin{array}{c}\text { PACKED } \\
\text { VOLLUME } \\
\text { (CUBIC M.) }\end{array}$ & $\begin{array}{c}\text { PU } \\
\text { (GRAMS) }\end{array}$ & $\begin{array}{l}\text { URANIUM } \\
\text { (GRANS) }\end{array}$ & $\begin{array}{l}\text { TOTAL } \\
\text { (CURIES) }\end{array}$ & $\begin{array}{l}\text { SR-90 } \\
\text { (CUR IES) }\end{array}$ & $\begin{array}{c}\text { RU- } 106 \\
\text { (CURIES) }\end{array}$ & $\begin{array}{c}\text { CS-137 } \\
\text { (CURIES) }\end{array}$ \\
\hline $\begin{array}{l}\text { RENCH } \\
\text { WHC }\end{array}$ & 2415 & & & & & & & & \\
\hline
\end{tabular}

$241 \mathrm{SY} \quad 2.990 \mathrm{E}-01 \quad 2.990 \mathrm{E}-01 \quad .000 \mathrm{E}+00 \quad .000 \mathrm{E}+00 \quad 2.091 \mathrm{E}-03 \quad 8.001 \mathrm{E}-04 \quad 3.475 \mathrm{E}-10 \quad 2.521 \mathrm{E}-0$

$241 \mathrm{SY} \quad 2.990 \mathrm{E}-01 \quad 2.990 \mathrm{E}-01 \quad .000 \mathrm{E}+00 \quad .000 \mathrm{E}+00 \quad 2.091 \mathrm{E}-03 \quad 8.001 \mathrm{E}-04 \quad 3.475 \mathrm{E}-10 \quad 2.521 \mathrm{E}-0$

$\begin{array}{ll}\text { CD-60 } & 1.925 E-06 \\ \text { EU-154 } & 1.124 E-06\end{array}$

$3.120 E-07$

AM-241 3.740E-09

PU-238 $\quad 9.290 E-09$

PU-239 3.100E-05

PU-240 $1.910 E-06$

PU-249 1.800E-07

PU-242 6.620E-09

$241 T$

2. 990E-01 2.990E-01

$.00 O E+C O$

$.000 E+00$

$2.150 E-04$

8.601E-05 4.931E-11

2.191E-0:

co- 60

$3.120 \mathrm{E}-07$

3.410E-07

$7.540 E-06$

AM-241 8.540E-10

PU- 238 2.110E-09

PU-239 7.060E-06

PU- 240 4.340E-07

PU-241 4.110E-D8

PU-242 1.500E-09

$241 T X \quad 2.990 E-01 \quad 2.990 E-01 \quad .000 E+00 \quad .000 E+00 \quad 4.910 E-04 \quad 1.731 E-04 \quad 5.612 E-10 \quad 7.421 E-05$

CO-60 $1.540 \mathrm{E}-07$

EU-154 1.870E-07

3.230E-07 1.218E-05

AM-241 1.380E-09

PU-238 3.420E-D9

PU-239 $1.140 E-05$

PU-240 7.050E-07

PU-241 6.640E-08

PU-242 2.420E-09

$241 \mathrm{U} \quad 2.990 \mathrm{E}-01 \quad 2.990 \mathrm{E}-01 \quad .000 \mathrm{E}+00 \quad .000 \mathrm{E}+00 \quad 3.770 \mathrm{E}-04 \quad 4.228 \mathrm{E}-05 \quad 2.555 \mathrm{E}-09 \quad 1.495 \mathrm{E}-04$

CO-60 1.360E-07

EU-156 $1.870 \mathrm{E}+07$

$1.270 \mathrm{E}-06$

AM- 241

$1.527 E-05$

PU $-238 \quad 4.260 E-09$

PU- $239 \quad 1.430 E-05$

PU-240 8.770E-07

PU-241 8.280E-08

PU -242 3.030E-09

CO-60 1.700E-07

EU- $154 \quad 1.100 E-06$

I PLANT $1.940 E+01 \quad 1.940 E+01 \quad .000 E+100 \quad .000 E+00 \quad 5.200 E-02 \quad 1.545 E-02 \quad 1.371 E-06 \quad 1.079 E-012$

PU-240 1.799E-03 
SWIR3280 - (Page 44 of 107)

RUN DATE: $03 / 15 / 96$

Land Disposal or storage of Solid Radioactive Waste from January 1 to December 31 , 1995 BURIAL GROUND 218W5, 200 WEST AREA, LOW-LEVEL NONINDUSTRIAL.

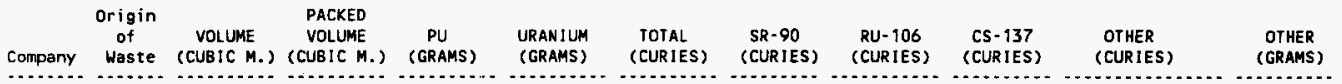

TRENCH 29

WHC

$2415 x$

$2415 Y$

$3.625 E+00 \quad 3.625 E+00$

$.000 E+100$

$.000 E+00 \quad 5.030 E-06 \quad T .931 E-06$

$2.818 E-12$

$6.006 \mathrm{E}-07$

$2411 x$

$3.625 E+00 \quad 3.625 E+00$

$.000 E+00$

$.000 E+00$

00

$.870 E-0$

$1.243 E-05$

1.

$350 E-10 \quad 2.253 E-05$

$241 \mathrm{U} \quad 7.250 E+00 \quad 7.250 E+00 \quad .000 E+00 \quad .000 E+00 \quad 6.024 E-04 \quad 2.304 E-04 \quad 8.139 E-10 \quad 7.274 E-05$

$2716 \quad 3.920 E+01 \quad 3.920 E+01 \quad .000 E+00$

308

$4.92016+013.920 E+01$

$.000 \mathrm{E}+00$

$\begin{array}{rr}.000 E+00 & 2.447 E-02 \\ .000 E+00 & 1.100 E-02\end{array}$

2.556E-03 5.446E-07 $\quad 9.923 E-03$

$.000 \mathrm{E}+00 \quad 1.100 \mathrm{E}-02$

2.197E-03

$1.174 E-05 \quad 2.374 E-03$

313

$2.538 E+01 \quad 2.538 E+01$

$.000 E+010$

$.000 E+00$

$2.076 \mathrm{E}-05$

$.000 E+0$

$.000 E+00$

$.000 \mathrm{E}+00$

333

$9.788 \mathrm{E}+01 \quad 9.788 \mathrm{E}+01$

$.000 E+010$

$.000 E+O D$

$2.994 E-05$

$.000 E+00$

$.000 E+00$

437

$3.361 E+01 \quad 3.361 E+0$

$0000+00$

$4.607 \cdot-01$

$1.414 \mathrm{E}-05$

7.555E-08 5.240E-03

$$
\mathrm{CO}
$$$$
\text { CS-134 }
$$$$
\text { MN }-54
$$

6290

$4.164 E-01 \quad 4.164 E-0$

$.000 \mathrm{E}+010$

$.000 E+00$

9.391E-06 2.5

$2.547 E-07 \quad 1.890 E-11$

4.564E-06

IC -9

TC- 99

EU- 154

1. $080 \mathrm{E}-06$

1.950E-07

$.000 E+00$

AM- 241 1.980E-12

PU-238 2.230E-11

PU-239 7.450E-08

PU-240 4.570E-09

PU-241 4.330E-10

PU-242 1.580E 11

$.000 E+00 \quad 4.047 E-06$

AM-241 4.580E-10

PU-238 1.130E-09

PU-239 3.790E-06

PU-240 2.330E-07

PU-241 2.200E-08

PU-242 8.040E-10

$.000 E+00 \quad 1.753 E-05$

AM-241 1.982E-09

PU-238 4.904E-09

PU-239 1.641E-05

PU-240 1.011E-06

PU-241 9.529E-08

PU-242 3.473E-09

$.000 E+00 \quad .000 E+00$

9.896E-04 3.400E +03

AM-241 $1.840 E-10$

PU-240 2.780E-09

TH-228 6.295E-07

U-235 2.248E+01

$U-238 \quad 3.377 \mathrm{E}+03$

$4.251 \mathrm{E}-04$

EU-152 5.573E-04

EU-154 4.453E-06

MN $-54 \quad 2.700 E-06$

$2.076 \mathrm{E}-05$

$\mathrm{U}-234$

$\mathrm{U}-235$

$\mathrm{U}-236$

U- 238

2.076E-05

2.994E-05

U- 234

U- 235

U- 236

U. 238

$2.994 \mathrm{E}-05$

4.504E-01

4. $281 \mathrm{E}-01$

$1.694 E-04$

2. 211 E-02

5.700E-05

$.000 E+00$

AM- 241

PU- 238

PU-239

PU-240

PU-241

PU-242

1.617E-04

AM- 241

$1.221 E+02$

8.195E-03

$1.157 \mathrm{E}+00$

$7.206 \mathrm{E}-02$

$1.208 E+02$

$1.761 E+02$

$1.178 \mathrm{E}-02$

$1.667 \mathrm{E}+00$

$1.038 E-0 ?$

$1.743 \mathrm{E}+02$

$.000 \mathrm{E}+00$

1.877E-06

2.129E- 10

5. $270 E-10$

1.758E-06

$1.081 E-07$

$1.022 E-08$

3. $740 \mathrm{E}-10$

1. $835 E-04$

$2.077 \mathrm{E}-08$ 
SWIR3280 - (Page 2 of 107)

RUN DATE: 03/15/96

Land Disposal or Storage of Solid Radioactive Waste from January 1 to December 31 , 1995

BURIAL GROUND 218W3A, 200 WEST AREA, LOW-LEVEL NONINDUSTRIAL-OFFSITE.

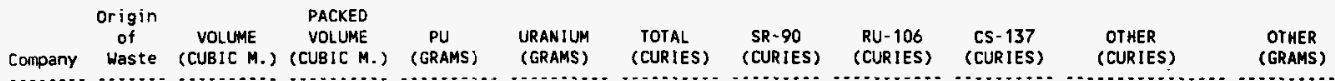

TRENCH 49

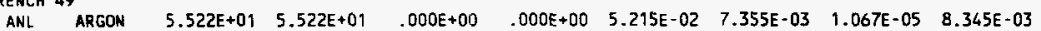

UCD DAVIS $\quad 1.091 E+01 \quad 1.091 E+01 \quad .000 E+00 \quad .000 E+00 \quad 2.272 E-03 \quad 7.600 E-04 \quad .000 E+00 \quad .000 E+013$

BA- $133,1.000 E-06$

2.097E-02 4.708E-01

$A M-241 \quad 5.298 E-03$

AM- 243 3.050E-04

CM-243 2.055E-06

NP- 237 4.086E-02

PU-238 $9.243 E-06$

PU-239 7.588E-02

PU-240 7.442E-03

PU-241 2.339E-05

TH-232 3.95OE-02

U-233 3.840E-04

$U-235$ 1.060E-04

U-238 3.010E-01

BE-7 $9.390 E-05$

B1-214 $4.400 E-07$

CO-56 1.500E-06

CO-57 1.507E-03

CO-5B 1.500E-06

CO-60 4.785E-03

CS-134 2.820E-04

EU-152 2.989E-03

EU-154 $9.300 E-05$

FE-55 1.790E-04

H-3 1.000 E-06

MN-54 1.630E-06

HA-22 5.000E-05

NI-63 9.943E-03

SB-125 $1.036 \mathrm{E}-03$

SR-85 1.000E-06

TC-99 1.200E-06

ZN-65 1.500E-06

$7.525 \mathrm{E}-04$

RA- 226

TOTALS

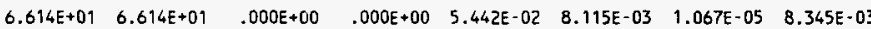

$Y-90$

7.525E-04

2.172E-02 4.713E-01

4.871E-04

$4.871 E-04$ 


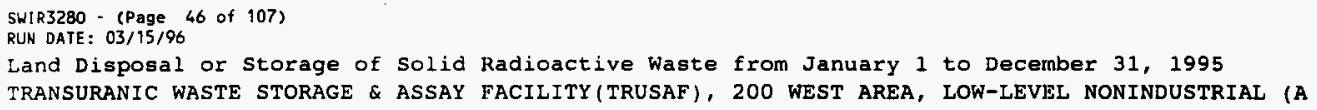


WHC-EP-0125-8

SWIR3280 - (Page 67 of 107 )

RUN DATE: $03 / 15 / 96$

Land Disposal or storage of Solid Radioactive Waste from January 1 to Decernber 31 , 1995

TRANSURANIC WASTE STORAGE \& ASSAY FACILITY (TRUSAF), 200 WEST AREA, LOW-LEVEL NONINDUSTRIAL.

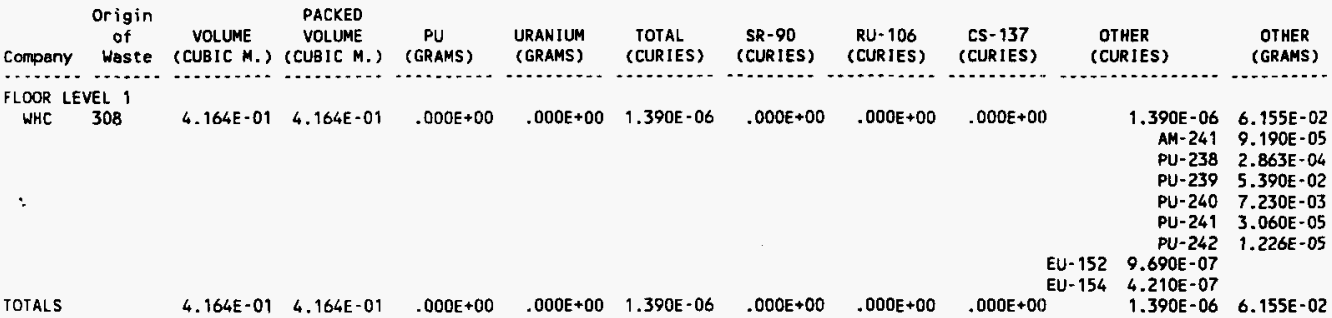


SHIR3280 - (Page 48 of 107)

RUN DATE: 03/15/96

Land Disposal or Storage of Solid Radioactive Waste from January 1 to December 31 , 1995

TRANSURANIC WASTE STORAGE \& ASSAY FACILITY(TRUSAF), 200 WEST AREA, TRANSURANIC (NOT PROCESSED

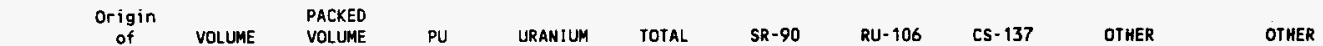

Company Haste (CUBIC M.) (CUBIC M.) (GRAMS) (GRAMS) (CURIES) (CURIES) (CURIES) (CURIES) (CURIES)

FLOOR LEVEL 3

HHC 23452

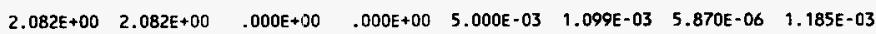

TOTALS

$2.082 E+00 \quad 2.082 E+00 \quad .000 E+130 \quad .000 E+00 \quad 5.000 E-03 \quad 1.099 E-03 \quad 5.870 E-06 \quad 1.185 E-03$

$.000 E+00 \quad 9.197 \mathrm{E}+02$

AM-241 2.171E+01

PU-238 5.128E-01

PU-239 6.748E+02

PU- 240 2.065E+02

PU-241 9.715E+00

PU- $242 \quad 6.545 E+00$ 


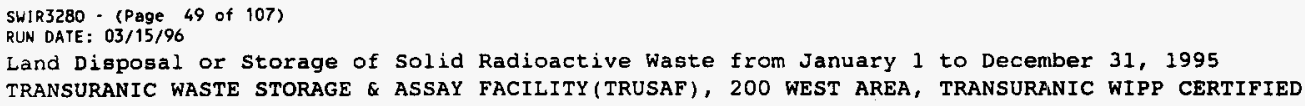


SWIR3280 - (Page 50 of 107)

RUN DATE: $03 / 15 / 96$

Land Disposal or storage of Solid Radioactive Waste from January 1 to December 31 , 1995

TRANSURANIC WASTE STORAGE \& ASSAY FACILITY(TRUSAF), 200 WEST AREA, TRANSURANIC WIPP UNCERTIFI

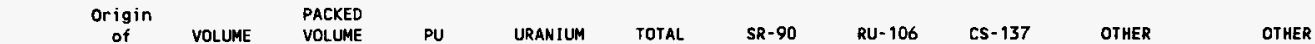

Company Waste (CUBIC M.) (CUBIC M.) (GRAMS) (GRAMS) (CURIES) (CURIES) (CURIES) (CURIES) (CURIES)
(GRAMS)

FLOOR LEVEL 1

PNL $325 \quad 2.082 E-01 \quad 2.082 E-01 \quad .000 E+00 \quad .000 E+00 \quad 5.000 E-03 \quad 1.099 E-03 \quad 5.870 E-06 \quad 1.185 E-03$

WHC $308 \quad 6.246 E-01 \quad 6.246 E-01 \quad .000 E+00 \quad .000 E+00 \quad 1.008 E-04 \quad 2.197 E-05 \quad 1.174 E-07 \quad 2.369 E-05$

FLOOR LEVEL 3

PNL 325
$2.290 E+00 \quad 2.290 E+00$
$.000+00$

$.000 \mathrm{E}$ 
WHC-EP-0125-8

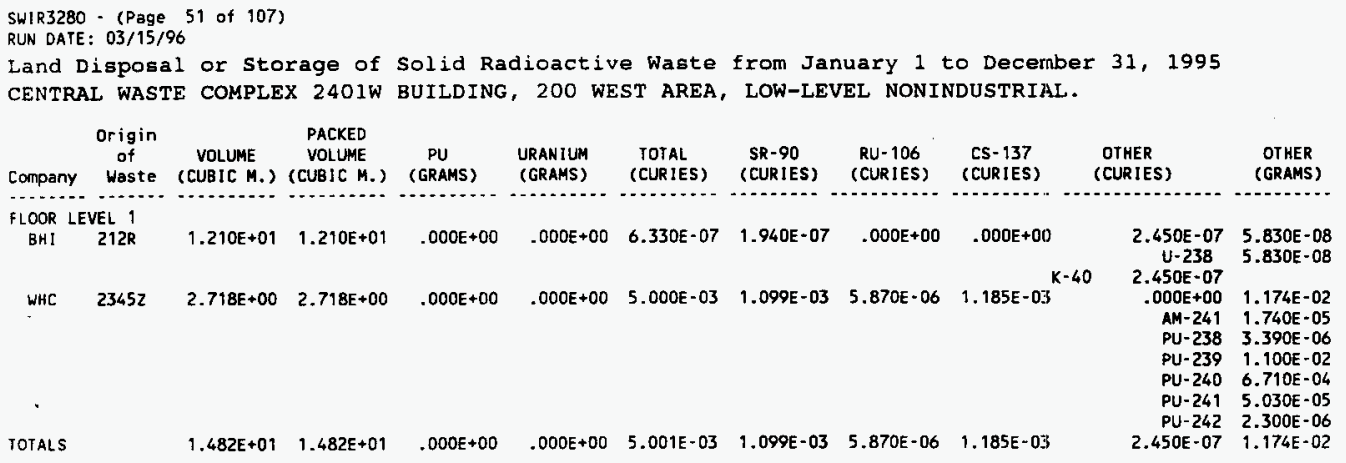


SWIR3280 - (Page 52 of 107 )

RUN DATE: 03/15/96

Land Disposal or Storage of Solid Radioactive Waste from January 1 to December 31 , 1995

CENTRAL WASTE COMPLEX $2402 \mathrm{~W}$ BUILDING, 200 WEST AREA, TRANSURANIC WIPP CERTIFIED.

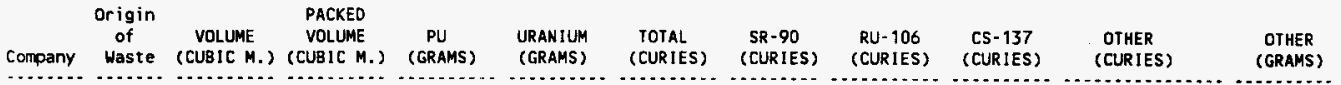

FLOOR LEVEL 1

PNL $\quad 325$

$4.372 E+00 \quad 4.372 E+00 \quad .000 E+100 \quad .000 E+00 \quad 5.390 E-02 \quad 1.180 E-02 \quad 6.305 E-05 \quad 1.282 E-02$

$23452 \quad 1.103 E+01 \quad 1.103 E+01 \quad .000 E+10 \quad .000 E+00 \quad 2.290 E-01 \quad 5.031 E-02 \quad 2.688 E-04 \quad 5.425 E-0 ?$

WHC 202A

$4.164 E-01 \quad 4.164 E-01$

$.000 E+010$

$.000 E+00$

$4.500 \mathrm{E}-03$

$.887 E-0$

$5.283 E-06 \quad 1.066 E-0.3$

1.

CO-60

EU-152 EU-154

$\begin{array}{ll} & 1 . \\ \text { CO-60 } & 7 . \\ \text { EU-152 } & 1 . \\ \text { EU-154 } & 1.2 \\ 0.3 & \end{array}$

$1.418 E-05 \quad 2.884 E+01$

AM-241 5.187E-01

AM-243 4.950E-05

CM-243 7.086E-05

CM-244 1.270E-03

NP-237 2.036E-01

PU-238 6.195E-03

PU-239 1.910E+01

PU-240 1.424E $\div 00$

PU-249 2.080E-01

PU-242 3.346E-01

TH-228 2.881E-07

U-235 $1.820 E+00$

U- $238 \quad 5.230 \mathrm{E}+00$

$7.460 \mathrm{E}-07$

$1.289 \mathrm{E}-06$

$1.214 E-05$

$.000 E+00 \quad 1.300 E+01$

AM-241 2.000E-04

PU-238 3.900E-03

PU-239 1.214E+01

PU-240 7.831E-01

PU-241 7.050E-02

$\mathrm{PU}-242$ 4.600E-03

$.000 E+00 \quad 1.326 E+02$

AM-241 2.006E-01

PU-238 3.901E-02

PU-239 $1.239 E+02$

$P U-240 \quad 7.598 E+00$

PU-241 8.738E-01

PU-242 2.681E-02

$\begin{array}{ll}1.418 E-05 & 1.744 E+02\end{array}$ 
WHC-EP-0125-8

SWIR3280 - (Page 53 of 107)

RUN DATE: 03/15/96

Land Disposal or storage of Solid Radioactive Waste from January 1 to December 31 , 1995

CENTRAL WASTE COMPLEX $2402 \mathrm{~W}$ BUILDING, 200 WEST AREA, TRANSURANIC WIPP UNCEIRTIFIED.

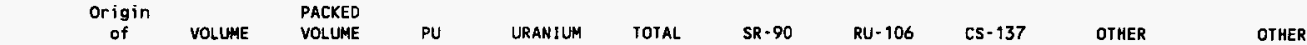

Company Waste (CUBIC M.) (CUBIC M.) (GRAMS) (GRAMS) (CURIES) (CURIES) (CURIES) (CURIES) (CURIES) (GRAMS)

FLOOR LEVEL 1

PNL $\quad 325$

$2.915 E+00 \quad 2.915 E+00$

$.000 E+00$

$.000 E+00 \quad 3.503 E-02 \quad 1.220 E-03 \quad 6.520 E-06 \quad 1.404 E-0 ?$

4.706E-03 1.130E+02

AM-24 $1.963 \mathrm{E}-01$

AN-243 4.870E-03

CM-243 $5.441 E-04$

CM- $244 \quad 1.443 E-03$

NP- $237 \quad 2.081 E-02$

PU-238 1.586E-03

PU -239 B. B52E+00

PU. $240 \quad 5.253 E-01$

PU-241 6.051E-02

PU-242 9.668E-04

TH-228 4.014E-05

U-235 $1.300 E+00$

$\mathrm{U}-238 \quad 1.020 E+02$

WHC 202A $\quad 1.041 E+00 \quad 1.041 E+00 \quad .000 E+00 \quad .000 E+00 \quad 2.500 E-02 \quad 3.296 E-03 \quad 1.761 E-05 \quad 3.871 E-0.3$

CS-134 5.520E-06

EU-152 3.640E-03

EU-154 1.060E-03

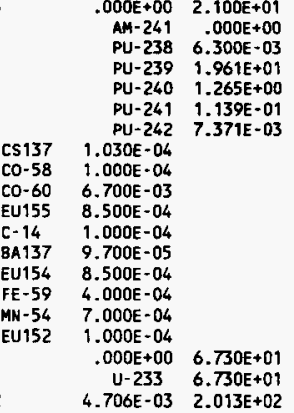




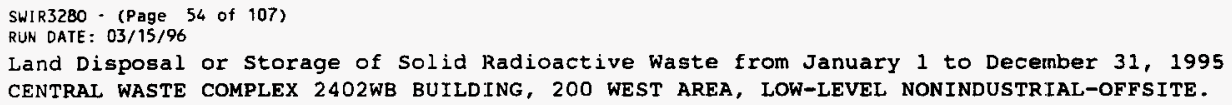

$\begin{array}{lrr} & 1.074 E-04 & 4.423 E+02 \\ & \text { AM-241 } & 2.980 E-05 \\ \text { CM-243 } & 2.590 E-07 \\ & P U-239 & 4.510 E-04 \\ & P U-244 & 1.333 E E+10 \\ & R A-226 & 6.270 E+05 \\ & T H-232 & 4.410 E+02 \\ & U-232 & 2.760 E-07 \\ & & \\ & & \\ B A-133 & 2.300 E-06 \\ C O-60 & 9.800 E-05 & \\ E U-154 & 7.100 E-06 & \\ & .000 E+00 & 1.991 E+03 \\ & T H-232 & 4.919 E+02 \\ & U-238 & 1.499 E+03 \\ & 1.074 E-04 & 2.433 E+03\end{array}$


WHC-EP-0125-8

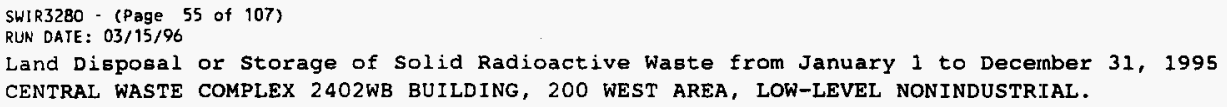


WHC-EP-0125-8

SWIR3280 - (Page 56 of 107)

RUN DATE : $03 / 15 / 96$

Land Disposal or storage of Solid Radioactive Waste from January 1 to December 31 , 1995

CENTRAL WASTE COMPLEX 2402WB BUILDING, 200 WEST AREA, TRANSURANIC WIPP CER'TIFIED.

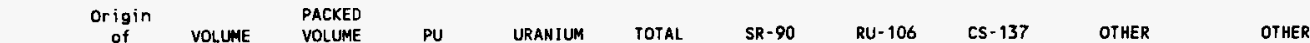

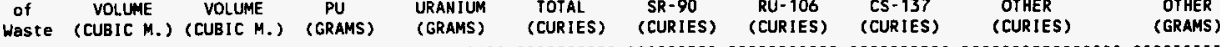

Company waste (CUBIC M.) (CUBIC $\left.M_{.}\right)$

(GRAMS)

FLOOR LEVEL 1

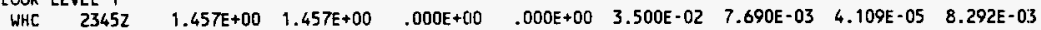


SWIR3280 - (Page 57 of 107 )

RUN DATE: 03/15/96

Land Disposal or Storage of Solid Radioactive Waste from January 1 to December 31 , 1995

CENTRAL WASTE COMPLEX 2402WC BUILDING, 200 WEST AREA, LOW-LEVEL NONINDUSTRIAL.

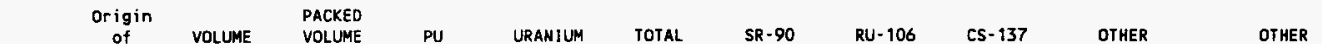

Company Waste (CUBIC M.) (CUBIC M.) (GRAMS) (GRAMS) (CURIES) (CURIES) (CURIES) (CURIES) (CURIES)
(GRAMS)

FLOOR LEVEL 1

PNL 324

325
$3.956 \mathrm{E}+00 \quad 3.956 \mathrm{E}+00$ $6.246 \mathrm{E}-01 \quad 6.246 \mathrm{E}-01$

$.000 E+00$

$.000 E+00 \quad 5.748 E-01 \quad 2.358 E-01 \quad 8.969 E-07 \quad 5.300 E-0.2$

$.000 E+00 \quad 1.434 E-03 \quad 1.714 E-06 \quad 4.405 E-09 \quad 6.792 E-0.4$
OOE+
329 $1.041 E+00 \quad 1.041 E+00$

$-000 E+00$

$.000 E+00$

2.909E-0

$1.114 E-04 \quad 1.731 E-07 \quad 7.433 E-0 \%$

WHC $222 \mathrm{~s}$
$224 \mathrm{U}$

23452

2.082E-01 2.082E-01

$.000 E+00$

$.000 \mathrm{E}+00$

2.082E-01 2.082E-01

$.000 E+00$

$.000 E+00$

$.000 E+00$

$00 \quad 6.120 E-0 B$

3.

.501E-04 2.709E-08 $\quad 1.735 E-04$

2.082E-01 2.082E-01

$.000 \mathrm{E}+00$

(n)

241

$5.300 E-01 \quad 5.300 E-0$

$.000 E+00$

$.000 E+00$

4. $113 E-05$

$05 \quad 1.364 E-05 \quad 8.688 E-11 \quad 7.323 E-06$
$.000 E+00 \quad 4.500 E-04 \quad 4.714 E-05 \quad 1.141 E-08 \quad 1.823 E-04$ $.000 E+00 \quad 5.584 E-05 \quad 3.976 E-06 \quad 3.287 E-11 \quad 2.461 E-05$

TOTALS
$7.192 E+00 \quad 7.192 E+00$

$.000 E+00$
TC-

C-99

\section{CS- 134 \\ EU- 154 EU-155}

Co- 60
(GRAMS)

$4.372 \mathrm{E}-06$

2.893E-01

AM- 24

PU-239

PU-242

u. 235

U-23B

AG-108 3.340E-02

CO-60 7.624E-02

CS- $\$ 34 \quad 4.600 E-09$

EU-152 2.100E-09

EU-154 1.700E-01

EU-155 1.950E-07

$\mathrm{H}-3$ 1.100E-08

MN-54 1.030E-04

NB-94 3.000E-05

NB-95 1.330E-08

NI $-63 \quad 9.420 E-03$

SB-125 5.580E-05

SR-85 3.000E-08

2R-95 2.200E-08

$.000 E+00$

AM- 241

$2.430 E-06$

120E-08 1.090E-01

U-234 9.000E-05

U-235 9.200E-03

U-236 $7.100 E-04$

U-238 $9.900 E-02$

$6.120 E-08$

$.000 \mathrm{E}+00$

AM-241

PU-238

$1.580 E-04$
$2.360 E-07$

PU-239 $1.480 E-04$

PU-240 9.010E-06

PU-241 6.730E-07

PU-242 3.090E-08

$.000 E+00 \quad 3.247 E-07$

AM-241 2.928E-11

PU-238 9.100E-11

PU-239 3.041E-07

PU-240 1.866E-08

PU-241 1.765E-09

PU-242 6.440E-11

$.000 E+00 \quad .000 E+00$

$.000 E+00 \quad 5.209 E-05$

AM- 241 4.700E-09

PU $=238 \quad 1.460 E-08$

PU-239 4.880E-05

PU-240 2.990E-06

PU-241 2.700E-07

PU-242 1.010E-08

2.894E-01 3.668E-01 
WHC-EP-0125-8

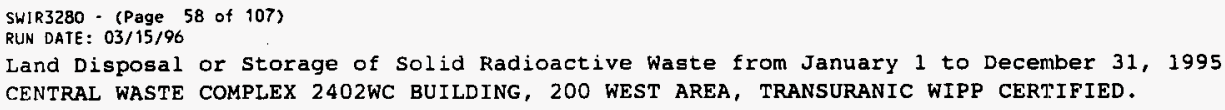

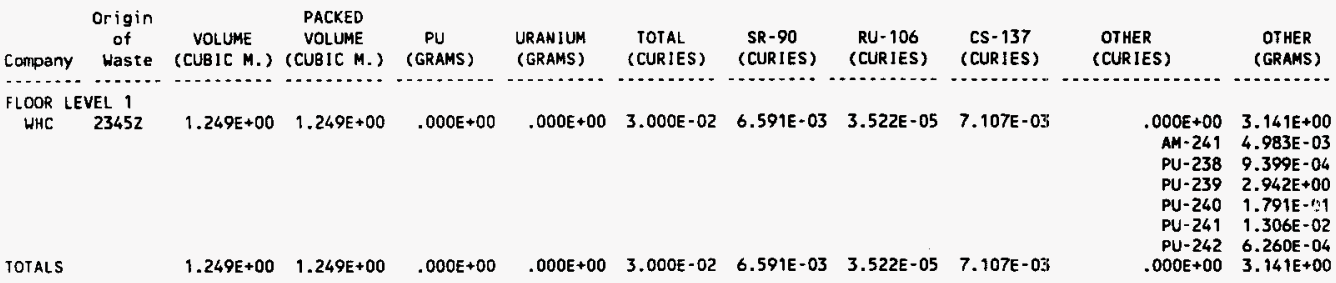




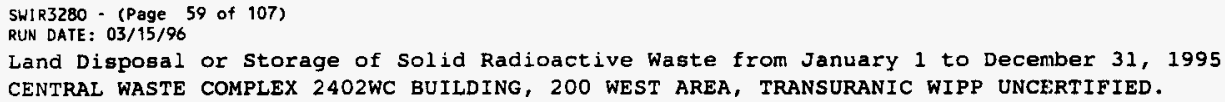

\begin{tabular}{|c|c|c|c|c|c|c|c|c|c|c|c|}
\hline Company & $\begin{array}{c}\text { Origin } \\
\text { of } \\
\text { Waste }\end{array}$ & $\begin{array}{c}\text { VOLUME } \\
\text { (CUBIC M.) }\end{array}$ & $\begin{array}{c}\text { PACKED } \\
\text { VOLUME } \\
\text { (CUBIC M.) }\end{array}$ & $\begin{array}{c}\text { PU } \\
\text { (GRAMS) }\end{array}$ & $\begin{array}{l}\text { URANIUM } \\
\text { (GRAMS) }\end{array}$ & $\begin{array}{l}\text { TOTAL } \\
\text { (CURIES) }\end{array}$ & $\begin{array}{l}\text { SR-90 } \\
\text { (CURIES) }\end{array}$ & $\begin{array}{l}\text { RU-106 } \\
\text { (CURIES) }\end{array}$ & $\begin{array}{l}\text { CS }-137 \\
\text { (CURIES) }\end{array}$ & $\begin{array}{l}\text { OTHER } \\
\text { (CURIES) }\end{array}$ & $\begin{array}{l}\text { OTHER } \\
\text { (GRAMS) }\end{array}$ \\
\hline FLOOR L & LEVEL 1 & & & & & & & & & & \\
\hline PNL & 325 & $4.164 E-01$ & $4.164 E-01$ & $.000 E+00$ & $.000 E+0.0$ & $1.000 \mathrm{E}-04$ & $2.197 E=05$ & $1.174 \mathrm{E}-07$ & $2.369 E-05$ & $\begin{array}{r}.000 E+00 \\
A M-241 \\
C M-244 \\
P U-238 \\
P U-239 \\
P U-240 \\
P U-241 \\
P U-262\end{array}$ & $\begin{array}{l}1.806 E+00 \\
1.335 E-01 \\
7.880 E-09 \\
3.630 E-04 \\
1.546 E+00 \\
1.027 \mathrm{E}-01 \\
2.293 E-02 \\
2.830 E-04\end{array}$ \\
\hline TOT \&LS & & 4.164E-01 & $4.164 \mathrm{E}-09$ & $.000 E+00$ & $.000 E+00$ & 1.000 E- 04 & $2.197 E-05$ & $1.174 \mathrm{E}-07$ & $2.369 E-05$ & $.000 E+00$ & $1.806 E+00$ \\
\hline
\end{tabular}


SWIR3280 - (Page 60 of 107)

RUN DATE: 03/15/96

Land Disposal or Storage of Solid Radioactive Waste from January 1 to December 31 , 1995

CENTRAL WASTE COMPLEX 2402WD BUILDING, 200 WEST AREA, LOW-LEVEL NONINDUSTRIAL-OFFSITE.

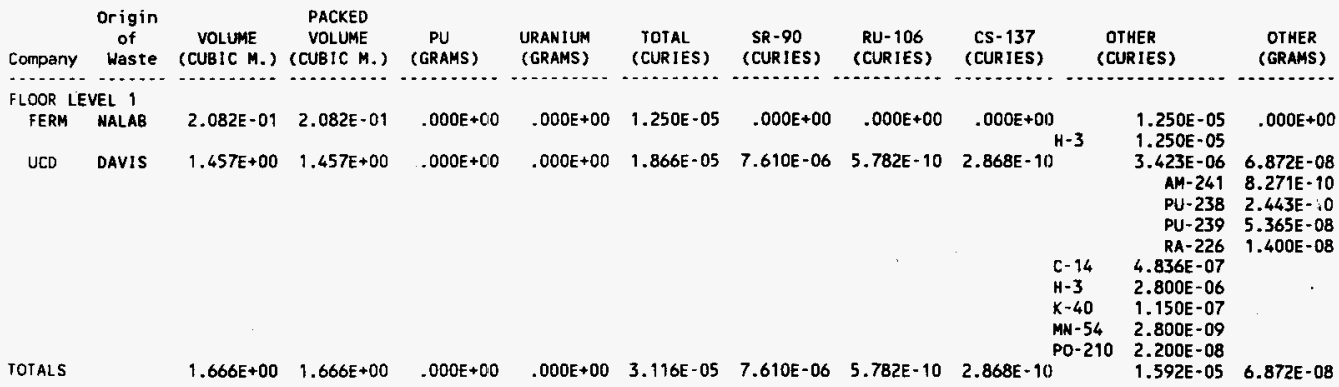


WHC-EP-0125-8

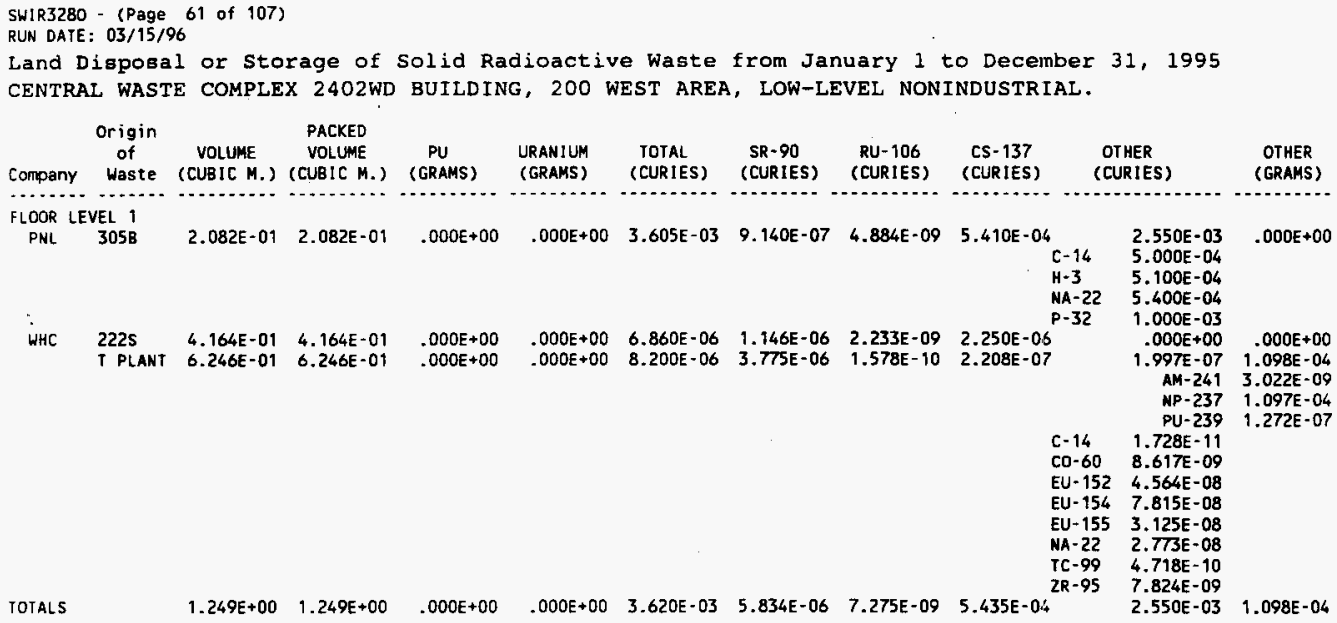


WHC-EP-0125-8

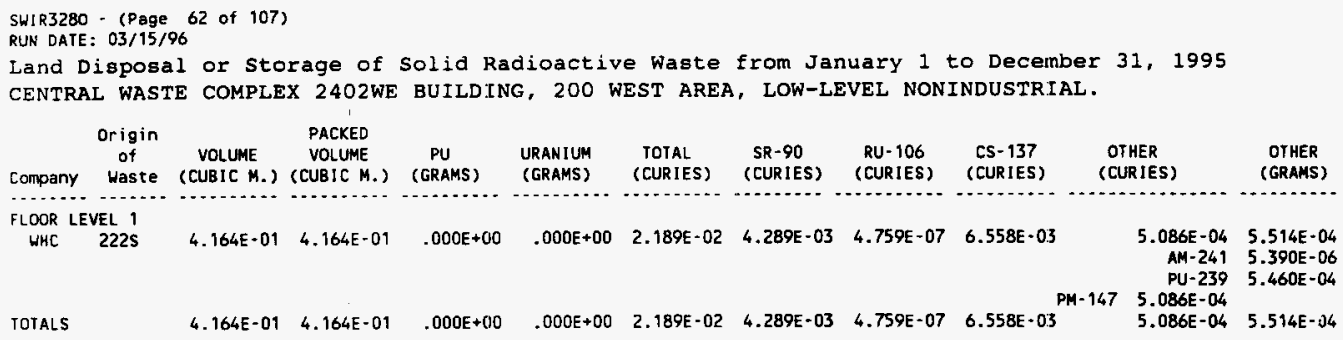


SWIR3280 - (Page 63 of 107 )

RUN DATE: 03/15/96

Land Disposal or Storage of Solid Radioactive Waste from January 1 to Decenber 31 , 1995

CENTRAL WASTE COMPLEX 2402WE BUILDING, 200 WEST AREA, TRANSURANIC WIPP UNCERTIFIED.

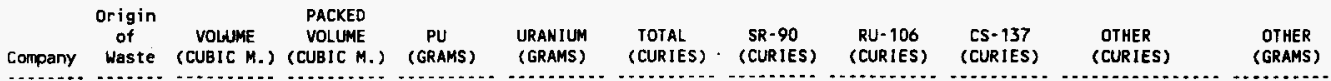

FLOOR LEVEL 1

PNL 325

$2.082 E-01 \quad 2.082 E-01 \quad .000 E+00 \quad .000 E+00 \quad 5.000 E-04 \quad 1.099 E-04 \quad 5.870 E-07 \quad 1.185 E-0.4$

$.000 E+00 \quad 9.001 E-03$

$A M-261 \quad 8.740 E-03$

AM- $243 \quad 1.810 E-04$

CM-243 3.800E-05

CM-244 4.190E-05

NP-237 9.350E-08

TOTALS

$2.082 E-01 \quad 2.082 E-01 \quad .000 E+00 \quad .000 E+00 \quad 5.000 E-04 \quad 1.099 E-04 \quad 5.870 E-07 \quad 1.185 E-04$

TH-228 8.830E-09

$000 E+00 \quad 5.000 E=04 \quad 1.099 E-04 \quad 5.870 E-07 \quad 1.185 E-04$

$.000 \mathrm{E}+00 \quad 9.001 \mathrm{E}-03$ 


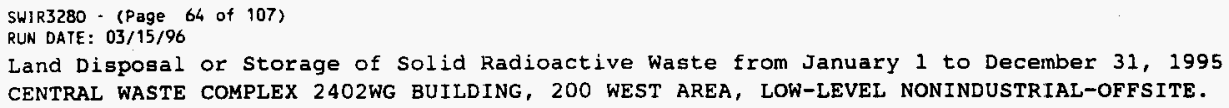

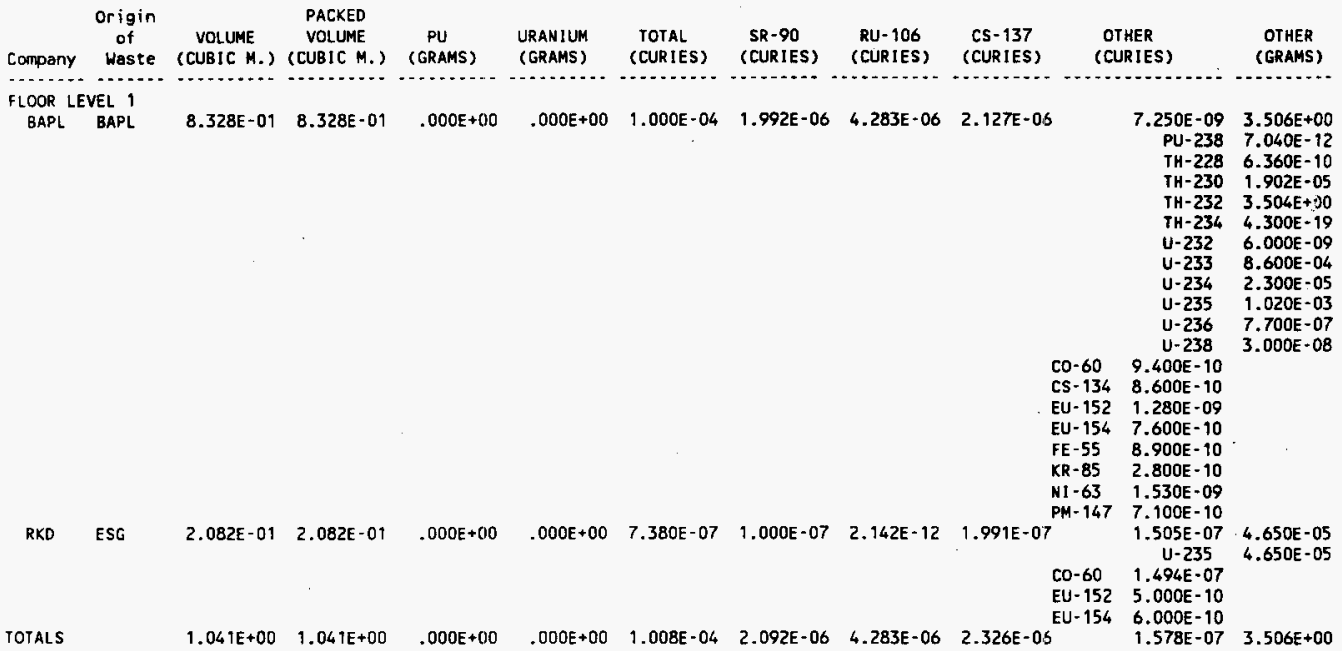


SWIR3280 - (Page 65 of 107)

RUN DATE: $03 / 15 / 96$

Land Disposal or storage of Solid Radioactive Waste from January 1 to December 31 , 1995

CENTRAL WASTE COMPLEX 2402WG BUILDING, 200 WEST AREA, LOW-LEVEL NONINDUSTRIAL.

$\begin{array}{ccccccccc}\begin{array}{c}\text { Origin } \\ \text { of }\end{array} & \text { VOLUME } & \text { PACKED } \\ \text { VOLUME } & \text { PU } & \text { URANIUM } & \text { TOTAL } & \text { SR-90 } & \text { RU-106 } & \text { CS-137 } & \text { OTHER } & \text { OTHER }\end{array}$ Company Waste (CUBIC M.) (CUBIC M.) (GRAMS) (GRAMS) (CURIES) (CURIES) (CURIES) (CURIES) (CURIES) FLOOR LEVEL 1

PNL 325

3.218E-01 3.218E-01

$.000 \mathrm{E}+00$

$\begin{array}{lllll}.000 E+00 & 9.440 E-05 & 6.152 E-09 & 3.287 E-11 & 4.701 E-05\end{array}$

(CURIES)

(GRAMS)

WHC 222

2244

$241 B Y$

2.082E-01 2.082E-01

$.000 E+00$

\begin{abstract}
2.082E-01 2.082E-01
$.000 E+00$
\end{abstract}

2.082E-01 2.082E-01

$.000 \mathrm{E}+0$

$.000 E+00 \quad 6.360 E-04 \quad 1.249 E-04 \quad 1.573 E-08 \quad 1.902 E-04$

$\begin{array}{llllll}.000 E+00 & 6.120 E-08 & .000 E+00 & .000 E+00 & .000 E+00\end{array}$

EU- 155

PM- 147

$-99$

$2415 Y \quad 2.082 E-01 \quad 2.082 E-01 \quad .000 E+00 \quad .000 E+00 \quad 1.040 E-06 \quad 4.509 E-07 \quad 4.931 E-12 \quad 7.079 E-08$

$242 \mathrm{~A}$

$2.082 E-01 \quad 2.082 E-01$

$.000 E+00$

$.000 \mathrm{E}+00$

4

$2448 X$

$3.218 E-01 \quad 3.218 E-01$

$.000 E+00$

$.000 \mathrm{E}+00$

$1.513 E-0$

$4.521 E-04$

$3.545 \mathrm{E}-10$

3.131E-04

308

9.464E-01 9.464E-01

$.000 E+00$

$.000 E+$

B-PLANT 2.082E-01 2.082E-01

$.000 E+00$

$.000 E+00$

$.000 \mathrm{E}+00$

$00 \quad 1.649 E-06 \quad 1.257 E-09 \quad 6.715 E-12 \quad 5.703 E-07$

I PLANT
$2.082 \mathrm{E}-01 \quad 2.082 \mathrm{E}-01$
$.000 E+00$

\section{$.660 \mathrm{E}-04 \quad 4.806 \mathrm{E}-05$}

$3.052 E-10$

$1.901 E-04$
$8.645 E-08$ 
SWIR3280 - (Page 66 of 107)

RUN DATE: $03 / 15 / 96$

Land Disposal or Storage of Solid Radioactive Waste from January 1 to December 31 , 1995

CENTRAL WASTE COMPLEX 2402WG BUILDING, 200 WEST AREA, TRANSURANIC WIPP UNCERTIFIED.

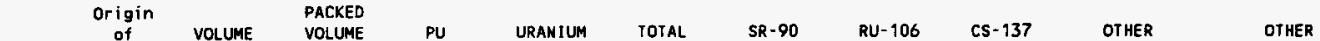

Company Wasțe (CUBIC M.) (CUBIC M.) (GRAMS) (GRAMS) (CURJES) (CURIES) (CURIES) (CURIES) (CURIES) (GRAMS)

.

FLOOR LEVEL 1

WHC $\quad 308$

$2.082 E-01 \quad 2.082 E-01 \quad .000 E+00$

$.000 E+00 \quad 5.000 E-05 \quad 1.099 E-05 \quad 5.870 E-08 \quad 1.185 E-05$

$.000 E+00 \quad 3.385 E+00$

$A M-241 \quad 9.820 E-02$

CM-244 3.120E-05

PU-238 2.950E-03

PU $-239 \quad 2.850 E+90$

PU-240 3.990E-01

PU-241 2.770E-02

PU-242 6.720E-03 
SWIR3280 - (Page 67 of 107)

RUN DATE: $03 / 15 / 96$

Land Disposal or Storage of Solid Radioactive Waste from January 1 to December 31 , 1995

CENTRAL WASTE COMPLEX 2402WH BUILDING, 200 WEST AREA, LOW-LEVEL NONINDUSTRIAL-OFFSITE.

\begin{tabular}{|c|c|c|c|c|c|c|c|c|c|c|}
\hline $\begin{array}{c}\text { Origin } \\
\text { of } \\
\text { Company Waste }\end{array}$ & $\begin{array}{c}\text { VOLUME } \\
\text { (CUBIC M.) }\end{array}$ & $\begin{array}{c}\text { PACKED } \\
\text { VOLUME } \\
\text { (CUBIC M.) }\end{array}$ & $\begin{array}{c}\text { PU } \\
\text { (GRAMS) }\end{array}$ & $\begin{array}{l}\text { URANIUM } \\
\text { (GRAMS) }\end{array}$ & $\begin{array}{l}\text { TOTAL } \\
\text { (CURIES) }\end{array}$ & $\begin{array}{l}\text { SR-90 } \\
\text { (CURIES) }\end{array}$ & $\begin{array}{l}\text { RU-106 } \\
\text { (CUR IES) }\end{array}$ & $\begin{array}{l}\text { CS-137 } \\
\text { (CURIES) }\end{array}$ & $\begin{array}{l}\text { OTHER } \\
\text { (CURIES) }\end{array}$ & $\begin{array}{l}\text { OTHER } \\
\text { (GRAMS) }\end{array}$ \\
\hline LOOR LEVEL 1 & & & & & & & & & & \\
\hline GASD & $2.707 E+00$ & $2.707 E+00$ & $.000 E+00$ & $.000 E+00$ & $6.500 E-02$ & $1.293 E-03$ & $2.784 E-03$ & $1.381 E-03$ & $\begin{array}{c}.000 E+00 \\
T H-232 \\
U-234 \\
U-235 \\
U-236 \\
U-238\end{array}$ & $\begin{array}{l}3.527 E+02 \\
3.470 E+02 \\
4.000 E-02 \\
5.330 E+00 \\
2.000 E-02 \\
3.300 E-01\end{array}$ \\
\hline TOIALS & $2.707 E+00$ & $2.707 E+00$ & $.000 E+00$ & $.000 E+00$ & $6.500 E-02$ & $1.293 \mathrm{E}-03$ & $2.784 \mathrm{E}-03$ & $1.381 \mathrm{E}-03$ & $.000 E+00$ & $3.527 \mathrm{E}+02$ \\
\hline
\end{tabular}


WHC-EP-0125-8

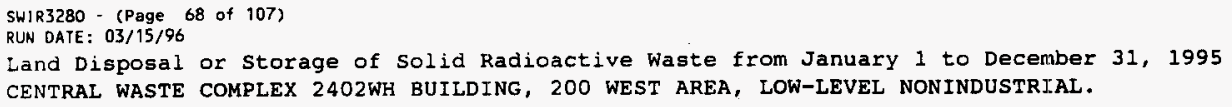

6. $120 E-08$

$.000 E+00 \quad .000 E+00 \quad .000 E+00$

23452

$2.082 E-01 \quad 2.082 E-01$

$.000 E+00$

$.000 \mathrm{E}+0$

IC- 99

9

271B

2.082E-01 2.082E-01

$.000 E+00$

$.000 E+0$

$8.800 E-05$

9.415E-06 2.219E-09 3.545E-0.5

$T$ PLANT

$4.164 \mathrm{E}-01 \quad 4.164 \mathrm{E}-01$

$.000 E+C O$

$.000 E+00$

5.737E-06 2.641E-06

$\begin{array}{ll}2.219 E-09 & 3.545 E-0.5 \\ 1.106 E-10 & 1.546 E-0.7\end{array}$

7


WHC-EP-0125-8

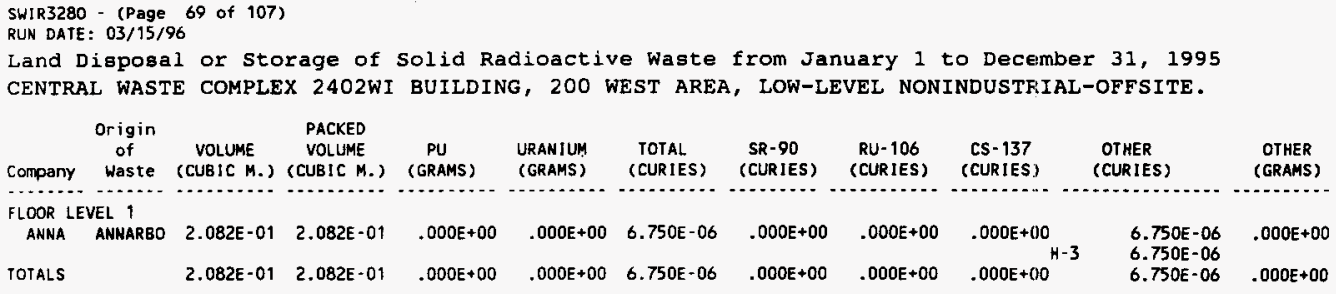

$6.750 E-06$ $6.750 \mathrm{E}-06$ 


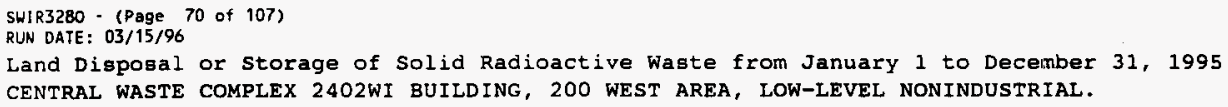

PU-238 1.910E-05

PU-239 6.210E-02

PU-240 3.790E-03

PU-241 2.780E-04

PU-242 1.300E-05 
SWIR3280 - (Page 71 of 107)

RUN DATE: 03/15/96

Land Disposal or Storage of Solid Radioactive Waste from January 1 to December 31 , 1995

CENTRAL WASTE COMPLEX 2402 WJ BUILDING, 200 WEST AREA, TRANSURANIC (NOT PROCESSED THROUGH TRUS

\begin{tabular}{|c|c|c|c|c|c|c|c|c|c|c|c|}
\hline $\begin{array}{c}\text { Origin } \\
\text { of } \\
\text { Company Waste }\end{array}$ & $\begin{array}{l}\text { VOLUME } \\
\text { (CUB IC M.) }\end{array}$ & $\begin{array}{l}\text { PACKED } \\
\text { VOLUME } \\
\text { (CUBIC M.) }\end{array}$ & $\begin{array}{c}\text { PU } \\
\text { (GRAMS) }\end{array}$ & $\begin{array}{l}\text { URANIUM } \\
\text { (GRAMS) }\end{array}$ & $\begin{array}{l}\text { TOTAL } \\
\text { (CURIES) }\end{array}$ & $\begin{array}{l}\text { SR-90 } \\
\text { (CURIES) }\end{array}$ & $\begin{array}{l}\text { RU-106 } \\
\text { (CURIES) }\end{array}$ & $\begin{array}{l}\text { CS-137 } \\
\text { (CURIES) }\end{array}$ & \multicolumn{2}{|c|}{$\begin{array}{l}\text { OTHER } \\
\text { (CUR IES) }\end{array}$} & $\begin{array}{c}\text { OTHER } \\
\text { (GRAMS) }\end{array}$ \\
\hline FLOOR LEVEL 1 & & & & & & & & & & & \\
\hline PML $\quad 325$ & $2.082 E-01$ & $2.082 \mathrm{E}-01$ & $.000 E+00$ & $.000 E+00$ & $5.573 \mathrm{E}-02$ & $1.338 E-06$ & $7.450 E-09$ & $2.740 \mathrm{E} \cdot 0 \mathrm{Z}$ & & $\begin{array}{c}2.404 E-03 \\
A M-241 \\
U-235 \\
U-238\end{array}$ & $\begin{array}{l}1.029 E+02 \\
1.320 E-02 \\
7.300 E-01 \\
1.022 E+02\end{array}$ \\
\hline : & & & & & & & & & $\begin{array}{l}\text { CO-60 } \\
C S-134 \\
E U-152 \\
E U-154\end{array}$ & $\begin{array}{l}7.510 E-06 \\
1.290 E-03 \\
1.690 E-04 \\
9.370 E-04\end{array}$ & \\
\hline TOTALS & $2.082 E-01$ & $2.082 E-01$ & $.000 E+00$ & $.000 E+00$ & $5.573 E-02$ & $1.338 E-06$ & $7.150 E-09$ & $2.740 \mathrm{E}-02$ & & $2.404 E-03$ & $1.029 E+02$ \\
\hline
\end{tabular}


SWIR3280 - (Page 72 of 107)

RUN DATE: $03 / 15 / 96$

Land Disposal or Storage of Solid Radioactive Waste from January 1 to December 31 , 1995

CENTRAL WASTE COMPLEX 2402 WJ BUILIING, 200 WEST AREA, TRANSURANIC WIPP CEFTIFIED.

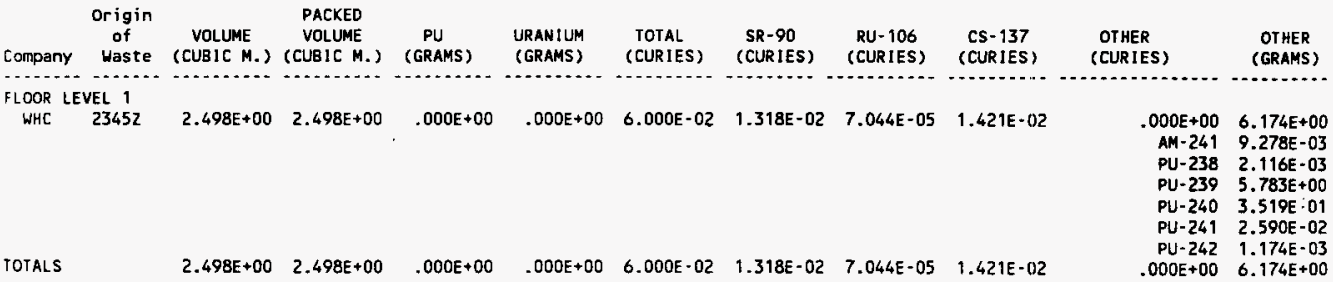


WHC-EP-0125-8

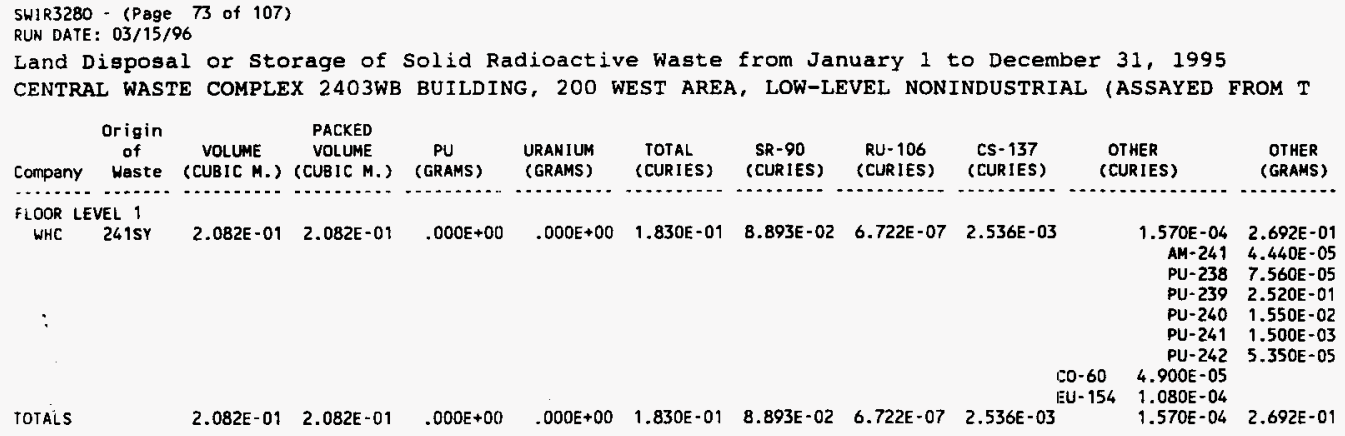

1.570E-04 2.692E-01 AM-241 4.440E-05 PU-238 7.560E-05 PU-239 2.520E-01 PU-240 $1.550 E-02$ PU-241 1.500E-03 PU-242 5.350E-05

4.900E-05

$1.080 E-04$

1.570E-04 2.692E-01 
SWIR3280 - (Page 74 of 107)

RUN DATE: $03 / 15 / 96$

Land Disposal or storage of Solid Radioactive Waste from January 1 to December 31 , 1995

CENTRAL WASTE COMPLEX 2403WB BUILDING, 200 WEST AREA, LOW-LEVEL NONINDUSTRIAL-OFFSITE.

$\begin{array}{ccccccccc}\text { Origin PACKED } & \text { POLUME } & \text { VOLUME } & \text { PU } & \text { URANIUM } & \text { TOTAL } & \text { SR-90 } & \text { RU-106 CS-137 } & \text { OTHER }\end{array}$ Company Waste (CUBIC M.) (CUBIC M.) (GRAMS) (GRAMS) (CURIES) (CURIES) (CURIES) (CURIES) (CURIES) (GRAMS) FLOOR LEVEL 1

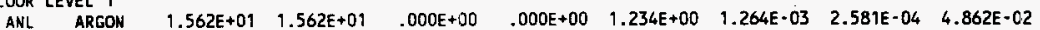

$\begin{array}{cc}1.132 E+00 & 1.497 E+04 \\ A M-241 & 1.970 E-04 \\ A M-243 & 4.441 E-04 \\ \text { CM-242 } & 2.351 E-08 \\ \text { CM-243 } & 7.696 E-06 \\ \text { CM-245 } & 1.445 E-03 \\ \text { NP-237 } & 1.402 E+00 \\ P U-238 & 1.215 E-10 \\ P U-239 & 1.047 E-02 \\ P U-240 & 6.680 E-05 \\ P U-241 & 2.000 E-06 \\ P U-244 & 1.366 E+01 \\ R A-226 & 1.785 E-04 \\ T H-228 & 3.600 E-07 \\ \text { TH-229 } & 6.523 E-04 \\ \text { TH-230 } & 3.140 E-03 \\ \text { TH-232 } & 6.184 E+03 \\ U-232 & 8.698 E-06 \\ U-235 & 1.020 E+02 \\ U-238 & 8.673 E+03 \\ 1.200 E-08 & \\ 1.000 E-05 & \\ 1.012 E-03 & \\ 3.255 E-04 & \\ 3.331 E-05 & \\ 8.705 E-05 & \\ 4.870 E-06 & \\ 1.130 E+00 & \\ 2.861 E-08 & \\ 1.989 E-05 & \\ 1.659 E-05 & \\ .000 E+00 & 2.601 E+03 \\ \text { TH-232 } & 6.586 E+02 \\ U-238 & 1.942 E+03 \\ 1.132 E+00 & 1.757 E+04\end{array}$

TOTALS $\quad 1.874 E+01 \quad 1.874 E+01 \quad .000 E+00 \quad .000 E+00 \quad 1.309 E+00 \quad 2.755 E-03 \quad 3.470 E-03 \quad 5.022 E-02$ 
SWIR3280 - (Page 75 of 107)

RUN DATE: 03/15/96

Land Disposal or storage of Solid Radioactive Waste from January 1 to December 31 , 1995 CENTRAL WASTE COMPLEX 2403WB BUILDING, 200 WEST AREA, LOW-LEVEL NONINDUSTRIAL.

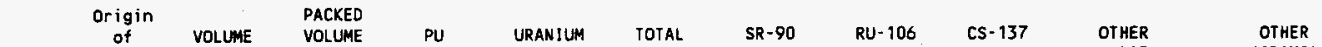
Company Waste (CU8IC M.) (CUBIC M.) (GRAMS) (GRAMS) (CURIES) (CURIES) (CURIES) (CURIES) (CURIES) (GRAMS) F......

FLOOR LEVEL 1

BHI $105 \mathrm{C}$

$\begin{array}{llllllll}2.082 E-01 & 2.082 E-01 & .000 E+00 & .000 E+00 & 1.628 E-08 & 7.190 E-10 & .000 E+00 & 2.160 E-09\end{array}$

$1.064 \mathrm{E}-08 \quad .000 \mathrm{E}+00$

CO-60 $\quad 4.310 E-09$

EU- $152 \quad 2.880 E-09$

EU- 154 1.440E-09

$1304 N \quad 1.912 E+00 \quad 1.912 E+00 \quad .000 E+00 \quad .000 E+00 \quad 3.057 E-01 \quad 8.735 E-03 \quad 3.675 E-08 \quad 2.591 E-02 E$

N1-63 2.010E-09

$2.378 E-01$

AM-241 7.189E-04

MP- 237 4.505E-01

PU- 239 1.364E-01

PU-240 3.145E-03

TH-232 $9.854 \mathrm{E}+02$

U- $235 \quad 2.668 E+01$

U- $238 \quad 1.102 E+03$

2335

$2.800 E+00 \quad 2.800 E+00 \quad .000 E+00 \quad .000 E+00 \quad 1.713 E-10 \quad 4.394 E-16 \quad 2.348 E-18 \quad 8.800 E-11$

CO-60 $2.378 E-0$

$.378 E-01$

4.070E-06

AM-241 4.900E-07

PU-238 2.640E-06

PU-239 4.700E-07

PU. $240 \quad 4.700 E-07$

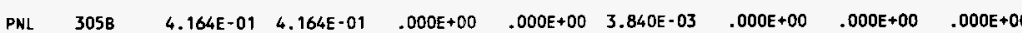

$3.840 \mathrm{E}-03$

$H-3 \quad 3.740 E-03$

P-32 1.000E-04

$324 \quad 5.716 E+00 \quad 5.716 E+00 \quad .000 E+00 \quad .000 E+00 \quad 2.642 E-01 \quad 7.457 E-02 \quad 1.245 E-07 \quad 5.906 E-0 \%$

CS- $134 \quad 2.000 E-06$

$.000 E+00$

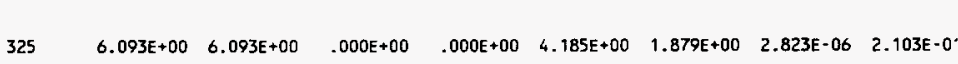
EU-154

(1)

co-

$1.202 E-04$

$1.852 E-02$

PU -239

$1.610 E-06$

$1.610 E-06$

CS-134 $3.350 \mathrm{E}-04$

EU-154 9.181E-03

MN $-54 \quad 3.300 E-07$

SB-125 3.140E-06

$\begin{array}{llllllllll}326 & 6.246 E-01 & 6.246 E-01 & .000 E+00 & .000 E+00 & 6.900 E-07 & 3.449 E-07 & 4.696 E-13 & 9.476 E-1 \\ 327 & 2.234 E+00 & 2.234 E+00 & .000 E+00 & .000 E+00 & 5.371 E-01 & 1.992 E-02 & 4.187 E-07 & 2.550 E-0\end{array}$

$.000 E+00$

PU 239

(327.

$C O-60$
EU -154

$1.060 E-03$

$3.265 E-06$

$3.265 E-06$

$.000 \mathrm{E}+00$

329

$\begin{array}{llllllll}2.290 E+00 & 2.290 E+00 & .000 E+00 & .000 E+00 & 4.302 E-07 & 4.789 E-11 & 2.559 E-13 & 1.121 E-0\end{array}$

7

- 04

2.120E-07

$1.120 E-07$

NI $-63 \quad 5.000 E-08$

PB-210 5.000E-08

$3718 \quad 5.300 E+00 \quad 5.300 E+00 \quad .000 E+00 \quad .000 E+00 \quad 1.370 E+00 \quad 3.142 E-05 \quad 3.000 E-02 \quad 3.388 E-05$

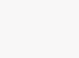

300E +00

AM- 241

PU -238

PU -239

CE-144 1.000E-02

CO-60 $1.090 \mathrm{E}+00$

MN-54 2.000E-02

NB-95 3.000E-02

SB-125 6.000E-02

PSL $\quad 3.840 E+00 \quad 3.840 E+00 \quad .000 E+00 \quad .000 E+00 \quad 1.821 E-06 \quad 5.002 E-07 \quad 9.392 E-13 \quad 1.519 E-0.9$

ZN-65 9.000E-02

$7.910 E-07$

$\mathrm{TH}-230$

$\mathrm{U}-235$

$\mathrm{U}-238$

C- $14 \quad 3.000 E-09$

CO-60 1.800E-08

$\mathrm{PB}-210 \quad 5.000 \mathrm{E}-07$

RA-228 $2.700 E-07$
$-000 E+00$

WHC I52ER $\quad 8.300 E+00 \quad 8.300 E+00 \quad .000 E+00 \quad .000 E+00 \quad 2.590 E+00 \quad 1.260 E+00 \quad 2.219 E-06 \quad 3.545 E-02$

$3.030 E+00$

$6.000 \mathrm{E}-05$

$3.030 E-02$

$3.000 E+00$

.210E-02

$2.000 E-03$

$3.000 \mathrm{E}-02$

$.000 E+00$

4.752E-04 
SWIR3280 - (Page 76 of 107)

RUN DATE: $03 / 15 / 96$

Land Disposal or Storage of Solid Radioactive Waste from January 1 to December 31 , 1995 CENTRAL WASTE COMPLEX 2403WB BUILDING, 200 WEST AREA, LOW-LEVEL NONINDUSTRIAL.

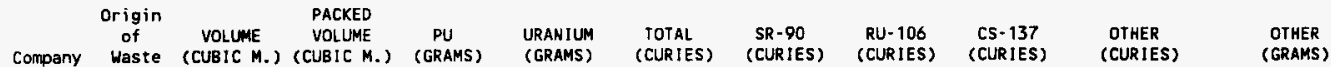

FLOOR LEVEL 1

WHC 152ER $\begin{array}{lllllll}4.997 E+00 & 4.997 E+00 \quad .000 E+00 & .000 E+00 & 8.903 E-02 & 1.600 E-02 & 8.452 E-08 & 1.848 E-02\end{array}$

.......

CCURIES

- . - .
(GRAMS) 更

$\begin{array}{lllllllllll}209 E & 4.164 E-01 & 4.164 E-01 & .000 E+00 & .000 E+00 & 8.698 E-07 & 2.471 E-07 & 7.161 E-13 & 1.929 E-07 \\ 216 \mathrm{~A} & 2.082 E-01 & 2.082 E-01 & .000 E+00 & .000 E+00 & 6.970 E-04 & 9.720 E-05 & 1.094 E-09 & 2.582 E-04 \\ 222 S & 2.763 E+01 & 2.763 E+01 & .000 E+00 & .000 E+00 & 1.235 E+01 & 1.085 E+00 & 5.123 E-04 & 5.193 E+00\end{array}$

\begin{abstract}
2241
$.000 E+00$

$.000 E+00 \quad 1.000 E-02 \quad 2.197 E-03 \quad 1.174 E-05 \quad 2.369 E-03$
\end{abstract}

$.000 \mathrm{E}+00$
$.000 E+00 \quad 5.000 E-02 \quad 1.099 E-02 \quad 5.870 E-05 \quad 1.185 E-0 ?$
$1-129$

$\mathrm{K}-40$

NB- 94

PM- 147

TC -99

CS137

$\mathrm{CO}-58$

Co- 60

EU155

C- 14

BA 137

EU154

FE-59

MN-54

EU152 
SWIR3280 - (Page 77 of 107 )

RUN DATE: 03/15/96

Land Disposal or storage of Solid Radioactive Waste from January 1 to December 31 , 1995

CENTRAL WASTE COMPLEX 2403WB BUILDING, 200 WEST AREA, LOW-LEVEL NONINDUSTRIAL.

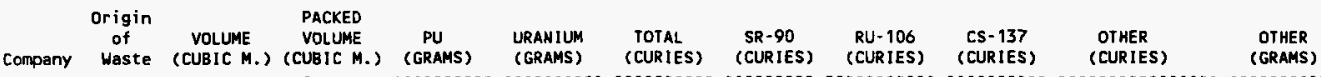

FLOOR LEVEL 1
WHE 23452

$241 A$

$.000 E+00 \quad 1.384 E-04 \quad 4.903 E-05 \quad 1.385 E-10 \quad 2.073 E-05$

241 AN

$1.861 E+00 \quad 1.861 E+00 \quad .000 E+00$

$.000 E+00 \quad 3.450 E-02 \quad 1.556 E-02 \quad 1.086 E-07 \quad 1.729 E-03$

241 AP

$\begin{array}{lllllll}8.508 E+00 & 8.508 E+00 \quad .000 E+00 \quad .000 E+00 & 1.001 E+00 & 4.588 E-01 & 1.315 E-06 & 4.266 E-02\end{array}$

$241 A Y \quad 2.161 E+00 \quad 2.161 E+00 \quad .000 E+00 \quad .000 E+00 \quad 3.693 E-02 \quad 6.978 E-03 \quad 2.492 E-08 \quad 1.173 E-02$.

$241 \mathrm{AW} \quad 1.016 \mathrm{E}+01 \quad 1.016 \mathrm{E}+01 \quad .000 \mathrm{E}+00 \quad .000 \mathrm{E}+00 \quad 1.948 \mathrm{E}+00 \quad 7.861 \mathrm{E}-01 \quad 2.783 \mathrm{E}-06 \quad 1.928 \mathrm{E}-01 \quad$ EU

$000+00$

\begin{tabular}{|c|c|c|c|c|c|}
\hline & & & & & $\begin{array}{l}E U-154 \\
K=40\end{array}$ \\
\hline $241 \mathrm{AZ}$ & $1.304 E+01 \quad 1.304 E+01$ & $.000 E+00$ & $8.485 E-01 \quad 4.060 E-01$ & $4.012 E-04$ & $1.741 E-0 ?$ \\
\hline
\end{tabular}

2418
$4.530 E+00 \quad 4.530 E+00 \quad .000 E+00$

$.000 E+00$
PU-238 7.980E-05
PU-239 2.586E-01
PU-240 1.577E-02
PU-241 1.173E-03
PU-242 5.416E-05
$.000 E+00 \quad 3.011 E-06$
AM-241 2.710E-10
PU-238 $8.430 E-10$
PU-239 2.820E-06
PU-240 1.730E-07
PU-241 1.670E-08
PU-242 5.970E-10
$.000 E+00$ 1.003E-03
AM-241 6.427E-08
PU-23B 2.002E-07
PU-239 9.396E-04
PU-240 5.770E-05
PU-261 5.381E-06
PU-242 1.412E-0?
1.809E-04 4.723E-02
PU-238 2.260E-06
PU-239 4.426E-02
PU-240 2.714E-03
PU-241 2.570E-04

$1.650 \mathrm{E}-04$

$.000 E+00 \quad 3.948 E-02$

AM-241 3.553E-06

PU-238 1.106E-05

PU-239 3.697E-02

PU-240 2.276E-03

PU-241 2.158E-04

PU-242 7.809E-06

1.407E-04 8.101E-04

AM-241 2.678E-09

PU-238 8.318E-09

PU-239 7.591E-04

PU-240 4.665E-05

PU-241 4.418E-06

PU-242 5.895E-09

$1.310 E-04$

9.700E-06

$1.187 \mathrm{E}-03 \quad 6.076 \mathrm{E}-03$

AM-241 5.070E-09

PU-238 1.580E-08

PU-239 5.693E-03

PU-240 3.493E-04

PU-241 3.311E-05

$P U-242$
$C E-144 \quad 5.900 E-04$

CO-60 2.45OE-05

CS- 134 6.810E-05

EU-154 2.181E-04

SB- $125 \quad 2.860 \mathrm{E}-04$

8.216E-05 8.397E-03

AM- $2413.160 E-08$

PU-238 9.812E-08

PU- 239 7.868E-03

PU-240 4.825E-04

PU-241 4.569E-OS 
SWIR3280 - (Page 78 of 107)

RUN DATE : $03 / 15 / 96$

Land Dispogal or Storage of Solid Radioactive Waste from January 1 to December 31 , 1995

CENTRAL WASTE COMPLEX 2403WB BUILDING, 200 WEST AREA, LOW-LEVEL NONINDUSTRIAL.

Origin
of VOLUME VACKED VOLUME PU URANIUM TOIAL SR-90 RU-106 CS-137 OTHER

Company Waste (CUBIC M.) (CUBIC M.) (GRAMS) (GRAMS) (CURIES) (CURIES) (CURIES) (CURIES) (CURIES)

FLOOR LEVEL 1 (

WHC 241B PU-242 $6.910 E-08$

$\begin{array}{lllllllllllll}2418 X & 1.621 E+01 & 1.621 E+01 & .000 E+00 & .000 E+00 & 4.817 E-01 & 1.701 E-01 & 2.962 E-07 & 7.268 E-02 & E U-154 & 8.216 E-05 & 2.052 E-05 & 1.868 E-03\end{array}$

$\begin{array}{ll}A M-241 & 5.639 E-08\end{array}$

PU-238 3.260E-07

PU-239 $1.750 E-03$

PU-240 1.075E-04

PU-241 1.028E-05

PU-242 2.309E-nT

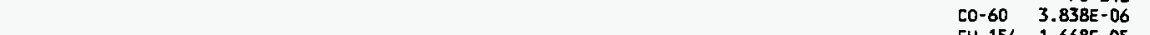

$241 \mathrm{BY} \quad 1.057 E+01 \quad 1.057 E+01 \quad .000 E+00 \quad .000 E+00 \quad 1.662 E+00 \quad 5.869 E-01 \quad 3.684 E-06 \quad 2.509 E-01^{E U-154} \quad \begin{array}{lllllll}1.668 E-05 & 2.626 E-05 & 5.470 E-03 & \end{array}$

AM- $241 \quad 2.430 E-07$

PU- $238 \quad 7.545 E-07$

PU-239 5.123E-03

PU-240 3.155E-04

PU-241 2.989E-05

PU-242 5.339E-07

$241 \mathrm{C}$

$\begin{array}{llllllll}3.929 E+01 & 3.929 E+01 & .000 E+00 & .000 E+00 & 5.276 E+01 & 2.163 E+01 & 2.845 E-04 & 4.773 E+00\end{array}$

EU-154 2.626E-05

$2.626 E-05$
$1.819 E-01$

AM-241 2.197E-05

PU-238 $6.815 E-05$

PU-239 2.329E-01

PU-240 1.424E-02

PU-241 1.348E-03

PU-242 4.841E-05

(10)

CO-60 9.251E-03

CS-134 2.280E-07

EU-154 3.478E-02

$241 \mathrm{~S} \quad 1.106 \mathrm{E}+01 \quad 1.106 \mathrm{E}+01 \quad .000 \mathrm{E}+00 \quad .000 \mathrm{E}+00 \quad 1.897 \mathrm{E}-01 \quad 6.209 \mathrm{E}-02 \quad 1.459 \mathrm{E}-06 \quad 3.359 \mathrm{E}-02$

TC- $99 \quad 1.371 \mathrm{E}-01$

8.601E-05

AM-241

PU-238

PU-239

PU-240

PU-241

PU-262

CO-60 5.107E-05

EU-154 2.773E-05

$\mathrm{K}-40 \quad 7.210 \mathrm{E}-06$

$2415 X \quad 1.681 E+01 \quad 1.681 E+01 \quad .000 E+00 \quad .000 E+00 \quad 7.909 E-02 \quad 2.954 E-02 \quad 1.721 E-07 \quad 1.028 E-02$

$1.759 E-06$

AM- 241

PU-238

$7.480 \mathrm{E}-03$

PU- 239 7.008E-03

PU-240 4.305E-04

PU-241 4.077E-05

PU-242 2.340E-08

CO-60 9.940E-07

EU-154 7.650E-07

$241 S Y$

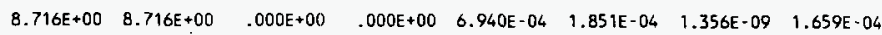

$\begin{array}{rr}8.319 E-07 & 5.988 E-04 \\ \text { AM-241 } & 1.290 E-05 \\ \text { CM-242 } & 8.220 E-16 \\ \text { CM-243 } & 1.210 E-13 \\ \text { CM-244 } & 1.350 E-12 \\ \text { NP-237 } & 1.280 E-06 \\ \text { PU-238 } & 8.860 E-13 \\ \text { PU- } 239 & 5.475 E-04 \\ \text { PU-240 } & 3.354 E-05 \\ \text { PU-241 } & 6.081 E-08 \\ \text { PU-242 } & 8.630 E-13\end{array}$ 
SWIR3280 - (Page 79 of 107)

RUN DATE: 03/15/96

Land Disposal or Storage of Solid Radioactive Waste from January 1 to December 31 , 1995

CENTRAL WASTE COMPLEX 2403 WB BUILDING, 200 WEST AREA, LOW-LEVEL NONINDUSTRIAL.

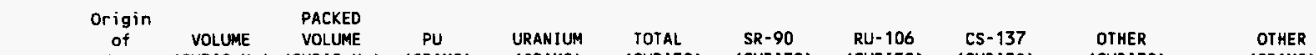

company Waste (CUBIC M.) (CUBIC M.) (GRAMS) (GRAMS) (CURIES) (CURIES) (CURIES) (CURIES) (CURIES) (GRAMS)

FLOOR LEVEL 1

WHC $241 \mathrm{SY}$
$2411 \quad 5.700 E+00 \quad 5.700 E+00 \quad .000 E+00 \quad-000 E+00 \quad 1.490 E-01 \quad 6.326 E-02 \quad 4.267 E-07 \quad 1.152 E-02$

$2617 x$

\begin{abstract}
$2.822 \mathrm{E}+00 \quad 2.822 \mathrm{E}+00$
\end{abstract}

(n)

$241 T Y$

$6.246 \mathrm{E}-01 \quad 6.246 \mathrm{E}-01$

$.000 \mathrm{E}+00$

$.000 \mathrm{E}+00$

$1.184 E-02$

5.005E-03 3.253E-08 9.369E-04

$$
2411 Y
$$

2411

$5.571 E+00-5.571 E+00$

$000 \mathrm{E}+00$

$3.333 E-02$

$-02 \quad 7.095 E-03 \quad 8.739 E-08 \quad 9.833 E-03$

$242 \mathrm{~A} \quad 1.874 \mathrm{E}+00 \quad 1.874 \mathrm{E}+00 \quad .000 \mathrm{E}+00 \quad .000 \mathrm{E}+00 \quad 6.095 \mathrm{E}-02 \quad 9.638 \mathrm{E}-03 \quad 1.403 \mathrm{E}-07 \quad 1.213 \mathrm{E}-03$

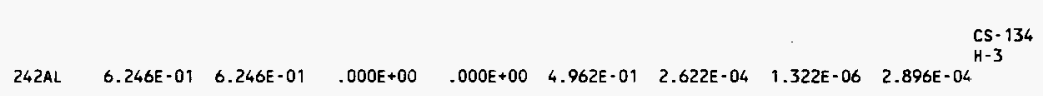

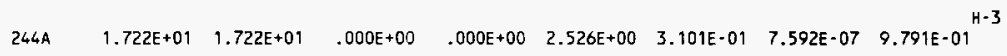

EU-

TC

$\begin{array}{lcr} & U-235 & 5.860 E-08 \\ & U-236 & 1.330 E-09 \\ & U-238 & 3.430 E-06 \\ C-14 & 2.090 E-11 & \\ \text { CO-60 } & 1.130 E-08 & \\ \text { EU-154 } & 8.116 E-07 & \end{array}$

NB-93M 8.670E-11

NB-94 1.010E-12

N) $-59 \quad 2.900 E-12$

N1-63 4.640E-10

SE-79 7.250E-12

TC-99 8.120E-09

$4.455 E-05$

PU- 238

PU-239

PU-240

PU- 241

PU-242

EU-154 4.455E-05

$4.455 E-05$
$2.970 E-06$

AN-241

PU- 238

PJ- 239

PU-240

PU-241

PU-242

2.970E-06

$9.300 E-07$

PU-239

PU-240

PU-241

9.300E-07

ODOE +00

AN- 241

PU -238

PU-239

PU -240

PU-241

PU-242

$3.930 \mathrm{E}-0$

AM- 241

PU -238

PU-240 3.281E-05

PU-241 3.109E-06

PU- 242 8.750E-08

4.740E-07

$3.930 E-02$

4.950E-01 1.038E-05

PU-239 9.730E-06

PU-240 5.980E-07

PU-241 5.660E-08

$4.950 \mathrm{E}-01$

1.780E-05 3.924E-03

$A M-241 \quad 3.519 \mathrm{E}-07$

PU-238 1.093E-06

PU-239 3.675E-03

PU-240 2.251E-04 
SWIR3280 - (Page 80 of 107)

RUN DATE: $03 / 15 / 96$

Land Disposal or Storage of Solid Radioactive Waste from January 1 to Decenber 31 , 1995

CENTRAL WASTE COMPLEX 2403WB BUILDING, 200 WEST AREA, LOW-LEVEL NONINDUSTRIAL.

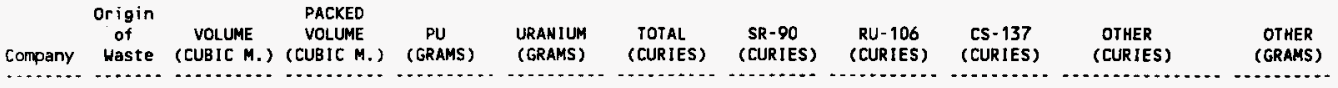

\section{FLOOR LEVEL 1}

WHC $244 \mathrm{~A}$

244A

304

$1.382 E+00 \quad 1.382 E+00 \quad .000 E+01$

$.000 E+00 \quad 7.194 E-06$

$6.042 E-10$

$3.229 E-12 \quad 6.515 E-10$

308

$9.464 \mathrm{E}-01 \quad 9.464 \mathrm{E}-01$

$.000 E+00$

$1.005 \mathrm{E}-0$

2. 197E-0

1

$1.174 \mathrm{E}-0$

$2.369 E-03$
EU-154
$2.330 E-05$

2.330E-05

7.191E-06

CO-60 8.842E-07

N8-95 $\quad 6.307 E-06$

4.723E-05

BA- $133 \quad 4.630 E-05$

CO-60 2.550E-07

EU-154 6.700E-07

377

$2.082 E-01 \quad 2.082 E-01 \quad .000 E+00$
$.000 E+0$

$4.560 E-08$

$2.197 \mathrm{E}-11$

$1.174 E-13 \quad 2.369 E-11$

(1)

6266

$6.246 E-01 \quad 6.246 E-01$

$.000 E+00$

$.000 E+00$

$2.748 \mathrm{E} \cdot 06$
$5.675 E-08 \quad 2.465 E-13 \quad 1.092 E-07$
IC-
$4.550 \mathrm{E}-08$

$4.550 \mathrm{E}-08$

2.422E-06

BA- $\mathrm{HM}$

$\begin{array}{ll}B A-133 & 8.520 E-07 \\ H=3 & 2.274 E-07\end{array}$

PM- $147 \quad 1.998 E-07$

RA-228 3.420E-08

SR-85 8.520E-07

SR-89 5.670E-08

TC-99 1.998E-07

$701 \mathrm{~A}$

$4.164 E-01 \quad 4.164 E-01 \quad .000 E+00$

$.000 E+00$

$1.310 \mathrm{E}-05$

$3.320 E-06$

$1.644 E-12 \quad 3.320 E-06$

$702 A$

\section{$8.300 E+00 \quad 8.300 E+00$}

$.000 E+00$
PU-241

PU-242

$.780 \mathrm{E}-05$

PJ-239

PU-240

PU-241

TH- 228

$\mathrm{TH}-234$

$\mathrm{U}-235$

U- 238

AM- 241

CM-243

CM- 244

HP -237

PU-238

PU-239

PU-240

PU-241

PU-262

u- 235

U- 238

U-234

U-235

U- 236

U-238

AM-241

AM- 243

PU- 239

PU-242

RA- 226

$\mathrm{U}-232$

U- 238

2. $110 \mathrm{E}-05$

8.169E-07

$2.156 \mathrm{E}-04$ $2.020 \mathrm{E}-\mathrm{C} 4$ $1.240 E-05$ 1.170 E-06

$2.576 E+33$ $5.878 E-07$ $7.669 E-08$ $3.226 E+01$ $2.544 E+03$

$3.639 E+01$ $2.230 E-02$ $1.170 E-08$ 2.04BE-05 $1.480 E-01$ $4.490 E-04$ $4.120 E-01$ 6.240E-02

$1.323 E-02$

$1.034 E-04$

5.467E-01

$3.518 E+01$

1.415E-02 $1.270 E-06$ $1.420 E-04$ $6.210 E-06$ 1.400 E- 02

6.870E-02 8. $400 E-09$ 5. $940 E-08$ 1. $755 \mathrm{E}-07$ 2. $949 E-06$ 2.310E-OB $1.032 E-09$ 6.870E-02

$.000 E+00$ AM-241 3.940E-29 PU-238 1.222E-08 PU-239 4.080E-05 PU-240 2.500E-06 PU-241 2.260E-07 PU-242 8.460E-09 $.000 \mathrm{E}+00 \quad 3.693 \mathrm{E}+05$ PU-239 3.460E-05 
WHC-EP-0125-8

SWIR3280 - (Page 81 of 107)

RUN DATE: 03/15/96

Land Disposal or Storage of Solid Radioactive Waste from January 1 to December 31 , 1995 CENTRAL WASTE COMPLEX 2403 WB BUILDING, 200 WEST AREA, LOW-LEVEL NONINDUSTRIAL.

\begin{tabular}{|c|c|c|c|c|c|c|c|c|c|c|c|}
\hline Company & $\begin{array}{l}\text { Origin } \\
\text { of } \\
\text { Waste }\end{array}$ & $\begin{array}{c}\text { VOLUME } \\
\text { (CUBIC M.) }\end{array}$ & $\begin{array}{l}\text { PACKED } \\
\text { VOLUME } \\
\text { (CUBIC M.) }\end{array}$ & $\begin{array}{c}\text { PU } \\
\text { (GRAMS) }\end{array}$ & $\begin{array}{l}\text { URANIUM } \\
\text { (GRAMS) }\end{array}$ & $\begin{array}{l}\text { TOTAL } \\
\text { (CURIES) }\end{array}$ & $\begin{array}{l}\text { SR-90 } \\
\text { (CURIES) }\end{array}$ & $\begin{array}{c}\text { RU-106 } \\
\text { (CURIES) }\end{array}$ & $\begin{array}{l}\text { CS }-137 \\
\text { (CURIES) }\end{array}$ & $\begin{array}{l}\text { OTHER } \\
\text { (CUR IES) }\end{array}$ & $\begin{array}{l}\text { OTHER } \\
\text { (GRAMS) }\end{array}$ \\
\hline $\begin{array}{l}\text { LOOR LE } \\
\text { WHC }\end{array}$ & $\begin{array}{l}\text { VEL } 1 \\
702 A\end{array}$ & & & & & & & & & $\begin{array}{l}P U-240 \\
P U-241\end{array}$ & $\begin{array}{l}2.130 E-06 \\
2.010 E-07\end{array}$ \\
\hline BIALS & I PLANT & $\begin{array}{l}1.041 E+00 \\
2.780 E+02\end{array}$ & $\begin{array}{l}1.041 E+00 \\
2.780 E+02\end{array}$ & $\begin{array}{l}.000 E+00 \\
.000 E+00\end{array}$ & $\begin{array}{l}.000 E+00 \\
.000 E+00\end{array}$ & $\begin{array}{l}2.995 E-03 \\
8.449 E+01\end{array}$ & $\begin{array}{l}3.712 E-04 \\
2.907 E+01\end{array}$ & $\begin{array}{l}1.267 \mathrm{E}-08 \\
3.130 \mathrm{E}-02\end{array}$ & $\begin{array}{l}1.157 \mathrm{E}-03 \\
1.227 \mathrm{E}+01\end{array}$ & $\begin{array}{r}.000 E+00 \\
2.309 E+00\end{array}$ & $\begin{array}{r}.000 E+00 \\
5.716 E+03\end{array}$ \\
\hline
\end{tabular}


WHC-EP-0125-8

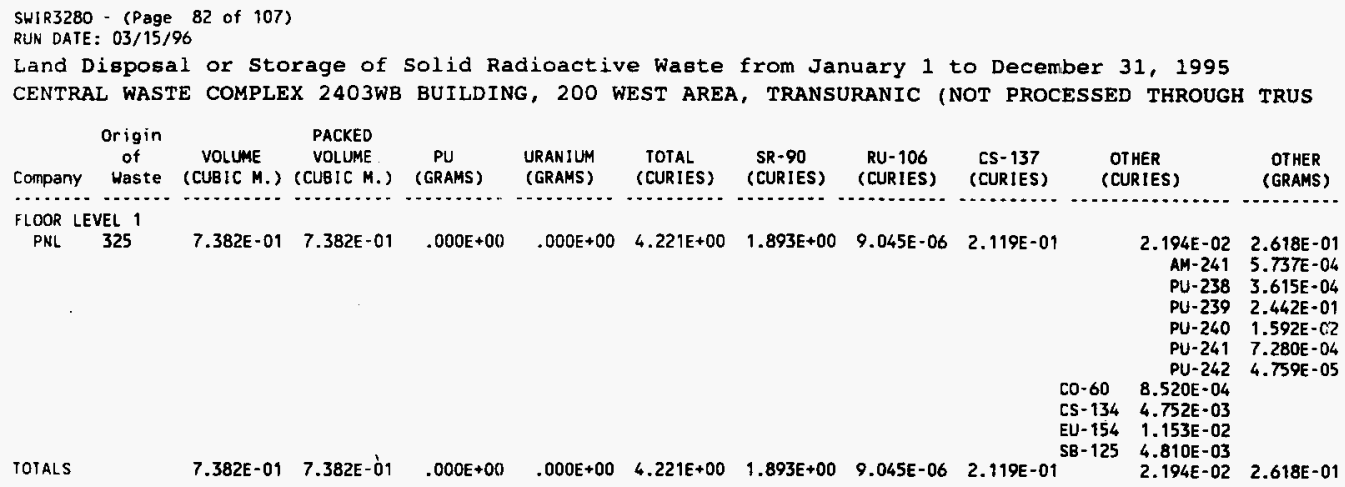


SWIR3280 - (Page 83 of 107)

RUN DATE : 03/15/96

Land Disposal or storage of Solid Radioactive Waste from January 1 to December 31 , 1995

CENTRAL WASTE COMPLEX 2403WB BUILDING, 200 WEST AREA, TRANSURANIC WIPP CERTIFIED.

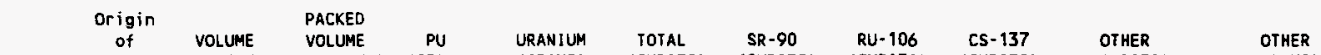

Company Waste (CUBIC M.) (CUBIC M.) (GRAMS) (GRAMS) (CURIES) (CURIES) (CURIES) (CURIES) (CURIES)

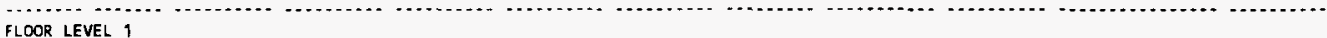

WHC LEVE

$\begin{array}{lllllll}6.246 E-01 & 6.246 E-01 \quad .000 E+00 & .000 E+00 & 1.350 E-02 & 2.197 E-03 & 1.174 E-05 & 2.475 E-03\end{array}$

3.

$.000 E+00 \quad 1.940 E+01$

AM-241 3.786E-04

PU-238 $5.811 E-03$

PU-239 1.812E+01

PU-240 1.167E +00

PU-241 1.050E-01

PU-242 6.852E-03

C\$137 3.605E-05

CO-58 3.500E-05

CO-60 2.345E-03

EU155 2.975E-04

[- 14 3.500E-05

BA137 3.395E-05

EU154 2.975E-04

FE-59 1.400E-04

MN-54 2.450E-04

$23452 \quad 7.169 E+00 \quad 7.169 E+00 \quad .000 E+00 \quad .000 E+00 \quad 3.600 E-02 \quad 7.909 E-03 \quad 4.226 E-05 \quad 8.528 E-03$

EU152 $3.500 E-05$

$.000 E+00 \quad 2.716 E+02$

AN-241 3.884E-01

PU-238 7.743E-02

PU-239 $2.544 E+02$

$P U-240 \quad 1.549 E+01$

PU- $241 \quad 1.148 E+00$

PU-242 5.257E-02

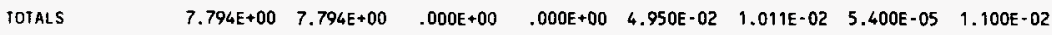

$.000 E+00 \quad 2.910 E+02$ 
WHC-EP-0125-8

SWIR3280 - (Page 84 of 107 )

RUN DATE: $03 / 15 / 96$

Land Disposal or storage of Solid Radioactive Waste from January 1 to Decenber 31 , 1995

CENTRAL WASTE COMPLEX 2403 WB BUILDING, 200 WEST AREA, TRANSURANIC WIPP UNCERTIFIED.

\begin{tabular}{|c|c|c|c|c|c|c|c|c|c|c|c|}
\hline$m$ & $\begin{array}{l}\text { of } \\
\text { Waste }\end{array}$ & $\begin{array}{l}\text { VOLUME } \\
\text { (CUBIC } M_{2} \text { ) }\end{array}$ & $\begin{array}{c}\text { VOLUME } \\
\text { (CUBIC M.) }\end{array}$ & $\begin{array}{l}\text { PU } \\
\text { (GRAMS) }\end{array}$ & $\begin{array}{l}\text { URANIUM } \\
\text { (GRANS) }\end{array}$ & $\begin{array}{l}\text { TOTAL } \\
\text { (CURIES) }\end{array}$ & $\begin{array}{l}\text { SR-90 } \\
\text { (CURIES) }\end{array}$ & $\begin{array}{c}\text { RU-106 } \\
\text { (CUR IES) }\end{array}$ & $\begin{array}{c}\text { CS-137 } \\
\text { (CURIES) }\end{array}$ & $\begin{array}{l}\text { OTHER } \\
\text { (CURIES) }\end{array}$ & $\begin{array}{c}\text { OTHER } \\
\text { (GRAMS) }\end{array}$ \\
\hline
\end{tabular}

FLOOR LEVEL 1

PNL 325

$\begin{array}{lllllll}4.200 E+00 & 4.200 E+00 \quad .000 E+00 & .000 E+00 & 1.937 E+01 & 8.103 E+00 & 1.615 E-05 & 1.603 E+00\end{array}$

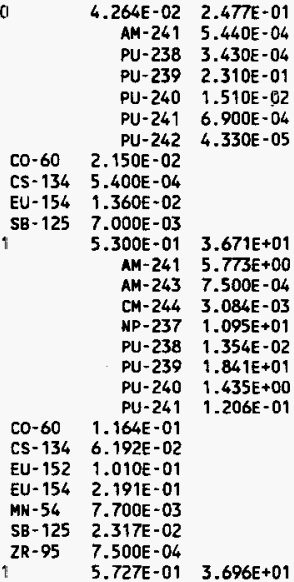


SWIR3280 - (Page 85 of 107 )

RUN DATE: $03 / 15 / 96$

Land Disposal or Storage of Solid Radioactive Waste from January 1 to Decenber 31 , 1995

CENTRAL WASTE COMPLEX 2403 WC BUILDING, 200 WEST AREA, LOW-LEVEL NONINDUSTRIAL-OFFSITE.

Origin
of voLUME VACKED VOLUME PU URANIUM TOTAL SR-90 RU-106 CS-137 OTHER

Company Haste (CUBIC M.) (CUBIC M.) (GRAMS) (GRAMS) (CURIES) (CURIES) (CURIES) (CURIES) (CURIES)
(GRAMS)

FLOOR LEVEL 1

$\begin{array}{lllllllll}\text { ANL ARGON } \quad 6.038 E+00 & 6.038 E+00 & .000 E+00 & .000 E+00 & 1.820 E-03 & 8.037 E-08 & 1.731 E-07 & 4.116 E-06\end{array}$

$1.809 E-03 \quad 2.547 E+02$

AM-241 6.764E-06

AM-243 2.301E-04

PU-239 1.600E-02

RA-226 6.330E-05

TH-228 $1.586 E-09$

TH-232 6.204E+01

U-232 1.872E-06

U-233 7.134E-02

U-234 1.010E-02

U-235 1.570E+00

U-238 $1.910 E+02$

BI-214 2.550E-08

CO-60 3.746E-05

$\mathrm{H}-3 \quad 1.536 \mathrm{E}-03$

K-40 3.196E-05

PB-214 3.800E-09

PO-210 1.039E-05

S-35 1.660E-05

IC- $99 \quad 1.760 \mathrm{E}-06$

$\begin{array}{llllllllll}\text { ANNA ANNARBO } & 1.874 E+00 & 1.874 E+00 & .000 E+00 & .000 E+00 & 7.936 E-02 & -000 E+00 & .000 E+00 & .000 E+00\end{array}$

$\begin{array}{lllllllll}\text { BATC BATCO } \quad 2.707 E+00 & 2.707 E+00 & .000 E+00 & .000 E+00 & 5.000 E-04 & 9.944 E-06 & 2.142 E-05 & 1.062 E-05\end{array}$

TM-170 $1.700 \mathrm{E}-07$

$7.936 \mathrm{E}-02$

BNL BNLAB $\quad 2.269 E+01 \quad 2.269 E+01 \quad-000 E+00 \quad .000 E+00 \quad 1.758 E-01 \quad 3.608 E-03 \quad 1.520 E-04 \quad 2.982 E-02$

7.936E-02

TH-232

$5.634 E+02$

$U-235 \quad 1.046 E-01$

$U-238 \quad 4.241 E+02$

$1.073 E-01 \quad 1.084 E+03$

AM-241 5.478E-04

TH-228 8.267E-04

$T H-230 \quad 4.786 E-02$

$T H-232 \quad 1.895 E+01$

$\mathrm{U}-233 \quad 6.745 \mathrm{E}-03$

U- $235 \quad 2.262 \mathrm{E}+00$

U-238 $1.063 E+03$

\begin{tabular}{|c|c|c|c|c|c|c|c|c|c|c|c|c|}
\hline & & & & & & & & & & $\begin{array}{l}C O-60 \\
E U-152 \\
E U-154 \\
E U-155 \\
H-3\end{array}$ & $\begin{array}{l}1.068 E-01 \\
2.857 E-05 \\
3.708 E-05 \\
7.260 E-06 \\
3.862 E-04\end{array}$ & \\
\hline FERM & NALAB & $2.498 E+00$ & $2.698 \mathrm{E}+00$ & $.000 E+00$ & $.000 E+00$ & $2.461 E-03$ & $8.876 E-08$ & $1.912 E-07$ & $9.480 E-08$ & $\begin{array}{l}\text { AU- } 195 \\
\text { BE- } 7 \\
\text { CO-56 } \\
\text { CO-57 } \\
\text { CO-58 } \\
\text { CO-60 } \\
H-3 \\
H F-175 \\
H G-203 \\
M N-54 \\
N A-22 \\
Z N-65\end{array}$ & $\begin{array}{l}2.457 E-03 \\
4.270 E-07 \\
2.116 E-03 \\
1.272 E-06 \\
6.985 E-05 \\
5.886 E-05 \\
2.349 E-05 \\
2.640 E-05 \\
8.850 E-05 \\
5.900 E-05 \\
1.259 E-05 \\
2.150 E-07 \\
4.530 E-07\end{array}$ & $.000 E+00$ \\
\hline GAS & GASD & $8.290 E+00$ & $8.290 E+00$ & $.000 E+00$ & $.000 E+00$ & $1.203 \mathrm{E}+00$ & $2.114 E-03$ & $4.553 E-03$ & $7.648 E-0.3$ & $G=11$ & $\begin{array}{c}1.086 E+00 \\
T H-232 \\
U-234 \\
U-235 \\
U-236 \\
U-238 \\
4.809 E-05\end{array}$ & $\begin{array}{l}2.236 E+03 \\
2.185 E+03 \\
4.700 E-01 \\
4.793 E+01 \\
1.600 E-01 \\
2.930 E+00\end{array}$ \\
\hline
\end{tabular}




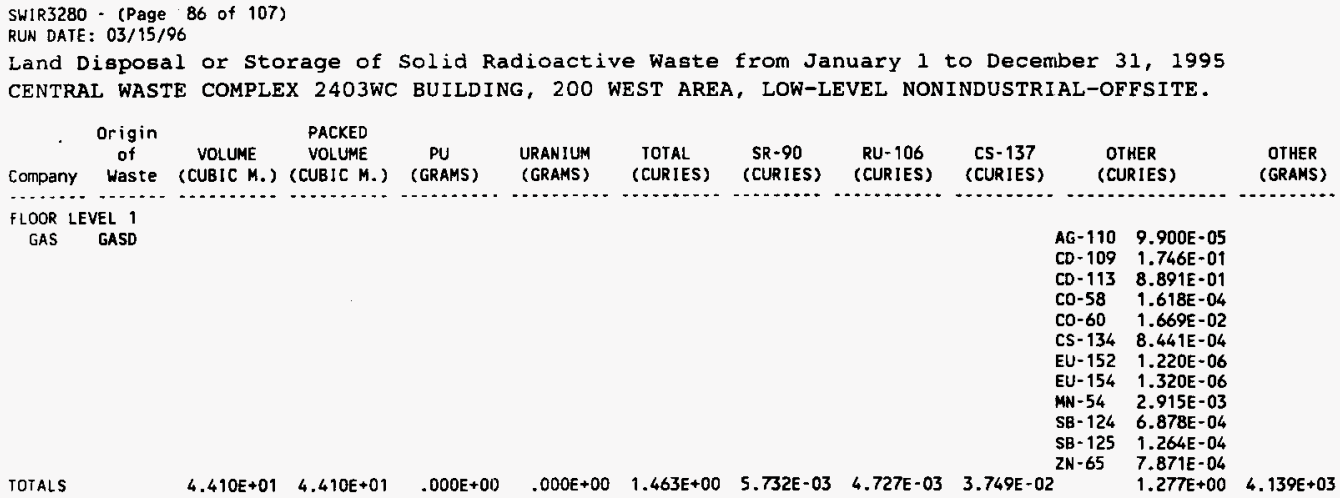


SWIR3280 - (Page 87 of 107)

RUN DATE: $03 / 15 / 96$

Land Disposal or storage of Solid Radioactive Waste from January 1 to Decernber 31 , 1995

CENTRAL WASTE COMPLEX 2403 WC BUILDING, 200 WEST AREA, LOW-LEVEI NONINDUSTREAL.

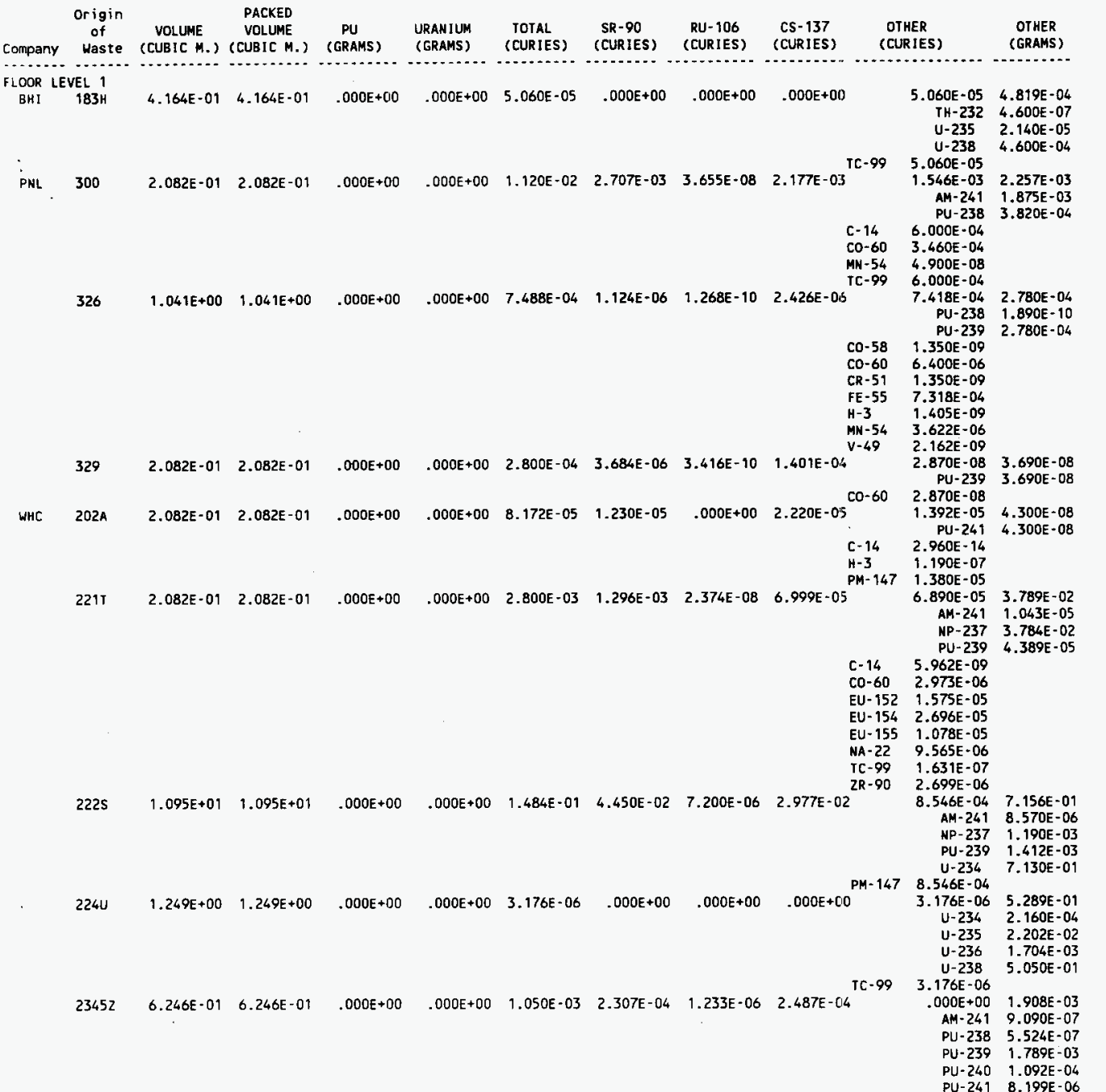


SWIR3280 - (Page 88 of 107)

RUN DATE : 03/15/96

Land Disposal or storage of solid Radioactive waste from January 1 to Decenber 31 , 1995

CENTRAL WASTE COMPLEX 2403WC BUILDING, 200 WEST AREA, LOW-LEVEL NONINDUSTRJAL.

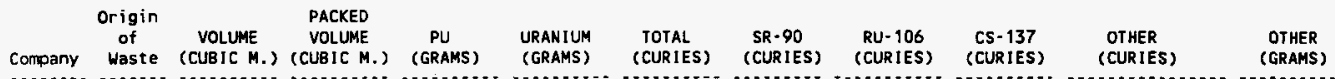

FLOOR LEVEL 1

WHC 23452

$241 \mathrm{~A} \quad 4.164 E-01 \quad 4.164 E-01 \quad .000 E+00 \quad .000 E+00 \quad 5.125 E-04 \quad 1.691 E-04 \quad 3.017 E-90 \quad 8.890 E-05$.

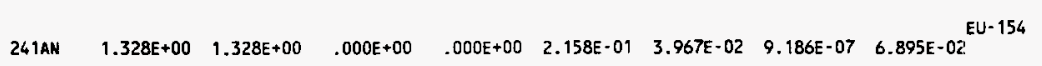

CS- 134

241AW $\quad 6.246 E-01 \quad 6.246 E-01 \quad .000 E+00 \quad .000 E+00 \quad 1.294 E-02 \quad 5.286 E-03 \quad 4.976 E-08 \quad 1.214 E-0.3$

CO-60 5.570E-05

$\mathrm{PU}-242 \quad 3.117 \mathrm{E}-07$

1.280E-06 5.474E-07

AM-241 4.945E-11

PU-238 1.535E-10

PU-239 5.126E-07

PU-240 3.150E-J8

PU-241 3.039E-09

PU- $242 \quad 1.083 E-10$

1.328

$1.328 \mathrm{0}$

.000

1.280E-06

2.211E-03 1.115E-03

AM-241 4.549E-05

PU-238 3.003E-0?

PU. 239 1.002E-03

PU-240 6.154E-05

PU-241 5.928E-06

PU. 242 2.125E-07

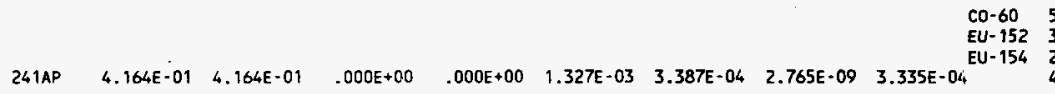

3.710E-05

24.

$241 A X \quad 1.704 E+00 \quad 1.704 E+00 \quad .000 E+00 \quad .000 E+00 \quad 2.264 E-03 \quad 4.810 E-04 \quad 8.382 E-09 \quad 6.640 E-04 \quad k-40$

$4.220 E$
$2.740 E$

$118 E+03$

4.220E-07

1.163E-05

AM-241 $1.050 E-09$

PU-238 3.260E-09

PU-239 1.089E-05

PU-240 6.680E-07

PU-241 6.280E-08

PU-242 2.310E-09

$-40$

AM- 241

3.444E-04

PU-238 $9.648 E-08$

PU-239 3.226E-04

PU-240 1.980E-05

PU-241 1.816E-06

PU-242 6.822E-08

$2.740 \mathrm{E}-06$

$9.238 E-06 \quad 1.528 E-04$

AM-241 5.769E-0B

PU-238 2.108E-06

PU-239 1.491E-04

PU-240 1.175E-06

PU-241 2.942E-07

$P U-242 \quad 3.948 E-09$

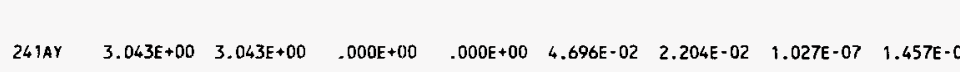

Co-60 $1.148 E-06$

EU- $154 \quad 8.090 E-06$

.

$3.0435+00$

(O)

(1)

03

3.983E-05

AM- $2412.004 E-06$

PU-238 6.148E-06

PU-239 2.056E-02

PU-240 1.264E-03

PU-241 1.214E-04

PU- 242 4.353E-06

\begin{tabular}{|c|c|c|c|c|c|c|c|c|c|c|c|}
\hline & & & & & & & & & $\begin{array}{l}C O-60 \\
E U-152 \\
E U-154 \\
E U-155 \\
K-40\end{array}$ & $\begin{array}{r}P U-242 \\
1.147 E-06 \\
5.540 E-06 \\
1.817 E-05 \\
1.380 E-05 \\
1.170 E-06\end{array}$ & $4.353 E-06$ \\
\hline $241 A Z$ & $6.246 \mathrm{E}-01$ & $6.246 E-01$ & $.000 E+00$ & $-000 E+00$ & $8.974 E-03$ & $1.283 E-04$ & 3. $731 E-08$ & $4.471 E-013$ & & $\begin{array}{r}1.313 E-05 \\
A M-241 \\
P U-238 \\
P U-239\end{array}$ & $\begin{array}{l}1.339 E-04 \\
1.206 E-08 \\
3.733 E-08 \\
1.255 E-04\end{array}$ \\
\hline
\end{tabular}


SWIR3280 - (Page 89 of 107)

RUN DATE: 03/15/96

Land Disposal or storage of Solid Radioactive Waste from January 1 to Decenber 31 , 1995

CENTRAL WASTE COMPLEX 2403WC BUILDING, 200 WEST AREA, LOW-LEVEL NONINDUSTRJAL.

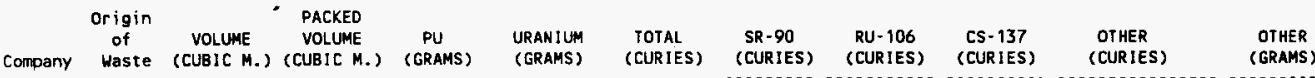

FLOOR LEVEL 1

WHC 241AZ
$241 \mathrm{BX} \quad 8.328 \mathrm{E}-01 \quad 8.328 \mathrm{E}-01 \quad .000 E+00 \quad .000 E+00 \quad 1.216 \mathrm{E}-02 \quad 3.186 E-03 \quad 1.923 E-08 \quad 2.976 E-03$

241BY

4.164E-01 4.164E-01 .000E+00 -000E+00 4.579E-02 $1.190 E-02 \quad 1.260 E-07 \quad 1.129 E-02 !$
EU-154

$\begin{array}{rr}134 & 1 \\ \mathrm{C}-154 & 6\end{array}$

PU -240

PU-241 6.952E-07

PU-242 2.600E-08

(1)

(2006

(4.579e

$241 C$

$.000 E+00$

$.000 E+0$

$\begin{array}{llll}1.534 E-02 & 4.422 E-03 & 1.329 E-07 & 3.332 E-0.3\end{array}$

\begin{abstract}
8. 199E+00
8. $199 E+00$

$.000 E+00$

$.000 \mathrm{E}+0$

$6.272 E-01$

$1 \quad 2.520 E-01 \quad 2.630 E-06 \quad 6.289 E-02$
\end{abstract}

\section{co-60 \\ EU-154}

250E-05

OOOE +00

PU-238 2.017E-06

PU-239 6.739E-03

PU-240 4.137E-04

PU-241 3.994E-05

PU-242 $1.434 \mathrm{E}-06$

1.152E-06 8.186E-04

AM-241 7.384E-08

PU-238 2.297E-07

PU-239 7.665E-04

$P U-240 \quad 4.705 E-05$

PU-241 4.535E-06

PU-242 1.625E-07

$5.640 \mathrm{E}-07$

$5.880 E-07$

6.711E-04 2.649E-01

AM- 241 1.668E-05

CM-243 6.846E-09

CM-244 7.619E-08

NP- $237 \quad 2.047 E-01$

PU-238 1.689E-05

PU- $239 \quad 5.636 E-02$

PU-240 3.462E-03

PU-241 3.341E-04

PU- $242 \quad 1.192 E-05$

C- $14 \quad 1.790 \mathrm{E}-06$

CO-60 8.512E-05

EU-154 3.845E-04

$\mathrm{H}=3$ 5.159E-06

$\mathrm{I}-129 \quad 3.480 \mathrm{E}-06$

K-40 4.090E-06

IC-99 $\quad 1.870 \mathrm{E}-06$

2.072E-06
$A M-241$

PU-238

PU-239

PU-240

PU- 241

PU -242

CO-60 5.020E-07

EU-154 1.570E-06

$2415 X \quad 6.246 E-01 \quad 6.246 E-01 \quad .000 E+00 \quad .000 E+00 \quad 7.336 E-05 \quad 2.562 E-05 \quad 9.040 E-11 \quad 9.510 E-0.6$

$$
2415 Y
$$

$3.876 \mathrm{E}+00 \quad 3.876 \mathrm{E}+00$
$1.570 \mathrm{E}-06$
$3.610 \mathrm{E}-06$

AM-26

PU- 238

PU-239

PU -240

PU -241

PU-242

$3.610 \mathrm{E}-06$

$1.051 E-04 \quad 1.405 E-02$

$A M-241 \quad 2.241 E-06$

CM-242 6.769E-13

CM-243 9.992E- 11

CM-244 1.112E-09 
SWIR3280 - (Page 90 of 107 )

RUN DATE: $03 / 15 / 96$

Land Disposal or Storage of solid Radioactive Waste from January 1 to December 31 , 1995 CENTRAL WASTE COMPLEX 2403WC BUILDING, 200 WEST AREA, LOW-LEVEL NONINDUSTRIAL.

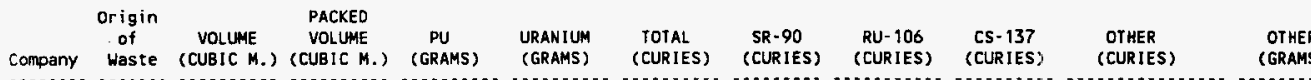

FLOOR LEVEL 1

WHC $2415 Y$

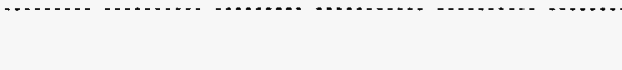

NP-237 $1.053 E-03$ PU-238 2.835E-06 $\mathrm{PU}-239 \quad 9.485 \mathrm{E}-03$ PU-240 5.821E-04 PU-241 5.610E-05 PU-242 2.009E-06 U-235 4.821E-05 $\mathrm{U}-236 \quad 1.092 \mathrm{E}-06$ U-238 $2.821 T-03$

C-14 1.721E-08 CO-60 7.422E-06

EU-154 $9.028 E-05$

$\begin{array}{ll}1-129 & 2.630 E-07\end{array}$

NB-93M 7.135E-08

NB-94 8.351E-10

NI-59 2.379E-09

NI $-63 \quad 3.820 E-07$

SE-79 5.971E-09

TC-99 6.679E-06

$.000 E+00$

AM- 241

PU- 238

PU- 239

PU -240

PU-241

PU -242

$1.078 E-06$

AM- 241

PU- 238

PU-239

PU-240

PU- 241

PU-242

CO-60 3.880E-07

EU-154 6.900E-07

2410

$2.082 E-01 \quad 2.082 E-01 \quad .000 E+00$

$.000 E+00 \quad 1.510 E-03 \quad 3.857 E-04 \quad 3.959 E-09 \quad 3.788 E-04$

$1.040 \mathrm{E}-06$

AM-241

PU -238

$\mathrm{PU}-239$

PU- 240

PU-242

EU-154 1.040E-06

$242 \mathrm{~A}$

$4.164 E-01 \quad 4.164 E-01 \quad .000 E+00$

$.000 E+00 \quad 3.480 E-03 \quad 7.004 E-0$

$1.764 E-08 \quad 3.906 E-106$

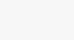

2718

$2.082 E-01 \quad 2.082 E-01$

$.000 E+00$

$000 E+00$

$.000 E+00$

$.000 E+00$

H-3

2ETF

2.082E-01 2.082E-01

9.200E-05

$9.439 E-06$

$62.346 \mathrm{E}-09 \quad 3.747 \mathrm{E}-105^{\mathrm{H}-3}$

$\begin{array}{llll}1.050 E-03 \quad 5.239 E-04 & 4.572 E-09 \quad 9.768 E-07\end{array}$

377
$2.082 E-01 \quad 2.082 E-01$
$.000 E+0$

(

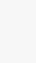

$\begin{array}{lllll}.000 E+00 & 1.920 E-06 & .000 E+00 \quad .000 E+00 & .000 E+100\end{array}$

$$
\begin{array}{rr}
1.040 E-06 & \\
2.070 E-03 & 2.285 E-02 \\
A M-241 & 2.060 E-06 \\
P U-238 & 6.410 E-06 \\
P U-239 & 2.140 E-02 \\
P U-240 & 1.310 E-03 \\
P U-241 & 1.270 E-04 \\
P U-242 & 4.540 E-06 \\
2.070 E-03 & \\
.000 E+00 & .000 E+00 \\
.000 E+00 & 4.389 E-03 \\
A M-241 & 3.960 E-07 \\
P U-238 & 1.230 E-06 \\
P U-239 & 4.110 E-03 \\
P U-240 & 2.520 E-04 \\
P U-241 & 2.430 E-05 \\
P U-242 & 8.710 E-07 \\
1.920 E-06 & 6.213 E-03
\end{array}
$$




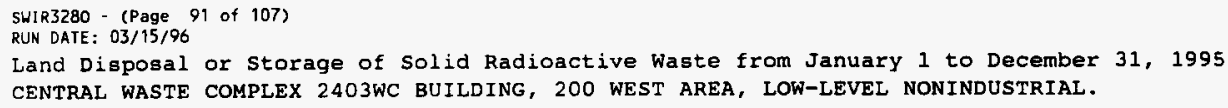

$701 \mathrm{~A} \quad 1.137 E+01 \quad 1.137 E+01 \quad .000 E+00 \quad .000 E+00 \quad 9.569 E-05 \quad 8.945 E-06 \quad 2.472 E-09 \quad 3.980 E-05$

IC.99 $\quad \begin{array}{r}\mathrm{U}-238 \\ 1.920 E-06\end{array}$

U-235 5.900E-03

U-236 2.600E-04

( )

\begin{tabular}{|c|c|c|c|c|c|c|c|c|}
\hline & & & & & & & & $c 0.60$ \\
\hline T PLANT & $1.457 E+00$ & $1.457 E+00$ & $.000 E+00$ & $.000 E+00$ & $1.758 \mathrm{E}-05$ & $8.047 E-06$ & $1.048 \mathrm{E}-10$ & $4.941 E-07$ \\
\hline
\end{tabular}

$1.920 \mathrm{E}-06$

AM-241 $1.257 \mathrm{E}-12$

PU-238 3.893E- 12

PU-239 1.295E-08

PU-240 7.983E-10

PU-241 7.697E-11

PU-242 $2.758 E-12$

T PLANT $1.457 E+00 \quad 1.457 E+00 \quad .000 E+00 \quad .000 E+00 \quad 1.758 E-05 \quad 8.047 E-06 \quad 1.048 E-10 \quad 4.941 E-07$

1.450E-07

4.997E-07 2.748E-04

AM-241 7.564E-09

NP- $237 \quad 2.745 E-04$

$[-14 \quad 4.369 \mathrm{E}-11$

CO-60 2.156E-08

EU-152 1.142E-07

EU-154 1.956E-07

EU- $155 \quad 7.818 E-08$

NA $-22 \quad 6.938 E-08$

TC-99 1.183E-09

$.000 E+00$

$1.302 E+00$

4.129E-01 1.264E-05 2.400E-01

2R-95 1.958E-08

$1.637 E+00$ 


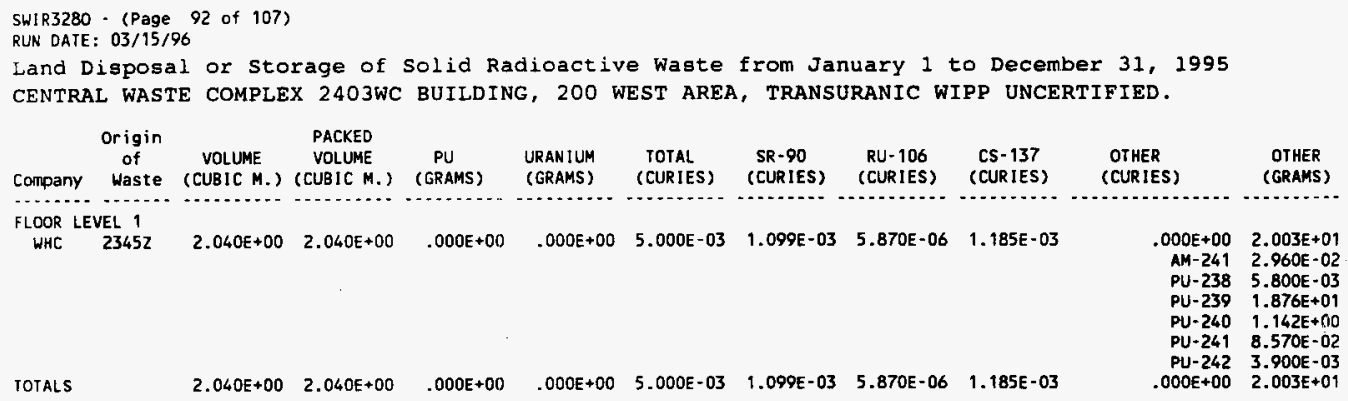


SWIR3280 - (Page 93 of 107 )

RUN DATE: $03 / 15 / 96$

Land Disposal or storage of solid Radioactive Waste from January 1 to December 31 , 1995

CENTRAL WASTE COMPLEX 2403WD BUILDING, 200 WEST AREA, LOW-LEVEL NONINDUSTRIAL.

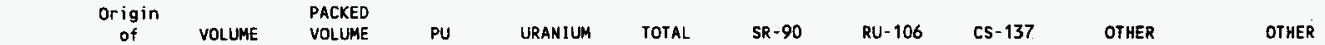
Company Waste (CUBIC M.) (CUBIC M.) (GRAMS) (GRAMS) (CURIES) (CURIES) (CURIES) (CURIES) (CURIES) FLOOR LEVEL 1

BHI $\quad 1304 \mathrm{~N}$

$\begin{array}{llllllll}9.480 E+00 & 9.480 E+00 \quad .000 E+00 & .000 E+00 & 6.918 E+00 & 1.976 E-01 & 1.054 E-06 & 5.872 E-01\end{array}$

1834

$2.082 E+00 \quad 2.082 E+00 \quad .000 E+00$

$.000 E+00 \quad 2.494 E-03 \quad 3.735 E-09 \quad 1.996 E-11 \quad 1.361 E-04$

$\begin{array}{llllllllllll}\text { WHC } \quad 23452 & 3.930 E+01 & 3.930 E+01 & .000 E+00 & .000 E+00 & 1.000 E-01 & 2.197 E-02 & 1.174 E-04 & 2.369 E-02 & \text { TC-99 }\end{array}$

$241 C \quad 1.162 E+01 \quad 1.162 E+01 \quad .000 E+00 \quad .000 E+00 \quad 7.583 E+00 \quad 3.110 E+00 \quad 1.118 E-06 \quad 6.892 E-01$

$2415 \quad 6.600 E+01 \quad 6.600 E+01 \quad .000 E+00 \quad .000 E+00 \quad 1.350 E+00 \quad 6.332 E-01 \quad 1.180 E-06 \quad 4.254 E-02$

377

$8.328 E-01 \quad 8.328 E-01 \quad .000 E+00$

$.000 E+00 \quad 5.990 E \cdot 04$

$.000 E+0$

$.000 E+00 \quad-.000 E+00$

EU- 154
$1.293 E+02 \quad 1.293 E+02 \quad .000 E+00$
$.000 E+00 \quad 1.595 E+01 \quad 3.963 E+00 \quad 1.208 E-04 \quad 1.343 E+00$

1.293

$10-60$

$-60$

$5.380 E+00 \quad 4.782 E+04$

AM-24i 1.627E-02

MP -237 1.018E+01

PU-239 3.091E+00

PU-240 7.110E-02

TH-232 2.231E+04

$U-235 \quad 5.550 E+02$

U-238 $\quad 2.494 E+04$

$5.380 \mathrm{E}+00$

2.229E-03

TH-234

U-232

v-233

U-236

$\mathrm{U}-235$

$\mathrm{u}-236$

U-238

$2.229 \mathrm{E}-03$

$.000 E+00$

AM- 241

PU-238

PU-239

PU-240

PU-241

PU-242

2.125E-02

AM-241

PU-238

Pu-239

PU-240

PU-241

PU- 242

CO-60 1.380E-04

EU-154 8.800E-04

K-40 3.600 E- 05

IC-99 2.020E-02

6. $790 \mathrm{E}-04$

PU-239

PU- 240

$\mathrm{PU}-241$

$6.790 \mathrm{E}-04$

$5.990 E-04$

U-234

$\mathrm{u}-235$

$\mathrm{u}-236$

U-238

TC- $99 \quad 5.990 \mathrm{E}-04$

$5.405 E+00 \quad 4.803 E+04$

$5.955 E+01$

$2.960 E-08$

$4.990 \mathrm{E}-03$

$1.863 \mathrm{E}-04$

$9.414 E-02$

$2.977 \mathrm{E}+00$

$1.927 \mathrm{E}+00$

$5.455 \mathrm{E}+01$

$1.924 E+00$

2.929E-03

$5.460 E-04$

$1.782 \mathrm{E}+00$

$8.000 E-03$

$3.720 \mathrm{E}-04$

2.93TE-02

8.230E-06

$2.750 E-02$

$1.690 \mathrm{E}-03$

$1.630 E-04$

$5.830 E-06$

1.366E-02

$1.280 E-02$

$7.840 E-04$

7.430E- 05

$1.496 \mathrm{E}+02$

$1.666 \mathrm{E}-02$

$1.866 \mathrm{E}+00$

$1.916 \mathrm{E}-01$

$1.475 E+02$

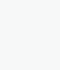

.

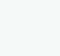

\begin{abstract}
.
\end{abstract}

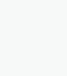

013.


WHC-EP-0125-8

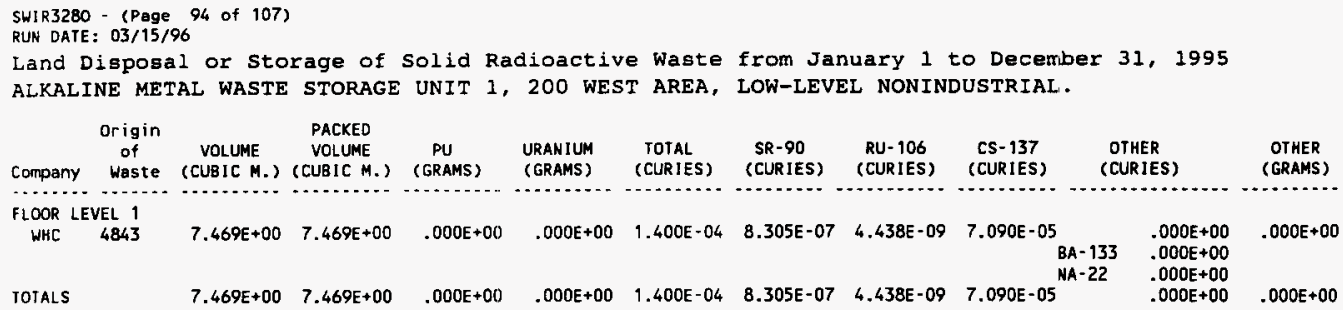


SWIR3280 - (Page 95 of 107 )

RUN DATE: 03/15/96

Land Disposal or Storage of Solid Radioactive Waste from January 1 to December 31 , 1995 ALKALINE METAL WASTE STORAGE UNIT 3, 200 WEST AREA, LOW-LEVEL NONINDUSTRIAL.

\begin{tabular}{|c|c|c|c|c|c|c|c|c|c|c|c|}
\hline $\begin{array}{c}\text { Origin } \\
\text { of } \\
\text { ompany waste }\end{array}$ & $\begin{array}{c}\text { VOLUME } \\
\text { (CUBIE M.) }\end{array}$ & $\begin{array}{l}\text { PACKED } \\
\text { VOLUME } \\
\text { (CUBIC M.) }\end{array}$ & $\begin{array}{c}\text { PU } \\
\text { (GRAMS) }\end{array}$ & $\begin{array}{l}\text { URANIUM } \\
\text { (GRAMS) }\end{array}$ & $\begin{array}{l}\text { TOTAL } \\
\text { (CURIES) }\end{array}$ & $\begin{array}{l}\text { SR-90 } \\
\text { (CURIES) }\end{array}$ & $\begin{array}{c}\text { RU- } 106 \\
\text { (CURIES) }\end{array}$ & $\begin{array}{l}\text { CS-137 } \\
\text { (CUR IES) }\end{array}$ & \multicolumn{2}{|c|}{$\begin{array}{l}\text { OTHER } \\
\text { (CURIES) }\end{array}$} & $\begin{array}{l}\text { OTHER } \\
\text { (GRAMS) }\end{array}$ \\
\hline LOOR LEVEL 1 & & & & & & & & & & & \\
\hline WHC & $6.371 E+00$ & $6.371 E+00$ & $.000 E+00$ & $.000 E+00$ & $2.310 \mathrm{E}-03$ & $4.152 E-06$ & 2.219E-08 & 3.545E-04 & & $\begin{array}{r}1.610 \mathrm{E}-03 \\
1.610 \mathrm{E}-03 \\
.000 \mathrm{E}+00\end{array}$ & $.000 E+00$ \\
\hline OTALS & $6.371 E+00$ & $6.371 E+00$ & $.000 E+00$ & $.000 E+00$ & $2.310 E-03$ & $4.152 E-06$ & $2.219 E-08$ & $3.545 E-04$ & & $1.610 \mathrm{E}-03$ & $000 E+00$ \\
\hline
\end{tabular}


WHC-EP-0125-8

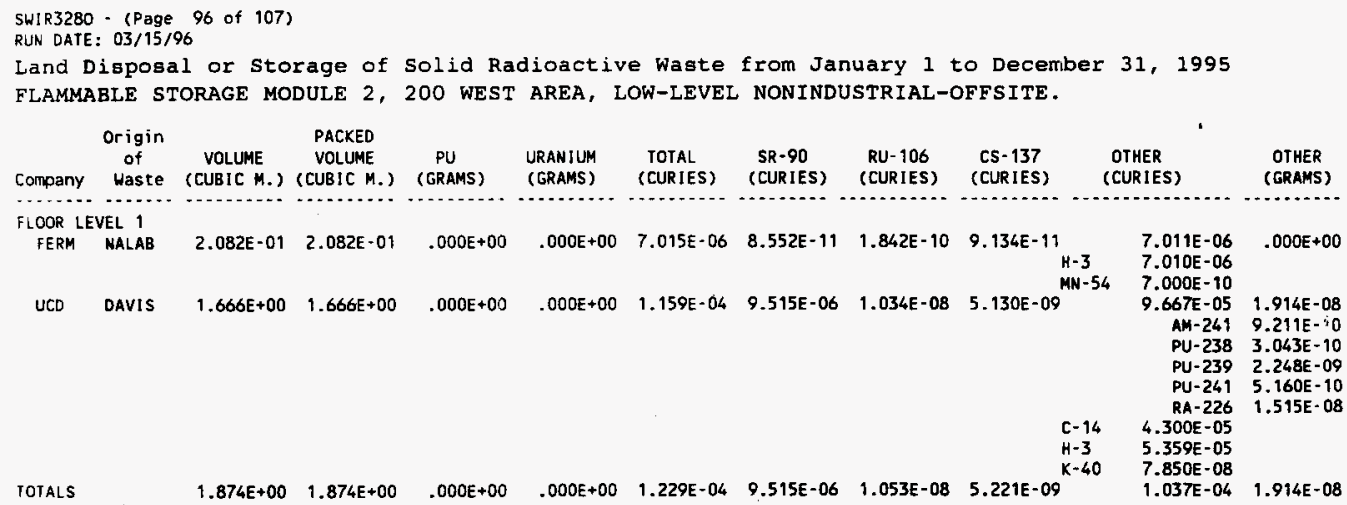


WHC-EP-0125-8

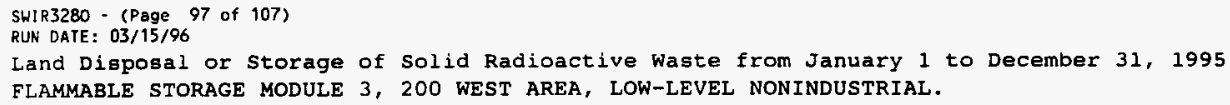

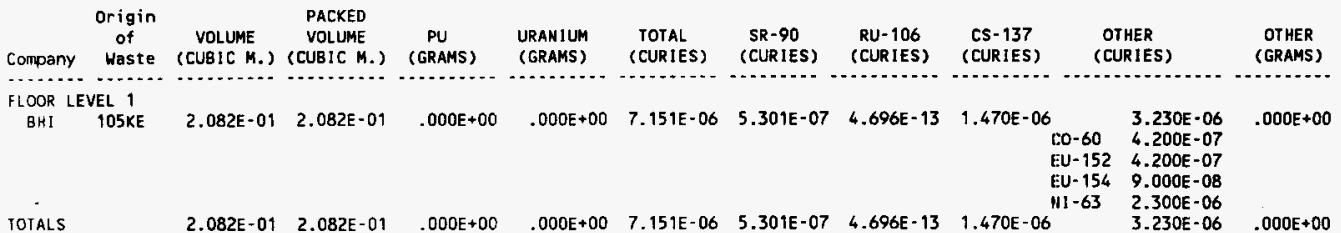




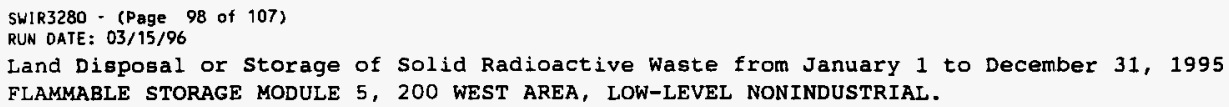


WHC-EP-0125-8

SWIR3280 - (Page 99 of 107)

RUN DATE: 03/15/96

Land Disposal or storage of Solid Radioactive Waste from January 1 to December 31 , 1995

FLAMMABLE STORAGE MODULE 6, 200 WEST AREA, LOW-LEVEL NONINDUSTRIAL-OFFSITE.

\begin{tabular}{|c|c|c|c|c|c|c|c|c|c|c|c|}
\hline Company & $\begin{array}{c}\text { Origin } \\
\text { of } \\
\text { Waste }\end{array}$ & $\begin{array}{c}\text { VOLUME } \\
\text { (CUBIC M.) }\end{array}$ & $\begin{array}{c}\text { PACKED } \\
\text { VOLUME } \\
\text { (CUBIC M.) }\end{array}$ & $\begin{array}{c}\text { PU } \\
\text { (GRAMS) }\end{array}$ & $\begin{array}{l}\text { URANIUM } \\
\text { (GRAMS) }\end{array}$ & $\begin{array}{l}\text { TOTAL } \\
\text { (CURIES) }\end{array}$ & $\begin{array}{l}\text { SR-90 } \\
\text { (CURIES) }\end{array}$ & $\begin{array}{l}\text { RU- } 106 \\
\text { (CURIES) }\end{array}$ & $\begin{array}{l}\text { CS }-137 \\
\text { (CURIES) }\end{array}$ & $\begin{array}{l}\text { OTHER } \\
\text { (CURIES) }\end{array}$ & $\begin{array}{l}\text { OTHER } \\
\text { (GRAMS) }\end{array}$ \\
\hline OOR LE & VEL 1 & & & & & & & & & & \\
\hline ANNA & ANMARBO & $2.082 E-01$ & $2.082 \mathrm{E}-01$ & $.000 E+00$ & $.000 E+00$ & $1.800 E-03$ & $.000 E+00$ & $.000 E+00$ & $.000 E+00_{H-3}$ & $\begin{array}{l}1.800 E-03 \\
1.800 E-03\end{array}$ & $.000 E+00$ \\
\hline GAS & GASD & $1.666 E+00$ & $1.666 E+00$ & $.000 E+00$ & $.000 E+00$ & 4.000E-02 & $7.955 E-04$ & $1.713 E-03$ & $8.496 E-04$ & $\begin{array}{c}.000 E+00 \\
T H-232 \\
U-234 \\
U-235 \\
U-238 \\
1.800 E-03\end{array}$ & $\begin{array}{l}1.703 E+02 \\
1.690 E+02 \\
1.000 E-02 \\
1.220 E+00 \\
8.000 E-02 \\
1.703 E+02\end{array}$ \\
\hline
\end{tabular}


SWIR3280 - (Page 100 of 107)

RUN DATE: $03 / 15 / 96$

Land Disposal or storage of Solid Radioactive Waste from January 1 to December 31 , 1995

FLAMMABLE STORAGE MODULE 6, 200 WEST AREA, LOW-LEVEL NONINDUSTRIAL.

\begin{tabular}{|c|c|c|c|c|c|c|c|c|c|c|c|}
\hline$y$ & $\begin{array}{l}\text { Origin } \\
\text { of } \\
\text { Waste }\end{array}$ & $\begin{array}{c}\text { VOLUME } \\
\text { (CUBIC M.) }\end{array}$ & $\begin{array}{c}\text { PACKED } \\
\text { VOLUME } \\
\text { (CUBIC M.) }\end{array}$ & $\begin{array}{c}\text { PU } \\
\text { (GRAMS) }\end{array}$ & $\begin{array}{l}\text { URANIUM } \\
\text { (GRAMS) }\end{array}$ & $\begin{array}{l}\text { TOTAL } \\
\text { (CURIES) }\end{array}$ & $\begin{array}{l}\text { SR-90 } \\
\text { (CURIES) }\end{array}$ & $\begin{array}{l}\text { RU-106 } \\
\text { (CURIES) }\end{array}$ & $\begin{array}{c}\text { CS-137 } \\
\text { (CURIES) }\end{array}$ & $\begin{array}{l}\text { OTHER } \\
\text { (CURIES) }\end{array}$ & $\begin{array}{c}\text { OTHER } \\
\text { (GRAMS) }\end{array}$ \\
\hline
\end{tabular}

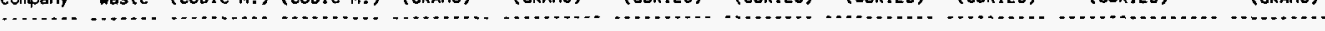

FLOOR LEVEL 1

$\begin{array}{llllllll}\text { WHC } \quad \text { 105KE } \quad 2.082 E-01 & 2.082 E-01 & .000 E+00 & .000 E+00 & 8.632 E-08 & 1.318 E-12 & 7.044 E-15 & 4.400 E-08\end{array}$

$202 \mathrm{~A} \quad 2.082 \mathrm{E}-01 \quad 2.082 \mathrm{E}-01 \quad .000 \mathrm{E}+00 \quad .000 \mathrm{E}+00 \quad 1.358 \mathrm{E}-05^{2} \quad 2.721 \mathrm{E}-06^{4} \quad 4.696 \mathrm{E}-12 \quad 3.161 \mathrm{E}-06^{100-60}$

$6.900 E-10 \quad 3.326 E-13$

$A M-241 \quad 3.326 E-13$

$2.0826-012.082-01000$

(2)

$5-14$

$6.900 \mathrm{E}-10$

$1.987 E-06 \quad 6.140 E-09$

PU-241 6.140E-09

202AL

$1.041 E+00 \quad 1.041 E+00 \quad .000 E+00$

$.000 E+00 \quad 7.326 E-05 \quad 1.236 E-05 \quad 2.348 E-12 \quad 1.433 E-05$

$H=3 \quad 1.700 E-08$

PM-147 1.970E-06

$1.970 E-06$

$5.700 E-03$

CM-242 1.585E-15

PU-241 3.778E-08

U-238 $5.700 E-03$

C-14 2.274E-14

$\mathrm{H}-3 \quad 7.700 \mathrm{E}-08$

PM- $167 \quad 2.056 \mathrm{E}-05$

CS $137 \quad 1.442 \mathrm{E}-10$

CO-58 $\quad 1.400 E-10$

CO-60 9.380E-09

EU155 1.190E-09

C-14 $\quad 1.400 E-10$

BA137 1.358E-10

EU154 1.190E-09

FE-59 5.600E-10

MN-54 $9.800 E-10$

$.000 E+00 \quad 2.750 E-06$

$.000 E+00 \quad .000 E+00 \quad .000 E+00$

EU152 
WHC-EP-0125-8

SWIR3280 - (Page 101 of 107)

RUN DATE: 03/15/96

Land Disposal or Storage of Solid Radioactive Waste from January 1 to December 31 , 1995

FLAMMABLE STORAGE MODULE 8, 200 WEST AREA, LOW-LEVEL NONINDUSTRIAL.

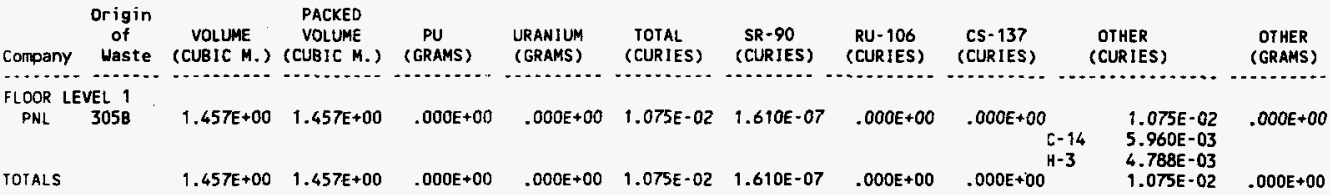


SWIR3280 - (Page 102 of 107 )

RUN DATE: 03/15/96

Land Disposal or Storage of Solid Radioactive Waste from January 1 to Deceraber 31 , 1995

FLAMMABLE STORAGE MODULE 10, 200 WEST AREA, LOW-LEVEL NONINDUSTRIAL-OFFSITE.

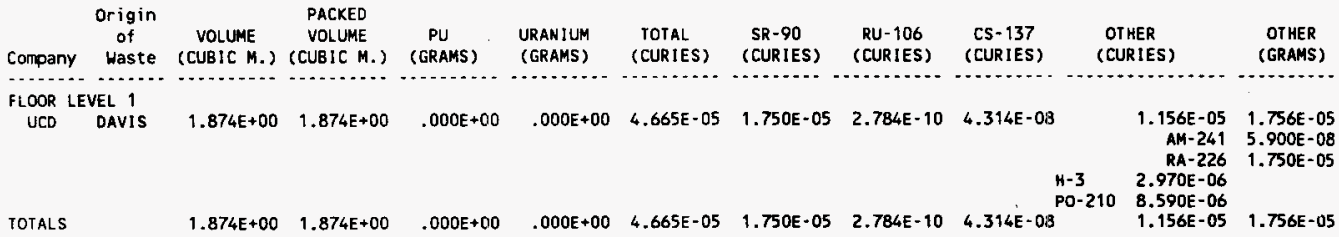


SWIR3280 - (Page 103 of 107)

RUN DATE: 03/15/96

Land Disposal or Storage of Solid Radioactive Waste from January 1 to December 31 , 1995 FLAMMABLE STORAGE MODULE 10, 200 WEST AREA, LOW-LEVEL NONINDUSTRIAL.

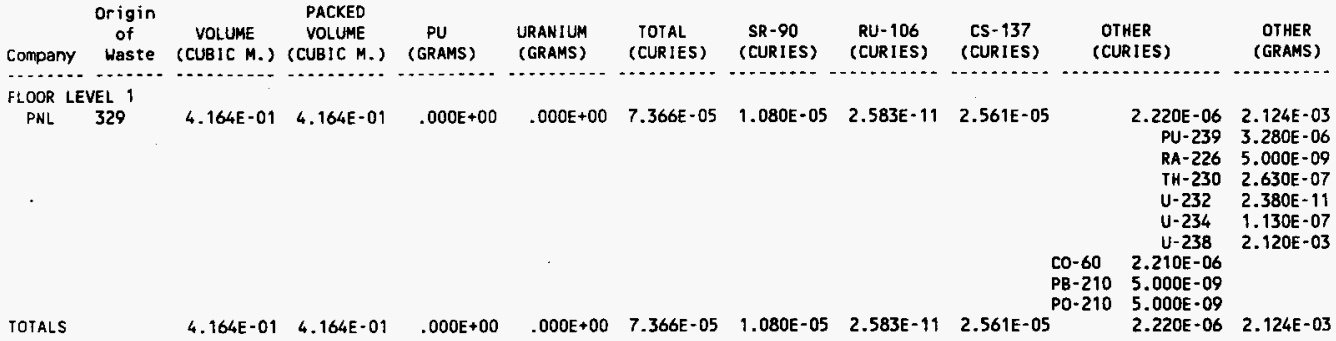


WHC-EP-0125-8

SWIR3280 - (Page 104 of 107 )

RUN DATE : 03/15/96

Land Disposal or Storage of Solid Radioactive Waste from January 1 to December 31 , 1995

FLAMMABLE STORAGE MODULE 15, 200 WEST AREA, LOW-LEVEL NONINDUSTRIAL-OFFSITIE.

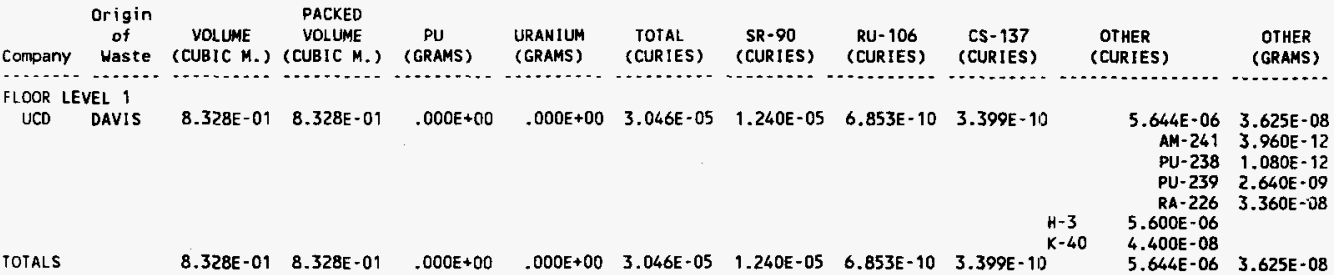


SWIR3280 - (Page 105 of 107) RUN DATE : $03 / 15 / 96$

Land Disposal or Storage of Solid Radioactive Waste from January 1 to December 31 , 1995 FLAMMABLE STORAGE MODULE 17, 200 WEST AREA, LOW-LEVEL NONINDUSTRIAL-OFFSITE.

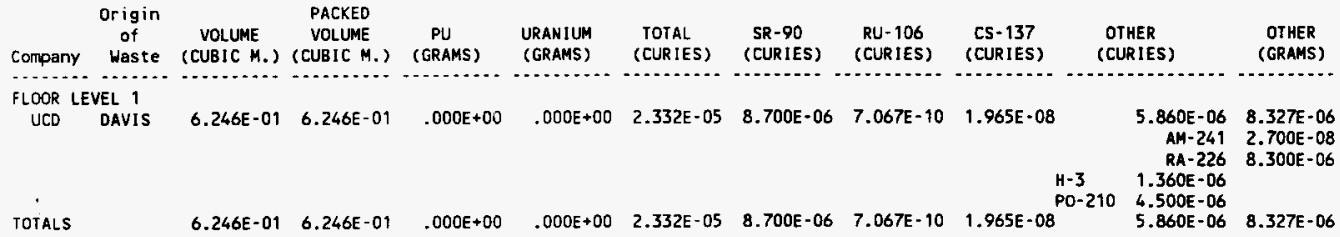




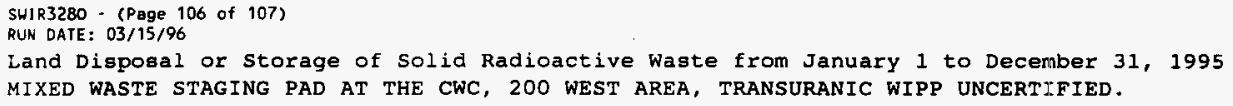

SH-126 1.450E-O6 
WHC-EP-0125-8

SWIR3280 - (Page 107 of 107)

RUN DATE: 03/15/96

SWIR3280 - (Page 107 of 107)

RUN DATE : 03/15/96

Grand Totals for Land Disposal or storage of Solid Radioactive Waste

from January 1 to December 31, 1995

\begin{tabular}{|c|c|c|c|c|c|c|c|c|c|c|c|}
\hline & $\begin{array}{c}\text { Origin } \\
\text { of } \\
\text { Waste }\end{array}$ & $\begin{array}{c}\text { VOLUME } \\
\text { (CUBIC M.) }\end{array}$ & $\begin{array}{c}\text { PACKED } \\
\text { VOLUME } \\
\text { (CUBIC M.) }\end{array}$ & $\begin{array}{c}\text { PL } \\
\text { (GRAMS) }\end{array}$ & $\begin{array}{l}\text { URANIUM } \\
\text { (GRAMS) }\end{array}$ & $\begin{array}{l}\text { TOTAL } \\
\text { (CURIES) }\end{array}$ & $\begin{array}{l}\text { SR-90 } \\
\text { (CURIES) }\end{array}$ & $\begin{array}{l}\text { RU-106 } \\
\text { (CURIES) }\end{array}$ & $\begin{array}{l}\text { CS-137 } \\
\text { (CURIES) }\end{array}$ & $\begin{array}{l}\text { OT HER } \\
\text { (CURIES) }\end{array}$ & $\begin{array}{l}\text { OTHER } \\
\text { (GRAMS) }\end{array}$ \\
\hline QAd & ALS & $1.507 E+04$ & $1.507 E+04$ & $.000 E+00$ & 2.000E-03 & $3.689 E+0 S$ & $1.140 \mathrm{E}+04$ & $1.192 E+01$ & $7.399 E+03$ & $3.312 E+05$ & $1.523 E+08$ \\
\hline
\end{tabular}




\subsection{REFERENCES}

WHC, 1991, Summary of Radioactive Solid Waste Received in the 200 Areas During Calendar Year 1990, WHC-EP-0125-3, Westinghouse Hanford Company, Richland, Washington.

WHC, 1992, Summary of Radioactive Solid Waste Received in the 200 Areas During Calendar Year 1991, WHC-EP-0125-4, Westinghouse Hanford Company, Richland, Washington.

Wiley-Interscience, 1978, Table of Isotopes, Seventh Edition, John Wiley and Sons, Wiley-Interscience, New York, New York.

\subsection{BIBLIOGRAPHY}

ARH, 1973, Summary of Radioactive Solid Waste Burials in the 200 Areas During 1972, ARH-2757-Part 2, Atlantic Richfield Hanford Company, Richland, Washington.

ARH, 1975, Summary of Radioactive Solid Waste Burials in the 200 Areas During 1975, ARH-CD-369-4Q, Atlantic Richfield Hanford Company, Richland, Washington.

RHO, 1978, Summary of Radioactive Solid Waste Burials in the 200 Areas During 1977, RHO-CD-27-3Q, Rockwe11 Hanford Operations, Richland, Washington.

RHO, 1979, Summary of Radioactive Solid Waste Burials in the 200 Areas During 1978, RH0-LD-78-24-4Q, Rockwell Hanford Operations, Richland, Washington.

RHO, 1981, Summary of Radioactive Solid Waste Burials in the 200 Areas During 1980, RHO-LD-80-24-4Q, Rockwell Hanford Operations, Richland, Washington.

RH0, 1981, Summary of Radioactive Solid Waste Burials in the 200 Areas During 1981, RH0-LD-81-24-4Q, Rockwell Hanford Operations, Richland, Washington.

RHO, 1986, Summary of Radioactive Solid Waste Burials in the 200 Areas During 1986, RH0-SR-86-9-4Q, Rockwel1 Hanford Operations, Richland, Washington.

WHC, 1988, Hanford Site Solid Waste Acceptance Criteria, WHC-EP-0063, Westinghouse Hanford Company, Richland, Washington.

WHC, 1989, Summary of Radioactive Solid Waste Burials in the 200 Areas During CY 1987, WHC-EP-0125, Westinghouse Hanford Company, Richland, Washington.

WHC, 1989, Summary of Radioactive Solid Waste Received in the 200 Areas During Calendar Year 1988, WHC-EP-0125-1, Westinghouse Hanford Company, Richland, Washington.

WHC, 1990, Summary of Radioactive Solid Waste Received in the 200 Areas During Calendar Year 1989, WHC-EP-0125-2, Westinghouse Hanford Company, Richland, Washington. 
WHC, 1993, Summary of Radioactive Solid Waste Received in the 200 Areas During Calendar Year 1992, WHC-EP-0125-5, Westinghouse Hanford Company, Richland, Washington.

WHC, 1994, Summary of Radioactive Solid Waste Received in the 200 Areas During Calendar Year 1993, WHC-EP-0125-6, Westinghouse Hanford Company, Richl and, Washington.

WHC, 1995, Summary of Radioactive Solid Waste Received in the 200 Areas During Calendar Year 1994, WHC-EP-0125-7, Westinghouse Hanford Company, Richland, Washington. 
WHC-EP-0125-8

This page intentionally left blank. 
WHC-EP-0125-8

\section{APPENDIX A}

\section{AREA SOLID WASTE STORAGE/DISPOSAL FACILITY COORDINATES}


WHC-EP-0125-8

This page intentionally left blank. 
WHC-EP-0125-8

APPENDIX A

200 AREA SOLID WASTE STORAGE/DISPOSAL FACILITY COORDINATES

\begin{tabular}{|c|c|c|}
\hline Burial Grounds & Coordinates & $\begin{array}{l}\text { Reference } \\
\text { Drawings }\end{array}$ \\
\hline \multicolumn{3}{|l|}{200 East Area } \\
\hline Burial Ground 218E1 & $\begin{array}{l}\text { N39821/W49847 NORTHWEST } \\
\text { N39817/449494 NORTHEAST } \\
\text { N39532/49850 SOUTHWEST } \\
\text { N39526/W49494 SOUTHEAST }\end{array}$ & $\begin{array}{l}\text { H-2-124 } \\
\text { REV. } 3\end{array}$ \\
\hline Burial Ground $218 \mathrm{E} 2$ & $\begin{array}{l}\text { N44826/W53909 NORTHWEST } \\
\text { N44827/W53425 NORTHEAST } \\
\text { N44407/W3426 SOUTHEAST } \\
\text { N44408/W53911 SOUTHWEST }\end{array}$ & $\begin{array}{l}\text { H-2-55534 } \\
\text { REV. } 16\end{array}$ \\
\hline $\begin{array}{l}\text { Burial Ground 218E4 } \\
\text { (MINOR CONSTR \#4) }\end{array}$ & $\begin{array}{l}\text { N44313/W54110 NORTHWEST } \\
\text { N43783//53503 NORTHEAST } \\
\text { N43685//53677 SOUTHEAST } \\
\text { N44241/W54142 SOUTHWEST }\end{array}$ & $\begin{array}{l}H-2-55534 \\
\text { REV. } 16\end{array}$ \\
\hline Burial Ground 218E5 & $\begin{array}{l}\text { N44826/W54209 NORTHWEST } \\
\text { N44826/W53909 NORTHEAST } \\
\text { N44408/W53911 SOUTHEAST } \\
\text { N44407/W54165 SOUTHWEST }\end{array}$ & $\begin{array}{l}\text { H-2-55534 } \\
\text { REV. } 16\end{array}$ \\
\hline Burial Ground 218E5A & $\begin{array}{ll}\text { N44827/W54309 } & \text { NORTHWEST } \\
\text { N44826/W54209 } & \text { NORTHEAST } \\
\text { N44495/W54164 } & \text { SOUTHEAST } \\
\text { N44494/W54309 } & \text { SOUTHWEST }\end{array}$ & $\begin{array}{l}H-2-55534 \\
\text { REV. } 16\end{array}$ \\
\hline $\begin{array}{l}\text { Burial Ground 218E8 } \\
\text { (CONSTRUCTION \#8) }\end{array}$ & $\begin{array}{l}\text { N45285/W48527 NORTHWEST } \\
\text { N45281/N48409 } \\
\text { NORTHEAST } \\
\text { N44882/W48418 } \\
\text { SOUTHEAST } \\
\text { N4485/W48534 SOUTHWEST }\end{array}$ & $\begin{array}{l}\text { H-2-33276 } \\
\text { SH } 1 \text { REV. } 15\end{array}$ \\
\hline Burial Ground 218EC9 & $\begin{array}{l}\text { N42717/W50106 NORTHWEST } \\
\text { N42716/449855 NORTHEAST } \\
\text { N42471/449857 SOUTHEAST } \\
\text { N42473/W50108 SOUTHWEST }\end{array}$ & \\
\hline Burial Ground 218E10 & $\begin{array}{l}\text { N45880/W56725 NORTHWEST } \\
\text { N45880/W54700 NORTHEAST } \\
\text { N44398/W54660 SOUTHEAST } \\
\text { N44473/W55475 SOUTH } \\
\text { N45180/W56725 SOUTHWEST. }\end{array}$ & $\begin{array}{l}H-2-92004 \\
\text { SH } 1 \text { REV. } 6 \\
\text { SH } 2 \text { REV. } 4\end{array}$ \\
\hline Burial Ground 218E12A & $\begin{array}{l}\text { N44136/W48500 NORTHEAST } \\
\text { N43211/W48531 SOUTHEAST } \\
\text { N43201/W49519 SOUTHWEST } \\
\text { N43272/W49580 SOUTHWEST } \\
\text { N44246/W49582 NORTHWEST }\end{array}$ & $\begin{array}{l}\mathrm{H}-2-32560 \\
\text { REV. } 2\end{array}$ \\
\hline & $A-3$ & \\
\hline
\end{tabular}


WHC-EP-0125-8

Burial Grounds

200 East Area

Burial Ground 218E12B

$218 E 14$

(PUREX TUNNEL \#1)

$218 \mathrm{E} 15$

(PUREX TUNNEL \#2)

222B VAULTS

CENTER POINT

(DRY WASTE E7)

\section{Hest Area}

Burial Ground 218Wl

Buriat Ground 218W1A

Burial Ground 218W2

$$
\begin{aligned}
& \text { N46775/W51475 NORTHWEST } \\
& \text { N46775/W47445 NORTHEAST } \\
& \text { N46675/W47345 NORTHEAST } \\
& \text { N45523/W47345 SOUTHEAST } \\
& \text { N45485/W48523 SOUTHEAST } \\
& \text { N44485/W48542 SOUTHEAST } \\
& \text { N44504/W49661 SOUTHWEST } \\
& \text { N45220/W51475 SOUTHWEST }
\end{aligned}
$$

Reference Drawings

N39196/W48020 NORTH

N39555/W48020 SOUTH

N37670/W47960 NORTH

N39366/W47960 SOUTH

N42269/W53826 VAULT 1

N42269/W53806 VAULT 2

N43364/W53776 VAULT 3

H-2-33276

SH 1 REV. 15

SH 2 REV. 7

SH 3 REV. 6

H-2-94665

REV. 0

H-2-94665

REV. 0

H-2-94662

REV. 0

H-2-75149

REV , 1

N42099/W77458 NORTHEAST

N41641/W77458 SOUTHEAST

N41641/W77979 SOUTHWEST

N45464/W74930 NORTH

N45114/W74431 NORTHEAST N45072/W74460 NORTHEAST N45053/W74668 NORTHEAST N44597/W74844 SOUTHEAST N44624/W75113 SOUTHWEST N44781/W75175 SOUTHWEST N45102/W75209 NORTHWEST

N41641/W77979 NORTHWEST N41641/W77458 NORTHEAST N41052/W77458 SOUTHEAST N41052/W77979 SOUTHWEST
$\mathrm{H}-2-2516$

REV. 3

$\mathrm{H}-2-2.503$

REV. 3 
WHC-EP-0125-8

Burial Grounds

Coordinates

Reference

Drawings

200 West Area (cont)

Burial Ground 218W2A

N44940/W77151 NORTHWEST

N44563/W76079 NORTHEAST

N43230/W76944 SOUTHEAST

N43227/W77600 SOUTHWEST

N43947/W77659 NORTHWEST

N44340/W77515 NORTHWEST

N44580/W77515 NORTHWEST

N44580/W77150 NORTHWEST

Burial Ground 218W3

N43945/W78090 NORTHWEST N43945/W77600 NORTHEAST N43227/W77600 SOUTHEAST N43227/W78038 SOUTHWEST N43312/W78090 SOUTHWEST

Burial Ground 218W3A

N46505/W78090 NORTHWEST N46505/W77120 NORTHEAST N44995/W77120 NORTHEAST N44580/W77150 NORTHEAST N44580/W77515 SOUTHEAST N44340/W77515 SOUTHEAST N43947/W77658 SOUTHEAST N43945/W78090 SOUTHWEST

Burial Ground 218W3AE N46505/W77070 NORTHWEST N46505/W75872 NORTHEAST N45650/W75605 EAST N44680/W76050 SOUTHEAST N45215/W77070 SOUTHWEST

H-2-32095

SH 1 REV. 11

SH 2 REV. 1

H-2-32095

SH 1 REV. 11

SH 2 REV. 1

$\mathrm{H}-2-34880$

SH 1 REV. 16

SH 2 REV. 8

$\mathrm{H}-2-75351$

SH 1 REV. 8

SH 2 REV. 5

N43158/W78079 NORTHWEST

N43158/W77012 NORTHEAST

N42278/W77431 SOUTHEAST

N42278/W78079 SOUTHWEST

$\mathrm{H}-2-32487$

REV. 9

N41052/W78079 NORTHWEST

N41052/W77458 NORTHEAST

N40450/W77458 SOUTHEAST

$\mathrm{H}-2-33055$

Burial Ground $218 W 4 B$

N40450/W78079 SOUTHWEST

REV. 14 
WHC-EP-0125-8

$\begin{array}{lll}\text { Burial Grounds } & \text { Coordinates } & \text { Reference } \\ \text { Drawings }\end{array}$


WHC-EP-0125-8

Burial Grounds

Coordinates

Reference

Drawings

\section{West Area Central Waste Complex Facilities}

213W COMPACTOR

Building 2401W

Building 2402W

Building 2402WB

Building 2402WC

Building 2402WD

Building 2402WE

Building 2402WF

Building 2402WG

Building 2402WH

Building 2402WI

Building 2402WJ

Building 2402WK

FLAMMABLE STORAGE

MODULES

MIXED WASTE

STORAGE PAD

STAGING AREA
N40608/W78722

N40716/W78515

N41310/W78530

N41310/W78690

N41410/W78530

N41410/W78690

N41510/W78530

N41510/W78690

N41610/W78530

N41610/W78690

N41710/W78530

N41710/W78690

N41810/W78530

N40900/W78700

N41560/W78250

N41310/W78400

N41510/W78250
H-2-93691

REV. I

H-2-80543

REV. 3

H-2-80605

REV. 2

H-2-80737

REV. 3

H-2-80737

REV. 3

H-2-80737

REV. 3

H-2-80737

REV. 3

H-2-80737

REV. 3

H-2-80737

REV. 3

H-2-80737

REV. 3

H-2-80737

REV. 3

H-2-810737

REV. 3

H-2-810737

REV. 3

H-2-81296

REV. 1

H-2-80580

REV. 4

H-2-90264

REV. 0 


\section{A1.0 REFERENCES}

WHC, 1944, Concrete Foundation Plan and Details, Drawing W-71323, Rev. 5, Westinghouse Hanford Company, Richland, Washington.

WHC, 1944, Concrete Plan Above Grade and Details, Drawing W-71324, Rev. 9, Westinghouse Hanford Company, Richland, Washington.

WHC, 1944, Concrete Sections and Details Sheet No. 1, Drawing W-71325, Rev. 9, Westinghouse Hanford Company, Richland, Washington.

WHC, 1962, Building Modifications for Solid Contaminated Waste St, Drawing H-2-32307, Rev. 0, Westinghouse Hanford Company, Richland, Washington.

WHC, 1964, North Area Liquid Waste Disposal Sites, Drawing H-2-32524, Rev. 1, West inghouse Hanford Company, Richland, Washington.

WHC, 1982, Civil Asphalt Storage Pad 200W Area, Drawing H-2-90264, Rev. 0, West inghouse Hanford Company, Richland, Washington.

WHC, 1984, 218-E-1 Dry Waste Burial Ground, Drawing H-2-124, Rev. 3, Westinghouse Hanford Company, Richland, Washington.

WHC, 1984, Dry Waste Burial Site 218-W-8 Plot Plan, Drawing H-2-2322, Rev. 3, Westinghouse Hanford Company, Richland, Washington.

WHC, 1984, Industrial Burial Ground 218-W-1A, Drawing H-2-2516, Rev. 3, Westinghouse Hanford Company, Richland, Washington.

WHC, 1984, Dry Waste Burial Ground 218-W-7, Drawing H-2-5170, Rev. 4, Westinghouse Hanford Company, Richland, Washington.

WHC, 1984, As-Built Dry Waste Burial Site \#218-E-12A, Drawing H-2-32560, Rev. 2, Westinghouse Hanford Company, Richland, Washington.

WHC, 1984, 218-E2, E2A, E4, E5, E5A, and E9 Industrial Burial Ground P1, Drawing H-2-55534, Rev. 16, Westinghouse Hanford Company, Richland, Washington.

WHC, 1984, Dry Waste Burial Ground 218-W-1, Drawing H-2-75149, Rev. I, Westinghouse Hanford Company, Richland, Washington.

WHC, 1984, Dry Waste Burial Ground 218-W-9, Drawing H-2-94261, Rev. 0, West inghouse Hanford Company, Richland, Washington.

WHC, 1984, Dry Waste Burial Ground 218-E7, Drawing H-2-94662, Rev. 0, Westinghouse Hanford Company, Richland, Washington.

WHC, 1984, RR Burial Tunnels 218-E-14, 218-E-15, Drawing H-2-94665, Rev. 0, Westinghouse Hanford Company, Richland, Washington.

WHC, 1985, Civil Profile and Details, Drawing H-2-93691, Rev. 1, Westinghouse Hanford Company, Richland, Washington. 
WHC, 1987, DOE/ER Building Layout, Drawing SK-2-62470, Rev. 0, Westinghouse Hanford Company, Richland, Washington.

WHC, 1991, Arch-Strl Plan, El, Sections, and Details, Drawing H-2-80605, Rev. 2, Westinghouse Hanford Company, Richland, Washington.

WHC, 1991, Electrical Storage Modules 7 and 8 Plans and Detail, Drawing H-2-81296, Rev. 1, Westinghouse Hanford Company, Richland, Washington.

WHC, 1992, Dry Waste Burial Ground 218-W-4B, Drawing H-2-33055, Rev. 14, Westinghouse Hanford Company, Richland, Washington.

WHC, 1992, Dry Waste Burial Ground 218-E-12B, Drawing H-2-33276, Sh. 3, Rev. 6, Westinghouse Hanford Company, Richland, Washington.

WHC, 1992, Dry Waste Burial Ground 218-W-5, Drawing H-2-94677, Sh. 2, Rev. 4, Westinghouse Hanford Company, Richland, Washington.

WHC, 1993, 218-W-2 Dry Waste Burial Ground, Drawing H-2-2503, Rev. 3, Westinghouse Hanford Company, Richland, Washington.

WHC, 1993, 218-W-2A Industrial Burial Ground and 218-W-3 Dry Waste, Drawing H-2-32095, Sh. 1, Rev. 11, Sh. 2, Rev. 1, Westinghouse Hanford Company, Richland, Washington.

WHC, 1993, 218-W-4A Dry Waste Burial Ground, Drawing H-2-32487, Rev. 9, Westinghouse Hanford Company, Richland, Washington.

WHC, 1993, Architectural Floor and Roof Plans and Details, Drawing H-2-36215, Rev. 7, Westinghouse Hanford Company, Richland, Washington.

WHC, 1993, Dry Waste Burial Ground 218-W-4C, Drawing H-2-37437, Sh. 2, Rev. 4, Sh. 3, Rev. 3, Westinghouse Hanford Company, Richiand, Washington.

WHC, 1993, Dry Waste Burial Ground 218-W-1, Drawing H-2-75149, Rev. 1, West inghouse Hanford Company, Richland, Washington.

WHC, 1993, Dry Waste Burial Ground 218-W-3A-E, Drawing H-2-75351, Sh. 2, Rev. 5, Westinghouse Hanford Company, Richland, Washington.

WHC, 1993, Civil Storage Pad, Plan, Sect, and Details, Drawing H-2-80580, Rev. 4, Westinghouse Hanford Company, Richland, Washington.

WHC, 1993, Industrial Burial Ground 218-E10 Site - Plan and Detail, Drawing H-2-92004, Sh. 2, Rev. 4, Westinghouse Hanford Company, Richland, Washington.

WHC, 1993, Dry Waste Burial Ground 218-W-11, Drawing H-2-94250, Rev. 1, Westinghouse Hanford Company, Richland, Washington.

WHC, 1994, M Dwg List-Civil Site Plan Rmw Grading and Roadways, Drawing H-2-80737, Rev. 3, Westinghouse Hanford Company, Richland, Washington. 
WHC, 1995, Civil/Arch/Strl/HVAC Plans, El, Sect, and Details, Drawing H-2-80543, Rev. 3, Westinghouse Hanford Company, Richland, Washington.

WHC, 1996, Dry Waste Burial Ground 218-E-12B, Drawing H-2-33276, Sh. I, Rev. 15, Sh. 2, Rev. 7, Westinghouse Hanford Company, Richiand, Washington.

WHC, 1996, Dry Waste Burial Ground 218-W-3A, Drawing H-2-34880, Sh. 1, Rev. 16, Sh. 2, Rev. 8, Westinghouse Hanford Company, Richland, Washington.

WHC, 1996, Dry Waste Buria7 Ground 218-W-4C, Drawing H-2-37437, Sh. 1, Rev. 11, Westinghouse Hanford Company, Richland, Washington.

WHC, 1996, Dry Waste Burial Ground 218-W-3A-E, Drawing H-2-75351, Sh. 1, Rev. 8 , Westinghouse Hanford Company, Richland, Washington.

WHC, 1996, Industrial Burial Ground 218-E10 Site - Plan and Detail, Drawing H-2-92004, Sh. 1, Rev. 6, Westinghouse Hanford Company, Richland, Washington.

WHC, 1996, Dry Waste Burial Ground 218-W-5, Drawing H-2-94677, Sh. 1, Rev. 6, Westinghouse Hanford Company, Richland, Washington. 
WHC-EP-0125-8

APPENDIX B

BURIAL GROUND/STORAGE FACILITY LAND USAGE

B-1 
WHC-EP-0125-8

This page intentionally left blank. 
WHC-EP-0125-8

\section{APPENDIX B}

BURIAL GROUND/STORAGE FACILITY LAND USAGE

Solid Waste Management Facilities Acreage Report Disposed and Stored Acres in the 200 Areas from Startup to December 31, 1995, Includes all Waste Received. (2 sheets)

\begin{tabular}{|c|c|c|c|c|}
\hline Burial Ground & $\begin{array}{l}\text { Total } \\
\text { Acres }\end{array}$ & $\begin{array}{c}\text { Total } \\
\text { Acres } \\
\text { Used }\end{array}$ & $\begin{array}{c}\text { Acres } \\
\text { Remaining }\end{array}$ & $\begin{array}{c}\text { Acres } \\
\text { Used CY } \\
1995\end{array}$ \\
\hline $218-E-01$ & 2.38 & 2.38 & 0 & 0 \\
\hline $218-E-02,2 A \& 9$ & 5.87 & 5.87 & 0 & 0 \\
\hline $218-E-4$ & 2.75 & 2.75 & 0 & 0 \\
\hline 218-E-5. & 2.31 & 2.31 & 0 & 0 \\
\hline $218-E-5 A$ & 1.11 & 1.11 & 0 & 0 \\
\hline $218-E-7$ & 0.05 & 0.05 & 0 & 0 \\
\hline $218-E-8$ & 1.15 & 1.15 & 0 & 0 \\
\hline $218-E-010$ & 56.64 & 40.30 & 16.34 & 0.49 \\
\hline $218-E-10 B$ & 32.54 & 0 & 32.54 & 0 \\
\hline $218-E-12 A$ & 24.56 & 24.56 & 0 & 0 \\
\hline $218-E-12 B$ & 169.80 & 39.19 & 130.61 & 1.83 \\
\hline $218-E-14$ & 0.50 & 0.50 & 0 & 0 \\
\hline $218-E-15$ & 2.34 & 2.34 & 0 & 0 \\
\hline $218-E-C 9$ & 4.20 & 4.20 & 0 & 0 \\
\hline Sint-totart East & 306.24 & $(126.11)$ & 179.49 & 2.32 \\
\hline $218 W-01$ & 5.48 & 5.48 & 0 & 0 \\
\hline 218-W-01A & 8.31 & 8.31 & 0 & 0 \\
\hline $218-W-2$ & 7.04 & 7.04 & 0 & 0 \\
\hline $218-W-3$ & 8.01 & 8.01 & 0 & 0 \\
\hline $218-W-2 A$ & 39.76 & 39.76 & 0 & 0 \\
\hline $218-W-3 A$ & 50.3 & 46.52 & 3.78 & 0.46 \\
\hline $218-W-3 A E$ & 49.4 & 7.12 & 39.38 & 0.33 \\
\hline 218-W-04A & 17.03 & 17.03 & 0 & 0 \\
\hline $218-W-04 B$ & 8.58 & 8.58 & 0 & 0 \\
\hline $218-W-04 C$ & 51.7 & 10.14 & 41.56 & 0 \\
\hline $218-W-05$ & 84.00 & 13.00 & 69.33 & 1.18 \\
\hline
\end{tabular}


Solid Waste Management Facilities Acreage Report Disposed and Stored Acres in the 200 Areas from Startup to December 31, 1995, Includes all Waste Received. (2 sheets)

\begin{tabular}{|c|c|c|c|c|}
\hline Burial Ground & $\begin{array}{l}\text { Total } \\
\text { Acres }\end{array}$ & $\begin{array}{c}\text { Total } \\
\text { Acres } \\
\text { Used }\end{array}$ & $\begin{array}{c}\text { Acres } \\
\text { Remaining }\end{array}$ & $\begin{array}{c}\text { Acres } \\
\text { Used CY } \\
1995\end{array}$ \\
\hline $218-W-06$ & 44.50 & 0 & 44.50 & 0 \\
\hline 218-W-07 & 0.01 & 0.01 & 0 & 0 \\
\hline $218-W-08$ & 0.08 & 0.08 & 0 & 0 \\
\hline $218-W-09$ & 0.31 & 0.31 & 0 & 0 \\
\hline $218-W-11$ & 2.14 & 2.14 & 0 & 0 \\
\hline 212-N Building & 0.36 & 0.36 & 0 & 0 \\
\hline 224-T Building & 0.14 & 0.14 & 0 & 0 \\
\hline CWC Facilities & 100.00 & 100.00 & 0 & 0 \\
\hline 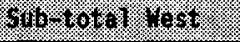 & $187 \times 3$ & 274.08. & 198,56 & 18.97 \\
\hline $\begin{array}{l}\text { Gond rotal } \\
200 \text { r }\end{array}$ & 786.96 & 400.74 & 180.04 & 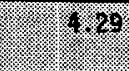 \\
\hline
\end{tabular}


Post-1970 Segregated Nontransuranic and Transuranic Acres used through December 31, 1995, within the Burial Grounds.

\begin{tabular}{|c|c|c|c|}
\hline Burial Ground & Waste Type & Square Feet & Used Acre \\
\hline \multirow[t]{4}{*}{$218-E-12 B$} & TRU & $2.048 E+04$ & 0.47 \\
\hline & UNSEGTRU & $4.078 E+05$ & 9.36 \\
\hline & LLW & $1.279 E+06$ & 29.36 \\
\hline & Total & $1.707 \mathrm{E}+06$ & 39.19 \\
\hline \multirow[t]{4}{*}{$218-W-03 A$} & TRU & $1.671 E+05$ & 3.84 \\
\hline & UNSEGTRU & $1.952 E+04$ & 0.45 \\
\hline & LLW & $1.839 E+06$ & 42.23 \\
\hline & Total & $2.026 \mathrm{E}+06$ & 46.52 \\
\hline \multirow[t]{6}{*}{$218-W-04 B$} & TRU & $4.968 \mathrm{E}+04$ & 1.14 \\
\hline & TRUCAIS & $1.728 E+04$ & 0.39 \\
\hline & LLW & $3.348 E+03$ & 0.77 \\
\hline & UNSEGTRUCAIS & $1.134 E+04$ & 0.26 \\
\hline & UNSEGTRU & $2.621 E+04$ & 6.02 \\
\hline & Total & $3.738 \mathrm{E}+05$ & 8.58 \\
\hline \multirow[t]{2}{*}{$218-W-04 C$} & TRU & $2.390 E+05$ & 5.49 \\
\hline & LLW & $2.026 E+05$ & 4.65 \\
\hline . & $700 \%$ & $1.1178 \times 83$ & 10.14 \\
\hline
\end{tabular}


Solid Waste Operations Storage Facilities Storage Status as of December 31, 1995

\begin{tabular}{|c|c|c|c|c|c|}
\hline $\begin{array}{l}\text { Building } \\
\text { Number }\end{array}$ & Square Feet & $\begin{array}{l}\text { Total } \\
\text { Acres }\end{array}$ & $\begin{array}{l}\text { Storage* } \\
\text { Capacity }\end{array}$ & $\begin{array}{l}\text { Storage* } \\
\text { Capacity } \\
\text { Used }\end{array}$ & $\begin{array}{l}\text { Storage* } \\
\text { Capacity } \\
\text { Remaining }\end{array}$ \\
\hline $212 N$ & $1.576 E+04$ & 0.36 & 1,032 & 1,032 & 0 \\
\hline $224 \mathrm{~T}$ & $5.910 E+03$ & 0.14 & 1,750 & 1,500 & 250 \\
\hline $2401 W$ & $4.000 E+03$ & 0.09 & 1,000 & 400 & 600 \\
\hline $2402 W$ & $4.000 E+03$ & 0.09 & 1,000 & 1,000 & 0 \\
\hline $2402 W B$ & $4.000 E+03$ & 0.09 & 1,000 & 675 & 325 \\
\hline $2402 W C$ & $4.000 E+03$ & 0.09 & 1,000 & 361 & 639 \\
\hline $2402 W D$ & $4.000 E+03$ & 0.09 & 1,000 & 405 & 595 \\
\hline 2402WE & $4.000 E+03$ & 0.09 & 1,000 & 894 & 106 \\
\hline $2402 W F$ & $4.000 E+03$ & 0.09 & 1,000 & 180 & 820 \\
\hline 2402WG & $4.000 E+03$ & 0.09 & 1,000 & 447 & 553 \\
\hline 2402WH & $4.000 E+03$ & 0.09 & 1,000 & 161 & 839 \\
\hline $2402 W I$ & $4.000 E+03$ & 0.09 & 1,000 & 1,000 & 0 \\
\hline $2402 W J$ & $4.000 E+03$ & 0.09 & 1,000 & 780 & 220 \\
\hline $2402 W K$ & $4.000 E+03$ & 0.09 & 1,000 & 1,000 & 0 \\
\hline $2402 W L$ & $4.000 E+03$ & 0.09 & 1,000 & 14 & 986 \\
\hline $2403 W A$ & $3.400 E+04$ & 0.78 & 11,000 & 8,980 & 2,020 \\
\hline $2403 \mathrm{WB}$ & $3.400 \mathrm{E}+04$ & 0.78 & 11,000 & 4,920 & 6,080 \\
\hline $2403 W C$ & $3.400 E+04$ & 0.78 & 11,000 & 7,200 & 3,800 \\
\hline $2403 W D$ & $5.525 \mathrm{E}+04$ & 1.27 & 15,500 & 9,100 & 6,400 \\
\hline FS 1 & 1. $509 E+02$ & 0.003 & 17 & 16 & 1 \\
\hline FS 2 & $1.600 E+02$ & 0.004 & 18 & 18 & 0 \\
\hline FS 3 & $1.965 E+02$ & 0.005 & 36 & 36 & 0 \\
\hline FS 4 & $2.289 E+02$ & 0.005 & 18 & 11 & 7 \\
\hline FS 5 & $2.400 E+02$ & 0.006 & 23 & 23 & 0 \\
\hline FS 6 & $4.800 E+02$ & 0.011 & 26 & 26 & 0 \\
\hline FS 7 & $1.953 E+02$ & 0.005 & 26 & 26 & 0 \\
\hline FS 8 & $2.070 \mathrm{E}+02$ & 0.005 & 17 & 17 & 0 \\
\hline FS 9 & $1.411 \mathrm{E}+02$ & 0.003 & $N / A$ & 0 & Non Op. \\
\hline FS 10 & $3.200 E+02$ & 0.007 & 23 & 22 & 1 \\
\hline
\end{tabular}


WHC-EP-0125-8

Solid Waste Operations Storage Facilities Storage Status as of December 31, 1995

\begin{tabular}{|c|c|c|c|c|c|}
\hline $\begin{array}{l}\text { Building } \\
\text { Number }\end{array}$ & Square Feet & $\begin{array}{l}\text { Total } \\
\text { Acres }\end{array}$ & $\begin{array}{l}\text { Storage* } \\
\text { Capacity }\end{array}$ & $\begin{array}{l}\text { Storage* } \\
\text { Capacity } \\
\text { Used }\end{array}$ & $\begin{array}{l}\text { Storage* } \\
\text { Capacity } \\
\text { Remaining }\end{array}$ \\
\hline FS 11 & $1.411 \mathrm{E}+02$ & 0.003 & 21 & 21 & 0 \\
\hline FS 12 & $3.200 \mathrm{E}+02$ & 0.007 & 24 & 24 & 0 \\
\hline FS 13 & $1.965 \mathrm{E}+02$ & 0.005 & 18 & 18 & 0 \\
\hline FS 14 & $1.965 E+02$ & 0.005 & 34 & 34 & 0 \\
\hline FS 15 & $1.965 E+02$ & 0.005 & 22 & 22 & 0 \\
\hline FS 16 & $1.965 \mathrm{E}+02$ & 0.005 & 30 & 30 & 0 \\
\hline FS 17 & $1.965 E+02$ & 0.005 & 29 & 28 & 1 \\
\hline FS 18 & $1.965 \mathrm{E}+02$ & 0.005 & 36 & 36 & 0 \\
\hline FS 19 & $1.411 E+02$ & 0.003 & 18 & 0 & 18 \\
\hline FS 20 & $1.965 \mathrm{E}+02$ & 0.005 & 21 & 9 & 12 \\
\hline AMW 1 & $2.299 E+02$ & 0.005 & 24 & 2 boxes & 0 \\
\hline AMW 2 & $2.299 E+02$ & 0.005 & 24 & 24 & 0 \\
\hline AMW 3 & $2.299 \mathrm{E}+02$ & 0.005 & 24 & 12 & 12 \\
\hline AMW 4 & $2.299 \mathrm{E}+02$ & 0.005 & 26 & 26 & 0 \\
\hline 1068 & 2.3 .611480 .5 & 5.48 & 54.841 & 40,486 & 24.356 \\
\hline
\end{tabular}

*Number of 55-gallon drums or drum equivalents. 
WHC-EP-0125-8

This page intentionally left blank.

B-8 
WHC-EP-0125-8

\section{DISTRIBUTION}

Number of Copies

OFFSITE

1

U.S. Department of Energy -

Headquarters

Germantown, Maryl and

R. Martinez EM-322

ONSITE

7

U.S. Department of Energy, Richland Operations office

D. W. Claussen $\$ 7-55$

R. M. Gordon $\$ 7-55$

R. F. Guercia (3) $57-55$

R. D. Izatt

$A 7-50$

Public Reading Room H?:-53

1

Bechtel Hanford. Inc.

L. A. Dietz

H.9-10

4

Pacific Northwest National Laboratory

D. A. Dickman

B. W. Killand

KB-58

M. W. McCoy

PNNL Technical Files

P7-75

P7-79

PI3-55

Westinghouse Hanford Company

J. D. Anderson (2)

T3-04

H. C. Boynton

T3-01

F. M. Coony

T3-01

P. J. Crane

T4-03

L. P. Diediker

T1-30

J. J. Dorian

H6-20

G. E. Fitzgibbon

H6-07

C. J. Geier

R. J. Giroir

B. P. Gleckler

R2-36

D. L. Hagel (3)

T4-05

\$3-95

W. H. Hamilton

T3-01

P. L. Hapke

T3-01

K. L. Hladek

T4-05

J. H. Hummer

T3-01

D. E. McKenney

G2-02

S. R. Nelson

T3-01

B4-55 
WHC-EP-0125-8

\section{DISTRIBUTION (cont)}

Number of Copies

ONSITE

Westinghouse Hanford Company (cont)

D. B. Powell, Jr.

T4-03

D. A. Pratt

N. P. Willis

T4-03

Document Processing Center

Central Files

T3-04

A3-94

A3-88 